Prepared for the U.S. Department of Energy under Contract DE-AC05-76RL01830

\title{
Analytical Data Report for Sediment Samples Collected from Borehole C5856 (BP-5 D Well)
}

Michael Lindberg

August 2009

Pacific Northwest

NATIONAL LABORATORY 


\title{
DISCLAIMER
}

This report was prepared as an account of work sponsored by an agency of the United States Government. Neither the United States Government nor any agency thereof, nor Battelle Memorial Institute, nor any of their employees, makes any warranty, express or implied, or assumes any legal liability or responsibility for the accuracy, completeness, or usefulness of any information, apparatus, product, or process disclosed, or represents that its use would not infringe privately owned rights. Reference herein to any specific commercial product, process, or service by trade name, trademark, manufacturer, or otherwise does not necessarily constitute or imply its endorsement, recommendation, or favoring by the United States Government or any agency thereof, or Battelle Memorial Institute. The views and opinions of authors expressed herein do not necessarily state or reflect those of the United States Government or any agency thereof.

\author{
PACIFIC NORTHWEST NATIONAL LABORATORY \\ operated by \\ BATTELLE \\ for the \\ UNITED STATES DEPARTMENT OF ENERGY \\ under Contract DE-AC05-76RL01830
}

Printed in the United States of America

Available to DOE and DOE contractors from the

Office of Scientific and Technical Information,

P.O. Box 62, Oak Ridge, TN 37831-0062;

ph: (865) 576-8401

fax: $(865) 576-5728$

email: reports@adonis.osti.gov

\footnotetext{
Available to the public from the National Technical Information Service, U.S. Department of Commerce, 5285 Port Royal Rd., Springfield, VA 22161 ph: (800) 553-6847 fax: $(703) 605-6900$

email: orders@ntis.fedworld.gov

online ordering: http://www.ntis.gov/ordering.htm
}

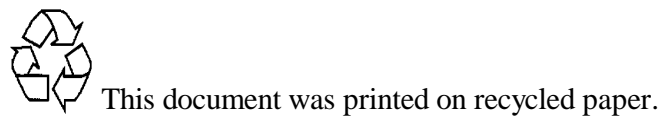


08/11/09 10:02

To: Dale Dyekman

From: Michael J. Lindberg

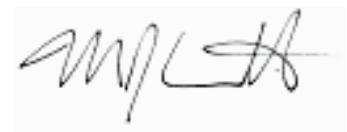

Environmental Sciences Laboratory

Energy and Environment Directorate, Pacific Northwest National Laboratory

Subject: Analytical Data Report for Sediment Samples Collected from Borehole C5856 (BP-5 D Well), Sample Delivery Group ESL080016, SAF Number F08-075

This letter contains the following information for sample delivery group ESL080016

- Cover Sheet

- Narrative

- Analytical Results

- Quality Control

- Geologic Logs

- Geologic Photos

- Chain of Custodies 


\section{Introduction}

Between April 30, 2008 and June 4, 2008 sediment samples were received from Borehole C5856 (BP-5 D Well) for geochemical studies.

\section{Analytical Results/Methodology}

The analyses for this project were performed at the 325 building located in the 300 Area of the Hanford Site. The analyses were performed according to Pacific Northwest National Laboratory (PNNL) approved procedures and/or nationally recognized test procedures. The data sets include the sample identification numbers, analytical results, estimated quantification limits (EQL), and quality control data.

\section{Quality Control}

The preparatory and analytical quality control requirements, calibration requirements, acceptance criteria, and failure actions are defined in the on-line QA plan “Conducting Analytical Work in Support of Regulatory Programs” (CAW). This QA plan implements the Hanford Analytical Services Quality Assurance Requirements Documents (HASQARD) for PNNL.

\section{Definitions}

Dup Duplicate

RPD Relative Percent Difference

NR No Recovery (percent recovery less than zero)

ND Non-Detectable

\%REC Percent Recovery

\section{Sample Receipt}

Samples were received with a chain of custody (COC) and were analyzed according to the sample identification numbers supplied by the client. All Samples were refrigerated upon receipt until prepared for analysis.

All samples were received with custody seals intact unless noted in the Case Narrative.

\section{Holding Times}

Holding time is defined as the time from sample preparation to the time of analyses. The prescribed holding times were met for all analytes unless noted in the Case Narrative.

\section{Analytical Results}

All reported analytical results meet the requirements of the CAW or client specified SOW unless noted in the case narrative. 


\section{Case Narrative Report}

\section{Hold Time:}

Due to the requirements of the statement of work and sampling events in the field, the 28 day and the 48 hr requirements could not be met. The statement of work requires samples to be selected at the completion of the borehole. All applicable hold times were started from the time of preparation and not the time of sampling.

\section{Preparation Blank (PB):}

No Discrepancies were noted.

\section{Duplicate (DUP):}

No Discrepancies were noted.

\section{Laboratory Control Samples (LCS):}

The ICV is considered the blank spike for alkalinity, electric conductivity and pH measurements. The value measured for the ICV is maintained at the laboratory and not reported.

\section{Post Spike (PS):}

Post-Spike Recovery for Sulfate (61.6\%) was outside acceptance limits (75-125) in 8G09011-PS1 for Anions by IC-WE The native sample concentration was greater than 5 times the spike concentration. There should be no impact to data as reported.

Post-Spike Recovery for Phosphate (71.4\%) was outside acceptance limits (75-125) in 8G09011-PS1 for Anions by IC-WE The native sample concentration was greater than 5 times the spike concentration. There should be no impact to data as reported.

Post-Spike Recovery for Chromium 52 (69.3\%) was outside acceptance limits (75-125) in 8I18001-PS1 for ICPMS-RCRA-AE The native sample concentration was greater than 5 times the spike concentration. There should be no impact to data as reported.

Post-Spike Recovery for Calcium (333\%) was outside acceptance limits (75-125) in 8I23006-PS1 for ICP-OES Vadose-AE The native sample concentration was greater than 5 times the spike concentration. There should be no impact to data as reported.

Post-Spike Recovery for Iron (627\%) was outside acceptance limits (75-125) in 8I23006-PS1 for ICP-OES Vadose-AE The native sample concentration was greater than 5 times the spike concentration. There should be no impact to data as reported.

Post-Spike Recovery for Magnesium (510\%) was outside acceptance limits (75-125) in 8I23006-PS1 for ICP-OES Vadose-AE The native sample concentration was greater than 5 times the spike concentration. There should be no impact to data as reported.

Post-Spike Recovery for Sodium (158\%) was outside acceptance limits (75-125) in 8I23006-PS1 for ICP-OES Vadose-AE The native sample concentration was greater than 5 times the spike concentration. There should be no impact to data as reported.

Post-Spike Recovery for Calcium (NR) was outside acceptance limits (75-125) in 8I23007-PS1 for ICP-OES Vadose-AE The native sample concentration was greater than 5 times the spike concentration. There should be no impact to data as reported.

Post-Spike Recovery for Iron (185\%) was outside acceptance limits (75-125) in 8I23007-PS1 for ICP-OES Vadose-AE The native sample concentration of the sample was greater than 5 times the spike concentration. There should be no impact to 


\section{Case Narrative Report}

\section{Post Spike (PS):}

data as reported.

Post-Spike Recovery for Magnesium (329\%) was outside acceptance limits (75-125) in 8I23007-PS1 for ICP-OES Vadose-AE The native sample concentration was greater than 5 times the spike concentration. There should be no impact to data as reported.

Post-Spike Recovery for Sodium (NR) was outside acceptance limits (75-125) in 8I23007-PS1 for ICP-OES Vadose-AE The native sample concentration was greater than 5 times the spike concentration. There should be no impact to data as reported.

Post-Spike Recovery for Sodium (NR) was outside acceptance limits (75-125) in 8G22002-PS1 for ICP-OES Vadose-WE The native sample concentration was greater than 5 times the spike concentration. There should be no impact to data as reported.

Post-Spike Recovery for Calcium (387\%) was outside acceptance limits (75-125) in 8G22002-PS2 for ICP-OES Vadose-WE The native sample concentration was greater than 5 times the spike concentration. There should be no impact to data as reported.

Post-Spike Recovery for Magnesium (1950\%) was outside acceptance limits (75-125) in 8G22002-PS2 for ICP-OES Vadose-WE The native sample concentration was greater than 5 times the spike concentration. There should be no impact to data as reported.

Post-Spike Recovery for Sodium (717\%) was outside acceptance limits (75-125) in 8G22002-PS2 for ICP-OES Vadose-WE The native sample concentration was greater than 5 times the spike concentration. There should be no impact to data as reported.

Post-Spike Recovery for Chromium 52 (267\%) was outside acceptance limits (75-125) in 8I18002-PS1 for ICPMS-RCRA-AE The native sample concentration was greater than 5 times the spike concentration. There should be no impact to data as reported.

Post-Spike Recovery for Aluminum (518\%) was outside acceptance limits (75-125) in 8I23006-PS1 for ICP-OES Vadose-AE The native sample concentration was greater than 5 times the spike concentration. There should be no impact to data as reported.

Post-Spike Recovery for Aluminum (262\%) was outside acceptance limits (75-125) in 8I23007-PS1 for ICP-OES Vadose-AE The native sample concentration was greater than 5 times the spike concentration. There should be no impact to data as reported.

Post-Spike Recovery for Iodine-129 (46.6\%) was outside acceptance limits (75-125) in 8I16006-PS1 for ICP/ICPMS WE. The native sample concentrations were all reported as ND. There should be no impact to data as reported.

\section{Matrix Spike (MS):}

Not Applicable.

\section{Other QC Criteria:}

No Discrepancies were noted.

\section{DISCLAIMER}

This report was prepared as an account of work sponsored by an agency of the United States Government. Neither the United States Government nor any agency thereof, nor Battelle Memorial Institute, nor any of their employees, makes any warranty, express or implied, or assumes any legal liability or responsibility for the accuracy, completeness, or usefulness of any information, apparatus, product, or process disclosed, or represents that its use would not infringe privately owned rights. Reference herein to any specific commercial product, process, or service by trade name, trademark, manufacturer, or otherwise does not necessarily constitute or imply its endorsement, recommendation, or favoring by the United States Government or any agency thereof, or Battelle Memorial Institute. The views and opinions of authors expressed herein do not necessarily state or reflect those of the United States Government or any agency thereof. 


\section{SAMPLES INCLUDED IN THIS REPORT}

\section{BP 5 OU, C5856 D-Well VZ}

\begin{tabular}{|c|c|c|}
\hline $\begin{array}{l}\text { HEIS No. } \\
\text { B1TP23 }\end{array}$ & $\begin{array}{l}\text { Laboratory ID } \\
0804025-01\end{array}$ & $\begin{array}{l}\text { Matrix } \\
\text { SOIL }\end{array}$ \\
\hline В1ТР24 & 0804025-04 & SOIL \\
\hline В1ТР28 & 0804025-07 & SOIL \\
\hline B1TP29 & 0804025-08 & SOIL \\
\hline В1TP30 & 0804025-09 & SOIL \\
\hline B1TNV9 & 0804025-10 & SOIL \\
\hline B1TNV6-2 & 0804025-11 & SOIL \\
\hline B1TNV6-3 & 0804025-12 & SOIL \\
\hline B1TP22 & 0804025-13 & SOIL \\
\hline B1TNV7-2 & 0804025-14 & SOIL \\
\hline B1TNV7-3 & 0804025-15 & SOIL \\
\hline B1TP26 & 0804025-16 & SOIL \\
\hline B1TNW0 & 0804025-17 & SOIL \\
\hline B1TNW1 & 0804025-18 & SOIL \\
\hline B1TNW3 & 0804025-20 & SOIL \\
\hline B1TNW5 & 0804025-22 & SOIL \\
\hline B1TP35 & 0804025-37 & SOIL \\
\hline B1TP40 & 0804025-48 & SOIL \\
\hline B1TP42 & 0804025-50 & SOIL \\
\hline В1TР43 & 0804025-51 & SOIL \\
\hline B1TP45 & 0804025-53 & SOIL \\
\hline В1TP47 & 0804025-57 & SOIL \\
\hline В1ТР49 & 0804025-61 & SOIL \\
\hline B1TP51 & 0804025-69 & SOIL \\
\hline B1TP52 & 0804025-72 & SOIL \\
\hline B1TP53 & 0804025-75 & SOIL \\
\hline B1TP55 & 0804025-81 & SOIL \\
\hline В1TP59 & 0804025-87 & SOIL \\
\hline B1TP61 & 0804025-91 & SOIL \\
\hline В1TP62 & 0804025-94 & SOIL \\
\hline В1TP64 & 0804025-AA & SOIL \\
\hline В1ТР67 & 0804025-AI & SOIL \\
\hline В1TP69 & 0804025-AP & SOIL \\
\hline B1TP71 & 0804025-AV & SOIL \\
\hline B1TP77 & 0804025-BK & SOIL \\
\hline B1TP78 & 0804025-BР & SOIL \\
\hline B1TP82 & 0804025-BZ & SOIL \\
\hline В1TP84 & 0804025-CF & SOIL \\
\hline B1TNX1 & 0804025-CI & SOIL \\
\hline В1TP86 & 0804025-СО & SOIL \\
\hline В1ТР89 & 0804025-CX & SOIL \\
\hline B1TP91 & 0804025-DF & SOIL \\
\hline В1ТР93 & 0804025-DL & SOIL \\
\hline В1ТР94 & 0804025-DM & SOIL \\
\hline
\end{tabular}

\begin{tabular}{|c|c|c|c|}
\hline $\begin{array}{l}\text { Date C } \\
4 / 29 / 08\end{array}$ & $\begin{array}{l}\text { ollected } \\
10: 05\end{array}$ & $\begin{array}{l}\text { Date R } \\
4 / 30 / 08\end{array}$ & $\begin{array}{l}\text { leceived } \\
14: 30\end{array}$ \\
\hline $4 / 29 / 08$ & 3 11:05 & 4/30/08 & $14: 30$ \\
\hline $4 / 29 / 08$ & 13:55 & $4 / 30 / 08$ & $14: 30$ \\
\hline $4 / 29 / 08$ & $\begin{array}{ll}34: 50\end{array}$ & $4 / 30 / 08$ & $14: 30$ \\
\hline 4/29/08 & 15:00 & 4/30/08 & $14: 30$ \\
\hline $4 / 29 / 08$ & $15: 15$ & $4 / 30 / 08$ & $14: 30$ \\
\hline 4/29/08 & 09:30 & $5 / 1 / 08$ & $14: 30$ \\
\hline $4 / 29 / 08$ & 09:30 & $5 / 1 / 08$ & $14: 30$ \\
\hline 4/29/08 & B 08:35 & $5 / 2 / 08$ & $14: 30$ \\
\hline $4 / 29 / 08$ & $10: 20$ & $5 / 1 / 08$ & $14: 30$ \\
\hline $4 / 29 / 08$ & $10: 20$ & $5 / 1 / 08$ & $14: 30$ \\
\hline 4/29/08 & 13:20 & $5 / 2 / 08$ & $14: 30$ \\
\hline $4 / 30 / 08$ & 08:00 & $5 / 2 / 08$ & $14: 30$ \\
\hline $4 / 30 / 08$ & 08:30 & $5 / 2 / 08$ & $14: 30$ \\
\hline $4 / 30 / 08$ & 09:15 & $5 / 2 / 08$ & $14: 30$ \\
\hline $4 / 30 / 08$ & $310: 25$ & $5 / 2 / 08$ & $14: 30$ \\
\hline 4/30/08 & 13:55 & $5 / 1 / 08$ & $14: 30$ \\
\hline $5 / 1 / 08$ & $10: 45$ & $5 / 6 / 08$ & $13: 10$ \\
\hline $5 / 1 / 08$ & 12:35 & $5 / 6 / 08$ & $13: 10$ \\
\hline $5 / 1 / 08$ & $12: 45$ & $5 / 6 / 08$ & $13: 10$ \\
\hline $5 / 1 / 08$ & $13: 40$ & $5 / 6 / 08$ & $13: 10$ \\
\hline $5 / 1 / 08$ & $14: 12$ & 5/6/08 & $13: 10$ \\
\hline $5 / 1 / 08$ & $15: 08$ & $5 / 6 / 08$ & $13: 10$ \\
\hline 5/8/08 & 08:45 & 5/9/08 & $14: 00$ \\
\hline $5 / 8 / 08$ & 09:10 & $5 / 9 / 08$ & $14: 00$ \\
\hline $5 / 8 / 08$ & 09:55 & 5/9/08 & $14: 00$ \\
\hline $5 / 8 / 08$ & $14: 00$ & 5/9/08 & $14: 00$ \\
\hline $5 / 9 / 08$ & 08:30 & $5 / 14 / 08$ & 8 11:45 \\
\hline 5/9/08 & 09:00 & $5 / 15 / 08$ & 8 09:00 \\
\hline $5 / 9 / 08$ & 09:20 & $5 / 15 / 08$ & $\begin{array}{ll}8 & 09: 00\end{array}$ \\
\hline 5/9/08 & 10:38 & $5 / 15 / 08$ & 8 09:00 \\
\hline 5/9/08 & 13:00 & $5 / 15 / 08$ & 8 09:00 \\
\hline $5 / 9 / 08$ & 13:55 & $5 / 15 / 08$ & $\begin{array}{ll}3 & 09: 00\end{array}$ \\
\hline $5 / 12 / 08$ & 10:25 & $5 / 15 / 08$ & $\begin{array}{ll}8 & 09: 00\end{array}$ \\
\hline $5 / 13 / 08$ & 08:42 & $5 / 15 / 08$ & 8 09:00 \\
\hline $5 / 13 / 08$ & 09:55 & $5 / 20 / 08$ & $\begin{array}{ll}8 & 13: 20\end{array}$ \\
\hline $5 / 13 / 08$ & 34:11 & $5 / 20 / 08$ & 8 13:20 \\
\hline $5 / 16 / 08$ & 3 08:07 & $5 / 22 / 08$ & $\begin{array}{ll}3 & 14: 00\end{array}$ \\
\hline $5 / 16 / 08$ & 09:35 & $5 / 22 / 08$ & $\begin{array}{ll}8 & 14: 00\end{array}$ \\
\hline $5 / 16 / 08$ & $14: 10$ & $5 / 22 / 08$ & $\begin{array}{ll}3 & 14: 00\end{array}$ \\
\hline $5 / 20 / 08$ & 10:25 & $5 / 22 / 08$ & $\begin{array}{ll}8 & 14: 00\end{array}$ \\
\hline $5 / 20 / 08$ & 3 14:35 & $5 / 28 / 08$ & $\begin{array}{ll}3 & 13: 00\end{array}$ \\
\hline $5 / 22 / 08$ & 14:11 & $6 / 4 / 08$ & $13: 15$ \\
\hline $5 / 22 / 08$ & 14:55 & $6 / 4 / 08$ & 13:15 \\
\hline
\end{tabular}


The following analyses were performed on the following samples included in this report:

Metals 1:1 DI Water Extract by ICPMS

Metals Acid Extract by ICPMS

1:1 DI Water Extract

AGG-TOC-001

Alkalinity, Titrimetic ( $\mathrm{pH} 4.5)$

Anions By Ion Chromatography

Carbon, Total, Combustion or Oxidation

Cyanide by Mircodistillation/Colorimetric

GEA No Preparation

Geological Description

Inorganic Carbon, Total, Combustion or Oxidation

Iodine-129 1:1 DI Water Extract by ICPMS

Metals 1:1 Water Extract by ICPOES

Metals Acid Extract by ICPOES

Moisture Content

Nitric Acid Digestion

Percent Solids

pH of Waters By Electrode

Specific Conductance

Tc_U Acid Extract by ICPMS

Tc_U 1:1 DI Water Extract by ICPMS

Total Alpha Total Beta Acid Extract By LSC 


\begin{tabular}{|c|c|c|}
\hline $\begin{array}{l}\text { HEIS No. } \\
\text { B1TP23 }\end{array}$ & $\begin{array}{l}\text { Laboratory ID } \\
0804025-01\end{array}$ & $\begin{array}{l}\text { Matrix } \\
\text { SOIL }\end{array}$ \\
\hline В1ТР24 & 0804025-04 & SOIL \\
\hline B1TP28 & 0804025-07 & SOIL \\
\hline В1ТР29 & 0804025-08 & SOIL \\
\hline В1ТР30 & 0804025-09 & SOIL \\
\hline B1TNV9 & 0804025-10 & SOIL \\
\hline B1TNV6-2 & 0804025-11 & SOIL \\
\hline B1TNV6-3 & 0804025-12 & SOIL \\
\hline B1TP22 & 0804025-13 & SOIL \\
\hline B1TNV7-2 & 0804025-14 & SOIL \\
\hline B1TNV7-3 & 0804025-15 & SOIL \\
\hline В1ТР26 & 0804025-16 & SOIL \\
\hline B1TNW0 & 0804025-17 & SOIL \\
\hline B1TNW1 & 0804025-18 & SOIL \\
\hline B1TNW3 & 0804025-20 & SOIL \\
\hline B1TNW5 & 0804025-22 & SOIL \\
\hline В1TP35 & 0804025-37 & SOIL \\
\hline B1TP40 & 0804025-48 & SOIL \\
\hline B1TP42 & 0804025-50 & SOIL \\
\hline В1ТР43 & 0804025-51 & SOIL \\
\hline B1TP45 & 0804025-53 & SOIL \\
\hline В1TP47 & 0804025-57 & SOIL \\
\hline В1ТР49 & 0804025-61 & SOIL \\
\hline B1TP51 & 0804025-69 & SOIL \\
\hline B1TP52 & 0804025-72 & SOIL \\
\hline В1TP53 & 0804025-75 & SOIL \\
\hline B1TP55 & 0804025-81 & SOIL \\
\hline B1TP59 & 0804025-87 & SOIL \\
\hline B1TP61 & 0804025-91 & SOIL \\
\hline B1TP62 & 0804025-94 & SOIL \\
\hline B1TP64 & 0804025-AA & SOIL \\
\hline В1ТР67 & 0804025-AI & SOIL \\
\hline В1ТР69 & 0804025-AP & SOIL \\
\hline B1TP71 & 0804025-AV & SOIL \\
\hline B1TP77 & 0804025-BK & SOIL \\
\hline В1ТР78 & 0804025-BР & SOIL \\
\hline В1TP82 & 0804025-BZ & SOIL \\
\hline В1ТР84 & 0804025-CF & SOIL \\
\hline B1TNX1 & 0804025-CI & SOIL \\
\hline В1TP86 & 0804025-CO & SOIL \\
\hline В1ТР89 & 0804025-CX & SOIL \\
\hline В1ТР91 & 0804025-DF & SOIL \\
\hline В1ТР93 & 0804025-DL & SOIL \\
\hline В1TP94 & 0804025-DM & SOIL \\
\hline
\end{tabular}

\begin{tabular}{|c|c|c|c|}
\hline $\begin{array}{l}\text { Date C } \\
4 / 29 / 08\end{array}$ & $\begin{array}{l}\text { ollected } \\
10: 05\end{array}$ & $\begin{array}{l}\text { Date Re } \\
4 / 30 / 08\end{array}$ & $\begin{array}{l}\text { eceived } \\
14: 30\end{array}$ \\
\hline $4 / 29 / 08$ & 11:05 & 4/30/08 & $14: 30$ \\
\hline $4 / 29 / 08$ & $13: 55$ & 4/30/08 & $14: 30$ \\
\hline $4 / 29 / 08$ & $14: 50$ & $4 / 30 / 08$ & $14: 30$ \\
\hline $4 / 29 / 08$ & $15: 00$ & 4/30/08 & $14: 30$ \\
\hline $4 / 29 / 08$ & $15: 15$ & $4 / 30 / 08$ & $14: 30$ \\
\hline $4 / 29 / 08$ & 09:30 & $5 / 1 / 08$ & $14: 30$ \\
\hline $4 / 29 / 08$ & 09:30 & $5 / 1 / 08$ & 14:30 \\
\hline $4 / 29 / 08$ & 08:35 & $5 / 2 / 08$ & $14: 30$ \\
\hline $4 / 29 / 08$ & $10: 20$ & $5 / 1 / 08$ & 14:30 \\
\hline $4 / 29 / 08$ & $10: 20$ & $5 / 1 / 08$ & 14:30 \\
\hline $4 / 29 / 08$ & $13: 20$ & $5 / 2 / 08$ & $14: 30$ \\
\hline 4/30/08 & 08:00 & $5 / 2 / 08$ & 14:30 \\
\hline $4 / 30 / 08$ & 08:30 & $5 / 2 / 08$ & 14:30 \\
\hline 4/30/08 & 09:15 & $5 / 2 / 08$ & 14:30 \\
\hline $4 / 30 / 08$ & $10: 25$ & $5 / 2 / 08$ & 14:30 \\
\hline 4/30/08 & 13:55 & $5 / 1 / 08$ & 14:30 \\
\hline 5/1/08 & 10:45 & $5 / 6 / 08$ & 13:10 \\
\hline 5/1/08 & 12:35 & $5 / 6 / 08$ & 13:10 \\
\hline $5 / 1 / 08$ & 12:45 & $5 / 6 / 08$ & 13:10 \\
\hline 5/1/08 & 13:40 & $5 / 6 / 08$ & 13:10 \\
\hline $5 / 1 / 08$ & $14: 12$ & $5 / 6 / 08$ & 13:10 \\
\hline 5/1/08 & 15:08 & $5 / 6 / 08$ & 13:10 \\
\hline 5/8/08 & 08:45 & 5/9/08 & 14:00 \\
\hline $5 / 8 / 08$ & 09:10 & $5 / 9 / 08$ & 14:00 \\
\hline $5 / 8 / 08$ & 09:55 & 5/9/08 & 14:00 \\
\hline $5 / 8 / 08$ & 14:00 & $5 / 9 / 08$ & 14:00 \\
\hline $5 / 9 / 08$ & 08:30 & $5 / 14 / 08$ & $11: 45$ \\
\hline 5/9/08 & 09:00 & $5 / 15 / 08$ & 09:00 \\
\hline 5/9/08 & 09:20 & $5 / 15 / 08$ & 09:00 \\
\hline 5/9/08 & 10:38 & $5 / 15 / 08$ & 09:00 \\
\hline 5/9/08 & 13:00 & $5 / 15 / 08$ & 09:00 \\
\hline $5 / 9 / 08$ & 13:55 & $5 / 15 / 08$ & 09:00 \\
\hline $5 / 12 / 08$ & $10: 25$ & $5 / 15 / 08$ & 09:00 \\
\hline $5 / 13 / 08$ & 08:42 & $5 / 15 / 08$ & 09:00 \\
\hline $5 / 13 / 08$ & 09:55 & $5 / 20 / 08$ & $13: 20$ \\
\hline $5 / 13 / 08$ & 14:11 & $5 / 20 / 08$ & $13: 20$ \\
\hline $5 / 16 / 08$ & 08:07 & $5 / 22 / 08$ & $14: 00$ \\
\hline $5 / 16 / 08$ & 09:35 & $5 / 22 / 08$ & $14: 00$ \\
\hline $5 / 16 / 08$ & $14: 10$ & $5 / 22 / 08$ & $14: 00$ \\
\hline $5 / 20 / 08$ & $10: 25$ & $5 / 22 / 08$ & $14: 00$ \\
\hline $5 / 20 / 08$ & $14: 35$ & $5 / 28 / 08$ & $13: 00$ \\
\hline $5 / 22 / 08$ & 14:11 & $6 / 4 / 08$ & 13:15 \\
\hline $5 / 22 / 08$ & $14: 55$ & $6 / 4 / 08$ & $13: 15$ \\
\hline
\end{tabular}




\section{Wet Chemistry}

\begin{tabular}{|c|c|c|c|c|c|}
\hline \multicolumn{6}{|c|}{ Alkalinity as CaCO3 (ug/g dry) by Standard Methods 2320B } \\
\hline Lab ID & HEIS No. & Results & EQL & Analyzed & Batch \\
\hline 0804025-04 & В1ТР24 & 2.12E2 & 2.48E1 & $7 / 14 / 08$ & 8G14005 \\
\hline 0804025-07 & В1ТР28 & $2.90 \mathrm{E} 2$ & 2.34E1 & $7 / 14 / 08$ & 8G14005 \\
\hline 0804025-08 & B1TP29 & $2.28 \mathrm{E} 2$ & 2.35E1 & 9/02/08 & 8I02001 \\
\hline 0804025-09 & В1ТР30 & $2.38 \mathrm{E} 2$ & $2.35 \mathrm{E} 1$ & $9 / 02 / 08$ & 8I02001 \\
\hline $0804025-10$ & B1TNV9 & 7.75E1 & 2.35E1 & 9/02/08 & 8I02001 \\
\hline 0804025-13 & В1TP22 & $5.45 \mathrm{E} 1$ & 2.38E1 & 7/14/08 & 8G14005 \\
\hline 0804025-16 & В1ТР26 & 2.72E2 & 2.36E1 & 7/14/08 & 8G14005 \\
\hline 0804025-17 & B1TNW0 & 4.33E1 & 2.35E1 & $9 / 02 / 08$ & 8I02001 \\
\hline 0804025-18 & B1TNW1 & 2.94E1 & 2.33E1 & 7/14/08 & 8G14005 \\
\hline 0804025-20 & B1TNW3 & 2.97E1 & $2.35 \mathrm{E} 1$ & 9/02/08 & 8I02001 \\
\hline 0804025-22 & B1TNW5 & 2.47E1 & $2.31 \mathrm{E} 1$ & 7/14/08 & 8G14005 \\
\hline 0804025-37 & B1TP35 & $4.02 \mathrm{E} 1$ & $2.54 \mathrm{E} 1$ & 7/14/08 & 8G14005 \\
\hline 0804025-48 & В1TP40 & 3.88E1 & 2.35E1 & $7 / 14 / 08$ & $8 \mathrm{G} 14005$ \\
\hline 0804025-50 & В1TP42 & 3.88E1 & 2.35E1 & $9 / 02 / 08$ & $8 \mathrm{I} 02001$ \\
\hline 0804025-51 & В1TP43 & 3.65E1 & 2.35E1 & 7/14/08 & 8G14005 \\
\hline 0804025-53 & B1TP45 & 7.15E1 & $2.35 \mathrm{E} 1$ & $9 / 02 / 08$ & 8I02001 \\
\hline 0804025-57 & B1TP47 & $<2.33 \mathrm{E} 1$ & 2.33E1 & 7/14/08 & 8G14005 \\
\hline 0804025-61 & В1ТР49 & $<2.35 \mathrm{E} 1$ & $2.35 \mathrm{E} 1$ & 7/14/08 & 8G14006 \\
\hline 0804025-69 & B1TP51 & 2.77E1 & $2.31 \mathrm{E} 1$ & 7/14/08 & 8G14006 \\
\hline 0804025-72 & B1TP52 & $4.31 \mathrm{E} 1$ & 2.34E1 & $7 / 14 / 08$ & 8G14006 \\
\hline 0804025-75 & B1TP53 & $<2.35 \mathrm{E} 1$ & 2.35E1 & $7 / 14 / 08$ & 8G14006 \\
\hline 0804025-81 & B1TP55 & $3.28 \mathrm{E} 1$ & $2.36 \mathrm{E} 1$ & 7/14/08 & 8G14006 \\
\hline 0804025-87 & В1TP59 & 3.24E1 & 2.38E1 & $7 / 14 / 08$ & 8G14006 \\
\hline 0804025-91 & B1TP61 & 3.27E1 & $2.35 \mathrm{E} 1$ & 9/02/08 & 8I02001 \\
\hline 0804025-94 & B1TP62 & 3.15E1 & $2.32 \mathrm{E} 1$ & 7/14/08 & 8G14006 \\
\hline 0804025-AA & B1TP64 & $2.36 \mathrm{E} 1$ & $2.36 \mathrm{E} 1$ & $9 / 02 / 08$ & 8102001 \\
\hline 0804025-AI & B1TP67 & $3.42 \mathrm{E} 1$ & $2.30 \mathrm{E} 1$ & 7/14/08 & 8G14006 \\
\hline 0804025-AP & B1TP69 & 6.17E1 & $2.35 \mathrm{E} 1$ & $9 / 02 / 08$ & $8 \mathrm{I} 02001$ \\
\hline 0804025-AV & B1TP71 & 5.15E1 & 2.34E1 & $7 / 14 / 08$ & 8G14006 \\
\hline 0804025-BK & B1TP77 & 5.47E1 & $2.35 \mathrm{E} 1$ & 7/14/08 & 8G14006 \\
\hline 0804025-BP & B1TP78 & 4.03E1 & $2.35 \mathrm{E} 1$ & $7 / 14 / 08$ & 8G14006 \\
\hline 0804025-BZ & B1TP82 & $4.24 \mathrm{E} 1$ & $2.34 \mathrm{E} 1$ & $7 / 14 / 08$ & 8G14006 \\
\hline 0804025-CF & B1TP84 & $6.45 \mathrm{E} 1$ & 2.34E1 & 7/14/08 & 8G14006 \\
\hline 0804025-CI & B1TNX1 & $3.14 \mathrm{E} 1$ & $2.37 \mathrm{E} 1$ & 7/14/08 & 8G14006 \\
\hline 0804025-CO & B1TP86 & 3.34E1 & $2.35 \mathrm{E} 1$ & 7/14/08 & 8G14006 \\
\hline 0804025-CX & В1TP89 & 3.35E1 & $2.36 \mathrm{E} 1$ & $7 / 14 / 08$ & 8G14006 \\
\hline 0804025-DF & B1TP91 & 5.72E1 & $2.36 \mathrm{E} 1$ & $7 / 14 / 08$ & 8G14006 \\
\hline 0804025-DL & В1ТР93 & $3.59 \mathrm{E} 1$ & $2.36 \mathrm{E} 1$ & $7 / 14 / 08$ & 8G14006 \\
\hline 0804025-DM & В1ТР94 & 3.64E1 & 2.34E1 & $7 / 14 / 08$ & 8G14006 \\
\hline
\end{tabular}




\section{Wet Chemistry}

\begin{tabular}{|c|c|c|c|c|c|}
\hline \multicolumn{6}{|c|}{ Specific Conductance (EC) (mS/cm) by EPA 120.1} \\
\hline Lab ID & HEIS No. & Results & EQL & Analyzed & Batch \\
\hline 0804025-04 & B1TP24 & $5.00 \mathrm{E}-1$ & $1.00 \mathrm{E}-2$ & $7 / 10 / 08$ & 8G09003 \\
\hline 0804025-07 & B1TP28 & 6.37E-1 & $1.00 \mathrm{E}-2$ & $7 / 10 / 08$ & 8G09003 \\
\hline 0804025-08 & B1TP29 & $5.05 \mathrm{E}-1$ & $1.00 \mathrm{E}-2$ & $8 / 28 / 08$ & $8 \mathrm{H} 27004$ \\
\hline 0804025-09 & В1ТР30 & $6.54 \mathrm{E}-1$ & $1.00 \mathrm{E}-2$ & $8 / 28 / 08$ & 8H27004 \\
\hline $0804025-10$ & B1TNV9 & 3.04E0 & $1.00 \mathrm{E}-2$ & 8/28/08 & 8H27004 \\
\hline 0804025-13 & B1TP22 & $2.38 \mathrm{E}-1$ & $1.00 \mathrm{E}-2$ & 7/10/08 & 8G09003 \\
\hline 0804025-16 & B1TP26 & 6.09E-1 & $1.00 \mathrm{E}-2$ & 7/10/08 & 8G09003 \\
\hline $0804025-17$ & B1TNW0 & 7.27E0 & $1.00 \mathrm{E}-2$ & 8/28/08 & 8H27004 \\
\hline 0804025-18 & B1TNW1 & $1.12 \mathrm{E} 1$ & $1.00 \mathrm{E}-2$ & 7/10/08 & 8G09003 \\
\hline 0804025-20 & B1TNW3 & $1.57 \mathrm{E} 0$ & $1.00 \mathrm{E}-2$ & 8/28/08 & $8 \mathrm{H} 27004$ \\
\hline 0804025-22 & B1TNW5 & $2.71 \mathrm{E} 0$ & $1.00 \mathrm{E}-2$ & $7 / 10 / 08$ & 8G09003 \\
\hline 0804025-37 & B1TP35 & $2.17 \mathrm{E}-1$ & $1.00 \mathrm{E}-2$ & $7 / 10 / 08$ & 8G09003 \\
\hline 0804025-48 & B1TP40 & 2.09E-1 & $1.00 \mathrm{E}-2$ & $7 / 10 / 08$ & 8G09003 \\
\hline 0804025-50 & B1TP42 & 6.66E-1 & $1.00 \mathrm{E}-2$ & 8/28/08 & 8H27004 \\
\hline 0804025-51 & В1TP43 & $7.25 \mathrm{E} 0$ & $1.00 \mathrm{E}-2$ & $7 / 10 / 08$ & 8G09003 \\
\hline $0804025-53$ & B1TP45 & $2.54 \mathrm{E} 0$ & $1.00 \mathrm{E}-2$ & 8/28/08 & $8 \mathrm{H} 27004$ \\
\hline 0804025-57 & B1TP47 & $3.48 \mathrm{E} 0$ & $1.00 \mathrm{E}-2$ & $7 / 10 / 08$ & 8G09003 \\
\hline 0804025-61 & В1ТР49 & 2.82E0 & $1.00 \mathrm{E}-2$ & $7 / 10 / 08$ & 8G09004 \\
\hline 0804025-69 & B1TP51 & $1.03 \mathrm{E} 1$ & $1.00 \mathrm{E}-2$ & $7 / 10 / 08$ & 8G09004 \\
\hline 0804025-72 & B1TP52 & $2.24 \mathrm{E} 0$ & $1.00 \mathrm{E}-2$ & 7/10/08 & 8G09004 \\
\hline 0804025-75 & B1TP53 & $2.91 \mathrm{E} 0$ & $1.00 \mathrm{E}-2$ & 7/10/08 & 8G09004 \\
\hline 0804025-81 & B1TP55 & $4.81 \mathrm{E}-1$ & $1.00 \mathrm{E}-2$ & $7 / 10 / 08$ & 8G09004 \\
\hline 0804025-87 & B1TP59 & $1.65 \mathrm{E}-1$ & $1.00 \mathrm{E}-2$ & 7/10/08 & 8G09004 \\
\hline 0804025-91 & B1TP61 & 1.63E-1 & $1.00 \mathrm{E}-2$ & 8/28/08 & $8 \mathrm{H} 27004$ \\
\hline 0804025-94 & B1TP62 & $1.65 \mathrm{E} 0$ & $1.00 \mathrm{E}-2$ & 7/10/08 & 8G09004 \\
\hline 0804025-AA & B1TP64 & $1.90 \mathrm{E} 0$ & $1.00 \mathrm{E}-2$ & 8/28/08 & 8H27004 \\
\hline 0804025-AI & B1TP67 & 2.61E-1 & $1.00 \mathrm{E}-2$ & 7/10/08 & 8G09004 \\
\hline 0804025-AP & В1TP69 & $1.94 \mathrm{E}-1$ & $1.00 \mathrm{E}-2$ & 8/28/08 & 8H27004 \\
\hline 0804025-AV & B1TP71 & $1.78 \mathrm{E}-1$ & $1.00 \mathrm{E}-2$ & 7/10/08 & 8G09004 \\
\hline 0804025-BK & B1TP77 & $1.65 \mathrm{E}-1$ & $1.00 \mathrm{E}-2$ & 7/10/08 & 8G09004 \\
\hline 0804025-BP & B1TP78 & $1.65 \mathrm{E}-1$ & $1.00 \mathrm{E}-2$ & 7/10/08 & 8G09004 \\
\hline 0804025-BZ & B1TP82 & $4.88 \mathrm{E}-1$ & $1.00 \mathrm{E}-2$ & 7/10/08 & 8G09004 \\
\hline 0804025-CF & В1TP84 & 4.06E-1 & $1.00 \mathrm{E}-2$ & 7/10/08 & 8G09004 \\
\hline 0804025-CI & B1TNX1 & $2.48 \mathrm{E}-1$ & $1.00 \mathrm{E}-2$ & 7/10/08 & 8G09004 \\
\hline 0804025-CO & B1TP86 & $1.47 \mathrm{E}-1$ & $1.00 \mathrm{E}-2$ & 7/10/08 & 8G09004 \\
\hline 0804025-CX & В1TP89 & $1.74 \mathrm{E}-1$ & $1.00 \mathrm{E}-2$ & $7 / 10 / 08$ & 8G09004 \\
\hline 0804025-DF & В1ТР91 & $1.86 \mathrm{E}-1$ & $1.00 \mathrm{E}-2$ & 7/10/08 & 8G09004 \\
\hline 0804025-DL & В1ТР93 & $2.85 \mathrm{E}-1$ & $1.00 \mathrm{E}-2$ & 7/10/08 & 8G09004 \\
\hline 0804025-DM & B1TP94 & 2.43E-1 & $1.00 \mathrm{E}-2$ & 7/10/08 & 8G09004 \\
\hline
\end{tabular}




\section{Wet Chemistry}

\begin{tabular}{|c|c|c|c|c|c|}
\hline $\begin{array}{l}\text { Moisture C } \\
\text { Lab ID }\end{array}$ & $\begin{array}{c}\text { tent (\% } \\
\text { HEIS No. }\end{array}$ & $\begin{array}{l}\text { C-001 } \\
\text { Results }\end{array}$ & EQL & Analyzed & Batch \\
\hline 0804025-04 & B1TP24 & $6.04 \mathrm{E} 0$ & N/A & $6 / 13 / 08$ & $8 \mathrm{~F} 09001$ \\
\hline 0804025-07 & B1TP28 & 3.80Е0 & N/A & 6/13/08 & 8F09001 \\
\hline 0804025-08 & В1TP29 & 3.64E0 & N/A & 6/13/08 & 8F09001 \\
\hline 0804025-09 & В1ТР30 & 3.18E0 & N/A & 6/13/08 & 8F09001 \\
\hline 0804025-10 & B1TNV9 & $3.00 \mathrm{E} 0$ & N/A & 6/13/08 & 8F09001 \\
\hline 0804025-13 & B1TP22 & 3.69E0 & N/A & 6/13/08 & 8F09001 \\
\hline 0804025-16 & В1TP26 & $2.74 \mathrm{E} 0$ & N/A & 6/13/08 & 8F09001 \\
\hline 0804025-17 & B1TNW0 & 2.65E0 & N/A & 6/13/08 & 8F09001 \\
\hline 0804025-18 & B1TNW1 & 3.74E0 & N/A & 6/13/08 & 8F09001 \\
\hline 0804025-20 & B1TNW3 & 2.25E0 & N/A & 6/13/08 & 8F09001 \\
\hline 0804025-22 & B1TNW5 & 4.55E0 & N/A & 6/13/08 & 8F09001 \\
\hline 0804025-37 & B1TP35 & $2.40 \mathrm{E} 0$ & N/A & 6/13/08 & 8F09001 \\
\hline 0804025-48 & B1TP40 & $2.88 \mathrm{E} 0$ & N/A & 6/13/08 & 8F09001 \\
\hline 0804025-50 & B1TP42 & $3.00 \mathrm{E} 0$ & N/A & 6/13/08 & 8F09001 \\
\hline 0804025-51 & B1TP43 & $1.25 \mathrm{E} 1$ & N/A & 6/13/08 & 8F09001 \\
\hline 0804025-53 & B1TP45 & $3.08 \mathrm{E} 0$ & N/A & 6/13/08 & 8F09001 \\
\hline 0804025-57 & B1TP47 & 2.32E0 & N/A & 6/13/08 & 8F09001 \\
\hline 0804025-61 & В1TP49 & $2.78 \mathrm{E} 0$ & N/A & 6/13/08 & 8F09001 \\
\hline 0804025-69 & B1TP51 & $6.73 \mathrm{E} 0$ & N/A & 6/13/08 & 8F09001 \\
\hline 0804025-72 & B1TP52 & $2.46 \mathrm{E} 0$ & N/A & 6/13/08 & 8F09001 \\
\hline 0804025-75 & B1TP53 & 1.89E0 & N/A & 6/13/08 & 8F09001 \\
\hline 0804025-81 & B1TP55 & 2.32E0 & N/A & 6/13/08 & 8F09001 \\
\hline 0804025-87 & B1TP59 & $1.99 \mathrm{E} 0$ & N/A & 6/13/08 & 8F09001 \\
\hline 0804025-91 & B1TP61 & 2.29E0 & N/A & 6/13/08 & 8F09001 \\
\hline 0804025-94 & B1TP62 & 3.56E0 & N/A & 6/13/08 & 8F09001 \\
\hline 0804025-AA & B1TP64 & $3.10 \mathrm{E} 0$ & N/A & 6/13/08 & 8F09001 \\
\hline 0804025-AI & B1TP67 & $3.22 \mathrm{E} 0$ & N/A & 6/13/08 & 8F09001 \\
\hline 0804025-AP & В1TP69 & $2.74 \mathrm{E} 0$ & N/A & 6/13/08 & 8F09001 \\
\hline 0804025-AV & B1TP71 & $2.90 \mathrm{E} 0$ & N/A & 6/13/08 & 8F09001 \\
\hline 0804025-BK & B1TP77 & $3.22 \mathrm{E} 0$ & N/A & 6/13/08 & 8F09001 \\
\hline 0804025-ВР & B1TP78 & $2.68 \mathrm{E} 0$ & N/A & 6/13/08 & 8F09001 \\
\hline 0804025-BZ & B1TP82 & 4.03E0 & N/A & 6/13/08 & 8F09001 \\
\hline 0804025-CF & B1TP84 & 4.65E0 & N/A & 6/13/08 & 8F09001 \\
\hline 0804025-CI & B1TNX1 & $4.20 \mathrm{E} 0$ & N/A & 6/13/08 & 8F09001 \\
\hline 0804025-CO & В1TP86 & 3.31E0 & N/A & 6/13/08 & 8F09001 \\
\hline 0804025-CX & B1TP89 & 3.94E0 & N/A & 6/13/08 & 8F09001 \\
\hline 0804025-DF & В1ТР91 & 2.69E0 & N/A & 6/13/08 & 8F09001 \\
\hline 0804025-DL & В1ТР93 & 8.49E0 & N/A & 6/13/08 & 8F09001 \\
\hline
\end{tabular}




\section{Wet Chemistry}

pH (pH Units) by AGG-pH-001

\begin{tabular}{|c|c|c|c|c|c|}
\hline Lab ID & HEIS No. & Results & EQL & Analyzed & Batch \\
\hline 0804025-04 & B1TP24 & $9.71 \mathrm{E} 0$ & N/A & $7 / 10 / 08$ & 8G09001 \\
\hline 0804025-07 & B1TP28 & $1.02 \mathrm{E} 1$ & N/A & 7/10/08 & 8G09001 \\
\hline 0804025-08 & B1TP29 & $9.99 \mathrm{E} 0$ & N/A & 8/28/08 & 8Н27001 \\
\hline 0804025-09 & В1ТР30 & $1.01 \mathrm{E} 1$ & N/A & 8/28/08 & $8 \mathrm{H} 27001$ \\
\hline 0804025-10 & B1TNV9 & $9.26 \mathrm{E} 0$ & N/A & 8/28/08 & 8H27001 \\
\hline 0804025-13 & B1TP22 & 8.62E0 & N/A & 7/10/08 & 8G09001 \\
\hline 0804025-16 & B1TP26 & $1.02 \mathrm{E} 1$ & N/A & $7 / 10 / 08$ & 8G09001 \\
\hline 0804025-17 & B1TNW0 & 7.89E0 & N/A & 8/28/08 & 8H27001 \\
\hline 0804025-18 & B1TNW1 & 7.51E0 & N/A & $7 / 10 / 08$ & 8G09001 \\
\hline 0804025-20 & B1TNW3 & 7.41E0 & N/A & $8 / 28 / 08$ & 8H27001 \\
\hline 0804025-22 & B1TNW5 & 7.47E0 & N/A & $7 / 10 / 08$ & 8G09001 \\
\hline 0804025-37 & B1TP35 & 7.95E0 & N/A & $7 / 10 / 08$ & 8G09001 \\
\hline 0804025-48 & B1TP40 & 7.82E0 & N/A & $7 / 10 / 08$ & 8G09001 \\
\hline 0804025-50 & B1TP42 & 7.77E0 & N/A & 8/28/08 & 8H27001 \\
\hline 0804025-51 & B1TP43 & 7.65E0 & N/A & $7 / 10 / 08$ & 8G09001 \\
\hline 0804025-53 & B1TP45 & 8.97E0 & N/A & 8/28/08 & 8H27001 \\
\hline 0804025-57 & B1TP47 & 7.18E0 & N/A & 7/10/08 & 8G09001 \\
\hline 0804025-61 & В1ТР49 & 7.18E0 & N/A & 7/10/08 & 8G09002 \\
\hline 0804025-69 & B1TP51 & 7.29E0 & N/A & $7 / 10 / 08$ & 8G09002 \\
\hline 0804025-72 & B1TP52 & 7.67E0 & N/A & $7 / 10 / 08$ & 8G09002 \\
\hline 0804025-75 & B1TP53 & 7.05E0 & N/A & $7 / 10 / 08$ & 8G09002 \\
\hline 0804025-81 & B1TP55 & 7.56E0 & N/A & $7 / 10 / 08$ & 8G09002 \\
\hline 0804025-87 & B1TP59 & 7.52E0 & N/A & $7 / 10 / 08$ & 8G09002 \\
\hline 0804025-91 & B1TP61 & 7.68E0 & N/A & 8/28/08 & 8H27001 \\
\hline 0804025-94 & B1TP62 & 7.34E0 & N/A & $7 / 10 / 08$ & 8G09002 \\
\hline 0804025-AA & B1TP64 & 7.43E0 & N/A & 8/28/08 & 8H27001 \\
\hline 0804025-AI & B1TP67 & $7.51 \mathrm{E} 0$ & N/A & $7 / 10 / 08$ & 8G09002 \\
\hline 0804025-AP & В1TP69 & 8.04E0 & N/A & 8/28/08 & $8 \mathrm{H} 27001$ \\
\hline 0804025-AV & B1TP71 & 7.74E0 & N/A & $7 / 10 / 08$ & 8G09002 \\
\hline 0804025-BK & B1TP77 & 7.99E0 & N/A & $7 / 10 / 08$ & 8G09002 \\
\hline 0804025-BP & B1TP78 & 7.70E0 & N/A & $7 / 10 / 08$ & 8G09002 \\
\hline 0804025-BZ & В1TP82 & 7.41E0 & N/A & $7 / 10 / 08$ & 8G09002 \\
\hline 0804025-CF & B1TP84 & 7.90E0 & N/A & $7 / 10 / 08$ & 8G09002 \\
\hline 0804025-CI & B1TNX1 & 7.67E0 & N/A & $7 / 10 / 08$ & 8G09002 \\
\hline 0804025-CO & В1TP86 & 7.91E0 & N/A & $7 / 10 / 08$ & 8G09002 \\
\hline 0804025-CX & B1TP89 & 6.77E0 & N/A & $7 / 10 / 08$ & 8G09002 \\
\hline 0804025-DF & В1ТР91 & 7.87E0 & N/A & $7 / 10 / 08$ & 8G09002 \\
\hline 0804025-DL & В1ТР93 & 7.82E0 & N/A & $7 / 10 / 08$ & 8G09002 \\
\hline 0804025-DM & В1ТР94 & 7.95E0 & N/A & 7/10/08 & 8G09002 \\
\hline
\end{tabular}




\section{Anions by Ion Chromatography}

\begin{tabular}{|c|c|c|c|c|c|c|c|}
\hline CAS \# & Analyte & Results & Units & EQL & Analyzed & Batch & Method \\
\hline HEIS No. & B1TP24 & \multicolumn{3}{|c|}{ Lab ID: $\quad$ 0804025-04 } & & & \\
\hline 16984-48-8 & Fluoride & $<2.11 \mathrm{E}-1$ & ug/g dry & $2.11 \mathrm{E}-1$ & $7 / 10 / 08$ & 8G09011 & AGG-IC-001 \\
\hline 16887-00-6 & Chloride & $1.43 \mathrm{E} 0$ & ug/g dry & $5.27 \mathrm{E}-1$ & 7/10/08 & 8G09011 & AGG-IC-001 \\
\hline 14797-65-0 & Nitrite & $<1.05 \mathrm{E} 0$ & ug/g dry & $1.05 \mathrm{E} 0$ & $7 / 10 / 08$ & 8G09011 & AGG-IC-001 \\
\hline 14797-55-8 & Nitrate & $1.47 \mathrm{E} 1$ & ug/g dry & $1.05 \mathrm{E} 0$ & $7 / 10 / 08$ & 8G09011 & AGG-IC-001 \\
\hline 14808-79-8 & Sulfate & 4.83E1 & ug/g dry & $1.58 \mathrm{E} 0$ & $7 / 10 / 08$ & 8G09011 & AGG-IC-001 \\
\hline $14265-44-2$ & Phosphate & $3.81 \mathrm{E} 1$ & ug/g dry & $1.58 \mathrm{E} 0$ & 7/10/08 & 8G09011 & AGG-IC-001 \\
\hline HEIS No. & B1TP28 & \multicolumn{3}{|c|}{ Lab ID: $\quad$ 0804025-07 } & & & \\
\hline 16984-48-8 & Fluoride & 3.12E-1 & ug/g dry & 2.00E-1 & $7 / 10 / 08$ & 8G09011 & AGG-IC-001 \\
\hline $16887-00-6$ & Chloride & $6.93 \mathrm{E}-1$ & ug/g dry & 4.99E-1 & $7 / 10 / 08$ & 8G09011 & AGG-IC-001 \\
\hline 14797-65-0 & Nitrite & $<9.98 \mathrm{E}-1$ & ug/g dry & $9.98 \mathrm{E}-1$ & 7/10/08 & 8G09011 & AGG-IC-001 \\
\hline 14797-55-8 & Nitrate & $1.71 \mathrm{E} 1$ & ug/g dry & $9.98 \mathrm{E}-1$ & 7/10/08 & 8G09011 & AGG-IC-001 \\
\hline 14808-79-8 & Sulfate & 3.11E1 & ug/g dry & $1.50 \mathrm{E} 0$ & $7 / 10 / 08$ & 8G09011 & AGG-IC-001 \\
\hline 14265-44-2 & Phosphate & 1.07E1 & ug/g dry & $1.50 \mathrm{E} 0$ & $7 / 10 / 08$ & 8G09011 & AGG-IC-001 \\
\hline HEIS No. & B1TP29 & \multicolumn{3}{|c|}{ Lab ID: $\quad$ 0804025-08 } & & & \\
\hline 16984-48-8 & Fluoride & $6.14 \mathrm{E}-1$ & ug/g dry & $2.00 \mathrm{E}-1$ & 8/28/08 & $8 \mathrm{H} 28003$ & AGG-IC-001 \\
\hline 16887-00-6 & Chloride & 8.96E-1 & ug/g dry & 4.99E-1 & 8/28/08 & 8H28003 & AGG-IC-001 \\
\hline 14797-65-0 & Nitrite & $<9.98 \mathrm{E}-1$ & ug/g dry & $9.98 \mathrm{E}-1$ & 8/28/08 & 8H28003 & AGG-IC-001 \\
\hline 14797-55-8 & Nitrate & 1.89E1 & ug/g dry & $9.98 \mathrm{E}-1$ & 8/28/08 & $8 \mathrm{H} 28003$ & AGG-IC-001 \\
\hline 14808-79-8 & Sulfate & $3.10 \mathrm{E} 1$ & ug/g dry & $1.50 \mathrm{E} 0$ & 8/28/08 & 8H28003 & AGG-IC-001 \\
\hline 14265-44-2 & Phosphate & $<1.50 \mathrm{E} 0$ & ug/g dry & $1.50 \mathrm{E} 0$ & 8/28/08 & 8H28003 & AGG-IC-001 \\
\hline HEIS No. & В1ТР30 & \multicolumn{3}{|c|}{ Lab ID: $\quad$ 0804025-09 } & & & \\
\hline 16984-48-8 & Fluoride & $5.39 \mathrm{E}-1$ & ug/g dry & 2.00E-1 & 8/28/08 & $8 \mathrm{H} 28003$ & AGG-IC-001 \\
\hline 16887-00-6 & Chloride & $1.31 \mathrm{E} 0$ & ug/g dry & $5.00 \mathrm{E}-1$ & 8/28/08 & 8H28003 & AGG-IC-001 \\
\hline 14797-65-0 & Nitrite & $<1.00 \mathrm{E} 0$ & ug/g dry & $1.00 \mathrm{E} 0$ & 8/28/08 & 8H28003 & AGG-IC-001 \\
\hline 14797-55-8 & Nitrate & $4.01 \mathrm{E} 1$ & ug/g dry & $1.00 \mathrm{E} 0$ & 8/28/08 & $8 \mathrm{H} 28003$ & AGG-IC-001 \\
\hline 14808-79-8 & Sulfate & $6.84 \mathrm{E} 1$ & ug/g dry & $1.50 \mathrm{E} 0$ & 8/28/08 & 8H28003 & AGG-IC-001 \\
\hline $14265-44-2$ & Phosphate & $<1.50 \mathrm{E} 0$ & ug/g dry & $1.50 \mathrm{E} 0$ & 8/28/08 & 8H28003 & AGG-IC-001 \\
\hline HEIS No. & B1TNV9 & \multicolumn{3}{|c|}{ Lab ID: $\quad$ 0804025-10 } & & & \\
\hline 16984-48-8 & Fluoride & $<2.00 \mathrm{E} 1$ & ug/g dry & $2.00 \mathrm{E} 1$ & 8/28/08 & 8H28003 & AGG-IC-001 \\
\hline 16887-00-6 & Chloride & $<5.00 \mathrm{E} 1$ & ug/g dry & $5.00 \mathrm{E} 1$ & 8/28/08 & 8H28003 & AGG-IC-001 \\
\hline 14797-65-0 & Nitrite & $<1.00 \mathrm{E} 2$ & ug/g dry & $1.00 \mathrm{E} 2$ & 8/28/08 & $8 \mathrm{H} 28003$ & AGG-IC-001 \\
\hline 14797-55-8 & Nitrate & 5.32E2 & ug/g dry & $1.00 \mathrm{E} 2$ & 8/28/08 & 8H28003 & AGG-IC-001 \\
\hline 14808-79-8 & Sulfate & 1.07E3 & ug/g dry & $1.50 \mathrm{E} 2$ & 8/28/08 & 8H28003 & AGG-IC-001 \\
\hline 14265-44-2 & Phosphate & $<1.50 \mathrm{E} 2$ & ug/g dry & $1.50 \mathrm{E} 2$ & 8/28/08 & $8 \mathrm{H} 28003$ & AGG-IC-001 \\
\hline HEIS No. & B1TP22 & \multicolumn{3}{|c|}{ Lab ID: $\quad 0804025-13$} & & & \\
\hline 16984-48-8 & Fluoride & $5.95 \mathrm{E}-1$ & ug/g dry & $2.02 \mathrm{E}-1$ & 7/10/08 & 8G09011 & AGG-IC-001 \\
\hline 16887-00-6 & Chloride & $7.20 \mathrm{E}-1$ & ug/g dry & $5.05 \mathrm{E}-1$ & $7 / 10 / 08$ & 8G09011 & AGG-IC-001 \\
\hline 14797-65-0 & Nitrite & $<1.01 \mathrm{E} 0$ & ug/g dry & $1.01 \mathrm{E} 0$ & $7 / 10 / 08$ & 8G09011 & AGG-IC-001 \\
\hline 14797-55-8 & Nitrate & 7.54E0 & ug/g dry & $1.01 \mathrm{E} 0$ & 7/10/08 & 8G09011 & AGG-IC-001 \\
\hline 14808-79-8 & Sulfate & 5.58E1 & ug/g dry & $1.52 \mathrm{E} 0$ & 7/10/08 & 8G09011 & AGG-IC-001 \\
\hline $14265-44-2$ & Phosphate & $<1.52 \mathrm{E} 0$ & ug/g dry & $1.52 \mathrm{E} 0$ & 7/10/08 & 8G09011 & AGG-IC-001 \\
\hline HEIS No. & B1TP26 & \multicolumn{3}{|c|}{ Lab ID: $\quad 0804025-16$} & & & \\
\hline 16984-48-8 & Fluoride & $2.46 \mathrm{E}-1$ & ug/g dry & $2.01 \mathrm{E}-1$ & 7/10/08 & 8G09011 & AGG-IC-001 \\
\hline 16887-00-6 & Chloride & $<5.02 \mathrm{E}-1$ & ug/g dry & $5.02 \mathrm{E}-1$ & 7/10/08 & 8G09011 & AGG-IC-001 \\
\hline $14797-65-0$ & Nitrite & $<1.00 \mathrm{E} 0$ & ug/g dry & $1.00 \mathrm{E} 0$ & 7/10/08 & 8G09011 & AGG-IC-001 \\
\hline 14797-55-8 & Nitrate & 5.57E0 & ug/g dry & $1.00 \mathrm{E} 0$ & 7/10/08 & 8G09011 & AGG-IC-001 \\
\hline 14808-79-8 & Sulfate & $2.16 \mathrm{E} 1$ & ug/g dry & $1.51 \mathrm{E} 0$ & $7 / 10 / 08$ & 8G09011 & AGG-IC-001 \\
\hline 14265-44-2 & Phosphate & $1.26 \mathrm{E} 1$ & ug/g dry & $1.51 \mathrm{E} 0$ & $7 / 10 / 08$ & 8G09011 & AGG-IC-001 \\
\hline
\end{tabular}




\section{Anions by Ion Chromatography}

\begin{tabular}{|c|c|c|c|c|c|c|c|}
\hline CAS \# & Analyte & Results & Units & EQL & Analyzed & Batch & Method \\
\hline HEIS No. & B1TNW0 & \multicolumn{3}{|c|}{ Lab ID: $\quad$ 0804025-17 } & & & \\
\hline 16984-48-8 & Fluoride & $<2.00 \mathrm{E} 1$ & ug/g dry & $2.00 \mathrm{E} 1$ & 8/28/08 & $8 \mathrm{H} 28003$ & AGG-IC-001 \\
\hline 16887-00-6 & Chloride & $<5.00 \mathrm{E} 1$ & ug/g dry & $5.00 \mathrm{E} 1$ & 8/28/08 & $8 \mathrm{H} 28003$ & AGG-IC-001 \\
\hline 14797-65-0 & Nitrite & $<1.00 \mathrm{E} 2$ & ug/g dry & $1.00 \mathrm{E} 2$ & 8/28/08 & $8 \mathrm{H} 28003$ & AGG-IC-001 \\
\hline 14797-55-8 & Nitrate & $4.12 \mathrm{E} 3$ & ug/g dry & $1.00 \mathrm{E} 2$ & 8/28/08 & $8 \mathrm{H} 28003$ & AGG-IC-001 \\
\hline 14808-79-8 & Sulfate & 4.61E2 & ug/g dry & $1.50 \mathrm{E} 2$ & 8/28/08 & 8H28003 & AGG-IC-001 \\
\hline $14265-44-2$ & Phosphate & $<1.50 \mathrm{E} 2$ & ug/g dry & $1.50 \mathrm{E} 2$ & 8/28/08 & 8H28003 & AGG-IC-001 \\
\hline HEIS No. & B1TNW1 & \multicolumn{3}{|c|}{ Lab ID: $\quad$ 0804025-18 } & & & \\
\hline 16984-48-8 & Fluoride & $<1.98 \mathrm{E} 1$ & ug/g dry & $1.98 \mathrm{E} 1$ & $7 / 10 / 08$ & 8G09011 & AGG-IC-001 \\
\hline $16887-00-6$ & Chloride & $<4.95 \mathrm{E} 1$ & ug/g dry & 4.95E1 & $7 / 10 / 08$ & 8G09011 & AGG-IC-001 \\
\hline 14797-65-0 & Nitrite & $<9.91 \mathrm{E} 1$ & ug/g dry & 9.91E1 & 7/10/08 & 8G09011 & AGG-IC-001 \\
\hline 14797-55-8 & Nitrate & 6.97E3 & ug/g dry & $9.91 \mathrm{E} 1$ & 7/10/08 & 8G09011 & AGG-IC-001 \\
\hline 14808-79-8 & Sulfate & $1.66 \mathrm{E} 2$ & ug/g dry & $1.49 \mathrm{E} 2$ & $7 / 10 / 08$ & 8G09011 & AGG-IC-001 \\
\hline 14265-44-2 & Phosphate & $<1.49 \mathrm{E} 2$ & ug/g dry & $1.49 \mathrm{E} 2$ & $7 / 10 / 08$ & 8G09011 & AGG-IC-001 \\
\hline HEIS No. & B1TNW3 & \multicolumn{3}{|c|}{ Lab ID: $\quad$ 0804025-20 } & & & \\
\hline 16984-48-8 & Fluoride & $<2.00 \mathrm{E} 1$ & ug/g dry & $2.00 \mathrm{E} 1$ & 8/28/08 & 8H28003 & AGG-IC-001 \\
\hline 16887-00-6 & Chloride & $<5.00 \mathrm{E} 1$ & ug/g dry & $5.00 \mathrm{E} 1$ & 8/28/08 & 8H28003 & AGG-IC-001 \\
\hline 14797-65-0 & Nitrite & $<1.00 \mathrm{E} 2$ & ug/g dry & $1.00 \mathrm{E} 2$ & 8/28/08 & 8H28003 & AGG-IC-001 \\
\hline 14797-55-8 & Nitrate & 8.64E2 & ug/g dry & $1.00 \mathrm{E} 2$ & 8/28/08 & $8 \mathrm{H} 28003$ & AGG-IC-001 \\
\hline 14808-79-8 & Sulfate & $<1.50 \mathrm{E} 2$ & ug/g dry & $1.50 \mathrm{E} 2$ & 8/28/08 & 8H28003 & AGG-IC-001 \\
\hline 14265-44-2 & Phosphate & $<1.50 \mathrm{E} 2$ & ug/g dry & $1.50 \mathrm{E} 2$ & 8/28/08 & 8H28003 & AGG-IC-001 \\
\hline HEIS No. & B1TNW5 & \multicolumn{3}{|c|}{ Lab ID: $\quad$ 0804025-22 } & & & \\
\hline 16984-48-8 & Fluoride & $<1.97 \mathrm{E} 0$ & ug/g dry & 1.97E0 & 7/10/08 & 8G09011 & AGG-IC-001 \\
\hline 16887-00-6 & Chloride & $1.34 \mathrm{E} 1$ & ug/g dry & 4.92E0 & $7 / 10 / 08$ & 8G09011 & AGG-IC-001 \\
\hline 14797-65-0 & Nitrite & $<9.84 \mathrm{E} 0$ & ug/g dry & $9.84 \mathrm{E} 0$ & $7 / 10 / 08$ & 8G09011 & AGG-IC-001 \\
\hline 14797-55-8 & Nitrate & $1.45 \mathrm{E} 1$ & ug/g dry & $9.84 \mathrm{E}-1$ & $7 / 14 / 08$ & 8G09011 & AGG-IC-001 \\
\hline 14808-79-8 & Sulfate & $5.83 \mathrm{E} 1$ & ug/g dry & $1.48 \mathrm{E} 1$ & $7 / 10 / 08$ & 8G09011 & AGG-IC-001 \\
\hline $14265-44-2$ & Phosphate & $<1.48 \mathrm{E} 1$ & ug/g dry & $1.48 \mathrm{E} 1$ & 7/10/08 & 8G09011 & AGG-IC-001 \\
\hline HEIS No. & B1TP35 & \multicolumn{3}{|c|}{ Lab ID: $\quad$ 0804025-37 } & & & \\
\hline 16984-48-8 & Fluoride & 3.36E-1 & ug/g dry & 2.16E-1 & $7 / 10 / 08$ & 8G09011 & AGG-IC-001 \\
\hline 16887-00-6 & Chloride & 3.98E0 & ug/g dry & $5.40 \mathrm{E}-1$ & $7 / 10 / 08$ & 8G09011 & AGG-IC-001 \\
\hline 14797-65-0 & Nitrite & $<1.08 \mathrm{E} 0$ & ug/g dry & $1.08 \mathrm{E} 0$ & 7/10/08 & 8G09011 & AGG-IC-001 \\
\hline 14797-55-8 & Nitrate & $1.32 \mathrm{E} 1$ & ug/g dry & $1.08 \mathrm{E} 0$ & $7 / 10 / 08$ & 8G09011 & AGG-IC-001 \\
\hline 14808-79-8 & Sulfate & 5.53E1 & ug/g dry & $1.62 \mathrm{E} 0$ & $7 / 10 / 08$ & 8G09011 & AGG-IC-001 \\
\hline 14265-44-2 & Phosphate & $<1.62 \mathrm{E} 0$ & ug/g dry & 1.62E0 & $7 / 10 / 08$ & 8G09011 & AGG-IC-001 \\
\hline HEIS No. & B1TP40 & \multicolumn{3}{|c|}{ Lab ID: $\quad 0804025-48$} & & & \\
\hline 16984-48-8 & Fluoride & $3.44 \mathrm{E}-1$ & ug/g dry & $2.00 \mathrm{E}-1$ & 7/10/08 & 8G09011 & AGG-IC-001 \\
\hline 16887-00-6 & Chloride & $1.95 \mathrm{E} 0$ & ug/g dry & $5.01 \mathrm{E}-1$ & 7/10/08 & 8G09011 & AGG-IC-001 \\
\hline 14797-65-0 & Nitrite & $<1.00 \mathrm{E} 0$ & ug/g dry & $1.00 \mathrm{E} 0$ & $7 / 10 / 08$ & 8G09011 & AGG-IC-001 \\
\hline 14797-55-8 & Nitrate & $1.59 \mathrm{E} 1$ & ug/g dry & $1.00 \mathrm{E} 0$ & 7/10/08 & 8G09011 & AGG-IC-001 \\
\hline 14808-79-8 & Sulfate & 4.62E1 & ug/g dry & $1.50 \mathrm{E} 0$ & 7/10/08 & 8G09011 & AGG-IC-001 \\
\hline $14265-44-2$ & Phosphate & $<1.50 \mathrm{E} 0$ & ug/g dry & $1.50 \mathrm{E} 0$ & 7/10/08 & 8G09011 & AGG-IC-001 \\
\hline HEIS No. & B1TP42 & \multicolumn{3}{|c|}{ Lab ID: $\quad$ 0804025-50 } & & & \\
\hline 16984-48-8 & Fluoride & 2.68E-1 & ug/g dry & $2.00 \mathrm{E}-1$ & 8/28/08 & 8Н28003 & AGG-IC-001 \\
\hline 16887-00-6 & Chloride & 4.16Е0 & ug/g dry & $5.00 \mathrm{E}-1$ & 8/28/08 & $8 \mathrm{H} 28003$ & AGG-IC-001 \\
\hline $14797-65-0$ & Nitrite & $<1.00 \mathrm{E} 0$ & ug/g dry & $1.00 \mathrm{E} 0$ & 8/28/08 & 8Н28003 & AGG-IC-001 \\
\hline 14797-55-8 & Nitrate & 2.56E2 & ug/g dry & $1.00 \mathrm{E} 2$ & 9/02/08 & 8Н28003 & AGG-IC-001 \\
\hline 14808-79-8 & Sulfate & 7.46E1 & ug/g dry & $1.50 \mathrm{E} 0$ & 8/28/08 & $8 \mathrm{H} 28003$ & AGG-IC-001 \\
\hline 14265-44-2 & Phosphate & $<1.50 \mathrm{E} 0$ & ug/g dry & $1.50 \mathrm{E} 0$ & 8/28/08 & $8 \mathrm{H} 28003$ & AGG-IC-001 \\
\hline
\end{tabular}




\section{Anions by Ion Chromatography}

\begin{tabular}{|c|c|c|c|c|c|c|c|}
\hline CAS \# & Analyte & Results & Units & EQL & Analyzed & Batch & Method \\
\hline HEIS No. & B1TP43 & \multicolumn{3}{|c|}{ Lab ID: $\quad$ 0804025-51 } & & & \\
\hline 16984-48-8 & Fluoride & $<2.00 \mathrm{E} 0$ & ug/g dry & $2.00 \mathrm{E} 0$ & $7 / 10 / 08$ & 8G09011 & AGG-IC-001 \\
\hline 16887-00-6 & Chloride & 7.27E1 & ug/g dry & $5.01 \mathrm{E} 0$ & 7/10/08 & 8G09011 & AGG-IC-001 \\
\hline 14797-65-0 & Nitrite & $<1.00 \mathrm{E} 1$ & ug/g dry & $1.00 \mathrm{E} 1$ & $7 / 10 / 08$ & 8G09011 & AGG-IC-001 \\
\hline 14797-55-8 & Nitrate & 3.61E1 & ug/g dry & $1.00 \mathrm{E} 0$ & $7 / 16 / 08$ & 8G09011 & AGG-IC-001 \\
\hline 14808-79-8 & Sulfate & 7.47E2 & ug/g dry & $1.50 \mathrm{E} 1$ & $7 / 10 / 08$ & 8G09011 & AGG-IC-001 \\
\hline $14265-44-2$ & Phosphate & $<1.50 \mathrm{E} 1$ & ug/g dry & $1.50 \mathrm{E} 1$ & 7/10/08 & 8G09011 & AGG-IC-001 \\
\hline HEIS No. & B1TP45 & \multicolumn{3}{|c|}{ Lab ID: $\quad$ 0804025-53 } & & & \\
\hline 16984-48-8 & Fluoride & $<2.00 \mathrm{E} 1$ & ug/g dry & $2.00 \mathrm{E} 1$ & 8/28/08 & 8H28003 & AGG-IC-001 \\
\hline $16887-00-6$ & Chloride & $<5.00 \mathrm{E} 1$ & ug/g dry & $5.00 \mathrm{E} 1$ & 8/28/08 & $8 \mathrm{H} 28003$ & AGG-IC-001 \\
\hline 14797-65-0 & Nitrite & $<1.00 \mathrm{E} 2$ & ug/g dry & $1.00 \mathrm{E} 2$ & 8/28/08 & 8H28003 & AGG-IC-001 \\
\hline 14797-55-8 & Nitrate & $1.06 \mathrm{E} 3$ & ug/g dry & $1.00 \mathrm{E} 2$ & 8/28/08 & $8 \mathrm{H} 28003$ & AGG-IC-001 \\
\hline 14808-79-8 & Sulfate & $3.28 \mathrm{E} 2$ & ug/g dry & $1.50 \mathrm{E} 2$ & 8/28/08 & $8 \mathrm{H} 28003$ & AGG-IC-001 \\
\hline 14265-44-2 & Phosphate & $<1.50 \mathrm{E} 2$ & ug/g dry & $1.50 \mathrm{E} 2$ & 8/28/08 & 8H28003 & AGG-IC-001 \\
\hline HEIS No. & B1TP47 & \multicolumn{3}{|c|}{ Lab ID: $\quad$ 0804025-57 } & & & \\
\hline 16984-48-8 & Fluoride & $<1.98 \mathrm{E} 0$ & ug/g dry & $1.98 \mathrm{E} 0$ & $7 / 10 / 08$ & 8G09011 & AGG-IC-001 \\
\hline 16887-00-6 & Chloride & $1.56 \mathrm{E} 1$ & ug/g dry & 4.96E0 & $7 / 10 / 08$ & 8G09011 & AGG-IC-001 \\
\hline 14797-65-0 & Nitrite & $<9.91 \mathrm{E} 0$ & ug/g dry & $9.91 \mathrm{E} 0$ & $7 / 10 / 08$ & 8G09011 & AGG-IC-001 \\
\hline 14797-55-8 & Nitrate & $2.00 \mathrm{E} 1$ & ug/g dry & $9.91 \mathrm{E}-1$ & 7/14/08 & 8G09011 & AGG-IC-001 \\
\hline 14808-79-8 & Sulfate & 3.63E1 & ug/g dry & $1.49 \mathrm{E} 1$ & $7 / 10 / 08$ & 8G09011 & AGG-IC-001 \\
\hline 14265-44-2 & Phosphate & $<1.49 \mathrm{E} 1$ & ug/g dry & 1.49E1 & $7 / 10 / 08$ & 8G09011 & AGG-IC-001 \\
\hline HEIS No. & В1ТР49 & \multicolumn{3}{|c|}{ Lab ID: $\quad$ 0804025-61 } & & & \\
\hline 16984-48-8 & Fluoride & $<2.00 \mathrm{E} 1$ & ug/g dry & $2.00 \mathrm{E} 1$ & $7 / 14 / 08$ & 8G10007 & AGG-IC-001 \\
\hline 16887-00-6 & Chloride & $<5.01 \mathrm{E} 1$ & ug/g dry & $5.01 \mathrm{E} 1$ & $7 / 14 / 08$ & 8G10007 & AGG-IC-001 \\
\hline 14797-65-0 & Nitrite & $<1.00 \mathrm{E} 2$ & ug/g dry & $1.00 \mathrm{E} 2$ & $7 / 14 / 08$ & 8G10007 & AGG-IC-001 \\
\hline 14797-55-8 & Nitrate & $1.59 \mathrm{E} 3$ & ug/g dry & $1.00 \mathrm{E} 2$ & $7 / 14 / 08$ & 8G10007 & AGG-IC-001 \\
\hline 14808-79-8 & Sulfate & $2.10 \mathrm{E} 2$ & ug/g dry & $1.50 \mathrm{E} 2$ & $7 / 14 / 08$ & 8G10007 & AGG-IC-001 \\
\hline $14265-44-2$ & Phosphate & $<1.50 \mathrm{E} 2$ & ug/g dry & $1.50 \mathrm{E} 2$ & $7 / 14 / 08$ & 8G10007 & AGG-IC-001 \\
\hline HEIS No. & B1TP51 & \multicolumn{3}{|c|}{ Lab ID: $\quad$ 0804025-69 } & & & \\
\hline 16984-48-8 & Fluoride & $<1.97 \mathrm{E} 1$ & ug/g dry & $1.97 \mathrm{E} 1$ & $7 / 11 / 08$ & 8G10007 & AGG-IC-001 \\
\hline 16887-00-6 & Chloride & 4.96E1 & ug/g dry & 4.92E1 & 7/11/08 & 8G10007 & AGG-IC-001 \\
\hline 14797-65-0 & Nitrite & $<9.85 \mathrm{E} 1$ & ug/g dry & $9.85 \mathrm{E} 1$ & 7/11/08 & 8G10007 & AGG-IC-001 \\
\hline 14797-55-8 & Nitrate & 6.36E3 & ug/g dry & $9.85 \mathrm{E} 1$ & $7 / 11 / 08$ & 8G10007 & AGG-IC-001 \\
\hline 14808-79-8 & Sulfate & 1.63E2 & ug/g dry & $1.48 \mathrm{E} 2$ & $7 / 11 / 08$ & 8G10007 & AGG-IC-001 \\
\hline 14265-44-2 & Phosphate & $<1.48 \mathrm{E} 2$ & ug/g dry & $1.48 \mathrm{E} 2$ & $7 / 11 / 08$ & 8G10007 & AGG-IC-001 \\
\hline HEIS No. & B1TP52 & \multicolumn{3}{|c|}{ Lab ID: $\quad 0804025-72$} & & & \\
\hline 16984-48-8 & Fluoride & $<1.99 \mathrm{E} 0$ & ug/g dry & 1.99E0 & 7/11/08 & 8G10007 & AGG-IC-001 \\
\hline 16887-00-6 & Chloride & 8.38E0 & ug/g dry & 4.97E0 & $7 / 11 / 08$ & $8 G 10007$ & AGG-IC-001 \\
\hline 14797-65-0 & Nitrite & $<9.94 \mathrm{E} 0$ & ug/g dry & 9.94E0 & 7/11/08 & 8G10007 & AGG-IC-001 \\
\hline 14797-55-8 & Nitrate & $1.16 \mathrm{E} 3$ & ug/g dry & $9.94 \mathrm{E} 1$ & 7/14/08 & 8G10007 & AGG-IC-001 \\
\hline 14808-79-8 & Sulfate & 7.30E1 & ug/g dry & 1.49E1 & 7/11/08 & 8G10007 & AGG-IC-001 \\
\hline 14265-44-2 & Phosphate & $<1.49 \mathrm{E} 1$ & ug/g dry & 1.49E1 & 7/11/08 & 8G10007 & AGG-IC-001 \\
\hline HEIS No. & B1TP53 & \multicolumn{3}{|c|}{ Lab ID: $\quad$ 0804025-75 } & & & \\
\hline 16984-48-8 & Fluoride & $<2.00 \mathrm{E} 0$ & ug/g dry & $2.00 \mathrm{E} 0$ & 7/11/08 & 8G10007 & AGG-IC-001 \\
\hline 16887-00-6 & Chloride & $1.05 \mathrm{E} 1$ & ug/g dry & 5.01E0 & 7/11/08 & 8G10007 & AGG-IC-001 \\
\hline $14797-65-0$ & Nitrite & $<1.00 \mathrm{E} 1$ & ug/g dry & $1.00 \mathrm{E} 1$ & 7/11/08 & 8G10007 & AGG-IC-001 \\
\hline 14797-55-8 & Nitrate & $1.68 \mathrm{E} 3$ & ug/g dry & $1.00 \mathrm{E} 2$ & 7/15/08 & 8G10007 & AGG-IC-001 \\
\hline 14808-79-8 & Sulfate & 2.63E1 & ug/g dry & $1.50 \mathrm{E} 1$ & 7/11/08 & 8G10007 & AGG-IC-001 \\
\hline 14265-44-2 & Phosphate & $<1.50 \mathrm{E} 1$ & ug/g dry & $1.50 \mathrm{E} 1$ & 7/11/08 & 8G10007 & AGG-IC-001 \\
\hline
\end{tabular}




\section{Anions by Ion Chromatography}

\begin{tabular}{|c|c|c|c|c|c|c|c|}
\hline CAS \# & Analyte & Results & Units & EQL & Analyzed & Batch & Method \\
\hline HEIS No. & B1TP55 & \multicolumn{3}{|c|}{ Lab ID: $\quad 0804025-81$} & & & \\
\hline 16984-48-8 & Fluoride & 2.33E-1 & ug/g dry & 2.01E-1 & 7/11/08 & 8G10007 & AGG-IC-001 \\
\hline 16887-00-6 & Chloride & $2.18 \mathrm{E} 0$ & ug/g dry & 5.01E-1 & 7/11/08 & 8G10007 & AGG-IC-001 \\
\hline 14797-65-0 & Nitrite & $<1.00 \mathrm{E} 0$ & ug/g dry & $1.00 \mathrm{E} 0$ & 7/11/08 & 8G10007 & AGG-IC-001 \\
\hline 14797-55-8 & Nitrate & $1.88 \mathrm{E} 2$ & ug/g dry & $1.00 \mathrm{E} 1$ & $7 / 15 / 08$ & 8G10007 & AGG-IC-001 \\
\hline 14808-79-8 & Sulfate & $3.40 \mathrm{E} 1$ & ug/g dry & $1.50 \mathrm{E} 0$ & $7 / 11 / 08$ & 8G10007 & AGG-IC-001 \\
\hline $14265-44-2$ & Phosphate & $<1.50 \mathrm{E} 0$ & ug/g dry & $1.50 \mathrm{E} 0$ & 7/11/08 & 8G10007 & AGG-IC-001 \\
\hline HEIS No. & B1TP59 & \multicolumn{3}{|c|}{ Lab ID: $\quad 0804025-87$} & & & \\
\hline 16984-48-8 & Fluoride & 2.13E-1 & ug/g dry & 2.03E-1 & $7 / 11 / 08$ & 8G10007 & AGG-IC-001 \\
\hline $16887-00-6$ & Chloride & 1.09E0 & ug/g dry & $5.07 \mathrm{E}-1$ & $7 / 11 / 08$ & 8G10007 & AGG-IC-001 \\
\hline 14797-65-0 & Nitrite & $<1.01 \mathrm{E} 0$ & ug/g dry & $1.01 \mathrm{E} 0$ & 7/11/08 & 8G10007 & AGG-IC-001 \\
\hline 14797-55-8 & Nitrate & 7.84E0 & ug/g dry & $1.01 \mathrm{E} 0$ & 7/11/08 & 8G10007 & AGG-IC-001 \\
\hline 14808-79-8 & Sulfate & 4.06E1 & ug/g dry & $1.52 \mathrm{E} 0$ & 7/11/08 & 8G10007 & AGG-IC-001 \\
\hline 14265-44-2 & Phosphate & $<1.52 \mathrm{E} 0$ & ug/g dry & $1.52 \mathrm{E} 0$ & $7 / 11 / 08$ & 8G10007 & AGG-IC-001 \\
\hline HEIS No. & B1TP61 & \multicolumn{3}{|c|}{ Lab ID: $\quad 0804025-91$} & & & \\
\hline 16984-48-8 & Fluoride & $<2.00 \mathrm{E}-1$ & ug/g dry & $2.00 \mathrm{E}-1$ & 8/28/08 & 8H28003 & AGG-IC-001 \\
\hline 16887-00-6 & Chloride & $1.41 \mathrm{E} 0$ & ug/g dry & $5.01 \mathrm{E}-1$ & 8/28/08 & 8H28003 & AGG-IC-001 \\
\hline 14797-65-0 & Nitrite & $<1.00 \mathrm{E} 0$ & ug/g dry & $1.00 \mathrm{E} 0$ & 8/28/08 & 8H28003 & AGG-IC-001 \\
\hline 14797-55-8 & Nitrate & 1.03E1 & ug/g dry & $1.00 \mathrm{E} 0$ & 8/28/08 & $8 \mathrm{H} 28003$ & AGG-IC-001 \\
\hline 14808-79-8 & Sulfate & 3.93E1 & ug/g dry & $1.50 \mathrm{E} 0$ & 8/28/08 & 8H28003 & AGG-IC-001 \\
\hline 14265-44-2 & Phosphate & $<1.50 \mathrm{E} 0$ & ug/g dry & $1.50 \mathrm{E} 0$ & 8/28/08 & 8H28003 & AGG-IC-001 \\
\hline HEIS No. & B1TP62 & \multicolumn{3}{|c|}{ Lab ID: $\quad$ 0804025-94 } & & & \\
\hline 16984-48-8 & Fluoride & $<1.98 \mathrm{E} 0$ & ug/g dry & $1.98 \mathrm{E} 0$ & 7/11/08 & 8G10007 & AGG-IC-001 \\
\hline 16887-00-6 & Chloride & 7.21E0 & ug/g dry & 4.94E0 & $7 / 11 / 08$ & 8G10007 & AGG-IC-001 \\
\hline 14797-65-0 & Nitrite & $<9.88 \mathrm{E} 0$ & ug/g dry & $9.88 \mathrm{E} 0$ & $7 / 11 / 08$ & 8G10007 & AGG-IC-001 \\
\hline 14797-55-8 & Nitrate & 8.17E2 & ug/g dry & $9.88 \mathrm{E} 0$ & $7 / 11 / 08$ & 8G10007 & AGG-IC-001 \\
\hline 14808-79-8 & Sulfate & 4.46E1 & ug/g dry & $1.48 \mathrm{E} 1$ & 7/11/08 & 8G10007 & AGG-IC-001 \\
\hline $14265-44-2$ & Phosphate & $<1.48 \mathrm{E} 1$ & ug/g dry & $1.48 \mathrm{E} 1$ & 7/11/08 & 8G10007 & AGG-IC-001 \\
\hline HEIS No. & B1TP64 & \multicolumn{3}{|c|}{ Lab ID: $\quad$ 0804025-AA } & & & \\
\hline 16984-48-8 & Fluoride & $<2.01 \mathrm{E} 1$ & ug/g dry & $2.01 \mathrm{E} 1$ & 8/28/08 & 8H28003 & AGG-IC-001 \\
\hline 16887-00-6 & Chloride & $<5.01 \mathrm{E} 1$ & ug/g dry & $5.01 \mathrm{E} 1$ & 8/28/08 & 8H28003 & AGG-IC-001 \\
\hline 14797-65-0 & Nitrite & $<1.00 \mathrm{E} 2$ & ug/g dry & $1.00 \mathrm{E} 2$ & 8/28/08 & $8 \mathrm{H} 28003$ & AGG-IC-001 \\
\hline 14797-55-8 & Nitrate & 1.03E3 & ug/g dry & $1.00 \mathrm{E} 2$ & 8/28/08 & 8H28003 & AGG-IC-001 \\
\hline 14808-79-8 & Sulfate & $<1.50 \mathrm{E} 2$ & ug/g dry & $1.50 \mathrm{E} 2$ & 8/28/08 & 8H28003 & AGG-IC-001 \\
\hline 14265-44-2 & Phosphate & $<1.50 \mathrm{E} 2$ & ug/g dry & $1.50 \mathrm{E} 2$ & 8/28/08 & $8 \mathrm{H} 28003$ & AGG-IC-001 \\
\hline HEIS No. & B1TP67 & \multicolumn{3}{|c|}{ Lab ID: $\quad$ 0804025-AI } & & & \\
\hline 16984-48-8 & Fluoride & $3.78 \mathrm{E}-1$ & ug/g dry & $1.96 \mathrm{E}-1$ & 7/11/08 & 8G10007 & AGG-IC-001 \\
\hline 16887-00-6 & Chloride & 1.79E0 & ug/g dry & 4.89E-1 & 7/11/08 & $8 G 10007$ & AGG-IC-001 \\
\hline 14797-65-0 & Nitrite & $<9.78 \mathrm{E}-1$ & ug/g dry & $9.78 \mathrm{E}-1$ & 7/11/08 & 8G10007 & AGG-IC-001 \\
\hline 14797-55-8 & Nitrate & 4.82E1 & ug/g dry & $9.78 \mathrm{E}-1$ & 7/11/08 & 8G10007 & AGG-IC-001 \\
\hline 14808-79-8 & Sulfate & 4.05E1 & ug/g dry & $1.47 \mathrm{E} 0$ & 7/11/08 & 8G10007 & AGG-IC-001 \\
\hline $14265-44-2$ & Phosphate & $<1.47 \mathrm{E} 0$ & ug/g dry & $1.47 \mathrm{E} 0$ & 7/11/08 & $8 \mathrm{G} 10007$ & AGG-IC-001 \\
\hline HEIS No. & B1TP69 & \multicolumn{3}{|c|}{ Lab ID: $\quad$ 0804025-AP } & & & \\
\hline 16984-48-8 & Fluoride & $4.82 \mathrm{E}-1$ & ug/g dry & $2.00 \mathrm{E}-1$ & 8/28/08 & 8Н28003 & AGG-IC-001 \\
\hline 16887-00-6 & Chloride & $1.85 \mathrm{E} 0$ & ug/g dry & $5.01 \mathrm{E}-1$ & 8/28/08 & $8 \mathrm{H} 28003$ & AGG-IC-001 \\
\hline $14797-65-0$ & Nitrite & $<1.00 \mathrm{E} 0$ & ug/g dry & $1.00 \mathrm{E} 0$ & 8/28/08 & 8Н28003 & AGG-IC-001 \\
\hline 14797-55-8 & Nitrate & $<1.00 \mathrm{E} 0$ & ug/g dry & $1.00 \mathrm{E} 0$ & 8/28/08 & 8Н28003 & AGG-IC-001 \\
\hline 14808-79-8 & Sulfate & 3.94E1 & ug/g dry & $1.50 \mathrm{E} 0$ & 8/28/08 & $8 \mathrm{H} 28003$ & AGG-IC-001 \\
\hline 14265-44-2 & Phosphate & $<1.50 \mathrm{E} 0$ & ug/g dry & $1.50 \mathrm{E} 0$ & 8/28/08 & $8 \mathrm{H} 28003$ & AGG-IC-001 \\
\hline
\end{tabular}




\section{Anions by Ion Chromatography}

\begin{tabular}{|c|c|c|c|c|c|c|c|}
\hline CAS \# & Analyte & Results & Units & EQL & Analyzed & Batch & Method \\
\hline HEIS No. & B1TP71 & \multicolumn{3}{|c|}{ Lab ID: $\quad$ 0804025-AV } & & & \\
\hline 16984-48-8 & Fluoride & $4.21 \mathrm{E}-1$ & ug/g dry & 1.99E-1 & 7/11/08 & 8G10007 & AGG-IC-001 \\
\hline 16887-00-6 & Chloride & $1.18 \mathrm{E} 0$ & ug/g dry & 4.98E-1 & 7/11/08 & 8G10007 & AGG-IC-001 \\
\hline 14797-65-0 & Nitrite & $<9.97 \mathrm{E}-1$ & ug/g dry & 9.97E-1 & $7 / 11 / 08$ & 8G10007 & AGG-IC-001 \\
\hline 14797-55-8 & Nitrate & $<9.97 \mathrm{E}-1$ & ug/g dry & $9.97 \mathrm{E}-1$ & 7/11/08 & 8G10007 & AGG-IC-001 \\
\hline 14808-79-8 & Sulfate & 3.42E1 & ug/g dry & $1.50 \mathrm{E} 0$ & $7 / 11 / 08$ & 8G10007 & AGG-IC-001 \\
\hline $14265-44-2$ & Phosphate & $<1.50 \mathrm{E} 0$ & ug/g dry & $1.50 \mathrm{E} 0$ & 7/11/08 & 8G10007 & AGG-IC-001 \\
\hline HEIS No. & B1TP77 & \multicolumn{3}{|c|}{ Lab ID: $\quad$ 0804025-BK } & & & \\
\hline 16984-48-8 & Fluoride & 4.66E-1 & ug/g dry & $2.00 \mathrm{E}-1$ & $7 / 11 / 08$ & 8G10007 & AGG-IC-001 \\
\hline $16887-00-6$ & Chloride & $1.01 \mathrm{E} 0$ & ug/g dry & $5.00 \mathrm{E}-1$ & $7 / 11 / 08$ & 8G10007 & AGG-IC-001 \\
\hline 14797-65-0 & Nitrite & $<9.99 \mathrm{E}-1$ & ug/g dry & 9.99E-1 & 7/11/08 & 8G10007 & AGG-IC-001 \\
\hline 14797-55-8 & Nitrate & $<9.99 \mathrm{E}-1$ & ug/g dry & 9.99E-1 & $7 / 11 / 08$ & 8G10007 & AGG-IC-001 \\
\hline 14808-79-8 & Sulfate & 2.69E1 & ug/g dry & $1.50 \mathrm{E} 0$ & 7/11/08 & 8G10007 & AGG-IC-001 \\
\hline 14265-44-2 & Phosphate & $<1.50 \mathrm{E} 0$ & ug/g dry & $1.50 \mathrm{E} 0$ & $7 / 11 / 08$ & 8G10007 & AGG-IC-001 \\
\hline HEIS No. & B1TP78 & \multicolumn{3}{|c|}{ Lab ID: $\quad$ 0804025-BP } & & & \\
\hline 16984-48-8 & Fluoride & $3.67 \mathrm{E}-1$ & ug/g dry & $2.00 \mathrm{E}-1$ & 7/11/08 & 8G10007 & AGG-IC-001 \\
\hline 16887-00-6 & Chloride & $1.22 \mathrm{E} 0$ & ug/g dry & $5.00 \mathrm{E}-1$ & $7 / 11 / 08$ & 8G10007 & AGG-IC-001 \\
\hline 14797-65-0 & Nitrite & $<1.00 \mathrm{E} 0$ & ug/g dry & $1.00 \mathrm{E} 0$ & $7 / 11 / 08$ & 8G10007 & AGG-IC-001 \\
\hline 14797-55-8 & Nitrate & 6.16Е0 & ug/g dry & $1.00 \mathrm{E} 0$ & 7/11/08 & 8G10007 & AGG-IC-001 \\
\hline 14808-79-8 & Sulfate & $3.45 \mathrm{E} 1$ & ug/g dry & $1.50 \mathrm{E} 0$ & 7/11/08 & 8G10007 & AGG-IC-001 \\
\hline 14265-44-2 & Phosphate & $<1.50 \mathrm{E} 0$ & ug/g dry & $1.50 \mathrm{E} 0$ & $7 / 11 / 08$ & 8G10007 & AGG-IC-001 \\
\hline HEIS No. & B1TP82 & \multicolumn{3}{|c|}{ Lab ID: $\quad$ 0804025-BZ } & & & \\
\hline 16984-48-8 & Fluoride & $4.47 \mathrm{E}-1$ & ug/g dry & 1.99E-1 & 7/11/08 & 8G10007 & AGG-IC-001 \\
\hline 16887-00-6 & Chloride & 4.19E0 & ug/g dry & 4.99E-1 & $7 / 11 / 08$ & 8G10007 & AGG-IC-001 \\
\hline 14797-65-0 & Nitrite & $<9.97 \mathrm{E}-1$ & ug/g dry & $9.97 \mathrm{E}-1$ & $7 / 11 / 08$ & 8G10007 & AGG-IC-001 \\
\hline 14797-55-8 & Nitrate & $2.76 \mathrm{E} 1$ & ug/g dry & $9.97 \mathrm{E}-1$ & $7 / 11 / 08$ & 8G10007 & AGG-IC-001 \\
\hline 14808-79-8 & Sulfate & $1.70 \mathrm{E} 2$ & ug/g dry & $1.50 \mathrm{E} 1$ & $7 / 15 / 08$ & 8G10007 & AGG-IC-001 \\
\hline $14265-44-2$ & Phosphate & $<1.50 \mathrm{E} 0$ & ug/g dry & $1.50 \mathrm{E} 0$ & 7/11/08 & 8G10007 & AGG-IC-001 \\
\hline HEIS No. & B1TP84 & \multicolumn{3}{|c|}{ Lab ID: $\quad$ 0804025-CF } & & & \\
\hline 16984-48-8 & Fluoride & $7.04 \mathrm{E}-1$ & ug/g dry & $2.00 \mathrm{E}-1$ & $7 / 11 / 08$ & 8G10007 & AGG-IC-001 \\
\hline 16887-00-6 & Chloride & $5.10 \mathrm{E} 0$ & ug/g dry & 4.99E-1 & 7/11/08 & 8G10007 & AGG-IC-001 \\
\hline 14797-65-0 & Nitrite & 4.32E0 & ug/g dry & $9.98 \mathrm{E}-1$ & $7 / 11 / 08$ & 8G10007 & AGG-IC-001 \\
\hline 14797-55-8 & Nitrate & $1.82 \mathrm{E} 1$ & ug/g dry & $9.98 \mathrm{E}-1$ & $7 / 11 / 08$ & 8G10007 & AGG-IC-001 \\
\hline 14808-79-8 & Sulfate & 9.82E1 & ug/g dry & $1.50 \mathrm{E} 0$ & $7 / 11 / 08$ & 8G10007 & AGG-IC-001 \\
\hline $14265-44-2$ & Phosphate & $<1.50 \mathrm{E} 0$ & ug/g dry & $1.50 \mathrm{E} 0$ & 7/11/08 & 8G10007 & AGG-IC-001 \\
\hline HEIS No. & B1TNX1 & \multicolumn{3}{|c|}{ Lab ID: $\quad$ 0804025-CI } & & & \\
\hline 16984-48-8 & Fluoride & 4.53E-1 & ug/g dry & $2.02 \mathrm{E}-1$ & 7/11/08 & 8G10007 & AGG-IC-001 \\
\hline 16887-00-6 & Chloride & $1.50 \mathrm{E} 0$ & ug/g dry & $5.04 \mathrm{E}-1$ & $7 / 11 / 08$ & $8 G 10007$ & AGG-IC-001 \\
\hline 14797-65-0 & Nitrite & $<1.01 \mathrm{E} 0$ & ug/g dry & $1.01 \mathrm{E} 0$ & 7/11/08 & 8G10007 & AGG-IC-001 \\
\hline 14797-55-8 & Nitrate & 6.12E0 & ug/g dry & $1.01 \mathrm{E} 0$ & $7 / 11 / 08$ & $8 G 10007$ & AGG-IC-001 \\
\hline 14808-79-8 & Sulfate & 7.64E1 & ug/g dry & $1.51 \mathrm{E} 0$ & 7/11/08 & 8G10007 & AGG-IC-001 \\
\hline $14265-44-2$ & Phosphate & $<1.51 \mathrm{E} 0$ & ug/g dry & $1.51 \mathrm{E} 0$ & 7/11/08 & $8 \mathrm{G} 10007$ & AGG-IC-001 \\
\hline HEIS No. & B1TP86 & \multicolumn{3}{|c|}{ Lab ID: $\quad 0804025-C O$} & & & \\
\hline 16984-48-8 & Fluoride & $4.20 \mathrm{E}-1$ & ug/g dry & $2.00 \mathrm{E}-1$ & 7/11/08 & 8G10007 & AGG-IC-001 \\
\hline 16887-00-6 & Chloride & 7.64E-1 & ug/g dry & $5.00 \mathrm{E}-1$ & 7/11/08 & 8G10007 & AGG-IC-001 \\
\hline 14797-65-0 & Nitrite & $<1.00 \mathrm{E} 0$ & ug/g dry & $1.00 \mathrm{E} 0$ & 7/11/08 & 8G10007 & AGG-IC-001 \\
\hline 14797-55-8 & Nitrate & 2.01E0 & ug/g dry & $1.00 \mathrm{E} 0$ & 7/11/08 & 8G10007 & AGG-IC-001 \\
\hline 14808-79-8 & Sulfate & 3.36E1 & ug/g dry & $1.50 \mathrm{E} 0$ & 7/11/08 & 8G10007 & AGG-IC-001 \\
\hline 14265-44-2 & Phosphate & $<1.50 \mathrm{E} 0$ & ug/g dry & $1.50 \mathrm{E} 0$ & 7/11/08 & 8G10007 & AGG-IC-001 \\
\hline
\end{tabular}




\section{Anions by Ion Chromatography}

\begin{tabular}{|c|c|c|c|c|c|c|c|}
\hline CAS \# & Analyte & Results & Units & EQL & Analyzed & Batch & Method \\
\hline HEIS No. & B1TP89 & \multicolumn{3}{|c|}{ Lab ID: $\quad$ 0804025-CX } & & & \\
\hline 16984-48-8 & Fluoride & 3.47E-1 & ug/g dry & $2.01 \mathrm{E}-1$ & $7 / 11 / 08$ & 8G10007 & AGG-IC-001 \\
\hline $16887-00-6$ & Chloride & 9.33E-1 & ug/g dry & $5.01 \mathrm{E}-1$ & $7 / 11 / 08$ & 8G10007 & AGG-IC-001 \\
\hline 14797-65-0 & Nitrite & $<1.00 \mathrm{E} 0$ & ug/g dry & $1.00 \mathrm{E} 0$ & 7/11/08 & 8G10007 & AGG-IC-001 \\
\hline 14797-55-8 & Nitrate & 1.97E0 & ug/g dry & $1.00 \mathrm{E} 0$ & 7/11/08 & 8G10007 & AGG-IC-001 \\
\hline $14808-79-8$ & Sulfate & 4.65E1 & ug/g dry & $1.50 \mathrm{E} 0$ & 7/11/08 & 8G10007 & AGG-IC-001 \\
\hline $14265-44-2$ & Phosphate & $<1.50 \mathrm{E} 0$ & ug/g dry & $1.50 \mathrm{E} 0$ & $7 / 11 / 08$ & 8G10007 & AGG-IC-001 \\
\hline HEIS No. & B1TP91 & \multicolumn{3}{|c|}{ Lab ID: $\quad$ 0804025-DF } & & & \\
\hline 16984-48-8 & Fluoride & 8.05E-1 & ug/g dry & $2.01 \mathrm{E}-1$ & $7 / 11 / 08$ & 8G10007 & AGG-IC-001 \\
\hline $16887-00-6$ & Chloride & $1.90 \mathrm{E} 0$ & ug/g dry & $5.01 \mathrm{E}-1$ & $7 / 11 / 08$ & 8G10007 & AGG-IC-001 \\
\hline $14797-65-0$ & Nitrite & $<1.00 \mathrm{E} 0$ & ug/g dry & $1.00 \mathrm{E} 0$ & $7 / 11 / 08$ & 8G10007 & AGG-IC-001 \\
\hline $14797-55-8$ & Nitrate & $<1.00 \mathrm{E} 0$ & ug/g dry & $1.00 \mathrm{E} 0$ & $7 / 11 / 08$ & 8G10007 & AGG-IC-001 \\
\hline 14808-79-8 & Sulfate & 2.91E1 & ug/g dry & $1.50 \mathrm{E} 0$ & $7 / 11 / 08$ & 8G10007 & AGG-IC-001 \\
\hline $14265-44-2$ & Phosphate & $<1.50 \mathrm{E} 0$ & ug/g dry & $1.50 \mathrm{E} 0$ & $7 / 11 / 08$ & 8G10007 & AGG-IC-001 \\
\hline HEIS No. & В1ТР93 & \multicolumn{3}{|c|}{ Lab ID: $\quad$ 0804025-DL } & & & \\
\hline 16984-48-8 & Fluoride & $2.98 \mathrm{E}-1$ & ug/g dry & $2.01 \mathrm{E}-1$ & 7/11/08 & 8G10007 & AGG-IC-001 \\
\hline $16887-00-6$ & Chloride & $4.84 \mathrm{E} 0$ & ug/g dry & $5.03 \mathrm{E}-1$ & $7 / 11 / 08$ & 8G10007 & AGG-IC-001 \\
\hline $14797-65-0$ & Nitrite & $1.61 \mathrm{E} 0$ & ug/g dry & $1.01 \mathrm{E} 0$ & $7 / 11 / 08$ & 8G10007 & AGG-IC-001 \\
\hline $14797-55-8$ & Nitrate & 8.10E1 & ug/g dry & $1.01 \mathrm{E} 0$ & $7 / 11 / 08$ & $8 G 10007$ & AGG-IC-001 \\
\hline 14808-79-8 & Sulfate & 2.38E1 & ug/g dry & $1.51 \mathrm{E} 0$ & 7/11/08 & 8G10007 & AGG-IC-001 \\
\hline $14265-44-2$ & Phosphate & $<1.51 \mathrm{E} 0$ & ug/g dry & $1.51 \mathrm{E} 0$ & $7 / 11 / 08$ & 8G10007 & AGG-IC-001 \\
\hline HEIS No. & B1TP94 & \multicolumn{3}{|c|}{ Lab ID: $\quad$ 0804025-DM } & & & \\
\hline 16984-48-8 & Fluoride & 4.24E-1 & ug/g dry & $1.99 \mathrm{E}-1$ & $7 / 11 / 08$ & 8G10007 & AGG-IC-001 \\
\hline $16887-00-6$ & Chloride & $3.15 \mathrm{E} 0$ & ug/g dry & $4.98 \mathrm{E}-1$ & 7/11/08 & 8G10007 & AGG-IC-001 \\
\hline $14797-65-0$ & Nitrite & $<9.97 \mathrm{E}-1$ & ug/g dry & $9.97 \mathrm{E}-1$ & 7/11/08 & 8G10007 & AGG-IC-001 \\
\hline 14797-55-8 & Nitrate & 6.93E1 & ug/g dry & $9.97 \mathrm{E}-1$ & 7/11/08 & 8G10007 & AGG-IC-001 \\
\hline 14808-79-8 & Sulfate & $1.52 \mathrm{E} 1$ & ug/g dry & $1.50 \mathrm{E} 0$ & 7/11/08 & 8G10007 & AGG-IC-001 \\
\hline $14265-44-2$ & Phosphate & $<1.50 \mathrm{E} 0$ & ug/g dry & $1.50 \mathrm{E} 0$ & 7/11/08 & 8G10007 & AGG-IC-001 \\
\hline
\end{tabular}




\section{Cyanide by Mircodistillation/Colorimetric}

Cyanide (ug/g dry) by MICRODIST Cyanide Method

\begin{tabular}{llcccc} 
Lab ID & HEIS No. & Results & EQL & Analyzed & Batch \\
\hline $0804025-04$ & B1TP24 & $<2.12 \mathrm{E}-1$ & $2.12 \mathrm{E}-1$ & $12 / 10 / 08$ & $9 \mathrm{H} 06008$ \\
$0804025-18$ & B1TNW1 & $5.80 \mathrm{E}-1$ & $2.07 \mathrm{E}-1$ & $12 / 10 / 08$ & $9 \mathrm{H} 06008$ \\
$0804025-51$ & B1TP43 & $2.22 \mathrm{E} 0$ & $2.25 \mathrm{E}-1$ & $12 / 10 / 08$ & $9 \mathrm{H} 06008$ \\
$0804025-53$ & B1TP45 & $3.86 \mathrm{E}-1$ & $2.06 \mathrm{E}-1$ & $12 / 10 / 08$ & $9 \mathrm{H} 06008$ \\
$0804025-69$ & B1TP51 & $1.66 \mathrm{E} 0$ & $2.13 \mathrm{E}-1$ & $12 / 10 / 08$ & $9 \mathrm{H} 06008$ \\
$0804025-D M$ & B1TP94 & $<2.14 \mathrm{E}-1$ & $2.14 \mathrm{E}-1$ & $12 / 10 / 08$ & $9 \mathrm{H} 06008$ \\
\hline
\end{tabular}


Total Metals by PNNL-AGG-ICP-AES/Water Extract

\begin{tabular}{|c|c|c|c|c|c|c|c|}
\hline CAS \# & Analyte & Results & Units & EQL & Analyzed & Batch & Method \\
\hline HEIS No. & B1TP24 & \multicolumn{3}{|c|}{ Lab ID: $\quad$ 0804025-04 } & & & \\
\hline $7429-90-5$ & Aluminum & $1.07 \mathrm{E} 0$ & ug/g dry & $1.51 \mathrm{E}-1$ & 7/30/08 & 8G22002 & PNNL-AGG-ICP-AES \\
\hline $7440-39-3$ & Barium & 2.47E-2 & ug/g dry & $1.54 \mathrm{E}-2$ & 7/30/08 & $8 G 22002$ & PNNL-AGG-ICP-AES \\
\hline $7440-70-2$ & Calcium & 2.85E0 & ug/g dry & $6.80 \mathrm{E}-1$ & 7/30/08 & $8 G 22002$ & PNNL-AGG-ICP-AES \\
\hline 7439-89-6 & Iron & 1.92E0 & ug/g dry & $2.50 \mathrm{E}-1$ & 7/30/08 & $8 G 22002$ & PNNL-AGG-ICP-AES \\
\hline 7440-09-7 & Potassium & $<4.09 \mathrm{E} 0$ & ug/g dry & 4.09E0 & 7/30/08 & $8 G 22002$ & PNNL-AGG-ICP-AES \\
\hline 7439-95-4 & Magnesium & $5.99 \mathrm{E}-1$ & ug/g dry & $1.47 \mathrm{E}-1$ & 7/30/08 & $8 G 22002$ & PNNL-AGG-ICP-AES \\
\hline $7439-96-5$ & Manganese & $<3.01 \mathrm{E}-2$ & ug/g dry & $3.01 \mathrm{E}-2$ & 7/30/08 & $8 G 22002$ & PNNL-AGG-ICP-AES \\
\hline $7440-02-0$ & Nickel & $<1.64 \mathrm{E}-1$ & ug/g dry & $1.64 \mathrm{E}-1$ & 7/30/08 & $8 G 22002$ & PNNL-AGG-ICP-AES \\
\hline $7440-28-0$ & Thallium & $<1.80 \mathrm{E} 0$ & ug/g dry & $1.80 \mathrm{E} 0$ & 7/30/08 & $8 G 22002$ & PNNL-AGG-ICP-AES \\
\hline $7440-23-5$ & Sodium & $1.23 \mathrm{E} 2$ & ug/g dry & $1.18 \mathrm{E} 0$ & 7/30/08 & $8 G 22002$ & PNNL-AGG-ICP-AES \\
\hline HEIS No. & B1TP28 & \multicolumn{3}{|c|}{ Lab ID: $\quad$ 0804025-07 } & & & \\
\hline $7429-90-5$ & Aluminum & $8.24 \mathrm{E}-1$ & ug/g dry & $1.43 \mathrm{E}-1$ & 7/30/08 & $8 G 22002$ & PNNL-AGG-ICP-AES \\
\hline $7440-39-3$ & Barium & $<1.46 \mathrm{E}-2$ & ug/g dry & $1.46 \mathrm{E}-2$ & 7/30/08 & $8 G 22002$ & PNNL-AGG-ICP-AES \\
\hline $7440-70-2$ & Calcium & $<6.43 \mathrm{E}-1$ & ug/g dry & $6.43 \mathrm{E}-1$ & 7/30/08 & $8 G 22002$ & PNNL-AGG-ICP-AES \\
\hline 7439-89-6 & Iron & $1.11 \mathrm{E} 0$ & ug/g dry & $2.36 \mathrm{E}-1$ & 7/30/08 & $8 G 22002$ & PNNL-AGG-ICP-AES \\
\hline $7440-09-7$ & Potassium & $<3.87 \mathrm{E} 0$ & ug/g dry & 3.87E0 & 7/30/08 & 8G22002 & PNNL-AGG-ICP-AES \\
\hline 7439-95-4 & Magnesium & $2.14 \mathrm{E}-1$ & ug/g dry & $1.39 \mathrm{E}-1$ & 7/30/08 & $8 G 22002$ & PNNL-AGG-ICP-AES \\
\hline 7439-96-5 & Manganese & $<2.85 \mathrm{E}-2$ & ug/g dry & $2.85 \mathrm{E}-2$ & 7/30/08 & $8 G 22002$ & PNNL-AGG-ICP-AES \\
\hline $7440-02-0$ & Nickel & $<1.55 \mathrm{E}-1$ & ug/g dry & $1.55 \mathrm{E}-1$ & 7/30/08 & $8 G 22002$ & PNNL-AGG-ICP-AES \\
\hline $7440-28-0$ & Thallium & $<1.71 \mathrm{E} 0$ & ug/g dry & $1.71 \mathrm{E} 0$ & 7/30/08 & $8 G 22002$ & PNNL-AGG-ICP-AES \\
\hline $7440-23-5$ & Sodium & $1.43 \mathrm{E} 2$ & ug/g dry & $1.11 \mathrm{E} 0$ & 7/30/08 & $8 G 22002$ & PNNL-AGG-ICP-AES \\
\hline HEIS No. & B1TP29 & \multicolumn{3}{|c|}{ Lab ID: $\quad$ 0804025-08 } & & & \\
\hline $7429-90-5$ & Aluminum & $1.16 \mathrm{E} 0$ & ug/g dry & $8.56 \mathrm{E}-2$ & $9 / 24 / 08$ & $8 \mathrm{I} 23005$ & PNNL-AGG-ICP-AES \\
\hline $7440-39-3$ & Barium & $1.76 \mathrm{E}-2$ & ug/g dry & $8.77 \mathrm{E}-3$ & $9 / 24 / 08$ & 8I23005 & PNNL-AGG-ICP-AES \\
\hline $7440-70-2$ & Calcium & $5.21 \mathrm{E}-1$ & ug/g dry & $3.86 \mathrm{E}-1$ & $9 / 24 / 08$ & $8 \mathrm{I} 23005$ & PNNL-AGG-ICP-AES \\
\hline 7439-89-6 & Iron & $1.73 \mathrm{E} 0$ & ug/g dry & $1.42 \mathrm{E}-1$ & $9 / 24 / 08$ & $8 \mathrm{I} 23005$ & PNNL-AGG-ICP-AES \\
\hline 7440-09-7 & Potassium & $<2.32 \mathrm{E} 0$ & ug/g dry & 2.32E0 & $9 / 24 / 08$ & $8 \mathrm{I} 23005$ & PNNL-AGG-ICP-AES \\
\hline $7439-95-4$ & Magnesium & 3.46E-1 & ug/g dry & $8.32 \mathrm{E}-2$ & $9 / 24 / 08$ & $8 \mathrm{I} 23005$ & PNNL-AGG-ICP-AES \\
\hline $7439-96-5$ & Manganese & $<1.71 \mathrm{E}-2$ & ug/g dry & $1.71 \mathrm{E}-2$ & $9 / 24 / 08$ & $8 \mathrm{I} 23005$ & PNNL-AGG-ICP-AES \\
\hline $7440-02-0$ & Nickel & $<9.31 \mathrm{E}-2$ & ug/g dry & $9.31 \mathrm{E}-2$ & $9 / 24 / 08$ & $8 \mathrm{I} 23005$ & PNNL-AGG-ICP-AES \\
\hline $7440-28-0$ & Thallium & $<1.02 \mathrm{E} 0$ & ug/g dry & $1.02 \mathrm{E} 0$ & $9 / 24 / 08$ & $8 \mathrm{I} 23005$ & PNNL-AGG-ICP-AES \\
\hline $7440-23-5$ & Sodium & $1.16 \mathrm{E} 2$ & ug/g dry & $6.68 \mathrm{E}-1$ & $9 / 24 / 08$ & $8 \mathrm{I} 23005$ & PNNL-AGG-ICP-AES \\
\hline HEIS No. & B1TP30 & \multicolumn{3}{|c|}{ Lab ID: $\quad$ 0804025-09 } & & & \\
\hline $7429-90-5$ & Aluminum & $1.12 \mathrm{E} 0$ & ug/g dry & $8.58 \mathrm{E}-2$ & $9 / 24 / 08$ & $8 \mathrm{I} 23005$ & PNNL-AGG-ICP-AES \\
\hline $7440-39-3$ & Barium & $1.17 \mathrm{E}-2$ & ug/g dry & $8.79 \mathrm{E}-3$ & $9 / 24 / 08$ & $8 \mathrm{I} 23005$ & PNNL-AGG-ICP-AES \\
\hline $7440-70-2$ & Calcium & 4.25E-1 & ug/g dry & $3.87 \mathrm{E}-1$ & $9 / 24 / 08$ & 8I23005 & PNNL-AGG-ICP-AES \\
\hline $7439-89-6$ & Iron & $1.62 \mathrm{E} 0$ & ug/g dry & $1.42 \mathrm{E}-1$ & $9 / 24 / 08$ & $8 \mathrm{I} 23005$ & PNNL-AGG-ICP-AES \\
\hline 7440-09-7 & Potassium & $<2.33 \mathrm{E} 0$ & ug/g dry & 2.33E0 & $9 / 24 / 08$ & $8 \mathrm{I} 23005$ & PNNL-AGG-ICP-AES \\
\hline 7439-95-4 & Magnesium & 3.07E-1 & ug/g dry & $8.34 \mathrm{E}-2$ & $9 / 24 / 08$ & $8 \mathrm{I} 23005$ & PNNL-AGG-ICP-AES \\
\hline $7439-96-5$ & Manganese & $<1.71 \mathrm{E}-2$ & ug/g dry & $1.71 \mathrm{E}-2$ & $9 / 24 / 08$ & $8 \mathrm{I} 23005$ & PNNL-AGG-ICP-AES \\
\hline $7440-02-0$ & Nickel & $<9.33 \mathrm{E}-2$ & ug/g dry & 9.33E-2 & $9 / 24 / 08$ & 8I23005 & PNNL-AGG-ICP-AES \\
\hline $7440-28-0$ & Thallium & $<1.03 \mathrm{E} 0$ & ug/g dry & 1.03E0 & $9 / 24 / 08$ & $8 \mathrm{I} 23005$ & PNNL-AGG-ICP-AES \\
\hline $7440-23-5$ & Sodium & $1.48 \mathrm{E} 2$ & ug/g dry & $6.69 \mathrm{E}-1$ & $9 / 24 / 08$ & $8 \mathrm{I} 23005$ & PNNL-AGG-ICP-AES \\
\hline HEIS No. & B1TNV9 & \multicolumn{3}{|c|}{ Lab ID: $\quad$ 0804025-10 } & & & \\
\hline $7429-90-5$ & Aluminum & 2.23E-1 & ug/g dry & $8.58 \mathrm{E}-2$ & $9 / 24 / 08$ & 8I23005 & PNNL-AGG-ICP-AES \\
\hline $7440-39-3$ & Barium & $9.96 \mathrm{E}-3$ & ug/g dry & $8.79 \mathrm{E}-3$ & $9 / 24 / 08$ & 8I23005 & PNNL-AGG-ICP-AES \\
\hline $7440-70-2$ & Calcium & 4.31E0 & ug/g dry & $3.87 \mathrm{E}-1$ & $9 / 24 / 08$ & $8 \mathrm{I} 23005$ & PNNL-AGG-ICP-AES \\
\hline 7439-89-6 & Iron & $1.02 \mathrm{E} 0$ & ug/g dry & $1.42 \mathrm{E}-1$ & $9 / 24 / 08$ & 8I23005 & PNNL-AGG-ICP-AES \\
\hline
\end{tabular}


Total Metals by PNNL-AGG-ICP-AES/Water Extract

\begin{tabular}{|c|c|c|c|c|c|c|c|}
\hline CAS \# & Analyte & Results & Units & EQL & Analyzed & Batch & Method \\
\hline HEIS No. & B1TNV9 & \multicolumn{3}{|c|}{ Lab ID: $\quad$ 0804025-10 } & & & \\
\hline 7440-09-7 & Potassium & $7.91 \mathrm{E} 0$ & ug/g dry & 2.33Е0 & 9/24/08 & $8 \mathrm{I} 23005$ & PNNL-AGG-ICP-AES \\
\hline 7439-95-4 & Magnesium & $7.02 \mathrm{E}-1$ & ug/g dry & $8.34 \mathrm{E}-2$ & $9 / 24 / 08$ & $8 I 23005$ & PNNL-AGG-ICP-AES \\
\hline 7439-96-5 & Manganese & $<1.71 \mathrm{E}-2$ & ug/g dry & $1.71 \mathrm{E}-2$ & $9 / 24 / 08$ & $8 \mathrm{I} 23005$ & PNNL-AGG-ICP-AES \\
\hline $7440-02-0$ & Nickel & $<9.33 \mathrm{E}-2$ & ug/g dry & $9.33 \mathrm{E}-2$ & $9 / 24 / 08$ & $8 \mathrm{I} 23005$ & PNNL-AGG-ICP-AES \\
\hline $7440-28-0$ & Thallium & $<1.03 \mathrm{E} 0$ & ug/g dry & $1.03 \mathrm{E} 0$ & $9 / 24 / 08$ & $8 \mathrm{I} 23005$ & PNNL-AGG-ICP-AES \\
\hline $7440-23-5$ & Sodium & 7.38E2 & ug/g dry & $6.69 \mathrm{E}-1$ & $9 / 24 / 08$ & $8 \mathrm{I} 23005$ & PNNL-AGG-ICP-AES \\
\hline HEIS No. & B1TP22 & \multicolumn{3}{|c|}{ Lab ID: $\quad 0804025-13$} & & & \\
\hline $7429-90-5$ & Aluminum & 4.46E-1 & ug/g dry & $1.45 \mathrm{E}-1$ & 7/30/08 & $8 G 22002$ & PNNL-AGG-ICP-AES \\
\hline $7440-39-3$ & Barium & $1.87 \mathrm{E}-2$ & ug/g dry & $1.48 \mathrm{E}-2$ & 7/30/08 & $8 G 22002$ & PNNL-AGG-ICP-AES \\
\hline $7440-70-2$ & Calcium & 4.26Е0 & ug/g dry & $6.52 \mathrm{E}-1$ & 7/30/08 & $8 G 22002$ & PNNL-AGG-ICP-AES \\
\hline 7439-89-6 & Iron & $6.75 \mathrm{E}-1$ & ug/g dry & $2.40 \mathrm{E}-1$ & 7/30/08 & $8 G 22002$ & PNNL-AGG-ICP-AES \\
\hline 7440-09-7 & Potassium & $<3.92 \mathrm{E} 0$ & ug/g dry & 3.92E0 & 7/30/08 & $8 G 22002$ & PNNL-AGG-ICP-AES \\
\hline 7439-95-4 & Magnesium & $7.68 \mathrm{E}-1$ & ug/g dry & $1.41 \mathrm{E}-1$ & 7/30/08 & $8 G 22002$ & PNNL-AGG-ICP-AES \\
\hline 7439-96-5 & Manganese & $<2.89 \mathrm{E}-2$ & ug/g dry & $2.89 \mathrm{E}-2$ & 7/30/08 & $8 G 22002$ & PNNL-AGG-ICP-AES \\
\hline $7440-02-0$ & Nickel & $<1.57 \mathrm{E}-1$ & ug/g dry & $1.57 \mathrm{E}-1$ & 7/30/08 & $8 G 22002$ & PNNL-AGG-ICP-AES \\
\hline $7440-28-0$ & Thallium & $<1.73 \mathrm{E} 0$ & ug/g dry & 1.73E0 & 7/30/08 & 8G22002 & PNNL-AGG-ICP-AES \\
\hline $7440-23-5$ & Sodium & 4.27E1 & ug/g dry & 1.13E0 & 7/30/08 & $8 G 22002$ & PNNL-AGG-ICP-AES \\
\hline HEIS No. & B1TP26 & \multicolumn{3}{|c|}{ Lab ID: $\quad$ 0804025-16 } & & & \\
\hline 7429-90-5 & Aluminum & 1.03E0 & ug/g dry & $1.43 \mathrm{E}-1$ & 7/30/08 & $8 G 22002$ & PNNL-AGG-ICP-AES \\
\hline $7440-39-3$ & Barium & $<1.47 \mathrm{E}-2$ & ug/g dry & $1.47 \mathrm{E}-2$ & 7/30/08 & $8 G 22002$ & PNNL-AGG-ICP-AES \\
\hline $7440-70-2$ & Calcium & $<6.47 \mathrm{E}-1$ & ug/g dry & $6.47 \mathrm{E}-1$ & 7/30/08 & $8 G 22002$ & PNNL-AGG-ICP-AES \\
\hline 7439-89-6 & Iron & $1.34 \mathrm{E} 0$ & ug/g dry & $2.38 \mathrm{E}-1$ & 7/30/08 & $8 G 22002$ & PNNL-AGG-ICP-AES \\
\hline 7440-09-7 & Potassium & $<3.89 \mathrm{E} 0$ & ug/g dry & 3.89E0 & 7/30/08 & $8 G 22002$ & PNNL-AGG-ICP-AES \\
\hline 7439-95-4 & Magnesium & $2.54 \mathrm{E}-1$ & ug/g dry & $1.39 \mathrm{E}-1$ & 7/30/08 & $8 G 22002$ & PNNL-AGG-ICP-AES \\
\hline $7439-96-5$ & Manganese & $<2.86 \mathrm{E}-2$ & ug/g dry & $2.86 \mathrm{E}-2$ & 7/30/08 & $8 G 22002$ & PNNL-AGG-ICP-AES \\
\hline $7440-02-0$ & Nickel & $<1.56 \mathrm{E}-1$ & ug/g dry & $1.56 \mathrm{E}-1$ & 7/30/08 & 8G22002 & PNNL-AGG-ICP-AES \\
\hline $7440-28-0$ & Thallium & $<1.72 \mathrm{E} 0$ & ug/g dry & $1.72 \mathrm{E} 0$ & 7/30/08 & $8 G 22002$ & PNNL-AGG-ICP-AES \\
\hline $7440-23-5$ & Sodium & $1.35 \mathrm{E} 2$ & ug/g dry & 1.12E0 & 7/30/08 & 8G22002 & PNNL-AGG-ICP-AES \\
\hline HEIS No. & B1TNW0 & \multicolumn{3}{|c|}{ Lab ID: $\quad 0804025-17$} & & & \\
\hline $7429-90-5$ & Aluminum & $<8.58 \mathrm{E}-2$ & ug/g dry & $8.58 \mathrm{E}-2$ & $9 / 24 / 08$ & $8 \mathrm{I} 23005$ & PNNL-AGG-ICP-AES \\
\hline $7440-39-3$ & Barium & $3.28 \mathrm{E}-2$ & ug/g dry & $8.79 \mathrm{E}-3$ & $9 / 24 / 08$ & $8 \mathrm{I} 23005$ & PNNL-AGG-ICP-AES \\
\hline $7440-70-2$ & Calcium & 3.19E1 & ug/g dry & $3.87 \mathrm{E}-1$ & $9 / 24 / 08$ & $8 \mathrm{I} 23005$ & PNNL-AGG-ICP-AES \\
\hline $7439-89-6$ & Iron & $2.30 \mathrm{E} 0$ & ug/g dry & $1.42 \mathrm{E}-1$ & $9 / 24 / 08$ & $8 \mathrm{I} 23005$ & PNNL-AGG-ICP-AES \\
\hline 7440-09-7 & Potassium & $2.40 \mathrm{E} 1$ & ug/g dry & 2.33Е0 & $9 / 24 / 08$ & $8 \mathrm{I} 23005$ & PNNL-AGG-ICP-AES \\
\hline 7439-95-4 & Magnesium & $1.98 \mathrm{E} 1$ & ug/g dry & $8.34 \mathrm{E}-2$ & $9 / 24 / 08$ & $8 \mathrm{I} 23005$ & PNNL-AGG-ICP-AES \\
\hline 7439-96-5 & Manganese & $<1.71 \mathrm{E}-2$ & ug/g dry & $1.71 \mathrm{E}-2$ & $9 / 24 / 08$ & 8I23005 & PNNL-AGG-ICP-AES \\
\hline $7440-02-0$ & Nickel & $<9.33 \mathrm{E}-2$ & ug/g dry & 9.33E-2 & $9 / 24 / 08$ & $8 \mathrm{I} 23005$ & PNNL-AGG-ICP-AES \\
\hline $7440-28-0$ & Thallium & $<1.03 \mathrm{E} 0$ & ug/g dry & 1.03E0 & $9 / 24 / 08$ & $8 \mathrm{I} 23005$ & PNNL-AGG-ICP-AES \\
\hline $7440-23-5$ & Sodium & $1.70 \mathrm{E} 3$ & ug/g dry & 2.23E1 & $9 / 24 / 08$ & $8 \mathrm{I} 23005$ & PNNL-AGG-ICP-AES \\
\hline HEIS No. & B1TNW1 & \multicolumn{3}{|c|}{$\begin{array}{ll}\text { Lab ID: } & 0804025-18\end{array}$} & & & \\
\hline $7429-90-5$ & Aluminum & $<1.42 \mathrm{E}-1$ & ug/g dry & $1.42 \mathrm{E}-1$ & 7/30/08 & 8G22002 & PNNL-AGG-ICP-AES \\
\hline $7440-39-3$ & Barium & $1.18 \mathrm{E}-1$ & ug/g dry & $1.45 \mathrm{E}-2$ & 7/30/08 & $8 G 22002$ & PNNL-AGG-ICP-AES \\
\hline $7440-70-2$ & Calcium & $1.49 \mathrm{E} 2$ & ug/g dry & $6.39 \mathrm{E}-1$ & 7/30/08 & $8 G 22002$ & PNNL-AGG-ICP-AES \\
\hline 7439-89-6 & Iron & 4.29E0 & ug/g dry & $2.35 \mathrm{E}-1$ & 7/30/08 & $8 G 22002$ & PNNL-AGG-ICP-AES \\
\hline 7440-09-7 & Potassium & 4.63E1 & ug/g dry & 3.84E0 & 7/30/08 & $8 G 22002$ & PNNL-AGG-ICP-AES \\
\hline 7439-95-4 & Magnesium & 8.29E1 & ug/g dry & $1.38 \mathrm{E}-1$ & 7/30/08 & $8 G 22002$ & PNNL-AGG-ICP-AES \\
\hline $7439-96-5$ & Manganese & $<2.83 \mathrm{E}-2$ & ug/g dry & 2.83E-2 & 7/30/08 & $8 G 22002$ & PNNL-AGG-ICP-AES \\
\hline $7440-02-0$ & Nickel & $<1.54 \mathrm{E}-1$ & ug/g dry & $1.54 \mathrm{E}-1$ & 7/30/08 & $8 G 22002$ & PNNL-AGG-ICP-AES \\
\hline
\end{tabular}


Total Metals by PNNL-AGG-ICP-AES/Water Extract

\begin{tabular}{|c|c|c|c|c|c|c|c|}
\hline CAS \# & Analyte & Results & Units & EQL & Analyzed & Batch & Method \\
\hline HEIS No. & B1TNW1 & \multicolumn{3}{|c|}{ Lab ID: $\quad 0804025-18$} & & & \\
\hline $7440-28-0$ & Thallium & $<1.69 \mathrm{E} 0$ & ug/g dry & $1.69 \mathrm{E} 0$ & $7 / 30 / 08$ & 8G22002 & PNNL-AGG-ICP-AES \\
\hline $7440-23-5$ & Sodium & $2.36 \mathrm{E} 3$ & ug/g dry & $1.10 \mathrm{E} 0$ & 7/30/08 & $8 G 22002$ & PNNL-AGG-ICP-AES \\
\hline HEIS No. & B1TNW3 & \multicolumn{3}{|c|}{ Lab ID: $\quad 0804025-20$} & & & \\
\hline $7429-90-5$ & Aluminum & $<8.58 \mathrm{E}-2$ & ug/g dry & $8.58 \mathrm{E}-2$ & $9 / 24 / 08$ & 8I23005 & PNNL-AGG-ICP-AES \\
\hline $7440-39-3$ & Barium & $1.34 \mathrm{E}-1$ & ug/g dry & 8.79E-3 & $9 / 24 / 08$ & $8 \mathrm{I} 23005$ & PNNL-AGG-ICP-AES \\
\hline $7440-70-2$ & Calcium & $1.60 \mathrm{E} 2$ & ug/g dry & $3.87 \mathrm{E}-1$ & $9 / 24 / 08$ & $8 \mathrm{I} 23005$ & PNNL-AGG-ICP-AES \\
\hline 7439-89-6 & Iron & 4.74E-1 & ug/g dry & $1.42 \mathrm{E}-1$ & $9 / 24 / 08$ & $8 \mathrm{I} 23005$ & PNNL-AGG-ICP-AES \\
\hline 7440-09-7 & Potassium & $1.55 \mathrm{E} 1$ & ug/g dry & $2.33 \mathrm{E} 0$ & $9 / 24 / 08$ & $8 \mathrm{I} 23005$ & PNNL-AGG-ICP-AES \\
\hline 7439-95-4 & Magnesium & $5.54 \mathrm{E} 1$ & ug/g dry & 8.34E-2 & $9 / 24 / 08$ & $8 \mathrm{I} 23005$ & PNNL-AGG-ICP-AES \\
\hline 7439-96-5 & Manganese & $4.12 \mathrm{E}-2$ & ug/g dry & $1.71 \mathrm{E}-2$ & $9 / 24 / 08$ & $8 \mathrm{I} 23005$ & PNNL-AGG-ICP-AES \\
\hline $7440-02-0$ & Nickel & $<9.33 \mathrm{E}-2$ & ug/g dry & 9.33E-2 & $9 / 24 / 08$ & $8 I 23005$ & PNNL-AGG-ICP-AES \\
\hline $7440-28-0$ & Thallium & $<1.03 \mathrm{E} 0$ & ug/g dry & 1.03E0 & $9 / 24 / 08$ & $8 \mathrm{I} 23005$ & PNNL-AGG-ICP-AES \\
\hline $7440-23-5$ & Sodium & 5.31E1 & ug/g dry & $6.69 \mathrm{E}-1$ & $9 / 24 / 08$ & $8 \mathrm{I} 23005$ & PNNL-AGG-ICP-AES \\
\hline HEIS No. & B1TNW5 & \multicolumn{3}{|c|}{ Lab ID: $\quad 0804025-22$} & & & \\
\hline $7429-90-5$ & Aluminum & $<1.41 \mathrm{E}-1$ & ug/g dry & $1.41 \mathrm{E}-1$ & 7/30/08 & 8G22002 & PNNL-AGG-ICP-AES \\
\hline $7440-39-3$ & Barium & $1.03 \mathrm{E}-1$ & ug/g dry & $1.44 \mathrm{E}-2$ & $7 / 30 / 08$ & $8 G 22002$ & PNNL-AGG-ICP-AES \\
\hline $7440-70-2$ & Calcium & $2.12 \mathrm{E} 2$ & ug/g dry & 6.34E-1 & 7/30/08 & $8 G 22002$ & PNNL-AGG-ICP-AES \\
\hline 7439-89-6 & Iron & $1.19 \mathrm{E} 0$ & ug/g dry & 2.33E-1 & 7/30/08 & 8G22002 & PNNL-AGG-ICP-AES \\
\hline 7440-09-7 & Potassium & $1.71 \mathrm{E} 1$ & ug/g dry & $3.82 \mathrm{E} 0$ & $7 / 30 / 08$ & $8 G 22002$ & PNNL-AGG-ICP-AES \\
\hline 7439-95-4 & Magnesium & 6.35E1 & ug/g dry & $1.37 \mathrm{E}-1$ & 7/30/08 & 8G22002 & PNNL-AGG-ICP-AES \\
\hline 7439-96-5 & Manganese & $<2.81 \mathrm{E}-2$ & ug/g dry & $2.81 \mathrm{E}-2$ & 7/30/08 & $8 G 22002$ & PNNL-AGG-ICP-AES \\
\hline $7440-02-0$ & Nickel & $<1.53 \mathrm{E}-1$ & ug/g dry & $1.53 \mathrm{E}-1$ & $7 / 30 / 08$ & 8G22002 & PNNL-AGG-ICP-AES \\
\hline $7440-28-0$ & Thallium & $<1.68 \mathrm{E} 0$ & ug/g dry & $1.68 \mathrm{E} 0$ & 7/30/08 & $8 G 22002$ & PNNL-AGG-ICP-AES \\
\hline 7440-23-5 & Sodium & $2.40 \mathrm{E} 2$ & ug/g dry & $1.10 \mathrm{E} 0$ & $7 / 30 / 08$ & 8G22002 & PNNL-AGG-ICP-AES \\
\hline HEIS No. & B1TP35 & \multicolumn{3}{|c|}{ Lab ID: $\quad 0804025-37$} & & & \\
\hline $7429-90-5$ & Aluminum & $2.56 \mathrm{E}-1$ & ug/g dry & $1.54 \mathrm{E}-1$ & $7 / 30 / 08$ & 8G22002 & PNNL-AGG-ICP-AES \\
\hline $7440-39-3$ & Barium & 2.09E-2 & ug/g dry & $1.58 \mathrm{E}-2$ & 7/30/08 & 8G22002 & PNNL-AGG-ICP-AES \\
\hline $7440-70-2$ & Calcium & $1.66 \mathrm{E} 1$ & ug/g dry & 6.96E-1 & $7 / 30 / 08$ & $8 G 22002$ & PNNL-AGG-ICP-AES \\
\hline 7439-89-6 & Iron & $<2.56 \mathrm{E}-1$ & ug/g dry & $2.56 \mathrm{E}-1$ & $7 / 30 / 08$ & 8G22002 & PNNL-AGG-ICP-AES \\
\hline 7440-09-7 & Potassium & $6.76 \mathrm{E} 0$ & ug/g dry & $4.19 \mathrm{E} 0$ & $7 / 30 / 08$ & $8 G 22002$ & PNNL-AGG-ICP-AES \\
\hline 7439-95-4 & Magnesium & $5.27 \mathrm{E} 0$ & ug/g dry & $1.50 \mathrm{E}-1$ & $7 / 30 / 08$ & $8 G 22002$ & PNNL-AGG-ICP-AES \\
\hline 7439-96-5 & Manganese & $<3.08 \mathrm{E}-2$ & ug/g dry & $3.08 \mathrm{E}-2$ & 7/30/08 & 8G22002 & PNNL-AGG-ICP-AES \\
\hline $7440-02-0$ & Nickel & $<1.68 \mathrm{E}-1$ & ug/g dry & $1.68 \mathrm{E}-1$ & $7 / 30 / 08$ & $8 G 22002$ & PNNL-AGG-ICP-AES \\
\hline $7440-28-0$ & Thallium & $<1.85 \mathrm{E} 0$ & ug/g dry & $1.85 \mathrm{E} 0$ & $7 / 30 / 08$ & $8 G 22002$ & PNNL-AGG-ICP-AES \\
\hline $7440-23-5$ & Sodium & $1.52 \mathrm{E} 1$ & ug/g dry & $1.20 \mathrm{E} 0$ & 7/30/08 & 8G22002 & PNNL-AGG-ICP-AES \\
\hline HEIS No. & B1TP40 & \multicolumn{3}{|c|}{ Lab ID: $\quad 0804025-48$} & & & \\
\hline 7429-90-5 & Aluminum & $1.49 \mathrm{E}-1$ & ug/g dry & $1.43 \mathrm{E}-1$ & 7/30/08 & $8 G 22002$ & PNNL-AGG-ICP-AES \\
\hline $7440-39-3$ & Barium & $<1.47 \mathrm{E}-2$ & ug/g dry & $1.47 \mathrm{E}-2$ & $7 / 30 / 08$ & 8G22002 & PNNL-AGG-ICP-AES \\
\hline $7440-70-2$ & Calcium & $1.62 \mathrm{E} 1$ & ug/g dry & $6.46 \mathrm{E}-1$ & $7 / 30 / 08$ & $8 G 22002$ & PNNL-AGG-ICP-AES \\
\hline 7439-89-6 & Iron & $<2.37 \mathrm{E}-1$ & ug/g dry & 2.37E-1 & $7 / 30 / 08$ & 8G22002 & PNNL-AGG-ICP-AES \\
\hline $7440-09-7$ & Potassium & 4.11E0 & ug/g dry & $3.88 \mathrm{E} 0$ & $7 / 30 / 08$ & 8G22002 & PNNL-AGG-ICP-AES \\
\hline 7439-95-4 & Magnesium & 4.35E0 & ug/g dry & $1.39 \mathrm{E}-1$ & $7 / 30 / 08$ & $8 G 22002$ & PNNL-AGG-ICP-AES \\
\hline 7439-96-5 & Manganese & $<2.86 \mathrm{E}-2$ & ug/g dry & $2.86 \mathrm{E}-2$ & $7 / 30 / 08$ & $8 G 22002$ & PNNL-AGG-ICP-AES \\
\hline $7440-02-0$ & Nickel & $<1.56 \mathrm{E}-1$ & ug/g dry & $1.56 \mathrm{E}-1$ & $7 / 30 / 08$ & 8G22002 & PNNL-AGG-ICP-AES \\
\hline $7440-28-0$ & Thallium & $<1.71 \mathrm{E} 0$ & ug/g dry & $1.71 \mathrm{E} 0$ & 7/30/08 & 8G22002 & PNNL-AGG-ICP-AES \\
\hline 7440-23-5 & Sodium & $1.23 \mathrm{E} 1$ & ug/g dry & $1.12 \mathrm{E} 0$ & $7 / 30 / 08$ & $8 G 22002$ & PNNL-AGG-ICP-AES \\
\hline HEIS No. & B1TP42 & \multicolumn{3}{|c|}{ Lab ID: $\quad$ 0804025-50 } & & & \\
\hline $7429-90-5$ & Aluminum & $<8.59 \mathrm{E}-2$ & ug/g dry & 8.59E-2 & $9 / 24 / 08$ & $8 \mathrm{I} 23005$ & PNNL-AGG-ICP-AES \\
\hline
\end{tabular}


Total Metals by PNNL-AGG-ICP-AES/Water Extract

\begin{tabular}{|c|c|c|c|c|c|c|c|}
\hline CAS \# & Analyte & Results & Units & EQL & Analyzed & Batch & Method \\
\hline HEIS No. & B1TP42 & \multicolumn{3}{|c|}{ Lab ID: $\quad 0804025-50$} & & & \\
\hline $7440-39-3$ & Barium & $1.57 \mathrm{E}-2$ & ug/g dry & $8.80 \mathrm{E}-3$ & 9/24/08 & $8 \mathrm{I} 23005$ & PNNL-AGG-ICP-AES \\
\hline $7440-70-2$ & Calcium & $1.92 \mathrm{E} 1$ & ug/g dry & 3.87E-1 & 9/24/08 & $8 \mathrm{I} 23005$ & PNNL-AGG-ICP-AES \\
\hline 7439-89-6 & Iron & $<1.42 \mathrm{E}-1$ & ug/g dry & $1.42 \mathrm{E}-1$ & $9 / 24 / 08$ & $8 \mathrm{I} 23005$ & PNNL-AGG-ICP-AES \\
\hline 7440-09-7 & Potassium & 4.30E0 & ug/g dry & 2.33Е0 & $9 / 24 / 08$ & $8 \mathrm{I} 23005$ & PNNL-AGG-ICP-AES \\
\hline 7439-95-4 & Magnesium & 4.99E0 & ug/g dry & 8.35E-2 & $9 / 24 / 08$ & 8I23005 & PNNL-AGG-ICP-AES \\
\hline 7439-96-5 & Manganese & $<1.71 \mathrm{E}-2$ & ug/g dry & $1.71 \mathrm{E}-2$ & 9/24/08 & $8 I 23005$ & PNNL-AGG-ICP-AES \\
\hline $7440-02-0$ & Nickel & $<9.34 \mathrm{E}-2$ & ug/g dry & $9.34 \mathrm{E}-2$ & $9 / 24 / 08$ & $8 \mathrm{I} 23005$ & PNNL-AGG-ICP-AES \\
\hline $7440-28-0$ & Thallium & $<1.03 \mathrm{E} 0$ & ug/g dry & 1.03E0 & 9/24/08 & $8 \mathrm{I} 23005$ & PNNL-AGG-ICP-AES \\
\hline $7440-23-5$ & Sodium & $1.11 \mathrm{E} 2$ & ug/g dry & $6.70 \mathrm{E}-1$ & $9 / 24 / 08$ & $8 \mathrm{I} 23005$ & PNNL-AGG-ICP-AES \\
\hline HEIS No. & В1TP43 & \multicolumn{3}{|c|}{ Lab ID: $\quad$ 0804025-51 } & & & \\
\hline $7429-90-5$ & Aluminum & $<1.43 \mathrm{E}-1$ & ug/g dry & 1.43E-1 & 7/30/08 & $8 G 22002$ & PNNL-AGG-ICP-AES \\
\hline $7440-39-3$ & Barium & $2.79 \mathrm{E}-2$ & ug/g dry & $1.47 \mathrm{E}-2$ & 7/30/08 & 8G22002 & PNNL-AGG-ICP-AES \\
\hline $7440-70-2$ & Calcium & $1.71 \mathrm{E} 2$ & ug/g dry & $6.46 \mathrm{E}-1$ & 7/30/08 & 8G22002 & PNNL-AGG-ICP-AES \\
\hline 7439-89-6 & Iron & $1.96 \mathrm{E} 0$ & ug/g dry & $2.37 \mathrm{E}-1$ & 7/30/08 & 8G22002 & PNNL-AGG-ICP-AES \\
\hline 7440-09-7 & Potassium & 1.94E1 & ug/g dry & 3.89E0 & 7/30/08 & 8G22002 & PNNL-AGG-ICP-AES \\
\hline 7439-95-4 & Magnesium & 4.63E1 & ug/g dry & $1.39 \mathrm{E}-1$ & 7/30/08 & 8G22002 & PNNL-AGG-ICP-AES \\
\hline 7439-96-5 & Manganese & $<2.86 \mathrm{E}-2$ & ug/g dry & $2.86 \mathrm{E}-2$ & 7/30/08 & 8G22002 & PNNL-AGG-ICP-AES \\
\hline 7440-02-0 & Nickel & $<1.56 \mathrm{E}-1$ & ug/g dry & $1.56 \mathrm{E}-1$ & 7/30/08 & $8 G 22002$ & PNNL-AGG-ICP-AES \\
\hline $7440-28-0$ & Thallium & $<1.71 \mathrm{E} 0$ & ug/g dry & $1.71 \mathrm{E} 0$ & 7/30/08 & $8 G 22002$ & PNNL-AGG-ICP-AES \\
\hline $7440-23-5$ & Sodium & $1.47 \mathrm{E} 3$ & ug/g dry & $1.12 \mathrm{E} 0$ & 7/30/08 & 8G22002 & PNNL-AGG-ICP-AES \\
\hline HEIS No. & B1TP45 & \multicolumn{3}{|c|}{ Lab ID: $\quad 0804025-53$} & & & \\
\hline $7429-90-5$ & Aluminum & $2.97 \mathrm{E}-1$ & ug/g dry & $8.58 \mathrm{E}-2$ & $9 / 24 / 08$ & 8I23005 & PNNL-AGG-ICP-AES \\
\hline $7440-39-3$ & Barium & $1.63 \mathrm{E}-2$ & ug/g dry & 8.79E-3 & $9 / 24 / 08$ & $8 \mathrm{I} 23005$ & PNNL-AGG-ICP-AES \\
\hline $7440-70-2$ & Calcium & 5.23E0 & ug/g dry & 3.87E-1 & $9 / 24 / 08$ & $8 \mathrm{I} 23005$ & PNNL-AGG-ICP-AES \\
\hline 7439-89-6 & Iron & $4.94 \mathrm{E}-1$ & ug/g dry & $1.42 \mathrm{E}-1$ & 9/24/08 & $8 \mathrm{I} 23005$ & PNNL-AGG-ICP-AES \\
\hline 7440-09-7 & Potassium & 7.42E0 & ug/g dry & 2.33Е0 & $9 / 24 / 08$ & $8 \mathrm{I} 23005$ & PNNL-AGG-ICP-AES \\
\hline 7439-95-4 & Magnesium & 1.69E0 & ug/g dry & 8.34E-2 & $9 / 24 / 08$ & 8I23005 & PNNL-AGG-ICP-AES \\
\hline 7439-96-5 & Manganese & $<1.71 \mathrm{E}-2$ & ug/g dry & $1.71 \mathrm{E}-2$ & $9 / 24 / 08$ & 8I23005 & PNNL-AGG-ICP-AES \\
\hline $7440-02-0$ & Nickel & $<9.33 \mathrm{E}-2$ & ug/g dry & $9.33 \mathrm{E}-2$ & 9/24/08 & $8 \mathrm{I} 23005$ & PNNL-AGG-ICP-AES \\
\hline $7440-28-0$ & Thallium & $<1.03 \mathrm{E} 0$ & ug/g dry & $1.03 \mathrm{E} 0$ & $9 / 24 / 08$ & 8I23005 & PNNL-AGG-ICP-AES \\
\hline $7440-23-5$ & Sodium & 5.67E2 & ug/g dry & $6.69 \mathrm{E}-1$ & $9 / 24 / 08$ & $8 \mathrm{I} 23005$ & PNNL-AGG-ICP-AES \\
\hline HEIS No. & B1TP47 & \multicolumn{3}{|c|}{ Lab ID: $\quad 0804025-57$} & & & \\
\hline $7429-90-5$ & Aluminum & $<1.42 \mathrm{E}-1$ & ug/g dry & $1.42 \mathrm{E}-1$ & 7/30/08 & 8G22002 & PNNL-AGG-ICP-AES \\
\hline $7440-39-3$ & Barium & $2.37 \mathrm{E}-1$ & ug/g dry & $1.45 \mathrm{E}-2$ & 7/30/08 & 8G22002 & PNNL-AGG-ICP-AES \\
\hline $7440-70-2$ & Calcium & 3.79E2 & ug/g dry & $6.39 \mathrm{E}-1$ & $7 / 30 / 08$ & $8 G 22002$ & PNNL-AGG-ICP-AES \\
\hline 7439-89-6 & Iron & $7.84 \mathrm{E}-1$ & ug/g dry & $2.35 \mathrm{E}-1$ & 7/30/08 & $8 G 22002$ & PNNL-AGG-ICP-AES \\
\hline 7440-09-7 & Potassium & 2.33E1 & ug/g dry & 3.85E0 & 7/30/08 & 8G22002 & PNNL-AGG-ICP-AES \\
\hline 7439-95-4 & Magnesium & 8.80E1 & ug/g dry & $1.38 \mathrm{E}-1$ & 7/30/08 & 8G22002 & PNNL-AGG-ICP-AES \\
\hline 7439-96-5 & Manganese & $<2.83 \mathrm{E}-2$ & ug/g dry & $2.83 \mathrm{E}-2$ & 7/30/08 & $8 G 22002$ & PNNL-AGG-ICP-AES \\
\hline $7440-02-0$ & Nickel & $<1.54 \mathrm{E}-1$ & ug/g dry & $1.54 \mathrm{E}-1$ & 7/30/08 & 8G22002 & PNNL-AGG-ICP-AES \\
\hline $7440-28-0$ & Thallium & $<1.69 \mathrm{E} 0$ & ug/g dry & 1.69E0 & 7/30/08 & 8G22002 & PNNL-AGG-ICP-AES \\
\hline $7440-23-5$ & Sodium & $1.40 \mathrm{E} 2$ & ug/g dry & $1.11 \mathrm{E} 0$ & 7/30/08 & 8G22002 & PNNL-AGG-ICP-AES \\
\hline HEIS No. & В1ТР49 & \multicolumn{3}{|c|}{ Lab ID: $\quad 0804025-61$} & & & \\
\hline 7429-90-5 & Aluminum & $<1.43 \mathrm{E}-1$ & ug/g dry & 1.43E-1 & 7/30/08 & $8 G 22002$ & PNNL-AGG-ICP-AES \\
\hline $7440-39-3$ & Barium & $2.35 \mathrm{E}-1$ & ug/g dry & $1.47 \mathrm{E}-2$ & 7/30/08 & 8G22002 & PNNL-AGG-ICP-AES \\
\hline $7440-70-2$ & Calcium & 3.47E2 & ug/g dry & $6.46 \mathrm{E}-1$ & 7/30/08 & 8G22002 & PNNL-AGG-ICP-AES \\
\hline 7439-89-6 & Iron & $1.08 \mathrm{E} 0$ & ug/g dry & 2.37E-1 & 7/30/08 & $8 G 22002$ & PNNL-AGG-ICP-AES \\
\hline 7440-09-7 & Potassium & 1.98E1 & ug/g dry & 3.88E0 & 7/30/08 & 8G22002 & PNNL-AGG-ICP-AES \\
\hline
\end{tabular}


Total Metals by PNNL-AGG-ICP-AES/Water Extract

\begin{tabular}{|c|c|c|c|c|c|c|c|}
\hline CAS \# & Analyte & Results & Units & EQL & Analyzed & Batch & Method \\
\hline HEIS No. & B1TP49 & \multicolumn{3}{|c|}{ Lab ID: $\quad$ 0804025-61 } & & & \\
\hline 7439-95-4 & Magnesium & $9.76 \mathrm{E} 1$ & ug/g dry & $1.39 \mathrm{E}-1$ & 7/30/08 & 8G22002 & PNNL-AGG-ICP-AES \\
\hline 7439-96-5 & Manganese & 7.46E-2 & ug/g dry & $2.86 \mathrm{E}-2$ & 7/30/08 & $8 G 22002$ & PNNL-AGG-ICP-AES \\
\hline $7440-02-0$ & Nickel & $<1.56 \mathrm{E}-1$ & ug/g dry & $1.56 \mathrm{E}-1$ & 7/30/08 & $8 G 22002$ & PNNL-AGG-ICP-AES \\
\hline $7440-28-0$ & Thallium & $<1.71 \mathrm{E} 0$ & ug/g dry & $1.71 \mathrm{E} 0$ & 7/30/08 & 8G22002 & PNNL-AGG-ICP-AES \\
\hline $7440-23-5$ & Sodium & 2.97E1 & ug/g dry & $1.12 \mathrm{E} 0$ & 7/30/08 & $8 G 22002$ & PNNL-AGG-ICP-AES \\
\hline HEIS No. & B1TP51 & \multicolumn{3}{|c|}{ Lab ID: $\quad$ 0804025-69 } & & & \\
\hline $7429-90-5$ & Aluminum & $<1.41 \mathrm{E}-1$ & ug/g dry & $1.41 \mathrm{E}-1$ & 7/30/08 & 8G22002 & PNNL-AGG-ICP-AES \\
\hline $7440-39-3$ & Barium & $1.85 \mathrm{E}-1$ & ug/g dry & $1.44 \mathrm{E}-2$ & 7/30/08 & $8 G 22002$ & PNNL-AGG-ICP-AES \\
\hline $7440-70-2$ & Calcium & 6.07E2 & ug/g dry & $6.35 \mathrm{E}-1$ & 7/30/08 & $8 G 22002$ & PNNL-AGG-ICP-AES \\
\hline 7439-89-6 & Iron & 2.87E0 & ug/g dry & $2.33 \mathrm{E}-1$ & 7/30/08 & $8 G 22002$ & PNNL-AGG-ICP-AES \\
\hline $7440-09-7$ & Potassium & 4.77E1 & ug/g dry & 3.82E0 & 7/30/08 & $8 G 22002$ & PNNL-AGG-ICP-AES \\
\hline 7439-95-4 & Magnesium & $1.80 \mathrm{E} 2$ & ug/g dry & $2.74 \mathrm{E} 0$ & 7/30/08 & $8 G 22002$ & PNNL-AGG-ICP-AES \\
\hline 7439-96-5 & Manganese & $2.94 \mathrm{E}-1$ & ug/g dry & $2.81 \mathrm{E}-2$ & 7/30/08 & $8 G 22002$ & PNNL-AGG-ICP-AES \\
\hline $7440-02-0$ & Nickel & $<1.53 \mathrm{E}-1$ & ug/g dry & $1.53 \mathrm{E}-1$ & 7/30/08 & $8 G 22002$ & PNNL-AGG-ICP-AES \\
\hline $7440-28-0$ & Thallium & $<1.68 \mathrm{E} 0$ & ug/g dry & $1.68 \mathrm{E} 0$ & 7/30/08 & $8 G 22002$ & PNNL-AGG-ICP-AES \\
\hline $7440-23-5$ & Sodium & $1.48 \mathrm{E} 3$ & ug/g dry & 1.10E0 & 7/30/08 & 8G22002 & PNNL-AGG-ICP-AES \\
\hline HEIS No. & B1TP52 & \multicolumn{3}{|c|}{ Lab ID: $\quad 0804025-72$} & & & \\
\hline $7429-90-5$ & Aluminum & $<1.42 \mathrm{E}-1$ & ug/g dry & $1.42 \mathrm{E}-1$ & 7/30/08 & $8 G 22002$ & PNNL-AGG-ICP-AES \\
\hline $7440-39-3$ & Barium & 7.63E-2 & ug/g dry & $1.46 \mathrm{E}-2$ & 7/30/08 & $8 G 22002$ & PNNL-AGG-ICP-AES \\
\hline $7440-70-2$ & Calcium & 7.04E1 & ug/g dry & $6.41 \mathrm{E}-1$ & 7/30/08 & $8 G 22002$ & PNNL-AGG-ICP-AES \\
\hline 7439-89-6 & Iron & $3.64 \mathrm{E}-1$ & ug/g dry & $2.36 \mathrm{E}-1$ & 7/30/08 & $8 G 22002$ & PNNL-AGG-ICP-AES \\
\hline 7440-09-7 & Potassium & $1.69 \mathrm{E} 1$ & ug/g dry & $3.86 \mathrm{E} 0$ & 7/30/08 & $8 G 22002$ & PNNL-AGG-ICP-AES \\
\hline 7439-95-4 & Magnesium & 2.39E1 & ug/g dry & $1.38 \mathrm{E}-1$ & 7/30/08 & $8 G 22002$ & PNNL-AGG-ICP-AES \\
\hline $7439-96-5$ & Manganese & $9.25 \mathrm{E}-2$ & ug/g dry & $2.84 \mathrm{E}-2$ & 7/30/08 & $8 G 22002$ & PNNL-AGG-ICP-AES \\
\hline $7440-02-0$ & Nickel & $<1.55 \mathrm{E}-1$ & ug/g dry & $1.55 \mathrm{E}-1$ & 7/30/08 & $8 G 22002$ & PNNL-AGG-ICP-AES \\
\hline $7440-28-0$ & Thallium & $<1.70 \mathrm{E} 0$ & ug/g dry & $1.70 \mathrm{E} 0$ & 7/30/08 & 8G22002 & PNNL-AGG-ICP-AES \\
\hline $7440-23-5$ & Sodium & 3.38E2 & ug/g dry & $1.11 \mathrm{E} 0$ & 7/30/08 & 8G22002 & PNNL-AGG-ICP-AES \\
\hline HEIS No. & B1TP53 & \multicolumn{3}{|c|}{ Lab ID: $\quad 0804025-75$} & & & \\
\hline $7429-90-5$ & Aluminum & $<1.43 \mathrm{E}-1$ & ug/g dry & $1.43 \mathrm{E}-1$ & 7/30/08 & 8G22002 & PNNL-AGG-ICP-AES \\
\hline $7440-39-3$ & Barium & $2.46 \mathrm{E}-1$ & ug/g dry & $1.47 \mathrm{E}-2$ & 7/30/08 & $8 G 22002$ & PNNL-AGG-ICP-AES \\
\hline $7440-70-2$ & Calcium & 3.43E2 & ug/g dry & $6.46 \mathrm{E}-1$ & 7/30/08 & $8 G 22002$ & PNNL-AGG-ICP-AES \\
\hline 7439-89-6 & Iron & 7.39E-1 & ug/g dry & $2.37 \mathrm{E}-1$ & 7/30/08 & $8 G 22002$ & PNNL-AGG-ICP-AES \\
\hline $7440-09-7$ & Potassium & $2.04 \mathrm{E} 1$ & ug/g dry & 3.89E0 & 7/30/08 & $8 G 22002$ & PNNL-AGG-ICP-AES \\
\hline 7439-95-4 & Magnesium & 7.48E1 & ug/g dry & $1.39 \mathrm{E}-1$ & 7/30/08 & $8 G 22002$ & PNNL-AGG-ICP-AES \\
\hline $7439-96-5$ & Manganese & $<2.86 \mathrm{E}-2$ & ug/g dry & $2.86 \mathrm{E}-2$ & 7/30/08 & $8 G 22002$ & PNNL-AGG-ICP-AES \\
\hline $7440-02-0$ & Nickel & $<1.56 \mathrm{E}-1$ & ug/g dry & $1.56 \mathrm{E}-1$ & 7/30/08 & $8 G 22002$ & PNNL-AGG-ICP-AES \\
\hline $7440-28-0$ & Thallium & $<1.71 \mathrm{E} 0$ & ug/g dry & $1.71 \mathrm{E} 0$ & 7/30/08 & 8G22002 & PNNL-AGG-ICP-AES \\
\hline $7440-23-5$ & Sodium & 9.63E1 & ug/g dry & 1.12E0 & 7/30/08 & 8G22002 & PNNL-AGG-ICP-AES \\
\hline HEIS No. & B1TP55 & \multicolumn{3}{|c|}{ Lab ID: $\quad$ 0804025-81 } & & & \\
\hline $7429-90-5$ & Aluminum & $<1.43 \mathrm{E}-1$ & ug/g dry & $1.43 \mathrm{E}-1$ & 7/30/08 & $8 G 22002$ & PNNL-AGG-ICP-AES \\
\hline $7440-39-3$ & Barium & $4.64 \mathrm{E}-2$ & ug/g dry & $1.47 \mathrm{E}-2$ & 7/30/08 & 8G22002 & PNNL-AGG-ICP-AES \\
\hline $7440-70-2$ & Calcium & 4.37E1 & ug/g dry & $6.47 \mathrm{E}-1$ & 7/30/08 & $8 G 22002$ & PNNL-AGG-ICP-AES \\
\hline 7439-89-6 & Iron & $<2.38 \mathrm{E}-1$ & ug/g dry & $2.38 \mathrm{E}-1$ & 7/30/08 & $8 G 22002$ & PNNL-AGG-ICP-AES \\
\hline 7440-09-7 & Potassium & $9.42 \mathrm{E} 0$ & ug/g dry & 3.89E0 & 7/30/08 & $8 G 22002$ & PNNL-AGG-ICP-AES \\
\hline 7439-95-4 & Magnesium & 1.39E1 & ug/g dry & $1.39 \mathrm{E}-1$ & 7/30/08 & $8 G 22002$ & PNNL-AGG-ICP-AES \\
\hline 7439-96-5 & Manganese & $<2.86 \mathrm{E}-2$ & ug/g dry & $2.86 \mathrm{E}-2$ & 7/30/08 & $8 G 22002$ & PNNL-AGG-ICP-AES \\
\hline $7440-02-0$ & Nickel & $<1.56 \mathrm{E}-1$ & ug/g dry & $1.56 \mathrm{E}-1$ & 7/30/08 & $8 G 22002$ & PNNL-AGG-ICP-AES \\
\hline $7440-28-0$ & Thallium & $<1.71 \mathrm{E} 0$ & ug/g dry & $1.71 \mathrm{E} 0$ & 7/30/08 & $8 G 22002$ & PNNL-AGG-ICP-AES \\
\hline
\end{tabular}


Total Metals by PNNL-AGG-ICP-AES/Water Extract

\begin{tabular}{|c|c|c|c|c|c|c|c|}
\hline CAS \# & Analyte & Results & Units & EQL & Analyzed & Batch & Method \\
\hline HEIS No. & B1TP55 & \multicolumn{3}{|c|}{ Lab ID: $\quad$ 0804025-81 } & & & \\
\hline $7440-23-5$ & Sodium & $1.78 \mathrm{E} 1$ & ug/g dry & $1.12 \mathrm{E} 0$ & 7/30/08 & $8 G 22002$ & PNNL-AGG-ICP-AES \\
\hline HEIS No. & B1TP59 & \multicolumn{3}{|c|}{ Lab ID: $\quad$ 0804025-87 } & & & \\
\hline 7429-90-5 & Aluminum & 3.05E-1 & ug/g dry & 1.45E-1 & 7/30/08 & 8G22002 & PNNL-AGG-ICP-AES \\
\hline $7440-39-3$ & Barium & $1.57 \mathrm{E}-2$ & ug/g dry & $1.48 \mathrm{E}-2$ & 7/30/08 & 8G22002 & PNNL-AGG-ICP-AES \\
\hline $7440-70-2$ & Calcium & $1.14 \mathrm{E} 1$ & ug/g dry & $6.54 \mathrm{E}-1$ & 7/30/08 & 8G22002 & PNNL-AGG-ICP-AES \\
\hline 7439-89-6 & Iron & $<2.40 \mathrm{E}-1$ & ug/g dry & $2.40 \mathrm{E}-1$ & $7 / 30 / 08$ & 8G22002 & PNNL-AGG-ICP-AES \\
\hline 7440-09-7 & Potassium & 4.76E0 & ug/g dry & 3.93E0 & 7/30/08 & 8G22002 & PNNL-AGG-ICP-AES \\
\hline 7439-95-4 & Magnesium & 3.39E0 & ug/g dry & $1.41 \mathrm{E}-1$ & 7/30/08 & 8G22002 & PNNL-AGG-ICP-AES \\
\hline 7439-96-5 & Manganese & $<2.89 \mathrm{E}-2$ & ug/g dry & 2.89E-2 & 7/30/08 & 8G22002 & PNNL-AGG-ICP-AES \\
\hline $7440-02-0$ & Nickel & $<1.58 \mathrm{E}-1$ & ug/g dry & 1.58E-1 & 7/30/08 & 8G22002 & PNNL-AGG-ICP-AES \\
\hline $7440-28-0$ & Thallium & $<1.73 \mathrm{E} 0$ & ug/g dry & $1.73 \mathrm{E} 0$ & 7/30/08 & $8 G 22002$ & PNNL-AGG-ICP-AES \\
\hline $7440-23-5$ & Sodium & $1.18 \mathrm{E} 1$ & ug/g dry & 1.13E0 & 7/30/08 & 8G22002 & PNNL-AGG-ICP-AES \\
\hline HEIS No. & B1TP61 & \multicolumn{3}{|c|}{ Lab ID: $\quad$ 0804025-91 } & & & \\
\hline 7429-90-5 & Aluminum & $3.74 \mathrm{E}-1$ & ug/g dry & 8.60E-2 & $9 / 24 / 08$ & $8 I 23005$ & PNNL-AGG-ICP-AES \\
\hline $7440-39-3$ & Barium & $1.28 \mathrm{E}-2$ & ug/g dry & 8.81E-3 & $9 / 24 / 08$ & $8 I 23005$ & PNNL-AGG-ICP-AES \\
\hline $7440-70-2$ & Calcium & 1.19E1 & ug/g dry & 3.88E-1 & $9 / 24 / 08$ & $8 \mathrm{I} 23005$ & PNNL-AGG-ICP-AES \\
\hline 7439-89-6 & Iron & $<1.42 \mathrm{E}-1$ & ug/g dry & $1.42 \mathrm{E}-1$ & $9 / 24 / 08$ & 8123005 & PNNL-AGG-ICP-AES \\
\hline 7440-09-7 & Potassium & 4.16E0 & ug/g dry & 2.33Е0 & $9 / 24 / 08$ & $8 \mathrm{I} 23005$ & PNNL-AGG-ICP-AES \\
\hline 7439-95-4 & Magnesium & 3.53E0 & ug/g dry & 8.35E-2 & 9/24/08 & $8 I 23005$ & PNNL-AGG-ICP-AES \\
\hline 7439-96-5 & Manganese & $<1.72 \mathrm{E}-2$ & ug/g dry & $1.72 \mathrm{E}-2$ & $9 / 24 / 08$ & 8I23005 & PNNL-AGG-ICP-AES \\
\hline $7440-02-0$ & Nickel & $<9.35 \mathrm{E}-2$ & ug/g dry & 9.35E-2 & $9 / 24 / 08$ & $8 \mathrm{I} 23005$ & PNNL-AGG-ICP-AES \\
\hline $7440-28-0$ & Thallium & $<1.03 \mathrm{E} 0$ & ug/g dry & $1.03 \mathrm{E} 0$ & $9 / 24 / 08$ & 8I23005 & PNNL-AGG-ICP-AES \\
\hline $7440-23-5$ & Sodium & $1.14 \mathrm{E} 1$ & ug/g dry & $6.70 \mathrm{E}-1$ & $9 / 24 / 08$ & 8I23005 & PNNL-AGG-ICP-AES \\
\hline HEIS No. & B1TP62 & \multicolumn{3}{|c|}{ Lab ID: $\quad$ 0804025-94 } & & & \\
\hline 7429-90-5 & Aluminum & $<1.41 \mathrm{E}-1$ & ug/g dry & $1.41 \mathrm{E}-1$ & 7/30/08 & 8G22002 & PNNL-AGG-ICP-AES \\
\hline $7440-39-3$ & Barium & $7.81 \mathrm{E}-2$ & ug/g dry & $1.45 \mathrm{E}-2$ & 7/30/08 & 8G22002 & PNNL-AGG-ICP-AES \\
\hline $7440-70-2$ & Calcium & 4.89E1 & ug/g dry & 6.37E-1 & $7 / 30 / 08$ & $8 G 22002$ & PNNL-AGG-ICP-AES \\
\hline 7439-89-6 & Iron & $3.06 \mathrm{E}-1$ & ug/g dry & $2.34 \mathrm{E}-1$ & 7/30/08 & 8G22002 & PNNL-AGG-ICP-AES \\
\hline 7440-09-7 & Potassium & 1.45E1 & ug/g dry & 3.83E0 & $7 / 30 / 08$ & $8 G 22002$ & PNNL-AGG-ICP-AES \\
\hline 7439-95-4 & Magnesium & 1.43E1 & ug/g dry & 1.37E-1 & 7/30/08 & 8G22002 & PNNL-AGG-ICP-AES \\
\hline 7439-96-5 & Manganese & $<2.82 \mathrm{E}-2$ & ug/g dry & 2.82E-2 & 7/30/08 & $8 G 22002$ & PNNL-AGG-ICP-AES \\
\hline $7440-02-0$ & Nickel & $<1.54 \mathrm{E}-1$ & ug/g dry & $1.54 \mathrm{E}-1$ & $7 / 30 / 08$ & 8G22002 & PNNL-AGG-ICP-AES \\
\hline $7440-28-0$ & Thallium & $<1.69 \mathrm{E} 0$ & ug/g dry & 1.69E0 & 7/30/08 & 8G22002 & PNNL-AGG-ICP-AES \\
\hline $7440-23-5$ & Sodium & 2.53E2 & ug/g dry & $1.10 \mathrm{E} 0$ & 7/30/08 & $8 G 22002$ & PNNL-AGG-ICP-AES \\
\hline HEIS No. & В1TP64 & \multicolumn{3}{|c|}{ Lab ID: $\quad$ 0804025-AA } & & & \\
\hline 7429-90-5 & Aluminum & $<8.60 \mathrm{E}-2$ & ug/g dry & 8.60E-2 & $9 / 24 / 08$ & 8I23005 & PNNL-AGG-ICP-AES \\
\hline 7440-39-3 & Barium & $1.48 \mathrm{E}-1$ & ug/g dry & 8.81E-3 & $9 / 24 / 08$ & 8I23005 & PNNL-AGG-ICP-AES \\
\hline $7440-70-2$ & Calcium & 2.39E2 & ug/g dry & 3.88E-1 & $9 / 24 / 08$ & $8 I 23005$ & PNNL-AGG-ICP-AES \\
\hline 7439-89-6 & Iron & 5.03E-1 & ug/g dry & $1.43 \mathrm{E}-1$ & 9/24/08 & $8 \mathrm{I} 23005$ & PNNL-AGG-ICP-AES \\
\hline 7440-09-7 & Potassium & $1.61 \mathrm{E} 1$ & ug/g dry & 2.33E0 & 9/24/08 & $8 \mathrm{I} 23005$ & PNNL-AGG-ICP-AES \\
\hline 7439-95-4 & Magnesium & 5.73E1 & ug/g dry & 8.36E-2 & $9 / 24 / 08$ & $8 \mathrm{I} 23005$ & PNNL-AGG-ICP-AES \\
\hline 7439-96-5 & Manganese & $1.93 \mathrm{E}-2$ & ug/g dry & $1.72 \mathrm{E}-2$ & 9/24/08 & $8 I 23005$ & PNNL-AGG-ICP-AES \\
\hline 7440-02-0 & Nickel & $<9.36 \mathrm{E}-2$ & ug/g dry & 9.36E-2 & $9 / 24 / 08$ & 8I23005 & PNNL-AGG-ICP-AES \\
\hline $7440-28-0$ & Thallium & $<1.03 \mathrm{E} 0$ & ug/g dry & $1.03 \mathrm{E} 0$ & $9 / 24 / 08$ & $8 \mathrm{I} 23005$ & PNNL-AGG-ICP-AES \\
\hline $7440-23-5$ & Sodium & $2.56 \mathrm{E} 1$ & ug/g dry & $6.71 \mathrm{E}-1$ & $9 / 24 / 08$ & $8 \mathrm{I} 23005$ & PNNL-AGG-ICP-AES \\
\hline HEIS No. & B1TP67 & \multicolumn{3}{|c|}{ Lab ID: $\quad$ 0804025-AI } & & & \\
\hline 7429-90-5 & Aluminum & 3.07E-1 & ug/g dry & $1.40 \mathrm{E}-1$ & 7/30/08 & 8G22002 & PNNL-AGG-ICP-AES \\
\hline $7440-39-3$ & Barium & $1.78 \mathrm{E}-2$ & ug/g dry & $1.43 \mathrm{E}-2$ & 7/30/08 & 8G22002 & PNNL-AGG-ICP-AES \\
\hline
\end{tabular}


Total Metals by PNNL-AGG-ICP-AES/Water Extract

\begin{tabular}{|c|c|c|c|c|c|c|c|}
\hline CAS \# & Analyte & Results & Units & EQL & Analyzed & Batch & Method \\
\hline HEIS No. & В1TP67 & \multicolumn{3}{|c|}{ Lab ID: $\quad$ 0804025-AI } & & & \\
\hline $7440-70-2$ & Calcium & $1.88 \mathrm{E} 1$ & ug/g dry & 6.31E-1 & 7/30/08 & 8G22002 & PNNL-AGG-ICP-AES \\
\hline 7439-89-6 & Iron & $<2.32 \mathrm{E}-1$ & ug/g dry & 2.32E-1 & 7/30/08 & 8G22002 & PNNL-AGG-ICP-AES \\
\hline 7440-09-7 & Potassium & 7.33E0 & ug/g dry & 3.79E0 & 7/30/08 & $8 G 22002$ & PNNL-AGG-ICP-AES \\
\hline 7439-95-4 & Magnesium & $5.22 \mathrm{E} 0$ & ug/g dry & 1.36E-1 & 7/30/08 & 8G22002 & PNNL-AGG-ICP-AES \\
\hline $7439-96-5$ & Manganese & $<2.79 \mathrm{E}-2$ & ug/g dry & 2.79E-2 & 7/30/08 & 8G22002 & PNNL-AGG-ICP-AES \\
\hline $7440-02-0$ & Nickel & $<1.52 \mathrm{E}-1$ & ug/g dry & $1.52 \mathrm{E}-1$ & 7/30/08 & $8 G 22002$ & PNNL-AGG-ICP-AES \\
\hline $7440-28-0$ & Thallium & $<1.67 \mathrm{E} 0$ & ug/g dry & $1.67 \mathrm{E} 0$ & 7/30/08 & $8 G 22002$ & PNNL-AGG-ICP-AES \\
\hline $7440-23-5$ & Sodium & 1.67E1 & ug/g dry & 1.09E0 & 7/30/08 & 8G22002 & PNNL-AGG-ICP-AES \\
\hline HEIS No. & В1TP69 & \multicolumn{3}{|c|}{ Lab ID: $\quad$ 0804025-AP } & & & \\
\hline 7429-90-5 & Aluminum & 4.73E-1 & ug/g dry & 8.59E-2 & 9/24/08 & $8 \mathrm{I} 23005$ & PNNL-AGG-ICP-AES \\
\hline $7440-39-3$ & Barium & $1.84 \mathrm{E}-2$ & ug/g dry & 8.80E-3 & $9 / 24 / 08$ & 8I23005 & PNNL-AGG-ICP-AES \\
\hline $7440-70-2$ & Calcium & 1.32E1 & ug/g dry & 3.88E-1 & $9 / 24 / 08$ & $8 \mathrm{I} 23005$ & PNNL-AGG-ICP-AES \\
\hline 7439-89-6 & Iron & $1.51 \mathrm{E}-1$ & ug/g dry & $1.42 \mathrm{E}-1$ & $9 / 24 / 08$ & 8I23005 & PNNL-AGG-ICP-AES \\
\hline $7440-09-7$ & Potassium & 7.30E0 & ug/g dry & 2.33E0 & $9 / 24 / 08$ & 8I23005 & PNNL-AGG-ICP-AES \\
\hline 7439-95-4 & Magnesium & 3.70E0 & ug/g dry & 8.35E-2 & $9 / 24 / 08$ & 8I23005 & PNNL-AGG-ICP-AES \\
\hline 7439-96-5 & Manganese & $<1.72 \mathrm{E}-2$ & ug/g dry & $1.72 \mathrm{E}-2$ & $9 / 24 / 08$ & 8I23005 & PNNL-AGG-ICP-AES \\
\hline $7440-02-0$ & Nickel & $<9.34 \mathrm{E}-2$ & ug/g dry & $9.34 \mathrm{E}-2$ & $9 / 24 / 08$ & 8I23005 & PNNL-AGG-ICP-AES \\
\hline $7440-28-0$ & Thallium & $<1.03 \mathrm{E} 0$ & ug/g dry & 1.03E0 & $9 / 24 / 08$ & 8I23005 & PNNL-AGG-ICP-AES \\
\hline $7440-23-5$ & Sodium & $1.81 \mathrm{E} 1$ & ug/g dry & $6.70 \mathrm{E}-1$ & $9 / 24 / 08$ & 8I23005 & PNNL-AGG-ICP-AES \\
\hline HEIS No. & B1TP71 & \multicolumn{3}{|c|}{ Lab ID: $\quad$ 0804025-AV } & & & \\
\hline $7429-90-5$ & Aluminum & $2.00 \mathrm{E}-1$ & ug/g dry & $1.43 \mathrm{E}-1$ & 7/30/08 & $8 G 22002$ & PNNL-AGG-ICP-AES \\
\hline $7440-39-3$ & Barium & $<1.46 \mathrm{E}-2$ & ug/g dry & $1.46 \mathrm{E}-2$ & 7/30/08 & 8G22002 & PNNL-AGG-ICP-AES \\
\hline $7440-70-2$ & Calcium & $1.23 \mathrm{E} 1$ & ug/g dry & $6.43 \mathrm{E}-1$ & 7/30/08 & $8 G 22002$ & PNNL-AGG-ICP-AES \\
\hline 7439-89-6 & Iron & $<2.36 \mathrm{E}-1$ & ug/g dry & 2.36E-1 & 7/30/08 & 8G22002 & PNNL-AGG-ICP-AES \\
\hline 7440-09-7 & Potassium & 5.64E0 & ug/g dry & 3.87E0 & 7/30/08 & $8 G 22002$ & PNNL-AGG-ICP-AES \\
\hline 7439-95-4 & Magnesium & 3.37E0 & ug/g dry & $1.39 \mathrm{E}-1$ & 7/30/08 & 8G22002 & PNNL-AGG-ICP-AES \\
\hline $7439-96-5$ & Manganese & $<2.85 \mathrm{E}-2$ & ug/g dry & 2.85E-2 & 7/30/08 & $8 G 22002$ & PNNL-AGG-ICP-AES \\
\hline $7440-02-0$ & Nickel & $<1.55 \mathrm{E}-1$ & ug/g dry & $1.55 \mathrm{E}-1$ & 7/30/08 & $8 G 22002$ & PNNL-AGG-ICP-AES \\
\hline $7440-28-0$ & Thallium & $<1.70 \mathrm{E} 0$ & ug/g dry & $1.70 \mathrm{E} 0$ & 7/30/08 & $8 G 22002$ & PNNL-AGG-ICP-AES \\
\hline $7440-23-5$ & Sodium & $1.38 \mathrm{E} 1$ & ug/g dry & $1.11 \mathrm{E} 0$ & 7/30/08 & $8 G 22002$ & PNNL-AGG-ICP-AES \\
\hline HEIS No. & B1TP77 & \multicolumn{3}{|c|}{ Lab ID: $\quad$ 0804025-BK } & & & \\
\hline $7429-90-5$ & Aluminum & $2.34 \mathrm{E}-1$ & ug/g dry & $1.43 \mathrm{E}-1$ & 7/30/08 & $8 G 22002$ & PNNL-AGG-ICP-AES \\
\hline $7440-39-3$ & Barium & $2.42 \mathrm{E}-2$ & ug/g dry & $1.46 \mathrm{E}-2$ & 7/30/08 & $8 G 22002$ & PNNL-AGG-ICP-AES \\
\hline $7440-70-2$ & Calcium & $1.14 \mathrm{E} 1$ & ug/g dry & $6.44 \mathrm{E}-1$ & 7/30/08 & 8G22002 & PNNL-AGG-ICP-AES \\
\hline 7439-89-6 & Iron & $<2.37 \mathrm{E}-1$ & ug/g dry & $2.37 \mathrm{E}-1$ & 7/30/08 & $8 G 22002$ & PNNL-AGG-ICP-AES \\
\hline $7440-09-7$ & Potassium & 5.33E0 & ug/g dry & $3.88 \mathrm{E} 0$ & 7/30/08 & $8 G 22002$ & PNNL-AGG-ICP-AES \\
\hline 7439-95-4 & Magnesium & 3.03E0 & ug/g dry & 1.39E-1 & 7/30/08 & $8 G 22002$ & PNNL-AGG-ICP-AES \\
\hline 7439-96-5 & Manganese & $<2.85 \mathrm{E}-2$ & ug/g dry & 2.85E-2 & 7/30/08 & 8G22002 & PNNL-AGG-ICP-AES \\
\hline $7440-02-0$ & Nickel & $<1.55 \mathrm{E}-1$ & ug/g dry & $1.55 \mathrm{E}-1$ & 7/30/08 & $8 G 22002$ & PNNL-AGG-ICP-AES \\
\hline $7440-28-0$ & Thallium & $<1.71 \mathrm{E} 0$ & ug/g dry & $1.71 \mathrm{E} 0$ & 7/30/08 & 8G22002 & PNNL-AGG-ICP-AES \\
\hline $7440-23-5$ & Sodium & $1.28 \mathrm{E} 1$ & ug/g dry & $1.11 \mathrm{E} 0$ & 7/30/08 & $8 G 22002$ & PNNL-AGG-ICP-AES \\
\hline HEIS No. & B1TP78 & \multicolumn{3}{|c|}{ Lab ID: $\quad$ 0804025-BP } & & & \\
\hline $7429-90-5$ & Aluminum & 3.83E-1 & ug/g dry & $1.43 \mathrm{E}-1$ & 7/30/08 & $8 G 22002$ & PNNL-AGG-ICP-AES \\
\hline $7440-39-3$ & Barium & $1.89 \mathrm{E}-2$ & ug/g dry & $1.47 \mathrm{E}-2$ & 7/30/08 & $8 G 22002$ & PNNL-AGG-ICP-AES \\
\hline $7440-70-2$ & Calcium & $1.22 \mathrm{E} 1$ & ug/g dry & $6.45 \mathrm{E}-1$ & 7/30/08 & 8G22002 & PNNL-AGG-ICP-AES \\
\hline 7439-89-6 & Iron & $<2.37 \mathrm{E}-1$ & ug/g dry & 2.37E-1 & 7/30/08 & $8 G 22002$ & PNNL-AGG-ICP-AES \\
\hline 7440-09-7 & Potassium & $5.06 \mathrm{E} 0$ & ug/g dry & $3.88 \mathrm{E} 0$ & 7/30/08 & $8 G 22002$ & PNNL-AGG-ICP-AES \\
\hline 7439-95-4 & Magnesium & 2.96E0 & ug/g dry & 1.39E-1 & 7/30/08 & $8 G 22002$ & PNNL-AGG-ICP-AES \\
\hline
\end{tabular}


Total Metals by PNNL-AGG-ICP-AES/Water Extract

\begin{tabular}{|c|c|c|c|c|c|c|c|}
\hline CAS \# & Analyte & Results & Units & EQL & Analyzed & Batch & Method \\
\hline HEIS No. & B1TP78 & \multicolumn{3}{|c|}{ Lab ID: $\quad$ 0804025-BP } & & & \\
\hline 7439-96-5 & Manganese & $<2.86 \mathrm{E}-2$ & ug/g dry & $2.86 \mathrm{E}-2$ & 7/30/08 & 8G22002 & PNNL-AGG-ICP-AES \\
\hline $7440-02-0$ & Nickel & $<1.56 \mathrm{E}-1$ & ug/g dry & $1.56 \mathrm{E}-1$ & 7/30/08 & $8 G 22002$ & PNNL-AGG-ICP-AES \\
\hline $7440-28-0$ & Thallium & $<1.71 \mathrm{E} 0$ & ug/g dry & $1.71 \mathrm{E} 0$ & 7/30/08 & $8 G 22002$ & PNNL-AGG-ICP-AES \\
\hline $7440-23-5$ & Sodium & $1.18 \mathrm{E} 1$ & ug/g dry & $1.12 \mathrm{E} 0$ & 7/30/08 & 8G22002 & PNNL-AGG-ICP-AES \\
\hline HEIS No. & B1TP82 & \multicolumn{3}{|c|}{ Lab ID: $\quad$ 0804025-BZ } & & & \\
\hline $7429-90-5$ & Aluminum & $<1.43 \mathrm{E}-1$ & ug/g dry & $1.43 \mathrm{E}-1$ & 7/30/08 & $8 G 22002$ & PNNL-AGG-ICP-AES \\
\hline $7440-39-3$ & Barium & $3.00 \mathrm{E}-2$ & ug/g dry & $1.46 \mathrm{E}-2$ & 7/30/08 & 8G22002 & PNNL-AGG-ICP-AES \\
\hline $7440-70-2$ & Calcium & $4.88 \mathrm{E} 1$ & ug/g dry & 6.43E-1 & 7/30/08 & $8 G 22002$ & PNNL-AGG-ICP-AES \\
\hline 7439-89-6 & Iron & $<2.36 \mathrm{E}-1$ & ug/g dry & $2.36 \mathrm{E}-1$ & 7/30/08 & $8 G 22002$ & PNNL-AGG-ICP-AES \\
\hline 7440-09-7 & Potassium & $1.12 \mathrm{E} 1$ & ug/g dry & 3.87E0 & 7/30/08 & $8 G 22002$ & PNNL-AGG-ICP-AES \\
\hline $7439-95-4$ & Magnesium & $1.10 \mathrm{E} 1$ & ug/g dry & $1.39 \mathrm{E}-1$ & 7/30/08 & $8 G 22002$ & PNNL-AGG-ICP-AES \\
\hline 7439-96-5 & Manganese & $<2.85 \mathrm{E}-2$ & ug/g dry & $2.85 \mathrm{E}-2$ & 7/30/08 & $8 G 22002$ & PNNL-AGG-ICP-AES \\
\hline $7440-02-0$ & Nickel & $<1.55 \mathrm{E}-1$ & ug/g dry & $1.55 \mathrm{E}-1$ & 7/30/08 & $8 G 22002$ & PNNL-AGG-ICP-AES \\
\hline $7440-28-0$ & Thallium & $<1.71 \mathrm{E} 0$ & ug/g dry & $1.71 \mathrm{E} 0$ & 7/30/08 & $8 G 22002$ & PNNL-AGG-ICP-AES \\
\hline $7440-23-5$ & Sodium & $2.42 \mathrm{E} 1$ & ug/g dry & $1.11 \mathrm{E} 0$ & 7/30/08 & $8 G 22002$ & PNNL-AGG-ICP-AES \\
\hline HEIS No. & B1TP84 & \multicolumn{3}{|c|}{ Lab ID: $\quad$ 0804025-CF } & & & \\
\hline $7429-90-5$ & Aluminum & $<1.43 \mathrm{E}-1$ & ug/g dry & $1.43 \mathrm{E}-1$ & 7/30/08 & $8 G 22002$ & PNNL-AGG-ICP-AES \\
\hline $7440-39-3$ & Barium & $3.55 \mathrm{E}-2$ & ug/g dry & $1.46 \mathrm{E}-2$ & 7/30/08 & $8 G 22002$ & PNNL-AGG-ICP-AES \\
\hline $7440-70-2$ & Calcium & $3.10 \mathrm{E} 1$ & ug/g dry & $6.44 \mathrm{E}-1$ & 7/30/08 & $8 G 22002$ & PNNL-AGG-ICP-AES \\
\hline 7439-89-6 & Iron & $<2.36 \mathrm{E}-1$ & ug/g dry & $2.36 \mathrm{E}-1$ & 7/30/08 & $8 G 22002$ & PNNL-AGG-ICP-AES \\
\hline 7440-09-7 & Potassium & $1.16 \mathrm{E} 1$ & ug/g dry & 3.87E0 & 7/30/08 & $8 G 22002$ & PNNL-AGG-ICP-AES \\
\hline 7439-95-4 & Magnesium & 7.83E0 & ug/g dry & $1.39 \mathrm{E}-1$ & 7/30/08 & $8 G 22002$ & PNNL-AGG-ICP-AES \\
\hline $7439-96-5$ & Manganese & $<2.85 \mathrm{E}-2$ & ug/g dry & $2.85 \mathrm{E}-2$ & 7/30/08 & $8 G 22002$ & PNNL-AGG-ICP-AES \\
\hline $7440-02-0$ & Nickel & $<1.55 \mathrm{E}-1$ & ug/g dry & $1.55 \mathrm{E}-1$ & 7/30/08 & $8 G 22002$ & PNNL-AGG-ICP-AES \\
\hline $7440-28-0$ & Thallium & $<1.71 \mathrm{E} 0$ & ug/g dry & $1.71 \mathrm{E} 0$ & 7/30/08 & $8 G 22002$ & PNNL-AGG-ICP-AES \\
\hline $7440-23-5$ & Sodium & 3.03E1 & ug/g dry & $1.11 \mathrm{E} 0$ & 7/30/08 & 8G22002 & PNNL-AGG-ICP-AES \\
\hline HEIS No. & B1TNX1 & \multicolumn{3}{|c|}{ Lab ID: $\quad$ 0804025-CI } & & & \\
\hline $7429-90-5$ & Aluminum & 3.81E-1 & ug/g dry & $1.44 \mathrm{E}-1$ & 7/30/08 & $8 G 22002$ & PNNL-AGG-ICP-AES \\
\hline $7440-39-3$ & Barium & $3.08 \mathrm{E}-2$ & ug/g dry & $1.48 \mathrm{E}-2$ & 7/30/08 & 8G22002 & PNNL-AGG-ICP-AES \\
\hline $7440-70-2$ & Calcium & 1.99E1 & ug/g dry & $6.50 \mathrm{E}-1$ & 7/30/08 & $8 G 22002$ & PNNL-AGG-ICP-AES \\
\hline 7439-89-6 & Iron & $<2.39 \mathrm{E}-1$ & ug/g dry & $2.39 \mathrm{E}-1$ & 7/30/08 & $8 G 22002$ & PNNL-AGG-ICP-AES \\
\hline $7440-09-7$ & Potassium & $6.78 \mathrm{E} 0$ & ug/g dry & 3.91E0 & 7/30/08 & $8 G 22002$ & PNNL-AGG-ICP-AES \\
\hline $7439-95-4$ & Magnesium & $5.28 \mathrm{E} 0$ & ug/g dry & $1.40 \mathrm{E}-1$ & 7/30/08 & $8 G 22002$ & PNNL-AGG-ICP-AES \\
\hline $7439-96-5$ & Manganese & $<2.88 \mathrm{E}-2$ & ug/g dry & $2.88 \mathrm{E}-2$ & 7/30/08 & $8 G 22002$ & PNNL-AGG-ICP-AES \\
\hline $7440-02-0$ & Nickel & $<1.57 \mathrm{E}-1$ & ug/g dry & $1.57 \mathrm{E}-1$ & 7/30/08 & $8 G 22002$ & PNNL-AGG-ICP-AES \\
\hline $7440-28-0$ & Thallium & $<1.72 \mathrm{E} 0$ & ug/g dry & $1.72 \mathrm{E} 0$ & 7/30/08 & $8 G 22002$ & PNNL-AGG-ICP-AES \\
\hline $7440-23-5$ & Sodium & $1.45 \mathrm{E} 1$ & ug/g dry & $1.12 \mathrm{E} 0$ & 7/30/08 & 8G22002 & PNNL-AGG-ICP-AES \\
\hline HEIS No. & B1TP86 & \multicolumn{3}{|c|}{ Lab ID: $\quad$ 0804025-CO } & & & \\
\hline $7429-90-5$ & Aluminum & 4.16E-1 & ug/g dry & $1.43 \mathrm{E}-1$ & 7/30/08 & $8 G 22002$ & PNNL-AGG-ICP-AES \\
\hline $7440-39-3$ & Barium & $1.81 \mathrm{E}-2$ & ug/g dry & $1.47 \mathrm{E}-2$ & 7/30/08 & $8 G 22002$ & PNNL-AGG-ICP-AES \\
\hline $7440-70-2$ & Calcium & $9.88 \mathrm{E} 0$ & ug/g dry & $6.45 \mathrm{E}-1$ & 7/30/08 & 8G22002 & PNNL-AGG-ICP-AES \\
\hline 7439-89-6 & Iron & $<2.37 \mathrm{E}-1$ & ug/g dry & 2.37E-1 & 7/30/08 & $8 G 22002$ & PNNL-AGG-ICP-AES \\
\hline 7440-09-7 & Potassium & $5.02 \mathrm{E} 0$ & ug/g dry & 3.88E0 & 7/30/08 & $8 G 22002$ & PNNL-AGG-ICP-AES \\
\hline $7439-95-4$ & Magnesium & 2.64E0 & ug/g dry & $1.39 \mathrm{E}-1$ & 7/30/08 & $8 G 22002$ & PNNL-AGG-ICP-AES \\
\hline 7439-96-5 & Manganese & $<2.86 \mathrm{E}-2$ & ug/g dry & $2.86 \mathrm{E}-2$ & 7/30/08 & $8 G 22002$ & PNNL-AGG-ICP-AES \\
\hline $7440-02-0$ & Nickel & $<1.56 \mathrm{E}-1$ & ug/g dry & $1.56 \mathrm{E}-1$ & 7/30/08 & $8 G 22002$ & PNNL-AGG-ICP-AES \\
\hline $7440-28-0$ & Thallium & $<1.71 \mathrm{E} 0$ & ug/g dry & $1.71 \mathrm{E} 0$ & 7/30/08 & $8 G 22002$ & PNNL-AGG-ICP-AES \\
\hline $7440-23-5$ & Sodium & $1.05 \mathrm{E} 1$ & ug/g dry & $1.12 \mathrm{E} 0$ & 7/30/08 & $8 G 22002$ & PNNL-AGG-ICP-AES \\
\hline
\end{tabular}


Total Metals by PNNL-AGG-ICP-AES/Water Extract

\begin{tabular}{|c|c|c|c|c|c|c|c|}
\hline CAS \# & Analyte & Results & Units & EQL & Analyzed & Batch & Method \\
\hline HEIS No. & B1TP89 & \multicolumn{3}{|c|}{ Lab ID: $\quad$ 0804025-CX } & & & \\
\hline 7429-90-5 & Aluminum & $4.42 \mathrm{E}-1$ & ug/g dry & $1.43 \mathrm{E}-1$ & $7 / 30 / 08$ & 8G22002 & PNNL-AGG-ICP-AES \\
\hline 7440-39-3 & Barium & 3.13E-2 & ug/g dry & $1.47 \mathrm{E}-2$ & 7/30/08 & $8 G 22002$ & PNNL-AGG-ICP-AES \\
\hline $7440-70-2$ & Calcium & $1.34 \mathrm{E} 1$ & ug/g dry & $6.47 \mathrm{E}-1$ & $7 / 30 / 08$ & $8 G 22002$ & PNNL-AGG-ICP-AES \\
\hline 7439-89-6 & Iron & $<2.38 \mathrm{E}-1$ & ug/g dry & $2.38 \mathrm{E}-1$ & 7/30/08 & $8 G 22002$ & PNNL-AGG-ICP-AES \\
\hline 7440-09-7 & Potassium & 4.53E0 & ug/g dry & 3.89E0 & 7/30/08 & $8 G 22002$ & PNNL-AGG-ICP-AES \\
\hline 7439-95-4 & Magnesium & $3.22 \mathrm{E} 0$ & ug/g dry & $1.39 \mathrm{E}-1$ & 7/30/08 & $8 G 22002$ & PNNL-AGG-ICP-AES \\
\hline 7439-96-5 & Manganese & $<2.86 \mathrm{E}-2$ & ug/g dry & $2.86 \mathrm{E}-2$ & 7/30/08 & 8G22002 & PNNL-AGG-ICP-AES \\
\hline $7440-02-0$ & Nickel & $<1.56 \mathrm{E}-1$ & ug/g dry & $1.56 \mathrm{E}-1$ & 7/30/08 & $8 G 22002$ & PNNL-AGG-ICP-AES \\
\hline $7440-28-0$ & Thallium & $<1.71 \mathrm{E} 0$ & ug/g dry & $1.71 \mathrm{E} 0$ & 7/30/08 & $8 G 22002$ & PNNL-AGG-ICP-AES \\
\hline $7440-23-5$ & Sodium & $1.20 \mathrm{E} 1$ & ug/g dry & $1.12 \mathrm{E} 0$ & $7 / 30 / 08$ & $8 G 22002$ & PNNL-AGG-ICP-AES \\
\hline HEIS No. & B1TP91 & \multicolumn{3}{|c|}{ Lab ID: $\quad$ 0804025-DF } & & & \\
\hline $7429-90-5$ & Aluminum & $2.98 \mathrm{E}-1$ & ug/g dry & 1.43E-1 & 7/30/08 & 8G22002 & PNNL-AGG-ICP-AES \\
\hline $7440-39-3$ & Barium & 3.93E-2 & ug/g dry & $1.47 \mathrm{E}-2$ & 7/30/08 & $8 G 22002$ & PNNL-AGG-ICP-AES \\
\hline $7440-70-2$ & Calcium & $1.17 \mathrm{E} 1$ & ug/g dry & $6.47 \mathrm{E}-1$ & 7/30/08 & $8 G 22002$ & PNNL-AGG-ICP-AES \\
\hline 7439-89-6 & Iron & $<2.38 \mathrm{E}-1$ & ug/g dry & $2.38 \mathrm{E}-1$ & 7/30/08 & $8 G 22002$ & PNNL-AGG-ICP-AES \\
\hline $7440-09-7$ & Potassium & $6.14 \mathrm{E} 0$ & ug/g dry & 3.89E0 & 7/30/08 & 8G22002 & PNNL-AGG-ICP-AES \\
\hline 7439-95-4 & Magnesium & $3.27 \mathrm{E} 0$ & ug/g dry & $1.39 \mathrm{E}-1$ & 7/30/08 & $8 G 22002$ & PNNL-AGG-ICP-AES \\
\hline 7439-96-5 & Manganese & $<2.86 \mathrm{E}-2$ & ug/g dry & $2.86 \mathrm{E}-2$ & $7 / 30 / 08$ & $8 G 22002$ & PNNL-AGG-ICP-AES \\
\hline $7440-02-0$ & Nickel & $<1.56 \mathrm{E}-1$ & ug/g dry & $1.56 \mathrm{E}-1$ & 7/30/08 & $8 G 22002$ & PNNL-AGG-ICP-AES \\
\hline $7440-28-0$ & Thallium & $<1.71 \mathrm{E} 0$ & ug/g dry & $1.71 \mathrm{E} 0$ & 7/30/08 & $8 G 22002$ & PNNL-AGG-ICP-AES \\
\hline $7440-23-5$ & Sodium & $1.77 \mathrm{E} 1$ & ug/g dry & $1.12 \mathrm{E} 0$ & 7/30/08 & $8 G 22002$ & PNNL-AGG-ICP-AES \\
\hline HEIS No. & В1ТР93 & \multicolumn{3}{|c|}{ Lab ID: $\quad$ 0804025-DL } & & & \\
\hline $7429-90-5$ & Aluminum & $<1.44 \mathrm{E}-1$ & ug/g dry & $1.44 \mathrm{E}-1$ & 7/30/08 & $8 G 22002$ & PNNL-AGG-ICP-AES \\
\hline $7440-39-3$ & Barium & 3.67E-2 & ug/g dry & $1.47 \mathrm{E}-2$ & 7/30/08 & $8 G 22002$ & PNNL-AGG-ICP-AES \\
\hline $7440-70-2$ & Calcium & $1.70 \mathrm{E} 1$ & ug/g dry & $6.48 \mathrm{E}-1$ & 7/30/08 & $8 G 22002$ & PNNL-AGG-ICP-AES \\
\hline 7439-89-6 & Iron & $<2.38 \mathrm{E}-1$ & ug/g dry & $2.38 \mathrm{E}-1$ & 7/30/08 & $8 G 22002$ & PNNL-AGG-ICP-AES \\
\hline 7440-09-7 & Potassium & $5.58 \mathrm{E} 0$ & ug/g dry & $3.90 \mathrm{E} 0$ & $7 / 30 / 08$ & $8 G 22002$ & PNNL-AGG-ICP-AES \\
\hline 7439-95-4 & Magnesium & $4.56 \mathrm{E} 0$ & ug/g dry & $1.40 \mathrm{E}-1$ & $7 / 30 / 08$ & 8G22002 & PNNL-AGG-ICP-AES \\
\hline 7439-96-5 & Manganese & $<2.87 \mathrm{E}-2$ & ug/g dry & $2.87 \mathrm{E}-2$ & 7/30/08 & $8 G 22002$ & PNNL-AGG-ICP-AES \\
\hline 7440-02-0 & Nickel & $<1.56 \mathrm{E}-1$ & ug/g dry & $1.56 \mathrm{E}-1$ & 7/30/08 & $8 G 22002$ & PNNL-AGG-ICP-AES \\
\hline $7440-28-0$ & Thallium & $<1.72 \mathrm{E} 0$ & ug/g dry & $1.72 \mathrm{E} 0$ & $7 / 30 / 08$ & $8 G 22002$ & PNNL-AGG-ICP-AES \\
\hline $7440-23-5$ & Sodium & $2.66 \mathrm{E} 1$ & ug/g dry & $1.12 \mathrm{E} 0$ & $7 / 30 / 08$ & $8 G 22002$ & PNNL-AGG-ICP-AES \\
\hline HEIS No. & B1TP94 & \multicolumn{3}{|c|}{ Lab ID: $\quad$ 0804025-DM } & & & \\
\hline $7429-90-5$ & Aluminum & $1.64 \mathrm{E}-1$ & ug/g dry & $1.43 \mathrm{E}-1$ & 7/30/08 & $8 G 22002$ & PNNL-AGG-ICP-AES \\
\hline $7440-39-3$ & Barium & $1.47 \mathrm{E}-2$ & ug/g dry & $1.46 \mathrm{E}-2$ & 7/30/08 & $8 G 22002$ & PNNL-AGG-ICP-AES \\
\hline $7440-70-2$ & Calcium & $9.29 \mathrm{E} 0$ & ug/g dry & 6.43E-1 & 7/30/08 & 8G22002 & PNNL-AGG-ICP-AES \\
\hline 7439-89-6 & Iron & $<2.36 \mathrm{E}-1$ & ug/g dry & $2.36 \mathrm{E}-1$ & 7/30/08 & 8G22002 & PNNL-AGG-ICP-AES \\
\hline 7440-09-7 & Potassium & $<3.87 \mathrm{E} 0$ & ug/g dry & 3.87E0 & 7/30/08 & $8 G 22002$ & PNNL-AGG-ICP-AES \\
\hline 7439-95-4 & Magnesium & $2.28 \mathrm{E} 0$ & ug/g dry & $1.39 \mathrm{E}-1$ & 7/30/08 & $8 G 22002$ & PNNL-AGG-ICP-AES \\
\hline 7439-96-5 & Manganese & $<2.85 \mathrm{E}-2$ & ug/g dry & $2.85 \mathrm{E}-2$ & 7/30/08 & 8G22002 & PNNL-AGG-ICP-AES \\
\hline $7440-02-0$ & Nickel & $<1.55 \mathrm{E}-1$ & ug/g dry & $1.55 \mathrm{E}-1$ & $7 / 30 / 08$ & $8 G 22002$ & PNNL-AGG-ICP-AES \\
\hline 7440-28-0 & Thallium & $<1.70 \mathrm{E} 0$ & ug/g dry & $1.70 \mathrm{E} 0$ & $7 / 30 / 08$ & $8 G 22002$ & PNNL-AGG-ICP-AES \\
\hline 7440-23-5 & Sodium & 3.17E1 & ug/g dry & $1.11 \mathrm{E} 0$ & 7/30/08 & $8 G 22002$ & PNNL-AGG-ICP-AES \\
\hline
\end{tabular}


Total Metals by PNNL-AGG-ICP-AES/Acid Extract

\begin{tabular}{|c|c|c|c|c|c|c|c|}
\hline CAS \# & Analyte & Results & Units & EQL & Analyzed & Batch & Method \\
\hline HEIS No. & В1TP24 & \multicolumn{3}{|c|}{ Lab ID: $\quad$ 0804025-04 } & & & \\
\hline 7429-90-5 & Aluminum & $4.41 \mathrm{E} 3$ & ug/g dry & 8.58E0 & 9/24/08 & $8 \mathrm{I} 23006$ & PNNL-AGG-ICP-AES \\
\hline $7440-39-3$ & Barium & 3.92E1 & ug/g dry & 8.17E-1 & 9/24/08 & $8 \mathrm{I} 23006$ & PNNL-AGG-ICP-AES \\
\hline $7440-70-2$ & Calcium & 3.33E3 & ug/g dry & 3.19E1 & $9 / 24 / 08$ & $8 \mathrm{I} 23006$ & PNNL-AGG-ICP-AES \\
\hline 7439-89-6 & Iron & $1.02 \mathrm{E} 4$ & ug/g dry & 8.72E1 & $9 / 24 / 08$ & $8 \mathrm{I} 23006$ & PNNL-AGG-ICP-AES \\
\hline 7440-09-7 & Potassium & 7.97E2 & ug/g dry & 2.02E1 & $9 / 24 / 08$ & $8 \mathrm{I} 23006$ & PNNL-AGG-ICP-AES \\
\hline 7439-95-4 & Magnesium & 2.57E3 & ug/g dry & $6.75 \mathrm{E} 0$ & 9/24/08 & $8 \mathrm{I} 23006$ & PNNL-AGG-ICP-AES \\
\hline 7439-96-5 & Manganese & $1.89 \mathrm{E} 2$ & ug/g dry & $2.55 \mathrm{E}-1$ & $9 / 24 / 08$ & 8123006 & PNNL-AGG-ICP-AES \\
\hline $7440-02-0$ & Nickel & 8.02E0 & ug/g dry & $1.75 \mathrm{E} 0$ & 9/24/08 & $8 \mathrm{I} 23006$ & PNNL-AGG-ICP-AES \\
\hline $7440-28-0$ & Thallium & $<9.64 \mathrm{E} 0$ & ug/g dry & $9.64 \mathrm{E} 0$ & $9 / 24 / 08$ & 8123006 & PNNL-AGG-ICP-AES \\
\hline $7440-23-5$ & Sodium & 8.72E2 & ug/g dry & 2.29E2 & $9 / 24 / 08$ & $8 \mathrm{I} 23006$ & PNNL-AGG-ICP-AES \\
\hline HEIS No. & В1TP28 & \multicolumn{3}{|c|}{ Lab ID: $\quad$ 0804025-07 } & & & \\
\hline 7429-90-5 & Aluminum & $4.56 \mathrm{E} 3$ & ug/g dry & 3.34E1 & $9 / 24 / 08$ & $8 \mathrm{I} 23006$ & PNNL-AGG-ICP-AES \\
\hline $7440-39-3$ & Barium & 5.59E1 & ug/g dry & 7.95E-1 & $9 / 24 / 08$ & $8 \mathrm{I} 23006$ & PNNL-AGG-ICP-AES \\
\hline $7440-70-2$ & Calcium & $5.81 \mathrm{E} 3$ & ug/g dry & $3.10 \mathrm{E} 1$ & $9 / 24 / 08$ & $8 \mathrm{I} 23006$ & PNNL-AGG-ICP-AES \\
\hline 7439-89-6 & Iron & $1.35 \mathrm{E} 4$ & ug/g dry & 8.48E1 & $9 / 24 / 08$ & $8 \mathrm{I} 23006$ & PNNL-AGG-ICP-AES \\
\hline $7440-09-7$ & Potassium & $7.28 \mathrm{E} 2$ & ug/g dry & $1.97 \mathrm{E} 1$ & $9 / 24 / 08$ & 8123006 & PNNL-AGG-ICP-AES \\
\hline 7439-95-4 & Magnesium & 3.17E3 & ug/g dry & 2.63E1 & 9/24/08 & $8 \mathrm{I} 23006$ & PNNL-AGG-ICP-AES \\
\hline 7439-96-5 & Manganese & $2.02 \mathrm{E} 2$ & ug/g dry & $2.48 \mathrm{E}-1$ & 9/24/08 & $8 \mathrm{I} 23006$ & PNNL-AGG-ICP-AES \\
\hline $7440-02-0$ & Nickel & $8.24 \mathrm{E} 0$ & ug/g dry & $1.71 \mathrm{E} 0$ & $9 / 24 / 08$ & $8 \mathrm{I} 23006$ & PNNL-AGG-ICP-AES \\
\hline $7440-28-0$ & Thallium & $<9.38 \mathrm{E} 0$ & ug/g dry & $9.38 \mathrm{E} 0$ & 9/24/08 & $8 \mathrm{I} 23006$ & PNNL-AGG-ICP-AES \\
\hline $7440-23-5$ & Sodium & $1.35 \mathrm{E} 3$ & ug/g dry & $2.22 \mathrm{E} 2$ & 9/24/08 & $8 \mathrm{I} 23006$ & PNNL-AGG-ICP-AES \\
\hline HEIS No. & В1TP29 & \multicolumn{3}{|c|}{ Lab ID: $\quad 0804025-08$} & & & \\
\hline $7429-90-5$ & Aluminum & $4.49 \mathrm{E} 3$ & ug/g dry & $8.28 \mathrm{E} 0$ & $9 / 24 / 08$ & $8 \mathrm{I} 23006$ & PNNL-AGG-ICP-AES \\
\hline 7440-39-3 & Barium & 5.38E1 & ug/g dry & $7.89 \mathrm{E}-1$ & $9 / 24 / 08$ & $8 \mathrm{I} 23006$ & PNNL-AGG-ICP-AES \\
\hline $7440-70-2$ & Calcium & 5.12E3 & ug/g dry & 3.08E1 & $9 / 24 / 08$ & $8 \mathrm{I} 23006$ & PNNL-AGG-ICP-AES \\
\hline 7439-89-6 & Iron & $1.43 \mathrm{E} 4$ & ug/g dry & $8.41 \mathrm{E} 1$ & $9 / 24 / 08$ & 8123006 & PNNL-AGG-ICP-AES \\
\hline 7440-09-7 & Potassium & 7.45E2 & ug/g dry & $1.95 \mathrm{E} 1$ & $9 / 24 / 08$ & $8 \mathrm{I} 23006$ & PNNL-AGG-ICP-AES \\
\hline 7439-95-4 & Magnesium & $3.00 \mathrm{E} 3$ & ug/g dry & $2.60 \mathrm{E} 1$ & $9 / 24 / 08$ & 8123006 & PNNL-AGG-ICP-AES \\
\hline 7439-96-5 & Manganese & 1.93E2 & ug/g dry & $2.46 \mathrm{E}-1$ & $9 / 24 / 08$ & $8 \mathrm{I} 23006$ & PNNL-AGG-ICP-AES \\
\hline 7440-02-0 & Nickel & $6.60 \mathrm{E} 0$ & ug/g dry & 1.69E0 & $9 / 24 / 08$ & $8 \mathrm{I} 23006$ & PNNL-AGG-ICP-AES \\
\hline $7440-28-0$ & Thallium & $<9.30 \mathrm{E} 0$ & ug/g dry & $9.30 \mathrm{E} 0$ & $9 / 24 / 08$ & $8 \mathrm{I} 23006$ & PNNL-AGG-ICP-AES \\
\hline $7440-23-5$ & Sodium & $1.13 \mathrm{E} 3$ & ug/g dry & $2.21 \mathrm{E} 2$ & $9 / 24 / 08$ & $8 \mathrm{I} 23006$ & PNNL-AGG-ICP-AES \\
\hline HEIS No. & В1TP30 & \multicolumn{3}{|c|}{ Lab ID: $\quad 0804025-09$} & & & \\
\hline $7429-90-5$ & Aluminum & $5.21 \mathrm{E} 3$ & ug/g dry & $3.42 \mathrm{E} 1$ & $9 / 24 / 08$ & 8123006 & PNNL-AGG-ICP-AES \\
\hline $7440-39-3$ & Barium & 4.93E1 & ug/g dry & 8.13E-1 & $9 / 24 / 08$ & $8 \mathrm{I} 23006$ & PNNL-AGG-ICP-AES \\
\hline $7440-70-2$ & Calcium & $6.52 \mathrm{E} 3$ & ug/g dry & 3.17E1 & $9 / 24 / 08$ & $8 \mathrm{I} 23006$ & PNNL-AGG-ICP-AES \\
\hline 7439-89-6 & Iron & $1.41 \mathrm{E} 4$ & ug/g dry & 8.68E1 & $9 / 24 / 08$ & $8 \mathrm{I} 23006$ & PNNL-AGG-ICP-AES \\
\hline $7440-09-7$ & Potassium & $8.46 \mathrm{E} 2$ & ug/g dry & $2.01 \mathrm{E} 1$ & $9 / 24 / 08$ & 8123006 & PNNL-AGG-ICP-AES \\
\hline 7439-95-4 & Magnesium & 3.75E3 & ug/g dry & 2.69E1 & $9 / 24 / 08$ & $8 \mathrm{I} 23006$ & PNNL-AGG-ICP-AES \\
\hline 7439-96-5 & Manganese & $2.12 \mathrm{E} 2$ & ug/g dry & $2.54 \mathrm{E}-1$ & $9 / 24 / 08$ & 8123006 & PNNL-AGG-ICP-AES \\
\hline $7440-02-0$ & Nickel & $1.03 \mathrm{E} 1$ & ug/g dry & $1.75 \mathrm{E} 0$ & $9 / 24 / 08$ & $8 \mathrm{I} 23006$ & PNNL-AGG-ICP-AES \\
\hline $7440-28-0$ & Thallium & $<9.59 \mathrm{E} 0$ & ug/g dry & 9.59E0 & 9/24/08 & $8 \mathrm{I} 23006$ & PNNL-AGG-ICP-AES \\
\hline $7440-23-5$ & Sodium & $1.03 \mathrm{E} 3$ & ug/g dry & $2.28 \mathrm{E} 2$ & $9 / 24 / 08$ & $8 \mathrm{I} 23006$ & PNNL-AGG-ICP-AES \\
\hline HEIS No. & B1TNV9 & \multicolumn{3}{|c|}{ Lab ID: $\quad$ 0804025-10 } & & & \\
\hline 7429-90-5 & Aluminum & $4.46 \mathrm{E} 3$ & ug/g dry & 8.29E0 & $9 / 24 / 08$ & $8 \mathrm{I} 23006$ & PNNL-AGG-ICP-AES \\
\hline $7440-39-3$ & Barium & $3.56 \mathrm{E} 1$ & ug/g dry & $7.89 \mathrm{E}-1$ & $9 / 24 / 08$ & 8123006 & PNNL-AGG-ICP-AES \\
\hline $7440-70-2$ & Calcium & $6.11 \mathrm{E} 3$ & ug/g dry & $3.08 \mathrm{E} 1$ & $9 / 24 / 08$ & $8 \mathrm{I} 23006$ & PNNL-AGG-ICP-AES \\
\hline 7439-89-6 & Iron & $1.19 \mathrm{E} 4$ & ug/g dry & 8.42E1 & $9 / 24 / 08$ & $8 \mathrm{I} 23006$ & PNNL-AGG-ICP-AES \\
\hline
\end{tabular}


Total Metals by PNNL-AGG-ICP-AES/Acid Extract

\begin{tabular}{|c|c|c|c|c|c|c|c|}
\hline CAS \# & Analyte & Results & Units & EQL & Analyzed & Batch & Method \\
\hline HEIS No. & B1TNV9 & \multicolumn{3}{|c|}{ Lab ID: $\quad$ 0804025-10 } & & & \\
\hline 7440-09-7 & Potassium & 7.47E2 & ug/g dry & $1.95 \mathrm{E} 1$ & $9 / 24 / 08$ & $8 \mathrm{I} 23006$ & PNNL-AGG-ICP-AES \\
\hline 7439-95-4 & Magnesium & 3.58E3 & ug/g dry & $2.61 \mathrm{E} 1$ & 9/24/08 & $8 \mathrm{I} 23006$ & PNNL-AGG-ICP-AES \\
\hline 7439-96-5 & Manganese & $1.95 \mathrm{E} 2$ & ug/g dry & $2.47 \mathrm{E}-1$ & $9 / 24 / 08$ & $8 \mathrm{I} 23006$ & PNNL-AGG-ICP-AES \\
\hline $7440-02-0$ & Nickel & $9.50 \mathrm{E} 0$ & ug/g dry & 1.69E0 & $9 / 24 / 08$ & $8 \mathrm{I} 23006$ & PNNL-AGG-ICP-AES \\
\hline $7440-28-0$ & Thallium & $<9.31 \mathrm{E} 0$ & ug/g dry & $9.31 \mathrm{E} 0$ & $9 / 24 / 08$ & $8 \mathrm{I} 23006$ & PNNL-AGG-ICP-AES \\
\hline $7440-23-5$ & Sodium & $1.44 \mathrm{E} 3$ & ug/g dry & $2.21 \mathrm{E} 2$ & 9/24/08 & $8 \mathrm{I} 23006$ & PNNL-AGG-ICP-AES \\
\hline HEIS No. & B1TP22 & \multicolumn{3}{|c|}{ Lab ID: $\quad$ 0804025-13 } & & & \\
\hline $7429-90-5$ & Aluminum & $4.04 \mathrm{E} 3$ & ug/g dry & 8.36Е0 & $9 / 24 / 08$ & $8 \mathrm{I} 23006$ & PNNL-AGG-ICP-AES \\
\hline $7440-39-3$ & Barium & $6.66 \mathrm{E} 1$ & ug/g dry & 7.96E-1 & 9/24/08 & $8 \mathrm{I} 23006$ & PNNL-AGG-ICP-AES \\
\hline $7440-70-2$ & Calcium & $5.70 \mathrm{E} 3$ & ug/g dry & $3.11 \mathrm{E} 1$ & 9/24/08 & $8 \mathrm{I} 23006$ & PNNL-AGG-ICP-AES \\
\hline 7439-89-6 & Iron & $1.87 \mathrm{E} 4$ & ug/g dry & 8.49E1 & $9 / 24 / 08$ & $8 \mathrm{I} 23006$ & PNNL-AGG-ICP-AES \\
\hline 7440-09-7 & Potassium & $6.16 \mathrm{E} 2$ & ug/g dry & 1.97E1 & $9 / 24 / 08$ & $8 \mathrm{I} 23006$ & PNNL-AGG-ICP-AES \\
\hline 7439-95-4 & Magnesium & 3.38E3 & ug/g dry & 2.63E1 & 9/24/08 & $8 \mathrm{I} 23006$ & PNNL-AGG-ICP-AES \\
\hline 7439-96-5 & Manganese & $2.40 \mathrm{E} 2$ & ug/g dry & $2.49 \mathrm{E}-1$ & $9 / 24 / 08$ & 8123006 & PNNL-AGG-ICP-AES \\
\hline $7440-02-0$ & Nickel & 6.64E0 & ug/g dry & $1.71 \mathrm{E} 0$ & 9/24/08 & $8 \mathrm{I} 23006$ & PNNL-AGG-ICP-AES \\
\hline $7440-28-0$ & Thallium & $<9.39 \mathrm{E} 0$ & ug/g dry & 9.39E0 & $9 / 24 / 08$ & $8 \mathrm{I} 23006$ & PNNL-AGG-ICP-AES \\
\hline $7440-23-5$ & Sodium & 4.72E2 & ug/g dry & $2.23 \mathrm{E} 2$ & $9 / 24 / 08$ & $8 \mathrm{I} 23006$ & PNNL-AGG-ICP-AES \\
\hline HEIS No. & B1TP26 & \multicolumn{3}{|c|}{ Lab ID: $\quad$ 0804025-16 } & & & \\
\hline 7429-90-5 & Aluminum & 4.61E3 & ug/g dry & 3.35E1 & 9/24/08 & $8 \mathrm{I} 23006$ & PNNL-AGG-ICP-AES \\
\hline $7440-39-3$ & Barium & $4.44 \mathrm{E} 1$ & ug/g dry & 7.97E-1 & $9 / 24 / 08$ & $8 \mathrm{I} 23006$ & PNNL-AGG-ICP-AES \\
\hline $7440-70-2$ & Calcium & $5.41 \mathrm{E} 3$ & ug/g dry & $3.11 \mathrm{E} 1$ & 9/24/08 & $8 \mathrm{I} 23006$ & PNNL-AGG-ICP-AES \\
\hline 7439-89-6 & Iron & $1.38 \mathrm{E} 4$ & ug/g dry & $8.51 \mathrm{E} 1$ & $9 / 24 / 08$ & 8123006 & PNNL-AGG-ICP-AES \\
\hline $7440-09-7$ & Potassium & 6.64E2 & ug/g dry & $1.97 \mathrm{E} 1$ & $9 / 24 / 08$ & $8 \mathrm{I} 23006$ & PNNL-AGG-ICP-AES \\
\hline 7439-95-4 & Magnesium & 3.43E3 & ug/g dry & 2.63E1 & $9 / 24 / 08$ & $8 \mathrm{I} 23006$ & PNNL-AGG-ICP-AES \\
\hline 7439-96-5 & Manganese & 2.03E2 & ug/g dry & 2.49E-1 & $9 / 24 / 08$ & $8 \mathrm{I} 23006$ & PNNL-AGG-ICP-AES \\
\hline 7440-02-0 & Nickel & 8.57E0 & ug/g dry & $1.71 \mathrm{E} 0$ & $9 / 24 / 08$ & $8 \mathrm{I} 23006$ & PNNL-AGG-ICP-AES \\
\hline $7440-28-0$ & Thallium & $<9.41 \mathrm{E} 0$ & ug/g dry & $9.41 \mathrm{E} 0$ & $9 / 24 / 08$ & $8 \mathrm{I} 23006$ & PNNL-AGG-ICP-AES \\
\hline $7440-23-5$ & Sodium & $1.04 \mathrm{E} 3$ & ug/g dry & $2.23 \mathrm{E} 2$ & $9 / 24 / 08$ & $8 \mathrm{I} 23006$ & PNNL-AGG-ICP-AES \\
\hline HEIS No. & B1TNW0 & \multicolumn{3}{|c|}{ Lab ID: $\quad$ 0804025-17 } & & & \\
\hline 7429-90-5 & Aluminum & 4.33E3 & ug/g dry & 8.43E0 & 9/24/08 & $8 \mathrm{I} 23006$ & PNNL-AGG-ICP-AES \\
\hline 7440-39-3 & Barium & 4.32E1 & ug/g dry & 8.03E-1 & $9 / 24 / 08$ & $8 \mathrm{I} 23006$ & PNNL-AGG-ICP-AES \\
\hline $7440-70-2$ & Calcium & $6.10 \mathrm{E} 3$ & ug/g dry & 3.13E1 & $9 / 24 / 08$ & $8 \mathrm{I} 23006$ & PNNL-AGG-ICP-AES \\
\hline 7439-89-6 & Iron & $1.16 \mathrm{E} 4$ & ug/g dry & 8.57E1 & 9/24/08 & $8 \mathrm{I} 23006$ & PNNL-AGG-ICP-AES \\
\hline $7440-09-7$ & Potassium & $7.88 \mathrm{E} 2$ & ug/g dry & $1.99 \mathrm{E} 1$ & $9 / 24 / 08$ & 8123006 & PNNL-AGG-ICP-AES \\
\hline 7439-95-4 & Magnesium & 3.09E3 & ug/g dry & $2.65 \mathrm{E} 1$ & $9 / 24 / 08$ & 8123006 & PNNL-AGG-ICP-AES \\
\hline 7439-96-5 & Manganese & $2.12 \mathrm{E} 2$ & ug/g dry & $2.51 \mathrm{E}-1$ & $9 / 24 / 08$ & 8123006 & PNNL-AGG-ICP-AES \\
\hline 7440-02-0 & Nickel & 6.86Е0 & ug/g dry & $1.72 \mathrm{E} 0$ & $9 / 24 / 08$ & $8 \mathrm{I} 23006$ & PNNL-AGG-ICP-AES \\
\hline $7440-28-0$ & Thallium & $<9.47 \mathrm{E} 0$ & ug/g dry & $9.47 \mathrm{E} 0$ & 9/24/08 & $8 I 23006$ & PNNL-AGG-ICP-AES \\
\hline 7440-23-5 & Sodium & $2.35 \mathrm{E} 3$ & ug/g dry & $2.25 \mathrm{E} 2$ & $9 / 24 / 08$ & $8 \mathrm{I} 23006$ & PNNL-AGG-ICP-AES \\
\hline HEIS No. & B1TNW1 & \multicolumn{3}{|c|}{ Lab ID: $\quad$ 0804025-18 } & & & \\
\hline 7429-90-5 & Aluminum & 4.97E3 & ug/g dry & 3.34E1 & $9 / 24 / 08$ & $8 \mathrm{I} 23006$ & PNNL-AGG-ICP-AES \\
\hline $7440-39-3$ & Barium & $5.06 \mathrm{E} 1$ & ug/g dry & 7.96E-1 & 9/24/08 & $8 \mathrm{I} 23006$ & PNNL-AGG-ICP-AES \\
\hline $7440-70-2$ & Calcium & 7.76E3 & ug/g dry & $3.10 \mathrm{E} 1$ & $9 / 24 / 08$ & 8123006 & PNNL-AGG-ICP-AES \\
\hline 7439-89-6 & Iron & $1.14 \mathrm{E} 4$ & ug/g dry & 8.49E1 & $9 / 24 / 08$ & $8 \mathrm{I} 23006$ & PNNL-AGG-ICP-AES \\
\hline 7440-09-7 & Potassium & $1.21 \mathrm{E} 3$ & ug/g dry & $1.97 \mathrm{E} 1$ & $9 / 24 / 08$ & $8 \mathrm{I} 23006$ & PNNL-AGG-ICP-AES \\
\hline 7439-95-4 & Magnesium & 3.97E3 & ug/g dry & 2.63E1 & 9/24/08 & $8 I 23006$ & PNNL-AGG-ICP-AES \\
\hline 7439-96-5 & Manganese & $2.09 \mathrm{E} 2$ & ug/g dry & 2.49E-1 & $9 / 24 / 08$ & $8 \mathrm{I} 23006$ & PNNL-AGG-ICP-AES \\
\hline $7440-02-0$ & Nickel & 8.55E0 & ug/g dry & $1.71 \mathrm{E} 0$ & $9 / 24 / 08$ & $8 \mathrm{I} 23006$ & PNNL-AGG-ICP-AES \\
\hline
\end{tabular}


Total Metals by PNNL-AGG-ICP-AES/Acid Extract

\begin{tabular}{|c|c|c|c|c|c|c|c|}
\hline CAS \# & Analyte & Results & Units & EQL & Analyzed & Batch & Method \\
\hline HEIS No. & B1TNW1 & \multicolumn{3}{|c|}{ Lab ID: $\quad$ 0804025-18 } & & & \\
\hline $7440-28-0$ & Thallium & $<9.39 \mathrm{E} 0$ & ug/g dry & 9.39E0 & 9/24/08 & $8 \mathrm{I} 23006$ & PNNL-AGG-ICP-AES \\
\hline $7440-23-5$ & Sodium & $2.81 \mathrm{E} 3$ & ug/g dry & $2.23 \mathrm{E} 2$ & 9/24/08 & $8 \mathrm{I} 23006$ & PNNL-AGG-ICP-AES \\
\hline HEIS No. & B1TNW3 & \multicolumn{3}{|c|}{ Lab ID: $\quad$ 0804025-20 } & & & \\
\hline $7429-90-5$ & Aluminum & 4.85E3 & ug/g dry & 3.35E1 & $9 / 24 / 08$ & $8 \mathrm{I} 23006$ & PNNL-AGG-ICP-AES \\
\hline $7440-39-3$ & Barium & $4.80 \mathrm{E} 1$ & ug/g dry & 7.98E-1 & $9 / 24 / 08$ & $8 \mathrm{I} 23006$ & PNNL-AGG-ICP-AES \\
\hline $7440-70-2$ & Calcium & $6.50 \mathrm{E} 3$ & ug/g dry & $3.11 \mathrm{E} 1$ & 9/24/08 & $8 \mathrm{I} 23006$ & PNNL-AGG-ICP-AES \\
\hline 7439-89-6 & Iron & $1.29 \mathrm{E} 4$ & ug/g dry & $8.51 \mathrm{E} 1$ & $9 / 24 / 08$ & $8 \mathrm{I} 23006$ & PNNL-AGG-ICP-AES \\
\hline 7440-09-7 & Potassium & 8.50E2 & ug/g dry & $1.98 \mathrm{E} 1$ & 9/24/08 & $8 \mathrm{I} 23006$ & PNNL-AGG-ICP-AES \\
\hline $7439-95-4$ & Magnesium & $3.78 \mathrm{E} 3$ & ug/g dry & 2.63E1 & $9 / 24 / 08$ & $8 \mathrm{I} 23006$ & PNNL-AGG-ICP-AES \\
\hline $7439-96-5$ & Manganese & $2.10 \mathrm{E} 2$ & ug/g dry & $2.49 \mathrm{E}-1$ & $9 / 24 / 08$ & $8 \mathrm{I} 23006$ & PNNL-AGG-ICP-AES \\
\hline $7440-02-0$ & Nickel & 8.99E0 & ug/g dry & $1.71 \mathrm{E} 0$ & $9 / 24 / 08$ & $8 \mathrm{I} 23006$ & PNNL-AGG-ICP-AES \\
\hline $7440-28-0$ & Thallium & $<9.41 \mathrm{E} 0$ & ug/g dry & $9.41 \mathrm{E} 0$ & $9 / 24 / 08$ & $8 \mathrm{I} 23006$ & PNNL-AGG-ICP-AES \\
\hline $7440-23-5$ & Sodium & $2.44 \mathrm{E} 2$ & ug/g dry & 2.23E2 & $9 / 24 / 08$ & $8 \mathrm{I} 23006$ & PNNL-AGG-ICP-AES \\
\hline HEIS No. & B1TNW5 & \multicolumn{3}{|c|}{ Lab ID: $\quad$ 0804025-22 } & & & \\
\hline $7429-90-5$ & Aluminum & 5.17E3 & ug/g dry & $3.38 \mathrm{E} 1$ & $9 / 24 / 08$ & $8 \mathrm{I} 23006$ & PNNL-AGG-ICP-AES \\
\hline $7440-39-3$ & Barium & $5.28 \mathrm{E} 1$ & ug/g dry & 8.04E-1 & 9/24/08 & $8 \mathrm{I} 23006$ & PNNL-AGG-ICP-AES \\
\hline $7440-70-2$ & Calcium & 6.97E3 & ug/g dry & $3.14 \mathrm{E} 1$ & $9 / 24 / 08$ & $8 \mathrm{I} 23006$ & PNNL-AGG-ICP-AES \\
\hline $7439-89-6$ & Iron & $1.02 \mathrm{E} 4$ & ug/g dry & $8.58 \mathrm{E} 1$ & 9/24/08 & $8 \mathrm{I} 23006$ & PNNL-AGG-ICP-AES \\
\hline 7440-09-7 & Potassium & $1.31 \mathrm{E} 3$ & ug/g dry & 1.99E1 & $9 / 24 / 08$ & $8 \mathrm{I} 23006$ & PNNL-AGG-ICP-AES \\
\hline 7439-95-4 & Magnesium & 3.79E3 & ug/g dry & $2.66 \mathrm{E} 1$ & 9/24/08 & $8 \mathrm{I} 23006$ & PNNL-AGG-ICP-AES \\
\hline $7439-96-5$ & Manganese & 2.19E2 & ug/g dry & $2.51 \mathrm{E}-1$ & 9/24/08 & 8123006 & PNNL-AGG-ICP-AES \\
\hline $7440-02-0$ & Nickel & 9.43E0 & ug/g dry & $1.73 \mathrm{E} 0$ & $9 / 24 / 08$ & $8 \mathrm{I} 23006$ & PNNL-AGG-ICP-AES \\
\hline $7440-28-0$ & Thallium & $<9.49 \mathrm{E} 0$ & ug/g dry & 9.49E0 & 9/24/08 & 8123006 & PNNL-AGG-ICP-AES \\
\hline $7440-23-5$ & Sodium & $3.64 \mathrm{E} 2$ & ug/g dry & $2.25 \mathrm{E} 2$ & $9 / 24 / 08$ & $8 \mathrm{I} 23006$ & PNNL-AGG-ICP-AES \\
\hline HEIS No. & B1TP35 & \multicolumn{3}{|c|}{ Lab ID: $\quad$ 0804025-37 } & & & \\
\hline $7429-90-5$ & Aluminum & 4.91E3 & ug/g dry & $3.36 \mathrm{E} 1$ & 9/24/08 & $8 \mathrm{I} 23006$ & PNNL-AGG-ICP-AES \\
\hline $7440-39-3$ & Barium & $5.46 \mathrm{E} 1$ & ug/g dry & $8.00 \mathrm{E}-1$ & 9/24/08 & $8 \mathrm{I} 23006$ & PNNL-AGG-ICP-AES \\
\hline $7440-70-2$ & Calcium & 6.82E3 & ug/g dry & 3.12E1 & $9 / 24 / 08$ & $8 \mathrm{I} 23006$ & PNNL-AGG-ICP-AES \\
\hline 7439-89-6 & Iron & $1.05 \mathrm{E} 4$ & ug/g dry & 8.54E1 & 9/24/08 & $8 \mathrm{I} 23006$ & PNNL-AGG-ICP-AES \\
\hline 7440-09-7 & Potassium & $9.71 \mathrm{E} 2$ & ug/g dry & $1.98 \mathrm{E} 1$ & 9/24/08 & $8 \mathrm{I} 23006$ & PNNL-AGG-ICP-AES \\
\hline 7439-95-4 & Magnesium & 3.93E3 & ug/g dry & $2.64 \mathrm{E} 1$ & $9 / 24 / 08$ & $8 \mathrm{I} 23006$ & PNNL-AGG-ICP-AES \\
\hline $7439-96-5$ & Manganese & 2.25E2 & ug/g dry & $2.50 \mathrm{E}-1$ & 9/24/08 & $8 \mathrm{I} 23006$ & PNNL-AGG-ICP-AES \\
\hline $7440-02-0$ & Nickel & $1.21 \mathrm{E} 1$ & ug/g dry & $1.72 \mathrm{E} 0$ & 9/24/08 & $8 \mathrm{I} 23006$ & PNNL-AGG-ICP-AES \\
\hline $7440-28-0$ & Thallium & $<9.44 \mathrm{E} 0$ & ug/g dry & $9.44 \mathrm{E} 0$ & 9/24/08 & $8 \mathrm{I} 23006$ & PNNL-AGG-ICP-AES \\
\hline $7440-23-5$ & Sodium & $<2.24 \mathrm{E} 2$ & ug/g dry & $2.24 \mathrm{E} 2$ & 9/24/08 & 8123006 & PNNL-AGG-ICP-AES \\
\hline HEIS No. & B1TP40 & \multicolumn{3}{|c|}{ Lab ID: $\quad$ 0804025-48 } & & & \\
\hline $7429-90-5$ & Aluminum & 5.39E3 & ug/g dry & 3.38E1 & 9/24/08 & $8 \mathrm{I} 23006$ & PNNL-AGG-ICP-AES \\
\hline $7440-39-3$ & Barium & $4.72 \mathrm{E} 1$ & ug/g dry & 8.05E-1 & 9/24/08 & 8123006 & PNNL-AGG-ICP-AES \\
\hline $7440-70-2$ & Calcium & $6.50 \mathrm{E} 3$ & ug/g dry & $3.14 \mathrm{E} 1$ & $9 / 24 / 08$ & $8 \mathrm{I} 23006$ & PNNL-AGG-ICP-AES \\
\hline 7439-89-6 & Iron & $1.06 \mathrm{E} 4$ & ug/g dry & 8.59E1 & $9 / 24 / 08$ & $8 \mathrm{I} 23006$ & PNNL-AGG-ICP-AES \\
\hline $7440-09-7$ & Potassium & $1.20 \mathrm{E} 3$ & ug/g dry & 1.99E1 & $9 / 24 / 08$ & $8 \mathrm{I} 23006$ & PNNL-AGG-ICP-AES \\
\hline 7439-95-4 & Magnesium & 4.07E3 & ug/g dry & $2.66 \mathrm{E} 1$ & $9 / 24 / 08$ & $8 \mathrm{I} 23006$ & PNNL-AGG-ICP-AES \\
\hline $7439-96-5$ & Manganese & $2.25 \mathrm{E} 2$ & ug/g dry & $2.52 \mathrm{E}-1$ & $9 / 24 / 08$ & $8 \mathrm{I} 23006$ & PNNL-AGG-ICP-AES \\
\hline $7440-02-0$ & Nickel & $1.22 \mathrm{E} 1$ & ug/g dry & 1.73E0 & 9/24/08 & $8 \mathrm{I} 23006$ & PNNL-AGG-ICP-AES \\
\hline $7440-28-0$ & Thallium & $<9.50 \mathrm{E} 0$ & ug/g dry & $9.50 \mathrm{E} 0$ & $9 / 24 / 08$ & $8 \mathrm{I} 23006$ & PNNL-AGG-ICP-AES \\
\hline $7440-23-5$ & Sodium & $<2.25 \mathrm{E} 2$ & ug/g dry & $2.25 \mathrm{E} 2$ & 9/24/08 & $8 \mathrm{I} 23006$ & PNNL-AGG-ICP-AES \\
\hline HEIS No. & B1TP42 & \multicolumn{3}{|c|}{ Lab ID: $\quad$ 0804025-50 } & & & \\
\hline $7429-90-5$ & Aluminum & $4.58 \mathrm{E} 3$ & ug/g dry & 3.27E1 & $9 / 24 / 08$ & $8 \mathrm{I} 23006$ & PNNL-AGG-ICP-AES \\
\hline
\end{tabular}


Total Metals by PNNL-AGG-ICP-AES/Acid Extract

\begin{tabular}{|c|c|c|c|c|c|c|c|}
\hline CAS \# & Analyte & Results & Units & EQL & Analyzed & Batch & Method \\
\hline HEIS No. & B1TP42 & \multicolumn{3}{|c|}{ Lab ID: $\quad 0804025-50$} & & & \\
\hline $7440-39-3$ & Barium & 3.77E1 & ug/g dry & $7.79 \mathrm{E}-1$ & 9/24/08 & $8 \mathrm{I} 23006$ & PNNL-AGG-ICP-AES \\
\hline $7440-70-2$ & Calcium & 6.25E3 & ug/g dry & 3.04E1 & 9/24/08 & $8 \mathrm{I} 23006$ & PNNL-AGG-ICP-AES \\
\hline 7439-89-6 & Iron & $9.08 \mathrm{E} 3$ & ug/g dry & 8.31E1 & $9 / 24 / 08$ & $8 \mathrm{I} 23006$ & PNNL-AGG-ICP-AES \\
\hline 7440-09-7 & Potassium & $1.02 \mathrm{E} 3$ & ug/g dry & $1.93 \mathrm{E} 1$ & $9 / 24 / 08$ & $8 \mathrm{I} 23006$ & PNNL-AGG-ICP-AES \\
\hline 7439-95-4 & Magnesium & $3.55 \mathrm{E} 3$ & ug/g dry & $2.57 \mathrm{E} 1$ & $9 / 24 / 08$ & $8 \mathrm{I} 23006$ & PNNL-AGG-ICP-AES \\
\hline 7439-96-5 & Manganese & $2.04 \mathrm{E} 2$ & ug/g dry & 2.43E-1 & 9/24/08 & $8 \mathrm{I} 23006$ & PNNL-AGG-ICP-AES \\
\hline $7440-02-0$ & Nickel & $1.12 \mathrm{E} 1$ & ug/g dry & 1.67E0 & $9 / 24 / 08$ & $8 \mathrm{I} 23006$ & PNNL-AGG-ICP-AES \\
\hline $7440-28-0$ & Thallium & $<9.19 \mathrm{E} 0$ & ug/g dry & 9.19E0 & 9/24/08 & $8 \mathrm{I} 23006$ & PNNL-AGG-ICP-AES \\
\hline $7440-23-5$ & Sodium & $2.50 \mathrm{E} 2$ & ug/g dry & 2.18E2 & $9 / 24 / 08$ & 8123006 & PNNL-AGG-ICP-AES \\
\hline HEIS No. & В1TP43 & \multicolumn{3}{|c|}{ Lab ID: $\quad$ 0804025-51 } & & & \\
\hline $7429-90-5$ & Aluminum & 5.57E3 & ug/g dry & 3.66E1 & $9 / 24 / 08$ & $8 \mathrm{I} 23006$ & PNNL-AGG-ICP-AES \\
\hline $7440-39-3$ & Barium & 4.33E1 & ug/g dry & $8.72 \mathrm{E}-1$ & $9 / 24 / 08$ & $8 \mathrm{I} 23006$ & PNNL-AGG-ICP-AES \\
\hline $7440-70-2$ & Calcium & 7.11E3 & ug/g dry & $3.40 \mathrm{E} 1$ & $9 / 24 / 08$ & $8 \mathrm{I} 23006$ & PNNL-AGG-ICP-AES \\
\hline 7439-89-6 & Iron & $1.10 \mathrm{E} 4$ & ug/g dry & $9.30 \mathrm{E} 1$ & $9 / 24 / 08$ & $8 \mathrm{I} 23006$ & PNNL-AGG-ICP-AES \\
\hline 7440-09-7 & Potassium & $1.43 \mathrm{E} 3$ & ug/g dry & $2.16 \mathrm{E} 1$ & 9/24/08 & $8 \mathrm{I} 23006$ & PNNL-AGG-ICP-AES \\
\hline 7439-95-4 & Magnesium & 3.84E3 & ug/g dry & $2.88 \mathrm{E} 1$ & $9 / 24 / 08$ & $8 \mathrm{I} 23006$ & PNNL-AGG-ICP-AES \\
\hline 7439-96-5 & Manganese & $2.46 \mathrm{E} 2$ & ug/g dry & $2.72 \mathrm{E}-1$ & 9/24/08 & $8 \mathrm{I} 23006$ & PNNL-AGG-ICP-AES \\
\hline 7440-02-0 & Nickel & $1.26 \mathrm{E} 1$ & ug/g dry & $1.87 \mathrm{E} 0$ & $9 / 24 / 08$ & $8 \mathrm{I} 23006$ & PNNL-AGG-ICP-AES \\
\hline $7440-28-0$ & Thallium & $<1.03 \mathrm{E} 1$ & ug/g dry & 1.03E1 & $9 / 24 / 08$ & $8 \mathrm{I} 23006$ & PNNL-AGG-ICP-AES \\
\hline $7440-23-5$ & Sodium & $1.75 \mathrm{E} 3$ & ug/g dry & $2.44 \mathrm{E} 2$ & $9 / 24 / 08$ & $8 \mathrm{I} 23006$ & PNNL-AGG-ICP-AES \\
\hline HEIS No. & B1TP45 & \multicolumn{3}{|c|}{ Lab ID: $\quad$ 0804025-53 } & & & \\
\hline $7429-90-5$ & Aluminum & $1.29 \mathrm{E} 3$ & ug/g dry & 8.36E0 & $9 / 24 / 08$ & $8 \mathrm{I} 23007$ & PNNL-AGG-ICP-AES \\
\hline $7440-39-3$ & Barium & 4.77E1 & ug/g dry & 7.97E-1 & $9 / 24 / 08$ & $8 \mathrm{I} 23007$ & PNNL-AGG-ICP-AES \\
\hline $7440-70-2$ & Calcium & $6.45 \mathrm{E} 3$ & ug/g dry & $3.11 \mathrm{E} 1$ & $9 / 24 / 08$ & $8 \mathrm{I} 23007$ & PNNL-AGG-ICP-AES \\
\hline 7439-89-6 & Iron & $1.39 \mathrm{E} 4$ & ug/g dry & 8.50E1 & $9 / 24 / 08$ & $8 \mathrm{I} 23007$ & PNNL-AGG-ICP-AES \\
\hline 7440-09-7 & Potassium & 8.77E2 & ug/g dry & $1.97 \mathrm{E} 1$ & $9 / 24 / 08$ & $8 \mathrm{I} 23007$ & PNNL-AGG-ICP-AES \\
\hline 7439-95-4 & Magnesium & $3.60 \mathrm{E} 3$ & ug/g dry & 2.63E1 & $9 / 24 / 08$ & $8 \mathrm{I} 23007$ & PNNL-AGG-ICP-AES \\
\hline 7439-96-5 & Manganese & $2.43 \mathrm{E} 2$ & ug/g dry & $2.49 \mathrm{E}-1$ & $9 / 24 / 08$ & $8 \mathrm{I} 23007$ & PNNL-AGG-ICP-AES \\
\hline $7440-02-0$ & Nickel & 7.75E0 & ug/g dry & $1.71 \mathrm{E} 0$ & 9/24/08 & $8 \mathrm{I} 23007$ & PNNL-AGG-ICP-AES \\
\hline $7440-28-0$ & Thallium & $<9.40 \mathrm{E} 0$ & ug/g dry & $9.40 \mathrm{E} 0$ & $9 / 24 / 08$ & $8 \mathrm{I} 23007$ & PNNL-AGG-ICP-AES \\
\hline $7440-23-5$ & Sodium & $1.30 \mathrm{E} 3$ & ug/g dry & 2.23E2 & $9 / 24 / 08$ & $8 \mathrm{I} 23007$ & PNNL-AGG-ICP-AES \\
\hline HEIS No. & B1TP47 & \multicolumn{3}{|c|}{ Lab ID: $\quad 0804025-57$} & & & \\
\hline $7429-90-5$ & Aluminum & $5.08 \mathrm{E} 3$ & ug/g dry & 3.32E1 & 9/24/08 & $8 \mathrm{I} 23007$ & PNNL-AGG-ICP-AES \\
\hline $7440-39-3$ & Barium & $5.02 \mathrm{E} 1$ & ug/g dry & $7.90 \mathrm{E}-1$ & 9/24/08 & $8 \mathrm{I} 23007$ & PNNL-AGG-ICP-AES \\
\hline $7440-70-2$ & Calcium & 7.63E3 & ug/g dry & $3.08 \mathrm{E} 1$ & $9 / 24 / 08$ & $8 \mathrm{I} 23007$ & PNNL-AGG-ICP-AES \\
\hline 7439-89-6 & Iron & $1.22 \mathrm{E} 4$ & ug/g dry & 8.43E1 & $9 / 24 / 08$ & $8 \mathrm{I} 23007$ & PNNL-AGG-ICP-AES \\
\hline 7440-09-7 & Potassium & $9.52 \mathrm{E} 2$ & ug/g dry & 1.96E1 & $9 / 24 / 08$ & $8 \mathrm{I} 23007$ & PNNL-AGG-ICP-AES \\
\hline 7439-95-4 & Magnesium & 4.10E3 & ug/g dry & $2.61 \mathrm{E} 1$ & 9/24/08 & $8 \mathrm{I} 23007$ & PNNL-AGG-ICP-AES \\
\hline 7439-96-5 & Manganese & $2.27 \mathrm{E} 2$ & ug/g dry & $2.47 \mathrm{E}-1$ & $9 / 24 / 08$ & $8 \mathrm{I} 23007$ & PNNL-AGG-ICP-AES \\
\hline $7440-02-0$ & Nickel & $1.08 \mathrm{E} 1$ & ug/g dry & $1.70 \mathrm{E} 0$ & $9 / 24 / 08$ & $8 \mathrm{I} 23007$ & PNNL-AGG-ICP-AES \\
\hline $7440-28-0$ & Thallium & $<9.32 \mathrm{E} 0$ & ug/g dry & 9.32E0 & 9/24/08 & $8 \mathrm{I} 23007$ & PNNL-AGG-ICP-AES \\
\hline $7440-23-5$ & Sodium & $3.22 \mathrm{E} 2$ & ug/g dry & $2.21 \mathrm{E} 2$ & $9 / 24 / 08$ & $8 \mathrm{I} 23007$ & PNNL-AGG-ICP-AES \\
\hline HEIS No. & В1ТР49 & \multicolumn{3}{|c|}{ Lab ID: $\quad 0804025-61$} & & & \\
\hline 7429-90-5 & Aluminum & 4.99E3 & ug/g dry & 3.30E1 & $9 / 24 / 08$ & $8 \mathrm{I} 23007$ & PNNL-AGG-ICP-AES \\
\hline $7440-39-3$ & Barium & 4.78E1 & ug/g dry & $7.85 \mathrm{E}-1$ & $9 / 24 / 08$ & $8 \mathrm{I} 23007$ & PNNL-AGG-ICP-AES \\
\hline $7440-70-2$ & Calcium & $9.28 \mathrm{E} 3$ & ug/g dry & 3.06E1 & 9/24/08 & $8 \mathrm{I} 23007$ & PNNL-AGG-ICP-AES \\
\hline 7439-89-6 & Iron & $1.26 \mathrm{E} 4$ & ug/g dry & 8.38E1 & $9 / 24 / 08$ & $8 \mathrm{I} 23007$ & PNNL-AGG-ICP-AES \\
\hline 7440-09-7 & Potassium & 9.03E2 & ug/g dry & $1.94 \mathrm{E} 1$ & $9 / 24 / 08$ & $8 \mathrm{I} 23007$ & PNNL-AGG-ICP-AES \\
\hline
\end{tabular}


Total Metals by PNNL-AGG-ICP-AES/Acid Extract

\begin{tabular}{|c|c|c|c|c|c|c|c|}
\hline CAS \# & Analyte & Results & Units & EQL & Analyzed & Batch & Method \\
\hline HEIS No. & В1ТР49 & \multicolumn{3}{|c|}{ Lab ID: $\quad 0804025-61$} & & & \\
\hline 7439-95-4 & Magnesium & 4.02E3 & ug/g dry & 2.59E1 & 9/24/08 & $8 \mathrm{I} 23007$ & PNNL-AGG-ICP-AES \\
\hline 7439-96-5 & Manganese & 2.27E2 & ug/g dry & $2.45 \mathrm{E}-1$ & 9/24/08 & $8 \mathrm{I} 23007$ & PNNL-AGG-ICP-AES \\
\hline $7440-02-0$ & Nickel & 9.73E0 & ug/g dry & 1.69E0 & $9 / 24 / 08$ & $8 \mathrm{I} 23007$ & PNNL-AGG-ICP-AES \\
\hline $7440-28-0$ & Thallium & $<9.27 \mathrm{E} 0$ & ug/g dry & $9.27 \mathrm{E} 0$ & $9 / 24 / 08$ & $8 \mathrm{I} 23007$ & PNNL-AGG-ICP-AES \\
\hline $7440-23-5$ & Sodium & $<2.20 \mathrm{E} 2$ & ug/g dry & $2.20 \mathrm{E} 2$ & $9 / 24 / 08$ & $8 \mathrm{I} 23007$ & PNNL-AGG-ICP-AES \\
\hline HEIS No. & B1TP51 & \multicolumn{3}{|c|}{ Lab ID: $\quad$ 0804025-69 } & & & \\
\hline 7429-90-5 & Aluminum & $5.34 \mathrm{E} 3$ & ug/g dry & 3.46E1 & 9/24/08 & $8 \mathrm{I} 23007$ & PNNL-AGG-ICP-AES \\
\hline $7440-39-3$ & Barium & $4.80 \mathrm{E} 1$ & ug/g dry & $8.24 \mathrm{E}-1$ & $9 / 24 / 08$ & $8 \mathrm{I} 23007$ & PNNL-AGG-ICP-AES \\
\hline $7440-70-2$ & Calcium & 7.39E3 & ug/g dry & $3.21 \mathrm{E} 1$ & 9/24/08 & $8 \mathrm{I} 23007$ & PNNL-AGG-ICP-AES \\
\hline 7439-89-6 & Iron & $1.11 \mathrm{E} 4$ & ug/g dry & 8.79E1 & 9/24/08 & $8 \mathrm{I} 23007$ & PNNL-AGG-ICP-AES \\
\hline 7440-09-7 & Potassium & $1.21 \mathrm{E} 3$ & ug/g dry & 2.04E1 & $9 / 24 / 08$ & $8 \mathrm{I} 23007$ & PNNL-AGG-ICP-AES \\
\hline 7439-95-4 & Magnesium & $4.01 \mathrm{E} 3$ & ug/g dry & 2.72E1 & $9 / 24 / 08$ & $8 \mathrm{I} 23007$ & PNNL-AGG-ICP-AES \\
\hline $7439-96-5$ & Manganese & $2.27 \mathrm{E} 2$ & ug/g dry & $2.57 \mathrm{E}-1$ & $9 / 24 / 08$ & $8 \mathrm{I} 23007$ & PNNL-AGG-ICP-AES \\
\hline $7440-02-0$ & Nickel & $1.23 \mathrm{E} 1$ & ug/g dry & $1.77 \mathrm{E} 0$ & $9 / 24 / 08$ & $8 \mathrm{I} 23007$ & PNNL-AGG-ICP-AES \\
\hline $7440-28-0$ & Thallium & $<9.72 \mathrm{E} 0$ & ug/g dry & $9.72 \mathrm{E} 0$ & $9 / 24 / 08$ & $8 \mathrm{I} 23007$ & PNNL-AGG-ICP-AES \\
\hline $7440-23-5$ & Sodium & $1.65 \mathrm{E} 3$ & ug/g dry & $2.30 \mathrm{E} 2$ & $9 / 24 / 08$ & 8123007 & PNNL-AGG-ICP-AES \\
\hline HEIS No. & B1TP52 & \multicolumn{3}{|c|}{ Lab ID: $\quad 0804025-72$} & & & \\
\hline $7429-90-5$ & Aluminum & 4.78E3 & ug/g dry & 3.35E1 & $9 / 24 / 08$ & $8 \mathrm{I} 23007$ & PNNL-AGG-ICP-AES \\
\hline 7440-39-3 & Barium & 4.34E1 & ug/g dry & 7.97E-1 & $9 / 24 / 08$ & $8 \mathrm{I} 23007$ & PNNL-AGG-ICP-AES \\
\hline $7440-70-2$ & Calcium & 6.09E3 & ug/g dry & $3.11 \mathrm{E} 1$ & $9 / 24 / 08$ & $8 \mathrm{I} 23007$ & PNNL-AGG-ICP-AES \\
\hline 7439-89-6 & Iron & $1.21 \mathrm{E} 4$ & ug/g dry & 8.51E1 & 9/24/08 & $8 \mathrm{I} 23007$ & PNNL-AGG-ICP-AES \\
\hline $7440-09-7$ & Potassium & 8.69E2 & ug/g dry & $1.97 \mathrm{E} 1$ & $9 / 24 / 08$ & $8 \mathrm{I} 23007$ & PNNL-AGG-ICP-AES \\
\hline 7439-95-4 & Magnesium & 3.76E3 & ug/g dry & 2.63E1 & $9 / 24 / 08$ & $8 \mathrm{I} 23007$ & PNNL-AGG-ICP-AES \\
\hline 7439-96-5 & Manganese & 2.09E2 & ug/g dry & $2.49 \mathrm{E}-1$ & $9 / 24 / 08$ & $8 \mathrm{I} 23007$ & PNNL-AGG-ICP-AES \\
\hline $7440-02-0$ & Nickel & $9.58 \mathrm{E} 0$ & ug/g dry & $1.71 \mathrm{E} 0$ & $9 / 24 / 08$ & $8 \mathrm{I} 23007$ & PNNL-AGG-ICP-AES \\
\hline $7440-28-0$ & Thallium & $<9.41 \mathrm{E} 0$ & ug/g dry & $9.41 \mathrm{E} 0$ & $9 / 24 / 08$ & $8 \mathrm{I} 23007$ & PNNL-AGG-ICP-AES \\
\hline $7440-23-5$ & Sodium & $5.21 \mathrm{E} 2$ & ug/g dry & $2.23 \mathrm{E} 2$ & $9 / 24 / 08$ & $8 \mathrm{I} 23007$ & PNNL-AGG-ICP-AES \\
\hline HEIS No. & В1TP53 & \multicolumn{3}{|c|}{ Lab ID: $\quad$ 0804025-75 } & & & \\
\hline 7429-90-5 & Aluminum & $4.99 \mathrm{E} 3$ & ug/g dry & 3.31E1 & 9/24/08 & $8 \mathrm{I} 23007$ & PNNL-AGG-ICP-AES \\
\hline $7440-39-3$ & Barium & 4.12E1 & ug/g dry & $7.88 \mathrm{E}-1$ & 9/24/08 & $8 \mathrm{I} 23007$ & PNNL-AGG-ICP-AES \\
\hline $7440-70-2$ & Calcium & $6.32 \mathrm{E} 3$ & ug/g dry & 3.07E1 & $9 / 24 / 08$ & $8 \mathrm{I} 23007$ & PNNL-AGG-ICP-AES \\
\hline 7439-89-6 & Iron & $1.22 \mathrm{E} 4$ & ug/g dry & $8.41 \mathrm{E} 1$ & $9 / 24 / 08$ & $8 \mathrm{I} 23007$ & PNNL-AGG-ICP-AES \\
\hline 7440-09-7 & Potassium & 8.55E2 & ug/g dry & $1.95 \mathrm{E} 1$ & 9/24/08 & $8 \mathrm{I} 23007$ & PNNL-AGG-ICP-AES \\
\hline 7439-95-4 & Magnesium & $3.94 \mathrm{E} 3$ & ug/g dry & $2.60 \mathrm{E} 1$ & $9 / 24 / 08$ & $8 \mathrm{I} 23007$ & PNNL-AGG-ICP-AES \\
\hline 7439-96-5 & Manganese & $2.15 \mathrm{E} 2$ & ug/g dry & $2.46 \mathrm{E}-1$ & $9 / 24 / 08$ & $8 \mathrm{I} 23007$ & PNNL-AGG-ICP-AES \\
\hline 7440-02-0 & Nickel & $1.07 \mathrm{E} 1$ & ug/g dry & $1.69 \mathrm{E} 0$ & $9 / 24 / 08$ & $8 \mathrm{I} 23007$ & PNNL-AGG-ICP-AES \\
\hline 7440-28-0 & Thallium & $<9.30 \mathrm{E} 0$ & ug/g dry & $9.30 \mathrm{E} 0$ & $9 / 24 / 08$ & $8 \mathrm{I} 23007$ & PNNL-AGG-ICP-AES \\
\hline $7440-23-5$ & Sodium & $2.47 \mathrm{E} 2$ & ug/g dry & $2.20 \mathrm{E} 2$ & $9 / 24 / 08$ & 8123007 & PNNL-AGG-ICP-AES \\
\hline HEIS No. & B1TP55 & \multicolumn{3}{|c|}{ Lab ID: $\quad$ 0804025-81 } & & & \\
\hline $7429-90-5$ & Aluminum & 4.77E3 & ug/g dry & 3.36E1 & $9 / 24 / 08$ & $8 \mathrm{I} 23007$ & PNNL-AGG-ICP-AES \\
\hline $7440-39-3$ & Barium & $4.38 \mathrm{E} 1$ & ug/g dry & $8.01 \mathrm{E}-1$ & $9 / 24 / 08$ & $8 \mathrm{I} 23007$ & PNNL-AGG-ICP-AES \\
\hline $7440-70-2$ & Calcium & 6.37E3 & ug/g dry & 3.12E1 & 9/24/08 & $8 \mathrm{I} 23007$ & PNNL-AGG-ICP-AES \\
\hline 7439-89-6 & Iron & $1.17 \mathrm{E} 4$ & ug/g dry & $8.55 \mathrm{E} 1$ & $9 / 24 / 08$ & $8 \mathrm{I} 23007$ & PNNL-AGG-ICP-AES \\
\hline 7440-09-7 & Potassium & $9.17 \mathrm{E} 2$ & ug/g dry & $1.98 \mathrm{E} 1$ & $9 / 24 / 08$ & $8 \mathrm{I} 23007$ & PNNL-AGG-ICP-AES \\
\hline 7439-95-4 & Magnesium & 3.73E3 & ug/g dry & 2.65E1 & $9 / 24 / 08$ & $8 \mathrm{I} 23007$ & PNNL-AGG-ICP-AES \\
\hline 7439-96-5 & Manganese & $2.12 \mathrm{E} 2$ & ug/g dry & $2.50 \mathrm{E}-1$ & $9 / 24 / 08$ & 8123007 & PNNL-AGG-ICP-AES \\
\hline $7440-02-0$ & Nickel & $1.03 \mathrm{E} 1$ & ug/g dry & $1.72 \mathrm{E} 0$ & $9 / 24 / 08$ & $8 \mathrm{I} 23007$ & PNNL-AGG-ICP-AES \\
\hline $7440-28-0$ & Thallium & $<9.45 \mathrm{E} 0$ & ug/g dry & $9.45 \mathrm{E} 0$ & $9 / 24 / 08$ & $8 \mathrm{I} 23007$ & PNNL-AGG-ICP-AES \\
\hline
\end{tabular}


Total Metals by PNNL-AGG-ICP-AES/Acid Extract

\begin{tabular}{|c|c|c|c|c|c|c|c|}
\hline CAS \# & Analyte & Results & Units & EQL & Analyzed & Batch & Method \\
\hline HEIS No. & B1TP55 & \multicolumn{3}{|c|}{ Lab ID: $\quad$ 0804025-81 } & & & \\
\hline $7440-23-5$ & Sodium & $<2.24 \mathrm{E} 2$ & ug/g dry & $2.24 \mathrm{E} 2$ & $9 / 24 / 08$ & $8 \mathrm{I} 23007$ & PNNL-AGG-ICP-AES \\
\hline HEIS No. & B1TP59 & \multicolumn{3}{|c|}{ Lab ID: $\quad$ 0804025-87 } & & & \\
\hline $7429-90-5$ & Aluminum & $5.16 \mathrm{E} 3$ & ug/g dry & 3.30E1 & 9/24/08 & $8 \mathrm{I} 23007$ & PNNL-AGG-ICP-AES \\
\hline $7440-39-3$ & Barium & 4.06E1 & ug/g dry & $7.86 \mathrm{E}-1$ & $9 / 24 / 08$ & $8 \mathrm{I} 23007$ & PNNL-AGG-ICP-AES \\
\hline $7440-70-2$ & Calcium & $5.86 \mathrm{E} 3$ & ug/g dry & 3.07E1 & $9 / 24 / 08$ & $8 \mathrm{I} 23007$ & PNNL-AGG-ICP-AES \\
\hline 7439-89-6 & Iron & $1.21 \mathrm{E} 4$ & ug/g dry & 8.39E1 & 9/24/08 & $8 \mathrm{I} 23007$ & PNNL-AGG-ICP-AES \\
\hline 7440-09-7 & Potassium & 8.60E2 & ug/g dry & $1.95 \mathrm{E} 1$ & $9 / 24 / 08$ & $8 I 23007$ & PNNL-AGG-ICP-AES \\
\hline $7439-95-4$ & Magnesium & 4.12E3 & ug/g dry & $2.60 \mathrm{E} 1$ & 9/24/08 & $8 \mathrm{I} 23007$ & PNNL-AGG-ICP-AES \\
\hline 7439-96-5 & Manganese & $2.14 \mathrm{E} 2$ & ug/g dry & $2.46 \mathrm{E}-1$ & $9 / 24 / 08$ & $8 I 23007$ & PNNL-AGG-ICP-AES \\
\hline $7440-02-0$ & Nickel & $1.31 \mathrm{E} 1$ & ug/g dry & 1.69E0 & $9 / 24 / 08$ & $8 \mathrm{I} 23007$ & PNNL-AGG-ICP-AES \\
\hline $7440-28-0$ & Thallium & $<9.27 \mathrm{E} 0$ & ug/g dry & $9.27 \mathrm{E} 0$ & $9 / 24 / 08$ & $8 \mathrm{I} 23007$ & PNNL-AGG-ICP-AES \\
\hline $7440-23-5$ & Sodium & $<2.20 \mathrm{E} 2$ & ug/g dry & $2.20 \mathrm{E} 2$ & 9/24/08 & $8 \mathrm{I} 23007$ & PNNL-AGG-ICP-AES \\
\hline HEIS No. & B1TP61 & \multicolumn{3}{|c|}{ Lab ID: $\quad$ 0804025-91 } & & & \\
\hline $7429-90-5$ & Aluminum & 4.95E3 & ug/g dry & 3.32E1 & 9/24/08 & $8 \mathrm{I} 23007$ & PNNL-AGG-ICP-AES \\
\hline $7440-39-3$ & Barium & $4.20 \mathrm{E} 1$ & ug/g dry & 7.91E-1 & 9/24/08 & $8 \mathrm{I} 23007$ & PNNL-AGG-ICP-AES \\
\hline $7440-70-2$ & Calcium & $5.48 \mathrm{E} 3$ & ug/g dry & $3.08 \mathrm{E} 1$ & 9/24/08 & $8 \mathrm{I} 23007$ & PNNL-AGG-ICP-AES \\
\hline 7439-89-6 & Iron & $1.05 \mathrm{E} 4$ & ug/g dry & $8.44 \mathrm{E} 1$ & $9 / 24 / 08$ & $8 \mathrm{I} 23007$ & PNNL-AGG-ICP-AES \\
\hline $7440-09-7$ & Potassium & 8.67E2 & ug/g dry & $1.96 \mathrm{E} 1$ & 9/24/08 & $8 \mathrm{I} 23007$ & PNNL-AGG-ICP-AES \\
\hline 7439-95-4 & Magnesium & 3.71E3 & ug/g dry & $2.61 \mathrm{E} 1$ & $9 / 24 / 08$ & $8 I 23007$ & PNNL-AGG-ICP-AES \\
\hline $7439-96-5$ & Manganese & $2.01 \mathrm{E} 2$ & ug/g dry & $2.47 \mathrm{E}-1$ & 9/24/08 & $8 \mathrm{I} 23007$ & PNNL-AGG-ICP-AES \\
\hline $7440-02-0$ & Nickel & $1.14 \mathrm{E} 1$ & ug/g dry & $1.70 \mathrm{E} 0$ & 9/24/08 & $8 \mathrm{I} 23007$ & PNNL-AGG-ICP-AES \\
\hline $7440-28-0$ & Thallium & $<9.33 \mathrm{E} 0$ & ug/g dry & $9.33 \mathrm{E} 0$ & $9 / 24 / 08$ & $8 \mathrm{I} 23007$ & PNNL-AGG-ICP-AES \\
\hline $7440-23-5$ & Sodium & $<2.21 \mathrm{E} 2$ & ug/g dry & $2.21 \mathrm{E} 2$ & 9/24/08 & $8 \mathrm{I} 23007$ & PNNL-AGG-ICP-AES \\
\hline HEIS No. & B1TP62 & \multicolumn{3}{|c|}{ Lab ID: $\quad 0804025-94$} & & & \\
\hline $7429-90-5$ & Aluminum & 5.32E3 & ug/g dry & $3.35 \mathrm{E} 1$ & 9/24/08 & $8 \mathrm{I} 23007$ & PNNL-AGG-ICP-AES \\
\hline $7440-39-3$ & Barium & $5.41 \mathrm{E} 1$ & ug/g dry & $7.98 \mathrm{E}-1$ & 9/24/08 & $8 \mathrm{I} 23007$ & PNNL-AGG-ICP-AES \\
\hline $7440-70-2$ & Calcium & 6.63E3 & ug/g dry & $3.11 \mathrm{E} 1$ & 9/24/08 & $8 \mathrm{I} 23007$ & PNNL-AGG-ICP-AES \\
\hline 7439-89-6 & Iron & $1.38 \mathrm{E} 4$ & ug/g dry & $8.51 \mathrm{E} 1$ & $9 / 24 / 08$ & $8 \mathrm{I} 23007$ & PNNL-AGG-ICP-AES \\
\hline $7440-09-7$ & Potassium & 9.39E2 & ug/g dry & $1.98 \mathrm{E} 1$ & 9/24/08 & $8 \mathrm{I} 23007$ & PNNL-AGG-ICP-AES \\
\hline $7439-95-4$ & Magnesium & 3.76E3 & ug/g dry & 2.63E1 & 9/24/08 & $8 \mathrm{I} 23007$ & PNNL-AGG-ICP-AES \\
\hline 7439-96-5 & Manganese & $2.44 \mathrm{E} 2$ & ug/g dry & 2.49E-1 & $9 / 24 / 08$ & $8 I 23007$ & PNNL-AGG-ICP-AES \\
\hline $7440-02-0$ & Nickel & $9.14 \mathrm{E} 0$ & ug/g dry & $1.71 \mathrm{E} 0$ & 9/24/08 & $8 \mathrm{I} 23007$ & PNNL-AGG-ICP-AES \\
\hline $7440-28-0$ & Thallium & $<9.41 \mathrm{E} 0$ & ug/g dry & $9.41 \mathrm{E} 0$ & $9 / 24 / 08$ & $8 \mathrm{I} 23007$ & PNNL-AGG-ICP-AES \\
\hline $7440-23-5$ & Sodium & $5.46 \mathrm{E} 2$ & ug/g dry & 2.23E2 & $9 / 24 / 08$ & $8 \mathrm{I} 23007$ & PNNL-AGG-ICP-AES \\
\hline HEIS No. & B1TP64 & \multicolumn{3}{|c|}{ Lab ID: $\quad$ 0804025-AA } & & & \\
\hline $7429-90-5$ & Aluminum & 4.83E3 & ug/g dry & 3.39E1 & 9/24/08 & $8 \mathrm{I} 23007$ & PNNL-AGG-ICP-AES \\
\hline $7440-39-3$ & Barium & 4.67E1 & ug/g dry & 8.06E-1 & 9/24/08 & $8 \mathrm{I} 23007$ & PNNL-AGG-ICP-AES \\
\hline $7440-70-2$ & Calcium & $6.00 \mathrm{E} 3$ & ug/g dry & $3.14 \mathrm{E} 1$ & 9/24/08 & $8 \mathrm{I} 23007$ & PNNL-AGG-ICP-AES \\
\hline 7439-89-6 & Iron & $1.29 \mathrm{E} 4$ & ug/g dry & $8.60 \mathrm{E} 1$ & $9 / 24 / 08$ & $8 \mathrm{I} 23007$ & PNNL-AGG-ICP-AES \\
\hline 7440-09-7 & Potassium & $9.31 \mathrm{E} 2$ & ug/g dry & $2.00 \mathrm{E} 1$ & $9 / 24 / 08$ & $8 \mathrm{I} 23007$ & PNNL-AGG-ICP-AES \\
\hline $7439-95-4$ & Magnesium & 3.81E3 & ug/g dry & $2.66 \mathrm{E} 1$ & $9 / 24 / 08$ & $8 \mathrm{I} 23007$ & PNNL-AGG-ICP-AES \\
\hline $7439-96-5$ & Manganese & 2.23E2 & ug/g dry & 2.52E-1 & $9 / 24 / 08$ & $8 \mathrm{I} 23007$ & PNNL-AGG-ICP-AES \\
\hline $7440-02-0$ & Nickel & $9.31 \mathrm{E} 0$ & ug/g dry & $1.73 \mathrm{E} 0$ & $9 / 24 / 08$ & $8 \mathrm{I} 23007$ & PNNL-AGG-ICP-AES \\
\hline $7440-28-0$ & Thallium & $<9.51 \mathrm{E} 0$ & ug/g dry & $9.51 \mathrm{E} 0$ & 9/24/08 & $8 \mathrm{I} 23007$ & PNNL-AGG-ICP-AES \\
\hline $7440-23-5$ & Sodium & $<2.26 \mathrm{E} 2$ & ug/g dry & $2.26 \mathrm{E} 2$ & 9/24/08 & $8 \mathrm{I} 23007$ & PNNL-AGG-ICP-AES \\
\hline HEIS No. & B1TP67 & \multicolumn{3}{|c|}{ Lab ID: $\quad$ 0804025-AI } & & & \\
\hline $7429-90-5$ & Aluminum & $5.15 \mathrm{E} 3$ & ug/g dry & $3.36 \mathrm{E} 1$ & 9/24/08 & $8 \mathrm{I} 23007$ & PNNL-AGG-ICP-AES \\
\hline $7440-39-3$ & Barium & $4.96 \mathrm{E} 1$ & ug/g dry & $8.00 \mathrm{E}-1$ & $9 / 24 / 08$ & $8 \mathrm{I} 23007$ & PNNL-AGG-ICP-AES \\
\hline
\end{tabular}


Total Metals by PNNL-AGG-ICP-AES/Acid Extract

\begin{tabular}{|c|c|c|c|c|c|c|c|}
\hline CAS \# & Analyte & Results & Units & EQL & Analyzed & Batch & Method \\
\hline HEIS No. & B1TP67 & \multicolumn{3}{|c|}{ Lab ID: $\quad$ 0804025-AI } & & & \\
\hline $7440-70-2$ & Calcium & $5.91 \mathrm{E} 3$ & ug/g dry & 3.12E1 & $9 / 24 / 08$ & $8 \mathrm{I} 23007$ & PNNL-AGG-ICP-AES \\
\hline 7439-89-6 & Iron & $1.32 \mathrm{E} 4$ & ug/g dry & $8.54 \mathrm{E} 1$ & $9 / 24 / 08$ & $8 \mathrm{I} 23007$ & PNNL-AGG-ICP-AES \\
\hline 7440-09-7 & Potassium & 9.59E2 & ug/g dry & $1.98 \mathrm{E} 1$ & $9 / 24 / 08$ & $8 \mathrm{I} 23007$ & PNNL-AGG-ICP-AES \\
\hline 7439-95-4 & Magnesium & 3.73E3 & ug/g dry & $2.64 \mathrm{E} 1$ & $9 / 24 / 08$ & $8 \mathrm{I} 23007$ & PNNL-AGG-ICP-AES \\
\hline 7439-96-5 & Manganese & 2.17E2 & ug/g dry & $2.50 \mathrm{E}-1$ & $9 / 24 / 08$ & 8I23007 & PNNL-AGG-ICP-AES \\
\hline $7440-02-0$ & Nickel & $1.02 \mathrm{E} 1$ & ug/g dry & $1.72 \mathrm{E} 0$ & $9 / 24 / 08$ & $8 \mathrm{I} 23007$ & PNNL-AGG-ICP-AES \\
\hline $7440-28-0$ & Thallium & $<9.44 \mathrm{E} 0$ & ug/g dry & $9.44 \mathrm{E} 0$ & $9 / 24 / 08$ & $8 \mathrm{I} 23007$ & PNNL-AGG-ICP-AES \\
\hline $7440-23-5$ & Sodium & $<2.24 \mathrm{E} 2$ & ug/g dry & $2.24 \mathrm{E} 2$ & $9 / 24 / 08$ & $8 \mathrm{I} 23007$ & PNNL-AGG-ICP-AES \\
\hline HEIS No. & B1TP69 & \multicolumn{3}{|c|}{ Lab ID: $\quad$ 0804025-AP } & & & \\
\hline $7429-90-5$ & Aluminum & 4.99E3 & ug/g dry & 3.38E1 & $9 / 24 / 08$ & $8 \mathrm{I} 23007$ & PNNL-AGG-ICP-AES \\
\hline $7440-39-3$ & Barium & 4.64E1 & ug/g dry & 8.05E-1 & $9 / 24 / 08$ & $8 \mathrm{I} 23007$ & PNNL-AGG-ICP-AES \\
\hline $7440-70-2$ & Calcium & $6.08 \mathrm{E} 3$ & ug/g dry & $3.14 \mathrm{E} 1$ & $9 / 24 / 08$ & $8 \mathrm{I} 23007$ & PNNL-AGG-ICP-AES \\
\hline 7439-89-6 & Iron & $1.33 \mathrm{E} 4$ & ug/g dry & $8.59 \mathrm{E} 1$ & $9 / 24 / 08$ & 8I23007 & PNNL-AGG-ICP-AES \\
\hline $7440-09-7$ & Potassium & $9.07 \mathrm{E} 2$ & ug/g dry & 1.99E1 & $9 / 24 / 08$ & $8 \mathrm{I} 23007$ & PNNL-AGG-ICP-AES \\
\hline 7439-95-4 & Magnesium & $3.49 \mathrm{E} 3$ & ug/g dry & $2.66 \mathrm{E} 1$ & $9 / 24 / 08$ & $8 \mathrm{I} 23007$ & PNNL-AGG-ICP-AES \\
\hline $7439-96-5$ & Manganese & $2.17 \mathrm{E} 2$ & ug/g dry & $2.52 \mathrm{E}-1$ & $9 / 24 / 08$ & $8 \mathrm{I} 23007$ & PNNL-AGG-ICP-AES \\
\hline $7440-02-0$ & Nickel & $1.02 \mathrm{E} 1$ & ug/g dry & 1.73E0 & $9 / 24 / 08$ & $8 \mathrm{I} 23007$ & PNNL-AGG-ICP-AES \\
\hline $7440-28-0$ & Thallium & $<9.50 \mathrm{E} 0$ & ug/g dry & $9.50 \mathrm{E} 0$ & $9 / 24 / 08$ & $8 \mathrm{I} 23007$ & PNNL-AGG-ICP-AES \\
\hline $7440-23-5$ & Sodium & $2.55 \mathrm{E} 2$ & ug/g dry & $2.25 \mathrm{E} 2$ & $9 / 24 / 08$ & $8 \mathrm{I} 23007$ & PNNL-AGG-ICP-AES \\
\hline HEIS No. & B1TP82 & \multicolumn{3}{|c|}{ Lab ID: $\quad$ 0804025-BZ } & & & \\
\hline $7429-90-5$ & Aluminum & 5.63E3 & ug/g dry & 3.38E1 & $9 / 24 / 08$ & $8 \mathrm{I} 23007$ & PNNL-AGG-ICP-AES \\
\hline $7440-39-3$ & Barium & $5.05 \mathrm{E} 1$ & ug/g dry & 8.04E-1 & $9 / 24 / 08$ & $8 \mathrm{I} 23007$ & PNNL-AGG-ICP-AES \\
\hline $7440-70-2$ & Calcium & $6.28 \mathrm{E} 3$ & ug/g dry & $3.14 \mathrm{E} 1$ & $9 / 24 / 08$ & $8 \mathrm{I} 23007$ & PNNL-AGG-ICP-AES \\
\hline 7439-89-6 & Iron & $1.32 \mathrm{E} 4$ & ug/g dry & $8.58 \mathrm{E} 1$ & $9 / 24 / 08$ & $8 \mathrm{I} 23007$ & PNNL-AGG-ICP-AES \\
\hline 7440-09-7 & Potassium & $1.10 \mathrm{E} 3$ & ug/g dry & $1.99 \mathrm{E} 1$ & $9 / 24 / 08$ & $8 \mathrm{I} 23007$ & PNNL-AGG-ICP-AES \\
\hline $7439-95-4$ & Magnesium & 3.65E3 & ug/g dry & $2.66 \mathrm{E} 1$ & $9 / 24 / 08$ & $8 \mathrm{I} 23007$ & PNNL-AGG-ICP-AES \\
\hline 7439-96-5 & Manganese & $2.24 \mathrm{E} 2$ & ug/g dry & $2.51 \mathrm{E}-1$ & $9 / 24 / 08$ & $8 \mathrm{I} 23007$ & PNNL-AGG-ICP-AES \\
\hline $7440-02-0$ & Nickel & $1.00 \mathrm{E} 1$ & ug/g dry & 1.73Е0 & $9 / 24 / 08$ & $8 \mathrm{I} 23007$ & PNNL-AGG-ICP-AES \\
\hline $7440-28-0$ & Thallium & $<9.49 \mathrm{E} 0$ & ug/g dry & $9.49 \mathrm{E} 0$ & $9 / 24 / 08$ & $8 \mathrm{I} 23007$ & PNNL-AGG-ICP-AES \\
\hline $7440-23-5$ & Sodium & 2.49E2 & ug/g dry & $2.25 \mathrm{E} 2$ & $9 / 24 / 08$ & $8 \mathrm{I} 23007$ & PNNL-AGG-ICP-AES \\
\hline HEIS No. & В1ТР93 & \multicolumn{3}{|c|}{ Lab ID: $\quad$ 0804025-DL } & & & \\
\hline $7429-90-5$ & Aluminum & $3.54 \mathrm{E} 3$ & ug/g dry & 8.86E0 & $9 / 24 / 08$ & $8 \mathrm{I} 23007$ & PNNL-AGG-ICP-AES \\
\hline $7440-39-3$ & Barium & $4.40 \mathrm{E} 1$ & ug/g dry & 8.44E-1 & $9 / 24 / 08$ & $8 \mathrm{I} 23007$ & PNNL-AGG-ICP-AES \\
\hline $7440-70-2$ & Calcium & 3.91E3 & ug/g dry & $3.29 \mathrm{E} 1$ & $9 / 24 / 08$ & $8 \mathrm{I} 23007$ & PNNL-AGG-ICP-AES \\
\hline 7439-89-6 & Iron & $1.09 \mathrm{E} 4$ & ug/g dry & $9.00 \mathrm{E} 1$ & $9 / 24 / 08$ & $8 \mathrm{I} 23007$ & PNNL-AGG-ICP-AES \\
\hline 7440-09-7 & Potassium & 4.17E2 & ug/g dry & 2.09E1 & $9 / 24 / 08$ & $8 \mathrm{I} 23007$ & PNNL-AGG-ICP-AES \\
\hline 7439-95-4 & Magnesium & $1.74 \mathrm{E} 3$ & ug/g dry & $6.97 \mathrm{E} 0$ & $9 / 24 / 08$ & $8 \mathrm{I} 23007$ & PNNL-AGG-ICP-AES \\
\hline $7439-96-5$ & Manganese & $1.48 \mathrm{E} 2$ & ug/g dry & $2.64 \mathrm{E}-1$ & $9 / 24 / 08$ & $8 \mathrm{I} 23007$ & PNNL-AGG-ICP-AES \\
\hline $7440-02-0$ & Nickel & 4.35E0 & ug/g dry & $1.81 \mathrm{E} 0$ & $9 / 24 / 08$ & $8 \mathrm{I} 23007$ & PNNL-AGG-ICP-AES \\
\hline $7440-28-0$ & Thallium & $<9.95 \mathrm{E} 0$ & ug/g dry & $9.95 \mathrm{E} 0$ & $9 / 24 / 08$ & $8 \mathrm{I} 23007$ & PNNL-AGG-ICP-AES \\
\hline $7440-23-5$ & Sodium & $3.20 \mathrm{E} 2$ & ug/g dry & $2.36 \mathrm{E} 2$ & $9 / 24 / 08$ & $8 \mathrm{I} 23007$ & PNNL-AGG-ICP-AES \\
\hline HEIS No. & B1TP94 & \multicolumn{3}{|c|}{ Lab ID: $\quad$ 0804025-DM } & & & \\
\hline $7429-90-5$ & Aluminum & 4.66E3 & ug/g dry & 8.61E0 & $9 / 24 / 08$ & 8I23007 & PNNL-AGG-ICP-AES \\
\hline $7440-39-3$ & Barium & 5.63E1 & ug/g dry & $8.20 \mathrm{E}-1$ & 9/24/08 & $8 \mathrm{I} 23007$ & PNNL-AGG-ICP-AES \\
\hline $7440-70-2$ & Calcium & 4.05E3 & ug/g dry & $3.20 \mathrm{E} 1$ & $9 / 24 / 08$ & $8 \mathrm{I} 23007$ & PNNL-AGG-ICP-AES \\
\hline 7439-89-6 & Iron & $1.47 \mathrm{E} 4$ & ug/g dry & 8.75E1 & $9 / 24 / 08$ & 8I23007 & PNNL-AGG-ICP-AES \\
\hline 7440-09-7 & Potassium & $5.60 \mathrm{E} 2$ & ug/g dry & 2.03E1 & $9 / 24 / 08$ & $8 \mathrm{I} 23007$ & PNNL-AGG-ICP-AES \\
\hline 7439-95-4 & Magnesium & $2.78 \mathrm{E} 3$ & ug/g dry & $6.77 \mathrm{E} 0$ & $9 / 24 / 08$ & $8 \mathrm{I} 23007$ & PNNL-AGG-ICP-AES \\
\hline
\end{tabular}


Total Metals by PNNL-AGG-ICP-AES/Acid Extract

\begin{tabular}{|c|c|c|c|c|c|c|c|}
\hline CAS \# & Analyte & Results & Units & EQL & Analyzed & Batch & Method \\
\hline HEIS No. & B1TP94 & & b ID: & 0804025-DM & & & \\
\hline 7439-96-5 & Manganese & 1.99E2 & ug/g dry & $2.56 \mathrm{E}-1$ & $9 / 24 / 08$ & $8 \mathrm{I} 23007$ & PNNL-AGG-ICP-AES \\
\hline 7440-02-0 & Nickel & 8.17E0 & ug/g dry & 1.76E0 & $9 / 24 / 08$ & $8 \mathrm{I} 23007$ & PNNL-AGG-ICP-AES \\
\hline 7440-28-0 & Thallium & $<9.67 \mathrm{E} 0$ & ug/g dry & $9.67 \mathrm{E} 0$ & $9 / 24 / 08$ & $8 \mathrm{I} 23007$ & PNNL-AGG-ICP-AES \\
\hline $7440-23-5$ & Sodium & $4.11 \mathrm{E} 2$ & ug/g dry & $2.29 \mathrm{E} 2$ & $9 / 24 / 08$ & 8123007 & PNNL-AGG-ICP-AES \\
\hline
\end{tabular}




\section{Radionuclides by ICP-MS/Acid Extract}

\begin{tabular}{|c|c|c|c|c|c|c|c|}
\hline CAS \# & Analyte & Results & Units & EQL & Analyzed & Batch & Method \\
\hline HEIS No. & В1TP24 & \multicolumn{3}{|c|}{ Lab ID: $\quad$ 0804025-04 } & & & \\
\hline \multirow[t]{2}{*}{$14133-76-7$} & Technetium-99 & $<4.14 \mathrm{E}-3$ & ug/g dry & $4.14 \mathrm{E}-3$ & $9 / 22 / 08$ & 8I22001 & PNNL-AGG-415 \\
\hline & Uranium 238 & 2.24E1 & ug/g dry & $3.00 \mathrm{E}-2$ & $9 / 22 / 08$ & 8I22001 & PNNL-AGG-415 \\
\hline HEIS No. & В1TP28 & \multicolumn{3}{|c|}{ Lab ID: $\quad$ 0804025-07 } & & & \\
\hline \multirow[t]{2}{*}{ 14133-76-7 } & Technetium-99 & $<4.02 \mathrm{E}-3$ & ug/g dry & 4.02E-3 & $9 / 22 / 08$ & 8I22001 & PNNL-AGG-415 \\
\hline & Uranium 238 & $3.52 \mathrm{E}-1$ & ug/g dry & $2.92 \mathrm{E}-2$ & $9 / 22 / 08$ & $8 \mathrm{I} 22001$ & PNNL-AGG-415 \\
\hline HEIS No. & В1TP29 & \multicolumn{3}{|c|}{ Lab ID: $\quad$ 0804025-08 } & & & \\
\hline \multirow[t]{2}{*}{$14133-76-7$} & Technetium-99 & $<3.99 \mathrm{E}-3$ & ug/g dry & $3.99 \mathrm{E}-3$ & $9 / 22 / 08$ & 8I22001 & PNNL-AGG-415 \\
\hline & Uranium 238 & 3.37E-1 & ug/g dry & $2.90 \mathrm{E}-2$ & $9 / 22 / 08$ & 8I22001 & PNNL-AGG-415 \\
\hline \multirow{3}{*}{$\begin{array}{l}\text { HEIS No. } \\
14133-76-7\end{array}$} & B1TP30 & \multicolumn{3}{|c|}{ Lab ID: $\quad$ 0804025-09 } & & & \\
\hline & Technetium-99 & $<4.11 \mathrm{E}-3$ & ug/g dry & 4.11E-3 & $9 / 22 / 08$ & 8I22001 & PNNL-AGG-415 \\
\hline & Uranium 238 & $3.45 \mathrm{E}-1$ & ug/g dry & $2.99 \mathrm{E}-2$ & $9 / 22 / 08$ & 8I22001 & PNNL-AGG-415 \\
\hline \multirow{3}{*}{$\begin{array}{l}\text { HEIS No. } \\
14133-76-7\end{array}$} & B1TNV9 & \multicolumn{3}{|c|}{ Lab ID: $\quad 0804025-10$} & & & \\
\hline & Technetium-99 & $<3.99 \mathrm{E}-3$ & ug/g dry & 3.99E-3 & $9 / 22 / 08$ & 8I22001 & PNNL-AGG-415 \\
\hline & Uranium 238 & 3.31E-1 & ug/g dry & $2.90 \mathrm{E}-2$ & $9 / 22 / 08$ & 8I22001 & PNNL-AGG-415 \\
\hline \multirow{3}{*}{$\begin{array}{l}\text { HEIS No. } \\
14133-76-7\end{array}$} & В1TP22 & \multicolumn{3}{|c|}{ Lab ID: $\quad 0804025-13$} & & & \\
\hline & Technetium-99 & $<4.03 \mathrm{E}-3$ & ug/g dry & 4.03E-3 & $9 / 22 / 08$ & 8I22001 & PNNL-AGG-415 \\
\hline & Uranium 238 & $4.48 \mathrm{E}-1$ & ug/g dry & $2.92 \mathrm{E}-2$ & 9/22/08 & 8I22001 & PNNL-AGG-415 \\
\hline \multirow{3}{*}{$\begin{array}{l}\text { HEIS No. } \\
14133-76-7\end{array}$} & В1TP26 & \multicolumn{3}{|c|}{ Lab ID: $\quad$ 0804025-16 } & & & \\
\hline & Technetium-99 & $<4.03 \mathrm{E}-3$ & ug/g dry & 4.03E-3 & $9 / 22 / 08$ & 8I22001 & PNNL-AGG-415 \\
\hline & Uranium 238 & 3.33E-1 & ug/g dry & 2.93E-2 & $9 / 22 / 08$ & 8I22001 & PNNL-AGG-415 \\
\hline \multirow{3}{*}{$\begin{array}{l}\text { HEIS No. } \\
\text { 14133-76-7 }\end{array}$} & B1TNW0 & \multicolumn{3}{|c|}{ Lab ID: $\quad$ 0804025-17 } & & & \\
\hline & Technetium-99 & 8.34E-3 & ug/g dry & 4.06E-3 & $9 / 22 / 08$ & 8I22001 & PNNL-AGG-415 \\
\hline & Uranium 238 & $2.95 \mathrm{E}-1$ & ug/g dry & $2.95 \mathrm{E}-2$ & $9 / 22 / 08$ & 8I22001 & PNNL-AGG-415 \\
\hline \multirow{3}{*}{$\begin{array}{l}\text { HEIS No. } \\
\text { 14133-76-7 }\end{array}$} & B1TNW1 & \multicolumn{3}{|c|}{ Lab ID: $\quad$ 0804025-18 } & & & \\
\hline & Technetium-99 & $1.17 \mathrm{E}-2$ & ug/g dry & 4.03E-3 & $9 / 22 / 08$ & 8I22001 & PNNL-AGG-415 \\
\hline & Uranium 238 & $3.73 E-1$ & ug/g dry & $2.92 \mathrm{E}-2$ & $9 / 22 / 08$ & $8 \mathrm{I} 22001$ & PNNL-AGG-415 \\
\hline \multirow{3}{*}{$\begin{array}{l}\text { HEIS No. } \\
\text { 14133-76-7 }\end{array}$} & B1TNW3 & \multicolumn{3}{|c|}{ Lab ID: $\quad$ 0804025-20 } & & & \\
\hline & Technetium-99 & $<4.03 \mathrm{E}-3$ & ug/g dry & 4.03E-3 & $9 / 22 / 08$ & 8I22001 & PNNL-AGG-415 \\
\hline & Uranium 238 & 3.57E-1 & ug/g dry & 2.93E-2 & 9/22/08 & 8I22001 & PNNL-AGG-415 \\
\hline \multirow{3}{*}{$\begin{array}{l}\text { HEIS No. } \\
14133-76-7\end{array}$} & B1TNW5 & & b ID: & $4025-22$ & & & \\
\hline & Technetium-99 & 4.53E-3 & ug/g dry & 4.07E-3 & $9 / 22 / 08$ & $8 I 22001$ & PNNL-AGG-415 \\
\hline & Uranium 238 & 3.73E-1 & ug/g dry & $2.95 \mathrm{E}-2$ & $9 / 22 / 08$ & 8I22001 & PNNL-AGG-415 \\
\hline HEIS No. & B1TP35 & & b ID: & $4025-37$ & & & \\
\hline 14133-76-7 & Technetium-99 & $<4.05 \mathrm{E}-3$ & ug/g dry & $4.05 \mathrm{E}-3$ & $9 / 22 / 08$ & $8 \mathrm{I} 22001$ & PNNL-AGG-415 \\
\hline & Uranium 238 & $3.93 E-1$ & ug/g dry & $2.94 \mathrm{E}-2$ & $9 / 22 / 08$ & $8 \mathrm{I} 22001$ & PNNL-AGG-415 \\
\hline HEIS No. & B1TP40 & & b ID: & $4025-48$ & & & \\
\hline 14133-76-7 & Technetium-99 & $<4.07 \mathrm{E}-3$ & ug/g dry & 4.07E-3 & $9 / 22 / 08$ & 8I22001 & PNNL-AGG-415 \\
\hline & Uranium 238 & $3.42 \mathrm{E}-1$ & ug/g dry & $2.96 \mathrm{E}-2$ & 9/22/08 & $8 \mathrm{I} 22001$ & PNNL-AGG-415 \\
\hline HEIS No. & B1TP42 & & b ID: & $4025-50$ & & & \\
\hline 14133-76-7 & Technetium-99 & $<3.94 \mathrm{E}-3$ & ug/g dry & $3.94 \mathrm{E}-3$ & $9 / 22 / 08$ & 8I22001 & PNNL-AGG-415 \\
\hline & Uranium 238 & 3.24E-1 & ug/g dry & $2.86 \mathrm{E}-2$ & 9/22/08 & 8I22001 & PNNL-AGG-415 \\
\hline HEIS No. & В1TP43 & & b ID: & $4025-51$ & & & \\
\hline 14133-76-7 & Technetium-99 & $6.96 \mathrm{E}-3$ & ug/g dry & $4.41 \mathrm{E}-3$ & $9 / 22 / 08$ & 8I22001 & PNNL-AGG-415 \\
\hline & Uranium 238 & 3.63E-1 & ug/g dry & $3.20 \mathrm{E}-2$ & $9 / 22 / 08$ & 8I22001 & PNNL-AGG-415 \\
\hline HEIS No. & B1TP45 & & b ID: & $4025-53$ & & & \\
\hline 14133-76-7 & Technetium-99 & $<4.03 \mathrm{E}-3$ & ug/g dry & 4.03E-3 & $9 / 22 / 08$ & $8 I 22002$ & PNNL-AGG-415 \\
\hline
\end{tabular}




\section{Radionuclides by ICP-MS/Acid Extract}

\begin{tabular}{|c|c|c|c|c|c|c|c|}
\hline CAS \# & Analyte & Results & Units & EQL & Analyzed & Batch & Method \\
\hline \multirow[t]{2}{*}{ HEIS No. } & В1TP45 & \multicolumn{3}{|c|}{ Lab ID: $\quad$ 0804025-53 } & & & \\
\hline & Uranium 238 & $3.15 \mathrm{E}-1$ & ug/g dry & 2.93E-2 & $9 / 22 / 08$ & 8I22002 & PNNL-AGG-415 \\
\hline \multirow{3}{*}{$\begin{array}{l}\text { HEIS No. } \\
14133-76-7\end{array}$} & B1TP47 & \multicolumn{3}{|c|}{ Lab ID: $\quad 0804025-57$} & & & \\
\hline & Technetium-99 & 4.99E-3 & ug/g dry & 3.99E-3 & $9 / 22 / 08$ & 8I22002 & PNNL-AGG-415 \\
\hline & Uranium 238 & $3.49 \mathrm{E}-1$ & ug/g dry & $2.90 \mathrm{E}-2$ & $9 / 22 / 08$ & 8I22002 & PNNL-AGG-415 \\
\hline \multirow{3}{*}{$\begin{array}{l}\text { HEIS No. } \\
14133-76-7\end{array}$} & В1ТР49 & \multicolumn{3}{|c|}{ Lab ID: $\quad$ 0804025-61 } & & & \\
\hline & Technetium-99 & 4.77E-3 & ug/g dry & $3.97 \mathrm{E}-3$ & $9 / 22 / 08$ & 8I22002 & PNNL-AGG-415 \\
\hline & Uranium 238 & $3.28 \mathrm{E}-1$ & ug/g dry & $2.88 \mathrm{E}-2$ & 9/22/08 & $8 \mathrm{I} 22002$ & PNNL-AGG-415 \\
\hline \multirow{3}{*}{$\begin{array}{l}\text { HEIS No. } \\
\text { 14133-76-7 }\end{array}$} & B1TP51 & \multicolumn{3}{|c|}{ Lab ID: $\quad$ 0804025-69 } & & & \\
\hline & Technetium-99 & $1.23 \mathrm{E}-2$ & ug/g dry & 4.17E-3 & $9 / 22 / 08$ & 8I22002 & PNNL-AGG-415 \\
\hline & Uranium 238 & 3.97E-1 & ug/g dry & $3.03 E-2$ & $9 / 22 / 08$ & 8I22002 & PNNL-AGG-415 \\
\hline \multirow{3}{*}{$\begin{array}{l}\text { HEIS No. } \\
\text { 14133-76-7 }\end{array}$} & B1TP52 & \multicolumn{3}{|c|}{ Lab ID: $\quad$ 0804025-72 } & & & \\
\hline & Technetium-99 & $<4.03 \mathrm{E}-3$ & ug/g dry & 4.03E-3 & $9 / 22 / 08$ & 8I22002 & PNNL-AGG-415 \\
\hline & Uranium 238 & $3.10 \mathrm{E}-1$ & ug/g dry & 2.93E-2 & $9 / 22 / 08$ & 8I22002 & PNNL-AGG-415 \\
\hline \multirow{3}{*}{$\begin{array}{l}\text { HEIS No. } \\
\text { 14133-76-7 }\end{array}$} & В1ТР53 & \multicolumn{3}{|c|}{ Lab ID: $\quad$ 0804025-75 } & & & \\
\hline & Technetium-99 & $5.40 \mathrm{E}-3$ & ug/g dry & 3.99E-3 & $9 / 22 / 08$ & 8I22002 & PNNL-AGG-415 \\
\hline & Uranium 238 & $3.44 \mathrm{E}-1$ & ug/g dry & $2.90 \mathrm{E}-2$ & $9 / 22 / 08$ & $8 \mathrm{I} 22002$ & PNNL-AGG-415 \\
\hline \multirow{3}{*}{$\begin{array}{l}\text { HEIS No. } \\
\text { 14133-76-7 }\end{array}$} & B1TP55 & \multicolumn{3}{|c|}{ Lab ID: $\quad$ 0804025-81 } & & & \\
\hline & Technetium-99 & $<4.05 \mathrm{E}-3$ & ug/g dry & 4.05E-3 & $9 / 22 / 08$ & 8I22002 & PNNL-AGG-415 \\
\hline & Uranium 238 & 3.62E-1 & ug/g dry & $2.94 \mathrm{E}-2$ & 9/22/08 & $8 I 22002$ & PNNL-AGG-415 \\
\hline \multirow{3}{*}{$\begin{array}{l}\text { HEIS No. } \\
\text { 14133-76-7 }\end{array}$} & B1TP59 & \multicolumn{3}{|c|}{ Lab ID: $\quad 0804025-87$} & & & \\
\hline & Technetium-99 & $<3.98 \mathrm{E}-3$ & ug/g dry & $3.98 \mathrm{E}-3$ & $9 / 22 / 08$ & 8I22002 & PNNL-AGG-415 \\
\hline & Uranium 238 & 3.03E-1 & ug/g dry & $2.89 \mathrm{E}-2$ & 9/22/08 & $8 \mathrm{I} 22002$ & PNNL-AGG-415 \\
\hline \multirow{3}{*}{$\begin{array}{l}\text { HEIS No. } \\
14133-76-7\end{array}$} & B1TP61 & \multicolumn{3}{|c|}{ Lab ID: $\quad 0804025-91$} & & & \\
\hline & Technetium-99 & $<4.00 \mathrm{E}-3$ & ug/g dry & $4.00 \mathrm{E}-3$ & $9 / 22 / 08$ & 8I22002 & PNNL-AGG-415 \\
\hline & Uranium 238 & $3.98 \mathrm{E}-1$ & ug/g dry & $2.90 \mathrm{E}-2$ & $9 / 22 / 08$ & 8I22002 & PNNL-AGG-415 \\
\hline \multirow{3}{*}{$\begin{array}{l}\text { HEIS No. } \\
14133-76-7\end{array}$} & B1TP62 & \multicolumn{3}{|c|}{ Lab ID: $\quad$ 0804025-94 } & & & \\
\hline & Technetium-99 & $<4.04 \mathrm{E}-3$ & ug/g dry & 4.04E-3 & $9 / 22 / 08$ & $8 \mathrm{I} 22002$ & PNNL-AGG-415 \\
\hline & Uranium 238 & $3.42 \mathrm{E}-1$ & ug/g dry & 2.93E-2 & $9 / 22 / 08$ & $8 \mathrm{I} 22002$ & PNNL-AGG-415 \\
\hline \multirow{3}{*}{$\begin{array}{l}\text { HEIS No. } \\
\text { 14133-76-7 }\end{array}$} & B1TP64 & & b ID: & 4025-AA & & & \\
\hline & Technetium-99 & $<4.08 \mathrm{E}-3$ & ug/g dry & $4.08 \mathrm{E}-3$ & $9 / 22 / 08$ & 8I22002 & PNNL-AGG-415 \\
\hline & Uranium 238 & 3.54E-1 & ug/g dry & $2.96 \mathrm{E}-2$ & $9 / 22 / 08$ & $8 I 22002$ & PNNL-AGG-415 \\
\hline HEIS No. & B1TP67 & & b ID: & 4025-AI & & & \\
\hline $14133-76-7$ & Technetium-99 & $<4.05 \mathrm{E}-3$ & ug/g dry & 4.05E-3 & $9 / 22 / 08$ & 8I22002 & PNNL-AGG-415 \\
\hline & Uranium 238 & $3.17 \mathrm{E}-1$ & ug/g dry & $2.94 \mathrm{E}-2$ & $9 / 22 / 08$ & $8 \mathrm{I} 22002$ & PNNL-AGG-415 \\
\hline HEIS No. & В1TP69 & & b ID: & 4025-AP & & & \\
\hline $14133-76-7$ & Technetium-99 & $<4.07 \mathrm{E}-3$ & ug/g dry & 4.07E-3 & $9 / 22 / 08$ & 8I22002 & PNNL-AGG-415 \\
\hline & Uranium 238 & $3.91 \mathrm{E}-1$ & ug/g dry & $2.96 \mathrm{E}-2$ & $9 / 22 / 08$ & $8 \mathrm{I} 22002$ & PNNL-AGG-415 \\
\hline HEIS No. & B1TP82 & & b ID: & 4025-BZ & & & \\
\hline $14133-76-7$ & Technetium-99 & $<4.07 \mathrm{E}-3$ & ug/g dry & 4.07E-3 & $9 / 22 / 08$ & 8I22002 & PNNL-AGG-415 \\
\hline & Uranium 238 & $3.23 E-1$ & ug/g dry & $2.95 \mathrm{E}-2$ & $9 / 22 / 08$ & 8I22002 & PNNL-AGG-415 \\
\hline HEIS No. & В1TP93 & & b ID: & 4025-DL & & & \\
\hline 14133-76-7 & Technetium-99 & $<4.27 \mathrm{E}-3$ & ug/g dry & 4.27E-3 & $9 / 22 / 08$ & 8I22002 & PNNL-AGG-415 \\
\hline & Uranium 238 & $2.72 \mathrm{E}-1$ & ug/g dry & $3.10 \mathrm{E}-2$ & 9/22/08 & $8 I 22002$ & PNNL-AGG-415 \\
\hline HEIS No. & В1TP94 & & b ID: & 4025-DM & & & \\
\hline 14133-76-7 & Technetium-99 & $<4.15 \mathrm{E}-3$ & ug/g dry & $4.15 \mathrm{E}-3$ & $9 / 22 / 08$ & $8 I 22002$ & PNNL-AGG-415 \\
\hline & Uranium 238 & $3.35 \mathrm{E}-1$ & ug/g dry & $3.01 \mathrm{E}-2$ & $9 / 22 / 08$ & $8 I 22002$ & PNNL-AGG-415 \\
\hline
\end{tabular}




\section{Radionuclides by ICP-MS/Water Extract}

\begin{tabular}{|c|c|c|c|c|c|c|}
\hline CAS \# & Analyte & Results & EQL & Analyzed & Batch & Method \\
\hline HEIS No. & B1TP24 & \multicolumn{2}{|c|}{ Lab ID: $\quad$ 0804025-04 } & & & \\
\hline $15046-84-1$ & Iodine-129 & $<3.98 \mathrm{E}-4 \quad$ ug/g dry & $3.98 E-4$ & 9/19/08 & 8I16005 & PNNL-AGG-415 \\
\hline HEIS No. & В1TP28 & \multicolumn{2}{|c|}{ Lab ID: $\quad$ 0804025-07 } & & & \\
\hline 15046-84-1 & Iodine-129 & $<3.77 \mathrm{E}-4$ ug/g dry & 3.77E-4 & 9/19/08 & $8 \mathrm{I} 16005$ & PNNL-AGG-415 \\
\hline HEIS No. & В1TP29 & \multicolumn{2}{|c|}{ Lab ID: $\quad$ 0804025-08 } & & & \\
\hline $15046-84-1$ & Iodine-129 & $<3.77 E-4$ ug/g dry & $3.77 \mathrm{E}-4$ & 9/19/08 & 8I16004 & PNNL-AGG-415 \\
\hline HEIS No. & B1TP30 & \multicolumn{2}{|c|}{ Lab ID: $\quad$ 0804025-09 } & & & \\
\hline 15046-84-1 & Iodine-129 & $<3.77 E-4 \quad u g / g$ dry & $3.77 \mathrm{E}-4$ & $9 / 22 / 08$ & 8I16004 & PNNL-AGG-415 \\
\hline HEIS No. & B1TNV9 & \multicolumn{2}{|c|}{ Lab ID: $\quad$ 0804025-10 } & & & \\
\hline $15046-84-1$ & Iodine-129 & $<3.78 \mathrm{E}-4$ ug/g dry & $3.78 \mathrm{E}-4$ & 9/19/08 & 8I16004 & PNNL-AGG-415 \\
\hline HEIS No. & B1TP22 & \multicolumn{2}{|c|}{ Lab ID: $\quad$ 0804025-13 } & & & \\
\hline 15046-84-1 & Iodine-129 & $<3.82 E-4 \quad$ ug/g dry & $3.82 \mathrm{E}-4$ & 9/19/08 & 8I16005 & PNNL-AGG-415 \\
\hline HEIS No. & B1TP26 & \multicolumn{2}{|c|}{ Lab ID: $\quad 0804025-16$} & & & \\
\hline 15046-84-1 & Iodine-129 & $<3.79$ E-4 ug/g dry & $3.79 E-4$ & $9 / 22 / 08$ & $8 \mathrm{I} 16005$ & PNNL-AGG-415 \\
\hline HEIS No. & B1TNW0 & \multicolumn{2}{|c|}{ Lab ID: $\quad$ 0804025-17 } & & & \\
\hline 15046-84-1 & Iodine-129 & $<3.78 \mathrm{E}-4 \quad$ ug/g dry & $3.78 \mathrm{E}-4$ & $9 / 19 / 08$ & 8I16004 & PNNL-AGG-415 \\
\hline HEIS No. & B1TNW1 & \multicolumn{2}{|c|}{ Lab ID: $\quad$ 0804025-18 } & & & \\
\hline $15046-84-1$ & Iodine-129 & $<3.74 \mathrm{E}-4$ ug/g dry & $3.74 \mathrm{E}-4$ & $9 / 22 / 08$ & 8I16005 & PNNL-AGG-415 \\
\hline HEIS No. & B1TNW3 & \multicolumn{2}{|c|}{ Lab ID: $\quad$ 0804025-20 } & & & \\
\hline 15046-84-1 & Iodine-129 & $<3.78 \mathrm{E}-4 \quad$ ug/g dry & $3.78 \mathrm{E}-4$ & 9/19/08 & 8I16004 & PNNL-AGG-415 \\
\hline HEIS No. & B1TNW5 & \multicolumn{2}{|c|}{ Lab ID: $\quad$ 0804025-22 } & & & \\
\hline 15046-84-1 & Iodine-129 & $<3.71 \mathrm{E}-4$ ug/g dry & $3.71 \mathrm{E}-4$ & 9/19/08 & $8 \mathrm{I} 16005$ & PNNL-AGG-415 \\
\hline HEIS No. & В1TP35 & \multicolumn{2}{|c|}{ Lab ID: $\quad$ 0804025-37 } & & & \\
\hline $15046-84-1$ & Iodine-129 & $<4.08 \mathrm{E}-4$ ug/g dry & $4.08 \mathrm{E}-4$ & 9/19/08 & $8 \mathrm{I} 16005$ & PNNL-AGG-415 \\
\hline HEIS No. & B1TP40 & \multicolumn{2}{|c|}{ Lab ID: $\quad 0804025-48$} & & & \\
\hline $15046-84-1$ & Iodine-129 & $<3.78 \mathrm{E}-4$ ug/g dry & $3.78 \mathrm{E}-4$ & 9/19/08 & 8I16005 & PNNL-AGG-415 \\
\hline HEIS No. & В1TP42 & \multicolumn{2}{|c|}{ Lab ID: $\quad 0804025-50$} & & & \\
\hline 15046-84-1 & Iodine-129 & $<3.78 \mathrm{E}-4 \quad$ ug/g dry & $3.78 \mathrm{E}-4$ & 9/19/08 & 8I16004 & PNNL-AGG-415 \\
\hline HEIS No. & В1TР43 & \multicolumn{2}{|c|}{ Lab ID: $\quad 0804025-51$} & & & \\
\hline 15046-84-1 & Iodine-129 & $<3.78 \mathrm{E}-4 \quad$ ug/g dry & $3.78 \mathrm{E}-4$ & $9 / 22 / 08$ & 8I16005 & PNNL-AGG-415 \\
\hline HEIS No. & B1TP45 & \multicolumn{2}{|c|}{ Lab ID: $\quad$ 0804025-53 } & & & \\
\hline 15046-84-1 & Iodine-129 & $<3.78 \mathrm{E}-4 \quad$ ug/g dry & $3.78 \mathrm{E}-4$ & 9/19/08 & 8I16004 & PNNL-AGG-415 \\
\hline HEIS No. & B1TP47 & \multicolumn{2}{|c|}{ Lab ID: $\quad$ 0804025-57 } & & & \\
\hline 15046-84-1 & Iodine-129 & $<3.74 \mathrm{E}-4$ ug/g dry & $3.74 \mathrm{E}-4$ & $9 / 22 / 08$ & $8 \mathrm{I} 16005$ & PNNL-AGG-415 \\
\hline HEIS No. & В1ТР49 & \multicolumn{2}{|c|}{ Lab ID: $\quad$ 0804025-61 } & & & \\
\hline $15046-84-1$ & Iodine-129 & $<3.78 \mathrm{E}-4$ ug/g dry & $3.78 \mathrm{E}-4$ & 9/19/08 & 8I16006 & PNNL-AGG-415 \\
\hline HEIS No. & B1TP51 & \multicolumn{2}{|c|}{ Lab ID: $\quad 0804025-69$} & & & \\
\hline 15046-84-1 & Iodine-129 & $<3.72$ E-4 ug/g dry & 3.72E-4 & $9 / 22 / 08$ & 8I16006 & PNNL-AGG-415 \\
\hline HEIS No. & В1TP52 & \multicolumn{2}{|c|}{ Lab ID: $\quad 0804025-72$} & & & \\
\hline 15046-84-1 & Iodine-129 & $<3.75 E-4$ ug/g dry & 3.75E-4 & $9 / 19 / 08$ & 8I16006 & PNNL-AGG-415 \\
\hline HEIS No. & В1ТР53 & \multicolumn{2}{|c|}{ Lab ID: $\quad 0804025-75$} & & & \\
\hline $15046-84-1$ & Iodine-129 & $<3.78 \mathrm{E}-4 \quad$ ug/g dry & $3.78 \mathrm{E}-4$ & 9/19/08 & $8 \mathrm{I} 16006$ & PNNL-AGG-415 \\
\hline HEIS No. & B1TP55 & \multicolumn{2}{|c|}{ Lab ID: $\quad$ 0804025-81 } & & & \\
\hline 15046-84-1 & Iodine-129 & $<3.79$ E-4 ug/g dry & 3.79E-4 & 9/19/08 & $8 \mathrm{I} 16006$ & PNNL-AGG-415 \\
\hline HEIS No. & B1TP59 & Lab ID: & $4025-87$ & & & \\
\hline 15046-84-1 & Iodine-129 & $<3.83 E-4 \quad$ ug/g dry & 3.83E-4 & 9/19/08 & 8I16006 & PNNL-AGG-415 \\
\hline
\end{tabular}




\section{Radionuclides by ICP-MS/Water Extract}

\begin{tabular}{|c|c|c|c|c|c|c|}
\hline CAS \# & Analyte & Results & EQL & Analyzed & Batch & Method \\
\hline HEIS No. & B1TP61 & \multicolumn{2}{|c|}{ Lab ID: $\quad 0804025-91$} & & & \\
\hline $15046-84-1$ & Iodine-129 & $<3.78 \mathrm{E}-4$ ug/g dry & $3.78 \mathrm{E}-4$ & 9/19/08 & $8 \mathrm{I} 16004$ & PNNL-AGG-415 \\
\hline HEIS No. & B1TP62 & \multicolumn{2}{|c|}{ Lab ID: $\quad$ 0804025-94 } & & & \\
\hline $15046-84-1$ & Iodine-129 & $<3.73 E-4$ ug/g dry & 3.73E-4 & 9/19/08 & $8 \mathrm{I} 16006$ & PNNL-AGG-415 \\
\hline HEIS No. & B1TP64 & \multicolumn{2}{|c|}{ Lab ID: $\quad$ 0804025-AA } & & & \\
\hline 15046-84-1 & Iodine-129 & $<3.79$ E-4 ug/g dry & 3.79E-4 & 9/19/08 & 8I16004 & PNNL-AGG-415 \\
\hline HEIS No. & B1TP67 & \multicolumn{2}{|c|}{ Lab ID: $\quad$ 0804025-AI } & & & \\
\hline $15046-84-1$ & Iodine-129 & $<3.69 \mathrm{E}-4$ ug/g dry & $3.69 \mathrm{E}-4$ & 9/19/08 & $8 \mathrm{I} 16006$ & PNNL-AGG-415 \\
\hline HEIS No. & B1TP69 & \multicolumn{2}{|c|}{ Lab ID: $\quad$ 0804025-AP } & & & \\
\hline 15046-84-1 & Iodine-129 & $<3.78 \mathrm{E}-4 \quad$ ug/g dry & $3.78 \mathrm{E}-4$ & 9/19/08 & 8I16004 & PNNL-AGG-415 \\
\hline HEIS No. & B1TP71 & \multicolumn{2}{|c|}{ Lab ID: $\quad$ 0804025-AV } & & & \\
\hline 15046-84-1 & Iodine-129 & $<3.76 \mathrm{E}-4 \quad$ ug/g dry & $3.76 \mathrm{E}-4$ & $9 / 22 / 08$ & 8I16006 & PNNL-AGG-415 \\
\hline HEIS No. & B1TP77 & \multicolumn{2}{|c|}{ Lab ID: $\quad$ 0804025-BK } & & & \\
\hline $15046-84-1$ & Iodine-129 & $<3.77 \mathrm{E}-4 \quad$ ug/g dry & 3.77E-4 & $9 / 22 / 08$ & $8 \mathrm{I} 16006$ & PNNL-AGG-415 \\
\hline HEIS No. & B1TP78 & \multicolumn{2}{|c|}{ Lab ID: $\quad$ 0804025-BP } & & & \\
\hline $15046-84-1$ & Iodine-129 & $<3.78 \mathrm{E}-4 \quad$ ug/g dry & $3.78 \mathrm{E}-4$ & 9/22/08 & $8 \mathrm{I} 16006$ & PNNL-AGG-415 \\
\hline HEIS No. & B1TP82 & \multicolumn{2}{|c|}{ Lab ID: $\quad$ 0804025-BZ } & & & \\
\hline $15046-84-1$ & Iodine-129 & $<3.76 \mathrm{E}-4$ ug/g dry & $3.76 \mathrm{E}-4$ & $9 / 22 / 08$ & $8 \mathrm{I} 16006$ & PNNL-AGG-415 \\
\hline HEIS No. & B1TP84 & \multicolumn{2}{|c|}{ Lab ID: $\quad$ 0804025-CF } & & & \\
\hline 15046-84-1 & Iodine-129 & $<3.77 \mathrm{E}-4 \quad$ ug/g dry & 3.77E-4 & $9 / 22 / 08$ & 8I16006 & PNNL-AGG-415 \\
\hline HEIS No. & B1TNX1 & \multicolumn{2}{|c|}{ Lab ID: $\quad$ 0804025-CI } & & & \\
\hline $15046-84-1$ & Iodine-129 & $<3.80 \mathrm{E}-4 \quad$ ug/g dry & $3.80 \mathrm{E}-4$ & $9 / 22 / 08$ & $8 \mathrm{I} 16006$ & PNNL-AGG-415 \\
\hline HEIS No. & B1TP86 & \multicolumn{2}{|c|}{ Lab ID: $\quad$ 0804025-CO } & & & \\
\hline 15046-84-1 & Iodine-129 & $<3.78 \mathrm{E}-4 \quad$ ug/g dry & $3.78 \mathrm{E}-4$ & $9 / 22 / 08$ & $8 \mathrm{I} 16006$ & PNNL-AGG-415 \\
\hline HEIS No. & B1TP89 & \multicolumn{2}{|c|}{ Lab ID: $\quad$ 0804025-CX } & & & \\
\hline $15046-84-1$ & Iodine-129 & $<3.79 \mathrm{E}-4 \quad$ ug/g dry & $3.79 E-4$ & $9 / 22 / 08$ & $8 \mathrm{I} 16006$ & PNNL-AGG-415 \\
\hline HEIS No. & B1TP91 & \multicolumn{2}{|c|}{ Lab ID: $\quad$ 0804025-DF } & & & \\
\hline 15046-84-1 & Iodine-129 & $<3.79 \mathrm{E}-4$ ug/g dry & $3.79 \mathrm{E}-4$ & $9 / 22 / 08$ & $8 \mathrm{I} 16006$ & PNNL-AGG-415 \\
\hline HEIS No. & В1TP93 & \multicolumn{2}{|c|}{ Lab ID: $\quad$ 0804025-DL } & & & \\
\hline $15046-84-1$ & Iodine-129 & $<3.79 \mathrm{E}-4 \quad$ ug/g dry & $3.79 \mathrm{E}-4$ & $9 / 22 / 08$ & $8 \mathrm{I} 16006$ & PNNL-AGG-415 \\
\hline HEIS No. & В1TP94 & \multicolumn{2}{|c|}{ Lab ID: $\quad$ 0804025-DM } & & & \\
\hline 15046-84-1 & Iodine-129 & $<3.76 \mathrm{E}-4$ ug/g dry & $3.76 \mathrm{E}-4$ & 9/22/08 & $8 \mathrm{I} 16006$ & PNNL-AGG-415 \\
\hline
\end{tabular}




\section{Radionuclides by ICP-MS/Water Extract}

\begin{tabular}{|c|c|c|c|c|c|c|c|}
\hline CAS \# & Analyte & Results & Units & EQL & Analyzed & Batch & Method \\
\hline HEIS No. & B1TP24 & \multicolumn{3}{|c|}{ Lab ID: $\quad$ 0804025-04 } & & & \\
\hline \multirow[t]{2}{*}{ 14133-76-7 } & Technetium-99 & $<2.43 \mathrm{E}-5$ & ug/g dry & $2.43 \mathrm{E}-5$ & 7/15/08 & 8G15001 & PNNL-AGG-415 \\
\hline & Uranium 238 & $1.39 \mathrm{E}-1$ & ug/g dry & $5.94 \mathrm{E}-4$ & $7 / 15 / 08$ & 8G15001 & PNNL-AGG-415 \\
\hline HEIS No. & B1TP28 & \multicolumn{3}{|c|}{ Lab ID: $\quad$ 0804025-07 } & & & \\
\hline \multirow[t]{2}{*}{$14133-76-7$} & Technetium-99 & $2.56 \mathrm{E}-5$ & ug/g dry & $2.29 \mathrm{E}-5$ & $7 / 15 / 08$ & 8G15001 & PNNL-AGG-415 \\
\hline & Uranium 238 & 3.49E-3 & ug/g dry & $5.62 \mathrm{E}-4$ & $7 / 15 / 08$ & 8G15001 & PNNL-AGG-415 \\
\hline \multirow{3}{*}{$\begin{array}{l}\text { HEIS No. } \\
14133-76-7\end{array}$} & B1TP29 & \multicolumn{3}{|c|}{ Lab ID: $\quad 0804025-08$} & & & \\
\hline & Technetium-99 & $5.29 \mathrm{E}-5$ & ug/g dry & $2.30 \mathrm{E}-5$ & 9/09/08 & 8I09003 & PNNL-AGG-415 \\
\hline & Uranium 238 & $5.89 \mathrm{E}-3$ & ug/g dry & $5.62 \mathrm{E}-4$ & 9/09/08 & 8I09003 & PNNL-AGG-415 \\
\hline \multirow{3}{*}{$\begin{array}{l}\text { HEIS No. } \\
14133-76-7\end{array}$} & B1TP30 & \multicolumn{3}{|c|}{ Lab ID: $\quad$ 0804025-09 } & & & \\
\hline & Technetium-99 & $1.48 \mathrm{E}-4$ & ug/g dry & $2.30 \mathrm{E}-5$ & 9/09/08 & 8I09003 & PNNL-AGG-415 \\
\hline & Uranium 238 & $6.80 \mathrm{E}-3$ & ug/g dry & 5.63E-4 & 9/09/08 & 8I09003 & PNNL-AGG-415 \\
\hline \multirow{3}{*}{$\begin{array}{l}\text { HEIS No. } \\
14133-76-7\end{array}$} & B1TNV9 & \multicolumn{3}{|c|}{ Lab ID: $\quad$ 0804025-10 } & & & \\
\hline & Technetium-99 & $5.87 \mathrm{E}-4$ & ug/g dry & $2.30 \mathrm{E}-5$ & 9/09/08 & 8I09003 & PNNL-AGG-415 \\
\hline & Uranium 238 & $2.59 \mathrm{E}-3$ & ug/g dry & $5.64 \mathrm{E}-4$ & 9/09/08 & 8I09003 & PNNL-AGG-415 \\
\hline \multirow{3}{*}{$\begin{array}{l}\text { HEIS No. } \\
14133-76-7\end{array}$} & B1TP22 & \multicolumn{3}{|c|}{ Lab ID: $\quad$ 0804025-13 } & & & \\
\hline & Technetium-99 & $<2.33 \mathrm{E}-5$ & ug/g dry & 2.33E-5 & $7 / 15 / 08$ & 8G15001 & PNNL-AGG-415 \\
\hline & Uranium 238 & 2.93E-3 & ug/g dry & $5.70 \mathrm{E}-4$ & $7 / 15 / 08$ & 8G15001 & PNNL-AGG-415 \\
\hline \multirow{3}{*}{$\begin{array}{l}\text { HEIS No. } \\
14133-76-7\end{array}$} & B1TP26 & \multicolumn{3}{|c|}{ Lab ID: $\quad$ 0804025-16 } & & & \\
\hline & Technetium-99 & $<2.31 \mathrm{E}-5$ & ug/g dry & $2.31 \mathrm{E}-5$ & $7 / 15 / 08$ & 8G15001 & PNNL-AGG-415 \\
\hline & Uranium 238 & $2.64 \mathrm{E}-3$ & ug/g dry & $5.65 \mathrm{E}-4$ & $7 / 15 / 08$ & 8G15001 & PNNL-AGG-415 \\
\hline \multirow{3}{*}{$\begin{array}{l}\text { HEIS No. } \\
14133-76-7\end{array}$} & B1TNW0 & \multicolumn{3}{|c|}{ Lab ID: $\quad$ 0804025-17 } & & & \\
\hline & Technetium-99 & 4.36E-3 & ug/g dry & $2.30 \mathrm{E}-5$ & 9/09/08 & 8109003 & PNNL-AGG-415 \\
\hline & Uranium 238 & $<5.64 \mathrm{E}-4$ & ug/g dry & $5.64 \mathrm{E}-4$ & 9/09/08 & 8109003 & PNNL-AGG-415 \\
\hline \multirow{3}{*}{$\begin{array}{l}\text { HEIS No. } \\
14133-76-7\end{array}$} & B1TNW1 & \multicolumn{3}{|c|}{ Lab ID: $\quad$ 0804025-18 } & & & \\
\hline & Technetium-99 & $1.16 \mathrm{E}-2$ & ug/g dry & $2.28 \mathrm{E}-5$ & $7 / 15 / 08$ & 8G15001 & PNNL-AGG-415 \\
\hline & Uranium 238 & $<5.58 \mathrm{E}-4$ & ug/g dry & $5.58 \mathrm{E}-4$ & $7 / 15 / 08$ & $8 G 15001$ & PNNL-AGG-415 \\
\hline \multirow{3}{*}{$\begin{array}{l}\text { HEIS No. } \\
14133-76-7\end{array}$} & B1TNW3 & \multicolumn{3}{|c|}{ Lab ID: $\quad$ 0804025-20 } & & & \\
\hline & Technetium-99 & $1.04 \mathrm{E}-3$ & ug/g dry & $2.30 \mathrm{E}-5$ & 9/09/08 & 8I09003 & PNNL-AGG-415 \\
\hline & Uranium 238 & $7.25 \mathrm{E}-4$ & ug/g dry & $5.64 \mathrm{E}-4$ & $9 / 09 / 08$ & 8109003 & PNNL-AGG-415 \\
\hline \multirow{3}{*}{$\begin{array}{l}\text { HEIS No. } \\
14133-76-7\end{array}$} & B1TNW5 & & b ID: & 4025-22 & & & \\
\hline & Technetium-99 & $2.57 \mathrm{E}-3$ & ug/g dry & $2.26 \mathrm{E}-5$ & 7/15/08 & 8G15001 & PNNL-AGG-415 \\
\hline & Uranium 238 & $8.14 \mathrm{E}-4$ & ug/g dry & $5.54 \mathrm{E}-4$ & $7 / 15 / 08$ & 8G15001 & PNNL-AGG-415 \\
\hline HEIS No. & B1TP35 & & b ID: & 4025-37 & & & \\
\hline $14133-76-7$ & Technetium-99 & $<2.48 \mathrm{E}-5$ & ug/g dry & $2.48 \mathrm{E}-5$ & $7 / 15 / 08$ & 8G15001 & PNNL-AGG-415 \\
\hline & Uranium 238 & $<6.08 \mathrm{E}-4$ & ug/g dry & $6.08 \mathrm{E}-4$ & 7/15/08 & 8G15001 & PNNL-AGG-415 \\
\hline HEIS No. & B1TP40 & & b ID: & 4025-48 & & & \\
\hline $14133-76-7$ & Technetium-99 & $<2.30 \mathrm{E}-5$ & ug/g dry & $2.30 \mathrm{E}-5$ & 7/15/08 & 8G15001 & PNNL-AGG-415 \\
\hline & Uranium 238 & $<5.64 \mathrm{E}-4$ & ug/g dry & $5.64 \mathrm{E}-4$ & $7 / 15 / 08$ & 8G15001 & PNNL-AGG-415 \\
\hline HEIS No. & B1TP42 & & b ID: & 4025-50 & & & \\
\hline 14133-76-7 & Technetium-99 & $3.02 \mathrm{E}-4$ & ug/g dry & $2.30 \mathrm{E}-5$ & 9/09/08 & 8I09003 & PNNL-AGG-415 \\
\hline & Uranium 238 & 7.26E-4 & ug/g dry & $5.64 \mathrm{E}-4$ & 9/09/08 & 8I09003 & PNNL-AGG-415 \\
\hline HEIS No. & B1TP43 & & b ID: & 4025-51 & & & \\
\hline $14133-76-7$ & Technetium-99 & $6.21 \mathrm{E}-3$ & ug/g dry & $2.30 \mathrm{E}-5$ & $7 / 15 / 08$ & $8 G 15001$ & PNNL-AGG-415 \\
\hline & Uranium 238 & $1.51 \mathrm{E}-3$ & ug/g dry & $5.65 \mathrm{E}-4$ & $7 / 15 / 08$ & 8G15001 & PNNL-AGG-415 \\
\hline HEIS No. & B1TP45 & & b ID: & 4025-53 & & & \\
\hline 14133-76-7 & Technetium-99 & $1.08 \mathrm{E}-3$ & ug/g dry & $2.30 \mathrm{E}-5$ & 9/09/08 & 8I09003 & PNNL-AGG-415 \\
\hline
\end{tabular}




\section{Radionuclides by ICP-MS/Water Extract}

\begin{tabular}{|c|c|c|c|c|c|c|c|}
\hline CAS \# & Analyte & Results & Units & EQL & Analyzed & Batch & Method \\
\hline \multirow[t]{2}{*}{ HEIS No. } & B1TP45 & \multicolumn{3}{|c|}{ Lab ID: $\quad$ 0804025-53 } & & & \\
\hline & Uranium 238 & 7.29E-4 & ug/g dry & $5.64 \mathrm{E}-4$ & 9/09/08 & 8109003 & PNNL-AGG-415 \\
\hline \multirow{3}{*}{$\begin{array}{l}\text { HEIS No. } \\
\text { 14133-76-7 }\end{array}$} & B1TP47 & \multicolumn{3}{|c|}{ Lab ID: $\quad$ 0804025-57 } & & & \\
\hline & Technetium-99 & $2.88 \mathrm{E}-3$ & ug/g dry & $2.28 \mathrm{E}-5$ & 7/15/08 & 8G15001 & PNNL-AGG-415 \\
\hline & Uranium 238 & $6.80 \mathrm{E}-4$ & ug/g dry & $5.58 \mathrm{E}-4$ & $7 / 15 / 08$ & 8G15001 & PNNL-AGG-415 \\
\hline \multirow{3}{*}{$\begin{array}{l}\text { HEIS No. } \\
14133-76-7\end{array}$} & B1TP49 & \multicolumn{3}{|c|}{ Lab ID: ～0804025-61 } & & & \\
\hline & Technetium-99 & $2.61 \mathrm{E}-3$ & ug/g dry & $2.30 \mathrm{E}-5$ & 7/15/08 & 8G15002 & PNNL-AGG-415 \\
\hline & Uranium 238 & 8.04E-4 & ug/g dry & $5.64 \mathrm{E}-4$ & 7/15/08 & 8G15002 & PNNL-AGG-415 \\
\hline \multirow{3}{*}{$\begin{array}{l}\text { HEIS No. } \\
14133-76-7\end{array}$} & B1TP51 & \multicolumn{3}{|c|}{ Lab ID: $\quad$ 0804025-69 } & & & \\
\hline & Technetium-99 & $1.06 \mathrm{E}-2$ & ug/g dry & $2.27 \mathrm{E}-5$ & $7 / 15 / 08$ & 8G15002 & PNNL-AGG-415 \\
\hline & Uranium 238 & $1.53 \mathrm{E}-3$ & ug/g dry & $5.55 E-4$ & $7 / 15 / 08$ & 8G15002 & PNNL-AGG-415 \\
\hline \multirow{3}{*}{$\begin{array}{l}\text { HEIS No. } \\
14133-76-7\end{array}$} & B1TP52 & \multicolumn{3}{|c|}{ Lab ID: $\quad$ 0804025-72 } & & & \\
\hline & Technetium-99 & $1.60 \mathrm{E}-3$ & ug/g dry & $2.29 \mathrm{E}-5$ & $7 / 15 / 08$ & $8 G 15002$ & PNNL-AGG-415 \\
\hline & Uranium 238 & $1.67 \mathrm{E}-3$ & ug/g dry & $5.60 \mathrm{E}-4$ & $7 / 15 / 08$ & 8G15002 & PNNL-AGG-415 \\
\hline \multirow{3}{*}{$\begin{array}{l}\text { HEIS No. } \\
14133-76-7\end{array}$} & B1TP53 & \multicolumn{3}{|c|}{ Lab ID: $\quad$ 0804025-75 } & & & \\
\hline & Technetium-99 & $2.13 \mathrm{E}-3$ & ug/g dry & $2.30 \mathrm{E}-5$ & $7 / 15 / 08$ & 8G15002 & PNNL-AGG-415 \\
\hline & Uranium 238 & $<5.64 \mathrm{E}-4$ & ug/g dry & $5.64 \mathrm{E}-4$ & 7/15/08 & 8G15002 & PNNL-AGG-415 \\
\hline \multirow{3}{*}{$\begin{array}{l}\text { HEIS No. } \\
14133-76-7\end{array}$} & B1TP55 & \multicolumn{3}{|c|}{ Lab ID: $\quad$ 0804025-81 } & & & \\
\hline & Technetium-99 & $1.98 \mathrm{E}-4$ & ug/g dry & $2.31 \mathrm{E}-5$ & $7 / 15 / 08$ & 8G15002 & PNNL-AGG-415 \\
\hline & Uranium 238 & 5.93E-4 & ug/g dry & $5.65 \mathrm{E}-4$ & 7/15/08 & 8G15002 & PNNL-AGG-415 \\
\hline \multirow{3}{*}{$\begin{array}{l}\text { HEIS No. } \\
14133-76-7\end{array}$} & B1TP59 & \multicolumn{3}{|c|}{ Lab ID: $\quad 0804025-87$} & & & \\
\hline & Technetium-99 & $<2.33 \mathrm{E}-5$ & ug/g dry & 2.33E-5 & 7/15/08 & 8G15002 & PNNL-AGG-415 \\
\hline & Uranium 238 & $<5.71 \mathrm{E}-4$ & ug/g dry & $5.71 \mathrm{E}-4$ & $7 / 15 / 08$ & 8G15002 & PNNL-AGG-415 \\
\hline \multirow{3}{*}{$\begin{array}{l}\text { HEIS No. } \\
14133-76-7\end{array}$} & B1TP61 & \multicolumn{3}{|c|}{ Lab ID: $\quad 0804025-91$} & & & \\
\hline & Technetium-99 & $5.72 \mathrm{E}-5$ & ug/g dry & $2.30 \mathrm{E}-5$ & 9/09/08 & 8I09003 & PNNL-AGG-415 \\
\hline & Uranium 238 & $<5.64 \mathrm{E}-4$ & ug/g dry & $5.64 \mathrm{E}-4$ & $9 / 09 / 08$ & 8I09003 & PNNL-AGG-415 \\
\hline \multirow{3}{*}{$\begin{array}{l}\text { HEIS No. } \\
14133-76-7\end{array}$} & В1TP62 & \multicolumn{3}{|c|}{ Lab ID: $\quad$ 0804025-94 } & & & \\
\hline & Technetium-99 & $1.12 \mathrm{E}-3$ & ug/g dry & $2.27 \mathrm{E}-5$ & $7 / 15 / 08$ & 8G15002 & PNNL-AGG-415 \\
\hline & Uranium 238 & $<5.57 \mathrm{E}-4$ & ug/g dry & 5.57E-4 & $7 / 15 / 08$ & 8G15002 & PNNL-AGG-415 \\
\hline \multirow{3}{*}{$\begin{array}{l}\text { HEIS No. } \\
14133-76-7\end{array}$} & B1TP64 & & ab ID: & 4025-AA & & & \\
\hline & Technetium-99 & $1.41 \mathrm{E}-3$ & ug/g dry & $2.31 \mathrm{E}-5$ & 9/09/08 & 8I09003 & PNNL-AGG-415 \\
\hline & Uranium 238 & $5.91 \mathrm{E}-4$ & ug/g dry & $5.65 E-4$ & 9/09/08 & 8I09003 & PNNL-AGG-415 \\
\hline HEIS No. & В1TP67 & & ab ID: & 4025-AI & & & \\
\hline 14133-76-7 & Technetium-99 & $4.08 \mathrm{E}-5$ & ug/g dry & $2.25 \mathrm{E}-5$ & 7/15/08 & 8G15002 & PNNL-AGG-415 \\
\hline & Uranium 238 & $<5.51 \mathrm{E}-4$ & ug/g dry & 5.51E-4 & $7 / 15 / 08$ & 8G15002 & PNNL-AGG-415 \\
\hline HEIS No. & В1TP69 & & ab ID: & 4025-AP & & & \\
\hline 14133-76-7 & Technetium-99 & 5.39E-5 & ug/g dry & $2.30 \mathrm{E}-5$ & $9 / 09 / 08$ & 8I09003 & PNNL-AGG-415 \\
\hline & Uranium 238 & $5.70 \mathrm{E}-4$ & ug/g dry & $5.64 \mathrm{E}-4$ & $9 / 09 / 08$ & 8I09003 & PNNL-AGG-415 \\
\hline HEIS No. & B1TP71 & & b ID: & 4025-AV & & & \\
\hline 14133-76-7 & Technetium-99 & $<2.29 \mathrm{E}-5$ & ug/g dry & $2.29 \mathrm{E}-5$ & 7/15/08 & 8G15002 & PNNL-AGG-415 \\
\hline & Uranium 238 & $<5.62 \mathrm{E}-4$ & ug/g dry & $5.62 \mathrm{E}-4$ & $7 / 15 / 08$ & 8G15002 & PNNL-AGG-415 \\
\hline HEIS No. & B1TP77 & & b ID: & 4025-BK & & & \\
\hline 14133-76-7 & Technetium-99 & $<2.30 \mathrm{E}-5$ & ug/g dry & $2.30 \mathrm{E}-5$ & 7/15/08 & 8G15002 & PNNL-AGG-415 \\
\hline & Uranium 238 & $<5.63 \mathrm{E}-4$ & ug/g dry & $5.63 \mathrm{E}-4$ & 7/15/08 & 8G15002 & PNNL-AGG-415 \\
\hline HEIS No. & B1TP78 & & ab ID: & 4025-BP & & & \\
\hline 14133-76-7 & Technetium-99 & $<2.30 \mathrm{E}-5$ & ug/g dry & $2.30 \mathrm{E}-5$ & 7/15/08 & 8G15002 & PNNL-AGG-415 \\
\hline & Uranium 238 & $<5.64 \mathrm{E}-4$ & ug/g dry & $5.64 \mathrm{E}-4$ & 7/15/08 & 8G15002 & PNNL-AGG-415 \\
\hline
\end{tabular}




\section{Radionuclides by ICP-MS/Water Extract}

\begin{tabular}{|c|c|c|c|c|c|c|c|}
\hline CAS \# & Analyte & Results & Units & EQL & Analyzed & Batch & Method \\
\hline \multirow{3}{*}{$\begin{array}{l}\text { HEIS No. } \\
\text { 14133-76-7 }\end{array}$} & B1TP82 & \multicolumn{3}{|c|}{ Lab ID: $\quad$ 0804025-BZ } & & & \\
\hline & Technetium-99 & $<2.29 \mathrm{E}-5$ & ug/g dry & $2.29 \mathrm{E}-5$ & $7 / 15 / 08$ & $8 G 15002$ & PNNL-AGG-415 \\
\hline & Uranium 238 & $<5.62 \mathrm{E}-4$ & ug/g dry & $5.62 \mathrm{E}-4$ & $7 / 15 / 08$ & 8G15002 & PNNL-AGG-415 \\
\hline \multirow{3}{*}{$\begin{array}{l}\text { HEIS No. } \\
\text { 14133-76-7 }\end{array}$} & B1TP84 & \multicolumn{3}{|c|}{ Lab ID: $\quad$ 0804025-CF } & & & \\
\hline & Technetium-99 & $<2.30 \mathrm{E}-5$ & ug/g dry & $2.30 \mathrm{E}-5$ & $7 / 15 / 08$ & 8G15002 & PNNL-AGG-415 \\
\hline & Uranium 238 & $7.26 \mathrm{E}-4$ & ug/g dry & $5.62 \mathrm{E}-4$ & $7 / 15 / 08$ & 8G15002 & PNNL-AGG-415 \\
\hline \multirow{3}{*}{$\begin{array}{l}\text { HEIS No. } \\
14133-76-7\end{array}$} & B1TNX1 & \multicolumn{3}{|c|}{ Lab ID: $\quad$ 0804025-CI } & & & \\
\hline & Technetium-99 & $<2.32 \mathrm{E}-5$ & ug/g dry & $2.32 \mathrm{E}-5$ & $7 / 15 / 08$ & 8G15002 & PNNL-AGG-415 \\
\hline & Uranium 238 & $<5.68 \mathrm{E}-4$ & ug/g dry & $5.68 \mathrm{E}-4$ & $7 / 15 / 08$ & $8 G 15002$ & PNNL-AGG-415 \\
\hline \multirow{3}{*}{$\begin{array}{l}\text { HEIS No. } \\
14133-76-7\end{array}$} & B1TP86 & \multicolumn{3}{|c|}{ Lab ID: $\quad$ 0804025-CO } & & & \\
\hline & Technetium-99 & $<2.30 \mathrm{E}-5$ & ug/g dry & $2.30 \mathrm{E}-5$ & $7 / 15 / 08$ & 8G15002 & PNNL-AGG-415 \\
\hline & Uranium 238 & $<5.64 \mathrm{E}-4$ & ug/g dry & $5.64 \mathrm{E}-4$ & $7 / 15 / 08$ & 8G15002 & PNNL-AGG-415 \\
\hline \multirow{3}{*}{\begin{tabular}{|l} 
HEIS No. \\
$14133-76-7$
\end{tabular}} & B1TP89 & \multicolumn{3}{|c|}{ Lab ID: $\quad$ 0804025-CX } & & & \\
\hline & Technetium-99 & $<2.31 \mathrm{E}-5$ & ug/g dry & $2.31 \mathrm{E}-5$ & $7 / 15 / 08$ & $8 G 15002$ & PNNL-AGG-415 \\
\hline & Uranium 238 & $<5.65 \mathrm{E}-4$ & ug/g dry & 5.65E-4 & $7 / 15 / 08$ & $8 G 15002$ & PNNL-AGG-415 \\
\hline \multirow{3}{*}{\begin{tabular}{|l} 
HEIS No. \\
$14133-76-7$
\end{tabular}} & B1TP91 & \multicolumn{3}{|c|}{ Lab ID: $\quad$ 0804025-DF } & & & \\
\hline & Technetium-99 & $<2.31 \mathrm{E}-5$ & ug/g dry & $2.31 \mathrm{E}-5$ & $7 / 15 / 08$ & 8G15002 & PNNL-AGG-415 \\
\hline & Uranium 238 & $<5.65 \mathrm{E}-4$ & ug/g dry & 5.65E-4 & $7 / 15 / 08$ & $8 G 15002$ & PNNL-AGG-415 \\
\hline \multirow{3}{*}{\begin{tabular}{|l} 
HEIS No. \\
$14133-76-7$
\end{tabular}} & В1ТР93 & \multicolumn{3}{|c|}{ Lab ID: $\quad$ 0804025-DL } & & & \\
\hline & Technetium-99 & $<2.31 \mathrm{E}-5$ & ug/g dry & $2.31 \mathrm{E}-5$ & $7 / 15 / 08$ & $8 G 15002$ & PNNL-AGG-415 \\
\hline & Uranium 238 & $<5.66 \mathrm{E}-4$ & ug/g dry & $5.66 \mathrm{E}-4$ & $7 / 15 / 08$ & 8G15002 & PNNL-AGG-415 \\
\hline \multirow{3}{*}{$\begin{array}{l}\text { HEIS No. } \\
14133-76-7\end{array}$} & В1TP94 & \multicolumn{3}{|c|}{ Lab ID: $\quad$ 0804025-DM } & & & \\
\hline & Technetium-99 & $<2.29 \mathrm{E}-5$ & ug/g dry & $2.29 \mathrm{E}-5$ & $7 / 15 / 08$ & 8G15002 & PNNL-AGG-415 \\
\hline & Uranium 238 & $<5.62 \mathrm{E}-4$ & ug/g dry & $5.62 \mathrm{E}-4$ & $7 / 15 / 08$ & $8 G 15002$ & PNNL-AGG-415 \\
\hline
\end{tabular}


RCRA Metals By PNNL-AGG-415/Water Extract

\begin{tabular}{|c|c|c|c|c|c|c|c|}
\hline CAS \# & Analyte & Results & Units & EQL & Analyzed & Batch & Method \\
\hline HEIS No. & B1TP24 & \multicolumn{3}{|c|}{ Lab ID: $\quad$ 0804025-04 } & & & \\
\hline 13981-78-7 & Chromium & $<6.75 \mathrm{E}-3$ & ug/g dry & $6.75 \mathrm{E}-3$ & $7 / 10 / 08$ & 8G10003 & PNNL-AGG-415 \\
\hline 14191-84-5 & Copper & $<3.67 \mathrm{E}-3$ & ug/g dry & $3.67 \mathrm{E}-3$ & 7/10/08 & 8G10003 & PNNL-AGG-415 \\
\hline $7440-38-2$ & Arsenic & $2.18 \mathrm{E}-2$ & ug/g dry & $6.59 \mathrm{E}-3$ & 7/10/08 & 8G10003 & PNNL-AGG-415 \\
\hline 14687-58-2 & Selenium & $<1.17 \mathrm{E}-2$ & ug/g dry & $1.17 \mathrm{E}-2$ & 7/10/08 & 8G10003 & PNNL-AGG-415 \\
\hline 14378-37-1 & Silver & $<9.75 \mathrm{E}-4$ & ug/g dry & $9.75 \mathrm{E}-4$ & $7 / 10 / 08$ & 8G10003 & PNNL-AGG-415 \\
\hline 14336-64-2 & Cadmium & $<3.11 \mathrm{E}-4$ & ug/g dry & $3.11 \mathrm{E}-4$ & 7/10/08 & 8G10003 & PNNL-AGG-415 \\
\hline 14265-72-6 & Antimony & $2.98 \mathrm{E}-3$ & ug/g dry & $5.69 \mathrm{E}-4$ & 7/10/08 & 8G10003 & PNNL-AGG-415 \\
\hline 13966-28-4 & Lead & $6.88 \mathrm{E}-4$ & ug/g dry & $5.90 \mathrm{E}-4$ & 7/10/08 & 8G10003 & PNNL-AGG-415 \\
\hline HEIS No. & В1TP28 & \multicolumn{3}{|c|}{ Lab ID: $\quad$ 0804025-07 } & & & \\
\hline 13981-78-7 & Chromium & $<6.38 \mathrm{E}-3$ & ug/g dry & $6.38 \mathrm{E}-3$ & 7/10/08 & 8G10003 & PNNL-AGG-415 \\
\hline 14191-84-5 & Copper & $<3.47 \mathrm{E}-3$ & ug/g dry & $3.47 \mathrm{E}-3$ & $7 / 10 / 08$ & 8G10003 & PNNL-AGG-415 \\
\hline $7440-38-2$ & Arsenic & $1.26 \mathrm{E}-1$ & ug/g dry & $6.23 \mathrm{E}-3$ & 7/10/08 & 8G10003 & PNNL-AGG-415 \\
\hline $14687-58-2$ & Selenium & $<1.10 \mathrm{E}-2$ & ug/g dry & $1.10 \mathrm{E}-2$ & $7 / 10 / 08$ & 8G10003 & PNNL-AGG-415 \\
\hline 14378-37-1 & Silver & $<9.23 \mathrm{E}-4$ & ug/g dry & $9.23 \mathrm{E}-4$ & $7 / 10 / 08$ & 8G10003 & PNNL-AGG-415 \\
\hline 14336-64-2 & Cadmium & $<2.94 \mathrm{E}-4$ & ug/g dry & $2.94 \mathrm{E}-4$ & $7 / 10 / 08$ & 8G10003 & PNNL-AGG-415 \\
\hline 14265-72-6 & Antimony & $<5.39 \mathrm{E}-4$ & ug/g dry & 5.39E-4 & 7/10/08 & 8G10003 & PNNL-AGG-415 \\
\hline 13966-28-4 & Lead & $<5.59 \mathrm{E}-4$ & ug/g dry & $5.59 \mathrm{E}-4$ & 7/10/08 & 8G10003 & PNNL-AGG-415 \\
\hline HEIS No. & В1TP29 & \multicolumn{3}{|c|}{ Lab ID: $\quad$ 0804025-08 } & & & \\
\hline 13981-78-7 & Chromium & $<6.39 \mathrm{E}-3$ & ug/g dry & $6.39 \mathrm{E}-3$ & $9 / 17 / 08$ & $8 \mathrm{I} 11003$ & PNNL-AGG-415 \\
\hline 14191-84-5 & Copper & $7.22 \mathrm{E}-3$ & ug/g dry & $3.47 \mathrm{E}-3$ & 9/17/08 & $8 \mathrm{I} 11003$ & PNNL-AGG-415 \\
\hline $7440-38-2$ & Arsenic & 4.97E-2 & ug/g dry & $6.24 \mathrm{E}-3$ & 9/17/08 & $8 \mathrm{I} 11003$ & PNNL-AGG-415 \\
\hline 14687-58-2 & Selenium & $<1.10 \mathrm{E}-2$ & ug/g dry & $1.10 \mathrm{E}-2$ & 9/17/08 & $8 \mathrm{I} 11003$ & PNNL-AGG-415 \\
\hline 14378-37-1 & Silver & $<9.23 \mathrm{E}-4$ & ug/g dry & $9.23 \mathrm{E}-4$ & $9 / 17 / 08$ & $8 \mathrm{I} 11003$ & PNNL-AGG-415 \\
\hline 14336-64-2 & Cadmium & 7.49E-4 & ug/g dry & 2.94E-4 & 9/17/08 & $8 \mathrm{I} 11003$ & PNNL-AGG-415 \\
\hline $14265-72-6$ & Antimony & $6.03 \mathrm{E}-4$ & ug/g dry & $5.39 \mathrm{E}-4$ & $9 / 17 / 08$ & $8 \mathrm{I} 11003$ & PNNL-AGG-415 \\
\hline 13966-28-4 & Lead & $7.80 \mathrm{E}-4$ & ug/g dry & $5.59 \mathrm{E}-4$ & 9/17/08 & $8 \mathrm{I} 11003$ & PNNL-AGG-415 \\
\hline HEIS No. & В1TP30 & \multicolumn{3}{|c|}{ Lab ID: $\quad$ 0804025-09 } & & & \\
\hline 13981-78-7 & Chromium & $<6.40 \mathrm{E}-3$ & ug/g dry & $6.40 \mathrm{E}-3$ & 9/18/08 & $8 \mathrm{I} 11003$ & PNNL-AGG-415 \\
\hline 14191-84-5 & Copper & $6.67 \mathrm{E}-3$ & ug/g dry & $3.48 \mathrm{E}-3$ & 9/18/08 & 8I11003 & PNNL-AGG-415 \\
\hline $7440-38-2$ & Arsenic & $2.99 \mathrm{E}-2$ & ug/g dry & $6.25 \mathrm{E}-3$ & 9/18/08 & $8 \mathrm{I} 11003$ & PNNL-AGG-415 \\
\hline 14687-58-2 & Selenium & $<1.10 \mathrm{E}-2$ & ug/g dry & $1.10 \mathrm{E}-2$ & 9/18/08 & $8 \mathrm{II} 1003$ & PNNL-AGG-415 \\
\hline 14378-37-1 & Silver & $<9.25 \mathrm{E}-4$ & ug/g dry & $9.25 \mathrm{E}-4$ & 9/18/08 & $8 \mathrm{I} 11003$ & PNNL-AGG-415 \\
\hline 14336-64-2 & Cadmium & $4.57 \mathrm{E}-4$ & ug/g dry & 2.95E-4 & 9/18/08 & 8I11003 & PNNL-AGG-415 \\
\hline 14265-72-6 & Antimony & $<5.40 \mathrm{E}-4$ & ug/g dry & $5.40 \mathrm{E}-4$ & 9/18/08 & $8 \mathrm{I} 11003$ & PNNL-AGG-415 \\
\hline 13966-28-4 & Lead & 8.95E-4 & ug/g dry & $5.60 \mathrm{E}-4$ & 9/18/08 & $8 \mathrm{I} 11003$ & PNNL-AGG-415 \\
\hline HEIS No. & B1TNV9 & \multicolumn{3}{|c|}{ Lab ID: $\quad$ 0804025-10 } & & & \\
\hline 13981-78-7 & Chromium & $<6.40 \mathrm{E}-3$ & ug/g dry & $6.40 \mathrm{E}-3$ & 9/18/08 & 8I11003 & PNNL-AGG-415 \\
\hline 14191-84-5 & Copper & 2.13E-2 & ug/g dry & $3.48 \mathrm{E}-3$ & 9/18/08 & $8 \mathrm{I} 11003$ & PNNL-AGG-415 \\
\hline 7440-38-2 & Arsenic & $1.65 \mathrm{E}-2$ & ug/g dry & $6.25 \mathrm{E}-3$ & 9/18/08 & $8 \mathrm{I} 11003$ & PNNL-AGG-415 \\
\hline $14687-58-2$ & Selenium & $<1.11 \mathrm{E}-2$ & ug/g dry & $1.11 \mathrm{E}-2$ & 9/18/08 & $8 \mathrm{I} 11003$ & PNNL-AGG-415 \\
\hline 14378-37-1 & Silver & $<9.25 \mathrm{E}-4$ & ug/g dry & $9.25 \mathrm{E}-4$ & 9/18/08 & 8I11003 & PNNL-AGG-415 \\
\hline 14336-64-2 & Cadmium & $<2.95 \mathrm{E}-4$ & ug/g dry & 2.95E-4 & 9/18/08 & $8 \mathrm{I} 11003$ & PNNL-AGG-415 \\
\hline 14265-72-6 & Antimony & $<5.40 \mathrm{E}-4$ & ug/g dry & $5.40 \mathrm{E}-4$ & 9/18/08 & 8I11003 & PNNL-AGG-415 \\
\hline 13966-28-4 & Lead & $<5.60 \mathrm{E}-4$ & ug/g dry & $5.60 \mathrm{E}-4$ & 9/18/08 & $8 \mathrm{I} 11003$ & PNNL-AGG-415 \\
\hline HEIS No. & В1TP22 & \multicolumn{3}{|c|}{$\begin{array}{ll}\text { Lab ID: } & 0804025-13\end{array}$} & & & \\
\hline 13981-78-7 & Chromium & $<6.47 \mathrm{E}-3$ & ug/g dry & $6.47 \mathrm{E}-3$ & $7 / 10 / 08$ & 8G10003 & PNNL-AGG-415 \\
\hline 14191-84-5 & Copper & $<3.52 \mathrm{E}-3$ & ug/g dry & $3.52 \mathrm{E}-3$ & 7/10/08 & 8G10003 & PNNL-AGG-415 \\
\hline 7440-38-2 & Arsenic & $1.20 \mathrm{E}-2$ & ug/g dry & $6.32 \mathrm{E}-3$ & 7/10/08 & 8G10003 & PNNL-AGG-415 \\
\hline
\end{tabular}


RCRA Metals By PNNL-AGG-415/Water Extract

\begin{tabular}{|c|c|c|c|c|c|c|c|}
\hline CAS \# & Analyte & Results & Units & EQL & Analyzed & Batch & Method \\
\hline HEIS No. & B1TP22 & \multicolumn{3}{|c|}{ Lab ID: $\quad$ 0804025-13 } & & & \\
\hline $14687-58-2$ & Selenium & $<1.12 \mathrm{E}-2$ & ug/g dry & $1.12 \mathrm{E}-2$ & 7/10/08 & 8G10003 & PNNL-AGG-415 \\
\hline 14378-37-1 & Silver & $<9.35 \mathrm{E}-4$ & ug/g dry & $9.35 \mathrm{E}-4$ & $7 / 10 / 08$ & 8G10003 & PNNL-AGG-415 \\
\hline 14336-64-2 & Cadmium & $<2.98 \mathrm{E}-4$ & ug/g dry & $2.98 \mathrm{E}-4$ & 7/10/08 & 8G10003 & PNNL-AGG-415 \\
\hline $14265-72-6$ & Antimony & $<5.46 \mathrm{E}-4$ & ug/g dry & $5.46 \mathrm{E}-4$ & $7 / 10 / 08$ & 8G10003 & PNNL-AGG-415 \\
\hline 13966-28-4 & Lead & $<5.66 \mathrm{E}-4$ & ug/g dry & $5.66 \mathrm{E}-4$ & 7/10/08 & 8 G10003 & PNNL-AGG-415 \\
\hline HEIS No. & B1TP26 & \multicolumn{3}{|c|}{ Lab ID: $\quad$ 0804025-16 } & & & \\
\hline 13981-78-7 & Chromium & $<6.42 \mathrm{E}-3$ & ug/g dry & $6.42 \mathrm{E}-3$ & $7 / 10 / 08$ & 8G10003 & PNNL-AGG-415 \\
\hline 14191-84-5 & Copper & $<3.49 \mathrm{E}-3$ & ug/g dry & $3.49 \mathrm{E}-3$ & $7 / 10 / 08$ & 8G10003 & PNNL-AGG-415 \\
\hline $7440-38-2$ & Arsenic & $1.24 \mathrm{E}-1$ & ug/g dry & $6.27 \mathrm{E}-3$ & $7 / 10 / 08$ & 8G10003 & PNNL-AGG-415 \\
\hline 14687-58-2 & Selenium & $<1.11 \mathrm{E}-2$ & ug/g dry & $1.11 \mathrm{E}-2$ & $7 / 10 / 08$ & 8G10003 & PNNL-AGG-415 \\
\hline 14378-37-1 & Silver & $<9.28 \mathrm{E}-4$ & ug/g dry & $9.28 \mathrm{E}-4$ & 7/10/08 & 8G10003 & PNNL-AGG-415 \\
\hline 14336-64-2 & Cadmium & $<2.96 \mathrm{E}-4$ & ug/g dry & $2.96 \mathrm{E}-4$ & $7 / 10 / 08$ & 8G10003 & PNNL-AGG-415 \\
\hline $14265-72-6$ & Antimony & $<5.42 \mathrm{E}-4$ & ug/g dry & $5.42 \mathrm{E}-4$ & $7 / 10 / 08$ & 8G10003 & PNNL-AGG-415 \\
\hline 13966-28-4 & Lead & $<5.62 \mathrm{E}-4$ & ug/g dry & $5.62 \mathrm{E}-4$ & 7/10/08 & 8G10003 & PNNL-AGG-415 \\
\hline HEIS No. & B1TNW0 & \multicolumn{3}{|c|}{ Lab ID: $\quad$ 0804025-17 } & & & \\
\hline 13981-78-7 & Chromium & $<6.40 \mathrm{E}-3$ & ug/g dry & $6.40 \mathrm{E}-3$ & 9/18/08 & $8 I 11003$ & PNNL-AGG-415 \\
\hline 14191-84-5 & Copper & $4.91 \mathrm{E}-2$ & ug/g dry & $3.48 \mathrm{E}-3$ & $9 / 18 / 08$ & $8 \mathrm{I} 11003$ & PNNL-AGG-415 \\
\hline 7440-38-2 & Arsenic & $<6.25 \mathrm{E}-3$ & ug/g dry & $6.25 \mathrm{E}-3$ & 9/18/08 & 8I11003 & PNNL-AGG-415 \\
\hline 14687-58-2 & Selenium & $<1.11 \mathrm{E}-2$ & ug/g dry & $1.11 \mathrm{E}-2$ & 9/18/08 & $8 \mathrm{I} 11003$ & PNNL-AGG-415 \\
\hline 14378-37-1 & Silver & $<9.25 \mathrm{E}-4$ & ug/g dry & $9.25 \mathrm{E}-4$ & 9/18/08 & 8I11003 & PNNL-AGG-415 \\
\hline 14336-64-2 & Cadmium & 3.26E-4 & ug/g dry & $2.95 \mathrm{E}-4$ & 9/18/08 & $8 \mathrm{I} 11003$ & PNNL-AGG-415 \\
\hline 14265-72-6 & Antimony & $<5.40 \mathrm{E}-4$ & ug/g dry & $5.40 \mathrm{E}-4$ & 9/18/08 & 8I11003 & PNNL-AGG-415 \\
\hline 13966-28-4 & Lead & $<5.60 \mathrm{E}-4$ & ug/g dry & $5.60 \mathrm{E}-4$ & 9/18/08 & $8 \mathrm{I} 11003$ & PNNL-AGG-415 \\
\hline HEIS No. & B1TNW1 & \multicolumn{3}{|c|}{ Lab ID: $\quad$ 0804025-18 } & & & \\
\hline 13981-78-7 & Chromium & $<6.34 \mathrm{E}-3$ & ug/g dry & $6.34 \mathrm{E}-3$ & $7 / 10 / 08$ & 8G10003 & PNNL-AGG-415 \\
\hline 14191-84-5 & Copper & $2.04 \mathrm{E}-2$ & ug/g dry & 3.45E-3 & 7/10/08 & 8G10003 & PNNL-AGG-415 \\
\hline $7440-38-2$ & Arsenic & $<6.19 \mathrm{E}-3$ & ug/g dry & $6.19 \mathrm{E}-3$ & 7/10/08 & 8G10003 & PNNL-AGG-415 \\
\hline $14687-58-2$ & Selenium & $<1.09 \mathrm{E}-2$ & ug/g dry & 1.09E-2 & 7/10/08 & 8G10003 & PNNL-AGG-415 \\
\hline 14378-37-1 & Silver & $<9.16 \mathrm{E}-4$ & ug/g dry & $9.16 \mathrm{E}-4$ & 7/10/08 & 8G10003 & PNNL-AGG-415 \\
\hline $14336-64-2$ & Cadmium & $<2.92 \mathrm{E}-4$ & ug/g dry & $2.92 \mathrm{E}-4$ & 7/10/08 & 8G10003 & PNNL-AGG-415 \\
\hline 14265-72-6 & Antimony & $<5.35 \mathrm{E}-4$ & ug/g dry & $5.35 \mathrm{E}-4$ & 7/10/08 & 8G10003 & PNNL-AGG-415 \\
\hline 13966-28-4 & Lead & $<5.55 \mathrm{E}-4$ & ug/g dry & $5.55 \mathrm{E}-4$ & 7/10/08 & 8G10003 & PNNL-AGG-415 \\
\hline HEIS No. & B1TNW3 & \multicolumn{3}{|c|}{ Lab ID: $\quad 0804025-20$} & & & \\
\hline 13981-78-7 & Chromium & $<6.40 \mathrm{E}-3$ & ug/g dry & $6.40 \mathrm{E}-3$ & $9 / 17 / 08$ & $8 \mathrm{I} 11003$ & PNNL-AGG-415 \\
\hline 14191-84-5 & Copper & 7.91E-3 & ug/g dry & $3.48 \mathrm{E}-3$ & 9/17/08 & $8 \mathrm{I} 11003$ & PNNL-AGG-415 \\
\hline $7440-38-2$ & Arsenic & $<6.25 \mathrm{E}-3$ & ug/g dry & $6.25 \mathrm{E}-3$ & $9 / 17 / 08$ & $8 \mathrm{I} 11003$ & PNNL-AGG-415 \\
\hline 14687-58-2 & Selenium & $<1.11 \mathrm{E}-2$ & ug/g dry & $1.11 \mathrm{E}-2$ & $9 / 17 / 08$ & $8 I 11003$ & PNNL-AGG-415 \\
\hline 14378-37-1 & Silver & $<9.25 \mathrm{E}-4$ & ug/g dry & $9.25 \mathrm{E}-4$ & 9/17/08 & $8 \mathrm{I} 11003$ & PNNL-AGG-415 \\
\hline 14336-64-2 & Cadmium & $6.52 \mathrm{E}-4$ & ug/g dry & $2.95 \mathrm{E}-4$ & 9/17/08 & 8I11003 & PNNL-AGG-415 \\
\hline $14265-72-6$ & Antimony & $<5.40 \mathrm{E}-4$ & ug/g dry & $5.40 \mathrm{E}-4$ & 9/17/08 & $8 \mathrm{I} 11003$ & PNNL-AGG-415 \\
\hline 13966-28-4 & Lead & $<5.60 \mathrm{E}-4$ & ug/g dry & $5.60 \mathrm{E}-4$ & 9/17/08 & 8I11003 & PNNL-AGG-415 \\
\hline HEIS No. & B1TNW5 & \multicolumn{3}{|c|}{ Lab ID: $\quad 0804025-22$} & & & \\
\hline 13981-78-7 & Chromium & $<6.29 \mathrm{E}-3$ & ug/g dry & $6.29 \mathrm{E}-3$ & 7/10/08 & 8G10003 & PNNL-AGG-415 \\
\hline 14191-84-5 & Copper & $<3.42 \mathrm{E}-3$ & ug/g dry & $3.42 \mathrm{E}-3$ & 7/10/08 & 8G10003 & PNNL-AGG-415 \\
\hline $7440-38-2$ & Arsenic & $<6.15 \mathrm{E}-3$ & ug/g dry & $6.15 \mathrm{E}-3$ & 7/10/08 & 8G10003 & PNNL-AGG-415 \\
\hline 14687-58-2 & Selenium & $<1.09 \mathrm{E}-2$ & ug/g dry & $1.09 \mathrm{E}-2$ & 7/10/08 & 8G10003 & PNNL-AGG-415 \\
\hline 14378-37-1 & Silver & $<9.10 \mathrm{E}-4$ & ug/g dry & $9.10 \mathrm{E}-4$ & 7/10/08 & 8G10003 & PNNL-AGG-415 \\
\hline 14336-64-2 & Cadmium & $<2.90 \mathrm{E}-4$ & ug/g dry & $2.90 \mathrm{E}-4$ & 7/10/08 & 8G10003 & PNNL-AGG-415 \\
\hline
\end{tabular}


RCRA Metals By PNNL-AGG-415/Water Extract

\begin{tabular}{|c|c|c|c|c|c|c|c|}
\hline CAS \# & Analyte & Results & Units & EQL & Analyzed & Batch & Method \\
\hline HEIS No. & B1TNW5 & \multicolumn{3}{|c|}{ Lab ID: $\quad 0804025-22$} & & & \\
\hline $14265-72-6$ & Antimony & $<5.31 \mathrm{E}-4$ & ug/g dry & $5.31 \mathrm{E}-4$ & $7 / 10 / 08$ & 8G10003 & PNNL-AGG-415 \\
\hline 13966-28-4 & Lead & $<5.51 \mathrm{E}-4$ & ug/g dry & $5.51 \mathrm{E}-4$ & 7/10/08 & 8G10003 & PNNL-AGG-415 \\
\hline HEIS No. & B1TP35 & \multicolumn{3}{|c|}{ Lab ID: $\quad$ 0804025-37 } & & & \\
\hline 13981-78-7 & Chromium & $<6.91 \mathrm{E}-3$ & ug/g dry & $6.91 \mathrm{E}-3$ & $7 / 10 / 08$ & 8G10003 & PNNL-AGG-415 \\
\hline $14191-84-5$ & Copper & $<3.76 \mathrm{E}-3$ & ug/g dry & $3.76 \mathrm{E}-3$ & $7 / 10 / 08$ & 8G10003 & PNNL-AGG-415 \\
\hline $7440-38-2$ & Arsenic & $6.75 \mathrm{E}-3$ & ug/g dry & $6.75 \mathrm{E}-3$ & 7/10/08 & $8 G 10003$ & PNNL-AGG-415 \\
\hline $14687-58-2$ & Selenium & $<1.19 \mathrm{E}-2$ & ug/g dry & $1.19 \mathrm{E}-2$ & $7 / 10 / 08$ & 8G10003 & PNNL-AGG-415 \\
\hline $14378-37-1$ & Silver & $<9.99 \mathrm{E}-4$ & ug/g dry & $9.99 \mathrm{E}-4$ & $7 / 10 / 08$ & 8G10003 & PNNL-AGG-415 \\
\hline $14336-64-2$ & Cadmium & $<3.18 \mathrm{E}-4$ & ug/g dry & $3.18 \mathrm{E}-4$ & 7/10/08 & 8G10003 & PNNL-AGG-415 \\
\hline $14265-72-6$ & Antimony & $<5.83 \mathrm{E}-4$ & ug/g dry & $5.83 \mathrm{E}-4$ & 7/10/08 & 8G10003 & PNNL-AGG-415 \\
\hline 13966-28-4 & Lead & $<6.05 \mathrm{E}-4$ & ug/g dry & $6.05 \mathrm{E}-4$ & 7/10/08 & 8G10003 & PNNL-AGG-415 \\
\hline HEIS No. & B1TP40 & \multicolumn{3}{|c|}{ Lab ID: $\quad 0804025-48$} & & & \\
\hline 13981-78-7 & Chromium & $<6.41 \mathrm{E}-3$ & ug/g dry & $6.41 \mathrm{E}-3$ & 7/10/08 & $8 G 10003$ & PNNL-AGG-415 \\
\hline $14191-84-5$ & Copper & $<3.48 \mathrm{E}-3$ & ug/g dry & $3.48 \mathrm{E}-3$ & 7/10/08 & 8G10003 & PNNL-AGG-415 \\
\hline $7440-38-2$ & Arsenic & $8.07 \mathrm{E}-3$ & ug/g dry & $6.26 \mathrm{E}-3$ & 7/10/08 & 8G10003 & PNNL-AGG-415 \\
\hline $14687-58-2$ & Selenium & $<1.11 \mathrm{E}-2$ & ug/g dry & $1.11 \mathrm{E}-2$ & 7/10/08 & 8G10003 & PNNL-AGG-415 \\
\hline 14378-37-1 & Silver & $<9.26 \mathrm{E}-4$ & ug/g dry & $9.26 \mathrm{E}-4$ & 7/10/08 & 8G10003 & PNNL-AGG-415 \\
\hline $14336-64-2$ & Cadmium & $<2.95 \mathrm{E}-4$ & ug/g dry & $2.95 \mathrm{E}-4$ & 7/10/08 & $8 G 10003$ & PNNL-AGG-415 \\
\hline $14265-72-6$ & Antimony & $<5.41 \mathrm{E}-4$ & ug/g dry & $5.41 \mathrm{E}-4$ & $7 / 10 / 08$ & 8G10003 & PNNL-AGG-415 \\
\hline 13966-28-4 & Lead & $<5.61 \mathrm{E}-4$ & ug/g dry & $5.61 \mathrm{E}-4$ & $7 / 10 / 08$ & 8G10003 & PNNL-AGG-415 \\
\hline HEIS No. & B1TP42 & \multicolumn{3}{|c|}{ Lab ID: $\quad 0804025-50$} & & & \\
\hline $13981-78-7$ & Chromium & $<6.41 \mathrm{E}-3$ & ug/g dry & $6.41 \mathrm{E}-3$ & 9/17/08 & $8 \mathrm{I} 11003$ & PNNL-AGG-415 \\
\hline $14191-84-5$ & Copper & $8.38 \mathrm{E}-3$ & ug/g dry & $3.48 \mathrm{E}-3$ & 9/17/08 & 8I11003 & PNNL-AGG-415 \\
\hline 7440-38-2 & Arsenic & $<6.26 \mathrm{E}-3$ & ug/g dry & $6.26 \mathrm{E}-3$ & 9/17/08 & $8 \mathrm{I} 11003$ & PNNL-AGG-415 \\
\hline $14687-58-2$ & Selenium & $<1.11 \mathrm{E}-2$ & ug/g dry & $1.11 \mathrm{E}-2$ & 9/17/08 & $8 \mathrm{I} 11003$ & PNNL-AGG-415 \\
\hline 14378-37-1 & Silver & $<9.26 \mathrm{E}-4$ & ug/g dry & $9.26 \mathrm{E}-4$ & 9/17/08 & $8 \mathrm{I} 11003$ & PNNL-AGG-415 \\
\hline $14336-64-2$ & Cadmium & 7.19E-4 & ug/g dry & $2.95 \mathrm{E}-4$ & 9/17/08 & $8 \mathrm{I} 11003$ & PNNL-AGG-415 \\
\hline $14265-72-6$ & Antimony & $<5.40 \mathrm{E}-4$ & ug/g dry & $5.40 \mathrm{E}-4$ & 9/17/08 & $8 \mathrm{I} 11003$ & PNNL-AGG-415 \\
\hline 13966-28-4 & Lead & $<5.60 \mathrm{E}-4$ & ug/g dry & $5.60 \mathrm{E}-4$ & 9/17/08 & $8 \mathrm{I} 11003$ & PNNL-AGG-415 \\
\hline HEIS No. & B1TP43 & \multicolumn{3}{|c|}{ Lab ID: $\quad 0804025-51$} & & & \\
\hline 13981-78-7 & Chromium & $<6.41 \mathrm{E}-3$ & ug/g dry & $6.41 \mathrm{E}-3$ & 7/10/08 & 8G10003 & PNNL-AGG-415 \\
\hline 14119-06-3 & Copper & $5.75 \mathrm{E}-3$ & ug/g dry & $4.85 \mathrm{E}-3$ & $7 / 10 / 08$ & 8G10003 & PNNL-AGG-415 \\
\hline 7440-38-2 & Arsenic & $6.35 \mathrm{E}-3$ & ug/g dry & $6.26 \mathrm{E}-3$ & 7/10/08 & 8G10003 & PNNL-AGG-415 \\
\hline $14687-58-2$ & Selenium & $<1.11 \mathrm{E}-2$ & ug/g dry & $1.11 \mathrm{E}-2$ & 7/10/08 & $8 G 10003$ & PNNL-AGG-415 \\
\hline 14378-37-1 & Silver & $<9.27 \mathrm{E}-4$ & ug/g dry & $9.27 \mathrm{E}-4$ & 7/10/08 & 8G10003 & PNNL-AGG-415 \\
\hline 14336-64-2 & Cadmium & $<2.96 \mathrm{E}-4$ & ug/g dry & $2.96 \mathrm{E}-4$ & 7/10/08 & 8G10003 & PNNL-AGG-415 \\
\hline $14265-72-6$ & Antimony & $<5.41 \mathrm{E}-4$ & ug/g dry & $5.41 \mathrm{E}-4$ & 7/10/08 & 8G10003 & PNNL-AGG-415 \\
\hline 13966-28-4 & Lead & $<5.61 \mathrm{E}-4$ & ug/g dry & $5.61 \mathrm{E}-4$ & 7/10/08 & 8G10003 & PNNL-AGG-415 \\
\hline HEIS No. & B1TP45 & \multicolumn{3}{|c|}{ Lab ID: $\quad 0804025-53$} & & & \\
\hline 13981-78-7 & Chromium & $<6.40 \mathrm{E}-3$ & ug/g dry & $6.40 \mathrm{E}-3$ & 9/18/08 & $8 \mathrm{I} 11003$ & PNNL-AGG-415 \\
\hline 14191-84-5 & Copper & $2.54 \mathrm{E}-2$ & ug/g dry & $3.48 \mathrm{E}-3$ & 9/18/08 & 8I11003 & PNNL-AGG-415 \\
\hline $7440-38-2$ & Arsenic & $1.51 \mathrm{E}-2$ & ug/g dry & $6.25 \mathrm{E}-3$ & 9/18/08 & $8 \mathrm{I} 11003$ & PNNL-AGG-415 \\
\hline $14687-58-2$ & Selenium & $<1.11 \mathrm{E}-2$ & ug/g dry & $1.11 \mathrm{E}-2$ & 9/18/08 & $8 \mathrm{I} 11003$ & PNNL-AGG-415 \\
\hline 14378-37-1 & Silver & $<9.25 \mathrm{E}-4$ & ug/g dry & $9.25 \mathrm{E}-4$ & 9/18/08 & $8 I 11003$ & PNNL-AGG-415 \\
\hline $14336-64-2$ & Cadmium & $<2.95 \mathrm{E}-4$ & ug/g dry & 2.95E-4 & 9/18/08 & 8I11003 & PNNL-AGG-415 \\
\hline $14265-72-6$ & Antimony & $<5.40 \mathrm{E}-4$ & ug/g dry & $5.40 \mathrm{E}-4$ & 9/18/08 & $8 \mathrm{I} 11003$ & PNNL-AGG-415 \\
\hline $13966-28-4$ & Lead & $<5.60 \mathrm{E}-4$ & ug/g dry & $5.60 \mathrm{E}-4$ & 9/18/08 & $8 \mathrm{I} 11003$ & PNNL-AGG-415 \\
\hline HEIS No. & $\begin{array}{l}\text { B1TP47 } \\
\end{array}$ & $\mathbf{L a}$ & b ID: & 4025-57 & & & \\
\hline
\end{tabular}


RCRA Metals By PNNL-AGG-415/Water Extract

\begin{tabular}{|c|c|c|c|c|c|c|c|}
\hline CAS \# & Analyte & Results & Units & EQL & Analyzed & Batch & Method \\
\hline HEIS No. & B1TP47 & \multicolumn{3}{|c|}{ Lab ID: $\quad$ 0804025-57 } & & & \\
\hline 13981-78-7 & Chromium & $<6.34 \mathrm{E}-3$ & ug/g dry & $6.34 \mathrm{E}-3$ & $7 / 10 / 08$ & 8G10003 & PNNL-AGG-415 \\
\hline 14191-84-5 & Copper & $<3.45 \mathrm{E}-3$ & ug/g dry & $3.45 \mathrm{E}-3$ & 7/10/08 & 8G10003 & PNNL-AGG-415 \\
\hline $7440-38-2$ & Arsenic & $<6.19 \mathrm{E}-3$ & ug/g dry & $6.19 \mathrm{E}-3$ & 7/10/08 & 8G10003 & PNNL-AGG-415 \\
\hline 14687-58-2 & Selenium & $<1.10 \mathrm{E}-2$ & ug/g dry & $1.10 \mathrm{E}-2$ & 7/10/08 & 8G10003 & PNNL-AGG-415 \\
\hline 14378-37-1 & Silver & $<9.17 \mathrm{E}-4$ & ug/g dry & $9.17 \mathrm{E}-4$ & $7 / 10 / 08$ & 8G10003 & PNNL-AGG-415 \\
\hline 14336-64-2 & Cadmium & $<2.92 \mathrm{E}-4$ & ug/g dry & $2.92 \mathrm{E}-4$ & $7 / 10 / 08$ & 8G10003 & PNNL-AGG-415 \\
\hline 14265-72-6 & Antimony & $<5.35 \mathrm{E}-4$ & ug/g dry & $5.35 \mathrm{E}-4$ & 7/10/08 & 8G10003 & PNNL-AGG-415 \\
\hline 13966-28-4 & Lead & $<5.55 \mathrm{E}-4$ & ug/g dry & $5.55 \mathrm{E}-4$ & 7/10/08 & 8G10003 & PNNL-AGG-415 \\
\hline HEIS No. & В1TP49 & \multicolumn{3}{|c|}{ Lab ID: $\quad$ 0804025-61 } & & & \\
\hline 13981-78-7 & Chromium & $<6.41 \mathrm{E}-3$ & ug/g dry & $6.41 \mathrm{E}-3$ & 7/10/08 & 8G10004 & PNNL-AGG-415 \\
\hline 14191-84-5 & Copper & $<3.48 \mathrm{E}-3$ & ug/g dry & $3.48 \mathrm{E}-3$ & $7 / 10 / 08$ & 8G10004 & PNNL-AGG-415 \\
\hline $7440-38-2$ & Arsenic & $<6.26 \mathrm{E}-3$ & ug/g dry & $6.26 \mathrm{E}-3$ & 7/10/08 & 8G10004 & PNNL-AGG-415 \\
\hline $14687-58-2$ & Selenium & $<1.11 \mathrm{E}-2$ & ug/g dry & $1.11 \mathrm{E}-2$ & $7 / 10 / 08$ & 8G10004 & PNNL-AGG-415 \\
\hline 14378-37-1 & Silver & $<9.26 \mathrm{E}-4$ & ug/g dry & $9.26 \mathrm{E}-4$ & 7/10/08 & 8G10004 & PNNL-AGG-415 \\
\hline 14336-64-2 & Cadmium & $<2.95 \mathrm{E}-4$ & ug/g dry & 2.95E-4 & $7 / 10 / 08$ & 8G10004 & PNNL-AGG-415 \\
\hline $14265-72-6$ & Antimony & $<5.41 \mathrm{E}-4$ & ug/g dry & $5.41 \mathrm{E}-4$ & $7 / 10 / 08$ & 8G10004 & PNNL-AGG-415 \\
\hline 13966-28-4 & Lead & $<5.61 \mathrm{E}-4$ & ug/g dry & $5.61 \mathrm{E}-4$ & $7 / 10 / 08$ & 8G10004 & PNNL-AGG-415 \\
\hline HEIS No. & B1TP51 & \multicolumn{3}{|c|}{ Lab ID: $\quad$ 0804025-69 } & & & \\
\hline 13981-78-7 & Chromium & $<6.30 \mathrm{E}-3$ & ug/g dry & $6.30 \mathrm{E}-3$ & $7 / 10 / 08$ & 8G10004 & PNNL-AGG-415 \\
\hline 14119-06-3 & Copper & $5.45 \mathrm{E}-3$ & ug/g dry & $4.77 \mathrm{E}-3$ & $7 / 10 / 08$ & 8G10004 & PNNL-AGG-415 \\
\hline $7440-38-2$ & Arsenic & $<6.16 \mathrm{E}-3$ & ug/g dry & $6.16 \mathrm{E}-3$ & $7 / 10 / 08$ & 8G10004 & PNNL-AGG-415 \\
\hline 14687-58-2 & Selenium & $1.24 \mathrm{E}-2$ & ug/g dry & $1.09 \mathrm{E}-2$ & 7/10/08 & 8G10004 & PNNL-AGG-415 \\
\hline 14378-37-1 & Silver & $<9.11 \mathrm{E}-4$ & ug/g dry & $9.11 \mathrm{E}-4$ & $7 / 10 / 08$ & 8G10004 & PNNL-AGG-415 \\
\hline 14336-64-2 & Cadmium & $<2.91 \mathrm{E}-4$ & ug/g dry & $2.91 \mathrm{E}-4$ & 7/10/08 & 8G10004 & PNNL-AGG-415 \\
\hline $14265-72-6$ & Antimony & $<5.32 \mathrm{E}-4$ & ug/g dry & $5.32 \mathrm{E}-4$ & $7 / 10 / 08$ & 8G10004 & PNNL-AGG-415 \\
\hline 13966-28-4 & Lead & $<5.52 \mathrm{E}-4$ & ug/g dry & $5.52 \mathrm{E}-4$ & $7 / 10 / 08$ & 8G10004 & PNNL-AGG-415 \\
\hline HEIS No. & B1TP52 & \multicolumn{3}{|c|}{ Lab ID: $\quad 0804025-72$} & & & \\
\hline 13981-78-7 & Chromium & $<6.36 \mathrm{E}-3$ & ug/g dry & $6.36 \mathrm{E}-3$ & $7 / 10 / 08$ & 8G10004 & PNNL-AGG-415 \\
\hline 14191-84-5 & Copper & $5.60 \mathrm{E}-3$ & ug/g dry & $3.46 \mathrm{E}-3$ & 7/10/08 & 8G10004 & PNNL-AGG-415 \\
\hline $7440-38-2$ & Arsenic & $<6.21 \mathrm{E}-3$ & ug/g dry & $6.21 \mathrm{E}-3$ & $7 / 10 / 08$ & 8G10004 & PNNL-AGG-415 \\
\hline 14687-58-2 & Selenium & $<1.10 \mathrm{E}-2$ & ug/g dry & $1.10 \mathrm{E}-2$ & 7/10/08 & 8G10004 & PNNL-AGG-415 \\
\hline 14378-37-1 & Silver & $<9.20 \mathrm{E}-4$ & ug/g dry & $9.20 \mathrm{E}-4$ & $7 / 10 / 08$ & 8G10004 & PNNL-AGG-415 \\
\hline 14336-64-2 & Cadmium & $<2.93 \mathrm{E}-4$ & ug/g dry & 2.93E-4 & $7 / 10 / 08$ & 8G10004 & PNNL-AGG-415 \\
\hline $14265-72-6$ & Antimony & $<5.37 \mathrm{E}-4$ & ug/g dry & 5.37E-4 & $7 / 10 / 08$ & 8G10004 & PNNL-AGG-415 \\
\hline 13966-28-4 & Lead & $<5.57 \mathrm{E}-4$ & ug/g dry & $5.57 \mathrm{E}-4$ & 7/10/08 & 8G10004 & PNNL-AGG-415 \\
\hline HEIS No. & B1TP53 & \multicolumn{3}{|c|}{ Lab ID: $\quad 0804025-75$} & & & \\
\hline 13981-78-7 & Chromium & $<6.41 \mathrm{E}-3$ & ug/g dry & $6.41 \mathrm{E}-3$ & 7/10/08 & 8G10004 & PNNL-AGG-415 \\
\hline 14191-84-5 & Copper & $<3.49 \mathrm{E}-3$ & ug/g dry & $3.49 \mathrm{E}-3$ & $7 / 10 / 08$ & 8G10004 & PNNL-AGG-415 \\
\hline 7440-38-2 & Arsenic & $<6.26 \mathrm{E}-3$ & ug/g dry & $6.26 \mathrm{E}-3$ & $7 / 10 / 08$ & 8G10004 & PNNL-AGG-415 \\
\hline $14687-58-2$ & Selenium & $<1.11 \mathrm{E}-2$ & ug/g dry & $1.11 \mathrm{E}-2$ & $7 / 10 / 08$ & 8G10004 & PNNL-AGG-415 \\
\hline 14378-37-1 & Silver & $<9.26 \mathrm{E}-4$ & ug/g dry & $9.26 \mathrm{E}-4$ & 7/10/08 & 8G10004 & PNNL-AGG-415 \\
\hline 14336-64-2 & Cadmium & $<2.95 \mathrm{E}-4$ & ug/g dry & 2.95E-4 & $7 / 10 / 08$ & 8G10004 & PNNL-AGG-415 \\
\hline 14265-72-6 & Antimony & $<5.41 \mathrm{E}-4$ & ug/g dry & $5.41 \mathrm{E}-4$ & $7 / 10 / 08$ & 8G10004 & PNNL-AGG-415 \\
\hline 13966-28-4 & Lead & $<5.61 \mathrm{E}-4$ & ug/g dry & $5.61 \mathrm{E}-4$ & 7/10/08 & 8G10004 & PNNL-AGG-415 \\
\hline HEIS No. & B1TP55 & \multicolumn{3}{|c|}{$\begin{array}{ll}\text { Lab ID: } & 0804025-81\end{array}$} & & & \\
\hline 13981-78-7 & Chromium & $<6.42 \mathrm{E}-3$ & ug/g dry & $6.42 \mathrm{E}-3$ & $7 / 10 / 08$ & 8G10004 & PNNL-AGG-415 \\
\hline 14191-84-5 & Copper & $<3.49 \mathrm{E}-3$ & ug/g dry & $3.49 \mathrm{E}-3$ & $7 / 10 / 08$ & 8G10004 & PNNL-AGG-415 \\
\hline $7440-38-2$ & Arsenic & $<6.27 \mathrm{E}-3$ & ug/g dry & $6.27 \mathrm{E}-3$ & $7 / 10 / 08$ & 8G10004 & PNNL-AGG-415 \\
\hline
\end{tabular}


RCRA Metals By PNNL-AGG-415/Water Extract

\begin{tabular}{|c|c|c|c|c|c|c|c|}
\hline CAS \# & Analyte & Results & Units & EQL & Analyzed & Batch & Method \\
\hline HEIS No. & B1TP55 & \multicolumn{3}{|c|}{ Lab ID: $\quad$ 0804025-81 } & & & \\
\hline $14687-58-2$ & Selenium & $<1.11 \mathrm{E}-2$ & ug/g dry & $1.11 \mathrm{E}-2$ & 7/10/08 & 8G10004 & PNNL-AGG-415 \\
\hline 14378-37-1 & Silver & $<9.28 \mathrm{E}-4$ & ug/g dry & $9.28 \mathrm{E}-4$ & $7 / 10 / 08$ & 8G10004 & PNNL-AGG-415 \\
\hline 14336-64-2 & Cadmium & $<2.96 \mathrm{E}-4$ & ug/g dry & $2.96 \mathrm{E}-4$ & 7/10/08 & 8G10004 & PNNL-AGG-415 \\
\hline $14265-72-6$ & Antimony & $<5.42 \mathrm{E}-4$ & ug/g dry & $5.42 \mathrm{E}-4$ & $7 / 10 / 08$ & 8G10004 & PNNL-AGG-415 \\
\hline 13966-28-4 & Lead & $<5.62 \mathrm{E}-4$ & ug/g dry & $5.62 \mathrm{E}-4$ & 7/10/08 & 8 G10004 & PNNL-AGG-415 \\
\hline HEIS No. & B1TP59 & \multicolumn{3}{|c|}{ Lab ID: $\quad$ 0804025-87 } & & & \\
\hline 13981-78-7 & Chromium & $<6.49 \mathrm{E}-3$ & ug/g dry & $6.49 \mathrm{E}-3$ & $7 / 10 / 08$ & 8G10004 & PNNL-AGG-415 \\
\hline 14191-84-5 & Copper & $<3.53 \mathrm{E}-3$ & ug/g dry & 3.53E-3 & 7/10/08 & 8G10004 & PNNL-AGG-415 \\
\hline $7440-38-2$ & Arsenic & $<6.33 \mathrm{E}-3$ & ug/g dry & $6.33 \mathrm{E}-3$ & $7 / 10 / 08$ & 8G10004 & PNNL-AGG-415 \\
\hline 14687-58-2 & Selenium & $<1.12 \mathrm{E}-2$ & ug/g dry & $1.12 \mathrm{E}-2$ & $7 / 10 / 08$ & 8G10004 & PNNL-AGG-415 \\
\hline 14378-37-1 & Silver & $<9.38 \mathrm{E}-4$ & ug/g dry & $9.38 \mathrm{E}-4$ & 7/10/08 & 8G10004 & PNNL-AGG-415 \\
\hline 14336-64-2 & Cadmium & $<2.99 \mathrm{E}-4$ & ug/g dry & 2.99E-4 & $7 / 10 / 08$ & 8G10004 & PNNL-AGG-415 \\
\hline $14265-72-6$ & Antimony & $<5.47 \mathrm{E}-4$ & ug/g dry & $5.47 \mathrm{E}-4$ & $7 / 10 / 08$ & 8G10004 & PNNL-AGG-415 \\
\hline 13966-28-4 & Lead & $<5.68 \mathrm{E}-4$ & ug/g dry & $5.68 \mathrm{E}-4$ & 7/10/08 & 8G10004 & PNNL-AGG-415 \\
\hline HEIS No. & B1TP61 & \multicolumn{3}{|c|}{ Lab ID: $\quad$ 0804025-91 } & & & \\
\hline 13981-78-7 & Chromium & $<6.41 \mathrm{E}-3$ & ug/g dry & $6.41 \mathrm{E}-3$ & 9/18/08 & $8 I 11003$ & PNNL-AGG-415 \\
\hline 14191-84-5 & Copper & $5.81 \mathrm{E}-3$ & ug/g dry & $3.49 \mathrm{E}-3$ & $9 / 18 / 08$ & $8 \mathrm{I} 11003$ & PNNL-AGG-415 \\
\hline 7440-38-2 & Arsenic & $6.76 \mathrm{E}-3$ & ug/g dry & $6.26 \mathrm{E}-3$ & 9/18/08 & 8I11003 & PNNL-AGG-415 \\
\hline 14687-58-2 & Selenium & $1.23 \mathrm{E}-2$ & ug/g dry & $1.11 \mathrm{E}-2$ & 9/18/08 & $8 \mathrm{I} 11003$ & PNNL-AGG-415 \\
\hline 14378-37-1 & Silver & $<9.27 \mathrm{E}-4$ & ug/g dry & $9.27 \mathrm{E}-4$ & 9/18/08 & 8I11003 & PNNL-AGG-415 \\
\hline 14336-64-2 & Cadmium & $<2.96 \mathrm{E}-4$ & ug/g dry & $2.96 \mathrm{E}-4$ & 9/18/08 & $8 \mathrm{I} 11003$ & PNNL-AGG-415 \\
\hline 14265-72-6 & Antimony & $<5.41 \mathrm{E}-4$ & ug/g dry & $5.41 \mathrm{E}-4$ & 9/18/08 & 8I11003 & PNNL-AGG-415 \\
\hline 13966-28-4 & Lead & $<5.61 \mathrm{E}-4$ & ug/g dry & $5.61 \mathrm{E}-4$ & 9/18/08 & $8 \mathrm{I} 11003$ & PNNL-AGG-415 \\
\hline HEIS No. & B1TP62 & \multicolumn{3}{|c|}{ Lab ID: $\quad$ 0804025-94 } & & & \\
\hline 13981-78-7 & Chromium & $<6.32 \mathrm{E}-3$ & ug/g dry & $6.32 \mathrm{E}-3$ & $7 / 10 / 08$ & 8G10004 & PNNL-AGG-415 \\
\hline 14191-84-5 & Copper & $<3.44 \mathrm{E}-3$ & ug/g dry & $3.44 \mathrm{E}-3$ & 7/10/08 & 8G10004 & PNNL-AGG-415 \\
\hline $7440-38-2$ & Arsenic & $<6.17 \mathrm{E}-3$ & ug/g dry & 6.17E-3 & 7/10/08 & 8G10004 & PNNL-AGG-415 \\
\hline $14687-58-2$ & Selenium & $<1.09 \mathrm{E}-2$ & ug/g dry & $1.09 \mathrm{E}-2$ & 7/10/08 & 8G10004 & PNNL-AGG-415 \\
\hline 14378-37-1 & Silver & $<9.14 \mathrm{E}-4$ & ug/g dry & $9.14 \mathrm{E}-4$ & 7/10/08 & 8G10004 & PNNL-AGG-415 \\
\hline $14336-64-2$ & Cadmium & $<2.91 \mathrm{E}-4$ & ug/g dry & $2.91 \mathrm{E}-4$ & 7/10/08 & 8G10004 & PNNL-AGG-415 \\
\hline 14265-72-6 & Antimony & $<5.33 \mathrm{E}-4$ & ug/g dry & $5.33 \mathrm{E}-4$ & 7/10/08 & 8G10004 & PNNL-AGG-415 \\
\hline 13966-28-4 & Lead & $<5.53 \mathrm{E}-4$ & ug/g dry & $5.53 \mathrm{E}-4$ & 7/10/08 & 8G10004 & PNNL-AGG-415 \\
\hline HEIS No. & B1TP64 & \multicolumn{3}{|c|}{ Lab ID: $\quad$ 0804025-AA } & & & \\
\hline 13981-78-7 & Chromium & $<6.42 \mathrm{E}-3$ & ug/g dry & $6.42 \mathrm{E}-3$ & $9 / 18 / 08$ & $8 \mathrm{I} 11003$ & PNNL-AGG-415 \\
\hline 14191-84-5 & Copper & $5.19 \mathrm{E}-3$ & ug/g dry & $3.49 \mathrm{E}-3$ & $9 / 18 / 08$ & $8 \mathrm{I} 11003$ & PNNL-AGG-415 \\
\hline $7440-38-2$ & Arsenic & $<6.27 \mathrm{E}-3$ & ug/g dry & $6.27 \mathrm{E}-3$ & $9 / 18 / 08$ & $8 \mathrm{I} 11003$ & PNNL-AGG-415 \\
\hline 14687-58-2 & Selenium & $<1.11 \mathrm{E}-2$ & ug/g dry & $1.11 \mathrm{E}-2$ & 9/18/08 & $8 I 11003$ & PNNL-AGG-415 \\
\hline 14378-37-1 & Silver & $<9.28 \mathrm{E}-4$ & ug/g dry & $9.28 \mathrm{E}-4$ & $9 / 18 / 08$ & $8 \mathrm{I} 11003$ & PNNL-AGG-415 \\
\hline 14336-64-2 & Cadmium & $<2.96 \mathrm{E}-4$ & ug/g dry & $2.96 \mathrm{E}-4$ & 9/18/08 & 8I11003 & PNNL-AGG-415 \\
\hline $14265-72-6$ & Antimony & $<5.41 \mathrm{E}-4$ & ug/g dry & $5.41 \mathrm{E}-4$ & 9/18/08 & $8 \mathrm{I} 11003$ & PNNL-AGG-415 \\
\hline 13966-28-4 & Lead & $<5.62 \mathrm{E}-4$ & ug/g dry & $5.62 \mathrm{E}-4$ & 9/18/08 & 8I11003 & PNNL-AGG-415 \\
\hline HEIS No. & B1TP67 & \multicolumn{3}{|c|}{ Lab ID: $\quad$ 0804025-AI } & & & \\
\hline 13981-78-7 & Chromium & $<6.26 \mathrm{E}-3$ & ug/g dry & $6.26 \mathrm{E}-3$ & 7/10/08 & 8G10004 & PNNL-AGG-415 \\
\hline 14191-84-5 & Copper & $<3.40 \mathrm{E}-3$ & ug/g dry & $3.40 \mathrm{E}-3$ & 7/10/08 & 8G10004 & PNNL-AGG-415 \\
\hline $7440-38-2$ & Arsenic & $<6.11 \mathrm{E}-3$ & ug/g dry & $6.11 \mathrm{E}-3$ & 7/10/08 & 8G10004 & PNNL-AGG-415 \\
\hline 14687-58-2 & Selenium & $<1.08 \mathrm{E}-2$ & ug/g dry & $1.08 \mathrm{E}-2$ & 7/10/08 & 8G10004 & PNNL-AGG-415 \\
\hline 14378-37-1 & Silver & $<9.05 \mathrm{E}-4$ & ug/g dry & $9.05 \mathrm{E}-4$ & 7/10/08 & 8G10004 & PNNL-AGG-415 \\
\hline 14336-64-2 & Cadmium & $<2.88 \mathrm{E}-4$ & ug/g dry & $2.88 \mathrm{E}-4$ & 7/10/08 & 8G10004 & PNNL-AGG-415 \\
\hline
\end{tabular}


RCRA Metals By PNNL-AGG-415/Water Extract

\begin{tabular}{|c|c|c|c|c|c|c|c|}
\hline CAS \# & Analyte & Results & Units & EQL & Analyzed & Batch & Method \\
\hline HEIS No. & B1TP67 & \multicolumn{3}{|c|}{ Lab ID: $\quad$ 0804025-AI } & & & \\
\hline $14265-72-6$ & Antimony & $<5.28 \mathrm{E}-4$ & ug/g dry & $5.28 \mathrm{E}-4$ & 7/10/08 & 8G10004 & PNNL-AGG-415 \\
\hline 13966-28-4 & Lead & $<5.48 \mathrm{E}-4$ & ug/g dry & $5.48 \mathrm{E}-4$ & 7/10/08 & 8G10004 & PNNL-AGG-415 \\
\hline HEIS No. & В1TP69 & \multicolumn{3}{|c|}{ Lab ID: $\quad$ 0804025-AP } & & & \\
\hline 13981-78-7 & Chromium & $<6.41 \mathrm{E}-3$ & ug/g dry & $6.41 \mathrm{E}-3$ & 9/18/08 & $8 \mathrm{I} 11003$ & PNNL-AGG-415 \\
\hline $14191-84-5$ & Copper & $4.98 \mathrm{E}-3$ & ug/g dry & $3.49 \mathrm{E}-3$ & 9/18/08 & $8 I 11003$ & PNNL-AGG-415 \\
\hline $7440-38-2$ & Arsenic & $<6.26 \mathrm{E}-3$ & ug/g dry & $6.26 \mathrm{E}-3$ & 9/18/08 & $8 \mathrm{I} 11003$ & PNNL-AGG-415 \\
\hline $14687-58-2$ & Selenium & $<1.11 \mathrm{E}-2$ & ug/g dry & $1.11 \mathrm{E}-2$ & 9/18/08 & $8 I 11003$ & PNNL-AGG-415 \\
\hline $14378-37-1$ & Silver & $<9.26 \mathrm{E}-4$ & ug/g dry & $9.26 \mathrm{E}-4$ & 9/18/08 & 8I11003 & PNNL-AGG-415 \\
\hline $14336-64-2$ & Cadmium & $<2.95 \mathrm{E}-4$ & ug/g dry & $2.95 \mathrm{E}-4$ & 9/18/08 & $8 \mathrm{I} 11003$ & PNNL-AGG-415 \\
\hline $14265-72-6$ & Antimony & $6.15 \mathrm{E}-4$ & ug/g dry & $5.41 \mathrm{E}-4$ & 9/18/08 & $8 \mathrm{I} 11003$ & PNNL-AGG-415 \\
\hline 13966-28-4 & Lead & $<5.61 \mathrm{E}-4$ & ug/g dry & $5.61 \mathrm{E}-4$ & 9/18/08 & 8I11003 & PNNL-AGG-415 \\
\hline HEIS No. & B1TP71 & \multicolumn{3}{|c|}{ Lab ID: $\quad$ 0804025-AV } & & & \\
\hline 13981-78-7 & Chromium & $<6.38 \mathrm{E}-3$ & ug/g dry & $6.38 \mathrm{E}-3$ & 7/10/08 & 8G10004 & PNNL-AGG-415 \\
\hline $14191-84-5$ & Copper & $<3.47 \mathrm{E}-3$ & ug/g dry & $3.47 \mathrm{E}-3$ & 7/10/08 & 8G10004 & PNNL-AGG-415 \\
\hline $7440-38-2$ & Arsenic & $<6.23 \mathrm{E}-3$ & ug/g dry & $6.23 \mathrm{E}-3$ & 7/10/08 & 8G10004 & PNNL-AGG-415 \\
\hline $14687-58-2$ & Selenium & $<1.10 \mathrm{E}-2$ & ug/g dry & $1.10 \mathrm{E}-2$ & 7/10/08 & 8G10004 & PNNL-AGG-415 \\
\hline 14378-37-1 & Silver & $<9.22 \mathrm{E}-4$ & ug/g dry & $9.22 \mathrm{E}-4$ & 7/10/08 & 8G10004 & PNNL-AGG-415 \\
\hline $14336-64-2$ & Cadmium & $<2.94 \mathrm{E}-4$ & ug/g dry & $2.94 \mathrm{E}-4$ & 7/10/08 & 8G10004 & PNNL-AGG-415 \\
\hline $14265-72-6$ & Antimony & $<5.38 \mathrm{E}-4$ & ug/g dry & $5.38 \mathrm{E}-4$ & $7 / 10 / 08$ & 8G10004 & PNNL-AGG-415 \\
\hline 13966-28-4 & Lead & $<5.58 \mathrm{E}-4$ & ug/g dry & $5.58 \mathrm{E}-4$ & $7 / 10 / 08$ & 8G10004 & PNNL-AGG-415 \\
\hline HEIS No. & B1TP77 & \multicolumn{3}{|c|}{ Lab ID: $\quad$ 0804025-BK } & & & \\
\hline $13981-78-7$ & Chromium & $<6.39 \mathrm{E}-3$ & ug/g dry & $6.39 \mathrm{E}-3$ & 7/10/08 & 8G10004 & PNNL-AGG-415 \\
\hline $14191-84-5$ & Copper & $<3.48 \mathrm{E}-3$ & ug/g dry & $3.48 \mathrm{E}-3$ & 7/10/08 & 8G10004 & PNNL-AGG-415 \\
\hline 7440-38-2 & Arsenic & $<6.24 \mathrm{E}-3$ & ug/g dry & $6.24 \mathrm{E}-3$ & 7/10/08 & 8G10004 & PNNL-AGG-415 \\
\hline $14687-58-2$ & Selenium & $<1.10 \mathrm{E}-2$ & ug/g dry & $1.10 \mathrm{E}-2$ & 7/10/08 & $8 G 10004$ & PNNL-AGG-415 \\
\hline 14378-37-1 & Silver & $<9.24 \mathrm{E}-4$ & ug/g dry & $9.24 \mathrm{E}-4$ & 7/10/08 & 8G10004 & PNNL-AGG-415 \\
\hline $14336-64-2$ & Cadmium & $<2.95 \mathrm{E}-4$ & ug/g dry & $2.95 \mathrm{E}-4$ & 7/10/08 & 8G10004 & PNNL-AGG-415 \\
\hline $14265-72-6$ & Antimony & $<5.39 \mathrm{E}-4$ & ug/g dry & 5.39E-4 & 7/10/08 & 8G10004 & PNNL-AGG-415 \\
\hline 13966-28-4 & Lead & $<5.59 \mathrm{E}-4$ & ug/g dry & 5.59E-4 & 7/10/08 & 8G10004 & PNNL-AGG-415 \\
\hline HEIS No. & B1TP78 & \multicolumn{3}{|c|}{ Lab ID: $\quad$ 0804025-BP } & & & \\
\hline 13981-78-7 & Chromium & $<6.40 \mathrm{E}-3$ & ug/g dry & $6.40 \mathrm{E}-3$ & 7/10/08 & 8G10004 & PNNL-AGG-415 \\
\hline $14191-84-5$ & Copper & $<3.48 \mathrm{E}-3$ & ug/g dry & $3.48 \mathrm{E}-3$ & $7 / 10 / 08$ & 8G10004 & PNNL-AGG-415 \\
\hline 7440-38-2 & Arsenic & $<6.25 \mathrm{E}-3$ & ug/g dry & $6.25 \mathrm{E}-3$ & 7/10/08 & 8G10004 & PNNL-AGG-415 \\
\hline $14687-58-2$ & Selenium & $<1.11 \mathrm{E}-2$ & ug/g dry & $1.11 \mathrm{E}-2$ & 7/10/08 & 8G10004 & PNNL-AGG-415 \\
\hline 14378-37-1 & Silver & $<9.25 \mathrm{E}-4$ & ug/g dry & $9.25 \mathrm{E}-4$ & 7/10/08 & 8G10004 & PNNL-AGG-415 \\
\hline 14336-64-2 & Cadmium & $<2.95 \mathrm{E}-4$ & ug/g dry & $2.95 \mathrm{E}-4$ & 7/10/08 & 8G10004 & PNNL-AGG-415 \\
\hline $14265-72-6$ & Antimony & $<5.40 \mathrm{E}-4$ & ug/g dry & $5.40 \mathrm{E}-4$ & 7/10/08 & 8G10004 & PNNL-AGG-415 \\
\hline 13966-28-4 & Lead & $<5.60 \mathrm{E}-4$ & ug/g dry & $5.60 \mathrm{E}-4$ & 7/10/08 & 8G10004 & PNNL-AGG-415 \\
\hline HEIS No. & B1TP82 & \multicolumn{3}{|c|}{ Lab ID: $\quad$ 0804025-BZ } & & & \\
\hline 13981-78-7 & Chromium & $<6.38 \mathrm{E}-3$ & ug/g dry & $6.38 \mathrm{E}-3$ & 7/10/08 & 8G10004 & PNNL-AGG-415 \\
\hline 14191-84-5 & Copper & $<3.47 \mathrm{E}-3$ & ug/g dry & $3.47 \mathrm{E}-3$ & 7/10/08 & $8 G 10004$ & PNNL-AGG-415 \\
\hline $7440-38-2$ & Arsenic & $<6.23 \mathrm{E}-3$ & ug/g dry & $6.23 \mathrm{E}-3$ & 7/10/08 & 8G10004 & PNNL-AGG-415 \\
\hline $14687-58-2$ & Selenium & $1.26 \mathrm{E}-2$ & ug/g dry & $1.10 \mathrm{E}-2$ & 7/10/08 & $8 G 10004$ & PNNL-AGG-415 \\
\hline 14378-37-1 & Silver & $<9.23 \mathrm{E}-4$ & ug/g dry & $9.23 \mathrm{E}-4$ & $7 / 10 / 08$ & 8G10004 & PNNL-AGG-415 \\
\hline $14336-64-2$ & Cadmium & $<2.94 \mathrm{E}-4$ & ug/g dry & $2.94 \mathrm{E}-4$ & 7/10/08 & 8G10004 & PNNL-AGG-415 \\
\hline $14265-72-6$ & Antimony & $<5.39 \mathrm{E}-4$ & ug/g dry & $5.39 \mathrm{E}-4$ & 7/10/08 & 8G10004 & PNNL-AGG-415 \\
\hline $13966-28-4$ & Lead & $<5.58 \mathrm{E}-4$ & ug/g dry & $5.58 \mathrm{E}-4$ & 7/10/08 & 8G10004 & PNNL-AGG-415 \\
\hline HEIS No. & B1TP84 & $\mathbf{L a}$ & b ID: & $4025-C F$ & & & \\
\hline
\end{tabular}


RCRA Metals By PNNL-AGG-415/Water Extract

\begin{tabular}{|c|c|c|c|c|c|c|c|}
\hline CAS \# & Analyte & Results & Units & EQL & Analyzed & Batch & Method \\
\hline HEIS No. & В1TP84 & \multicolumn{3}{|c|}{ Lab ID: $\quad$ 0804025-CF } & & & \\
\hline 13981-78-7 & Chromium & $<6.39 \mathrm{E}-3$ & ug/g dry & 6.39E-3 & $7 / 10 / 08$ & 8G10004 & PNNL-AGG-415 \\
\hline 14191-84-5 & Copper & $<3.47 \mathrm{E}-3$ & ug/g dry & 3.47E-3 & 7/10/08 & 8G10004 & PNNL-AGG-415 \\
\hline $7440-38-2$ & Arsenic & $<6.24 \mathrm{E}-3$ & ug/g dry & $6.24 \mathrm{E}-3$ & 7/10/08 & 8G10004 & PNNL-AGG-415 \\
\hline 14687-58-2 & Selenium & $1.41 \mathrm{E}-2$ & ug/g dry & $1.10 \mathrm{E}-2$ & 7/10/08 & 8G10004 & PNNL-AGG-415 \\
\hline 14378-37-1 & Silver & $<9.23 \mathrm{E}-4$ & ug/g dry & $9.23 \mathrm{E}-4$ & $7 / 10 / 08$ & 8G10004 & PNNL-AGG-415 \\
\hline 14336-64-2 & Cadmium & $<2.94 \mathrm{E}-4$ & ug/g dry & $2.94 \mathrm{E}-4$ & $7 / 10 / 08$ & 8G10004 & PNNL-AGG-415 \\
\hline 14265-72-6 & Antimony & $<5.39 \mathrm{E}-4$ & ug/g dry & 5.39E-4 & 7/10/08 & 8G10004 & PNNL-AGG-415 \\
\hline 13966-28-4 & Lead & $<5.59 \mathrm{E}-4$ & ug/g dry & 5.59E-4 & 7/10/08 & 8G10004 & PNNL-AGG-415 \\
\hline HEIS No. & B1TNX1 & \multicolumn{3}{|c|}{ Lab ID: $\quad$ 0804025-CI } & & & \\
\hline 13981-78-7 & Chromium & $<6.45 \mathrm{E}-3$ & ug/g dry & $6.45 \mathrm{E}-3$ & 7/10/08 & 8G10004 & PNNL-AGG-415 \\
\hline 14191-84-5 & Copper & $<3.51 \mathrm{E}-3$ & ug/g dry & 3.51E-3 & $7 / 10 / 08$ & 8G10004 & PNNL-AGG-415 \\
\hline $7440-38-2$ & Arsenic & $<6.30 \mathrm{E}-3$ & ug/g dry & $6.30 \mathrm{E}-3$ & 7/10/08 & 8G10004 & PNNL-AGG-415 \\
\hline $14687-58-2$ & Selenium & $<1.11 \mathrm{E}-2$ & ug/g dry & $1.11 \mathrm{E}-2$ & $7 / 10 / 08$ & 8G10004 & PNNL-AGG-415 \\
\hline 14378-37-1 & Silver & $<9.32 \mathrm{E}-4$ & ug/g dry & $9.32 \mathrm{E}-4$ & 7/10/08 & 8G10004 & PNNL-AGG-415 \\
\hline 14336-64-2 & Cadmium & $<2.97 \mathrm{E}-4$ & ug/g dry & $2.97 \mathrm{E}-4$ & $7 / 10 / 08$ & 8G10004 & PNNL-AGG-415 \\
\hline 14265-72-6 & Antimony & $<5.44 \mathrm{E}-4$ & ug/g dry & $5.44 \mathrm{E}-4$ & $7 / 10 / 08$ & 8G10004 & PNNL-AGG-415 \\
\hline 13966-28-4 & Lead & $<5.64 \mathrm{E}-4$ & ug/g dry & $5.64 \mathrm{E}-4$ & $7 / 10 / 08$ & 8G10004 & PNNL-AGG-415 \\
\hline HEIS No. & B1TP86 & \multicolumn{3}{|c|}{ Lab ID: $\quad$ 0804025-CO } & & & \\
\hline 13981-78-7 & Chromium & $<6.40 \mathrm{E}-3$ & ug/g dry & $6.40 \mathrm{E}-3$ & $7 / 10 / 08$ & 8G10004 & PNNL-AGG-415 \\
\hline 14191-84-5 & Copper & $<3.48 \mathrm{E}-3$ & ug/g dry & $3.48 \mathrm{E}-3$ & $7 / 10 / 08$ & 8G10004 & PNNL-AGG-415 \\
\hline $7440-38-2$ & Arsenic & $<6.25 \mathrm{E}-3$ & ug/g dry & $6.25 \mathrm{E}-3$ & $7 / 10 / 08$ & 8G10004 & PNNL-AGG-415 \\
\hline 14687-58-2 & Selenium & $<1.11 \mathrm{E}-2$ & ug/g dry & $1.11 \mathrm{E}-2$ & $7 / 10 / 08$ & 8G10004 & PNNL-AGG-415 \\
\hline 14378-37-1 & Silver & $<9.25 \mathrm{E}-4$ & ug/g dry & $9.25 \mathrm{E}-4$ & $7 / 10 / 08$ & 8G10004 & PNNL-AGG-415 \\
\hline 14336-64-2 & Cadmium & $<2.95 \mathrm{E}-4$ & ug/g dry & 2.95E-4 & 7/10/08 & 8G10004 & PNNL-AGG-415 \\
\hline $14265-72-6$ & Antimony & $<5.40 \mathrm{E}-4$ & ug/g dry & $5.40 \mathrm{E}-4$ & $7 / 10 / 08$ & 8G10004 & PNNL-AGG-415 \\
\hline 13966-28-4 & Lead & $<5.60 \mathrm{E}-4$ & ug/g dry & $5.60 \mathrm{E}-4$ & $7 / 10 / 08$ & 8G10004 & PNNL-AGG-415 \\
\hline HEIS No. & B1TP89 & \multicolumn{3}{|c|}{ Lab ID: $\quad$ 0804025-CX } & & & \\
\hline 13981-78-7 & Chromium & $<6.42 \mathrm{E}-3$ & ug/g dry & $6.42 \mathrm{E}-3$ & $7 / 10 / 08$ & 8G10004 & PNNL-AGG-415 \\
\hline 14191-84-5 & Copper & $<3.49 \mathrm{E}-3$ & ug/g dry & $3.49 \mathrm{E}-3$ & 7/10/08 & 8G10004 & PNNL-AGG-415 \\
\hline $7440-38-2$ & Arsenic & $<6.27 \mathrm{E}-3$ & ug/g dry & $6.27 \mathrm{E}-3$ & $7 / 10 / 08$ & 8G10004 & PNNL-AGG-415 \\
\hline 14687-58-2 & Selenium & $<1.11 \mathrm{E}-2$ & ug/g dry & $1.11 \mathrm{E}-2$ & 7/10/08 & 8G10004 & PNNL-AGG-415 \\
\hline 14378-37-1 & Silver & $<9.28 \mathrm{E}-4$ & ug/g dry & $9.28 \mathrm{E}-4$ & $7 / 10 / 08$ & 8G10004 & PNNL-AGG-415 \\
\hline 14336-64-2 & Cadmium & $<2.96 \mathrm{E}-4$ & ug/g dry & $2.96 \mathrm{E}-4$ & $7 / 10 / 08$ & 8G10004 & PNNL-AGG-415 \\
\hline 14265-72-6 & Antimony & $<5.41 \mathrm{E}-4$ & ug/g dry & $5.41 \mathrm{E}-4$ & $7 / 10 / 08$ & 8G10004 & PNNL-AGG-415 \\
\hline 13966-28-4 & Lead & $<5.62 \mathrm{E}-4$ & ug/g dry & 5.62E-4 & 7/10/08 & 8G10004 & PNNL-AGG-415 \\
\hline HEIS No. & В1TP91 & \multicolumn{3}{|c|}{ Lab ID: $\quad$ 0804025-DF } & & & \\
\hline 13981-78-7 & Chromium & $<6.42 \mathrm{E}-3$ & ug/g dry & $6.42 \mathrm{E}-3$ & 7/10/08 & 8G10004 & PNNL-AGG-415 \\
\hline 14191-84-5 & Copper & $<3.49 \mathrm{E}-3$ & ug/g dry & $3.49 \mathrm{E}-3$ & $7 / 10 / 08$ & 8G10004 & PNNL-AGG-415 \\
\hline 7440-38-2 & Arsenic & $<6.27 \mathrm{E}-3$ & ug/g dry & $6.27 \mathrm{E}-3$ & $7 / 10 / 08$ & 8G10004 & PNNL-AGG-415 \\
\hline $14687-58-2$ & Selenium & $<1.11 \mathrm{E}-2$ & ug/g dry & $1.11 \mathrm{E}-2$ & $7 / 10 / 08$ & 8G10004 & PNNL-AGG-415 \\
\hline 14378-37-1 & Silver & $<9.28 \mathrm{E}-4$ & ug/g dry & $9.28 \mathrm{E}-4$ & 7/10/08 & 8G10004 & PNNL-AGG-415 \\
\hline 14336-64-2 & Cadmium & $<2.96 \mathrm{E}-4$ & ug/g dry & $2.96 \mathrm{E}-4$ & $7 / 10 / 08$ & 8G10004 & PNNL-AGG-415 \\
\hline 14265-72-6 & Antimony & $1.50 \mathrm{E}-3$ & ug/g dry & $5.42 \mathrm{E}-4$ & $7 / 10 / 08$ & 8G10004 & PNNL-AGG-415 \\
\hline 13966-28-4 & Lead & $<5.62 \mathrm{E}-4$ & ug/g dry & $5.62 \mathrm{E}-4$ & 7/10/08 & 8G10004 & PNNL-AGG-415 \\
\hline HEIS No. & В1ТР93 & \multicolumn{3}{|c|}{ Lab ID: $\quad$ 0804025-DL } & & & \\
\hline 13981-78-7 & Chromium & $<6.43 \mathrm{E}-3$ & ug/g dry & $6.43 \mathrm{E}-3$ & $7 / 10 / 08$ & 8G10004 & PNNL-AGG-415 \\
\hline 14191-84-5 & Copper & $<3.50 \mathrm{E}-3$ & ug/g dry & $3.50 \mathrm{E}-3$ & $7 / 10 / 08$ & 8G10004 & PNNL-AGG-415 \\
\hline 7440-38-2 & Arsenic & $<6.28 \mathrm{E}-3$ & ug/g dry & $6.28 \mathrm{E}-3$ & $7 / 10 / 08$ & 8G10004 & PNNL-AGG-415 \\
\hline
\end{tabular}


RCRA Metals By PNNL-AGG-415/Water Extract

\begin{tabular}{|c|c|c|c|c|c|c|c|}
\hline CAS \# & Analyte & Results & Units & EQL & Analyzed & Batch & Method \\
\hline HEIS No. & В1ТР93 & \multicolumn{3}{|c|}{ Lab ID: $\quad$ 0804025-DL } & & & \\
\hline $14687-58-2$ & Selenium & $<1.11 \mathrm{E}-2$ & ug/g dry & $1.11 \mathrm{E}-2$ & $7 / 10 / 08$ & 8G10004 & PNNL-AGG-415 \\
\hline $14378-37-1$ & Silver & $<9.30 \mathrm{E}-4$ & ug/g dry & $9.30 \mathrm{E}-4$ & 7/10/08 & 8G10004 & PNNL-AGG-415 \\
\hline $14336-64-2$ & Cadmium & $<2.97 \mathrm{E}-4$ & ug/g dry & 2.97E-4 & 7/10/08 & 8G10004 & PNNL-AGG-415 \\
\hline $14265-72-6$ & Antimony & $<5.43 \mathrm{E}-4$ & ug/g dry & $5.43 \mathrm{E}-4$ & $7 / 10 / 08$ & 8G10004 & PNNL-AGG-415 \\
\hline 13966-28-4 & Lead & $<5.63 \mathrm{E}-4$ & ug/g dry & 5.63E-4 & $7 / 10 / 08$ & 8G10004 & PNNL-AGG-415 \\
\hline HEIS No. & В1ТР94 & \multicolumn{3}{|c|}{ Lab ID: $\quad$ 0804025-DM } & & & \\
\hline $13981-78-7$ & Chromium & $<6.38 \mathrm{E}-3$ & ug/g dry & $6.38 \mathrm{E}-3$ & 7/10/08 & 8G10004 & PNNL-AGG-415 \\
\hline $14191-84-5$ & Copper & $<3.47 \mathrm{E}-3$ & ug/g dry & $3.47 \mathrm{E}-3$ & 7/10/08 & 8G10004 & PNNL-AGG-415 \\
\hline $7440-38-2$ & Arsenic & $<6.23 \mathrm{E}-3$ & ug/g dry & $6.23 \mathrm{E}-3$ & 7/10/08 & 8G10004 & PNNL-AGG-415 \\
\hline $14687-58-2$ & Selenium & $<1.10 \mathrm{E}-2$ & ug/g dry & $1.10 \mathrm{E}-2$ & 7/10/08 & 8G10004 & PNNL-AGG-415 \\
\hline 14378-37-1 & Silver & $<9.22 \mathrm{E}-4$ & ug/g dry & $9.22 \mathrm{E}-4$ & 7/10/08 & 8G10004 & PNNL-AGG-415 \\
\hline $14336-64-2$ & Cadmium & $<2.94 \mathrm{E}-4$ & ug/g dry & $2.94 \mathrm{E}-4$ & 7/10/08 & 8G10004 & PNNL-AGG-415 \\
\hline $14265-72-6$ & Antimony & $<5.38 \mathrm{E}-4$ & ug/g dry & $5.38 \mathrm{E}-4$ & 7/10/08 & 8G10004 & PNNL-AGG-415 \\
\hline 13966-28-4 & Lead & $<5.58 \mathrm{E}-4$ & ug/g dry & $5.58 \mathrm{E}-4$ & 7/10/08 & 8G10004 & PNNL-AGG-415 \\
\hline
\end{tabular}


RCRA Metals By PNNL-AGG-415/Acid Extract

\begin{tabular}{|c|c|c|c|c|c|c|c|}
\hline CAS \# & Analyte & Results & Units & EQL & Analyzed & Batch & Method \\
\hline HEIS No. & B1TP24 & \multicolumn{3}{|c|}{ Lab ID: $\quad$ 0804025-04 } & & & \\
\hline 14092-98-9 & Chromium & 8.02E0 & ug/g dry & $1.90 \mathrm{E}-1$ & $9 / 24 / 08$ & 8I18001 & PNNL-AGG-415 \\
\hline 14119-06-3 & Copper & 7.24E0 & ug/g dry & $5.98 \mathrm{E}-1$ & 9/24/08 & $8 \mathrm{I} 18001$ & PNNL-AGG-415 \\
\hline $7440-38-2$ & Arsenic & $1.22 \mathrm{E} 0$ & ug/g dry & 4.17E-1 & 9/24/08 & $8 \mathrm{I} 18001$ & PNNL-AGG-415 \\
\hline 14687-58-2 & Selenium & $<1.14 \mathrm{E} 0$ & ug/g dry & $1.14 \mathrm{E} 0$ & 9/24/08 & 8I18001 & PNNL-AGG-415 \\
\hline 14378-37-1 & Silver & $<6.92 \mathrm{E}-2$ & ug/g dry & $6.92 \mathrm{E}-2$ & $9 / 24 / 08$ & 8I18001 & PNNL-AGG-415 \\
\hline 14336-64-2 & Cadmium & $4.91 \mathrm{E}-2$ & ug/g dry & $4.90 \mathrm{E}-2$ & 9/24/08 & $8 \mathrm{I} 18001$ & PNNL-AGG-415 \\
\hline 14265-72-6 & Antimony & $<7.92 \mathrm{E}-2$ & ug/g dry & $7.92 \mathrm{E}-2$ & $9 / 24 / 08$ & 8I18001 & PNNL-AGG-415 \\
\hline 13966-28-4 & Lead & $2.40 \mathrm{E} 0$ & ug/g dry & $3.68 \mathrm{E}-2$ & 9/24/08 & 8I18001 & PNNL-AGG-415 \\
\hline HEIS No. & В1TP28 & \multicolumn{3}{|c|}{ Lab ID: $\quad$ 0804025-07 } & & & \\
\hline 14092-98-9 & Chromium & 5.95E0 & ug/g dry & $1.85 \mathrm{E}-1$ & 9/24/08 & 8I18001 & PNNL-AGG-415 \\
\hline 14119-06-3 & Copper & $8.31 \mathrm{E} 0$ & ug/g dry & $5.81 \mathrm{E}-1$ & $9 / 24 / 08$ & 8I18001 & PNNL-AGG-415 \\
\hline $7440-38-2$ & Arsenic & 9.97E-1 & ug/g dry & 4.06E-1 & 9/24/08 & 8I18001 & PNNL-AGG-415 \\
\hline $14687-58-2$ & Selenium & $<1.11 \mathrm{E} 0$ & ug/g dry & $1.11 \mathrm{E} 0$ & $9 / 24 / 08$ & 8I18001 & PNNL-AGG-415 \\
\hline 14378-37-1 & Silver & $<6.72 \mathrm{E}-2$ & ug/g dry & $6.72 \mathrm{E}-2$ & $9 / 24 / 08$ & $8 \mathrm{I} 18001$ & PNNL-AGG-415 \\
\hline 14336-64-2 & Cadmium & $6.18 \mathrm{E}-2$ & ug/g dry & 4.76E-2 & $9 / 24 / 08$ & 8I18001 & PNNL-AGG-415 \\
\hline 14265-72-6 & Antimony & $<7.70 \mathrm{E}-2$ & ug/g dry & $7.70 \mathrm{E}-2$ & 9/24/08 & 8I18001 & PNNL-AGG-415 \\
\hline 13966-28-4 & Lead & $2.58 \mathrm{E} 0$ & ug/g dry & $3.58 \mathrm{E}-2$ & $9 / 24 / 08$ & 8I18001 & PNNL-AGG-415 \\
\hline HEIS No. & В1TP29 & \multicolumn{3}{|c|}{ Lab ID: $\quad$ 0804025-08 } & & & \\
\hline 14092-98-9 & Chromium & $5.52 \mathrm{E} 0$ & ug/g dry & $1.83 \mathrm{E}-1$ & $9 / 24 / 08$ & 8I18001 & PNNL-AGG-415 \\
\hline 14119-06-3 & Copper & $8.76 \mathrm{E} 0$ & ug/g dry & $5.77 \mathrm{E}-1$ & $9 / 24 / 08$ & 8I18001 & PNNL-AGG-415 \\
\hline $7440-38-2$ & Arsenic & $9.34 \mathrm{E}-1$ & ug/g dry & $4.02 \mathrm{E}-1$ & $9 / 24 / 08$ & 8I18001 & PNNL-AGG-415 \\
\hline 14687-58-2 & Selenium & $<1.10 \mathrm{E} 0$ & ug/g dry & $1.10 \mathrm{E} 0$ & $9 / 24 / 08$ & 8I18001 & PNNL-AGG-415 \\
\hline 14378-37-1 & Silver & $<6.67 \mathrm{E}-2$ & ug/g dry & $6.67 \mathrm{E}-2$ & $9 / 24 / 08$ & 8I18001 & PNNL-AGG-415 \\
\hline 14336-64-2 & Cadmium & $5.79 \mathrm{E}-2$ & ug/g dry & 4.73E-2 & 9/24/08 & 8I18001 & PNNL-AGG-415 \\
\hline $14265-72-6$ & Antimony & $<7.64 \mathrm{E}-2$ & ug/g dry & $7.64 \mathrm{E}-2$ & $9 / 24 / 08$ & 8I18001 & PNNL-AGG-415 \\
\hline 13966-28-4 & Lead & $2.05 \mathrm{E} 0$ & ug/g dry & $3.55 \mathrm{E}-2$ & $9 / 24 / 08$ & 8I18001 & PNNL-AGG-415 \\
\hline HEIS No. & В1TP30 & \multicolumn{3}{|c|}{ Lab ID: $\quad$ 0804025-09 } & & & \\
\hline 14092-98-9 & Chromium & $7.90 \mathrm{E} 0$ & ug/g dry & $1.89 \mathrm{E}-1$ & $9 / 24 / 08$ & 8I18001 & PNNL-AGG-415 \\
\hline 14119-06-3 & Copper & $9.16 \mathrm{E} 0$ & ug/g dry & $5.95 \mathrm{E}-1$ & 9/24/08 & 8I18001 & PNNL-AGG-415 \\
\hline $7440-38-2$ & Arsenic & $1.45 \mathrm{E} 0$ & ug/g dry & 4.15E-1 & $9 / 24 / 08$ & 8I18001 & PNNL-AGG-415 \\
\hline 14687-58-2 & Selenium & $<1.13 \mathrm{E} 0$ & ug/g dry & $1.13 \mathrm{E} 0$ & $9 / 24 / 08$ & 8I18001 & PNNL-AGG-415 \\
\hline 14378-37-1 & Silver & $<6.88 \mathrm{E}-2$ & ug/g dry & $6.88 \mathrm{E}-2$ & $9 / 24 / 08$ & 8I18001 & PNNL-AGG-415 \\
\hline 14336-64-2 & Cadmium & $5.22 \mathrm{E}-2$ & ug/g dry & $4.88 \mathrm{E}-2$ & $9 / 24 / 08$ & 8I18001 & PNNL-AGG-415 \\
\hline 14265-72-6 & Antimony & $<7.88 \mathrm{E}-2$ & ug/g dry & $7.88 \mathrm{E}-2$ & $9 / 24 / 08$ & 8I18001 & PNNL-AGG-415 \\
\hline 13966-28-4 & Lead & 2.96E0 & ug/g dry & 3.67E-2 & 9/24/08 & $8 \mathrm{I} 18001$ & PNNL-AGG-415 \\
\hline HEIS No. & B1TNV9 & \multicolumn{3}{|c|}{ Lab ID: $\quad$ 0804025-10 } & & & \\
\hline 14092-98-9 & Chromium & 7.57E0 & ug/g dry & $1.83 \mathrm{E}-1$ & 9/24/08 & 8I18001 & PNNL-AGG-415 \\
\hline 14119-06-3 & Copper & 7.86E0 & ug/g dry & $5.77 \mathrm{E}-1$ & $9 / 24 / 08$ & 8I18001 & PNNL-AGG-415 \\
\hline 7440-38-2 & Arsenic & $1.86 \mathrm{E} 0$ & ug/g dry & 4.03E-1 & $9 / 24 / 08$ & 8I18001 & PNNL-AGG-415 \\
\hline $14687-58-2$ & Selenium & $<1.10 \mathrm{E} 0$ & ug/g dry & $1.10 \mathrm{E} 0$ & $9 / 24 / 08$ & 8I18001 & PNNL-AGG-415 \\
\hline 14378-37-1 & Silver & $<6.68 \mathrm{E}-2$ & ug/g dry & $6.68 \mathrm{E}-2$ & $9 / 24 / 08$ & 8I18001 & PNNL-AGG-415 \\
\hline 14336-64-2 & Cadmium & $4.80 \mathrm{E}-2$ & ug/g dry & 4.73E-2 & $9 / 24 / 08$ & 8I18001 & PNNL-AGG-415 \\
\hline 14265-72-6 & Antimony & $1.02 \mathrm{E}-1$ & ug/g dry & $7.65 \mathrm{E}-2$ & $9 / 24 / 08$ & 8I18001 & PNNL-AGG-415 \\
\hline 13966-28-4 & Lead & 2.85E0 & ug/g dry & $3.56 \mathrm{E}-2$ & 9/24/08 & $8 \mathrm{I} 18001$ & PNNL-AGG-415 \\
\hline HEIS No. & В1TP22 & \multicolumn{3}{|c|}{ Lab ID: $\quad 0804025-13$} & & & \\
\hline 14092-98-9 & Chromium & $3.34 \mathrm{E} 0$ & ug/g dry & $1.85 \mathrm{E}-1$ & $9 / 24 / 08$ & 8I18001 & PNNL-AGG-415 \\
\hline 14119-06-3 & Copper & $1.10 \mathrm{E} 1$ & ug/g dry & $5.82 \mathrm{E}-1$ & $9 / 24 / 08$ & 8I18001 & PNNL-AGG-415 \\
\hline 7440-38-2 & Arsenic & 8.83E-1 & ug/g dry & 4.06E-1 & $9 / 24 / 08$ & $8 \mathrm{I} 18001$ & PNNL-AGG-415 \\
\hline
\end{tabular}


RCRA Metals By PNNL-AGG-415/Acid Extract

\begin{tabular}{|c|c|c|c|c|c|c|c|}
\hline CAS \# & Analyte & Results & Units & EQL & Analyzed & Batch & Method \\
\hline HEIS No. & B1TP22 & \multicolumn{3}{|c|}{ Lab ID: $\quad$ 0804025-13 } & & & \\
\hline $14687-58-2$ & Selenium & $<1.11 \mathrm{E} 0$ & ug/g dry & $1.11 \mathrm{E} 0$ & $9 / 24 / 08$ & $8 \mathrm{I} 18001$ & PNNL-AGG-415 \\
\hline 14378-37-1 & Silver & $<6.74 \mathrm{E}-2$ & ug/g dry & $6.74 \mathrm{E}-2$ & $9 / 24 / 08$ & 8I18001 & PNNL-AGG-415 \\
\hline 14336-64-2 & Cadmium & $4.98 \mathrm{E}-2$ & ug/g dry & 4.77E-2 & 9/24/08 & 8I18001 & PNNL-AGG-415 \\
\hline $14265-72-6$ & Antimony & $<7.72 \mathrm{E}-2$ & ug/g dry & $7.72 \mathrm{E}-2$ & $9 / 24 / 08$ & 8I18001 & PNNL-AGG-415 \\
\hline 13966-28-4 & Lead & $1.77 \mathrm{E} 0$ & ug/g dry & $3.59 \mathrm{E}-2$ & 9/24/08 & $8 \mathrm{I} 18001$ & PNNL-AGG-415 \\
\hline HEIS No. & B1TP26 & \multicolumn{3}{|c|}{ Lab ID: $\quad$ 0804025-16 } & & & \\
\hline 14092-98-9 & Chromium & 6.99E0 & ug/g dry & $1.85 \mathrm{E}-1$ & 9/24/08 & 8I18001 & PNNL-AGG-415 \\
\hline 14119-06-3 & Copper & 9.39E0 & ug/g dry & 5.83E-1 & $9 / 24 / 08$ & 8I18001 & PNNL-AGG-415 \\
\hline $7440-38-2$ & Arsenic & $1.47 \mathrm{E} 0$ & ug/g dry & 4.07E-1 & $9 / 24 / 08$ & 8I18001 & PNNL-AGG-415 \\
\hline 14687-58-2 & Selenium & $<1.11 \mathrm{E} 0$ & ug/g dry & $1.11 \mathrm{E} 0$ & $9 / 24 / 08$ & 8I18001 & PNNL-AGG-415 \\
\hline 14378-37-1 & Silver & $<6.74 \mathrm{E}-2$ & ug/g dry & $6.74 \mathrm{E}-2$ & 9/24/08 & $8 \mathrm{I} 18001$ & PNNL-AGG-415 \\
\hline 14336-64-2 & Cadmium & $<4.78 \mathrm{E}-2$ & ug/g dry & 4.78E-2 & $9 / 24 / 08$ & 8I18001 & PNNL-AGG-415 \\
\hline $14265-72-6$ & Antimony & $<7.73 \mathrm{E}-2$ & ug/g dry & 7.73E-2 & $9 / 24 / 08$ & $8 \mathrm{I} 18001$ & PNNL-AGG-415 \\
\hline 13966-28-4 & Lead & 2.27E0 & ug/g dry & $3.59 \mathrm{E}-2$ & 9/24/08 & 8I18001 & PNNL-AGG-415 \\
\hline HEIS No. & B1TNW0 & \multicolumn{3}{|c|}{ Lab ID: $\quad$ 0804025-17 } & & & \\
\hline 14092-98-9 & Chromium & $6.22 \mathrm{E} 0$ & ug/g dry & 1.86E-1 & 9/24/08 & 8I18001 & PNNL-AGG-415 \\
\hline 14119-06-3 & Copper & 7.53E0 & ug/g dry & $5.87 \mathrm{E}-1$ & $9 / 24 / 08$ & $8 \mathrm{I} 18001$ & PNNL-AGG-415 \\
\hline 7440-38-2 & Arsenic & $1.10 \mathrm{E} 0$ & ug/g dry & $4.10 \mathrm{E}-1$ & $9 / 24 / 08$ & 8I18001 & PNNL-AGG-415 \\
\hline 14687-58-2 & Selenium & $<1.12 \mathrm{E} 0$ & ug/g dry & $1.12 \mathrm{E} 0$ & $9 / 24 / 08$ & 8I18001 & PNNL-AGG-415 \\
\hline 14378-37-1 & Silver & $<6.79 \mathrm{E}-2$ & ug/g dry & $6.79 \mathrm{E}-2$ & 9/24/08 & 8I18001 & PNNL-AGG-415 \\
\hline 14336-64-2 & Cadmium & $4.92 \mathrm{E}-2$ & ug/g dry & $4.81 \mathrm{E}-2$ & $9 / 24 / 08$ & 8I18001 & PNNL-AGG-415 \\
\hline 14265-72-6 & Antimony & $<7.78 \mathrm{E}-2$ & ug/g dry & $7.78 \mathrm{E}-2$ & $9 / 24 / 08$ & $8 \mathrm{I} 18001$ & PNNL-AGG-415 \\
\hline 13966-28-4 & Lead & $2.20 \mathrm{E} 0$ & ug/g dry & $3.62 \mathrm{E}-2$ & 9/24/08 & $8 \mathrm{I} 18001$ & PNNL-AGG-415 \\
\hline HEIS No. & B1TNW1 & \multicolumn{3}{|c|}{ Lab ID: $\quad$ 0804025-18 } & & & \\
\hline 14092-98-9 & Chromium & 8.23E0 & ug/g dry & $1.85 \mathrm{E}-1$ & $9 / 24 / 08$ & 8I18001 & PNNL-AGG-415 \\
\hline 14119-06-3 & Copper & 7.79E0 & ug/g dry & $5.82 \mathrm{E}-1$ & $9 / 24 / 08$ & $8 \mathrm{I} 18001$ & PNNL-AGG-415 \\
\hline $7440-38-2$ & Arsenic & 2.32E0 & ug/g dry & $4.06 \mathrm{E}-1$ & $9 / 24 / 08$ & $8 \mathrm{I} 18001$ & PNNL-AGG-415 \\
\hline $14687-58-2$ & Selenium & $<1.11 \mathrm{E} 0$ & ug/g dry & $1.11 \mathrm{E} 0$ & $9 / 24 / 08$ & 8I18001 & PNNL-AGG-415 \\
\hline 14378-37-1 & Silver & $<6.73 \mathrm{E}-2$ & ug/g dry & 6.73E-2 & 9/24/08 & $8 \mathrm{I} 18001$ & PNNL-AGG-415 \\
\hline $14336-64-2$ & Cadmium & $6.27 \mathrm{E}-2$ & ug/g dry & $4.77 \mathrm{E}-2$ & $9 / 24 / 08$ & $8 \mathrm{I} 18001$ & PNNL-AGG-415 \\
\hline $14265-72-6$ & Antimony & $<7.72 \mathrm{E}-2$ & ug/g dry & $7.72 \mathrm{E}-2$ & 9/24/08 & $8 \mathrm{I} 18001$ & PNNL-AGG-415 \\
\hline 13966-28-4 & Lead & 3.07E0 & ug/g dry & $3.59 \mathrm{E}-2$ & 9/24/08 & $8 \mathrm{I} 18001$ & PNNL-AGG-415 \\
\hline HEIS No. & B1TNW3 & \multicolumn{3}{|c|}{ Lab ID: $\quad 0804025-20$} & & & \\
\hline 14092-98-9 & Chromium & 7.29E0 & ug/g dry & $1.85 \mathrm{E}-1$ & $9 / 24 / 08$ & 8I18001 & PNNL-AGG-415 \\
\hline 14119-06-3 & Copper & $8.26 \mathrm{E} 0$ & ug/g dry & $5.83 \mathrm{E}-1$ & $9 / 24 / 08$ & $8 \mathrm{I} 18001$ & PNNL-AGG-415 \\
\hline $7440-38-2$ & Arsenic & $1.61 \mathrm{E} 0$ & ug/g dry & $4.07 \mathrm{E}-1$ & $9 / 24 / 08$ & 8I18001 & PNNL-AGG-415 \\
\hline 14687-58-2 & Selenium & $<1.11 \mathrm{E} 0$ & ug/g dry & $1.11 \mathrm{E} 0$ & $9 / 24 / 08$ & 8I18001 & PNNL-AGG-415 \\
\hline 14378-37-1 & Silver & $<6.75 \mathrm{E}-2$ & ug/g dry & $6.75 \mathrm{E}-2$ & $9 / 24 / 08$ & $8 \mathrm{I} 18001$ & PNNL-AGG-415 \\
\hline 14336-64-2 & Cadmium & $4.89 \mathrm{E}-2$ & ug/g dry & $4.78 \mathrm{E}-2$ & $9 / 24 / 08$ & $8 \mathrm{I} 18001$ & PNNL-AGG-415 \\
\hline $14265-72-6$ & Antimony & $<7.73 \mathrm{E}-2$ & ug/g dry & 7.73E-2 & 9/24/08 & $8 \mathrm{I} 18001$ & PNNL-AGG-415 \\
\hline 13966-28-4 & Lead & 2.39E0 & ug/g dry & $3.59 \mathrm{E}-2$ & 9/24/08 & $8 \mathrm{I} 18001$ & PNNL-AGG-415 \\
\hline HEIS No. & B1TNW5 & \multicolumn{3}{|c|}{ Lab ID: $\quad 0804025-22$} & & & \\
\hline 14092-98-9 & Chromium & $9.20 \mathrm{E} 0$ & ug/g dry & $1.87 \mathrm{E}-1$ & $9 / 24 / 08$ & $8 \mathrm{I} 18001$ & PNNL-AGG-415 \\
\hline 14119-06-3 & Copper & 7.12E0 & ug/g dry & $5.88 \mathrm{E}-1$ & 9/24/08 & $8 \mathrm{I} 18001$ & PNNL-AGG-415 \\
\hline $7440-38-2$ & Arsenic & 2.65E0 & ug/g dry & $4.10 \mathrm{E}-1$ & 9/24/08 & 8I18001 & PNNL-AGG-415 \\
\hline 14687-58-2 & Selenium & $<1.12 \mathrm{E} 0$ & ug/g dry & $1.12 \mathrm{E} 0$ & $9 / 24 / 08$ & 8I18001 & PNNL-AGG-415 \\
\hline 14378-37-1 & Silver & $<6.80 \mathrm{E}-2$ & ug/g dry & $6.80 \mathrm{E}-2$ & $9 / 24 / 08$ & $8 \mathrm{I} 18001$ & PNNL-AGG-415 \\
\hline 14336-64-2 & Cadmium & $6.51 \mathrm{E}-2$ & ug/g dry & $4.82 \mathrm{E}-2$ & $9 / 24 / 08$ & 8I18001 & PNNL-AGG-415 \\
\hline
\end{tabular}


RCRA Metals By PNNL-AGG-415/Acid Extract

\begin{tabular}{|c|c|c|c|c|c|c|c|}
\hline CAS \# & Analyte & Results & Units & EQL & Analyzed & Batch & Method \\
\hline HEIS No. & B1TNW5 & \multicolumn{3}{|c|}{ Lab ID: $\quad$ 0804025-22 } & & & \\
\hline $14265-72-6$ & Antimony & 8.87E-2 & ug/g dry & 7.79E-2 & 9/24/08 & 8I18001 & PNNL-AGG-415 \\
\hline 13966-28-4 & Lead & 3.09E0 & ug/g dry & $3.62 \mathrm{E}-2$ & 9/24/08 & $8 \mathrm{I} 18001$ & PNNL-AGG-415 \\
\hline HEIS No. & B1TP35 & \multicolumn{3}{|c|}{ Lab ID: $\quad$ 0804025-37 } & & & \\
\hline 14092-98-9 & Chromium & $1.09 \mathrm{E} 1$ & ug/g dry & $1.86 \mathrm{E}-1$ & 9/24/08 & $8 \mathrm{I} 18001$ & PNNL-AGG-415 \\
\hline 14119-06-3 & Copper & 7.47E0 & ug/g dry & $5.85 \mathrm{E}-1$ & $9 / 24 / 08$ & 8I18001 & PNNL-AGG-415 \\
\hline $7440-38-2$ & Arsenic & 2.17E0 & ug/g dry & $4.08 \mathrm{E}-1$ & 9/24/08 & $8 \mathrm{I} 18001$ & PNNL-AGG-415 \\
\hline 14687-58-2 & Selenium & $<1.12 \mathrm{E} 0$ & ug/g dry & $1.12 \mathrm{E} 0$ & $9 / 24 / 08$ & 8I18001 & PNNL-AGG-415 \\
\hline 14378-37-1 & Silver & $<6.77 \mathrm{E}-2$ & ug/g dry & $6.77 \mathrm{E}-2$ & 9/24/08 & 8I18001 & PNNL-AGG-415 \\
\hline 14336-64-2 & Cadmium & $5.11 \mathrm{E}-2$ & ug/g dry & 4.79E-2 & 9/24/08 & $8 \mathrm{I} 18001$ & PNNL-AGG-415 \\
\hline $14265-72-6$ & Antimony & $<7.75 \mathrm{E}-2$ & ug/g dry & $7.75 \mathrm{E}-2$ & $9 / 24 / 08$ & 8I18001 & PNNL-AGG-415 \\
\hline 13966-28-4 & Lead & 2.59E0 & ug/g dry & $3.60 \mathrm{E}-2$ & $9 / 24 / 08$ & $8 \mathrm{I} 18001$ & PNNL-AGG-415 \\
\hline HEIS No. & B1TP40 & \multicolumn{3}{|c|}{ Lab ID: $\quad 0804025-48$} & & & \\
\hline 14092-98-9 & Chromium & $1.04 \mathrm{E} 1$ & ug/g dry & $1.87 \mathrm{E}-1$ & 9/24/08 & $8 \mathrm{I} 18001$ & PNNL-AGG-415 \\
\hline 14119-06-3 & Copper & 7.27E0 & ug/g dry & $5.89 \mathrm{E}-1$ & $9 / 24 / 08$ & 8I18001 & PNNL-AGG-415 \\
\hline $7440-38-2$ & Arsenic & $2.45 \mathrm{E} 0$ & ug/g dry & $4.11 \mathrm{E}-1$ & $9 / 24 / 08$ & $8 \mathrm{I} 18001$ & PNNL-AGG-415 \\
\hline 14687-58-2 & Selenium & $<1.12 \mathrm{E} 0$ & ug/g dry & $1.12 \mathrm{E} 0$ & 9/24/08 & $8 \mathrm{I} 18001$ & PNNL-AGG-415 \\
\hline 14378-37-1 & Silver & $<6.81 \mathrm{E}-2$ & ug/g dry & $6.81 \mathrm{E}-2$ & 9/24/08 & $8 \mathrm{I} 18001$ & PNNL-AGG-415 \\
\hline $14336-64-2$ & Cadmium & $5.61 \mathrm{E}-2$ & ug/g dry & 4.83E-2 & $9 / 24 / 08$ & 8I18001 & PNNL-AGG-415 \\
\hline 14265-72-6 & Antimony & $4.89 \mathrm{E}-1$ & ug/g dry & $7.80 \mathrm{E}-2$ & $9 / 24 / 08$ & 8I18001 & PNNL-AGG-415 \\
\hline 13966-28-4 & Lead & $2.58 \mathrm{E} 0$ & ug/g dry & 3.63E-2 & $9 / 24 / 08$ & 8I18001 & PNNL-AGG-415 \\
\hline HEIS No. & B1TP42 & \multicolumn{3}{|c|}{ Lab ID: $\quad 0804025-50$} & & & \\
\hline 14092-98-9 & Chromium & $9.86 \mathrm{E} 0$ & ug/g dry & $1.81 \mathrm{E}-1$ & 9/24/08 & $8 \mathrm{I} 18001$ & PNNL-AGG-415 \\
\hline 14119-06-3 & Copper & 6.93E0 & ug/g dry & $5.70 \mathrm{E}-1$ & $9 / 24 / 08$ & 8I18001 & PNNL-AGG-415 \\
\hline $7440-38-2$ & Arsenic & 2.36E0 & ug/g dry & $3.98 \mathrm{E}-1$ & 9/24/08 & 8I18001 & PNNL-AGG-415 \\
\hline 14687-58-2 & Selenium & $<1.09 \mathrm{E} 0$ & ug/g dry & $1.09 \mathrm{E} 0$ & 9/24/08 & $8 \mathrm{I} 18001$ & PNNL-AGG-415 \\
\hline 14378-37-1 & Silver & $<6.59 \mathrm{E}-2$ & ug/g dry & $6.59 \mathrm{E}-2$ & $9 / 24 / 08$ & 8I18001 & PNNL-AGG-415 \\
\hline 14336-64-2 & Cadmium & $5.42 \mathrm{E}-2$ & ug/g dry & 4.67E-2 & 9/24/08 & $8 \mathrm{I} 18001$ & PNNL-AGG-415 \\
\hline $14265-72-6$ & Antimony & 8.05E-2 & ug/g dry & 7.55E-2 & 9/24/08 & 8I18001 & PNNL-AGG-415 \\
\hline 13966-28-4 & Lead & $2.44 \mathrm{E} 0$ & ug/g dry & $3.51 \mathrm{E}-2$ & 9/24/08 & $8 \mathrm{I} 18001$ & PNNL-AGG-415 \\
\hline HEIS No. & B1TP43 & \multicolumn{3}{|c|}{ Lab ID: $\quad 0804025-51$} & & & \\
\hline 14119-06-3 & Copper & 8.98E0 & ug/g dry & $6.37 \mathrm{E}-1$ & 9/24/08 & 8I18001 & PNNL-AGG-415 \\
\hline 14687-58-2 & Selenium & $<1.22 \mathrm{E} 0$ & ug/g dry & $1.22 \mathrm{E} 0$ & $9 / 24 / 08$ & $8 \mathrm{I} 18001$ & PNNL-AGG-415 \\
\hline 14336-64-2 & Cadmium & $6.60 \mathrm{E}-2$ & ug/g dry & $5.23 \mathrm{E}-2$ & 9/24/08 & $8 \mathrm{I} 18001$ & PNNL-AGG-415 \\
\hline $14265-72-6$ & Antimony & $1.14 \mathrm{E}-1$ & ug/g dry & $8.45 \mathrm{E}-2$ & $9 / 24 / 08$ & $8 \mathrm{I} 18001$ & PNNL-AGG-415 \\
\hline HEIS No. & B1TP45 & \multicolumn{3}{|c|}{ Lab ID: $\quad$ 0804025-53 } & & & \\
\hline 14092-98-9 & Chromium & 6.90E0 & ug/g dry & $1.85 \mathrm{E}-1$ & $9 / 24 / 08$ & 8I18002 & PNNL-AGG-415 \\
\hline 14119-06-3 & Copper & 7.68E0 & ug/g dry & $5.82 \mathrm{E}-1$ & 9/24/08 & $8 \mathrm{I} 18002$ & PNNL-AGG-415 \\
\hline $7440-38-2$ & Arsenic & $1.14 \mathrm{E} 0$ & ug/g dry & 4.06E-1 & $9 / 24 / 08$ & $8 \mathrm{I} 18002$ & PNNL-AGG-415 \\
\hline 14687-58-2 & Selenium & $<1.11 \mathrm{E} 0$ & ug/g dry & $1.11 \mathrm{E} 0$ & $9 / 24 / 08$ & 8I18002 & PNNL-AGG-415 \\
\hline 14378-37-1 & Silver & $<6.74 \mathrm{E}-2$ & ug/g dry & $6.74 \mathrm{E}-2$ & $9 / 24 / 08$ & $8 \mathrm{I} 18002$ & PNNL-AGG-415 \\
\hline 14336-64-2 & Cadmium & $<4.77 \mathrm{E}-2$ & ug/g dry & $4.77 \mathrm{E}-2$ & 9/24/08 & 8I18002 & PNNL-AGG-415 \\
\hline $14265-72-6$ & Antimony & $<7.72 \mathrm{E}-2$ & ug/g dry & $7.72 \mathrm{E}-2$ & 9/24/08 & $8 \mathrm{I} 18002$ & PNNL-AGG-415 \\
\hline 13966-28-4 & Lead & 2.13E0 & ug/g dry & $3.59 \mathrm{E}-2$ & 9/24/08 & $8 \mathrm{I} 18002$ & PNNL-AGG-415 \\
\hline HEIS No. & B1TP47 & \multicolumn{3}{|c|}{ Lab ID: $\quad$ 0804025-57 } & & & \\
\hline 14092-98-9 & Chromium & 9.39E0 & ug/g dry & $1.83 \mathrm{E}-1$ & $9 / 24 / 08$ & 8I18002 & PNNL-AGG-415 \\
\hline 14119-06-3 & Copper & 7.74E0 & ug/g dry & $5.77 \mathrm{E}-1$ & $9 / 24 / 08$ & 8I18002 & PNNL-AGG-415 \\
\hline $7440-38-2$ & Arsenic & $1.77 \mathrm{E} 0$ & ug/g dry & 4.03E-1 & $9 / 24 / 08$ & $8 \mathrm{I} 18002$ & PNNL-AGG-415 \\
\hline 14687-58-2 & Selenium & $<1.10 \mathrm{E} 0$ & ug/g dry & $1.10 \mathrm{E} 0$ & $9 / 24 / 08$ & 8I18002 & PNNL-AGG-415 \\
\hline
\end{tabular}


RCRA Metals By PNNL-AGG-415/Acid Extract

\begin{tabular}{|c|c|c|c|c|c|c|c|}
\hline CAS \# & Analyte & Results & Units & EQL & Analyzed & Batch & Method \\
\hline HEIS No. & B1TP47 & \multicolumn{3}{|c|}{ Lab ID: $\quad$ 0804025-57 } & & & \\
\hline 14378-37-1 & Silver & $<6.68 \mathrm{E}-2$ & ug/g dry & $6.68 \mathrm{E}-2$ & 9/24/08 & 8I18002 & PNNL-AGG-415 \\
\hline 14336-64-2 & Cadmium & $5.01 \mathrm{E}-2$ & ug/g dry & 4.73E-2 & $9 / 24 / 08$ & 8I18002 & PNNL-AGG-415 \\
\hline $14265-72-6$ & Antimony & $<7.65 \mathrm{E}-2$ & ug/g dry & 7.65E-2 & 9/24/08 & 8I18002 & PNNL-AGG-415 \\
\hline 13966-28-4 & Lead & $2.24 \mathrm{E} 0$ & ug/g dry & 3.56E-2 & $9 / 24 / 08$ & $8 \mathrm{I} 18002$ & PNNL-AGG-415 \\
\hline HEIS No. & В1TP49 & \multicolumn{3}{|c|}{ Lab ID: $\quad$ 0804025-61 } & & & \\
\hline 14092-98-9 & Chromium & 8.96Е0 & ug/g dry & $1.82 \mathrm{E}-1$ & $9 / 24 / 08$ & 8I18002 & PNNL-AGG-415 \\
\hline 14119-06-3 & Copper & 8.09E0 & ug/g dry & $5.74 \mathrm{E}-1$ & $9 / 24 / 08$ & 8I18002 & PNNL-AGG-415 \\
\hline $7440-38-2$ & Arsenic & $1.91 \mathrm{E} 0$ & ug/g dry & $4.01 \mathrm{E}-1$ & 9/24/08 & $8 \mathrm{I} 18002$ & PNNL-AGG-415 \\
\hline 14687-58-2 & Selenium & $<1.10 \mathrm{E} 0$ & ug/g dry & $1.10 \mathrm{E} 0$ & 9/24/08 & $8 \mathrm{I} 18002$ & PNNL-AGG-415 \\
\hline 14378-37-1 & Silver & $<6.64 \mathrm{E}-2$ & ug/g dry & $6.64 \mathrm{E}-2$ & $9 / 24 / 08$ & 8I18002 & PNNL-AGG-415 \\
\hline $14336-64-2$ & Cadmium & $4.72 \mathrm{E}-2$ & ug/g dry & $4.71 \mathrm{E}-2$ & $9 / 24 / 08$ & $8 \mathrm{I} 18002$ & PNNL-AGG-415 \\
\hline $14265-72-6$ & Antimony & $<7.61 \mathrm{E}-2$ & ug/g dry & 7.61E-2 & 9/24/08 & $8 \mathrm{I} 18002$ & PNNL-AGG-415 \\
\hline 13966-28-4 & Lead & 2.33E0 & ug/g dry & $3.54 \mathrm{E}-2$ & $9 / 24 / 08$ & $8 \mathrm{I} 18002$ & PNNL-AGG-415 \\
\hline HEIS No. & B1TP51 & \multicolumn{3}{|c|}{ Lab ID: $\quad 0804025-69$} & & & \\
\hline 14119-06-3 & Copper & 8.69E0 & ug/g dry & $6.02 \mathrm{E}-1$ & 9/24/08 & $8 \mathrm{I} 18002$ & PNNL-AGG-415 \\
\hline 14687-58-2 & Selenium & $<1.15 \mathrm{E} 0$ & ug/g dry & $1.15 \mathrm{E} 0$ & 9/24/08 & 8I18002 & PNNL-AGG-415 \\
\hline 14336-64-2 & Cadmium & $6.44 \mathrm{E}-2$ & ug/g dry & $4.94 \mathrm{E}-2$ & 9/24/08 & $8 \mathrm{I} 18002$ & PNNL-AGG-415 \\
\hline $14265-72-6$ & Antimony & $1.07 \mathrm{E}-1$ & ug/g dry & $7.99 \mathrm{E}-2$ & 9/24/08 & $8 \mathrm{I} 18002$ & PNNL-AGG-415 \\
\hline HEIS No. & B1TP52 & \multicolumn{3}{|c|}{ Lab ID: $\quad 0804025-72$} & & & \\
\hline 14092-98-9 & Chromium & 8.78E0 & ug/g dry & $1.85 \mathrm{E}-1$ & 9/24/08 & $8 \mathrm{I} 18002$ & PNNL-AGG-415 \\
\hline 14119-06-3 & Copper & 8.40E0 & ug/g dry & $5.83 \mathrm{E}-1$ & $9 / 24 / 08$ & $8 \mathrm{I} 18002$ & PNNL-AGG-415 \\
\hline $7440-38-2$ & Arsenic & $1.60 \mathrm{E} 0$ & ug/g dry & 4.07E-1 & 9/24/08 & $8 \mathrm{I} 18002$ & PNNL-AGG-415 \\
\hline 14687-58-2 & Selenium & $<1.11 \mathrm{E} 0$ & ug/g dry & $1.11 \mathrm{E} 0$ & $9 / 24 / 08$ & 8I18002 & PNNL-AGG-415 \\
\hline 14378-37-1 & Silver & $<6.75 \mathrm{E}-2$ & ug/g dry & $6.75 \mathrm{E}-2$ & 9/24/08 & 8I18002 & PNNL-AGG-415 \\
\hline 14336-64-2 & Cadmium & $5.44 \mathrm{E}-2$ & ug/g dry & $4.78 \mathrm{E}-2$ & 9/24/08 & $8 \mathrm{I} 18002$ & PNNL-AGG-415 \\
\hline $14265-72-6$ & Antimony & $8.82 \mathrm{E}-2$ & ug/g dry & 7.73E-2 & $9 / 24 / 08$ & 8I18002 & PNNL-AGG-415 \\
\hline 13966-28-4 & Lead & $2.11 \mathrm{E} 0$ & ug/g dry & $3.59 \mathrm{E}-2$ & 9/24/08 & $8 \mathrm{I} 18002$ & PNNL-AGG-415 \\
\hline HEIS No. & B1TP53 & \multicolumn{3}{|c|}{ Lab ID: $\quad 0804025-75$} & & & \\
\hline 14092-98-9 & Chromium & $9.62 \mathrm{E} 0$ & ug/g dry & $1.83 \mathrm{E}-1$ & 9/24/08 & $8 \mathrm{I} 18002$ & PNNL-AGG-415 \\
\hline 14119-06-3 & Copper & 7.98E0 & ug/g dry & $5.76 \mathrm{E}-1$ & $9 / 24 / 08$ & $8 \mathrm{I} 18002$ & PNNL-AGG-415 \\
\hline $7440-38-2$ & Arsenic & $1.66 \mathrm{E} 0$ & ug/g dry & $4.02 \mathrm{E}-1$ & 9/24/08 & $8 \mathrm{I} 18002$ & PNNL-AGG-415 \\
\hline 14687-58-2 & Selenium & $<1.10 \mathrm{E} 0$ & ug/g dry & $1.10 \mathrm{E} 0$ & $9 / 24 / 08$ & 8I18002 & PNNL-AGG-415 \\
\hline 14378-37-1 & Silver & $<6.67 \mathrm{E}-2$ & ug/g dry & $6.67 \mathrm{E}-2$ & 9/24/08 & $8 \mathrm{I} 18002$ & PNNL-AGG-415 \\
\hline 14336-64-2 & Cadmium & 5.37E-2 & ug/g dry & 4.72E-2 & 9/24/08 & $8 \mathrm{I} 18002$ & PNNL-AGG-415 \\
\hline $14265-72-6$ & Antimony & $<7.64 \mathrm{E}-2$ & ug/g dry & $7.64 \mathrm{E}-2$ & $9 / 24 / 08$ & 8I18002 & PNNL-AGG-415 \\
\hline 13966-28-4 & Lead & 2.16E0 & ug/g dry & 3.55E-2 & 9/24/08 & $8 \mathrm{I} 18002$ & PNNL-AGG-415 \\
\hline HEIS No. & B1TP55 & \multicolumn{3}{|c|}{ Lab ID: $\quad$ 0804025-81 } & & & \\
\hline 14092-98-9 & Chromium & 8.91E0 & ug/g dry & $1.86 \mathrm{E}-1$ & $9 / 24 / 08$ & $8 \mathrm{I} 18002$ & PNNL-AGG-415 \\
\hline 14119-06-3 & Copper & 8.13E0 & ug/g dry & $5.86 \mathrm{E}-1$ & $9 / 24 / 08$ & 8I18002 & PNNL-AGG-415 \\
\hline $7440-38-2$ & Arsenic & $1.47 \mathrm{E} 0$ & ug/g dry & $4.09 \mathrm{E}-1$ & $9 / 24 / 08$ & $8 \mathrm{I} 18002$ & PNNL-AGG-415 \\
\hline 14687-58-2 & Selenium & $<1.12 \mathrm{E} 0$ & ug/g dry & $1.12 \mathrm{E} 0$ & 9/24/08 & 8I18002 & PNNL-AGG-415 \\
\hline $14378-37-1$ & Silver & $<6.77 \mathrm{E}-2$ & ug/g dry & $6.77 \mathrm{E}-2$ & 9/24/08 & $8 \mathrm{I} 18002$ & PNNL-AGG-415 \\
\hline 14336-64-2 & Cadmium & $5.32 \mathrm{E}-2$ & ug/g dry & $4.80 \mathrm{E}-2$ & 9/24/08 & $8 \mathrm{I} 18002$ & PNNL-AGG-415 \\
\hline $14265-72-6$ & Antimony & $<7.76 \mathrm{E}-2$ & ug/g dry & 7.76E-2 & $9 / 24 / 08$ & 8I18002 & PNNL-AGG-415 \\
\hline 13966-28-4 & Lead & 2.26E0 & ug/g dry & $3.61 \mathrm{E}-2$ & 9/24/08 & 8I18002 & PNNL-AGG-415 \\
\hline HEIS No. & B1TP59 & \multicolumn{3}{|c|}{ Lab ID: $\quad$ 0804025-87 } & & & \\
\hline 14092-98-9 & Chromium & $1.09 \mathrm{E} 1$ & ug/g dry & $1.82 \mathrm{E}-1$ & 9/24/08 & $8 \mathrm{I} 18002$ & PNNL-AGG-415 \\
\hline 14119-06-3 & Copper & 8.36Е0 & ug/g dry & $5.75 \mathrm{E}-1$ & $9 / 24 / 08$ & 8I18002 & PNNL-AGG-415 \\
\hline
\end{tabular}


RCRA Metals By PNNL-AGG-415/Acid Extract

\begin{tabular}{|c|c|c|c|c|c|c|c|}
\hline CAS \# & Analyte & Results & Units & EQL & Analyzed & Batch & Method \\
\hline HEIS No. & B1TP59 & \multicolumn{3}{|c|}{ Lab ID: $\quad$ 0804025-87 } & & & \\
\hline $7440-38-2$ & Arsenic & $1.64 \mathrm{E} 0$ & ug/g dry & $4.01 \mathrm{E}-1$ & $9 / 24 / 08$ & 8I18002 & PNNL-AGG-415 \\
\hline 14687-58-2 & Selenium & $<1.10 \mathrm{E} 0$ & ug/g dry & $1.10 \mathrm{E} 0$ & $9 / 24 / 08$ & 8I18002 & PNNL-AGG-415 \\
\hline 14378-37-1 & Silver & $<6.65 \mathrm{E}-2$ & ug/g dry & $6.65 \mathrm{E}-2$ & 9/24/08 & 8I18002 & PNNL-AGG-415 \\
\hline 14336-64-2 & Cadmium & 4.93E-2 & ug/g dry & $4.71 \mathrm{E}-2$ & $9 / 24 / 08$ & 8I18002 & PNNL-AGG-415 \\
\hline 14265-72-6 & Antimony & $<7.62 \mathrm{E}-2$ & ug/g dry & $7.62 \mathrm{E}-2$ & $9 / 24 / 08$ & 8I18002 & PNNL-AGG-415 \\
\hline 13966-28-4 & Lead & $2.01 \mathrm{E} 0$ & ug/g dry & $3.54 \mathrm{E}-2$ & 9/24/08 & $8 \mathrm{I} 18002$ & PNNL-AGG-415 \\
\hline HEIS No. & B1TP61 & \multicolumn{3}{|c|}{ Lab ID: $\quad$ 0804025-91 } & & & \\
\hline 14092-98-9 & Chromium & $1.07 \mathrm{E} 1$ & ug/g dry & $1.84 \mathrm{E}-1$ & $9 / 24 / 08$ & 8I18002 & PNNL-AGG-415 \\
\hline 14119-06-3 & Copper & 7.17E0 & ug/g dry & $5.78 \mathrm{E}-1$ & $9 / 24 / 08$ & 8I18002 & PNNL-AGG-415 \\
\hline $7440-38-2$ & Arsenic & 1.77E0 & ug/g dry & $4.03 \mathrm{E}-1$ & $9 / 24 / 08$ & $8 \mathrm{I} 18002$ & PNNL-AGG-415 \\
\hline $14687-58-2$ & Selenium & $<1.10 \mathrm{E} 0$ & ug/g dry & $1.10 \mathrm{E} 0$ & 9/24/08 & $8 \mathrm{I} 18002$ & PNNL-AGG-415 \\
\hline 14378-37-1 & Silver & $<6.69 \mathrm{E}-2$ & ug/g dry & $6.69 \mathrm{E}-2$ & $9 / 24 / 08$ & 8I18002 & PNNL-AGG-415 \\
\hline $14336-64-2$ & Cadmium & $5.56 \mathrm{E}-2$ & ug/g dry & $4.74 \mathrm{E}-2$ & $9 / 24 / 08$ & $8 \mathrm{I} 18002$ & PNNL-AGG-415 \\
\hline 14265-72-6 & Antimony & $<7.67 \mathrm{E}-2$ & ug/g dry & 7.67E-2 & $9 / 24 / 08$ & $8 \mathrm{I} 18002$ & PNNL-AGG-415 \\
\hline 13966-28-4 & Lead & $2.10 \mathrm{E} 0$ & ug/g dry & $3.56 \mathrm{E}-2$ & 9/24/08 & $8 \mathrm{I} 18002$ & PNNL-AGG-415 \\
\hline HEIS No. & B1TP62 & \multicolumn{3}{|c|}{ Lab ID: $\quad$ 0804025-94 } & & & \\
\hline 14092-98-9 & Chromium & 9.38E0 & ug/g dry & $1.85 \mathrm{E}-1$ & $9 / 24 / 08$ & $8 \mathrm{I} 18002$ & PNNL-AGG-415 \\
\hline 14119-06-3 & Copper & $9.29 \mathrm{E} 0$ & ug/g dry & 5.83E-1 & $9 / 24 / 08$ & $8 \mathrm{I} 18002$ & PNNL-AGG-415 \\
\hline 7440-38-2 & Arsenic & 1.19E0 & ug/g dry & 4.07E-1 & $9 / 24 / 08$ & $8 \mathrm{I} 18002$ & PNNL-AGG-415 \\
\hline $14687-58-2$ & Selenium & $<1.11 \mathrm{E} 0$ & ug/g dry & $1.11 \mathrm{E} 0$ & 9/24/08 & 8I18002 & PNNL-AGG-415 \\
\hline 14378-37-1 & Silver & $<6.75 \mathrm{E}-2$ & ug/g dry & $6.75 \mathrm{E}-2$ & $9 / 24 / 08$ & 8I18002 & PNNL-AGG-415 \\
\hline 14336-64-2 & Cadmium & $5.42 \mathrm{E}-2$ & ug/g dry & $4.78 \mathrm{E}-2$ & $9 / 24 / 08$ & $8 \mathrm{I} 18002$ & PNNL-AGG-415 \\
\hline $14265-72-6$ & Antimony & $<7.73 \mathrm{E}-2$ & ug/g dry & 7.73E-2 & 9/24/08 & 8I18002 & PNNL-AGG-415 \\
\hline 13966-28-4 & Lead & $2.16 \mathrm{E} 0$ & ug/g dry & $3.59 \mathrm{E}-2$ & 9/24/08 & $8 \mathrm{I} 18002$ & PNNL-AGG-415 \\
\hline HEIS No. & B1TP64 & \multicolumn{3}{|c|}{ Lab ID: $\quad$ 0804025-AA } & & & \\
\hline 14092-98-9 & Chromium & $7.58 \mathrm{E} 0$ & ug/g dry & $1.87 \mathrm{E}-1$ & $9 / 24 / 08$ & $8 \mathrm{I} 18002$ & PNNL-AGG-415 \\
\hline 14119-06-3 & Copper & 8.69E0 & ug/g dry & $5.90 \mathrm{E}-1$ & $9 / 24 / 08$ & $8 \mathrm{I} 18002$ & PNNL-AGG-415 \\
\hline $7440-38-2$ & Arsenic & $1.25 \mathrm{E} 0$ & ug/g dry & 4.11E-1 & $9 / 24 / 08$ & 8I18002 & PNNL-AGG-415 \\
\hline $14687-58-2$ & Selenium & $<1.12 \mathrm{E} 0$ & ug/g dry & $1.12 \mathrm{E} 0$ & 9/24/08 & $8 \mathrm{I} 18002$ & PNNL-AGG-415 \\
\hline 14378-37-1 & Silver & $<6.82 \mathrm{E}-2$ & ug/g dry & $6.82 \mathrm{E}-2$ & 9/24/08 & 8I18002 & PNNL-AGG-415 \\
\hline 14336-64-2 & Cadmium & $5.22 \mathrm{E}-2$ & ug/g dry & $4.83 \mathrm{E}-2$ & $9 / 24 / 08$ & 8I18002 & PNNL-AGG-415 \\
\hline $14265-72-6$ & Antimony & $<7.82 \mathrm{E}-2$ & ug/g dry & 7.82E-2 & $9 / 24 / 08$ & $8 \mathrm{I} 18002$ & PNNL-AGG-415 \\
\hline 13966-28-4 & Lead & 2.18E0 & ug/g dry & 3.63E-2 & $9 / 24 / 08$ & 8I18002 & PNNL-AGG-415 \\
\hline HEIS No. & B1TP67 & \multicolumn{3}{|c|}{ Lab ID: $\quad$ 0804025-AI } & & & \\
\hline 14092-98-9 & Chromium & $1.00 \mathrm{E} 1$ & ug/g dry & $1.86 \mathrm{E}-1$ & $9 / 24 / 08$ & $8 \mathrm{I} 18002$ & PNNL-AGG-415 \\
\hline 14119-06-3 & Copper & 8.19E0 & ug/g dry & $5.85 \mathrm{E}-1$ & $9 / 24 / 08$ & $8 \mathrm{I} 18002$ & PNNL-AGG-415 \\
\hline $7440-38-2$ & Arsenic & $1.25 \mathrm{E} 0$ & ug/g dry & 4.08E-1 & $9 / 24 / 08$ & 8I18002 & PNNL-AGG-415 \\
\hline $14687-58-2$ & Selenium & $<1.12 \mathrm{E} 0$ & ug/g dry & 1.12E0 & $9 / 24 / 08$ & $8 \mathrm{I} 18002$ & PNNL-AGG-415 \\
\hline 14378-37-1 & Silver & $<6.77 \mathrm{E}-2$ & ug/g dry & $6.77 \mathrm{E}-2$ & $9 / 24 / 08$ & $8 \mathrm{I} 18002$ & PNNL-AGG-415 \\
\hline $14336-64-2$ & Cadmium & $5.16 \mathrm{E}-2$ & ug/g dry & $4.80 \mathrm{E}-2$ & 9/24/08 & $8 \mathrm{I} 18002$ & PNNL-AGG-415 \\
\hline $14265-72-6$ & Antimony & $<7.76 \mathrm{E}-2$ & ug/g dry & $7.76 \mathrm{E}-2$ & $9 / 24 / 08$ & $8 \mathrm{I} 18002$ & PNNL-AGG-415 \\
\hline 13966-28-4 & Lead & $2.34 \mathrm{E} 0$ & ug/g dry & $3.61 \mathrm{E}-2$ & 9/24/08 & $8 \mathrm{I} 18002$ & PNNL-AGG-415 \\
\hline HEIS No. & B1TP69 & \multicolumn{3}{|c|}{ Lab ID: $\quad$ 0804025-AP } & & & \\
\hline 14092-98-9 & Chromium & $1.19 \mathrm{E} 1$ & ug/g dry & $1.87 \mathrm{E}-1$ & 9/24/08 & $8 \mathrm{I} 18002$ & PNNL-AGG-415 \\
\hline 14119-06-3 & Copper & 8.67E0 & ug/g dry & $5.89 \mathrm{E}-1$ & 9/24/08 & 8I18002 & PNNL-AGG-415 \\
\hline $7440-38-2$ & Arsenic & $1.16 \mathrm{E} 0$ & ug/g dry & $4.11 \mathrm{E}-1$ & $9 / 24 / 08$ & 8I18002 & PNNL-AGG-415 \\
\hline 14687-58-2 & Selenium & $<1.12 \mathrm{E} 0$ & ug/g dry & $1.12 \mathrm{E} 0$ & $9 / 24 / 08$ & $8 \mathrm{I} 18002$ & PNNL-AGG-415 \\
\hline 14378-37-1 & Silver & $<6.81 \mathrm{E}-2$ & ug/g dry & $6.81 \mathrm{E}-2$ & $9 / 24 / 08$ & $8 \mathrm{I} 18002$ & PNNL-AGG-415 \\
\hline
\end{tabular}


RCRA Metals By PNNL-AGG-415/Acid Extract

\begin{tabular}{|c|c|c|c|c|c|c|c|}
\hline CAS \# & Analyte & Results & Units & EQL & Analyzed & Batch & Method \\
\hline HEIS No. & B1TP69 & \multicolumn{3}{|c|}{ Lab ID: $\quad$ 0804025-AP } & & & \\
\hline $14336-64-2$ & Cadmium & $5.34 \mathrm{E}-2$ & ug/g dry & $4.83 \mathrm{E}-2$ & $9 / 24 / 08$ & $8 \mathrm{II} 18002$ & PNNL-AGG-415 \\
\hline $14265-72-6$ & Antimony & $<7.80 \mathrm{E}-2$ & ug/g dry & $7.80 \mathrm{E}-2$ & $9 / 24 / 08$ & $8 \mathrm{I} 18002$ & PNNL-AGG-415 \\
\hline $13966-28-4$ & Lead & 2.01E0 & ug/g dry & 3.63E-2 & $9 / 24 / 08$ & $8 \mathrm{I} 18002$ & PNNL-AGG-415 \\
\hline HEIS No. & B1TP82 & \multicolumn{3}{|c|}{ Lab ID: $\quad$ 0804025-BZ } & & & \\
\hline $14092-98-9$ & Chromium & $1.07 \mathrm{E} 1$ & ug/g dry & $1.87 \mathrm{E}-1$ & $9 / 24 / 08$ & $8 \mathrm{I} 18002$ & PNNL-AGG-415 \\
\hline $14119-06-3$ & Copper & 8.05E0 & ug/g dry & $5.88 \mathrm{E}-1$ & $9 / 24 / 08$ & $8 \mathrm{I} 18002$ & PNNL-AGG-415 \\
\hline 7440-38-2 & Arsenic & $1.46 \mathrm{E} 0$ & ug/g dry & $4.10 \mathrm{E}-1$ & $9 / 24 / 08$ & $8 \mathrm{I} 18002$ & PNNL-AGG-415 \\
\hline $14687-58-2$ & Selenium & $<1.12 \mathrm{E} 0$ & ug/g dry & $1.12 \mathrm{E} 0$ & $9 / 24 / 08$ & $8 \mathrm{I} 18002$ & PNNL-AGG-415 \\
\hline $14378-37-1$ & Silver & $<6.80 \mathrm{E}-2$ & ug/g dry & $6.80 \mathrm{E}-2$ & $9 / 24 / 08$ & $8 \mathrm{I} 18002$ & PNNL-AGG-415 \\
\hline $14336-64-2$ & Cadmium & $5.86 \mathrm{E}-2$ & ug/g dry & $4.82 \mathrm{E}-2$ & $9 / 24 / 08$ & $8 \mathrm{I} 18002$ & PNNL-AGG-415 \\
\hline $14265-72-6$ & Antimony & $<7.79 \mathrm{E}-2$ & ug/g dry & $7.79 \mathrm{E}-2$ & $9 / 24 / 08$ & $8 \mathrm{I} 18002$ & PNNL-AGG-415 \\
\hline $13966-28-4$ & Lead & $2.41 \mathrm{E} 0$ & ug/g dry & $3.62 \mathrm{E}-2$ & $9 / 24 / 08$ & $8 \mathrm{I} 18002$ & PNNL-AGG-415 \\
\hline HEIS No. & В1ТР93 & \multicolumn{3}{|c|}{ Lab ID: $\quad$ 0804025-DL } & & & \\
\hline $14092-98-9$ & Chromium & 7.92E0 & ug/g dry & $1.96 \mathrm{E}-1$ & $9 / 24 / 08$ & $8 \mathrm{I} 18002$ & PNNL-AGG-415 \\
\hline $14119-06-3$ & Copper & 8.95E0 & ug/g dry & $6.17 \mathrm{E}-1$ & $9 / 24 / 08$ & $8 \mathrm{I} 18002$ & PNNL-AGG-415 \\
\hline 7440-38-2 & Arsenic & $<4.30 \mathrm{E}-1$ & ug/g dry & $4.30 \mathrm{E}-1$ & $9 / 24 / 08$ & $8 \mathrm{I} 18002$ & PNNL-AGG-415 \\
\hline $14687-58-2$ & Selenium & $<1.18 \mathrm{E} 0$ & ug/g dry & $1.18 \mathrm{E} 0$ & $9 / 24 / 08$ & $8 \mathrm{I} 18002$ & PNNL-AGG-415 \\
\hline 14378-37-1 & Silver & $<7.14 \mathrm{E}-2$ & ug/g dry & $7.14 \mathrm{E}-2$ & $9 / 24 / 08$ & $8 \mathrm{I} 18002$ & PNNL-AGG-415 \\
\hline $14336-64-2$ & Cadmium & $6.21 \mathrm{E}-2$ & ug/g dry & $5.06 \mathrm{E}-2$ & $9 / 24 / 08$ & $8 \mathrm{I} 18002$ & PNNL-AGG-415 \\
\hline $14265-72-6$ & Antimony & $<8.18 \mathrm{E}-2$ & ug/g dry & $8.18 \mathrm{E}-2$ & $9 / 24 / 08$ & $8 \mathrm{I} 18002$ & PNNL-AGG-415 \\
\hline $13966-28-4$ & Lead & $1.35 \mathrm{E} 0$ & ug/g dry & $3.80 \mathrm{E}-2$ & $9 / 24 / 08$ & $8 \mathrm{I} 18002$ & PNNL-AGG-415 \\
\hline HEIS No. & В1ТР94 & \multicolumn{3}{|c|}{ Lab ID: $\quad$ 0804025-DM } & & & \\
\hline $14092-98-9$ & Chromium & 6.95E0 & ug/g dry & $1.90 \mathrm{E}-1$ & $9 / 24 / 08$ & $8 \mathrm{I} 18002$ & PNNL-AGG-415 \\
\hline $14119-06-3$ & Copper & $9.55 \mathrm{E} 0$ & ug/g dry & $6.00 \mathrm{E}-1$ & $9 / 24 / 08$ & $8 \mathrm{I} 18002$ & PNNL-AGG-415 \\
\hline $7440-38-2$ & Arsenic & $<4.18 \mathrm{E}-1$ & ug/g dry & $4.18 \mathrm{E}-1$ & $9 / 24 / 08$ & $8 \mathrm{I} 18002$ & PNNL-AGG-415 \\
\hline $14687-58-2$ & Selenium & $<1.14 \mathrm{E} 0$ & ug/g dry & $1.14 \mathrm{E} 0$ & $9 / 24 / 08$ & $8 \mathrm{I} 18002$ & PNNL-AGG-415 \\
\hline $14378-37-1$ & Silver & $<6.94 \mathrm{E}-2$ & ug/g dry & $6.94 \mathrm{E}-2$ & $9 / 24 / 08$ & $8 \mathrm{I} 18002$ & PNNL-AGG-415 \\
\hline $14336-64-2$ & Cadmium & $5.18 \mathrm{E}-2$ & ug/g dry & $4.92 \mathrm{E}-2$ & $9 / 24 / 08$ & $8 \mathrm{I} 18002$ & PNNL-AGG-415 \\
\hline $14265-72-6$ & Antimony & $<7.95 \mathrm{E}-2$ & ug/g dry & $7.95 \mathrm{E}-2$ & $9 / 24 / 08$ & $8 \mathrm{I} 18002$ & PNNL-AGG-415 \\
\hline $13966-28-4$ & Lead & $1.86 \mathrm{E} 0$ & ug/g dry & $3.70 \mathrm{E}-2$ & $9 / 24 / 08$ & $8 \mathrm{I} 18002$ & PNNL-AGG-415 \\
\hline
\end{tabular}




\section{Carbon Analysis/Soil}

\begin{tabular}{|c|c|c|c|c|c|}
\hline $\begin{array}{l}\text { Total Orga } \\
\text { Lab ID }\end{array}$ & $\begin{array}{c}\text { c Carbon } \\
\text { HEIS No. }\end{array}$ & $\begin{array}{l}\mathbf{0 1} \\
\text { Results }\end{array}$ & EQL & Analyzed & Batch \\
\hline 0804025-04 & B1TP24 & $<2.00 \mathrm{E} 2$ & $2.00 \mathrm{E} 2$ & $10 / 29 / 08$ & [CALC] \\
\hline 0804025-07 & В1ТР28 & $<2.00 \mathrm{E} 2$ & $2.00 \mathrm{E} 2$ & 10/29/08 & [CALC] \\
\hline 0804025-08 & В1ТР29 & 2.93E2 & $2.00 \mathrm{E} 2$ & 10/29/08 & [CALC] \\
\hline 0804025-09 & В1ТР30 & $<2.00 \mathrm{E} 2$ & $2.00 \mathrm{E} 2$ & $10 / 29 / 08$ & [CALC] \\
\hline 0804025-10 & B1TNV9 & $<2.00 \mathrm{E} 2$ & $2.00 \mathrm{E} 2$ & 10/29/08 & [CALC] \\
\hline 0804025-13 & B1TP22 & $<2.00 \mathrm{E} 2$ & $2.00 \mathrm{E} 2$ & 10/29/08 & [CALC] \\
\hline 0804025-16 & B1TP26 & $<2.00 \mathrm{E} 2$ & $2.00 \mathrm{E} 2$ & $10 / 29 / 08$ & [CALC] \\
\hline 0804025-17 & B1TNW0 & $<2.00 \mathrm{E} 2$ & $2.00 \mathrm{E} 2$ & 10/29/08 & [CALC] \\
\hline 0804025-18 & B1TNW1 & $<2.00 \mathrm{E} 2$ & $2.00 \mathrm{E} 2$ & 10/29/08 & [CALC] \\
\hline 0804025-20 & B1TNW3 & $<2.00 \mathrm{E} 2$ & $2.00 \mathrm{E} 2$ & 10/29/08 & [CALC] \\
\hline $0804025-22$ & B1TNW5 & 2.17E2 & $2.00 \mathrm{E} 2$ & 10/29/08 & [CALC] \\
\hline 0804025-37 & B1TP35 & $<2.00 \mathrm{E} 2$ & $2.00 \mathrm{E} 2$ & 10/29/08 & [CALC] \\
\hline 0804025-48 & B1TP40 & $<2.00 \mathrm{E} 2$ & $2.00 \mathrm{E} 2$ & 10/30/08 & [CALC] \\
\hline 0804025-50 & B1TP42 & $<2.00 \mathrm{E} 2$ & $2.00 \mathrm{E} 2$ & 10/30/08 & [CALC] \\
\hline 0804025-51 & B1TР43 & $<2.00 \mathrm{E} 2$ & $2.00 \mathrm{E} 2$ & 10/30/08 & [CALC] \\
\hline 0804025-53 & B1TP45 & $<2.00 \mathrm{E} 2$ & $2.00 \mathrm{E} 2$ & 10/30/08 & [CALC] \\
\hline 0804025-57 & B1TP47 & $<2.00 \mathrm{E} 2$ & $2.00 \mathrm{E} 2$ & 10/30/08 & [CALC] \\
\hline 0804025-61 & В1ТР49 & $<2.00 \mathrm{E} 2$ & $2.00 \mathrm{E} 2$ & 10/30/08 & [CALC] \\
\hline 0804025-69 & B1TP51 & $<2.00 \mathrm{E} 2$ & $2.00 \mathrm{E} 2$ & 10/30/08 & [CALC] \\
\hline 0804025-72 & B1TP52 & 2.26E2 & $2.00 \mathrm{E} 2$ & 10/30/08 & [CALC] \\
\hline 0804025-75 & B1TP53 & 2.13E2 & $2.00 \mathrm{E} 2$ & 10/30/08 & [CALC] \\
\hline 0804025-81 & B1TP55 & 2.16E2 & $2.00 \mathrm{E} 2$ & 10/30/08 & [CALC] \\
\hline 0804025-87 & B1TP59 & $<2.00 \mathrm{E} 2$ & $2.00 \mathrm{E} 2$ & 10/30/08 & [CALC] \\
\hline 0804025-91 & B1TP61 & $<2.00 \mathrm{E} 2$ & $2.00 \mathrm{E} 2$ & 10/30/08 & [CALC] \\
\hline 0804025-94 & B1TP62 & $<2.00 \mathrm{E} 2$ & $2.00 \mathrm{E} 2$ & 10/30/08 & [CALC] \\
\hline 0804025-AA & B1TP64 & $<2.00 \mathrm{E} 2$ & $2.00 \mathrm{E} 2$ & 10/30/08 & [CALC] \\
\hline 0804025-AI & B1TP67 & $<2.00 \mathrm{E} 2$ & $2.00 \mathrm{E} 2$ & 10/31/08 & [CALC] \\
\hline 0804025-AP & B1TP69 & $<2.00 \mathrm{E} 2$ & $2.00 \mathrm{E} 2$ & 10/31/08 & [CALC] \\
\hline 0804025-BZ & B1TP82 & $<2.00 \mathrm{E} 2$ & $2.00 \mathrm{E} 2$ & 10/31/08 & [CALC] \\
\hline 0804025-DL & В1ТР93 & 4.85E2 & $2.00 \mathrm{E} 2$ & 10/31/08 & [CALC] \\
\hline 0804025-DM & В1ТР94 & $4.82 \mathrm{E} 2$ & $2.00 \mathrm{E} 2$ & $10 / 31 / 08$ & [CALC] \\
\hline
\end{tabular}




\section{Carbon Analysis/Soil}

\begin{tabular}{|c|c|c|c|c|c|}
\hline $\begin{array}{l}\text { Total Carb } \\
\text { Lab ID }\end{array}$ & $\begin{array}{l}\text { (ug/g) by } \\
\text { HEIS No. }\end{array}$ & Results & EQL & Analyzed & Batch \\
\hline 0804025-04 & B1TP24 & $1.02 \mathrm{E} 3$ & $2.00 \mathrm{E} 2$ & $10 / 20 / 08$ & $8 \mathrm{~J} 20001$ \\
\hline 0804025-07 & В1ТР28 & 2.27E3 & $2.00 \mathrm{E} 2$ & 10/20/08 & 8J20001 \\
\hline 0804025-08 & В1ТР29 & 1.55E3 & $2.00 \mathrm{E} 2$ & 10/20/08 & 8J20001 \\
\hline 0804025-09 & В1ТР30 & $1.83 \mathrm{E} 3$ & $2.00 \mathrm{E} 2$ & $10 / 20 / 08$ & 8J20001 \\
\hline 0804025-10 & B1TNV9 & $2.45 \mathrm{E} 3$ & $2.00 \mathrm{E} 2$ & $10 / 20 / 08$ & 8J20001 \\
\hline 0804025-13 & B1TP22 & 7.00E2 & $2.00 \mathrm{E} 2$ & 10/20/08 & 8J20001 \\
\hline 0804025-16 & B1TP26 & $1.38 \mathrm{E} 3$ & $2.00 \mathrm{E} 2$ & $10 / 20 / 08$ & 8J20001 \\
\hline 0804025-17 & B1TNW0 & $1.56 \mathrm{E} 3$ & $2.00 \mathrm{E} 2$ & 10/20/08 & 8J20001 \\
\hline 0804025-18 & B1TNW1 & $2.24 \mathrm{E} 3$ & $2.00 \mathrm{E} 2$ & $10 / 20 / 08$ & 8J20001 \\
\hline 0804025-20 & B1TNW3 & 2.05E3 & $2.00 \mathrm{E} 2$ & 10/20/08 & 8J20001 \\
\hline $0804025-22$ & B1TNW5 & 2.23E3 & $2.00 \mathrm{E} 2$ & $10 / 20 / 08$ & 8J20001 \\
\hline 0804025-37 & B1TP35 & 2.19E3 & $2.00 \mathrm{E} 2$ & 10/20/08 & 8J20001 \\
\hline 0804025-48 & B1TP40 & $2.18 \mathrm{E} 3$ & $2.00 \mathrm{E} 2$ & 10/21/08 & 8J20001 \\
\hline 0804025-50 & B1TP42 & $2.20 \mathrm{E} 3$ & $2.00 \mathrm{E} 2$ & 10/21/08 & 8J20001 \\
\hline 0804025-51 & B1TР43 & $2.28 \mathrm{E} 3$ & $2.00 \mathrm{E} 2$ & 10/21/08 & 8J20001 \\
\hline 0804025-53 & B1TP45 & 2.05E3 & $2.00 \mathrm{E} 2$ & 10/21/08 & 8J20001 \\
\hline 0804025-57 & B1TP47 & $2.04 \mathrm{E} 3$ & $2.00 \mathrm{E} 2$ & $10 / 21 / 08$ & 8J20001 \\
\hline 0804025-61 & В1ТР49 & $1.78 \mathrm{E} 3$ & $2.00 \mathrm{E} 2$ & $10 / 21 / 08$ & 8J20001 \\
\hline 0804025-69 & B1TP51 & 2.12E3 & $2.00 \mathrm{E} 2$ & 10/21/08 & 8J20001 \\
\hline 0804025-72 & B1TP52 & 2.03E3 & $2.00 \mathrm{E} 2$ & $10 / 21 / 08$ & 8J20001 \\
\hline 0804025-75 & B1TP53 & $1.74 \mathrm{E} 3$ & $2.00 \mathrm{E} 2$ & 10/21/08 & 8J20001 \\
\hline 0804025-81 & B1TP55 & $1.66 \mathrm{E} 3$ & $2.00 \mathrm{E} 2$ & $10 / 21 / 08$ & 8J20001 \\
\hline 0804025-87 & B1TP59 & $1.89 \mathrm{E} 3$ & $2.00 \mathrm{E} 2$ & $10 / 21 / 08$ & 8J20001 \\
\hline 0804025-91 & B1TP61 & $1.63 \mathrm{E} 3$ & $2.00 \mathrm{E} 2$ & 10/21/08 & 8J20001 \\
\hline 0804025-94 & B1TP62 & $1.66 \mathrm{E} 3$ & $2.00 \mathrm{E} 2$ & $10 / 21 / 08$ & 8J20001 \\
\hline 0804025-AA & B1TP64 & $1.66 \mathrm{E} 3$ & $2.00 \mathrm{E} 2$ & $10 / 21 / 08$ & 8J20001 \\
\hline 0804025-AI & B1TP67 & $1.62 \mathrm{E} 3$ & $2.00 \mathrm{E} 2$ & $10 / 21 / 08$ & 8J20001 \\
\hline 0804025-AP & B1TP69 & $1.49 \mathrm{E} 3$ & $2.00 \mathrm{E} 2$ & 10/21/08 & 8J20001 \\
\hline 0804025-BZ & B1TP82 & $1.58 \mathrm{E} 3$ & $2.00 \mathrm{E} 2$ & $10 / 21 / 08$ & 8J20001 \\
\hline 0804025-DL & В1ТР93 & 4.85E2 & $2.00 \mathrm{E} 2$ & $10 / 22 / 08$ & $8 \mathrm{~J} 20001$ \\
\hline 0804025-DM & В1ТР94 & $4.82 \mathrm{E} 2$ & $2.00 \mathrm{E} 2$ & $10 / 22 / 08$ & 8J20001 \\
\hline
\end{tabular}




\begin{tabular}{|c|c|c|c|c|c|}
\hline & & Carbon & alysis/S & & \\
\hline Total Inory & nic Carbo & -001 & & & \\
\hline Lab ID & HEIS No. & Results & EQL & Analyzed & Batch \\
\hline 0804025-04 & B1TP24 & 9.15E2 & $2.00 \mathrm{E} 2$ & 10/29/08 & 8J29001 \\
\hline 0804025-07 & В1TP28 & $2.41 \mathrm{E} 3$ & $2.00 \mathrm{E} 2$ & 10/29/08 & 8J29001 \\
\hline 0804025-08 & B1TP29 & $1.26 \mathrm{E} 3$ & $2.00 \mathrm{E} 2$ & 10/29/08 & 8J29001 \\
\hline 0804025-09 & В1ТР30 & $1.74 \mathrm{E} 3$ & $2.00 \mathrm{E} 2$ & 10/29/08 & 8J29001 \\
\hline 0804025-10 & B1TNV9 & $2.58 \mathrm{E} 3$ & $2.00 \mathrm{E} 2$ & 10/29/08 & 8J29001 \\
\hline 0804025-13 & В1TP22 & 6.31E2 & $2.00 \mathrm{E} 2$ & 10/29/08 & 8J29001 \\
\hline 0804025-16 & B1TP26 & $1.34 \mathrm{E} 3$ & $2.00 \mathrm{E} 2$ & 10/29/08 & 8J29001 \\
\hline 0804025-17 & B1TNW0 & $1.43 \mathrm{E} 3$ & $2.00 \mathrm{E} 2$ & 10/29/08 & 8J29001 \\
\hline 0804025-18 & B1TNW1 & 2.22E3 & $2.00 \mathrm{E} 2$ & 10/29/08 & 8J29001 \\
\hline 0804025-20 & B1TNW3 & $1.99 \mathrm{E} 3$ & $2.00 \mathrm{E} 2$ & 10/29/08 & 8J29001 \\
\hline 0804025-22 & B1TNW5 & $2.01 \mathrm{E} 3$ & $2.00 \mathrm{E} 2$ & 10/29/08 & 8J29001 \\
\hline 0804025-37 & B1TP35 & 2.33E3 & $2.00 \mathrm{E} 2$ & 10/29/08 & 8J29001 \\
\hline 0804025-48 & B1TP40 & $2.06 \mathrm{E} 3$ & $2.00 \mathrm{E} 2$ & 10/30/08 & 8J29001 \\
\hline 0804025-50 & B1TP42 & $2.24 \mathrm{E} 3$ & $2.00 \mathrm{E} 2$ & 10/30/08 & 8J29001 \\
\hline 0804025-51 & B1TP43 & 2.13E3 & $2.00 \mathrm{E} 2$ & 10/30/08 & 8J29001 \\
\hline 0804025-53 & B1TP45 & $2.14 \mathrm{E} 3$ & $2.00 \mathrm{E} 2$ & 10/30/08 & 8J29001 \\
\hline 0804025-57 & B1TP47 & 2.07E3 & $2.00 \mathrm{E} 2$ & 10/30/08 & 8J29001 \\
\hline 0804025-61 & В1TP49 & $1.78 \mathrm{E} 3$ & $2.00 \mathrm{E} 2$ & 10/30/08 & 8J29001 \\
\hline 0804025-69 & B1TP51 & $1.93 \mathrm{E} 3$ & $2.00 \mathrm{E} 2$ & 10/30/08 & 8J29001 \\
\hline 0804025-72 & B1TP52 & $1.81 \mathrm{E} 3$ & $2.00 \mathrm{E} 2$ & 10/30/08 & 8J29001 \\
\hline 0804025-75 & B1TP53 & $1.53 \mathrm{E} 3$ & $2.00 \mathrm{E} 2$ & 10/30/08 & 8J29001 \\
\hline 0804025-81 & B1TP55 & $1.44 \mathrm{E} 3$ & $2.00 \mathrm{E} 2$ & 10/30/08 & 8J29001 \\
\hline 0804025-87 & B1TP59 & $1.92 \mathrm{E} 3$ & $2.00 \mathrm{E} 2$ & 10/30/08 & 8J29001 \\
\hline 0804025-91 & B1TP61 & $1.55 \mathrm{E} 3$ & $2.00 \mathrm{E} 2$ & 10/30/08 & 8J29001 \\
\hline 0804025-94 & B1TP62 & $1.60 \mathrm{E} 3$ & $2.00 \mathrm{E} 2$ & 10/30/08 & 8J29001 \\
\hline 0804025-AA & B1TP64 & $1.66 \mathrm{E} 3$ & $2.00 \mathrm{E} 2$ & 10/30/08 & 8J29001 \\
\hline 0804025-AI & В1TP67 & $1.43 \mathrm{E} 3$ & $2.00 \mathrm{E} 2$ & 10/31/08 & 8J29001 \\
\hline 0804025-AP & В1ТР69 & $1.32 \mathrm{E} 3$ & $2.00 \mathrm{E} 2$ & 10/31/08 & 8J29001 \\
\hline 0804025-BZ & В1TP82 & $1.50 \mathrm{E} 3$ & $2.00 \mathrm{E} 2$ & 10/31/08 & 8J29001 \\
\hline 0804025-DL & В1ТР93 & $<2.00 \mathrm{E} 2$ & $2.00 \mathrm{E} 2$ & 10/31/08 & 8J29001 \\
\hline 0804025-DM & В1ТР94 & $<2.00 \mathrm{E} 2$ & $2.00 \mathrm{E} 2$ & 10/31/08 & 8J29001 \\
\hline
\end{tabular}




\section{GEA/Soil}

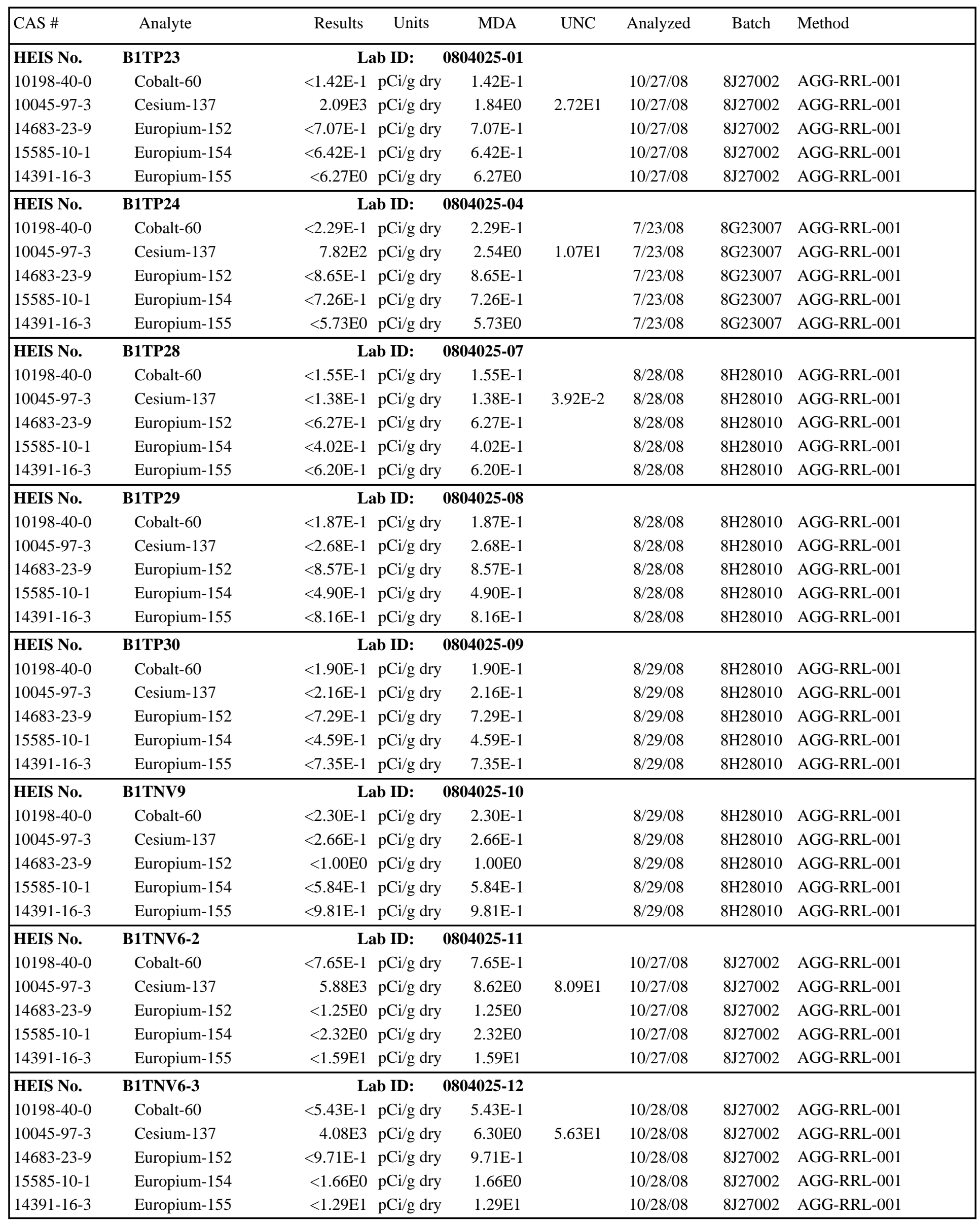




\section{GEA/Soil}

\begin{tabular}{|c|c|c|c|c|c|c|c|c|}
\hline CAS \# & Analyte & Results & Units & MDA & UNC & Analyzed & Batch & Method \\
\hline HEIS No. & B1TP22 & \multicolumn{3}{|c|}{ Lab ID: $\quad$ 0804025-13 } & & & & \\
\hline 10198-40-0 & Cobalt-60 & $<1.58 \mathrm{E}-1$ & pCi/g dry & $1.58 \mathrm{E}-1$ & & $7 / 23 / 08$ & $8 G 23007$ & AGG-RRL-001 \\
\hline $10045-97-3$ & Cesium-137 & $2.01 \mathrm{E} 1$ & pCi/g dry & 2.33E-1 & $3.26 \mathrm{E}-1$ & $7 / 23 / 08$ & $8 G 23007$ & AGG-RRL-001 \\
\hline 14683-23-9 & Europium-152 & $<7.18 \mathrm{E}-1$ & pCi/g dry & $7.18 \mathrm{E}-1$ & & $7 / 23 / 08$ & $8 G 23007$ & AGG-RRL-001 \\
\hline 15585-10-1 & Europium-154 & $<4.78 \mathrm{E}-1$ & pCi/g dry & $4.78 \mathrm{E}-1$ & & $7 / 23 / 08$ & $8 G 23007$ & AGG-RRL-001 \\
\hline 14391-16-3 & Europium-155 & $<8.61 \mathrm{E}-1$ & pCi/g dry & $8.61 \mathrm{E}-1$ & & $7 / 23 / 08$ & 8G23007 & AGG-RRL-001 \\
\hline HEIS No. & B1TNV7-2 & \multicolumn{3}{|c|}{ Lab ID: $\quad$ 0804025-14 } & & & & \\
\hline 10198-40-0 & Cobalt-60 & $<1.80 \mathrm{E}-1$ & pCi/g dry & $1.80 \mathrm{E}-1$ & & 10/28/08 & 8J27002 & AGG-RRL-001 \\
\hline 10045-97-3 & Cesium-137 & $3.48 \mathrm{E} 1$ & pCi/g dry & 3.33E-1 & $5.26 \mathrm{E}-1$ & 10/28/08 & 8J27002 & AGG-RRL-001 \\
\hline 14683-23-9 & Europium-152 & $<9.29 \mathrm{E}-1$ & pCi/g dry & $9.29 \mathrm{E}-1$ & & 10/28/08 & 8J27002 & AGG-RRL-001 \\
\hline $15585-10-1$ & Europium-154 & $<5.84 \mathrm{E}-1$ & pCi/g dry & $5.84 \mathrm{E}-1$ & & $10 / 28 / 08$ & $8 J 27002$ & AGG-RRL-001 \\
\hline 14391-16-3 & Europium-155 & $<1.13 \mathrm{E} 0$ & pCi/g dry & 1.13E0 & & 10/28/08 & 8J27002 & AGG-RRL-001 \\
\hline HEIS No. & B1TNV7-3 & \multicolumn{3}{|c|}{ Lab ID: $\quad$ 0804025-15 } & & & & \\
\hline 10198-40-0 & Cobalt-60 & $<2.68 \mathrm{E}-1$ & pCi/g dry & $2.68 \mathrm{E}-1$ & & 10/28/08 & 8J27002 & AGG-RRL-001 \\
\hline $10045-97-3$ & Cesium-137 & $1.26 \mathrm{E} 3$ & pCi/g dry & $1.75 \mathrm{E} 0$ & $1.56 \mathrm{E} 1$ & $10 / 28 / 08$ & 8J27002 & AGG-RRL-001 \\
\hline $14683-23-9$ & Europium-152 & $<1.27 \mathrm{E} 0$ & pCi/g dry & $1.27 \mathrm{E} 0$ & & $10 / 28 / 08$ & 8J27002 & AGG-RRL-001 \\
\hline 15585-10-1 & Europium-154 & $<9.65 \mathrm{E}-1$ & pCi/g dry & $9.65 \mathrm{E}-1$ & & $10 / 28 / 08$ & 8J27002 & AGG-RRL-001 \\
\hline 14391-16-3 & Europium-155 & $<6.02 \mathrm{E} 0$ & pCi/g dry & 6.02E0 & & $10 / 28 / 08$ & 8J27002 & AGG-RRL-001 \\
\hline HEIS No. & B1TP26 & \multicolumn{3}{|c|}{ Lab ID: $\quad 0804025-16$} & & & & \\
\hline $10198-40-0$ & Cobalt-60 & $<2.13 \mathrm{E}-1$ & pCi/g dry & $2.13 \mathrm{E}-1$ & & $7 / 23 / 08$ & $8 G 23007$ & AGG-RRL-001 \\
\hline $10045-97-3$ & Cesium-137 & $<2.19 \mathrm{E}-1$ & pCi/g dry & $2.19 \mathrm{E}-1$ & $5.83 \mathrm{E}-2$ & $7 / 23 / 08$ & $8 G 23007$ & AGG-RRL-001 \\
\hline 14683-23-9 & Europium-152 & $<7.86 \mathrm{E}-1$ & pCi/g dry & 7.86E-1 & & $7 / 23 / 08$ & $8 G 23007$ & AGG-RRL-001 \\
\hline 15585-10-1 & Europium-154 & $<5.11 \mathrm{E}-1$ & pCi/g dry & $5.11 \mathrm{E}-1$ & & $7 / 23 / 08$ & $8 G 23007$ & AGG-RRL-001 \\
\hline 14391-16-3 & Europium-155 & $<8.59 \mathrm{E}-1$ & pCi/g dry & $8.59 \mathrm{E}-1$ & & $7 / 23 / 08$ & $8 \mathrm{G} 23007$ & AGG-RRL-001 \\
\hline HEIS No. & B1TNW0 & \multicolumn{3}{|c|}{ Lab ID: $\quad$ 0804025-17 } & & & & \\
\hline $10198-40-0$ & Cobalt-60 & $<1.49 \mathrm{E}-1$ & pCi/g dry & $1.49 \mathrm{E}-1$ & $6.50 \mathrm{E}-2$ & 8/29/08 & $8 \mathrm{H} 28010$ & AGG-RRL-001 \\
\hline $10045-97-3$ & Cesium-137 & $<2.05 \mathrm{E}-1$ & pCi/g dry & 2.05E-1 & & $8 / 29 / 08$ & 8H28010 & AGG-RRL-001 \\
\hline $14683-23-9$ & Europium-152 & $<6.80 \mathrm{E}-1$ & pCi/g dry & $6.80 \mathrm{E}-1$ & & 8/29/08 & 8H28010 & AGG-RRL-001 \\
\hline 15585-10-1 & Europium-154 & $<4.38 \mathrm{E}-1$ & pCi/g dry & $4.38 \mathrm{E}-1$ & & 8/29/08 & 8H28010 & AGG-RRL-001 \\
\hline 14391-16-3 & Europium-155 & $<6.82 \mathrm{E}-1$ & pCi/g dry & $6.82 \mathrm{E}-1$ & & $8 / 29 / 08$ & $8 \mathrm{H} 28010$ & AGG-RRL-001 \\
\hline HEIS No. & B1TNW1 & \multicolumn{3}{|c|}{$\begin{array}{ll}\text { Lab ID: } & 0804025-18\end{array}$} & & & & \\
\hline 10198-40-0 & Cobalt-60 & $<2.03 \mathrm{E}-1$ & pCi/g dry & 2.03E-1 & $1.02 \mathrm{E}-1$ & 8/29/08 & 8H28010 & AGG-RRL-001 \\
\hline $10045-97-3$ & Cesium-137 & $<2.89 \mathrm{E}-1$ & pCi/g dry & $2.89 \mathrm{E}-1$ & & $8 / 29 / 08$ & 8H28010 & AGG-RRL-001 \\
\hline 14683-23-9 & Europium-152 & $<1.08 \mathrm{E} 0$ & pCi/g dry & $1.08 \mathrm{E} 0$ & & $8 / 29 / 08$ & $8 \mathrm{H} 28010$ & AGG-RRL-001 \\
\hline 15585-10-1 & Europium-154 & $<6.40 \mathrm{E}-1$ & pCi/g dry & $6.40 \mathrm{E}-1$ & & $8 / 29 / 08$ & 8H28010 & AGG-RRL-001 \\
\hline 14391-16-3 & Europium-155 & $<1.12 \mathrm{E} 0$ & pCi/g dry & $1.12 \mathrm{E} 0$ & & $8 / 29 / 08$ & $8 \mathrm{H} 28010$ & AGG-RRL-001 \\
\hline HEIS No. & B1TNW3 & \multicolumn{3}{|c|}{ Lab ID: $\quad$ 0804025-20 } & & & & \\
\hline $10198-40-0$ & Cobalt-60 & $<2.08 \mathrm{E}-1$ & pCi/g dry & $2.08 \mathrm{E}-1$ & & $8 / 29 / 08$ & 8H28010 & AGG-RRL-001 \\
\hline $10045-97-3$ & Cesium-137 & $<2.12 \mathrm{E}-1$ & pCi/g dry & $2.12 \mathrm{E}-1$ & & $8 / 29 / 08$ & $8 \mathrm{H} 28010$ & AGG-RRL-001 \\
\hline 14683-23-9 & Europium-152 & $<6.85 \mathrm{E}-1$ & pCi/g dry & $6.85 \mathrm{E}-1$ & & 8/29/08 & 8H28010 & AGG-RRL-001 \\
\hline $15585-10-1$ & Europium-154 & $<4.37 \mathrm{E}-1$ & pCi/g dry & $4.37 \mathrm{E}-1$ & & 8/29/08 & 8H28010 & AGG-RRL-001 \\
\hline 14391-16-3 & Europium-155 & $<6.87 \mathrm{E}-1$ & pCi/g dry & $6.87 \mathrm{E}-1$ & & $8 / 29 / 08$ & 8H28010 & AGG-RRL-001 \\
\hline HEIS No. & B1TNW5 & \multicolumn{3}{|c|}{ Lab ID: $\quad$ 0804025-22 } & & & & \\
\hline 10198-40-0 & Cobalt-60 & $<3.03 \mathrm{E}-1$ & pCi/g dry & 3.03E-1 & & 8/29/08 & 8H28010 & AGG-RRL-001 \\
\hline $10045-97-3$ & Cesium-137 & $<3.01 \mathrm{E}-1$ & pCi/g dry & $3.01 \mathrm{E}-1$ & & 8/29/08 & 8H28010 & AGG-RRL-001 \\
\hline $14683-23-9$ & Europium-152 & $<1.17 \mathrm{E} 0$ & pCi/g dry & $1.17 \mathrm{E} 0$ & & $8 / 29 / 08$ & 8H28010 & AGG-RRL-001 \\
\hline 15585-10-1 & Europium-154 & $<6.50 \mathrm{E}-1$ & pCi/g dry & $6.50 \mathrm{E}-1$ & & 8/29/08 & 8H28010 & AGG-RRL-001 \\
\hline 14391-16-3 & Europium-155 & $<1.12 \mathrm{E} 0$ & pCi/g dry & $1.12 \mathrm{E} 0$ & & $8 / 29 / 08$ & 8Н28010 & AGG-RRL-001 \\
\hline
\end{tabular}




\section{GEA/Soil}

\begin{tabular}{|c|c|c|c|c|c|c|c|c|}
\hline CAS \# & Analyte & Results & Units & MDA & UNC & Analyzed & Batch & Method \\
\hline HEIS No. & В1TP35 & \multicolumn{4}{|c|}{ Lab ID: $\quad$ 0804025-37 } & & & \\
\hline 10198-40-0 & Cobalt-60 & $<1.73 \mathrm{E}-1$ & pCi/g dry & 1.73E-1 & & 10/06/08 & 8H28010 & AGG-RRL-001 \\
\hline $10045-97-3$ & Cesium-137 & $<2.12 \mathrm{E}-1$ & pCi/g dry & 2.12E-1 & & 10/06/08 & 8H28010 & AGG-RRL-001 \\
\hline 14683-23-9 & Europium-152 & $<7.58 \mathrm{E}-1$ & pCi/g dry & $7.58 \mathrm{E}-1$ & & 10/06/08 & $8 \mathrm{H} 28010$ & AGG-RRL-001 \\
\hline 15585-10-1 & Europium-154 & $<4.74 \mathrm{E}-1$ & pCi/g dry & 4.74E-1 & & 10/06/08 & 8H28010 & AGG-RRL-001 \\
\hline 14391-16-3 & Europium-155 & $<7.29 \mathrm{E}-1$ & pCi/g dry & $7.29 \mathrm{E}-1$ & & $10 / 06 / 08$ & $8 \mathrm{H} 28010$ & AGG-RRL-001 \\
\hline HEIS No. & B1TP40 & \multicolumn{4}{|c|}{ Lab ID: $\quad$ 0804025-48 } & & & \\
\hline 10198-40-0 & Cobalt-60 & $<2.21 \mathrm{E}-1$ & pCi/g dry & $2.21 \mathrm{E}-1$ & & 10/06/08 & 8H28010 & AGG-RRL-001 \\
\hline $10045-97-3$ & Cesium-137 & $<2.56 \mathrm{E}-1$ & pCi/g dry & $2.56 \mathrm{E}-1$ & & $10 / 06 / 08$ & $8 \mathrm{H} 28010$ & AGG-RRL-001 \\
\hline 14683-23-9 & Europium-152 & $<9.58 \mathrm{E}-1$ & pCi/g dry & $9.58 \mathrm{E}-1$ & & 10/06/08 & 8H28010 & AGG-RRL-001 \\
\hline 15585-10-1 & Europium-154 & $<5.62 \mathrm{E}-1$ & pCi/g dry & $5.62 \mathrm{E}-1$ & & 10/06/08 & 8H28010 & AGG-RRL-001 \\
\hline 14391-16-3 & Europium-155 & $<9.52 \mathrm{E}-1$ & pCi/g dry & $9.52 \mathrm{E}-1$ & & $10 / 06 / 08$ & $8 \mathrm{H} 28010$ & AGG-RRL-001 \\
\hline HEIS No. & B1TP42 & \multicolumn{4}{|c|}{ Lab ID: $\quad$ 0804025-50 } & & & \\
\hline 10198-40-0 & Cobalt-60 & $<2.25 \mathrm{E}-1$ & pCi/g dry & 2.25E-1 & & 10/06/08 & 8H28010 & AGG-RRL-001 \\
\hline $10045-97-3$ & Cesium-137 & $<2.68 \mathrm{E}-1$ & pCi/g dry & $2.68 \mathrm{E}-1$ & & $10 / 06 / 08$ & 8H28010 & AGG-RRL-001 \\
\hline $14683-23-9$ & Europium-152 & $<8.87 \mathrm{E}-1$ & pCi/g dry & 8.87E-1 & & $10 / 06 / 08$ & 8H28010 & AGG-RRL-001 \\
\hline 15585-10-1 & Europium-154 & $<5.64 \mathrm{E}-1$ & pCi/g dry & $5.64 \mathrm{E}-1$ & & $10 / 06 / 08$ & $8 \mathrm{H} 28010$ & AGG-RRL-001 \\
\hline 14391-16-3 & Europium-155 & $<8.50 \mathrm{E}-1$ & pCi/g dry & $8.50 \mathrm{E}-1$ & & $10 / 06 / 08$ & 8H28010 & AGG-RRL-001 \\
\hline HEIS No. & B1TP43 & \multicolumn{4}{|c|}{ Lab ID: $\quad$ 0804025-51 } & & & \\
\hline $10198-40-0$ & Cobalt-60 & $<2.95 \mathrm{E}-1$ & pCi/g dry & 2.95E-1 & & $10 / 06 / 08$ & $8 \mathrm{H} 28010$ & AGG-RRL-001 \\
\hline $10045-97-3$ & Cesium-137 & $<3.03 \mathrm{E}-1$ & pCi/g dry & 3.03E-1 & & $10 / 06 / 08$ & $8 \mathrm{H} 28010$ & AGG-RRL-001 \\
\hline 14683-23-9 & Europium-152 & $<1.14 \mathrm{E} 0$ & pCi/g dry & $1.14 \mathrm{E} 0$ & & 10/06/08 & 8Н28010 & AGG-RRL-001 \\
\hline 15585-10-1 & Europium-154 & $<6.56 \mathrm{E}-1$ & pCi/g dry & $6.56 \mathrm{E}-1$ & & 10/06/08 & 8H28010 & AGG-RRL-001 \\
\hline 14391-16-3 & Europium-155 & $<1.10 \mathrm{E} 0$ & pCi/g dry & $1.10 \mathrm{E} 0$ & & 10/06/08 & $8 \mathrm{H} 28010$ & AGG-RRL-001 \\
\hline HEIS No. & B1TP45 & \multicolumn{4}{|c|}{ Lab ID: $\quad$ 0804025-53 } & & & \\
\hline $10198-40-0$ & Cobalt-60 & $<1.57 \mathrm{E}-1$ & pCi/g dry & $1.57 \mathrm{E}-1$ & & $10 / 07 / 08$ & $8 \mathrm{H} 28010$ & AGG-RRL-001 \\
\hline $10045-97-3$ & Cesium-137 & $<1.89 \mathrm{E}-1$ & pCi/g dry & $1.89 \mathrm{E}-1$ & & 10/07/08 & 8H28010 & AGG-RRL-001 \\
\hline $14683-23-9$ & Europium-152 & $<6.16 \mathrm{E}-1$ & pCi/g dry & $6.16 \mathrm{E}-1$ & & $10 / 07 / 08$ & 8H28010 & AGG-RRL-001 \\
\hline $15585-10-1$ & Europium-154 & $<3.83 \mathrm{E}-1$ & pCi/g dry & 3.83E-1 & & $10 / 07 / 08$ & $8 \mathrm{H} 28010$ & AGG-RRL-001 \\
\hline 14391-16-3 & Europium-155 & $<6.05 \mathrm{E}-1$ & pCi/g dry & $6.05 \mathrm{E}-1$ & & $10 / 07 / 08$ & $8 \mathrm{H} 28010$ & AGG-RRL-001 \\
\hline HEIS No. & B1TP47 & \multicolumn{4}{|c|}{ Lab ID: $\quad$ 0804025-57 } & & & \\
\hline 10198-40-0 & Cobalt-60 & $<2.49 \mathrm{E}-1$ & pCi/g dry & 2.49E-1 & & $10 / 07 / 08$ & 8H28010 & AGG-RRL-001 \\
\hline $10045-97-3$ & Cesium-137 & $<2.26 \mathrm{E}-1$ & pCi/g dry & $2.26 \mathrm{E}-1$ & & $10 / 07 / 08$ & 8H28010 & AGG-RRL-001 \\
\hline 14683-23-9 & Europium-152 & $<8.80 \mathrm{E}-1$ & pCi/g dry & $8.80 \mathrm{E}-1$ & & 10/07/08 & $8 \mathrm{H} 28010$ & AGG-RRL-001 \\
\hline 15585-10-1 & Europium-154 & $<5.31 \mathrm{E}-1$ & pCi/g dry & $5.31 \mathrm{E}-1$ & & 10/07/08 & 8H28010 & AGG-RRL-001 \\
\hline 14391-16-3 & Europium-155 & $<8.98 \mathrm{E}-1$ & pCi/g dry & 8.98E-1 & & 10/07/08 & $8 \mathrm{H} 28010$ & AGG-RRL-001 \\
\hline HEIS No. & B1TP49 & \multicolumn{4}{|c|}{ Lab ID: $\quad$ 0804025-61 } & & & \\
\hline $10198-40-0$ & Cobalt-60 & $<2.14 \mathrm{E}-1$ & pCi/g dry & $2.14 \mathrm{E}-1$ & & $10 / 07 / 08$ & 8H28010 & AGG-RRL-001 \\
\hline $10045-97-3$ & Cesium-137 & $<2.05 \mathrm{E}-1$ & pCi/g dry & 2.05E-1 & & $10 / 07 / 08$ & $8 \mathrm{H} 28010$ & AGG-RRL-001 \\
\hline 14683-23-9 & Europium-152 & $<7.09 \mathrm{E}-1$ & pCi/g dry & 7.09E-1 & & 10/07/08 & 8H28010 & AGG-RRL-001 \\
\hline $15585-10-1$ & Europium-154 & $<4.31 \mathrm{E}-1$ & pCi/g dry & $4.31 \mathrm{E}-1$ & & $10 / 07 / 08$ & 8H28010 & AGG-RRL-001 \\
\hline 14391-16-3 & Europium-155 & $<6.79 \mathrm{E}-1$ & pCi/g dry & $6.79 \mathrm{E}-1$ & & $10 / 07 / 08$ & 8H28010 & AGG-RRL-001 \\
\hline HEIS No. & B1TP51 & \multicolumn{3}{|c|}{ Lab ID: $\quad 0804025-69$} & & & & \\
\hline 10198-40-0 & Cobalt-60 & $<1.46 \mathrm{E}-1$ & pCi/g dry & 1.46E-1 & 1.37E-1 & $7 / 23 / 08$ & $8 G 23007$ & AGG-RRL-001 \\
\hline $10045-97-3$ & Cesium-137 & $<2.51 \mathrm{E}-1$ & pCi/g dry & $2.51 \mathrm{E}-1$ & & $7 / 23 / 08$ & 8G23007 & AGG-RRL-001 \\
\hline $14683-23-9$ & Europium-152 & $<8.37 \mathrm{E}-1$ & pCi/g dry & 8.37E-1 & & $7 / 23 / 08$ & $8 G 23007$ & AGG-RRL-001 \\
\hline 15585-10-1 & Europium-154 & $<4.99 \mathrm{E}-1$ & pCi/g dry & 4.99E-1 & & 7/23/08 & $8 G 23007$ & AGG-RRL-001 \\
\hline 14391-16-3 & Europium-155 & $<8.10 \mathrm{E}-1$ & pCi/g dry & $8.10 \mathrm{E}-1$ & & $7 / 23 / 08$ & $8 G 23007$ & AGG-RRL-001 \\
\hline
\end{tabular}




\section{GEA/Soil}

\begin{tabular}{|c|c|c|c|c|c|c|c|c|}
\hline CAS \# & Analyte & Results & Units & MDA & UNC & Analyzed & Batch & Method \\
\hline HEIS No. & B1TP52 & \multicolumn{4}{|c|}{ Lab ID: $\quad$ 0804025-72 } & & & \\
\hline $10198-40-0$ & Cobalt-60 & $<2.31 \mathrm{E}-1$ & pCi/g dry & $2.31 \mathrm{E}-1$ & & $7 / 23 / 08$ & $8 G 23007$ & AGG-RRL-001 \\
\hline $10045-97-3$ & Cesium-137 & $<2.48 \mathrm{E}-1$ & pCi/g dry & $2.48 \mathrm{E}-1$ & & $7 / 23 / 08$ & $8 G 23007$ & AGG-RRL-001 \\
\hline 14683-23-9 & Europium-152 & $<9.47 \mathrm{E}-1$ & pCi/g dry & $9.47 \mathrm{E}-1$ & & $7 / 23 / 08$ & $8 G 23007$ & AGG-RRL-001 \\
\hline 15585-10-1 & Europium-154 & $<5.19 \mathrm{E}-1$ & pCi/g dry & 5.19E-1 & & $7 / 23 / 08$ & $8 G 23007$ & AGG-RRL-001 \\
\hline 14391-16-3 & Europium-155 & $<9.02 \mathrm{E}-1$ & pCi/g dry & $9.02 \mathrm{E}-1$ & & 7/23/08 & 8G23007 & AGG-RRL-001 \\
\hline HEIS No. & B1TP53 & \multicolumn{4}{|c|}{ Lab ID: $\quad$ 0804025-75 } & & & \\
\hline $10198-40-0$ & Cobalt-60 & $<2.06 \mathrm{E}-1$ & pCi/g dry & $2.06 \mathrm{E}-1$ & & $7 / 23 / 08$ & $8 G 23007$ & AGG-RRL-001 \\
\hline $10045-97-3$ & Cesium-137 & $<2.09 \mathrm{E}-1$ & pCi/g dry & $2.09 \mathrm{E}-1$ & & $7 / 23 / 08$ & $8 G 23007$ & AGG-RRL-001 \\
\hline 14683-23-9 & Europium-152 & $<6.91 \mathrm{E}-1$ & pCi/g dry & $6.91 \mathrm{E}-1$ & & $7 / 23 / 08$ & $8 G 23007$ & AGG-RRL-001 \\
\hline 15585-10-1 & Europium-154 & $<4.43 \mathrm{E}-1$ & pCi/g dry & 4.43E-1 & & $7 / 23 / 08$ & $8 G 23007$ & AGG-RRL-001 \\
\hline HEIS No. & B1TP55 & \multicolumn{4}{|c|}{ Lab ID: $\quad$ 0804025-81 } & & & \\
\hline $10198-40-0$ & Cobalt-60 & $<2.10 \mathrm{E}-1$ & pCi/g dry & $2.10 \mathrm{E}-1$ & & 10/07/08 & $8 \mathrm{H} 28010$ & AGG-RRL-001 \\
\hline $10045-97-3$ & Cesium-137 & $<2.37 \mathrm{E}-1$ & pCi/g dry & 2.37E-1 & & 10/07/08 & 8H28010 & AGG-RRL-001 \\
\hline $14683-23-9$ & Europium-152 & $<8.39 \mathrm{E}-1$ & pCi/g dry & 8.39E-1 & & $10 / 07 / 08$ & 8H28010 & AGG-RRL-001 \\
\hline 15585-10-1 & Europium-154 & $<4.88 \mathrm{E}-1$ & pCi/g dry & $4.88 \mathrm{E}-1$ & & $10 / 07 / 08$ & $8 \mathrm{H} 28010$ & AGG-RRL-001 \\
\hline $14391-16-3$ & Europium-155 & $<8.27 \mathrm{E}-1$ & pCi/g dry & 8.27E-1 & & $10 / 07 / 08$ & 8H28010 & AGG-RRL-001 \\
\hline HEIS No. & B1TP59 & \multicolumn{4}{|c|}{ Lab ID: $\quad$ 0804025-87 } & & & \\
\hline $10198-40-0$ & Cobalt-60 & $<1.75 \mathrm{E}-1$ & pCi/g dry & $1.75 \mathrm{E}-1$ & & $10 / 07 / 08$ & 8H28010 & AGG-RRL-001 \\
\hline $10045-97-3$ & Cesium-137 & $<2.08 \mathrm{E}-1$ & pCi/g dry & $2.08 \mathrm{E}-1$ & & $10 / 07 / 08$ & $8 \mathrm{H} 28010$ & AGG-RRL-001 \\
\hline $14683-23-9$ & Europium-152 & $<7.11 \mathrm{E}-1$ & pCi/g dry & $7.11 \mathrm{E}-1$ & & $10 / 07 / 08$ & $8 \mathrm{H} 28010$ & AGG-RRL-001 \\
\hline $15585-10-1$ & Europium-154 & $<4.37 \mathrm{E}-1$ & pCi/g dry & 4.37E-1 & & $10 / 07 / 08$ & 8H28010 & AGG-RRL-001 \\
\hline $14391-16-3$ & Europium-155 & $<6.96 \mathrm{E}-1$ & pCi/g dry & $6.96 \mathrm{E}-1$ & & $10 / 07 / 08$ & 8H28010 & AGG-RRL-001 \\
\hline HEIS No. & B1TP61 & \multicolumn{4}{|c|}{ Lab ID: $\quad 0804025-91$} & & & \\
\hline $10198-40-0$ & Cobalt-60 & $<2.11 \mathrm{E}-1$ & pCi/g dry & $2.11 \mathrm{E}-1$ & & $10 / 07 / 08$ & $8 \mathrm{H} 28010$ & AGG-RRL-001 \\
\hline $10045-97-3$ & Cesium-137 & $<2.63 \mathrm{E}-1$ & pCi/g dry & 2.63E-1 & & 10/07/08 & 8Н28010 & AGG-RRL-001 \\
\hline 14683-23-9 & Europium-152 & $<9.63 \mathrm{E}-1$ & pCi/g dry & 9.63E-1 & & 10/07/08 & 8H28010 & AGG-RRL-001 \\
\hline $15585-10-1$ & Europium-154 & $<5.60 \mathrm{E}-1$ & pCi/g dry & $5.60 \mathrm{E}-1$ & & $10 / 07 / 08$ & 8H28010 & AGG-RRL-001 \\
\hline 14391-16-3 & Europium-155 & $<9.17 \mathrm{E}-1$ & pCi/g dry & 9.17E-1 & & $10 / 07 / 08$ & 8H28010 & AGG-RRL-001 \\
\hline HEIS No. & B1TP62 & \multicolumn{4}{|c|}{ Lab ID: $\quad$ 0804025-94 } & & & \\
\hline $10198-40-0$ & Cobalt-60 & $<1.82 \mathrm{E}-1$ & pCi/g dry & $1.82 \mathrm{E}-1$ & & 10/08/08 & 8Н28010 & AGG-RRL-001 \\
\hline $10045-97-3$ & Cesium-137 & $<1.96 \mathrm{E}-1$ & pCi/g dry & $1.96 \mathrm{E}-1$ & & $10 / 08 / 08$ & 8H28010 & AGG-RRL-001 \\
\hline 14683-23-9 & Europium-152 & $<6.62 \mathrm{E}-1$ & pCi/g dry & $6.62 \mathrm{E}-1$ & & 10/08/08 & $8 \mathrm{H} 28010$ & AGG-RRL-001 \\
\hline 15585-10-1 & Europium-154 & $<4.24 \mathrm{E}-1$ & pCi/g dry & $4.24 \mathrm{E}-1$ & & 10/08/08 & $8 \mathrm{H} 28010$ & AGG-RRL-001 \\
\hline 14391-16-3 & Europium-155 & $<6.68 \mathrm{E}-1$ & pCi/g dry & $6.68 \mathrm{E}-1$ & & $10 / 08 / 08$ & 8H28010 & AGG-RRL-001 \\
\hline HEIS No. & B1TP64 & \multicolumn{4}{|c|}{ Lab ID: $\quad$ 0804025-AA } & & & \\
\hline $10198-40-0$ & Cobalt-60 & $<2.15 \mathrm{E}-1$ & pCi/g dry & $2.15 \mathrm{E}-1$ & & 10/08/08 & 8H28010 & AGG-RRL-001 \\
\hline $10045-97-3$ & Cesium-137 & $<2.37 \mathrm{E}-1$ & pCi/g dry & $2.37 \mathrm{E}-1$ & & $10 / 08 / 08$ & 8H28010 & AGG-RRL-001 \\
\hline $14683-23-9$ & Europium-152 & $<9.03 \mathrm{E}-1$ & pCi/g dry & $9.03 \mathrm{E}-1$ & & 10/08/08 & 8Н28010 & AGG-RRL-001 \\
\hline 15585-10-1 & Europium-154 & $<5.24 \mathrm{E}-1$ & pCi/g dry & $5.24 \mathrm{E}-1$ & & 10/08/08 & 8H28010 & AGG-RRL-001 \\
\hline $14391-16-3$ & Europium-155 & $<8.76 \mathrm{E}-1$ & pCi/g dry & $8.76 \mathrm{E}-1$ & & $10 / 08 / 08$ & 8H28010 & AGG-RRL-001 \\
\hline HEIS No. & B1TP67 & \multicolumn{4}{|c|}{ Lab ID: $\quad$ 0804025-AI } & & & \\
\hline $10198-40-0$ & Cobalt-60 & $<1.58 \mathrm{E}-1$ & pCi/g dry & $1.58 \mathrm{E}-1$ & & $10 / 08 / 08$ & $8 \mathrm{H} 28010$ & AGG-RRL-001 \\
\hline $10045-97-3$ & Cesium-137 & $<1.89 \mathrm{E}-1$ & pCi/g dry & $1.89 \mathrm{E}-1$ & & 10/08/08 & 8Н28010 & AGG-RRL-001 \\
\hline $14683-23-9$ & Europium-152 & $<6.39 \mathrm{E}-1$ & pCi/g dry & $6.39 \mathrm{E}-1$ & & 10/08/08 & 8H28010 & AGG-RRL-001 \\
\hline $15585-10-1$ & Europium-154 & $<4.38 \mathrm{E}-1$ & pCi/g dry & $4.38 \mathrm{E}-1$ & & 10/08/08 & 8H28010 & AGG-RRL-001 \\
\hline $14391-16-3$ & Europium-155 & $<6.76 \mathrm{E}-1$ & pCi/g dry & $6.76 \mathrm{E}-1$ & & $10 / 08 / 08$ & $8 \mathrm{H} 28010$ & AGG-RRL-001 \\
\hline HEIS No. & B1TP69 & \multicolumn{4}{|c|}{ Lab ID: $\quad$ 0804025-AP } & & & \\
\hline
\end{tabular}


GEA/Soil

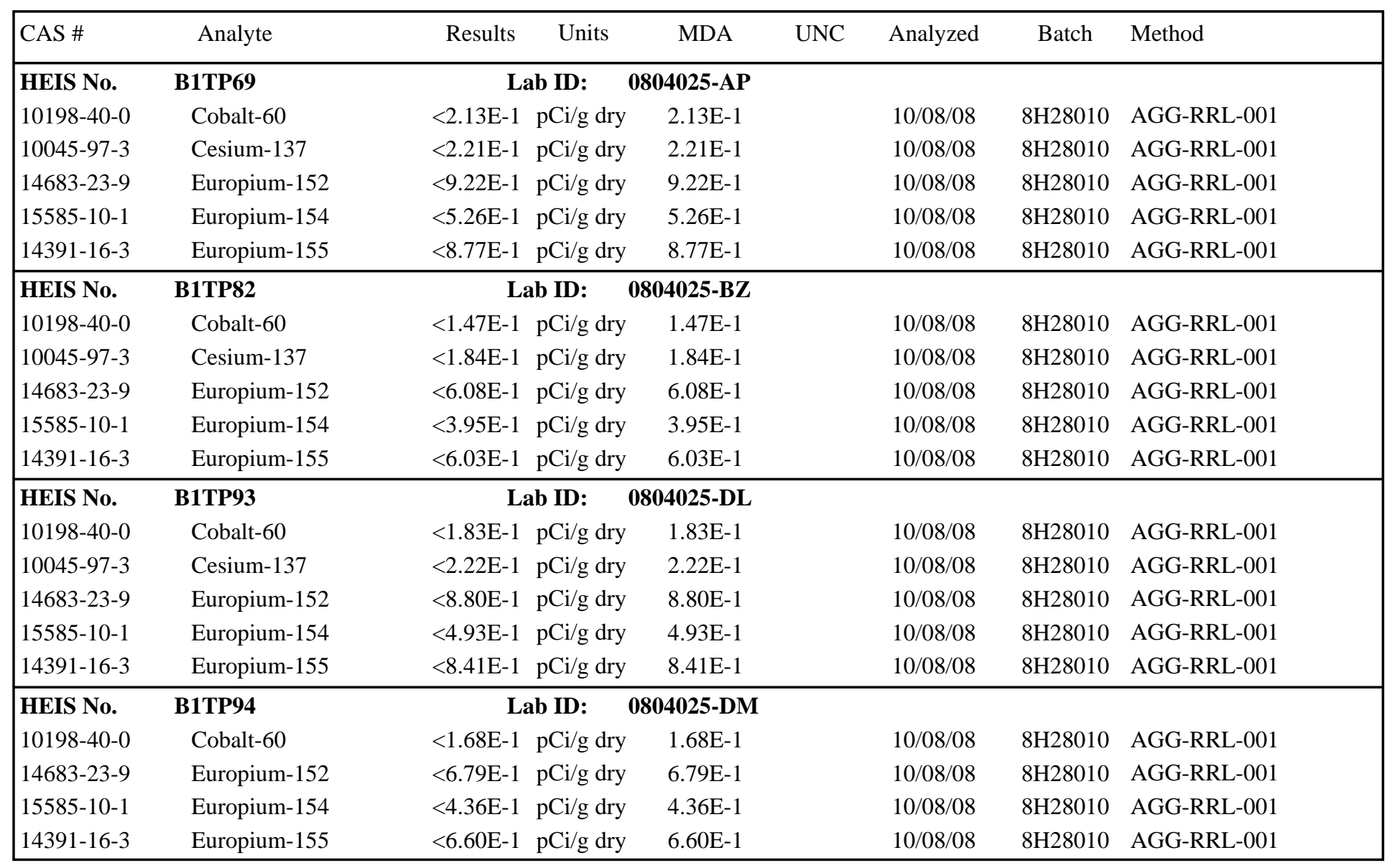


Total Alpha Total Beta/Acid Extract

\begin{tabular}{|c|c|c|c|c|c|c|c|c|}
\hline CAS \# & Analyte & Results & Units & MDA & UNC & Analyzed & Batch & Method \\
\hline HEIS No. & B1TP24 & \multicolumn{3}{|c|}{ Lab ID: $\quad$ 0804025-04 } & & & & \\
\hline $12587-47-2$ & Gross Beta & $9.94 \mathrm{E} 2$ & pCi/g dry & $1.53 \mathrm{E} 1$ & $1.25 \mathrm{E} 1$ & 10/06/08 & 8J07001 & AGG-RRL-002 \\
\hline $12587-46-1$ & Gross Alpha & $<6.55 \mathrm{E} 0$ & pCi/g dry & $6.55 \mathrm{E} 0$ & & $10 / 06 / 08$ & 8J07001 & AGG-RRL-002 \\
\hline HEIS No. & B1TP28 & \multicolumn{3}{|c|}{ Lab ID: $\quad$ 0804025-07 } & & & & \\
\hline $12587-47-2$ & Gross Beta & $<1.49 \mathrm{E} 1$ & pCi/g dry & 1.49E1 & & 10/06/08 & 8J07001 & AGG-RRL-002 \\
\hline $12587-46-1$ & Gross Alpha & $<6.37 \mathrm{E} 0$ & pCi/g dry & 6.37E0 & & $10 / 06 / 08$ & 8J07001 & AGG-RRL-002 \\
\hline HEIS No. & B1TP29 & \multicolumn{3}{|c|}{ Lab ID: $\quad$ 0804025-08 } & & & & \\
\hline $12587-47-2$ & Gross Beta & $<1.48 \mathrm{E} 1$ & pCi/g dry & $1.48 \mathrm{E} 1$ & & $10 / 06 / 08$ & 8J07001 & AGG-RRL-002 \\
\hline $12587-46-1$ & Gross Alpha & $<6.32 \mathrm{E} 0$ & pCi/g dry & $6.32 \mathrm{E} 0$ & & $10 / 06 / 08$ & 8J07001 & AGG-RRL-002 \\
\hline HEIS No. & B1TP30 & \multicolumn{3}{|c|}{ Lab ID: $\quad$ 0804025-09 } & & & & \\
\hline $12587-47-2$ & Gross Beta & $<1.52 \mathrm{E} 1$ & pCi/g dry & $1.52 \mathrm{E} 1$ & & $10 / 06 / 08$ & 8J07001 & AGG-RRL-002 \\
\hline $12587-46-1$ & Gross Alpha & $<6.52 \mathrm{E} 0$ & pCi/g dry & 6.52E0 & & $10 / 06 / 08$ & 8J07001 & AGG-RRL-002 \\
\hline HEIS No. & B1TNV9 & \multicolumn{3}{|c|}{ Lab ID: $\quad$ 0804025-10 } & & & & \\
\hline $12587-47-2$ & Gross Beta & $<1.48 \mathrm{E} 1$ & pCi/g dry & $1.48 \mathrm{E} 1$ & & 10/06/08 & 8J07001 & AGG-RRL-002 \\
\hline $12587-46-1$ & Gross Alpha & $<6.33 \mathrm{E} 0$ & pCi/g dry & 6.33Е0 & & 10/06/08 & 8J07001 & AGG-RRL-002 \\
\hline HEIS No. & B1TP22 & \multicolumn{3}{|c|}{ Lab ID: $\quad$ 0804025-13 } & & & & \\
\hline $12587-47-2$ & Gross Beta & $<1.49 \mathrm{E} 1$ & pCi/g dry & $1.49 \mathrm{E} 1$ & & $10 / 06 / 08$ & 8J07001 & AGG-RRL-002 \\
\hline $12587-46-1$ & Gross Alpha & $<6.38 \mathrm{E} 0$ & pCi/g dry & $6.38 \mathrm{E} 0$ & & $10 / 06 / 08$ & 8J07001 & AGG-RRL-002 \\
\hline HEIS No. & B1TP26 & \multicolumn{3}{|c|}{ Lab ID: $\quad$ 0804025-16 } & & & & \\
\hline $12587-47-2$ & Gross Beta & $<1.49 \mathrm{E} 1$ & pCi/g dry & $1.49 \mathrm{E} 1$ & & $10 / 06 / 08$ & 8J07001 & AGG-RRL-002 \\
\hline $12587-46-1$ & Gross Alpha & $<6.39 \mathrm{E} 0$ & pCi/g dry & 6.39E0 & & 10/06/08 & 8J07001 & AGG-RRL-002 \\
\hline HEIS No. & B1TNW0 & \multicolumn{3}{|c|}{ Lab ID: $\quad$ 0804025-17 } & & & & \\
\hline $12587-47-2$ & Gross Beta & $<1.50 \mathrm{E} 1$ & pCi/g dry & $1.50 \mathrm{E} 1$ & & $10 / 06 / 08$ & 8J07001 & AGG-RRL-002 \\
\hline $12587-46-1$ & Gross Alpha & $<6.44 \mathrm{E} 0$ & pCi/g dry & $6.44 \mathrm{E} 0$ & & $10 / 06 / 08$ & 8J07001 & AGG-RRL-002 \\
\hline HEIS No. & B1TNW1 & \multicolumn{3}{|c|}{ Lab ID: $\quad$ 0804025-18 } & & & & \\
\hline $12587-47-2$ & Gross Beta & $<1.49 \mathrm{E} 1$ & pCi/g dry & 1.49E1 & & 10/06/08 & 8J07001 & AGG-RRL-002 \\
\hline $12587-46-1$ & Gross Alpha & $<6.38 \mathrm{E} 0$ & pCi/g dry & 6.38E0 & & $10 / 06 / 08$ & 8J07001 & AGG-RRL-002 \\
\hline HEIS No. & B1TNW3 & \multicolumn{3}{|c|}{ Lab ID: $\quad$ 0804025-20 } & & & & \\
\hline $12587-47-2$ & Gross Beta & $<1.49 \mathrm{E} 1$ & pCi/g dry & $1.49 \mathrm{E} 1$ & & 10/06/08 & 8J07001 & AGG-RRL-002 \\
\hline $12587-46-1$ & Gross Alpha & $<6.40 \mathrm{E} 0$ & pCi/g dry & $6.40 \mathrm{E} 0$ & & $10 / 06 / 08$ & 8J07001 & AGG-RRL-002 \\
\hline HEIS No. & B1TNW5 & \multicolumn{3}{|c|}{ Lab ID: $\quad$ 0804025-22 } & & & & \\
\hline $12587-47-2$ & Gross Beta & $<1.51 \mathrm{E} 1$ & pCi/g dry & $1.51 \mathrm{E} 1$ & & $10 / 06 / 08$ & 8J07001 & AGG-RRL-002 \\
\hline $12587-46-1$ & Gross Alpha & $<6.45 \mathrm{E} 0$ & pCi/g dry & $6.45 \mathrm{E} 0$ & & $10 / 06 / 08$ & 8J07001 & AGG-RRL-002 \\
\hline HEIS No. & B1TP35 & \multicolumn{3}{|c|}{ Lab ID: $\quad$ 0804025-37 } & & & & \\
\hline $12587-47-2$ & Gross Beta & $<1.50 \mathrm{E} 1$ & pCi/g dry & $1.50 \mathrm{E} 1$ & & 10/06/08 & 8J07001 & AGG-RRL-002 \\
\hline $12587-46-1$ & Gross Alpha & $<6.41 \mathrm{E} 0$ & pCi/g dry & $6.41 \mathrm{E} 0$ & & $10 / 06 / 08$ & 8J07001 & AGG-RRL-002 \\
\hline HEIS No. & B1TP40 & \multicolumn{3}{|c|}{ Lab ID: $\quad$ 0804025-48 } & & & & \\
\hline $12587-47-2$ & Gross Beta & $<1.51 \mathrm{E} 1$ & pCi/g dry & $1.51 \mathrm{E} 1$ & & $10 / 06 / 08$ & 8J07001 & AGG-RRL-002 \\
\hline $12587-46-1$ & Gross Alpha & $<6.46 \mathrm{E} 0$ & pCi/g dry & 6.46E0 & & $10 / 06 / 08$ & 8J07001 & AGG-RRL-002 \\
\hline HEIS No. & B1TP42 & \multicolumn{3}{|c|}{ Lab ID: $\quad$ 0804025-50 } & & & & \\
\hline $12587-47-2$ & Gross Beta & $<1.46 \mathrm{E} 1$ & pCi/g dry & $1.46 \mathrm{E} 1$ & & 10/06/08 & 8J07001 & AGG-RRL-002 \\
\hline $12587-46-1$ & Gross Alpha & $<6.25 \mathrm{E} 0$ & pCi/g dry & $6.25 \mathrm{E} 0$ & & 10/06/08 & 8J07001 & AGG-RRL-002 \\
\hline HEIS No. & B1TP43 & \multicolumn{3}{|c|}{ Lab ID: $\quad 0804025-51$} & & & & \\
\hline $12587-47-2$ & Gross Beta & $<1.63 \mathrm{E} 1$ & pCi/g dry & $1.63 \mathrm{E} 1$ & & $10 / 06 / 08$ & 8J07001 & AGG-RRL-002 \\
\hline $12587-46-1$ & Gross Alpha & $<6.99 \mathrm{E} 0$ & pCi/g dry & 6.99E0 & & 10/06/08 & 8J07001 & AGG-RRL-002 \\
\hline HEIS No. & B1TP45 & \multicolumn{3}{|c|}{ Lab ID: $\quad 0804025-53$} & & & & \\
\hline $12587-47-2$ & Gross Beta & $<1.50 \mathrm{E} 1$ & pCi/g dry & $1.50 \mathrm{E} 1$ & & 10/07/08 & 8J07002 & AGG-RRL-002 \\
\hline
\end{tabular}


Total Alpha Total Beta/Acid Extract

\begin{tabular}{|c|c|c|c|c|c|c|c|c|}
\hline CAS \# & Analyte & Results & Units & MDA & UNC & Analyzed & Batch & Method \\
\hline HEIS No. & B1TP45 & \multicolumn{4}{|c|}{ Lab ID: $\quad$ 0804025-53 } & & & \\
\hline 12587-46-1 & Gross Alpha & $<6.31 \mathrm{E} 0$ & pCi/g dry & $6.31 \mathrm{E} 0$ & & 10/07/08 & 8J07002 & AGG-RRL-002 \\
\hline HEIS No. & B1TP47 & \multicolumn{4}{|c|}{ Lab ID: $\quad$ 0804025-57 } & & & \\
\hline $12587-47-2$ & Gross Beta & $<1.49 \mathrm{E} 1$ & pCi/g dry & $1.49 \mathrm{E} 1$ & & 10/07/08 & 8J07002 & AGG-RRL-002 \\
\hline 12587-46-1 & Gross Alpha & $<6.25 \mathrm{E} 0$ & pCi/g dry & $6.25 \mathrm{E} 0$ & & 10/07/08 & 8J07002 & AGG-RRL-002 \\
\hline HEIS No. & B1TP49 & \multicolumn{4}{|c|}{ Lab ID: $\quad 0804025-61$} & & & \\
\hline $12587-47-2$ & Gross Beta & $<1.48 \mathrm{E} 1$ & pCi/g dry & $1.48 \mathrm{E} 1$ & & 10/07/08 & 8J07002 & AGG-RRL-002 \\
\hline $12587-46-1$ & Gross Alpha & $<6.22 \mathrm{E} 0$ & pCi/g dry & $6.22 \mathrm{E} 0$ & & 10/07/08 & 8J07002 & AGG-RRL-002 \\
\hline HEIS No. & B1TP51 & \multicolumn{4}{|c|}{ Lab ID: $\quad$ 0804025-69 } & & & \\
\hline $12587-47-2$ & Gross Beta & $4.26 \mathrm{E} 1$ & pCi/g dry & $1.55 \mathrm{E} 1$ & & 10/07/08 & 8J07002 & AGG-RRL-002 \\
\hline $12587-46-1$ & Gross Alpha & $<6.52 \mathrm{E} 0$ & pCi/g dry & $6.52 \mathrm{E} 0$ & & $10 / 07 / 08$ & 8J07002 & AGG-RRL-002 \\
\hline HEIS No. & B1TP52 & \multicolumn{4}{|c|}{ Lab ID: $\quad 0804025-72$} & & & \\
\hline $12587-47-2$ & Gross Beta & $<1.50 \mathrm{E} 1$ & pCi/g dry & $1.50 \mathrm{E} 1$ & & $10 / 07 / 08$ & 8J07002 & AGG-RRL-002 \\
\hline $12587-46-1$ & Gross Alpha & $<6.31 \mathrm{E} 0$ & pCi/g dry & $6.31 \mathrm{E} 0$ & & $10 / 07 / 08$ & 8J07002 & AGG-RRL-002 \\
\hline HEIS No. & B1TP53 & \multicolumn{4}{|c|}{ Lab ID: $\quad 0804025-75$} & & & \\
\hline $12587-47-2$ & Gross Beta & $<1.48 \mathrm{E} 1$ & pCi/g dry & $1.48 \mathrm{E} 1$ & & $10 / 07 / 08$ & 8J07002 & AGG-RRL-002 \\
\hline $12587-46-1$ & Gross Alpha & $<6.24 \mathrm{E} 0$ & pCi/g dry & $6.24 \mathrm{E} 0$ & & $10 / 07 / 08$ & 8J07002 & AGG-RRL-002 \\
\hline HEIS No. & B1TP55 & \multicolumn{4}{|c|}{ Lab ID: $\quad$ 0804025-81 } & & & \\
\hline $12587-47-2$ & Gross Beta & $<1.51 \mathrm{E} 1$ & pCi/g dry & $1.51 \mathrm{E} 1$ & & 10/07/08 & 8J07002 & AGG-RRL-002 \\
\hline $12587-46-1$ & Gross Alpha & $<6.34 \mathrm{E} 0$ & pCi/g dry & $6.34 \mathrm{E} 0$ & & $10 / 07 / 08$ & 8J07002 & AGG-RRL-002 \\
\hline HEIS No. & B1TP59 & \multicolumn{4}{|c|}{ Lab ID: $\quad 0804025-87$} & & & \\
\hline $12587-47-2$ & Gross Beta & $<1.48 \mathrm{E} 1$ & pCi/g dry & $1.48 \mathrm{E} 1$ & & $10 / 07 / 08$ & 8J07002 & AGG-RRL-002 \\
\hline $12587-46-1$ & Gross Alpha & $<6.23 \mathrm{E} 0$ & pCi/g dry & $6.23 \mathrm{E} 0$ & & $10 / 07 / 08$ & 8J07002 & AGG-RRL-002 \\
\hline HEIS No. & B1TP61 & \multicolumn{4}{|c|}{ Lab ID: $\quad$ 0804025-91 } & & & \\
\hline $12587-47-2$ & Gross Beta & $<1.49 \mathrm{E} 1$ & pCi/g dry & $1.49 \mathrm{E} 1$ & & $10 / 07 / 08$ & 8J07002 & AGG-RRL-002 \\
\hline $12587-46-1$ & Gross Alpha & $<6.26 \mathrm{E} 0$ & pCi/g dry & $6.26 \mathrm{E} 0$ & & $10 / 07 / 08$ & 8J07002 & AGG-RRL-002 \\
\hline HEIS No. & B1TP62 & \multicolumn{4}{|c|}{ Lab ID: $\quad 0804025-94$} & & & \\
\hline $12587-47-2$ & Gross Beta & $<1.50 \mathrm{E} 1$ & pCi/g dry & $1.50 \mathrm{E} 1$ & & $10 / 07 / 08$ & 8J07002 & AGG-RRL-002 \\
\hline $12587-46-1$ & Gross Alpha & $<6.32 \mathrm{E} 0$ & pCi/g dry & $6.32 \mathrm{E} 0$ & & $10 / 07 / 08$ & 8J07002 & AGG-RRL-002 \\
\hline HEIS No. & B1TP64 & \multicolumn{4}{|c|}{ Lab ID: $\quad$ 0804025-AA } & & & \\
\hline $12587-47-2$ & Gross Beta & $<1.52 \mathrm{E} 1$ & pCi/g dry & $1.52 \mathrm{E} 1$ & & 10/07/08 & 8J07002 & AGG-RRL-002 \\
\hline $12587-46-1$ & Gross Alpha & $<6.39 \mathrm{E} 0$ & pCi/g dry & $6.39 \mathrm{E} 0$ & & $10 / 07 / 08$ & 8J07002 & AGG-RRL-002 \\
\hline HEIS No. & B1TP67 & \multicolumn{4}{|c|}{ Lab ID: $\quad$ 0804025-AI } & & & \\
\hline $12587-47-2$ & Gross Beta & $<1.54 \mathrm{E} 2$ & pCi/g dry & $1.54 \mathrm{E} 2$ & & $10 / 07 / 08$ & 8J07002 & AGG-RRL-002 \\
\hline $12587-46-1$ & Gross Alpha & $<6.45 \mathrm{E} 1$ & pCi/g dry & $6.45 \mathrm{E} 1$ & & $10 / 07 / 08$ & 8J07002 & AGG-RRL-002 \\
\hline HEIS No. & B1TP69 & \multicolumn{4}{|c|}{ Lab ID: $\quad$ 0804025-AP } & & & \\
\hline $12587-47-2$ & Gross Beta & $<1.52 \mathrm{E} 1$ & pCi/g dry & $1.52 \mathrm{E} 1$ & & $10 / 07 / 08$ & 8J07002 & AGG-RRL-002 \\
\hline $12587-46-1$ & Gross Alpha & $<6.38 \mathrm{E} 0$ & pCi/g dry & $6.38 \mathrm{E} 0$ & & $10 / 07 / 08$ & 8J07002 & AGG-RRL-002 \\
\hline HEIS No. & B1TP82 & \multicolumn{4}{|c|}{ Lab ID: $\quad$ 0804025-BZ } & & & \\
\hline $12587-47-2$ & Gross Beta & $<1.51 \mathrm{E} 1$ & pCi/g dry & $1.51 \mathrm{E} 1$ & & 10/07/08 & 8J07002 & AGG-RRL-002 \\
\hline $12587-46-1$ & Gross Alpha & $<6.37 \mathrm{E} 0$ & pCi/g dry & $6.37 \mathrm{E} 0$ & & $10 / 07 / 08$ & 8J07002 & AGG-RRL-002 \\
\hline HEIS No. & B1TP93 & \multicolumn{4}{|c|}{ Lab ID: $\quad$ 0804025-DL } & & & \\
\hline $12587-47-2$ & Gross Beta & $<1.59 \mathrm{E} 1$ & pCi/g dry & $1.59 \mathrm{E} 1$ & & $10 / 07 / 08$ & 8J07002 & AGG-RRL-002 \\
\hline $12587-46-1$ & Gross Alpha & $<6.68 \mathrm{E} 0$ & pCi/g dry & 6.68E0 & & 10/07/08 & 8J07002 & AGG-RRL-002 \\
\hline HEIS No. & B1TP94 & \multicolumn{4}{|c|}{ Lab ID: $\quad$ 0804025-DM } & & & \\
\hline $12587-47-2$ & Gross Beta & $<1.54 \mathrm{E} 1$ & pCi/g dry & $1.54 \mathrm{E} 1$ & & $10 / 07 / 08$ & 8J07002 & AGG-RRL-002 \\
\hline 12587-46-1 & Gross Alpha & $<6.49 \mathrm{E} 0$ & pCi/g dry & $6.49 \mathrm{E} 0$ & & 10/07/08 & 8J07002 & AGG-RRL-002 \\
\hline
\end{tabular}


Wet Chemistry - Quality Control

Environmental Science Laboratory

\begin{tabular}{|lrrrrrrrrrr}
\hline & & Reporting & & Spike & Source & & \%REC & & RPD & \\
Analyte & Result & Limit & Units & Level & Result & \%REC & Limits & RPD & Limit & Notes \\
\hline
\end{tabular}

Batch 8G09001 - 1:1 Water Extract (pH_EC_Alk)

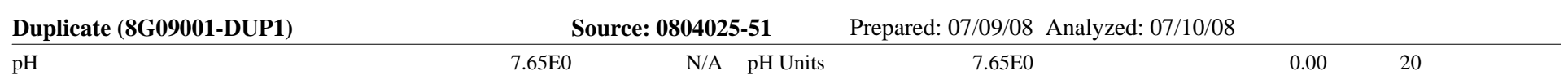

Batch 8G09002 - 1:1 Water Extract (pH_EC_Alk)

\begin{tabular}{|c|c|c|c|c|c|c|c|}
\hline Duplicate (8G09002-DUP1) & \multicolumn{3}{|c|}{ Source: 0804025-69 } & \multicolumn{4}{|c|}{ Prepared: 07/09/08 Analyzed: 07/10/08 } \\
\hline $\mathrm{pH}$ & 7.27E0 & N/A & pH Units & 7.29E0 & & 0.275 & 20 \\
\hline Duplicate (8G09002-DUP2) & \multicolumn{3}{|c|}{ Source: 0804025-87 } & Prepared: 07/09/08 & Analyzed: 07/10/08 & & \\
\hline $\mathrm{pH}$ & 7.48E0 & N/A & pH Units & 7.52E0 & & 0.533 & 20 \\
\hline
\end{tabular}

Batch 8G09003 - 1:1 Water Extract (pH_EC_Alk)

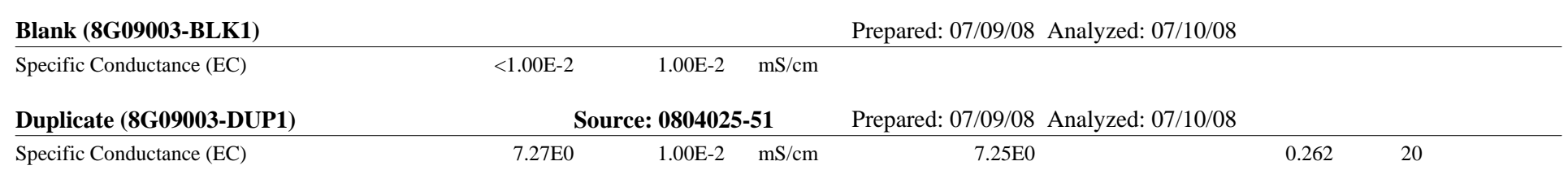

Batch 8G09004 - 1:1 Water Extract (pH_EC_Alk)

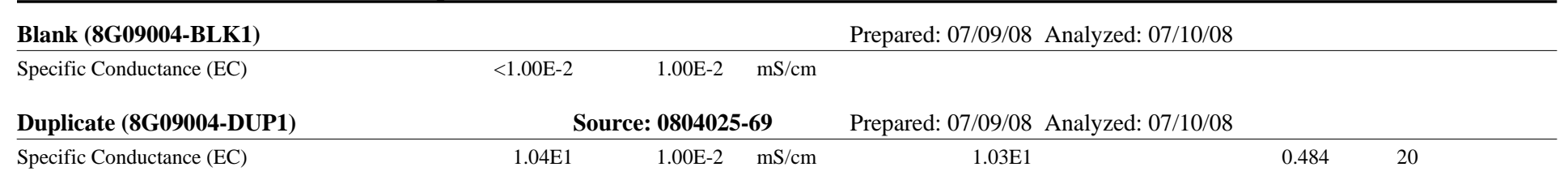


Wet Chemistry - Quality Control

Environmental Science Laboratory

\begin{tabular}{|lrrrrrrrrrrr}
\hline & & Reporting & & Spike & Source & & \%REC & & RPD & & \\
Analyte & Result & Limit & Units & Level & Result & \%REC & Limits & RPD & Limit & Notes \\
\hline
\end{tabular}

Batch 8G14005 - 1:1 Water Extract (pH_EC_Alk)

Blank (8G14005-BLK1)

Alkalinity as $\mathrm{CaCO} 3$

$<2.35 \mathrm{E} 1 \quad 2.35 \mathrm{E} 1 \mathrm{ug} / \mathrm{g}$ wet

Prepared \& Analyzed: 07/14/08

Duplicate (8G14005-DUP1)

Alkalinity as $\mathrm{CaCO} 3$

Source: 0804025-51

Prepared \& Analyzed: 07/14/08

3.39E1 2.44E1 ug/g dry 3.65E1

Prepared \& Analyzed: 07/14/08

Batch 8G14006 - 1:1 Water Extract (pH_EC_Alk)

Blank (8G14006-BLK1)

Alkalinity as $\mathrm{CaCO} 3$

$<2.35 \mathrm{E} 1$

$2.35 \mathrm{E} 1 \mathrm{ug} / \mathrm{g}$ wet

Duplicate (8G14006-DUP1)

Alkalinity as $\mathrm{CaCO} 3$ Source: 0804025-69

Prepared \& Analyzed: 07/14/08

2.58E1 2.35E1 ug/g dry 2.77E1

$6.98 \quad 20$

Batch 8H27001 - 1:1 Water Extract (pH_EC_Alk)

Duplicate (8H27001-DUP1)

$\mathrm{pH}$

$7.64 \mathrm{E}$

Source: 0804025-50 Prepared: 08/27/08 Analyzed: 08/28/08

N/A pH Units

7.77EO

1.69

35

Batch 8H27004 - 1:1 Water Extract (pH_EC_Alk)

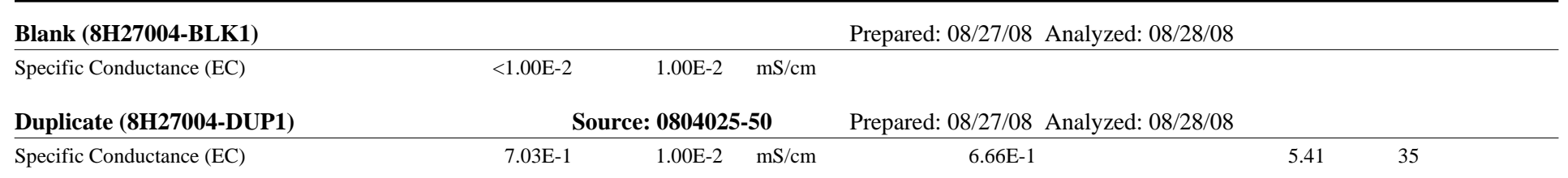


Wet Chemistry - Quality Control

Environmental Science Laboratory

\begin{tabular}{|lrrrrrrrrrrr}
\hline & & Reporting & & Spike & Source & & \%REC & & RPD & & \\
Analyte & Result & Limit & Units & Level & Result & \%REC & Limits & RPD & Limit & Notes \\
\hline
\end{tabular}

Batch 8I02001 - 1:1 Water Extract (pH_EC_Alk)

Blank (8I02001-BLK1)

Alkalinity as $\mathrm{CaCO} 3$

Prepared \& Analyzed: 09/02/08

Duplicate (8I02001-DUP1)

Alkalinity as $\mathrm{CaCO} 3$

Source: 0804025-50

Prepared \& Analyzed: 09/02/08

4.18E1 2.35E1 ug/g dry

3.88E1

7.48

35 
Anions by Ion Chromatography - Quality Control

Environmental Science Laboratory

\begin{tabular}{|lrrrrrrrrrr}
\hline & & Reporting & & Spike & Source & & \%REC & & RPD & \\
Analyte & Result & Limit & Units & Level & Result & \%REC & Limits & RPD & Limit & Notes \\
\hline
\end{tabular}

Batch 8G09011 - 1:1 Water Extract (IC)

\section{Blank (8G09011-BLK1)}

Fluoride

Chloride

Nitrite

Nitrate

Sulfate

Phosphate

LCS (8G09011-BS1)

Fluoride

Chloride

Nitrite

Nitrate

Sulfate

Phosphate

Duplicate (8G09011-DUP1)

\section{Fluoride}

Chloride

Nitrite

Nitrate

Sulfate

Phosphate

Post Spike (8G09011-PS1)

Fluoride

Chloride

Nitrite

Nitrate

Sulfate

Phosphate

(n)

$\begin{array}{rrc} & & \\ <2.00 \mathrm{E}-1 & 2.00 \mathrm{E}-1 & \text { ug/g wet } \\ <5.00 \mathrm{E}-1 & 5.00 \mathrm{E}-1 & " \\ <1.00 \mathrm{E} 0 & 1.00 \mathrm{E} 0 & " \\ <1.00 \mathrm{E} 0 & 1.00 \mathrm{E} 0 & " \\ <1.50 \mathrm{E} 0 & 1.50 \mathrm{E} 0 & " \\ <1.50 \mathrm{E} 0 & 1.50 \mathrm{E} 0 & "\end{array}$

Prepared: 07/09/08 Analyzed: 07/10/08

Prepared: 07/09/08 Analyzed: 07/10/08

$\begin{array}{lrcccc}2.13 \mathrm{E} 0 & 2.00 \mathrm{E}-1 & \text { ug/g wet } & 2.00 \mathrm{E} 0 & 107 & 80-120 \\ 5.08 \mathrm{E} 0 & 5.00 \mathrm{E}-1 & " & 5.00 \mathrm{E} 0 & 102 & 80-120 \\ 1.02 \mathrm{E} 1 & 1.00 \mathrm{E} 0 & " & 1.00 \mathrm{E} 1 & 102 & 80-120 \\ 1.08 \mathrm{E} 1 & 1.00 \mathrm{E} 0 & " & 1.00 \mathrm{E} 1 & 108 & 80-120 \\ 1.55 \mathrm{E} 1 & 1.50 \mathrm{E} 0 & " & 1.50 \mathrm{E} 1 & 103 & 80-120 \\ 1.53 \mathrm{E} 1 & 1.50 \mathrm{E} 0 & " & 1.50 \mathrm{E} 1 & 102 & 80-120\end{array}$

Source: 0804025-51 Prepared: 07/09/08 Analyzed: 07/10/08

\begin{tabular}{|c|c|c|c|c|c|c|}
\hline$<2.08 \mathrm{E} 0$ & $2.08 \mathrm{E} 0$ & ug/g dry & ND & & 20 & \\
\hline 7.61E1 & $5.19 \mathrm{E} 0$ & " & 7.27E1 & 4.61 & 20 & D \\
\hline$<1.04 \mathrm{E} 1$ & $1.04 \mathrm{E} 1$ & $"$ & ND & & 20 & \\
\hline 3.79E1 & $1.04 \mathrm{E} 0$ & $"$ & 3.61E1 & 4.83 & 20 & D \\
\hline 6.90E2 & $1.56 \mathrm{E} 1$ & $"$ & 7.47E2 & 7.98 & 20 & $\mathrm{D}$ \\
\hline$<1.56 \mathrm{E} 1$ & $1.56 \mathrm{E} 1$ & " & ND & & 20 & \\
\hline
\end{tabular}

Source: 0804025-04 Prepared: 07/09/08 Analyzed: 07/10/08

$\begin{array}{rcccccc}8.93 \mathrm{E}-1 & \text { N/A } & \text { ug/mL } & 8.00 \mathrm{E}-1 & 1.43 \mathrm{E}-1 & 93.8 & 75-125 \\ 3.23 \mathrm{E} 0 & \text { N/A } & " & 2.00 \mathrm{E} 0 & 1.35 \mathrm{E} 0 & 93.6 & 75-125 \\ 3.78 \mathrm{E} 0 & \text { N/A } & " & 4.00 \mathrm{E} 0 & 2.31 \mathrm{E}-1 & 88.8 & 75-125 \\ 1.74 \mathrm{E} 1 & \text { N/A } & " & 4.00 \mathrm{E} 0 & 1.40 \mathrm{E} 1 & 86.2 & 75-125 \\ 4.95 \mathrm{E} 1 & \text { N/A } & " & 6.00 \mathrm{E} 0 & 4.58 \mathrm{E} 1 & 61.6 & 75-125 \\ 4.04 \mathrm{E} 1 & \text { N/A } & " & 6.00 \mathrm{E} 0 & 3.62 \mathrm{E} 1 & 71.4 & 75-125\end{array}$


Anions by Ion Chromatography - Quality Control

Environmental Science Laboratory

\begin{tabular}{|c|c|c|c|c|c|c|c|c|c|c|}
\hline Analyte & Result & $\begin{array}{r}\text { orting } \\
\text { Limit }\end{array}$ & Units & $\begin{array}{l}\text { Spike } \\
\text { Level }\end{array}$ & $\begin{array}{l}\text { Source } \\
\text { Result }\end{array}$ & \%REC & $\begin{array}{l}\text { \%REC } \\
\text { Limits }\end{array}$ & RPD & $\begin{array}{l}\text { RPD } \\
\text { Limit }\end{array}$ & Notes \\
\hline
\end{tabular}

Batch 8G10007 - 1:1 Water Extract (IC)

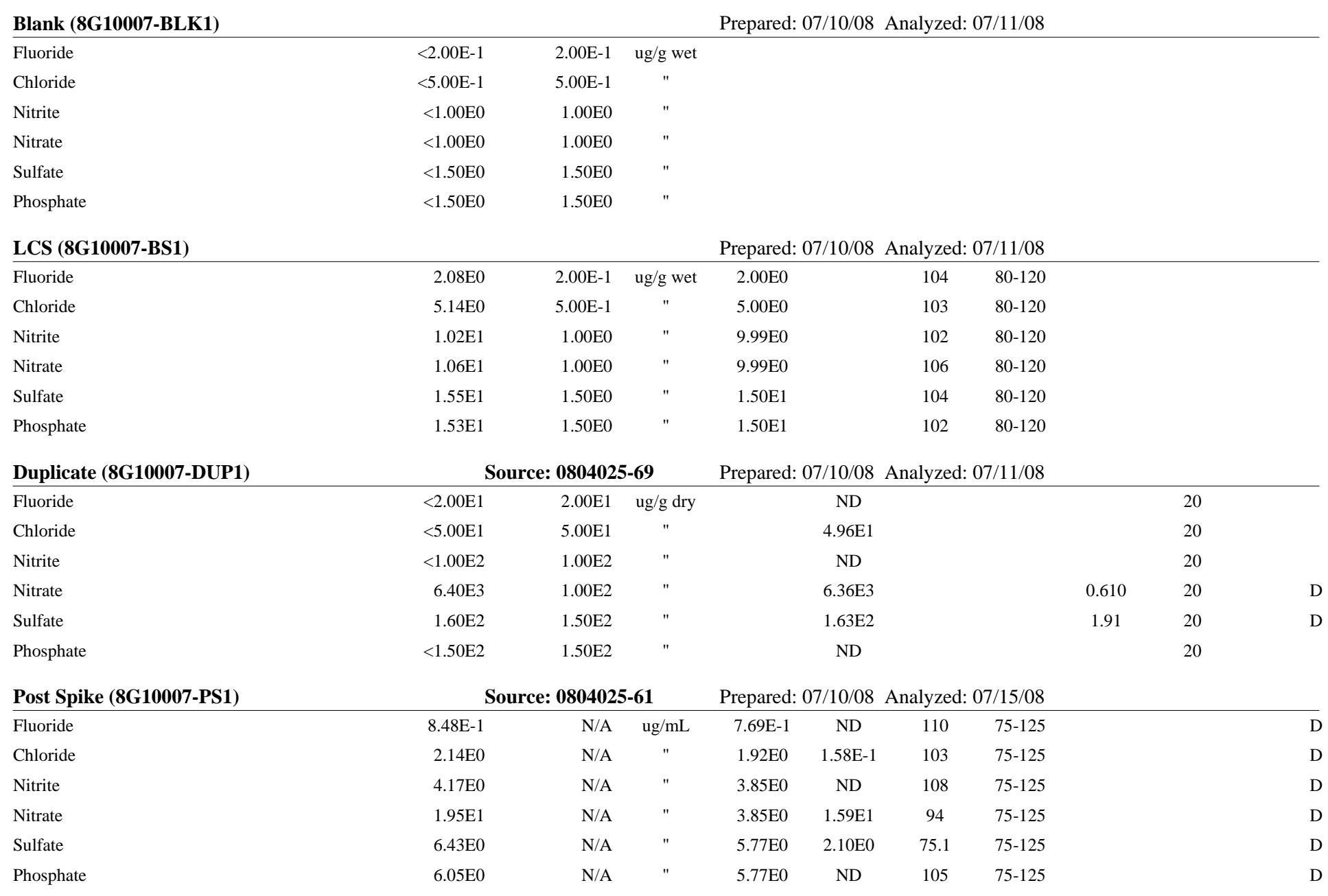


Anions by Ion Chromatography - Quality Control

Environmental Science Laboratory

\begin{tabular}{|c|c|c|c|c|c|c|c|c|c|c|}
\hline Analyte & Result & $\begin{array}{r}\text { orting } \\
\text { Limit }\end{array}$ & Units & $\begin{array}{l}\text { Spike } \\
\text { Level }\end{array}$ & $\begin{array}{l}\text { Source } \\
\text { Result }\end{array}$ & \%REC & $\begin{array}{l}\text { \%REC } \\
\text { Limits }\end{array}$ & RPD & $\begin{array}{l}\text { RPD } \\
\text { Limit }\end{array}$ & Notes \\
\hline
\end{tabular}

Batch 8H28003 - 1:1 Water Extract (IC)

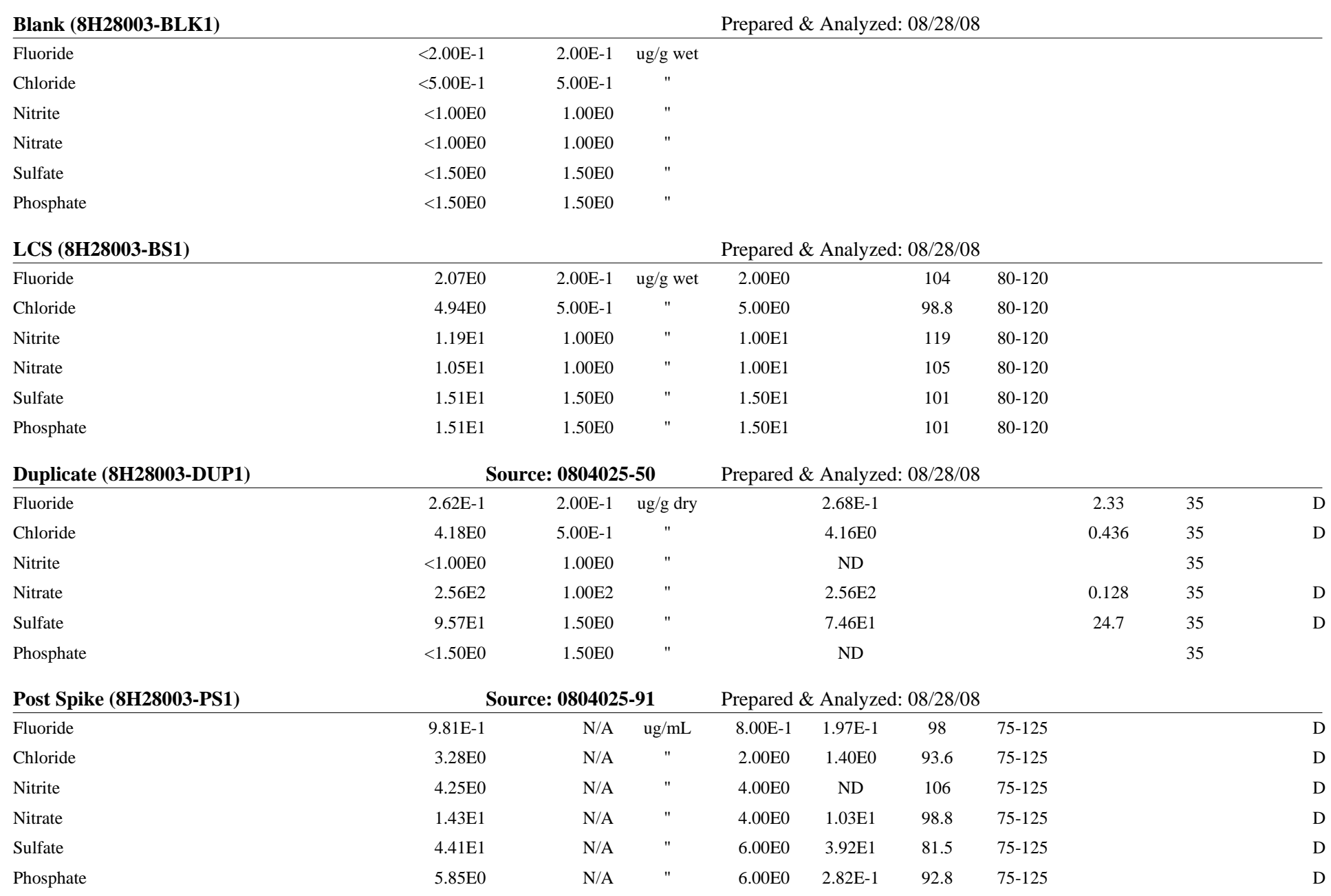


Cyanide by Mircodistillation/Colorimetric - Quality Control

Environmental Science Laboratory

\begin{tabular}{|lrrrrrrrrrrr}
\hline & & Reporting & & Spike & Source & & \%REC & & RPD & & \\
Analyte & Result & Limit & Units & Level & Result & \%REC & Limits & RPD & Limit & Notes
\end{tabular}

Batch 9H06008 - NO PREP

Blank (9H06008-BLK1)

Cyanide

$<2.00 \mathrm{E}-1 \quad 2.00 \mathrm{E}-1 \quad \mathrm{ug} / \mathrm{g}$ wet

Prepared \& Analyzed: 12/10/08

LCS (9H06008-BS1)

Prepared \& Analyzed: 12/10/08

Cyanide

$9.74 \mathrm{E}-1$

N/A ug/g $1.00 \mathrm{E} 0$

N97.4 80-120


Total Metals by PNNL-AGG-ICP-AES/Water Extract - Quality Control

Environmental Science Laboratory

\begin{tabular}{|c|c|c|c|c|c|c|c|c|c|c|}
\hline Analyte & Result & $\begin{array}{r}\text { porting } \\
\text { Limit }\end{array}$ & Units & $\begin{array}{l}\text { Spike } \\
\text { Level }\end{array}$ & $\begin{array}{l}\text { Source } \\
\text { Result }\end{array}$ & \%REC & $\begin{array}{l}\text { \%REC } \\
\text { Limits }\end{array}$ & RPD & $\begin{array}{l}\text { RPD } \\
\text { Limit }\end{array}$ & Notes \\
\hline
\end{tabular}

Batch 8G22002 - 1:1 Water Extract (ICP/ICPMS)

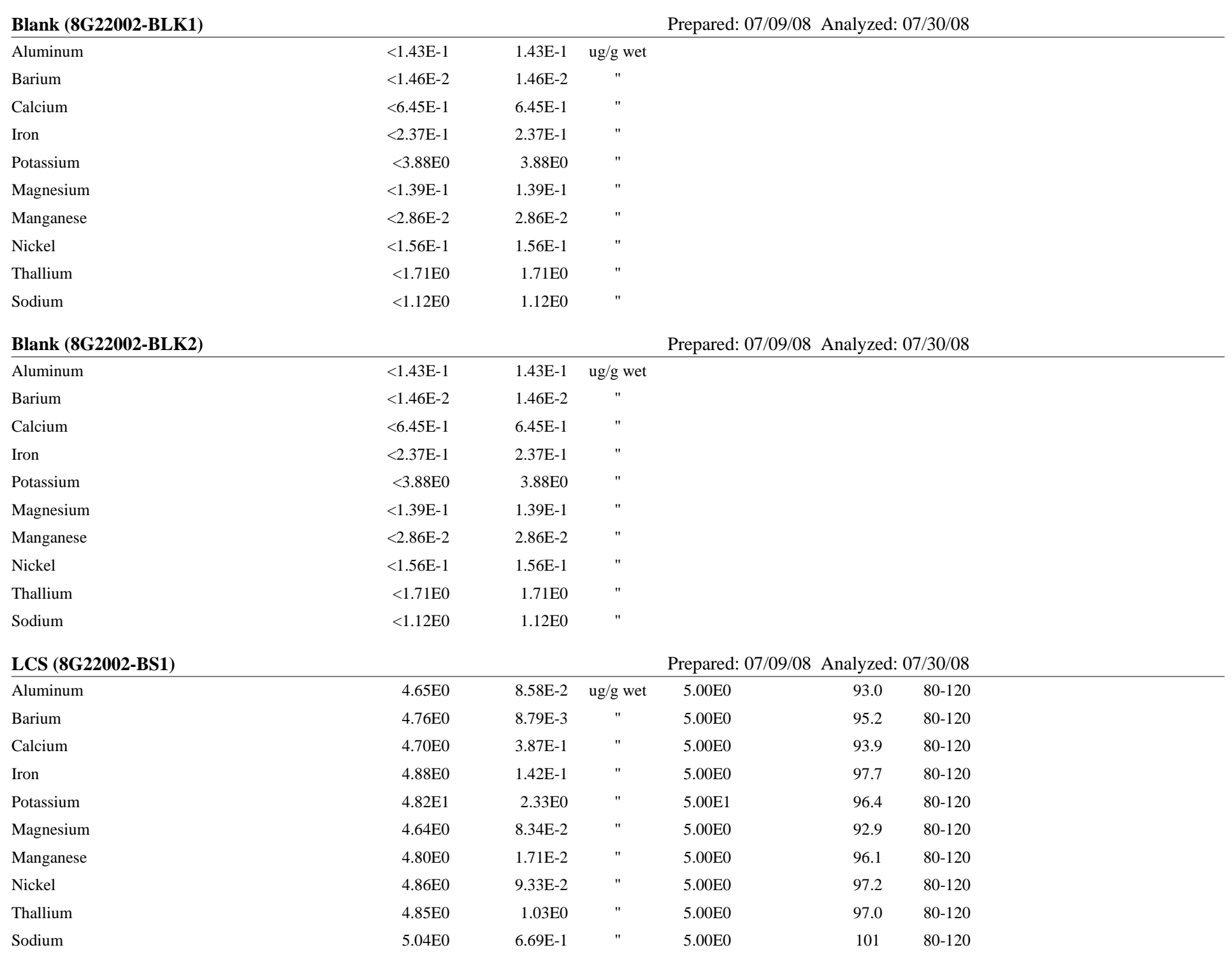


Total Metals by PNNL-AGG-ICP-AES/Water Extract - Quality Control

Environmental Science Laboratory

\begin{tabular}{|lrrrrrrrrrr}
\hline & & Reporting & & Spike & Source & & \%REC & & RPD \\
Analyte & Result & Limit & Units & Level & Result & \%REC & Limits & RPD & Limit & Notes \\
\hline
\end{tabular}

Batch 8G22002 - 1:1 Water Extract (ICP/ICPMS)

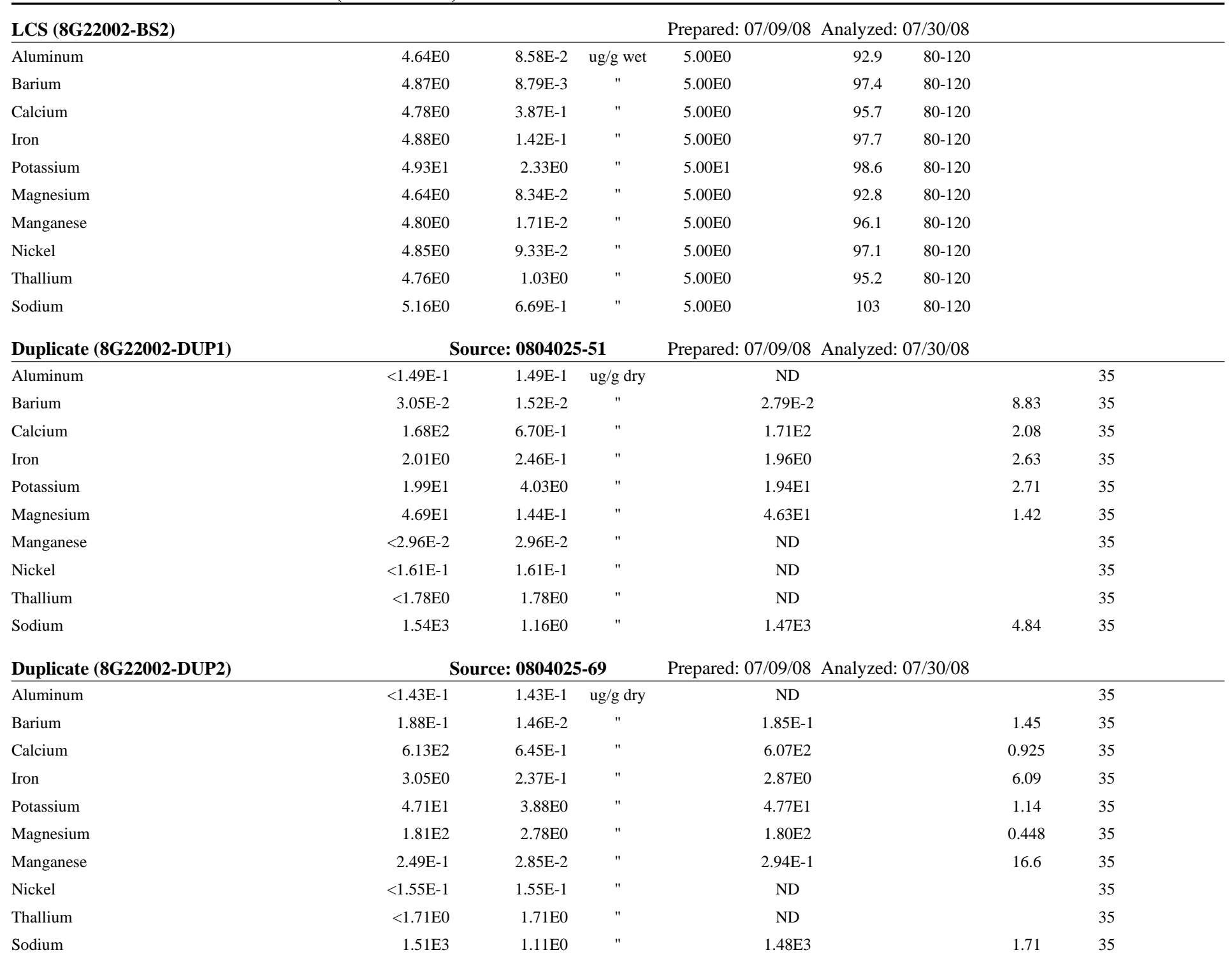


Total Metals by PNNL-AGG-ICP-AES/Water Extract - Quality Control

Environmental Science Laboratory

\begin{tabular}{|lrrrrrrrrrrr}
\hline & & Reporting & & Spike & Source & & \%REC & & RPD \\
Analyte & Result & Limit & Units & Level & Result & \%REC & Limits & RPD & Limit & Notes \\
\hline
\end{tabular}

Batch 8G22002 - 1:1 Water Extract (ICP/ICPMS)

\begin{tabular}{|c|c|c|c|c|c|c|}
\hline \multirow{2}{*}{$\frac{\text { Post Spike (8G22002-PS1) }}{\text { Aluminum }}$} & \multicolumn{3}{|c|}{ Source: 0804025-51 } & \multicolumn{2}{|c|}{ Prepared: 07/22/08 } & Analyzed: 07/30/08 \\
\hline & 5.23E2 & N/A & ug/L & $5.00 \mathrm{E} 2$ & 5.98E0 & $75-125$ \\
\hline Barium & 2.58E2 & N/A & $"$ & $2.50 \mathrm{E} 2$ & 5.58E0 & $75-125$ \\
\hline Calcium & $3.46 \mathrm{E} 4$ & N/A & $"$ & $5.00 \mathrm{E} 2$ & $3.42 \mathrm{E} 4$ & $75-125$ \\
\hline Iron & 8.97E2 & N/A & $"$ & $5.00 \mathrm{E} 2$ & 3.92E2 & $75-125$ \\
\hline Potassium & 5.17E3 & N/A & $"$ & $1.25 \mathrm{E} 3$ & 3.87E3 & $75-125$ \\
\hline Magnesium & 9.74E3 & N/A & $"$ & $5.00 \mathrm{E} 2$ & 9.23E3 & $75-125$ \\
\hline Manganese & $2.48 \mathrm{E} 2$ & N/A & $"$ & $2.50 \mathrm{E} 2$ & ND & $75-125$ \\
\hline Nickel & $4.96 \mathrm{E} 2$ & N/A & $"$ & $5.00 \mathrm{E} 2$ & $3.48 \mathrm{E}-1$ & $75-125$ \\
\hline Thallium & 3.77E2 & N/A & $"$ & $5.00 \mathrm{E} 2$ & ND & $75-125$ \\
\hline Sodium & 2.93E5 & N/A & $"$ & $5.00 \mathrm{E} 2$ & 2.94E5 & $75-125$ \\
\hline Post Spike (8G22002-PS2) & \multicolumn{3}{|c|}{ Source: 0804025-69 } & \multicolumn{2}{|c|}{ Prepared: 07/22/08 } & Analyzed: 07/30/08 \\
\hline Aluminum & $5.22 \mathrm{E} 2$ & N/A & $\mathrm{ug} / \mathrm{L}$ & $5.00 \mathrm{E} 2$ & ND & $75-125$ \\
\hline Barium & 2.92E2 & N/A & $"$ & $2.50 \mathrm{E} 2$ & $3.75 \mathrm{E} 1$ & $75-125$ \\
\hline Calcium & $1.25 \mathrm{E} 5$ & N/A & $"$ & $5.00 \mathrm{E} 2$ & $1.23 \mathrm{E} 5$ & $75-125$ \\
\hline Iron & $1.08 \mathrm{E} 3$ & N/A & $"$ & $5.00 \mathrm{E} 2$ & $5.82 \mathrm{E} 2$ & $75-125$ \\
\hline Potassium & $1.10 \mathrm{E} 4$ & N/A & $"$ & $1.25 \mathrm{E} 3$ & $9.68 \mathrm{E} 3$ & $75-125$ \\
\hline Magnesium & 2.32E3 & N/A & $"$ & $2.50 \mathrm{E} 1$ & 1.83E3 & $75-125$ \\
\hline Manganese & $3.08 \mathrm{E} 2$ & N/A & $"$ & $2.50 \mathrm{E} 2$ & $5.96 \mathrm{E} 1$ & $75-125$ \\
\hline Nickel & 4.83E2 & N/A & $"$ & $5.00 \mathrm{E} 2$ & $3.71 \mathrm{E} 0$ & $75-125$ \\
\hline Thallium & $2.50 \mathrm{E} 2$ & N/A & $"$ & $5.00 \mathrm{E} 2$ & ND & $75-125$ \\
\hline Sodium & $3.05 \mathrm{E} 5$ & N/A & $"$ & $5.00 \mathrm{E} 2$ & $3.01 \mathrm{E} 5$ & $75-125$ \\
\hline \multicolumn{7}{|c|}{ Batch 8I23005 - 1:1 Water Extract (ICP/ICPMS) } \\
\hline Blank (8I23005-BLK1) & & & & \multicolumn{3}{|c|}{ Prepared: 08/25/08 Analyzed: 09/24/08 } \\
\hline Aluminum & $<8.58 \mathrm{E}-2$ & $8.58 \mathrm{E}-2$ & ug/g wet & & & \\
\hline Barium & $<8.79 \mathrm{E}-3$ & 8.79E-3 & $"$ & & & \\
\hline Calcium & $<3.87 \mathrm{E}-1$ & 3.87E-1 & $"$ & & & \\
\hline Iron & $<1.42 \mathrm{E}-1$ & $1.42 \mathrm{E}-1$ & $"$ & & & \\
\hline Potassium & $<2.33 \mathrm{E} 0$ & 2.33E0 & $"$ & & & \\
\hline Magnesium & $<8.34 \mathrm{E}-2$ & 8.34E-2 & $"$ & & & \\
\hline Manganese & $<1.71 \mathrm{E}-2$ & $1.71 \mathrm{E}-2$ & $"$ & & & \\
\hline Nickel & $<9.33 \mathrm{E}-2$ & $9.33 \mathrm{E}-2$ & $"$ & & & \\
\hline Thallium & $<1.03 \mathrm{E} 0$ & 1.03E0 & $"$ & & & \\
\hline Sodium & $<6.69 \mathrm{E}-1$ & 6.69E-1 & $"$ & & & \\
\hline
\end{tabular}


Total Metals by PNNL-AGG-ICP-AES/Water Extract - Quality Control

Environmental Science Laboratory

\begin{tabular}{|lrrrrrrrrrrr}
\hline & & Reporting & & Spike & Source & & & \%REC & & RPD & \\
Analyte & Result & Limit & Units & Level & Result & \%REC & Limits & RPD & Limit & Notes \\
\hline
\end{tabular}

Batch 8I23005 - 1:1 Water Extract (ICP/ICPMS)

\begin{tabular}{|c|c|c|c|c|c|c|c|c|c|}
\hline \multirow{2}{*}{$\frac{\text { LCS (8I23005-BS1) }}{\text { Aluminum }}$} & \multicolumn{9}{|c|}{ Prepared: 08/25/08 Analyzed: 09/24/08 } \\
\hline & 4.77E0 & $8.58 \mathrm{E}-2$ & ug/g wet & $5.00 \mathrm{E} 0$ & & 95.5 & $80-120$ & & \\
\hline Barium & 4.76E0 & $8.79 \mathrm{E}-3$ & $"$ & $5.00 \mathrm{E} 0$ & & 95.3 & $80-120$ & & \\
\hline Calcium & 4.76E0 & $3.87 \mathrm{E}-1$ & $"$ & $5.00 \mathrm{E} 0$ & & 95.3 & $80-120$ & & \\
\hline Iron & 4.95E0 & $1.42 \mathrm{E}-1$ & $"$ & $5.00 \mathrm{E} 0$ & & 99.1 & $80-120$ & & \\
\hline Potassium & 4.83E1 & 2.33E0 & $"$ & $5.00 \mathrm{E} 1$ & & 96.8 & $80-120$ & & \\
\hline Magnesium & 4.76E0 & 8.34E-2 & $"$ & $5.00 \mathrm{E} 0$ & & 95.4 & $80-120$ & & \\
\hline Manganese & 4.88E0 & $1.71 \mathrm{E}-2$ & $"$ & $5.00 \mathrm{E} 0$ & & 97.8 & $80-120$ & & \\
\hline Nickel & 4.96E0 & $9.33 \mathrm{E}-2$ & $"$ & $5.00 \mathrm{E} 0$ & & 99.2 & $80-120$ & & \\
\hline Thallium & 4.92E0 & $1.03 \mathrm{E} 0$ & $"$ & $5.00 \mathrm{E} 0$ & & 98.5 & $80-120$ & & \\
\hline Sodium & 5.19E0 & $6.69 \mathrm{E}-1$ & $"$ & $5.00 \mathrm{E} 0$ & & 104 & $80-120$ & & \\
\hline Duplicate (8I23005-DUP1) & \multicolumn{3}{|c|}{ Source: 0804025-50 } & \multicolumn{4}{|c|}{ Prepared: 08/25/08 } & & \\
\hline Aluminum & $<8.58 \mathrm{E}-2$ & $8.58 \mathrm{E}-2$ & ug/g dry & & ND & & & & 35 \\
\hline Barium & $1.18 \mathrm{E}-2$ & 8.79E-3 & $"$ & & $1.57 \mathrm{E}-2$ & & & 28.3 & 35 \\
\hline Calcium & 2.30E1 & 3.87E-1 & $"$ & & $1.92 \mathrm{E} 1$ & & & 17.8 & 35 \\
\hline Iron & $<1.42 \mathrm{E}-1$ & $1.42 \mathrm{E}-1$ & $"$ & & ND & & & & 35 \\
\hline Potassium & 4.45E0 & 2.33E0 & $"$ & & 4.30E0 & & & 3.55 & 35 \\
\hline Magnesium & 5.91E0 & 8.34E-2 & $"$ & & 4.99E0 & & & 16.9 & 35 \\
\hline Manganese & $<1.71 \mathrm{E}-2$ & $1.71 \mathrm{E}-2$ & $"$ & & ND & & & & 35 \\
\hline Nickel & $<9.33 \mathrm{E}-2$ & $9.33 \mathrm{E}-2$ & $"$ & & ND & & & & 35 \\
\hline Thallium & $<1.03 \mathrm{E} 0$ & 1.03E0 & $"$ & & ND & & & & 35 \\
\hline Sodium & $1.11 \mathrm{E} 2$ & 6.69E-1 & $"$ & & $1.11 \mathrm{E} 2$ & & & 0.0347 & 35 \\
\hline Post Spike (8I23005-PS1) & \multicolumn{3}{|c|}{ Source: 0804025-50 } & \multicolumn{2}{|c|}{ Prepared: 09/23/08 } & \multicolumn{2}{|c|}{ Analyzed: 09/24/08 } & & \\
\hline Aluminum & $5.26 \mathrm{E} 2$ & N/A & $\mathrm{ug} / \mathrm{L}$ & $5.00 \mathrm{E} 2$ & $2.45 \mathrm{E} 1$ & 100 & $75-125$ & & \\
\hline Barium & 2.43E2 & N/A & $"$ & $2.50 \mathrm{E} 2$ & $5.24 \mathrm{E} 0$ & 95.2 & $75-125$ & & \\
\hline Calcium & $6.88 \mathrm{E} 3$ & N/A & $"$ & $5.00 \mathrm{E} 2$ & 6.39E3 & 96.7 & $75-125$ & & \\
\hline Iron & 5.11E2 & N/A & $"$ & $5.00 \mathrm{E} 2$ & $6.10 \mathrm{E} 0$ & 101 & $75-125$ & & \\
\hline Potassium & $2.66 \mathrm{E} 3$ & N/A & $"$ & $1.25 \mathrm{E} 3$ & 1.43E3 & 98.7 & $75-125$ & & \\
\hline Magnesium & $2.18 \mathrm{E} 3$ & N/A & $"$ & $5.00 \mathrm{E} 2$ & $1.66 \mathrm{E} 3$ & 103 & $75-125$ & & \\
\hline Manganese & $2.50 \mathrm{E} 2$ & N/A & $"$ & $2.50 \mathrm{E} 2$ & ND & 102 & $75-125$ & & \\
\hline Nickel & 4.95E2 & N/A & " & $5.00 \mathrm{E} 2$ & ND & 99.1 & $75-125$ & & \\
\hline Thallium & $4.66 \mathrm{E} 2$ & N/A & $"$ & $5.00 \mathrm{E} 2$ & ND & 101 & $75-125$ & & \\
\hline Sodium & $3.74 \mathrm{E} 4$ & N/A & $"$ & $5.00 \mathrm{E} 2$ & 3.69E4 & 107 & $75-125$ & & \\
\hline
\end{tabular}


Total Metals by PNNL-AGG-ICP-AES/Acid Extract - Quality Control

Environmental Science Laboratory

\begin{tabular}{|c|c|c|c|c|c|c|c|c|c|c|}
\hline Analyte & Result & $\begin{array}{r}\text { orting } \\
\text { Limit }\end{array}$ & Units & $\begin{array}{l}\text { Spike } \\
\text { Level }\end{array}$ & $\begin{array}{l}\text { Source } \\
\text { Result }\end{array}$ & \%REC & $\begin{array}{l}\text { \%REC } \\
\text { Limits }\end{array}$ & RPD & $\begin{array}{l}\text { RPD } \\
\text { Limit }\end{array}$ & Notes \\
\hline
\end{tabular}

Batch $8 I 23006$ - ASTM D 5198 (ICP/ICPMS)

Blank (8I23006-BLK1)

Aluminum

Barium

Calcium

Iron

Potassium

Magnesium

Manganese

Nickel

Thallium

Sodium

LCS (8I23006-BS1)

Aluminum

Barium

Calcium

Iron

Potassium

Magnesium

Manganese

Nickel

Thallium

Sodium

Duplicate (8I23006-DUP1)

Aluminum

Barium

Calcium

Iron

Potassium

Magnesium

Manganese

Nickel

Thallium

Sodium

23006-BS1)

$\begin{array}{lrc} & & \\ <2.30 \mathrm{E} 0 & 2.30 \mathrm{E} 0 & \text { ug/g wet } \\ <2.19 \mathrm{E}-1 & 2.19 \mathrm{E}-1 & " \\ <8.56 \mathrm{E} 0 & 8.56 \mathrm{E} 0 & " \\ <5.85 \mathrm{E} 0 & 5.85 \mathrm{E} 0 & " \\ <5.43 \mathrm{E} 0 & 5.43 \mathrm{E} 0 & " \\ <1.81 \mathrm{E} 0 & 1.81 \mathrm{E} 0 & " \\ <6.86 \mathrm{E}-2 & 6.86 \mathrm{E}-2 & " \\ <4.71 \mathrm{E}-1 & 4.71 \mathrm{E}-1 & " \\ <2.59 \mathrm{E} 0 & 2.59 \mathrm{E} 0 & " \\ <6.14 \mathrm{E} 1 & 6.14 \mathrm{E} 1 & "\end{array}$

Prepared: 08/26/08 Analyzed: 09/24/08

Prepared: 08/26/08 Analyzed: 09/24/08

$\begin{array}{lccccc}5.36 \mathrm{E} 0 & 1.30 \mathrm{E}-1 & \text { ug/g wet } & 5.71 \mathrm{E} 0 & 93.8 & 80-120 \\ 5.77 \mathrm{E} 0 & 1.24 \mathrm{E}-2 & " & 5.71 \mathrm{E} 0 & 101 & 80-120 \\ 5.55 \mathrm{E} 0 & 4.83 \mathrm{E}-1 & " & 5.71 \mathrm{E} 0 & 97.1 & 80-120 \\ 5.70 \mathrm{E} 0 & 3.30 \mathrm{E}-1 & " & 5.71 \mathrm{E} 0 & 99.7 & 80-120 \\ 5.66 \mathrm{E} 1 & 3.06 \mathrm{E}-1 & " & 5.71 \mathrm{E} 1 & 99.0 & 80-120 \\ 5.29 \mathrm{E} 0 & 1.02 \mathrm{E}-1 & " & 5.71 \mathrm{E} 0 & 92.5 & 80-120 \\ 5.66 \mathrm{E} 0 & 3.87 \mathrm{E}-3 & " & 5.71 \mathrm{E} 0 & 99.1 & 80-120 \\ 5.58 \mathrm{E} 0 & 2.66 \mathrm{E}-2 & " & 5.71 \mathrm{E} 0 & 97.6 & 80-120 \\ 5.48 \mathrm{E} 0 & 1.46 \mathrm{E}-1 & " & 5.71 \mathrm{E} 0 & 95.9 & 80-120 \\ 6.03 \mathrm{E} 0 & 3.46 \mathrm{E} 0 & " & 5.71 \mathrm{E} 0 & 105 & 80-120\end{array}$

Source: 0804025-51 Prepared: 08/26/08 Analyzed: 09/24/08

$\begin{array}{rrrcrr}5.87 \mathrm{E} 3 & 3.66 \mathrm{E} 1 & \text { ug/g dry } & 5.57 \mathrm{E} 3 & 5.31 & 35 \\ 4.45 \mathrm{E} 1 & 8.72 \mathrm{E}-1 & " & 4.33 \mathrm{E} 1 & 2.68 & 35 \\ 7.21 \mathrm{E} 3 & 3.40 \mathrm{E} 1 & " & 7.11 \mathrm{E} 3 & 1.40 & 35 \\ 1.15 \mathrm{E} 4 & 9.30 \mathrm{E} 1 & " & 1.10 \mathrm{E} 4 & 3.90 & 35 \\ 1.43 \mathrm{E} 3 & 2.16 \mathrm{E} 1 & " & 1.43 \mathrm{E} 3 & 0.0202 & 35 \\ 4.03 \mathrm{E} 3 & 2.88 \mathrm{E} 1 & " & 3.84 \mathrm{E} 3 & 4.98 & 35 \\ 2.39 \mathrm{E} 2 & 2.72 \mathrm{E}-1 & " & 2.46 \mathrm{E} 2 & 3.10 & 35 \\ 1.24 \mathrm{E} 1 & 1.87 \mathrm{E} 0 & " & 1.26 \mathrm{E} 1 & 1.04 & 35 \\ <1.03 \mathrm{E} 1 & 1.03 \mathrm{E} 1 & " & \text { ND } & & 35 \\ 1.77 \mathrm{E} 3 & 2.44 \mathrm{E} 2 & " & 1.75 \mathrm{E} 3 & 0.979 & 35\end{array}$


Total Metals by PNNL-AGG-ICP-AES/Acid Extract - Quality Control

Environmental Science Laboratory

\begin{tabular}{|c|c|c|c|c|c|c|c|c|c|c|}
\hline Analyte & Result & $\begin{array}{r}\text { orting } \\
\text { Limit }\end{array}$ & Units & $\begin{array}{l}\text { Spike } \\
\text { Level }\end{array}$ & $\begin{array}{l}\text { Source } \\
\text { Result }\end{array}$ & \%REC & $\begin{array}{l}\text { \%REC } \\
\text { Limits }\end{array}$ & RPD & $\begin{array}{l}\text { RPD } \\
\text { Limit }\end{array}$ & Notes \\
\hline
\end{tabular}

Batch 8123007 - ASTM D 5198 (ICP/ICPMS)

\begin{tabular}{|c|c|c|c|c|c|c|c|c|c|}
\hline \multirow{2}{*}{$\begin{array}{l}\text { Duplicate (8I23007-DUP1) } \\
\text { Aluminum }\end{array}$} & \multicolumn{3}{|c|}{ Source: 0804025-69 } & \multicolumn{4}{|c|}{ Prepared: 08/27/08 Analyzed: 09/24/08 } & \multirow[b]{2}{*}{1.80} & \multirow[b]{2}{*}{35} \\
\hline & $5.25 \mathrm{E} 3$ & $3.49 \mathrm{E} 1$ & ug/g dry & & $5.34 \mathrm{E} 3$ & & & & \\
\hline Barium & $5.14 \mathrm{E} 1$ & 8.31E-1 & $"$ & & 4.80E1 & & & 6.72 & 35 \\
\hline Calcium & $7.46 \mathrm{E} 3$ & $3.24 \mathrm{E} 1$ & $"$ & & 7.39E3 & & & 0.842 & 35 \\
\hline Iron & $1.06 \mathrm{E} 4$ & 8.87E1 & $"$ & & $1.11 \mathrm{E} 4$ & & & 4.63 & 35 \\
\hline Potassium & $1.22 \mathrm{E} 3$ & $2.06 \mathrm{E} 1$ & $"$ & & $1.21 \mathrm{E} 3$ & & & 0.994 & 35 \\
\hline Magnesium & $3.96 \mathrm{E} 3$ & $2.74 \mathrm{E} 1$ & $"$ & & 4.01E3 & & & 1.31 & 35 \\
\hline Manganese & 2.32E2 & $2.60 \mathrm{E}-1$ & $"$ & & 2.27E2 & & & 2.35 & 35 \\
\hline Nickel & $1.09 \mathrm{E} 1$ & $1.78 \mathrm{E} 0$ & $"$ & & $1.23 \mathrm{E} 1$ & & & 12.6 & 35 \\
\hline Thallium & $<9.80 \mathrm{E} 0$ & $9.80 \mathrm{E} 0$ & $"$ & & ND & & & & 35 \\
\hline Sodium & 1.77E3 & 2.32E2 & $"$ & & $1.65 \mathrm{E} 3$ & & & 6.55 & 35 \\
\hline Post Spike (8I23007-PS1) & \multicolumn{3}{|c|}{ Source: 0804025-69 } & \multicolumn{2}{|c|}{ Prepared: 09/23/08 } & \multicolumn{2}{|c|}{ Analyzed: 09/24/08 } & & \\
\hline Aluminum & $1.56 \mathrm{E} 4$ & N/A & ug/L & 1.25E2 & $1.53 \mathrm{E} 4$ & 262 & $75-125$ & & \\
\hline Barium & 7.77E2 & N/A & $"$ & 2.50E2 & 5.49E2 & 91.3 & $75-125$ & & \\
\hline Calcium & $8.26 \mathrm{E} 4$ & N/A & $"$ & $5.00 \mathrm{E} 2$ & 8.44E4 & NR & $75-125$ & & \\
\hline Iron & $3.20 \mathrm{E} 4$ & N/A & $"$ & 1.25E2 & $3.18 \mathrm{E} 4$ & 185 & $75-125$ & & \\
\hline Potassium & $1.49 \mathrm{E} 4$ & N/A & $"$ & $1.25 \mathrm{E} 3$ & $1.38 \mathrm{E} 4$ & 86 & $75-125$ & & \\
\hline Magnesium & $1.19 \mathrm{E} 4$ & N/A & $"$ & $1.25 \mathrm{E} 2$ & $1.14 \mathrm{E} 4$ & 329 & $75-125$ & & \\
\hline Manganese & 2.82E3 & N/A & $"$ & $2.50 \mathrm{E} 2$ & $2.59 \mathrm{E} 3$ & 92.9 & $75-125$ & & \\
\hline Nickel & $6.09 \mathrm{E} 2$ & N/A & $"$ & $5.00 \mathrm{E} 2$ & $1.41 \mathrm{E} 2$ & 93.7 & $75-125$ & & \\
\hline Thallium & 7.61E1 & N/A & $"$ & $5.00 \mathrm{E} 2$ & ND & 98.9 & $75-125$ & & \\
\hline Sodium & $1.89 \mathrm{E} 4$ & N/A & $"$ & $5.00 \mathrm{E} 2$ & $1.89 \mathrm{E} 4$ & NR & $75-125$ & & \\
\hline
\end{tabular}


Radionuclides by ICP-MS/Acid Extract - Quality Control

Environmental Science Laboratory

\begin{tabular}{|lrrrrrrrrrrr}
\hline & & Reporting & & Spike & Source & & \%REC & & RPD & & \\
Analyte & Result & Limit & Units & Level & Result & \%REC & Limits & RPD & Limit & Notes \\
\hline
\end{tabular}

Batch 8I22001 - ASTM D 5198 (ICP/ICPMS)

Blank (8I22001-BLK1)

Technetium-99

Uranium 238

$\begin{array}{ccc}<1.11 \mathrm{E}-3 & 1.11 \mathrm{E}-3 & \mathrm{ug} / \mathrm{g} \text { wet } \\ <8.06 \mathrm{E}-3 & 8.06 \mathrm{E}-3 & "\end{array}$

Prepared \& Analyzed: 09/22/08

Duplicate (8I22001-DUP1)

$<8.06 \mathrm{E}-3 \quad 8.06 \mathrm{E}-3$

Technetium-99

Source: 0804025-51

Prepared \& Analyzed: 09/22/08

$\begin{array}{llclll}7.03 \mathrm{E}-3 & 4.41 \mathrm{E}-3 & \text { ug/g dry } & 6.96 \mathrm{E}-3 & 1.00 & 35 \\ 3.71 \mathrm{E}-1 & 3.20 \mathrm{E}-2 & " & 3.63 \mathrm{E}-1 & 2.32 & 35\end{array}$

Uranium 238

Source: 0804025-51

Prepared \& Analyzed: 09/22/08

Post Spike (8I22001-PS1)

Technetium-99

5.45E-1

N/A ug/L

5.00E-1 1.88E-2 $105 \quad 75-125$

Uranium 238

$1.42 \mathrm{E} 0$

N/A "

$5.00 \mathrm{E}-1$

9.79E-1

$88.6 \quad 75-125$

Batch 8I22002 - ASTM D 5198 (ICP/ICPMS)

\begin{tabular}{|c|c|c|c|c|c|c|c|c|c|}
\hline \multirow{2}{*}{$\frac{\text { Blank (8I22002-BLK1) }}{\text { Technetium-99 }}$} & \multicolumn{9}{|c|}{ Prepared \& Analyzed: 09/22/08 } \\
\hline & $<1.14 \mathrm{E}-3$ & $1.14 \mathrm{E}-3$ & ug/g wet & & & & & & \\
\hline Uranium 238 & $<8.30 \mathrm{E}-3$ & $8.30 \mathrm{E}-3$ & $"$ & & & & & & \\
\hline Duplicate (8I22002-DUP1) & \multicolumn{3}{|c|}{ Source: 0804025-69 } & \multicolumn{6}{|c|}{ Prepared \& Analyzed: 09/22/08 } \\
\hline Technetium-99 & $1.29 \mathrm{E}-2$ & $4.20 \mathrm{E}-3$ & ug/g dry & & $1.23 \mathrm{E}-2$ & & & 4.51 & 35 \\
\hline Uranium 238 & 4.07E-1 & $3.05 E-2$ & $"$ & & 3.97E-1 & & & 2.35 & 35 \\
\hline Post Spike (8I22002-PS1) & \multicolumn{3}{|c|}{ Source: 0804025-69 } & \multicolumn{4}{|c|}{ Prepared \& Analyzed: 09/22/08 } & & \\
\hline Technetium-99 & $5.61 \mathrm{E}-1$ & N/A & $\mathrm{ug} / \mathrm{L}$ & $5.00 \mathrm{E}-1$ & $3.51 \mathrm{E}-2$ & 105 & $75-125$ & & \\
\hline Uranium 238 & $1.58 \mathrm{E} 0$ & N/A & $"$ & $5.00 \mathrm{E}-1$ & 1.13E0 & 88.8 & $75-125$ & & \\
\hline
\end{tabular}




\section{Radionuclides by ICP-MS/Water Extract - Quality Control \\ Environmental Science Laboratory}

\begin{tabular}{|c|c|c|c|c|c|c|c|c|c|c|}
\hline Analyte & Result & $\begin{array}{r}\text { orting } \\
\text { Limit }\end{array}$ & Units & $\begin{array}{l}\text { Spike } \\
\text { Level }\end{array}$ & $\begin{array}{l}\text { Source } \\
\text { Result }\end{array}$ & \%REC & $\begin{array}{l}\text { \%REC } \\
\text { Limits }\end{array}$ & RPD & $\begin{array}{l}\text { RPD } \\
\text { Limit }\end{array}$ & Notes \\
\hline
\end{tabular}

Batch 8G15001 - 1:1 Water Extract (ICP/ICPMS)

Blank (8G15001-BLK1)

Technetium-99

Uranium 238

Duplicate (8G15001-DUP1)

Technetium-99

Uranium 238

Post Spike (8G15001-PS1)

Technetium-99

Uranium 238

Batch 8G15002 - 1:1 Water Extract (ICP/ICPMS)

\section{Blank (8G15002-BLK1)}

Technetium-99

Uranium 238

Duplicate (8G15002-DUP1)

Technetium-99

Uranium 238

Post Spike (8G15002-PS1)

Technetium-99

Uranium 238

Batch 8I09003 - 1:1 Water Extract (ICP/ICPMS)

Blank (8I09003-BLK1)

Technetium-99

Uranium 238

$\begin{array}{llc}<2.30 \mathrm{E}-5 & 2.30 \mathrm{E}-5 & \text { ug/g wet } \\ <5.64 \mathrm{E}-4 & 5.64 \mathrm{E}-4 & "\end{array}$

$\begin{array}{llc}<2.30 \mathrm{E}-5 & 2.30 \mathrm{E}-5 & \text { ug } / \mathrm{g} \text { wet } \\ <5.64 \mathrm{E}-4 & 5.64 \mathrm{E}-4 & "\end{array}$

Source: 0804025-51

Source: 0804025-51

政

$<2.30 \mathrm{E}-5 \quad 2.30 \mathrm{E}-5 \quad \mathrm{ug} / \mathrm{g}$ wet

$<5.64 \mathrm{E}-4 \quad 5.64 \mathrm{E}-4 \quad "$

Prepared \& Analyzed: 07/15/08

Prepared \& Analyzed: 07/15/08

$\begin{array}{lccccc}6.25 \mathrm{E}-3 & 2.39 \mathrm{E}-5 & \text { ug/g dry } & 6.21 \mathrm{E}-3 & 0.642 & 20 \\ 1.53 \mathrm{E}-3 & 5.85 \mathrm{E}-4 & " & 1.51 \mathrm{E}-3 & 1.52 & 20\end{array}$

Prepared \& Analyzed: 07/15/08

$\begin{array}{lllllll}1.82 \mathrm{E} 0 & \mathrm{~N} / \mathrm{A} & \mathrm{ug} / \mathrm{L} & 5.00 \mathrm{E}-1 & 1.24 \mathrm{E} 0 & 116 & 75-125\end{array}$

\begin{tabular}{|c|c|}
\hline $8.46 \mathrm{E}-1$ & \\
\hline
\end{tabular}

Prepared \& Analyzed: 07/15/08

\begin{tabular}{lccccc} 
Source: & $\mathbf{0 8 0 4 0 2 5 - 6 9}$ & Prepared \& Analyzed: 07/15/08 \\
\hline $1.05 \mathrm{E}-2$ & $2.30 \mathrm{E}-5$ & $\mathrm{ug} / \mathrm{g}$ dry & $1.06 \mathrm{E}-2$ & 0.501 & 20 \\
$1.40 \mathrm{E}-3$ & $5.63 \mathrm{E}-4$ & $"$ & $1.53 \mathrm{E}-3$ & 8.82 & 20
\end{tabular}

\begin{tabular}{ccccccc}
\multicolumn{3}{c}{ Source: $0804025-69$} & \multicolumn{3}{c}{ Prepared \& Analyzed: $07 / 15 / 08$} \\
\hline $2.69 \mathrm{E} 0$ & N/A & ug/L & $5.00 \mathrm{E}-1$ & $2.15 \mathrm{E} 0$ & 108 & $75-125$ \\
$8.77 \mathrm{E}-1$ & N/A & $"$ & $5.00 \mathrm{E}-1$ & $3.10 \mathrm{E}-1$ & 113 & $75-125$
\end{tabular}

Prepared \& Analyzed: 09/09/08 


\section{Radionuclides by ICP-MS/Water Extract - Quality Control \\ Environmental Science Laboratory}

\begin{tabular}{|lrrrrrrrrrrr}
\hline & & Reporting & & Spike & Source & & & \%REC & & RPD & \\
Analyte & Result & Limit & Units & Level & Result & \%REC & Limits & RPD & Limit & Notes \\
\hline
\end{tabular}

Batch 8I09003 - 1:1 Water Extract (ICP/ICPMS)

Duplicate (8I09003-DUP1)

Technetium-99

Uranium 238

Post Spike (8I09003-PS1)

Technetium-99

Uranium 238

Batch 8I16004 - 1:1 Water Extract (ICP/ICPMS)

Blank (8I16004-BLK1)

Iodine-129

Duplicate (8I16004-DUP1)

Iodine-129

Post Spike (8I16004-PS1)

Iodine-129

\begin{tabular}{|c|c|c|c|c|c|c|}
\hline \multicolumn{3}{|c|}{ Source: 0804025-50 } & \multicolumn{4}{|c|}{ Prepared \& Analyzed: 09/09/08 } \\
\hline $2.84 \mathrm{E}-4$ & $2.30 \mathrm{E}-5$ & ug/g dry & & $3.02 \mathrm{E}-4$ & & \\
\hline $7.25 \mathrm{E}-4$ & $5.64 \mathrm{E}-4$ & " & & $7.26 \mathrm{E}-4$ & & \\
\hline \multicolumn{3}{|c|}{ Source: 0804025-50 } & \multicolumn{4}{|c|}{ Prepared \& Analyzed: 09/09/08 } \\
\hline 5.58E-1 & N/A & ug/L & $5.00 \mathrm{E}-1$ & $6.04 \mathrm{E}-2$ & 99.5 & $75-125$ \\
\hline 6.55E-1 & N/A & " & $5.00 \mathrm{E}-1$ & $1.45 \mathrm{E}-1$ & 102 & $75-125$ \\
\hline
\end{tabular}

Prepared \& Analyzed: 09/22/08

$<3.78 \mathrm{E}-4 \quad 3.78 \mathrm{E}-4 \quad \mathrm{ug} / \mathrm{g}$ wet

Source: 0804025-50 Prepared \& Analyzed: 09/19/08

$<3.78 \mathrm{E}-4 \quad 3.78 \mathrm{E}-4 \quad \mathrm{ug} / \mathrm{g}$ dry $\quad$ ND

35

Source: 0804025-50 Prepared \& Analyzed: 09/19/08

$\begin{array}{lllllll}5.03 \mathrm{E}-1 & \text { N/A } & \text { ug/L } & 5.00 \mathrm{E}-1 & 1.05 \mathrm{E}-2 & 98.5 & 75-125\end{array}$

Batch 8I16005 - 1:1 Water Extract (ICP/ICPMS)

Blank (8I16005-BLK1)

Prepared \& Analyzed: 09/19/08

Iodine-129

$<3.78 \mathrm{E}-4 \quad 3.78 \mathrm{E}-4 \quad \mathrm{ug} / \mathrm{g}$ wet

Duplicate (8I16005-DUP1)

Iodine-129

Source: 0804025-51

Prepared \& Analyzed: 09/22/08

$<3.92 \mathrm{E}-4 \quad 3.92 \mathrm{E}-4 \quad \mathrm{ug} / \mathrm{g}$ dry

ND 


\section{Radionuclides by ICP-MS/Water Extract - Quality Control \\ Environmental Science Laboratory}

\begin{tabular}{|lrrrrrrrrrrr}
\hline & & Reporting & & Spike & Source & & \%REC & & RPD & & \\
Analyte & Result & Limit & Units & Level & Result & \%REC & Limits & RPD & Limit & Notes \\
\hline
\end{tabular}

Batch 8I16005 - 1:1 Water Extract (ICP/ICPMS)

Post Spike (8I16005-PS1)

Source: 0804025-51 Prepared \& Analyzed: 09/22/08

\begin{tabular}{lllllll}
\hline Iodine-129 & $4.52 \mathrm{E}-1$ & $\mathrm{~N} / \mathrm{A}$ ug/L & $5.00 \mathrm{E}-1$ & $2.20 \mathrm{E}-2$ & 90 & $75-125$
\end{tabular}

Batch 8I16006 - 1:1 Water Extract (ICP/ICPMS)

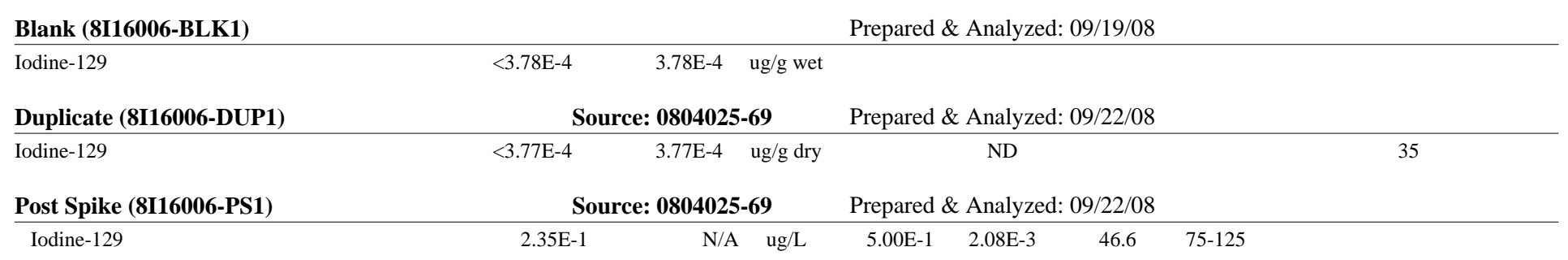


RCRA Metals By PNNL-AGG-415/Water Extract - Quality Control

Environmental Science Laboratory

\begin{tabular}{|c|c|c|c|c|c|c|c|c|c|c|}
\hline Analyte & Result & $\begin{array}{r}\text { orting } \\
\text { Limit }\end{array}$ & Units & $\begin{array}{l}\text { Spike } \\
\text { Level }\end{array}$ & $\begin{array}{l}\text { Source } \\
\text { Result }\end{array}$ & \%REC & $\begin{array}{l}\text { \%REC } \\
\text { Limits }\end{array}$ & RPD & $\begin{array}{l}\text { RPD } \\
\text { Limit }\end{array}$ & Notes \\
\hline
\end{tabular}

Batch 8G10003 - 1:1 Water Extract (ICP/ICPMS)

\begin{tabular}{|c|c|c|c|c|c|c|c|c|}
\hline \multirow{2}{*}{$\begin{array}{l}\text { Blank (8G10003-BLK1) } \\
\text { Chromium }\end{array}$} & \multicolumn{8}{|c|}{ Prepared \& Analyzed: 07/10/08 } \\
\hline & $<6.40 \mathrm{E}-3$ & $6.40 \mathrm{E}-3$ & ug/g wet & & & & & \\
\hline Copper & $<3.48 \mathrm{E}-3$ & $3.48 E-3$ & $"$ & & & & & \\
\hline Arsenic & $<6.25 \mathrm{E}-3$ & $6.25 \mathrm{E}-3$ & $"$ & & & & & \\
\hline Selenium & $<1.10 \mathrm{E}-2$ & $1.10 \mathrm{E}-2$ & $"$ & & & & & \\
\hline Silver & $<9.25 \mathrm{E}-4$ & $9.25 \mathrm{E}-4$ & $"$ & & & & & \\
\hline Cadmium & $<2.95 \mathrm{E}-4$ & $2.95 \mathrm{E}-4$ & $"$ & & & & & \\
\hline Antimony & $<5.40 \mathrm{E}-4$ & $5.40 \mathrm{E}-4$ & $"$ & & & & & \\
\hline Lead & $<5.60 \mathrm{E}-4$ & $5.60 \mathrm{E}-4$ & $"$ & & & & & \\
\hline LCS (8G10003-BS1) & \multicolumn{8}{|c|}{ Prepared \& Analyzed: 07/10/08 } \\
\hline Chromium & 5.09E0 & $3.20 \mathrm{E}-1$ & ug/g wet & $5.00 \mathrm{E} 0$ & 102 & $80-120$ & & \\
\hline Copper & 4.88E0 & $1.74 \mathrm{E}-1$ & $"$ & 5.00E0 & 97.6 & $80-120$ & & \\
\hline Arsenic & $5.05 \mathrm{E} 0$ & $3.12 \mathrm{E}-1$ & $"$ & $5.00 \mathrm{E} 0$ & 101 & $80-120$ & & \\
\hline Selenium & 4.93E0 & $5.52 \mathrm{E}-1$ & $"$ & $5.00 \mathrm{E} 0$ & 98.5 & $80-120$ & & \\
\hline Silver & 4.94E0 & 4.62E-2 & $"$ & $5.00 \mathrm{E} 0$ & 98.8 & $80-120$ & & \\
\hline Cadmium & $4.92 \mathrm{E} 0$ & $1.48 \mathrm{E}-2$ & $"$ & $5.00 \mathrm{E} 0$ & 98.5 & $80-120$ & & \\
\hline Lead & 4.70E0 & $2.80 \mathrm{E}-2$ & $"$ & $5.00 \mathrm{E} 0$ & 94.0 & $80-120$ & & \\
\hline Duplicate (8G10003-DUP1) & \multicolumn{3}{|c|}{ Source: 0804025-51 } & \multicolumn{5}{|c|}{ Prepared \& Analyzed: 07/10/08 } \\
\hline Chromium & $<6.65 \mathrm{E}-3$ & $6.65 \mathrm{E}-3$ & ug/g dry & & & & & 20 \\
\hline Copper & $1.28 \mathrm{E}-2$ & $3.61 \mathrm{E}-3$ & $"$ & & & & 14.0 & 20 \\
\hline Arsenic & $<6.49 \mathrm{E}-3$ & $6.49 \mathrm{E}-3$ & $"$ & & & & & 20 \\
\hline Selenium & $<1.15 \mathrm{E}-2$ & $1.15 \mathrm{E}-2$ & $"$ & & & & & 20 \\
\hline Silver & $<9.61 \mathrm{E}-4$ & $9.61 \mathrm{E}-4$ & $"$ & & & & & 20 \\
\hline Cadmium & $<3.06 \mathrm{E}-4$ & $3.06 \mathrm{E}-4$ & $"$ & & & & & 20 \\
\hline Antimony & $<5.61 \mathrm{E}-4$ & $5.61 \mathrm{E}-4$ & " & & & & & 20 \\
\hline Lead & $<5.82 \mathrm{E}-4$ & $5.82 \mathrm{E}-4$ & $"$ & & & & & 20 \\
\hline
\end{tabular}


RCRA Metals By PNNL-AGG-415/Water Extract - Quality Control

Environmental Science Laboratory

\begin{tabular}{|c|c|c|c|c|c|c|c|c|c|c|}
\hline Analyte & Result & $\begin{array}{r}\text { orting } \\
\text { Limit }\end{array}$ & Units & $\begin{array}{l}\text { Spike } \\
\text { Level }\end{array}$ & $\begin{array}{l}\text { Source } \\
\text { Result }\end{array}$ & \%REC & $\begin{array}{l}\text { \%REC } \\
\text { Limits }\end{array}$ & RPD & $\begin{array}{l}\text { RPD } \\
\text { Limit }\end{array}$ & Notes \\
\hline
\end{tabular}

Batch 8G10003 - 1:1 Water Extract (ICP/ICPMS)

\begin{tabular}{|c|c|c|c|c|c|c|c|}
\hline \multirow{2}{*}{$\begin{array}{l}\text { Post Spike (8G10003-PS1) } \\
\text { Chromium }\end{array}$} & \multicolumn{3}{|c|}{ Source: 0804025-51 } & \multicolumn{4}{|c|}{ Prepared \& Analyzed: 07/10/08 } \\
\hline & $6.25 \mathrm{E} 0$ & N/A & ug/L & $5.00 \mathrm{E} 0$ & 1.16E0 & 102 & $75-125$ \\
\hline Arsenic & $6.24 \mathrm{E} 0$ & N/A & $"$ & $5.00 \mathrm{E} 0$ & 1.27E0 & 99.4 & $75-125$ \\
\hline Selenium & 6.61E0 & N/A & $"$ & $5.00 \mathrm{E} 0$ & 1.73E0 & 97.5 & $75-125$ \\
\hline Silver & 4.57E0 & N/A & $"$ & $5.00 \mathrm{E} 0$ & $1.67 \mathrm{E}-1$ & 88 & $75-125$ \\
\hline Cadmium & 4.86E0 & N/A & $"$ & $5.00 \mathrm{E} 0$ & 4.23E-2 & 96.4 & $75-125$ \\
\hline Antimony & $5.11 \mathrm{E} 0$ & N/A & $"$ & $5.00 \mathrm{E} 0$ & $4.88 \mathrm{E}-2$ & 101 & $75-125$ \\
\hline Lead & 5.19E0 & N/A & $"$ & $5.00 \mathrm{E} 0$ & $2.30 \mathrm{E}-2$ & 103 & $75-125$ \\
\hline
\end{tabular}

Batch 8G10004 - 1:1 Water Extract (ICP/ICPMS)

\begin{tabular}{|c|c|c|c|c|c|c|}
\hline Blank (8G10004-BLK1) & \multicolumn{6}{|c|}{ Prepared \& Analyzed: 07/10/08 } \\
\hline Chromium & $<6.40 \mathrm{E}-3$ & $6.40 \mathrm{E}-3$ & ug/g wet & & & \\
\hline Copper & $<3.48 \mathrm{E}-3$ & $3.48 \mathrm{E}-3$ & $"$ & & & \\
\hline Arsenic & $<6.25 \mathrm{E}-3$ & $6.25 \mathrm{E}-3$ & $"$ & & & \\
\hline Selenium & $<1.10 \mathrm{E}-2$ & $1.10 \mathrm{E}-2$ & $"$ & & & \\
\hline Silver & $<9.25 \mathrm{E}-4$ & $9.25 \mathrm{E}-4$ & $"$ & & & \\
\hline Cadmium & $<2.95 \mathrm{E}-4$ & $2.95 \mathrm{E}-4$ & $"$ & & & \\
\hline Antimony & $<5.40 \mathrm{E}-4$ & $5.40 \mathrm{E}-4$ & $"$ & & & \\
\hline Lead & $<5.60 \mathrm{E}-4$ & $5.60 \mathrm{E}-4$ & $"$ & & & \\
\hline LCS (8G10004-BS1) & \multicolumn{6}{|c|}{ Prepared \& Analyzed: 07/10/08 } \\
\hline Chromium & 5.11E0 & $3.20 \mathrm{E}-1$ & ug/g wet & $5.00 \mathrm{E} 0$ & 102 & $80-120$ \\
\hline Copper & 4.78E0 & $1.74 \mathrm{E}-1$ & $"$ & $5.00 \mathrm{E} 0$ & 95.6 & $80-120$ \\
\hline Arsenic & 4.94E0 & $3.12 \mathrm{E}-1$ & $"$ & $5.00 \mathrm{E} 0$ & 98.9 & $80-120$ \\
\hline Selenium & 4.76E0 & $5.52 \mathrm{E}-1$ & $"$ & $5.00 \mathrm{E} 0$ & 95.3 & $80-120$ \\
\hline Silver & 4.87E0 & 4.62E-2 & $"$ & 5.00E0 & 97.5 & $80-120$ \\
\hline Cadmium & 4.89E0 & $1.48 \mathrm{E}-2$ & $"$ & $5.00 \mathrm{E} 0$ & 97.8 & $80-120$ \\
\hline Lead & 4.75E0 & $2.80 \mathrm{E}-2$ & $"$ & $5.00 \mathrm{E} 0$ & 95.1 & $80-120$ \\
\hline
\end{tabular}


RCRA Metals By PNNL-AGG-415/Water Extract - Quality Control

Environmental Science Laboratory

\begin{tabular}{|c|c|c|c|c|c|c|c|c|c|c|}
\hline Analyte & Result & $\begin{array}{r}\text { orting } \\
\text { Limit }\end{array}$ & Units & $\begin{array}{l}\text { Spike } \\
\text { Level }\end{array}$ & $\begin{array}{l}\text { Source } \\
\text { Result }\end{array}$ & \%REC & $\begin{array}{l}\text { \%REC } \\
\text { Limits }\end{array}$ & RPD & $\begin{array}{l}\text { RPD } \\
\text { Limit }\end{array}$ & Notes \\
\hline
\end{tabular}

Batch 8G10004 - 1:1 Water Extract (ICP/ICPMS)

Duplicate (8G10004-DUP1)

Chromium

Copper

Arsenic

Selenium

Silver

Cadmium

Antimony

Lead

Post Spike (8G10004-PS1)

Chromium

Arsenic

Selenium

Silver

Cadmium

Antimony

Lead
Source: 0804025-69

$<6.40 \mathrm{E}-3 \quad 6.40 \mathrm{E}-3 \quad$ ug/g dry

$1.46 \mathrm{E}-2$

$<6.25 \mathrm{E}-3$

$1.31 \mathrm{E}-2$

$<9.25 \mathrm{E}-4$

$<2.95 \mathrm{E}-4$

$<5.40 \mathrm{E}-4$

$<5.60 \mathrm{E}-4$

$3.48 \mathrm{E}-3 \quad "$

6.25E-3 "

$1.10 \mathrm{E}-2 \quad "$

$9.25 \mathrm{E}-4 \quad "$

$2.95 \mathrm{E}-4 \quad "$

$5.40 \mathrm{E}-4$

$5.60 \mathrm{E}-4 \quad "$
Prepared \& Analyzed: 07/10/08

$\begin{array}{ccc}\text { ND } & & 20 \\ 1.46 \mathrm{E}-2 & 0.639 & 20 \\ \text { ND } & & 20 \\ 1.24 \mathrm{E}-2 & 5.75 & 20 \\ \text { ND } & & 20 \\ \text { ND } & & 20 \\ \text { ND } & & 20 \\ \text { ND } & 20\end{array}$

Batch 8I11003 - 1:1 Water Extract (ICP/ICPMS)

\begin{tabular}{lllc}
\hline Blank (8I11003-BLK1) & & & \\
\hline Chromium & $<6.40 \mathrm{E}-3$ & $6.40 \mathrm{E}-3$ & ug/g wet \\
Copper & $<3.48 \mathrm{E}-3$ & $3.48 \mathrm{E}-3$ & $"$ \\
Arsenic & $<6.25 \mathrm{E}-3$ & $6.25 \mathrm{E}-3$ & $"$ \\
Selenium & $<1.10 \mathrm{E}-2$ & $1.10 \mathrm{E}-2$ & $"$ \\
Silver & $<9.25 \mathrm{E}-4$ & $9.25 \mathrm{E}-4$ & $"$ \\
Cadmium & $<2.95 \mathrm{E}-4$ & $2.95 \mathrm{E}-4$ & $"$ \\
Antimony & $<5.40 \mathrm{E}-4$ & $5.40 \mathrm{E}-4$ & $"$ \\
Lead & $<5.60 \mathrm{E}-4$ & $5.60 \mathrm{E}-4$ & $"$
\end{tabular}

Prepared \& Analyzed: 07/10/08

$\begin{array}{lcccccc}5.23 \mathrm{E} 0 & \text { N/A } & \text { ug/L } & 5.00 \mathrm{E} 0 & 2.43 \mathrm{E}-1 & 99.8 & 75-125 \\ 5.19 \mathrm{E} 0 & \text { N/A } & " & 5.00 \mathrm{E} 0 & 3.10 \mathrm{E}-1 & 97.6 & 75-125 \\ 7.31 \mathrm{E} 0 & \text { N/A } & " & 5.00 \mathrm{E} 0 & 2.51 \mathrm{E} 0 & 96 & 75-125 \\ 4.38 \mathrm{E} 0 & \text { N/A } & " & 5.00 \mathrm{E} 0 & 1.01 \mathrm{E}-1 & 85.6 & 75-125 \\ 4.74 \mathrm{E} 0 & \text { N/A } & " & 5.00 \mathrm{E} 0 & 1.57 \mathrm{E}-2 & 94.4 & 75-125 \\ 4.99 \mathrm{E} 0 & \text { N/A } & " & 5.00 \mathrm{E} 0 & 3.07 \mathrm{E}-2 & 99.2 & 75-125 \\ 5.30 \mathrm{E} 0 & \text { N/A } & " & 5.00 \mathrm{E} 0 & 1.36 \mathrm{E}-2 & 106 & 75-125\end{array}$


RCRA Metals By PNNL-AGG-415/Water Extract - Quality Control

Environmental Science Laboratory

\begin{tabular}{|c|c|c|c|c|c|c|c|c|c|c|}
\hline Analyte & Result & $\begin{array}{r}\text { orting } \\
\text { Limit }\end{array}$ & Units & $\begin{array}{l}\text { Spike } \\
\text { Level }\end{array}$ & $\begin{array}{l}\text { Source } \\
\text { Result }\end{array}$ & \%REC & $\begin{array}{l}\text { \%REC } \\
\text { Limits }\end{array}$ & RPD & $\begin{array}{l}\text { RPD } \\
\text { Limit }\end{array}$ & Notes \\
\hline
\end{tabular}

Batch 8I11003 - 1:1 Water Extract (ICP/ICPMS)

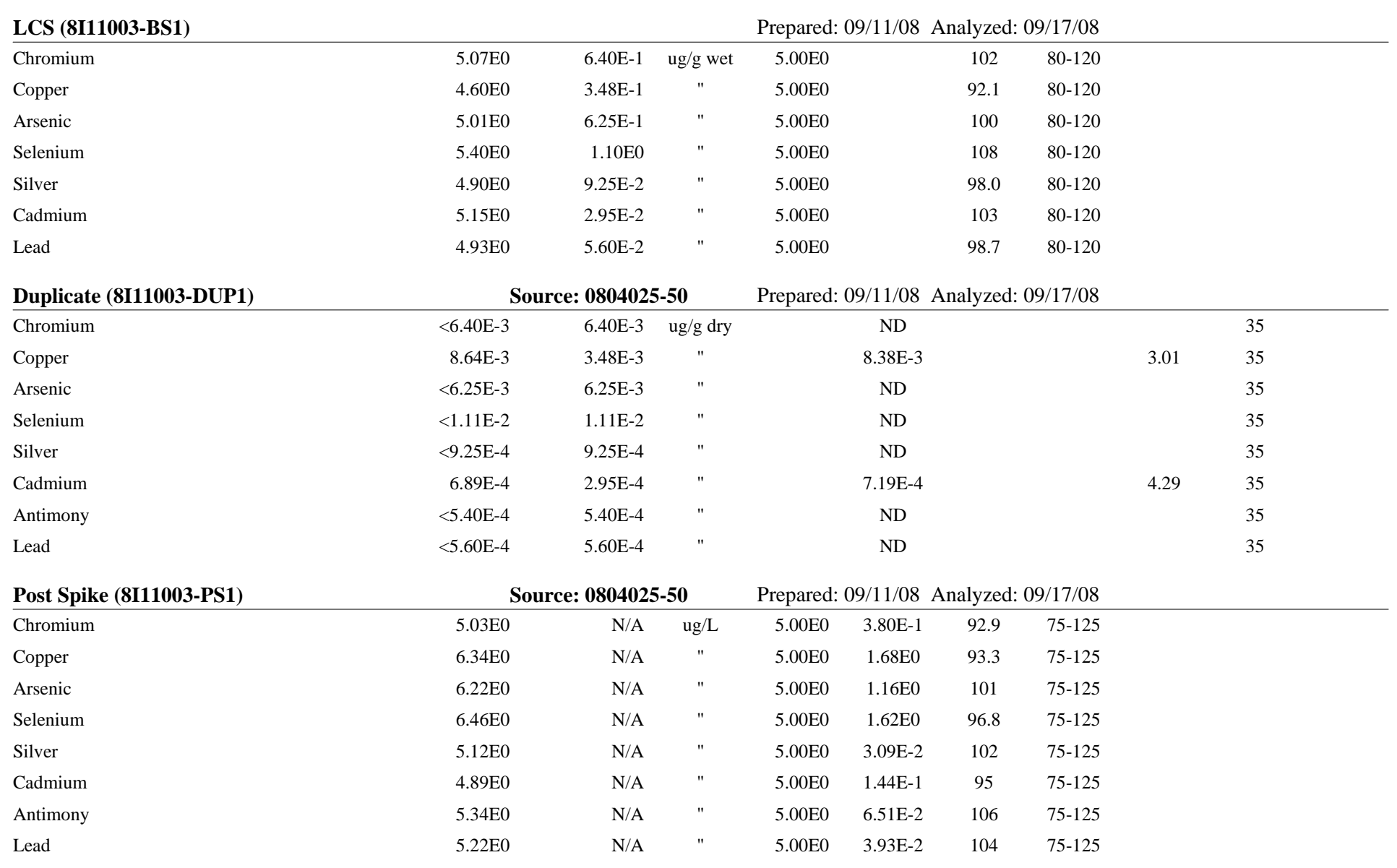


RCRA Metals By PNNL-AGG-415/Acid Extract - Quality Control

Environmental Science Laboratory

\begin{tabular}{|c|c|c|c|c|c|c|c|c|c|c|}
\hline Analyte & Result & $\begin{array}{r}\text { orting } \\
\text { Limit }\end{array}$ & Units & $\begin{array}{l}\text { Spike } \\
\text { Level }\end{array}$ & $\begin{array}{l}\text { Source } \\
\text { Result }\end{array}$ & \%REC & $\begin{array}{l}\text { \%REC } \\
\text { Limits }\end{array}$ & RPD & $\begin{array}{l}\text { RPD } \\
\text { Limit }\end{array}$ & Notes \\
\hline
\end{tabular}

Batch 8I18001 - ASTM D 5198 (ICP/ICPMS)

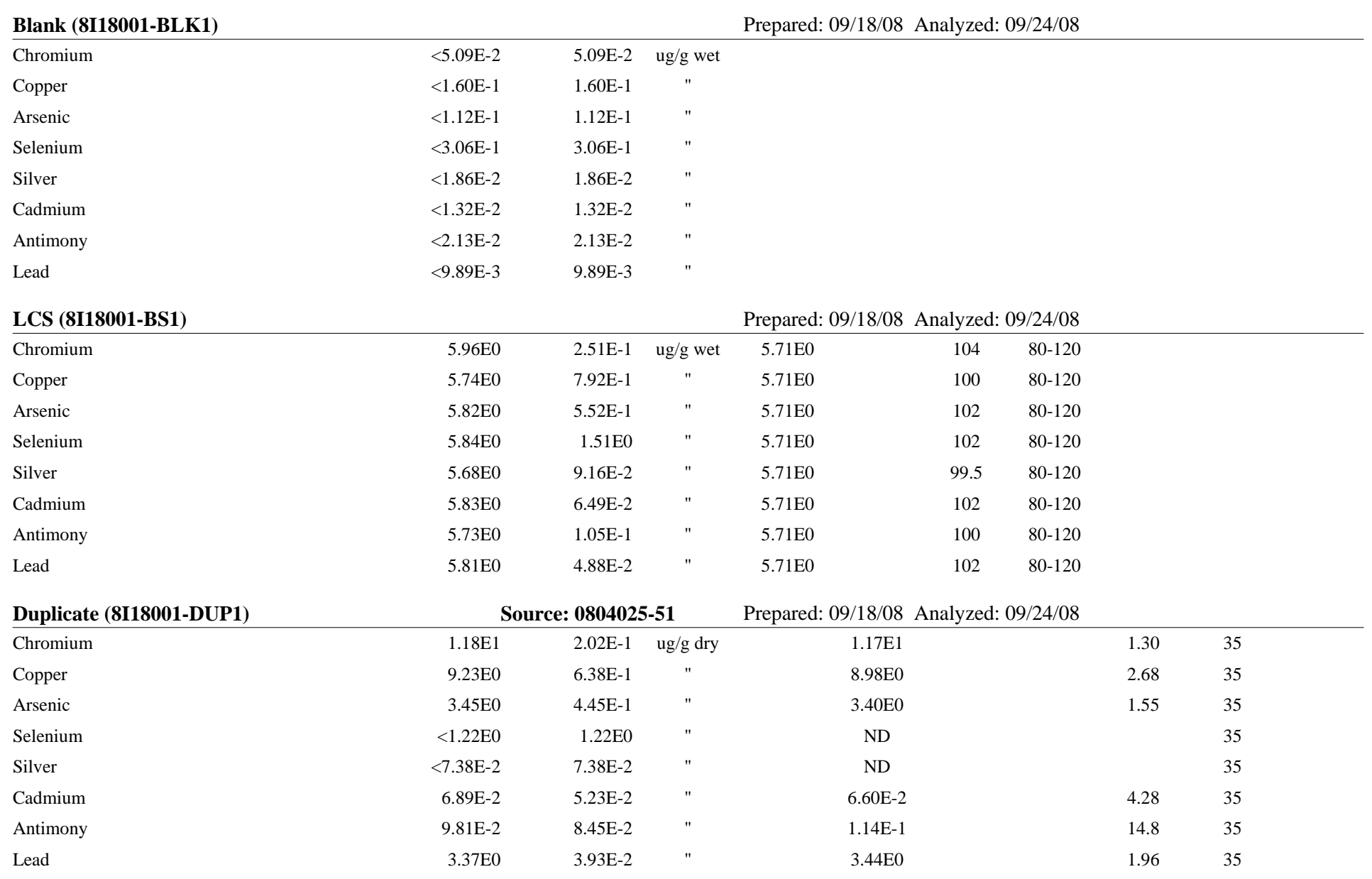


RCRA Metals By PNNL-AGG-415/Acid Extract - Quality Control

Environmental Science Laboratory

\begin{tabular}{|c|c|c|c|c|c|c|c|c|c|c|}
\hline Analyte & Result & $\begin{array}{r}\text { orting } \\
\text { Limit }\end{array}$ & Units & $\begin{array}{l}\text { Spike } \\
\text { Level }\end{array}$ & $\begin{array}{l}\text { Source } \\
\text { Result }\end{array}$ & \%REC & $\begin{array}{l}\text { \%REC } \\
\text { Limits }\end{array}$ & RPD & $\begin{array}{l}\text { RPD } \\
\text { Limit }\end{array}$ & Notes \\
\hline
\end{tabular}

Batch 8I18001 - ASTM D 5198 (ICP/ICPMS)

\begin{tabular}{llllllll}
\hline Post Spike (8I18001-PS1) & \multicolumn{3}{c}{ Source: 0804025-51 } & \multicolumn{2}{c}{ Prepared: 09/18/08 } & Analyzed: 09/24/08 \\
\hline Copper & $2.85 \mathrm{E} 1$ & N/A & ug/L & $5.00 \mathrm{E} 0$ & $2.42 \mathrm{E} 1$ & 85.3 & $75-125$ \\
Selenium & $4.22 \mathrm{E} 0$ & N/A & $"$ & $5.00 \mathrm{E} 0$ & ND & 89.9 & $75-125$ \\
Cadmium & $4.87 \mathrm{E} 0$ & N/A & $"$ & $5.00 \mathrm{E} 0$ & $1.78 \mathrm{E}-1$ & 93.8 & $75-125$ \\
Antimony & $4.94 \mathrm{E} 0$ & N/A & $"$ & $5.00 \mathrm{E} 0$ & $3.07 \mathrm{E}-1$ & 92.6 & $75-125$
\end{tabular}

Batch 8I18002 - ASTM D 5198 (ICP/ICPMS)

Blank (8I18002-BLK1)

Chromium

Copper

Arsenic

Selenium

Silver

Cadmium

Antimony

Lead

LCS (8I18002-BS1)

Chromium

Copper

Arsenic

Selenium

Silver

Cadmium

Antimony

Lead
Prepared: 09/18/08 Analyzed: 09/24/08

$\begin{array}{llc}<5.25 \mathrm{E}-2 & 5.25 \mathrm{E}-2 & \text { ug/g wet } \\ <1.65 \mathrm{E}-1 & 1.65 \mathrm{E}-1 & " \\ <1.15 \mathrm{E}-1 & 1.15 \mathrm{E}-1 & " \\ <3.15 \mathrm{E}-1 & 3.15 \mathrm{E}-1 & " \\ <1.91 \mathrm{E}-2 & 1.91 \mathrm{E}-2 & " \\ <1.36 \mathrm{E}-2 & 1.36 \mathrm{E}-2 & " \\ <2.19 \mathrm{E}-2 & 2.19 \mathrm{E}-2 & " \\ <1.02 \mathrm{E}-2 & 1.02 \mathrm{E}-2\end{array}$

Prepared: 09/18/08 Analyzed: 09/24/08

$\begin{array}{lccccc}6.00 \mathrm{E} 0 & 7.06 \mathrm{E}-2 & \text { ug/g wet } & 5.71 \mathrm{E} 0 & 105 & 80-120 \\ 5.76 \mathrm{E} 0 & 2.29 \mathrm{E}-1 & " & 5.71 \mathrm{E} 0 & 101 & 80-120 \\ 5.97 \mathrm{E} 0 & 1.55 \mathrm{E}-1 & " & 5.71 \mathrm{E} 0 & 105 & 80-120 \\ 5.90 \mathrm{E} 0 & 4.24 \mathrm{E}-1 & " & 5.71 \mathrm{E} 0 & 103 & 80-120 \\ 5.87 \mathrm{E} 0 & 2.57 \mathrm{E}-2 & " & 5.71 \mathrm{E} 0 & 103 & 80-120 \\ 6.22 \mathrm{E} 0 & 1.82 \mathrm{E}-2 & " & 5.71 \mathrm{E} 0 & 109 & 80-120 \\ 6.16 \mathrm{E} 0 & 2.95 \mathrm{E}-2 & " & 5.71 \mathrm{E} 0 & 108 & 80-120 \\ 6.02 \mathrm{E} 0 & 1.37 \mathrm{E}-2 & " & 5.71 \mathrm{E} 0 & 105 & 80-120\end{array}$


RCRA Metals By PNNL-AGG-415/Acid Extract - Quality Control

Environmental Science Laboratory

\begin{tabular}{|lrrrrrrrrrrr}
\hline & & Reporting & & Spike & Source & & \%REC & & RPD & & \\
Analyte & Result & Limit & Units & Level & Result & \%REC & Limits & RPD & Limit & Notes \\
\hline
\end{tabular}

Batch $8 I 18002$ - ASTM D 5198 (ICP/ICPMS)

Duplicate (8I18002-DUP1)

Chromium

Source: 0804025-69

Prepared: 09/18/08 Analyzed: 09/24/08

Copper

Arsenic

Selenium

Silver

Cadmium

Antimony

Lead

$1.16 \mathrm{E} 1 \quad 1.93 \mathrm{E}-1 \quad$ ug/g dry

$8.64 \mathrm{E} 0$

$6.08 \mathrm{E}-1$

$1.43 \mathrm{E} 1$

8.69E0

2.72E0

$<1.16 \mathrm{E} 0$

4.24E-1

ND

$<7.03 \mathrm{E}-2$

$1.16 \mathrm{E} 0$

ND

$6.93 \mathrm{E}-2$

7.03E-2

$6.44 \mathrm{E}-2$

9.90E-2

$4.98 \mathrm{E}-2$

$1.07 \mathrm{E}-1$

2.61E0

3.74E-2

$2.88 \mathrm{E} 0$

$20.8 \quad 35$

$0.624 \quad 35$

$5.32 \quad 35$

$5.32 \quad 35$

35
35

$7.30 \quad 35$
8.11

$8.11 \quad 35$

$\begin{array}{ll}8.11 & 35 \\ 9.63 & 35\end{array}$

Post Spike (8I18002-PS1)

Copper

Source: 0804025-69

Prepared: 09/18/08 Analyzed: 09/24/08

Selenium

2.90E1

N/A ug/

$5.00 \mathrm{E} 0 \quad 2.48 \mathrm{E} 1$

$\begin{array}{cr}82.8 & 75-125 \\ 89.9 & 75-125 \\ 95.3 & 75-125 \\ 96.2 & 75-125\end{array}$

Antimony

4.24E0

N/A

N/A

$5.00 \mathrm{E} 0$

ND

$5.00 \mathrm{E} 0 \quad 1.84 \mathrm{E}-1$

$96.2 \quad 75-125$ 
Total Alpha Total Beta/Acid Extract - Quality Control

Environmental Science Laboratory

\begin{tabular}{|lrrrrrrrrrr}
\hline & & Reporting & & Spike & Source & & \%REC & & RPD & \\
Analyte & Result & Limit & Units & Level & Result & \%REC & Limits & RPD & Limit & Notes \\
\hline
\end{tabular}

Batch 8J07001 - ASTM D 5198 (RadChem)

Blank (8J07001-BLK1)

Gross Beta

Gross Alpha

Duplicate (8J07001-DUP1)

Gross Beta

Gross Alpha

+

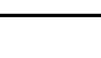

$\begin{array}{lll}<4.11 \mathrm{E} 0 & 4.11 \mathrm{E} 0 & \mathrm{pCi} / \mathrm{g} \text { wet } \\ <1.76 \mathrm{E} 0 & 1.76 \mathrm{E} 0 & "\end{array}$

Prepared: 10/05/08 Analyzed: 10/06/08

Batch 8J07002 - ASTM D 5198 (RadChem)

\section{Blank (8J07002-BLK1)}

Gross Beta

Gross Alpha

Source: 0804025-51

Prepared: 10/05/08 Analyzed: 10/06/08

$\begin{array}{llcll}<1.63 \mathrm{E} 1 & 1.63 \mathrm{E} 1 & \mathrm{pCi} / \mathrm{g} \text { dry } & \mathrm{ND} & 35 \\ <6.99 \mathrm{E} 0 & 6.99 \mathrm{E} 0 & " & \mathrm{ND} & 35\end{array}$

Duplicate (8J07002-DUP1)

Gross Beta

Gross Alpha

(8J07002-DUP1)

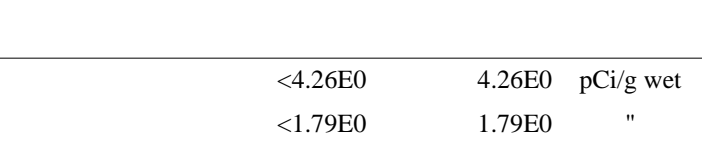

Prepared: 10/06/08 Analyzed: 10/07/08

Source: 0804025-69 Prepared: 10/06/08 Analyzed: 10/07/08

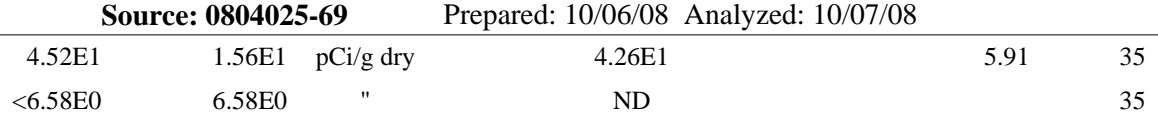




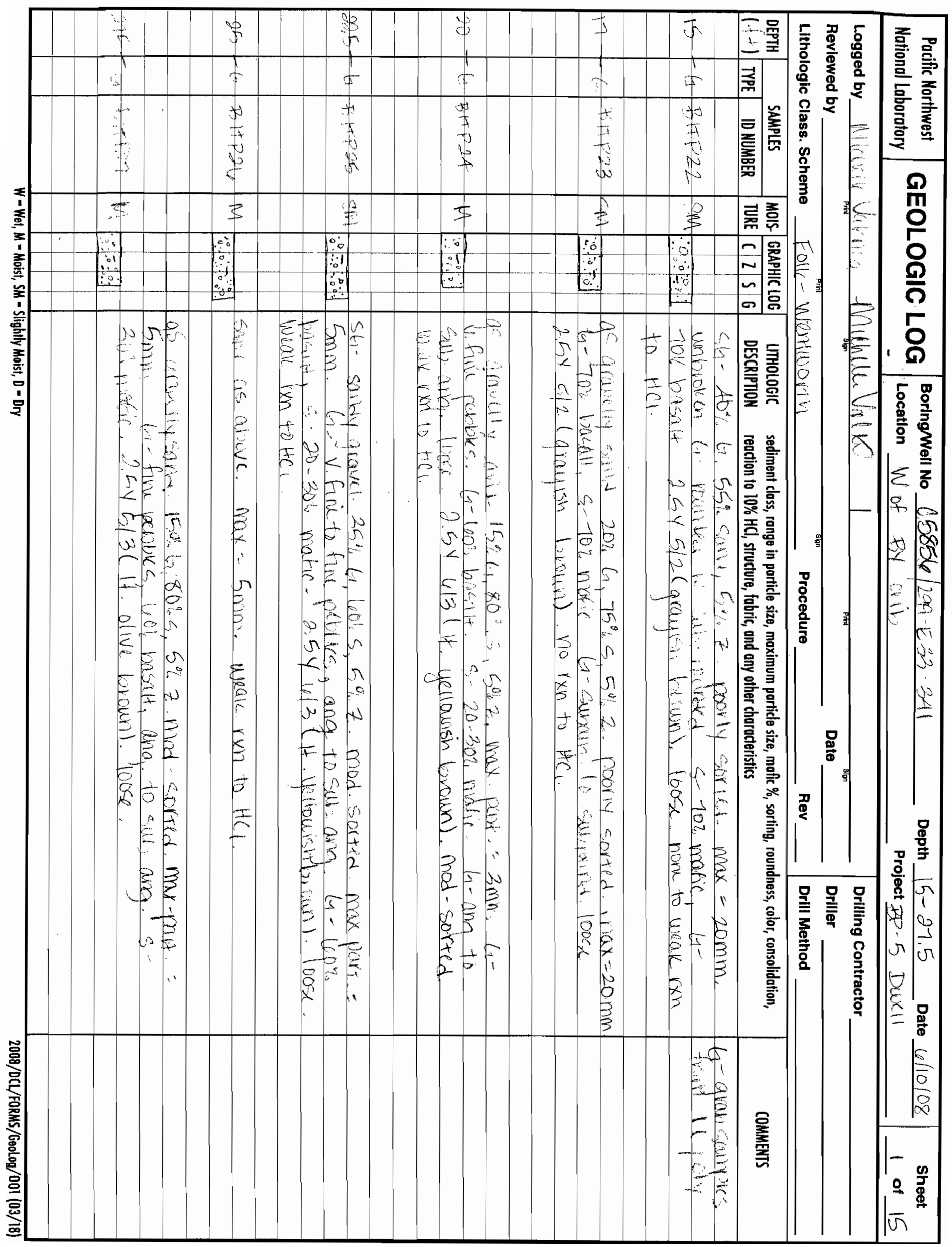




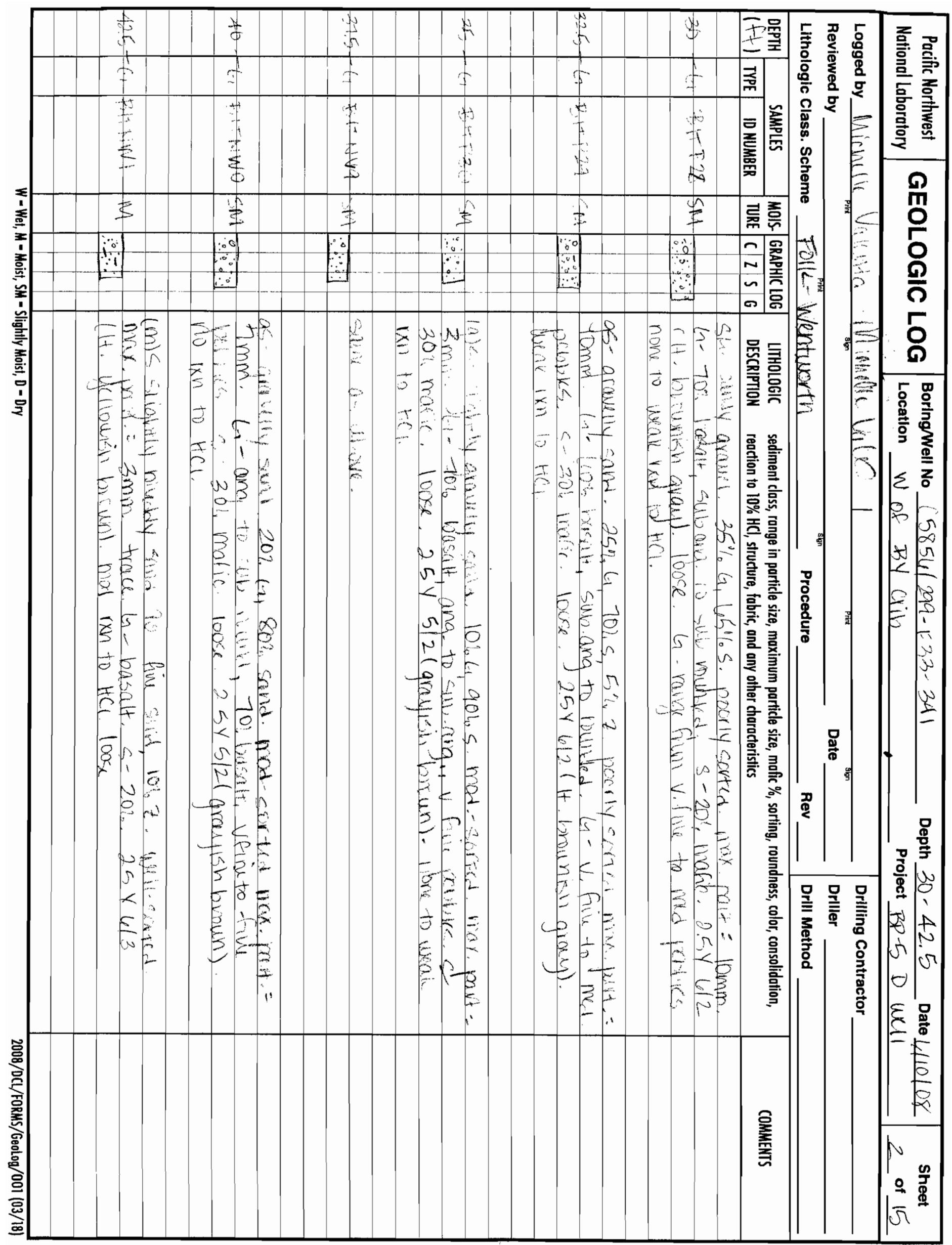




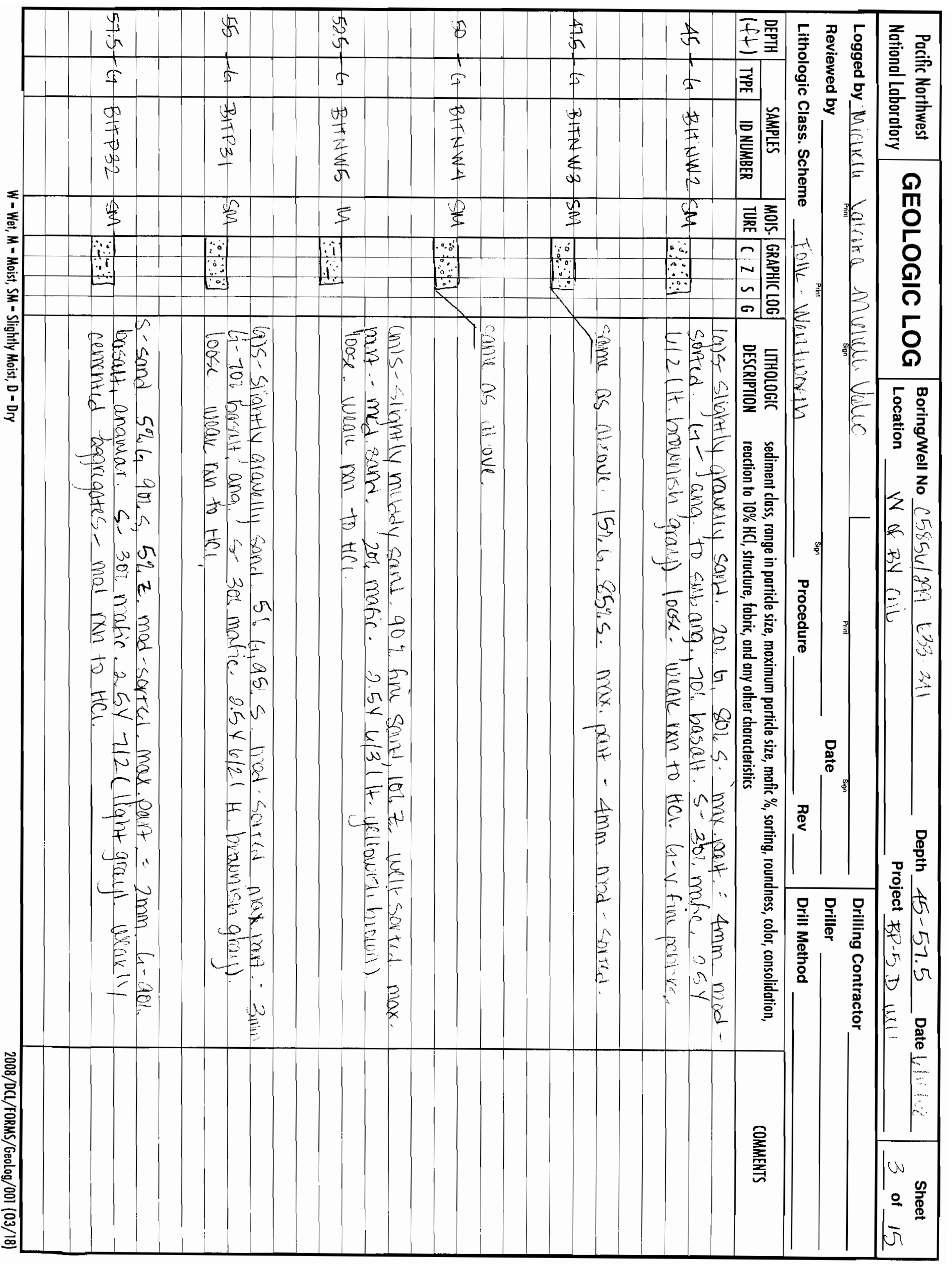




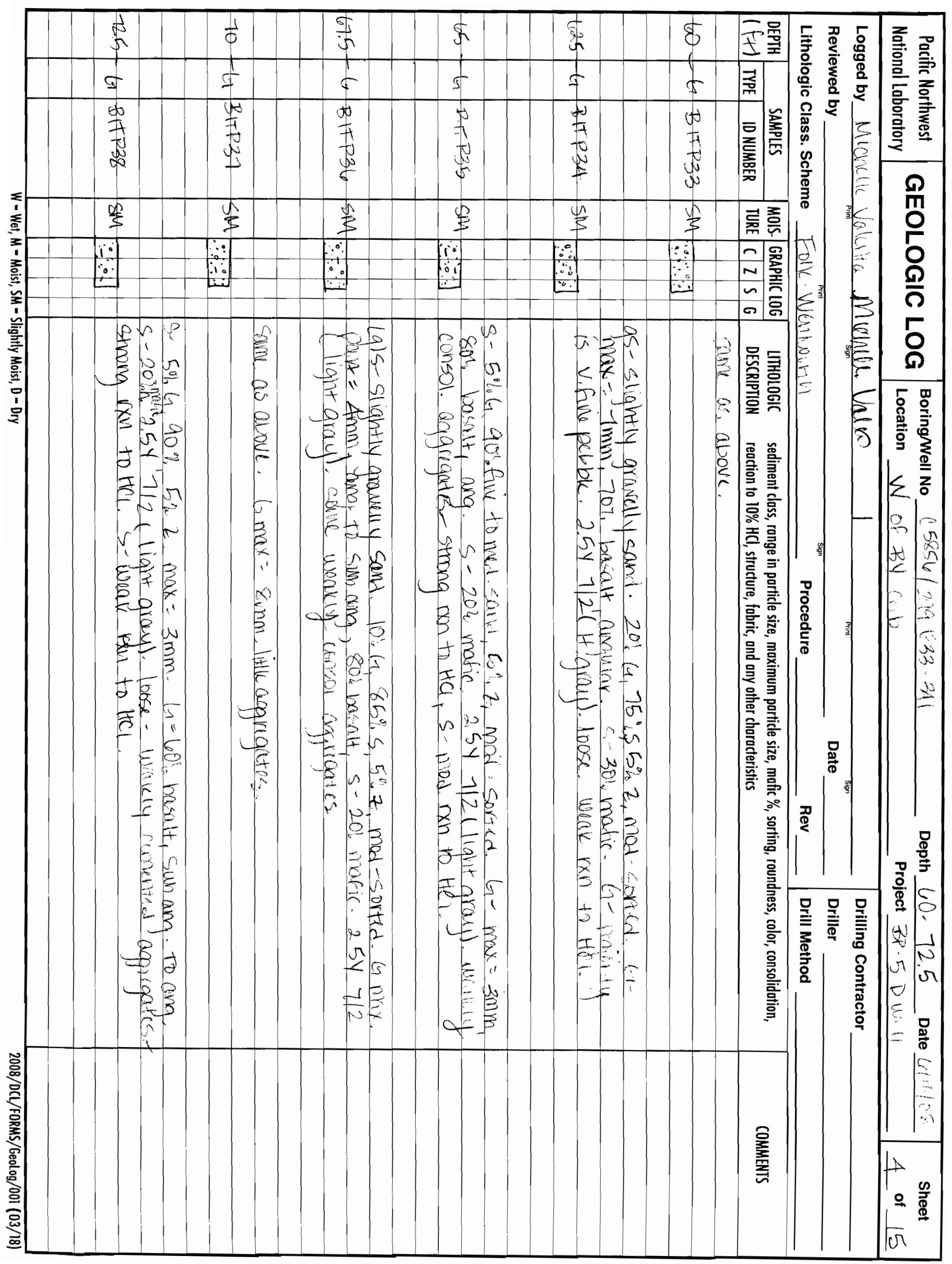




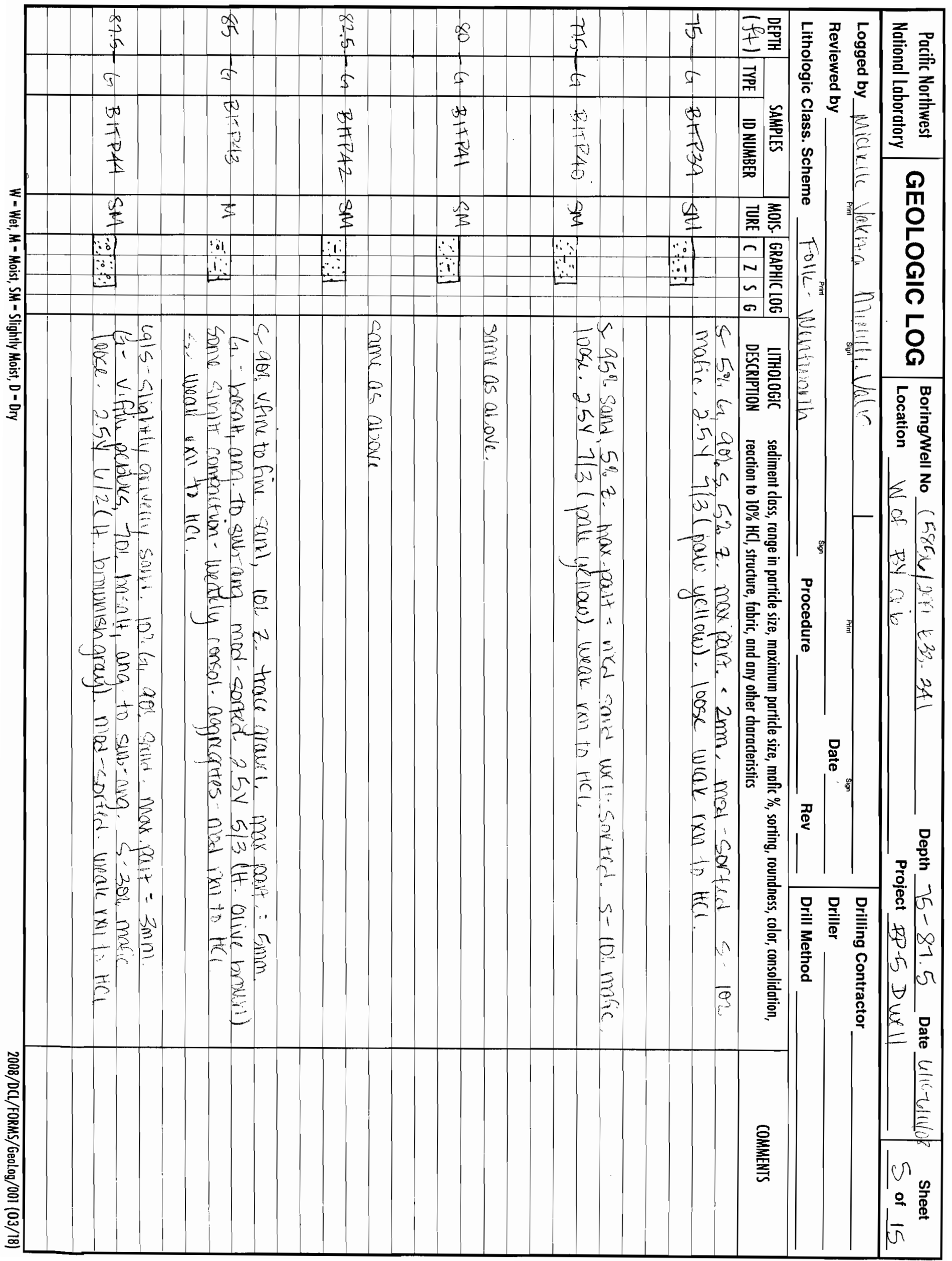




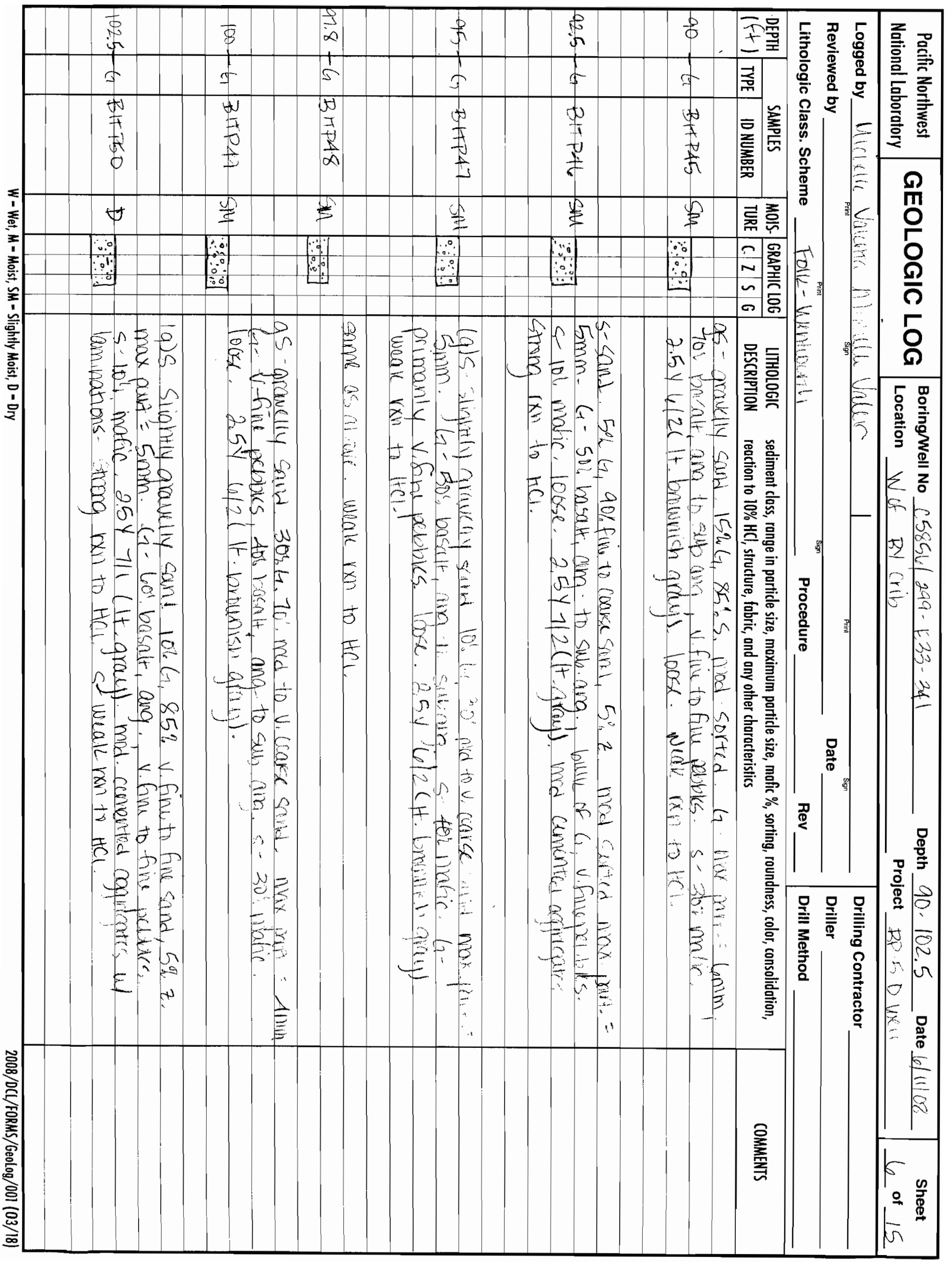




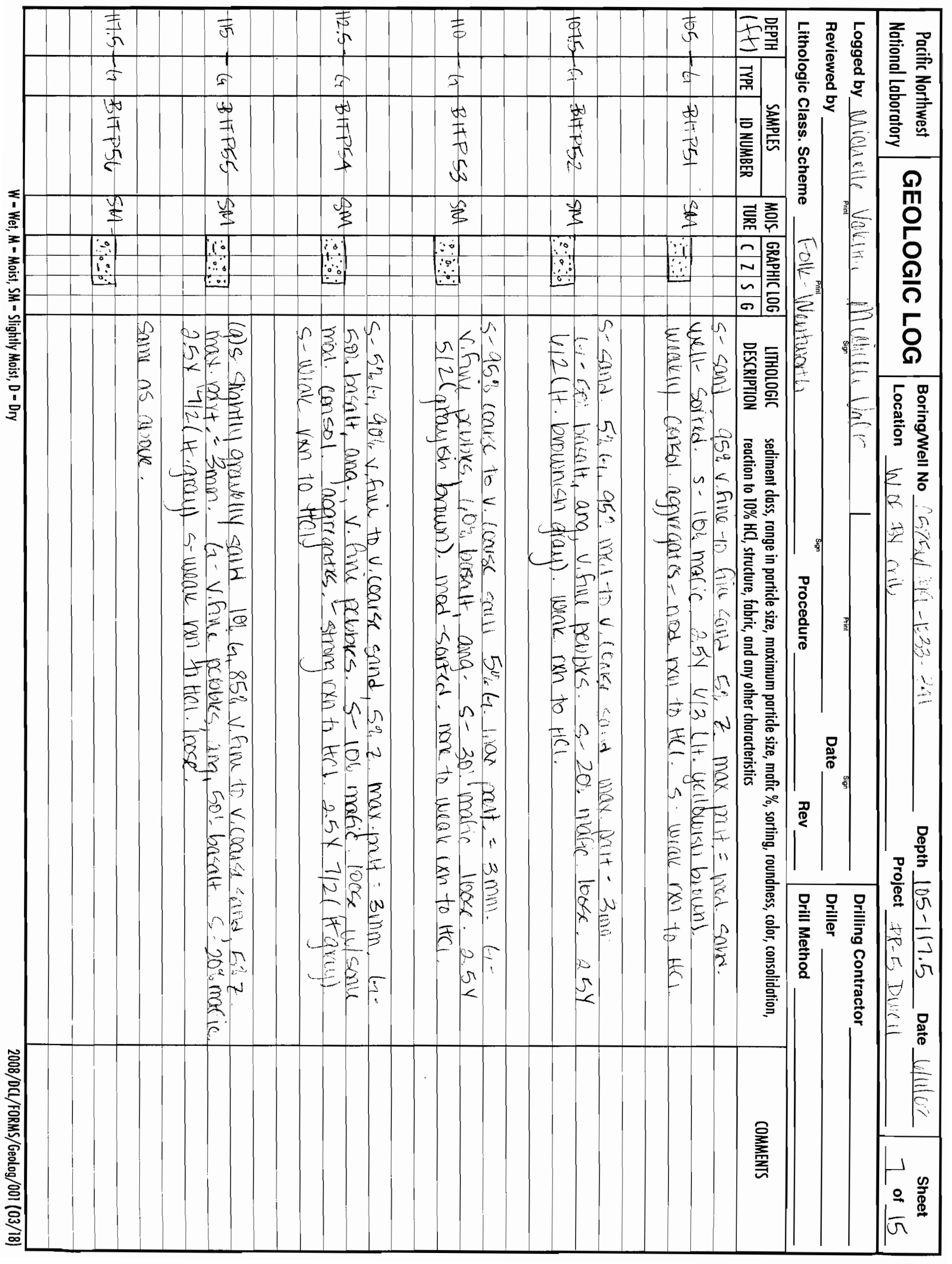




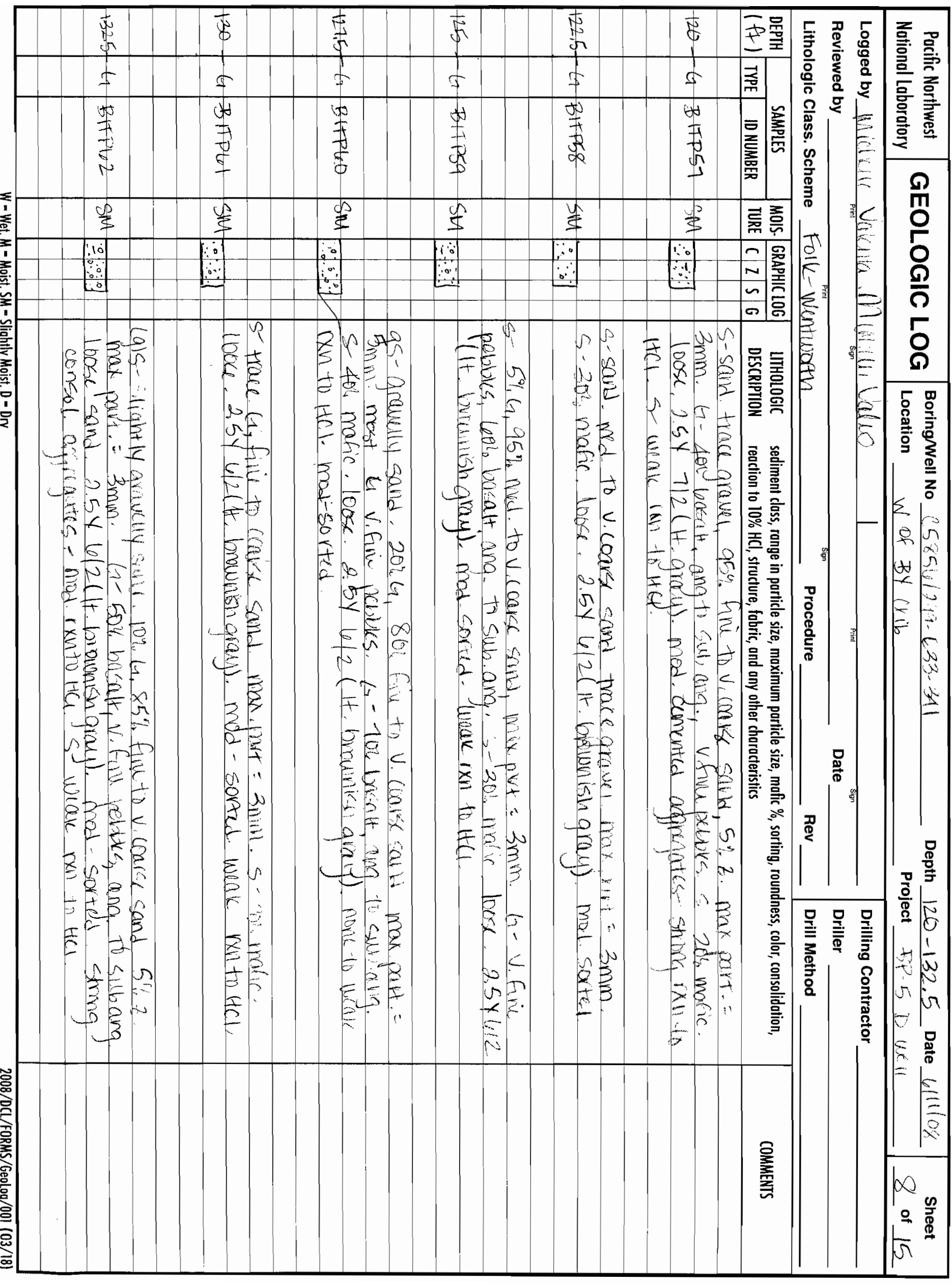




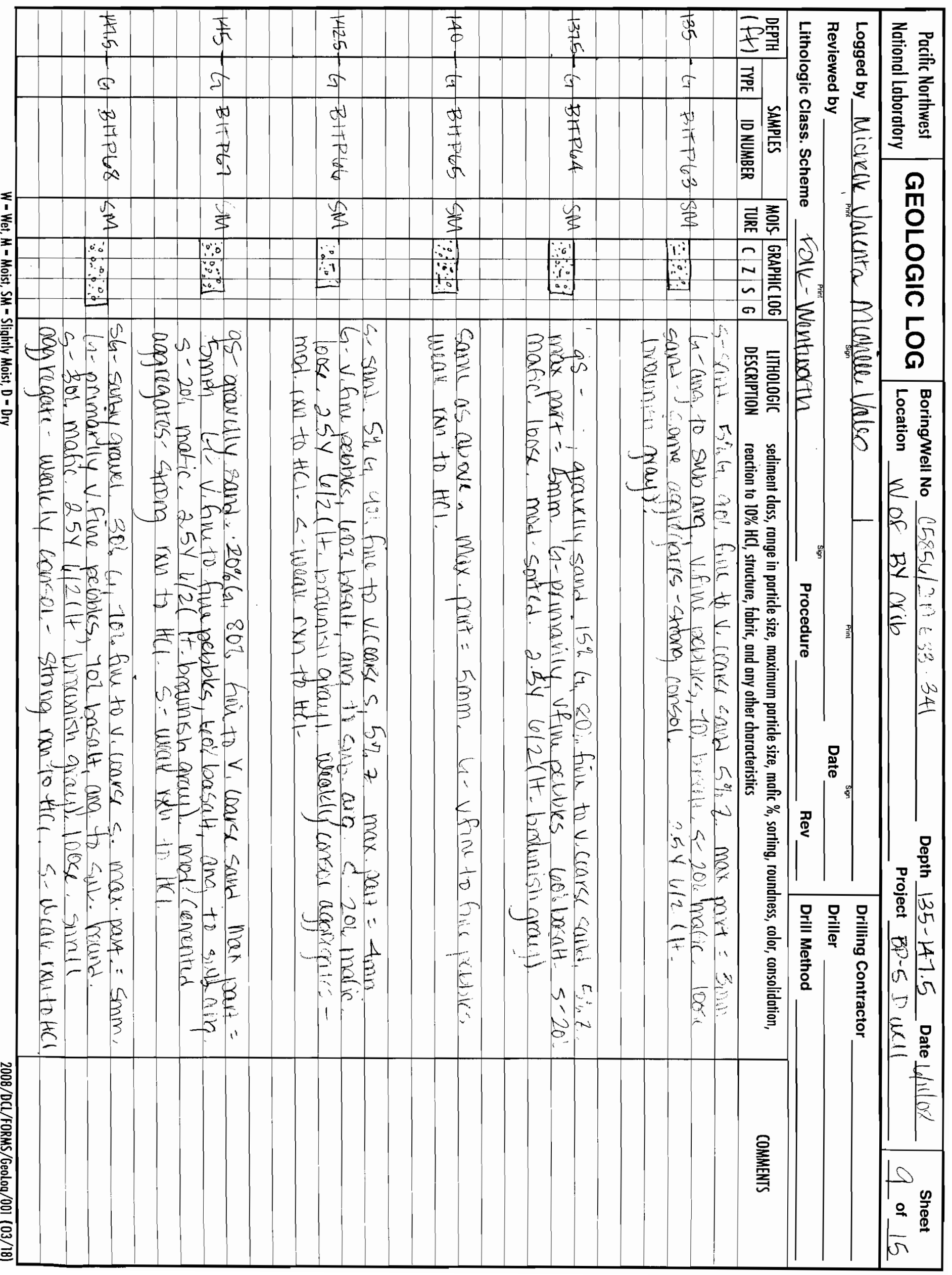




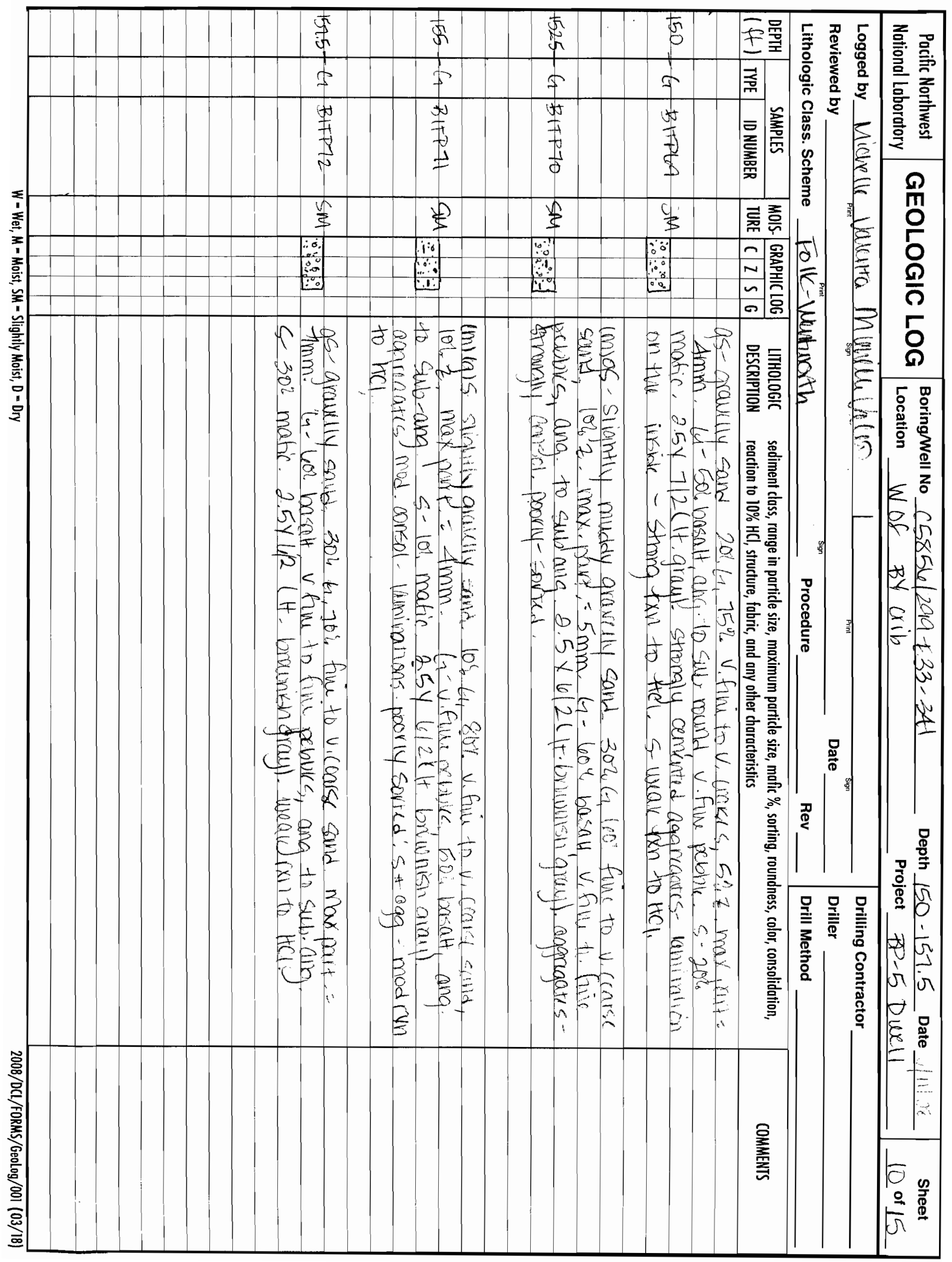




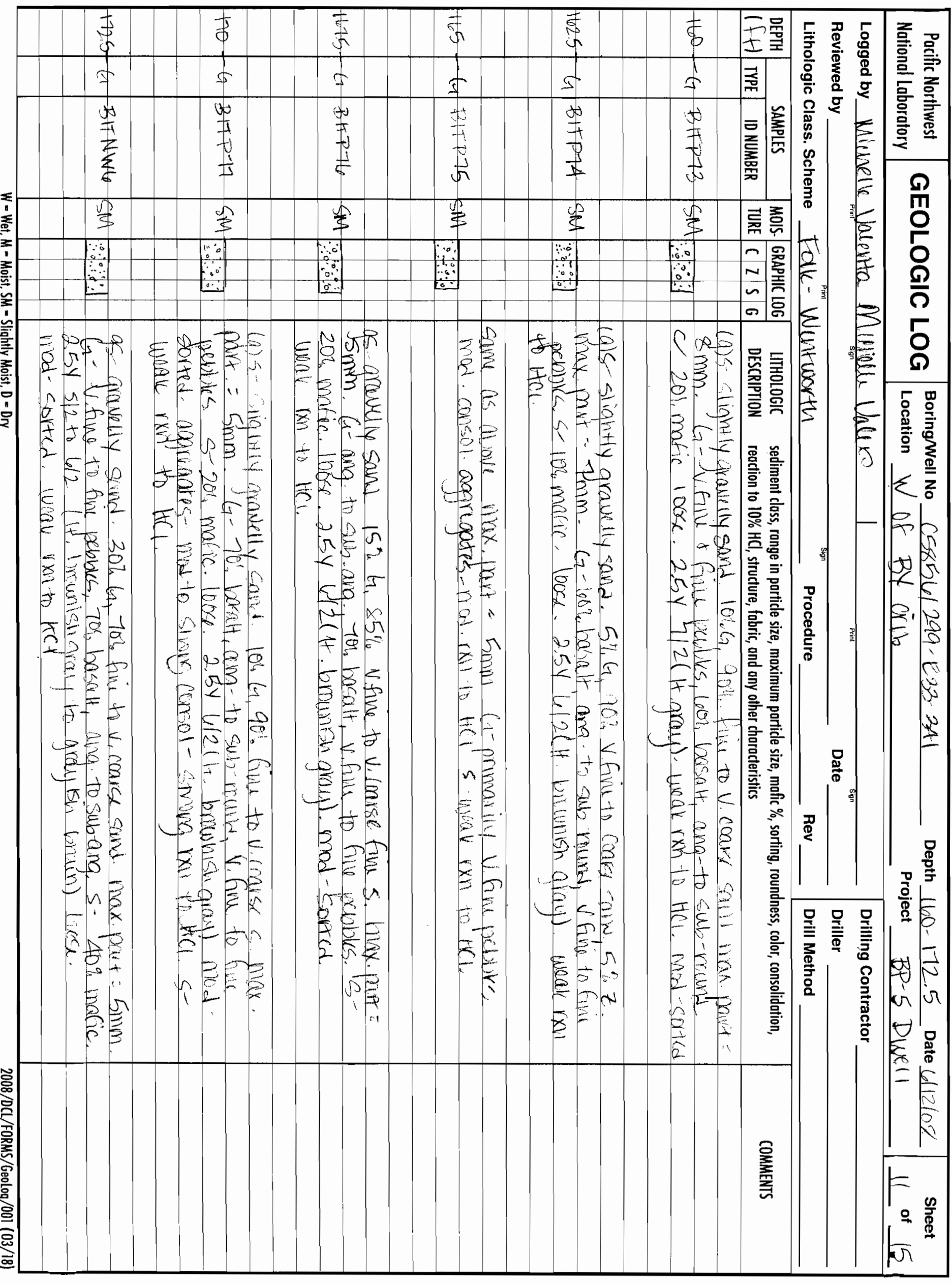




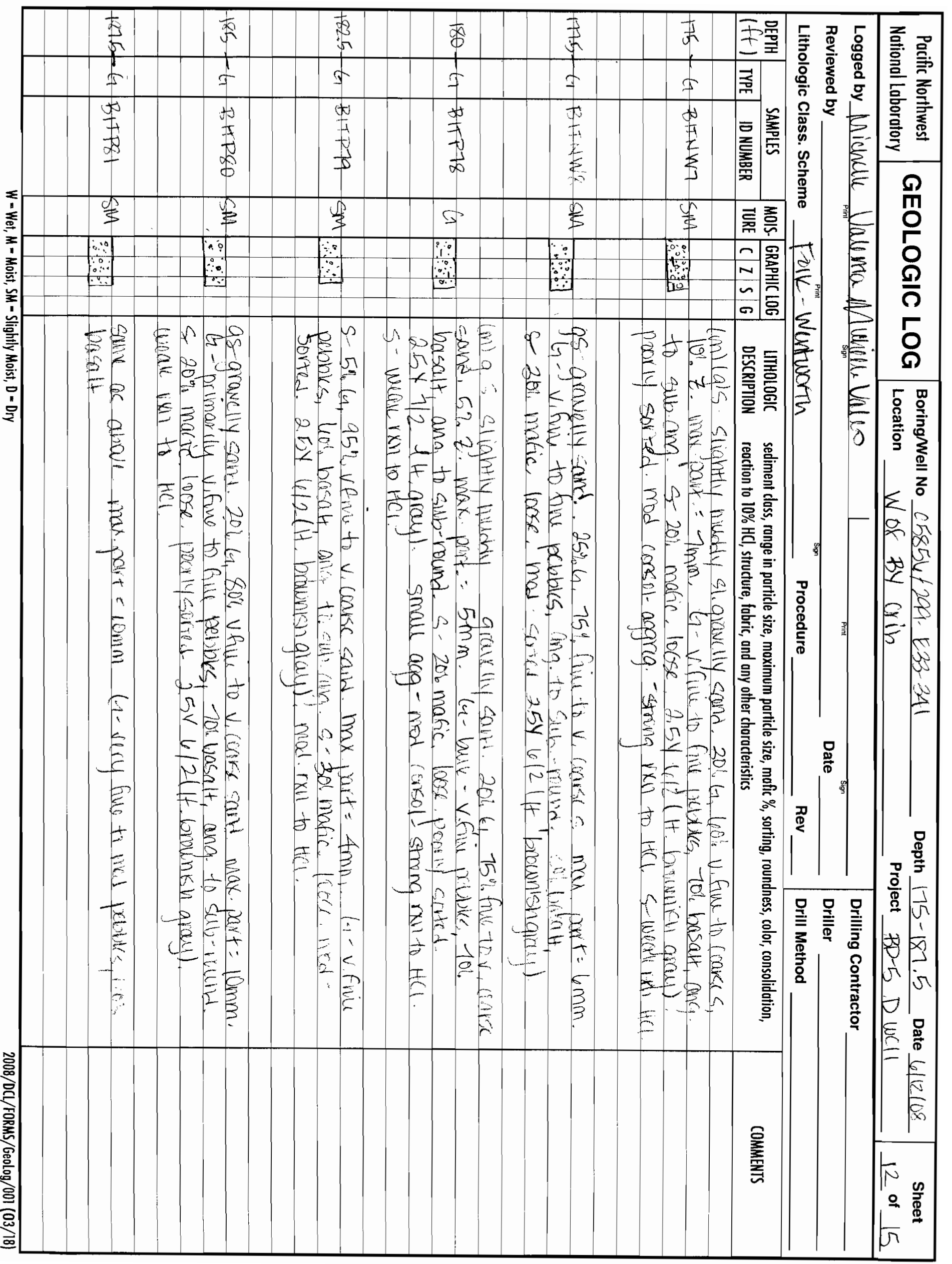




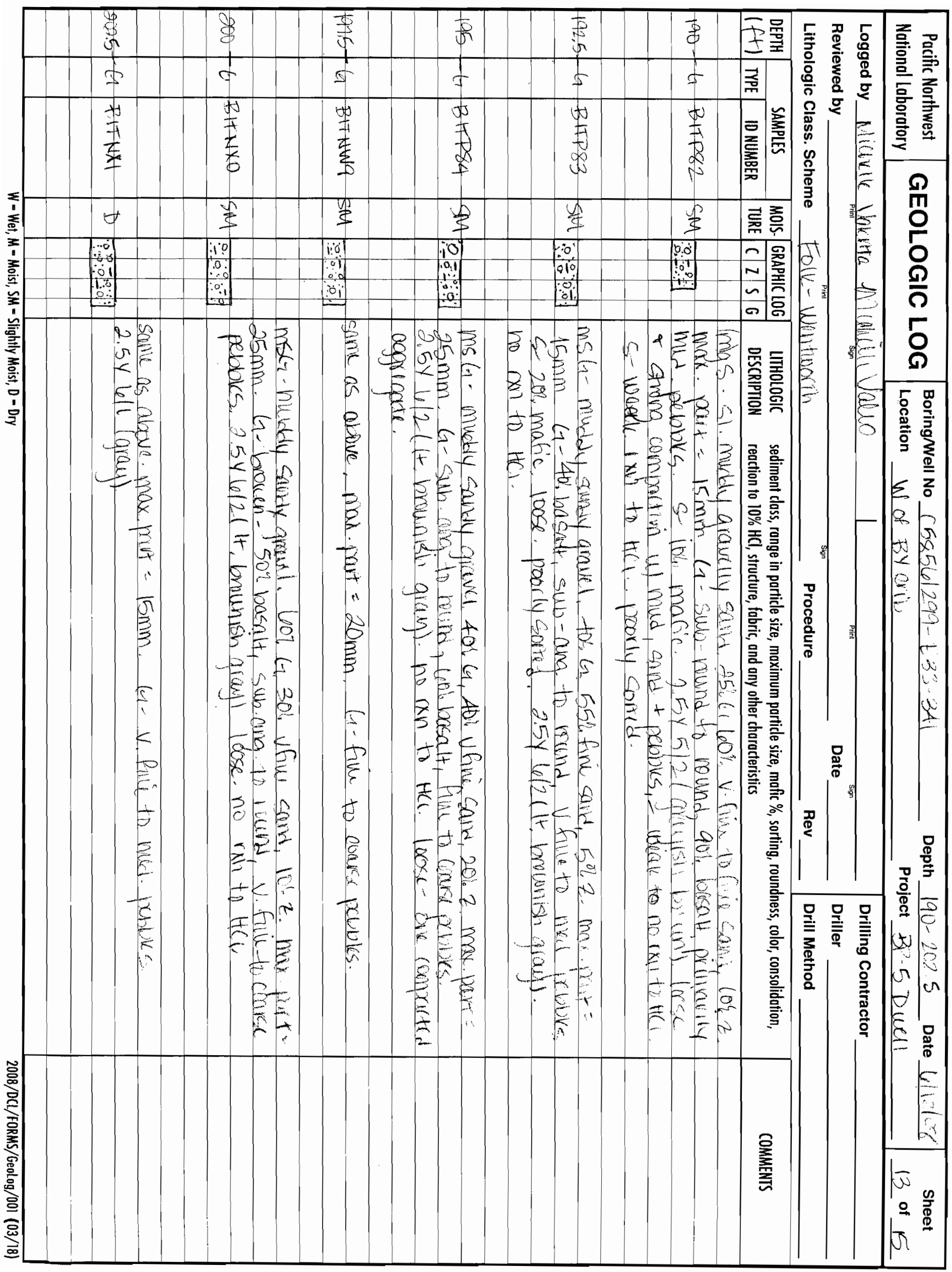




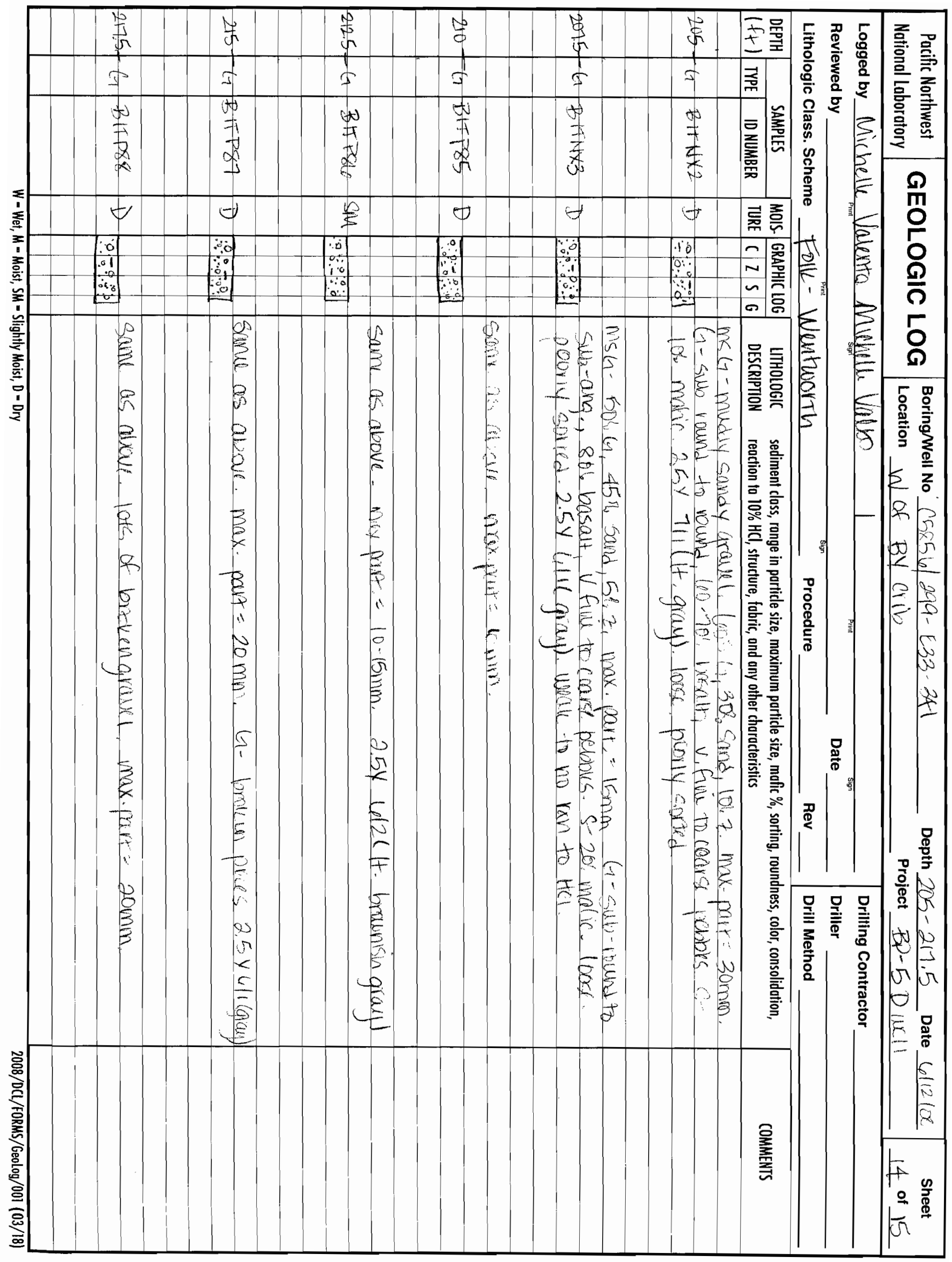




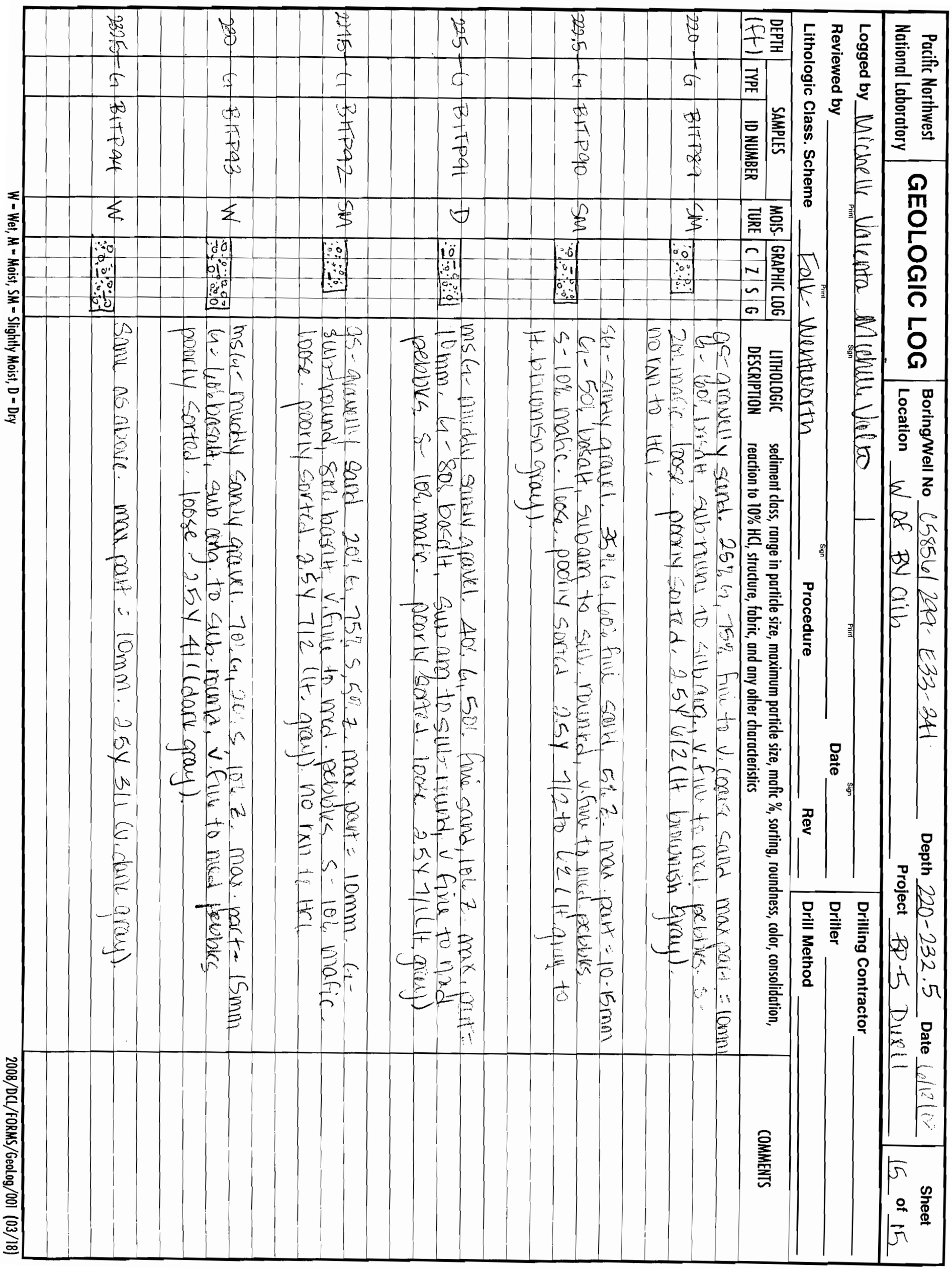




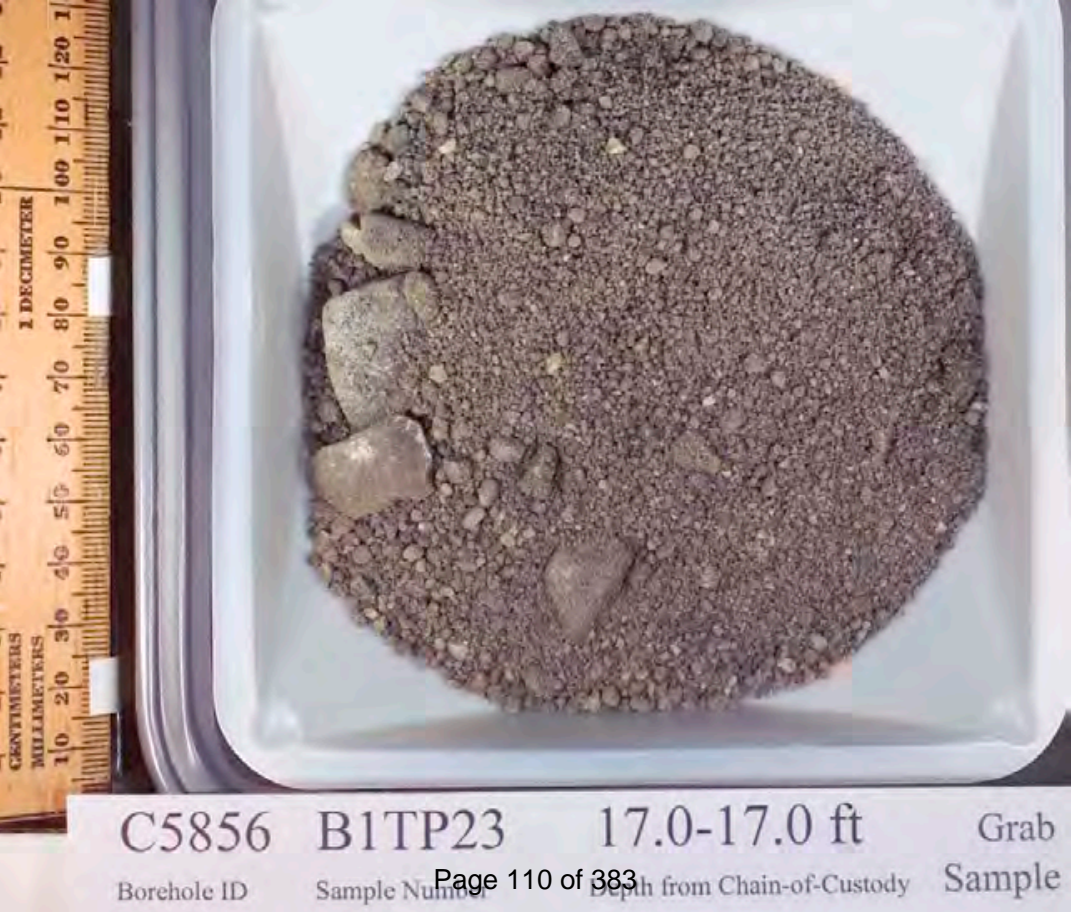




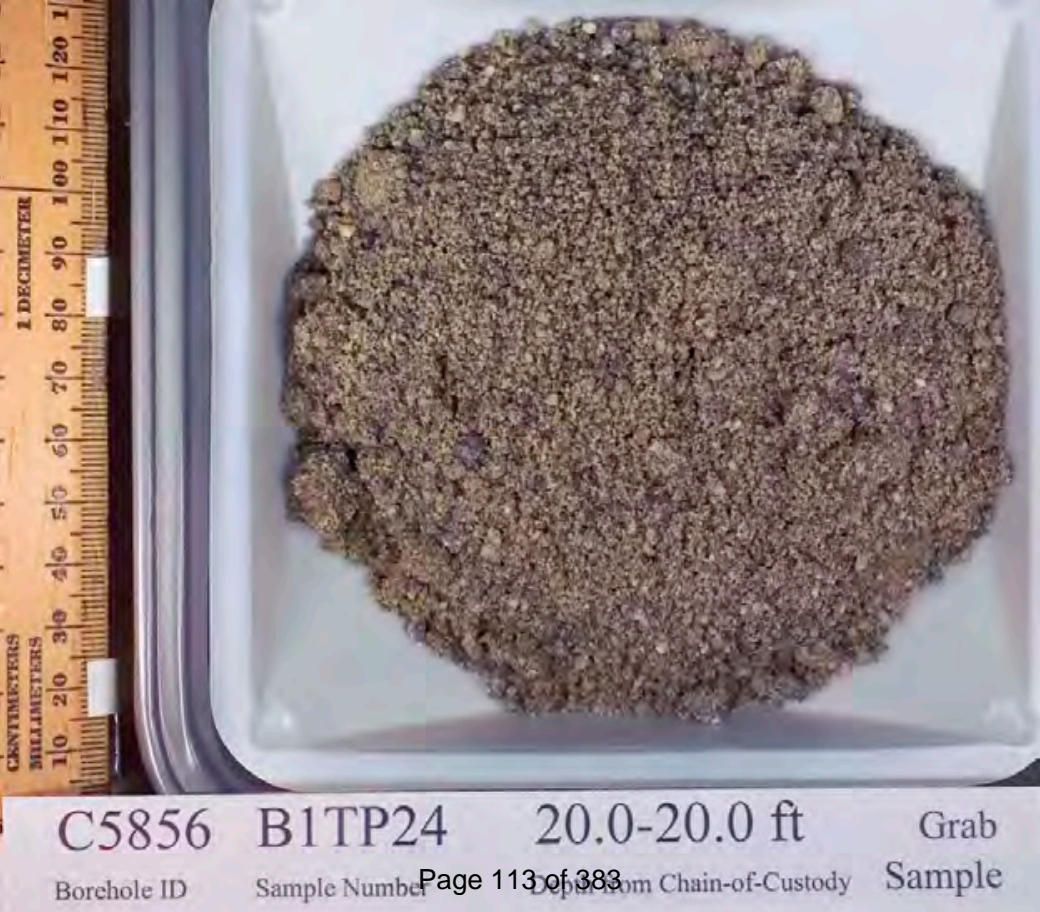




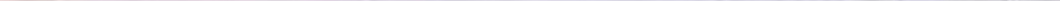




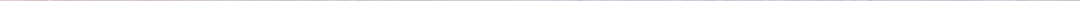




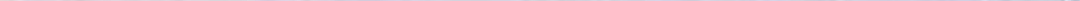




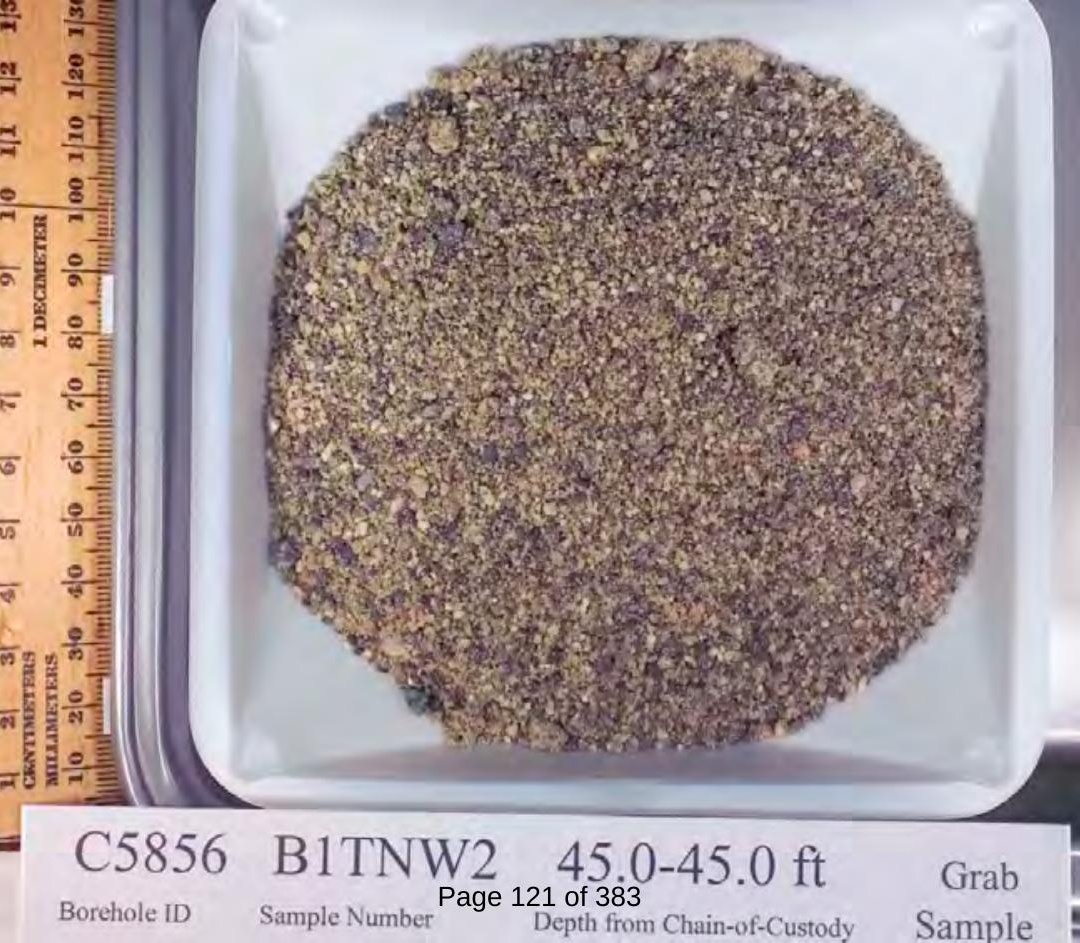




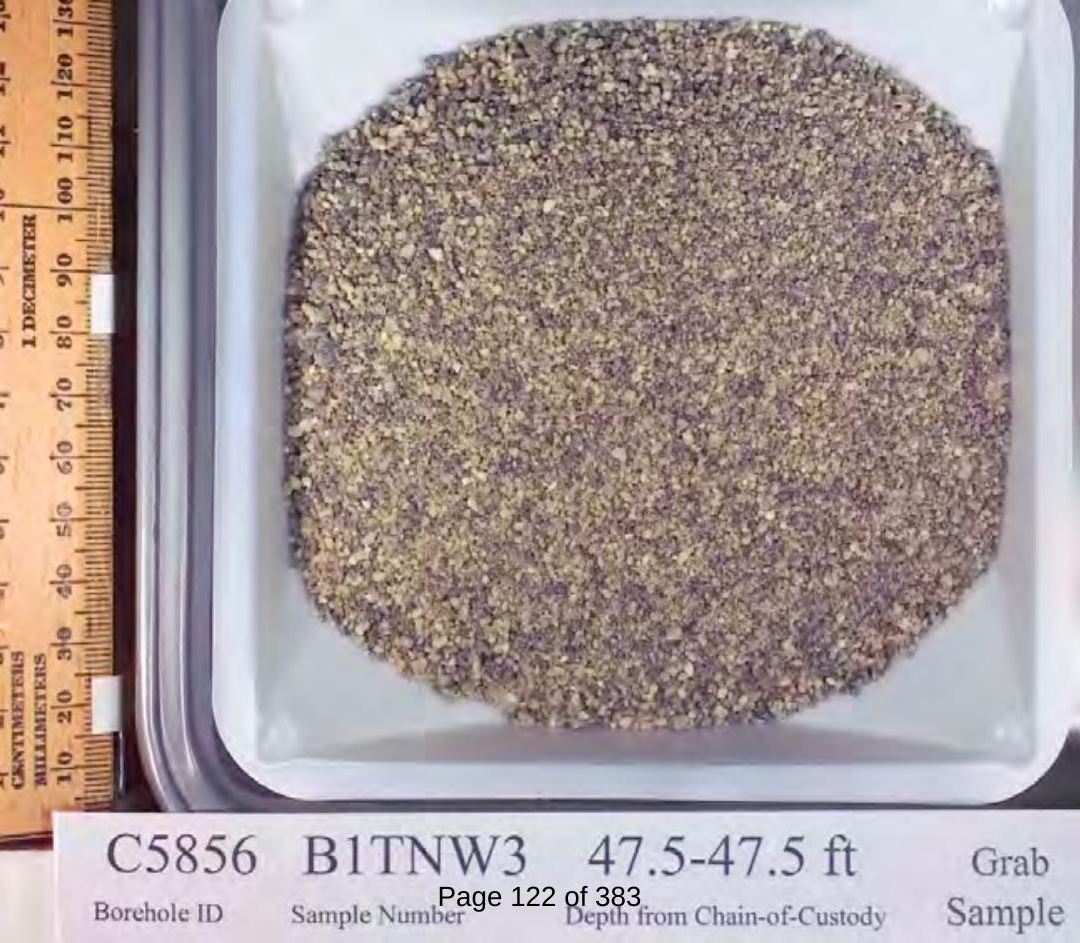




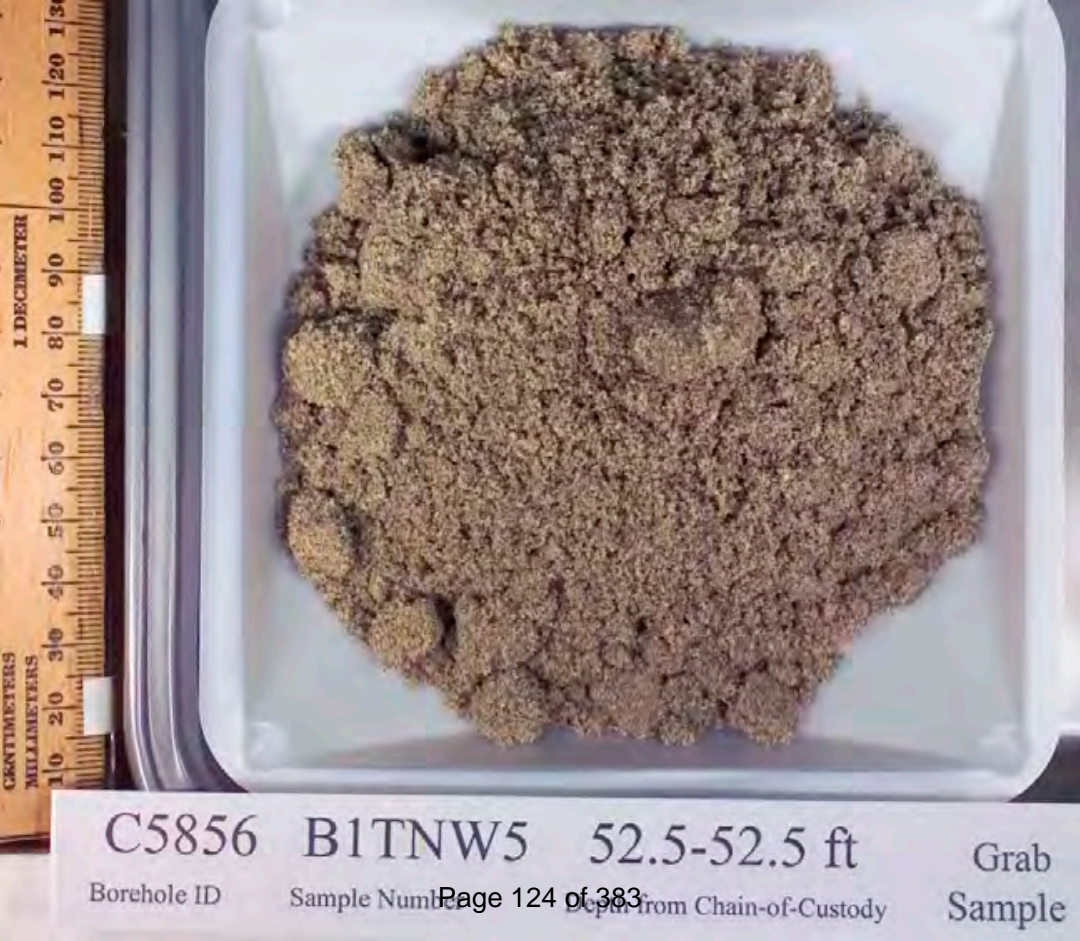




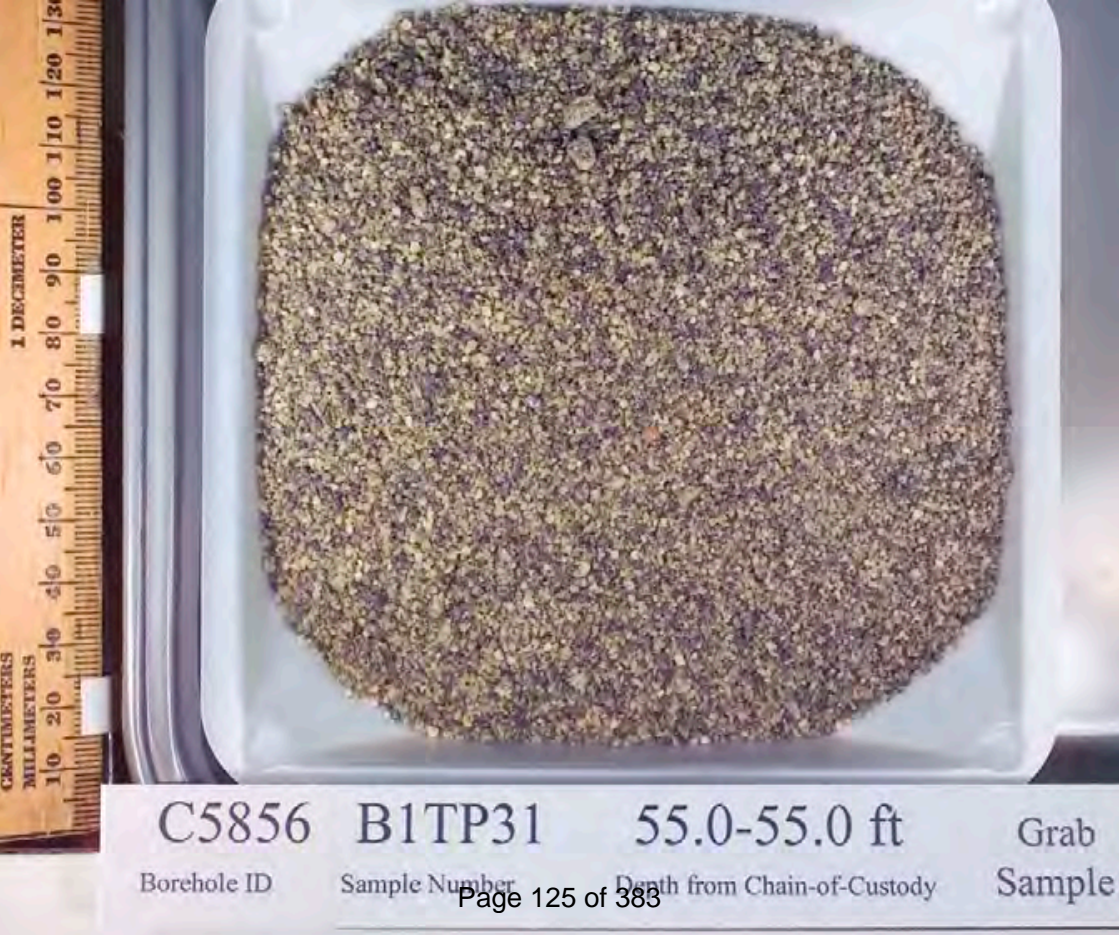




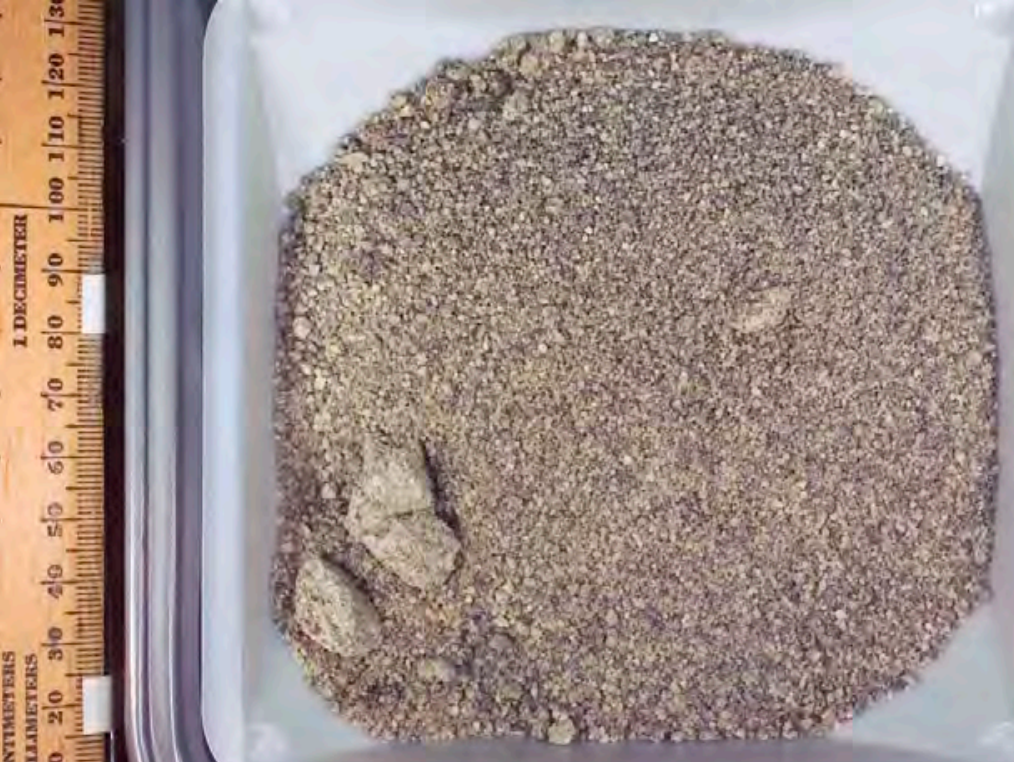

\section{C5856 B1TP32 $57.5-57.5 \mathrm{ft}$}

Borehole ID
Sample NunPage 126 of $\mathbf{3 8 3}$ from Chain-of-Custody
Grab

Sample 


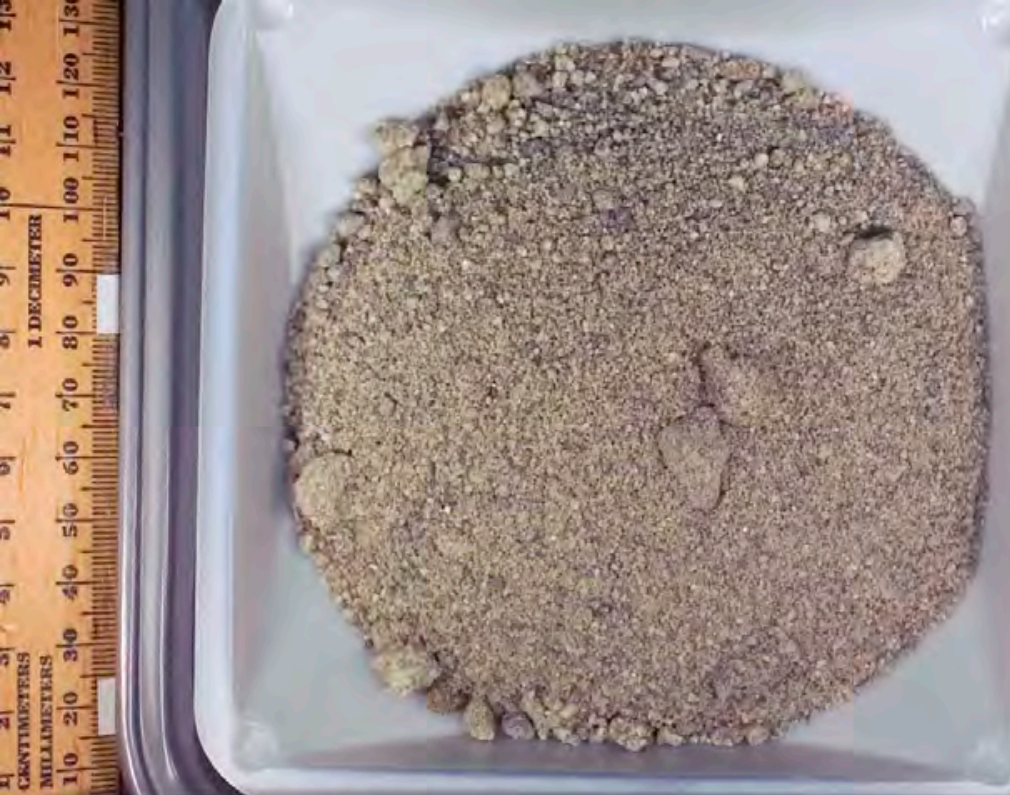

\section{C5856 B1TP36 $67.5-67.5 \mathrm{ft}$}

Borehole ID
Sample NumbRage 130 of 383 rom Chain-of-Custody

\section{Grab \\ Sample}




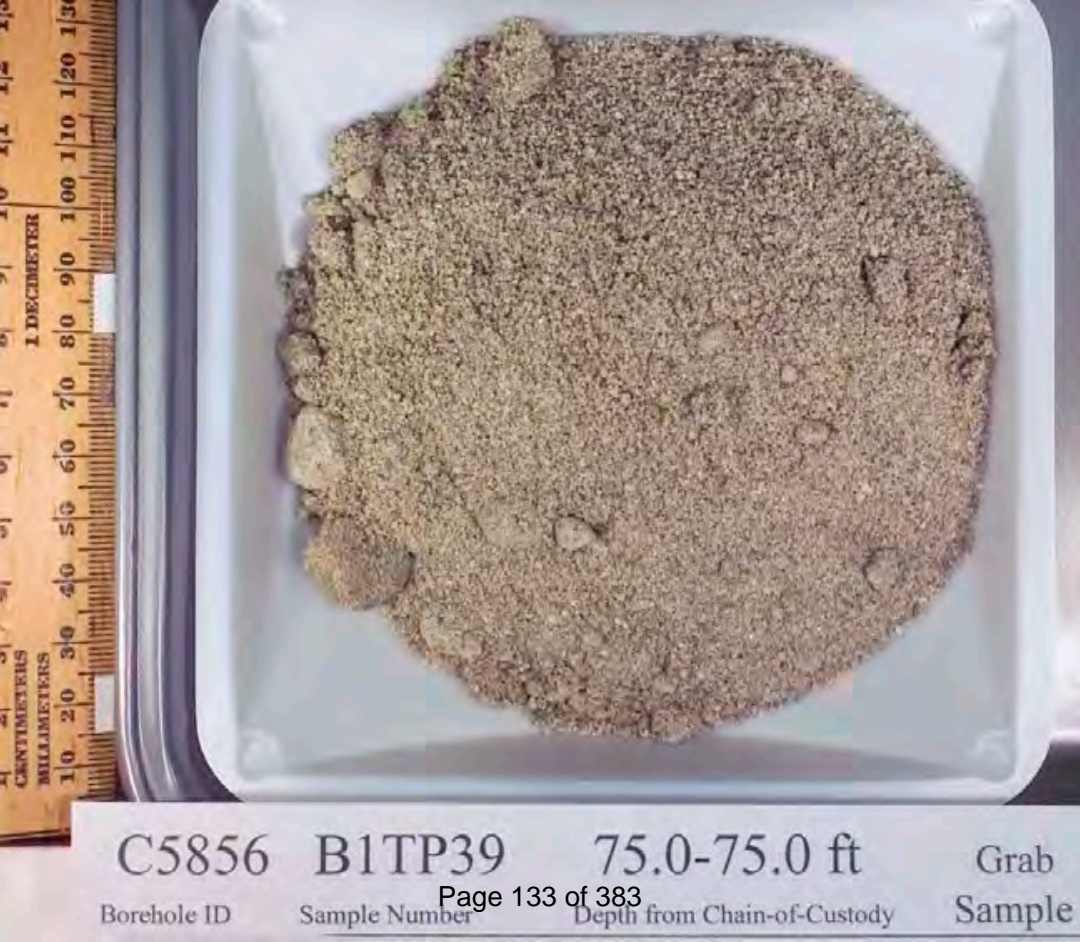




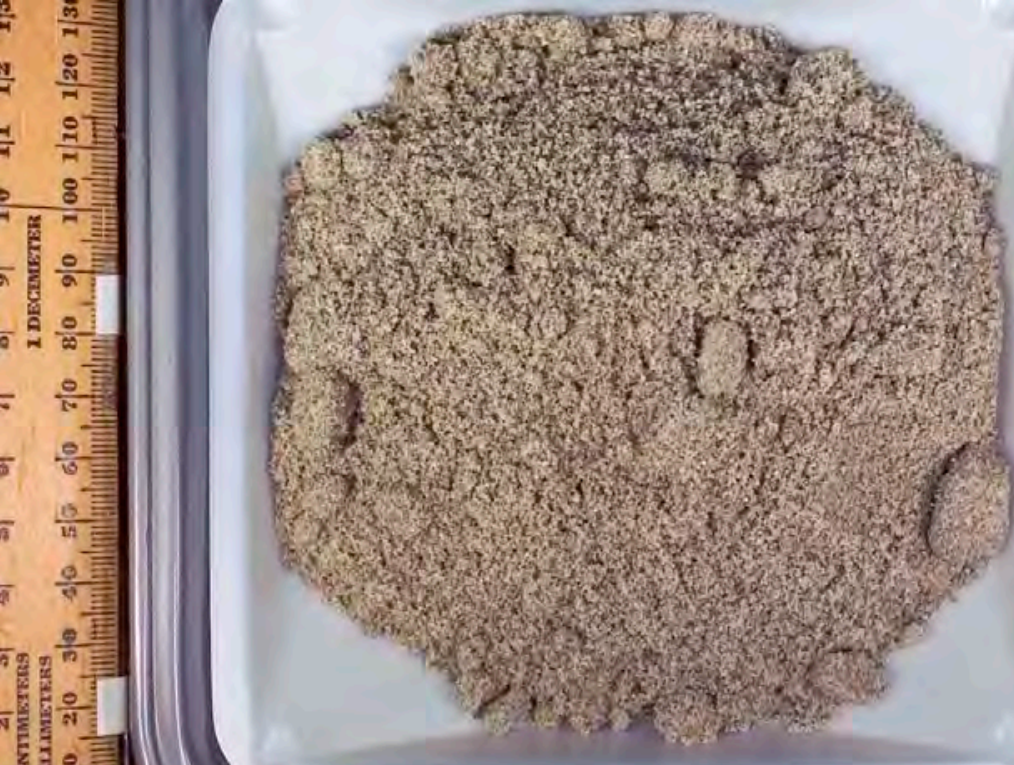

\section{C5856 B1TP41 $80.0-80.0 \mathrm{ft}$}

Borehole ID 


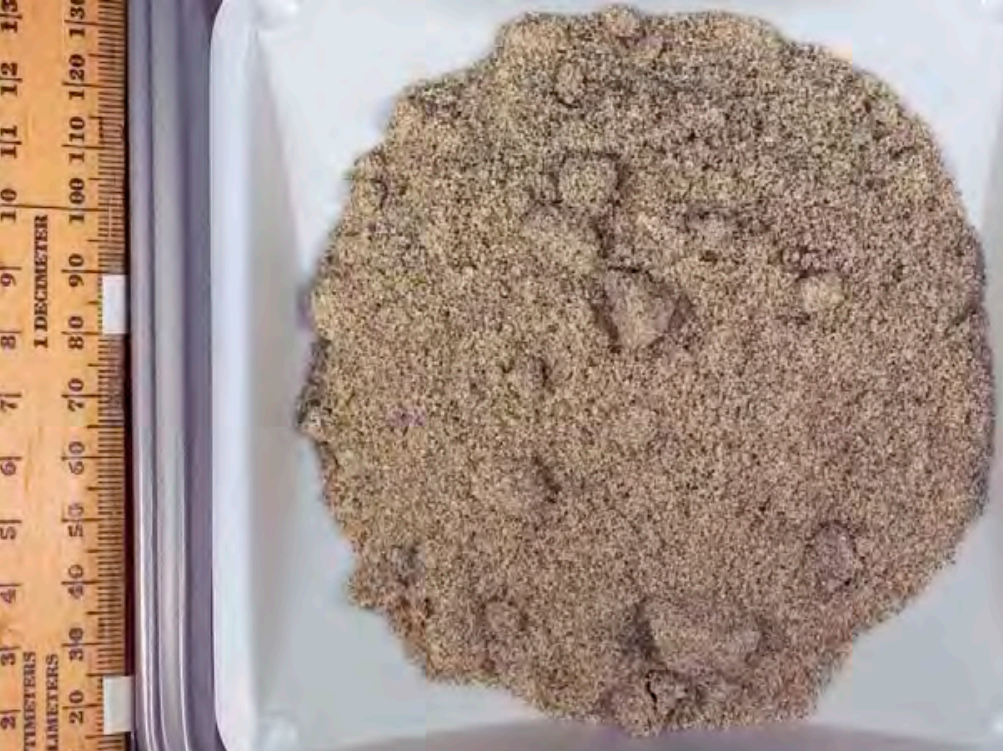

C5856 B1TP42 82.5-82.5 ft

Borehole ID
Sample Number

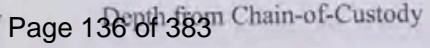

Grab Sample 

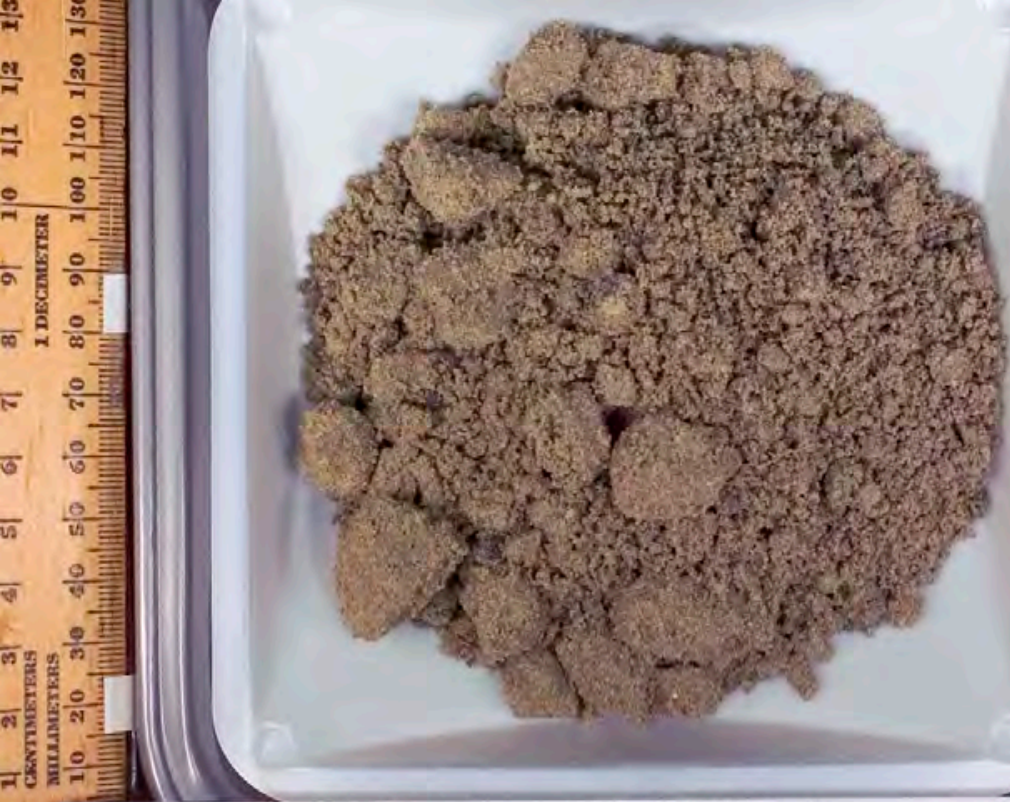

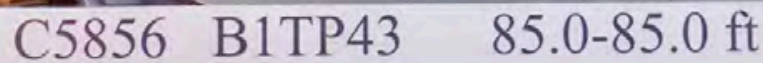

Borchole ID
Sample Numbepage 1370f $3830 m$ Chain-of-Custody
Grab Sample 


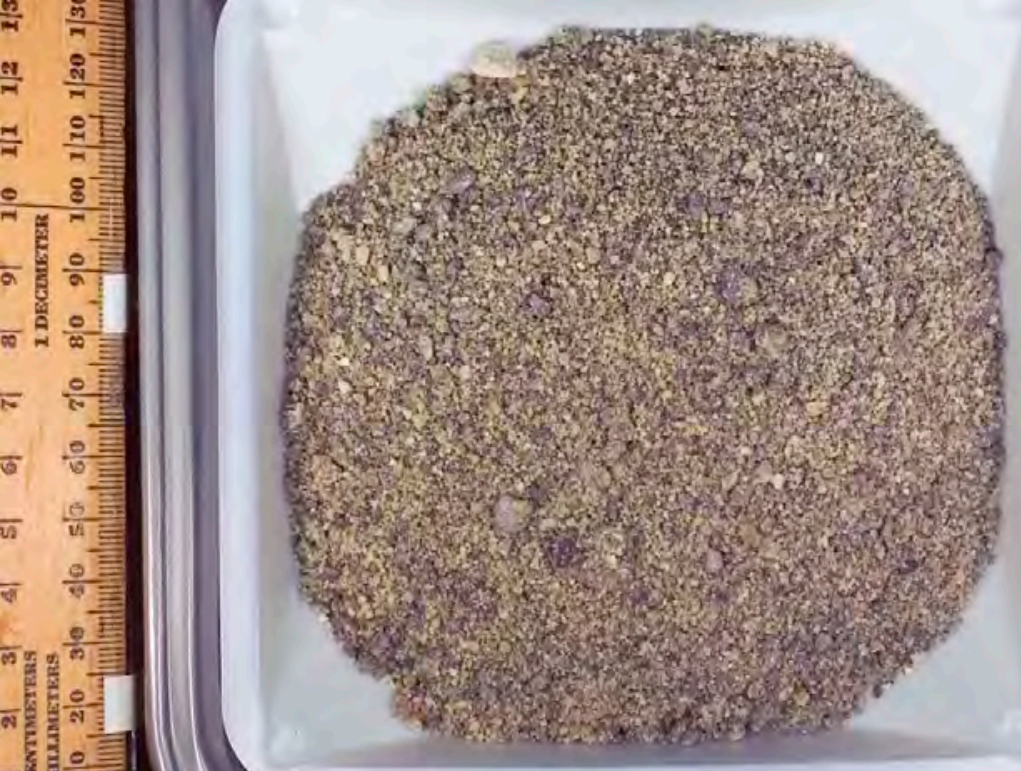

\section{C5856 B1TP45 90.0-90.0 ft}

Borehole ID
Sample NumtRage 139 of 383 rom Chain-of-Custody
Grab

Sample 


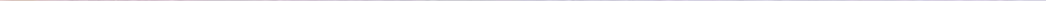




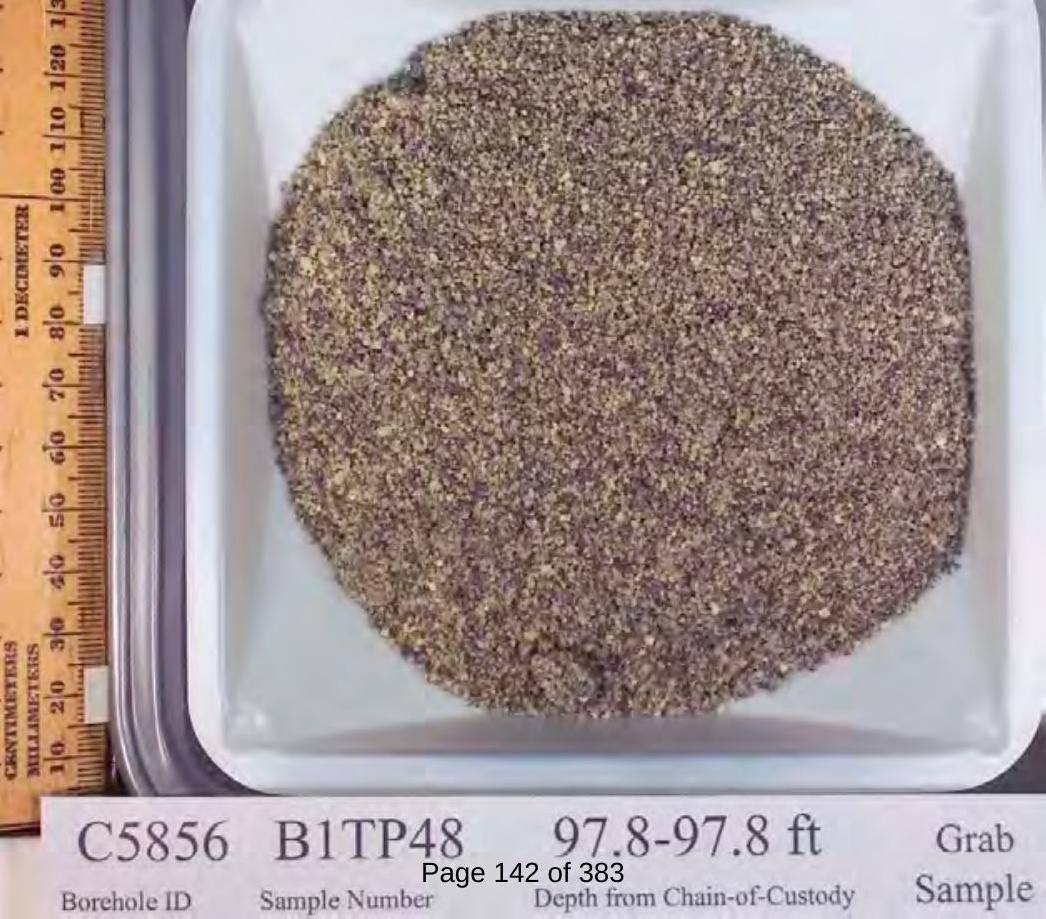






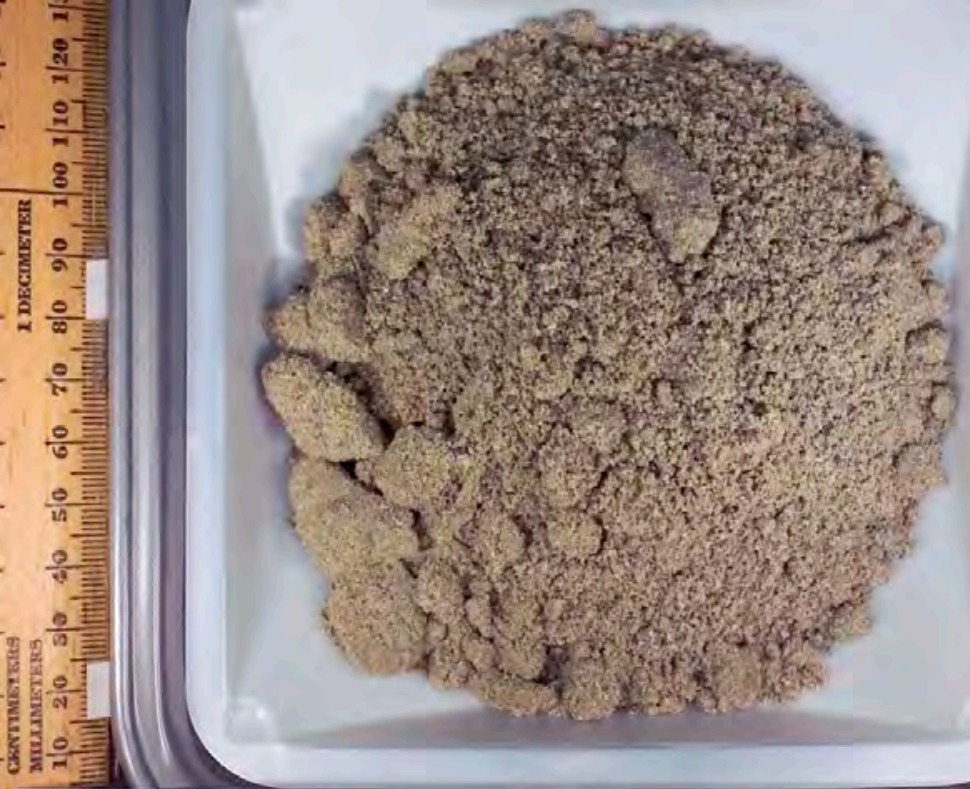
C5856
B1TP51
$105.0-105.0 \mathrm{ft}$
Borehole ID
Sample Number
Page 145 of 383 Chain-of-Custody
Grab Sample 


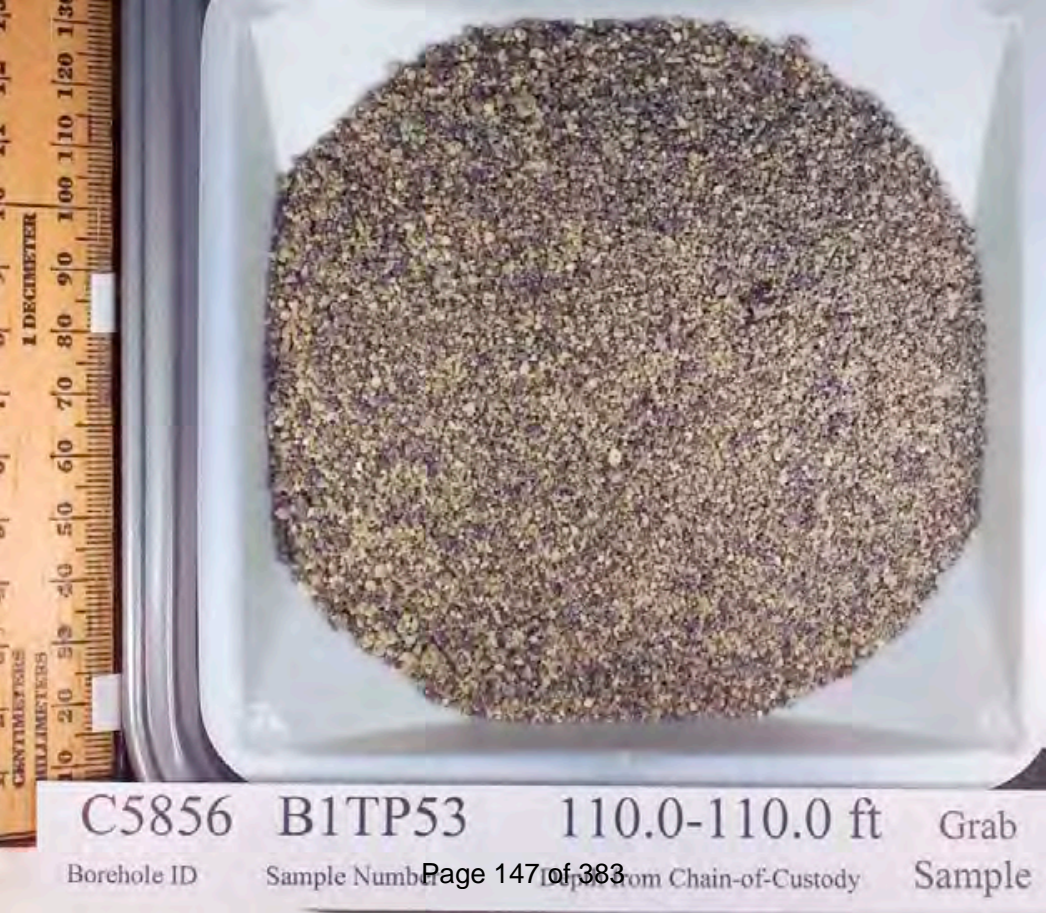




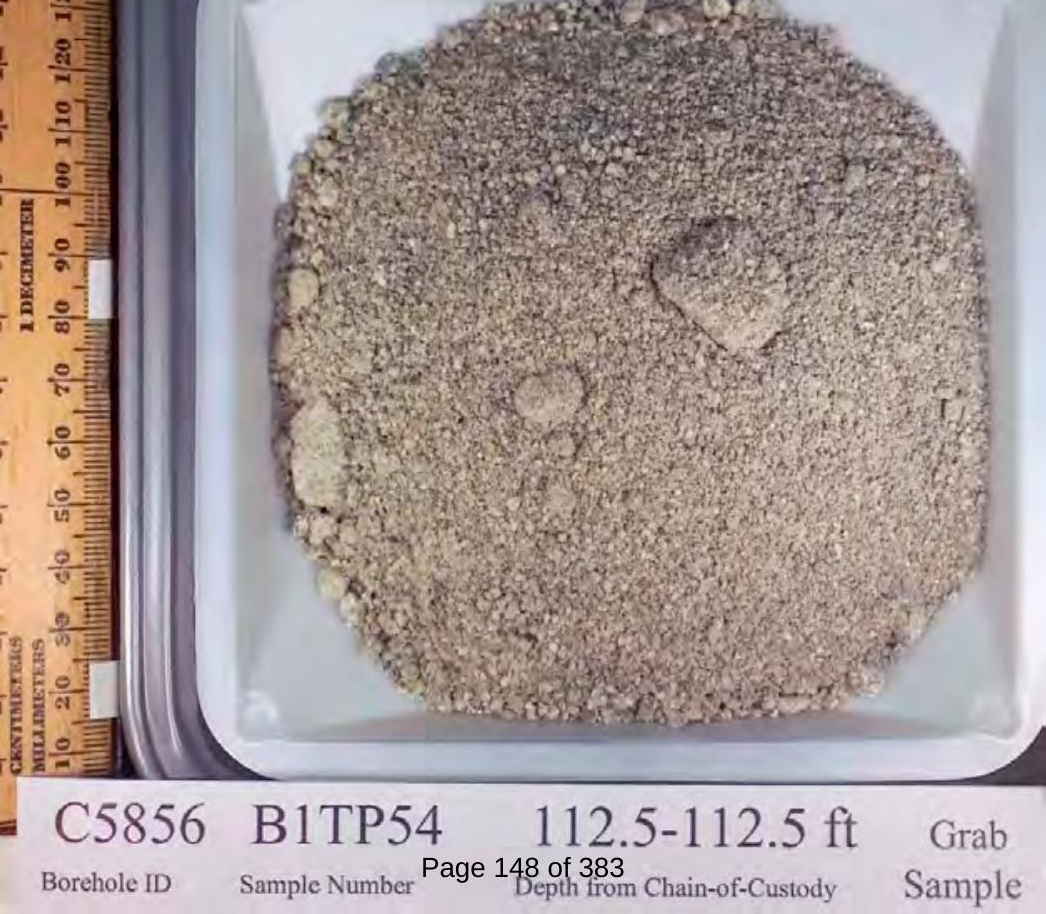




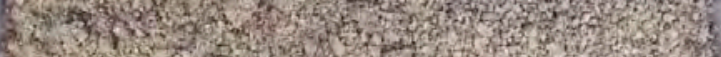

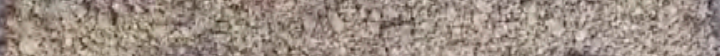

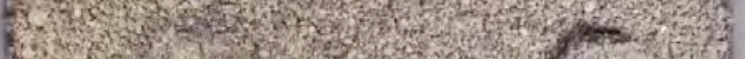
835 3. 20. in

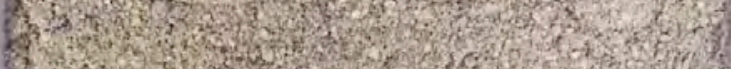

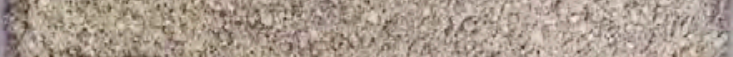
B.7.0. 3. 1 .

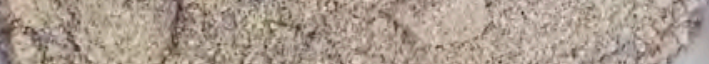

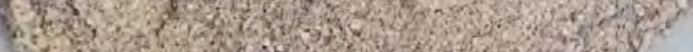
2.

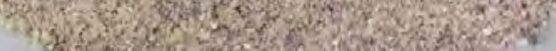

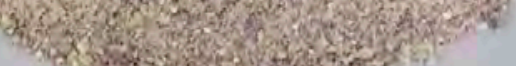

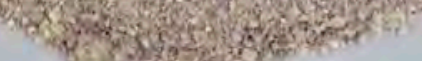

C5856 B1TP55 115.0-115.0 ft

Borehole ID
Sample Number
Page 149 of 383

49 of 383 m Chain-of-Custody
Grab Sample 


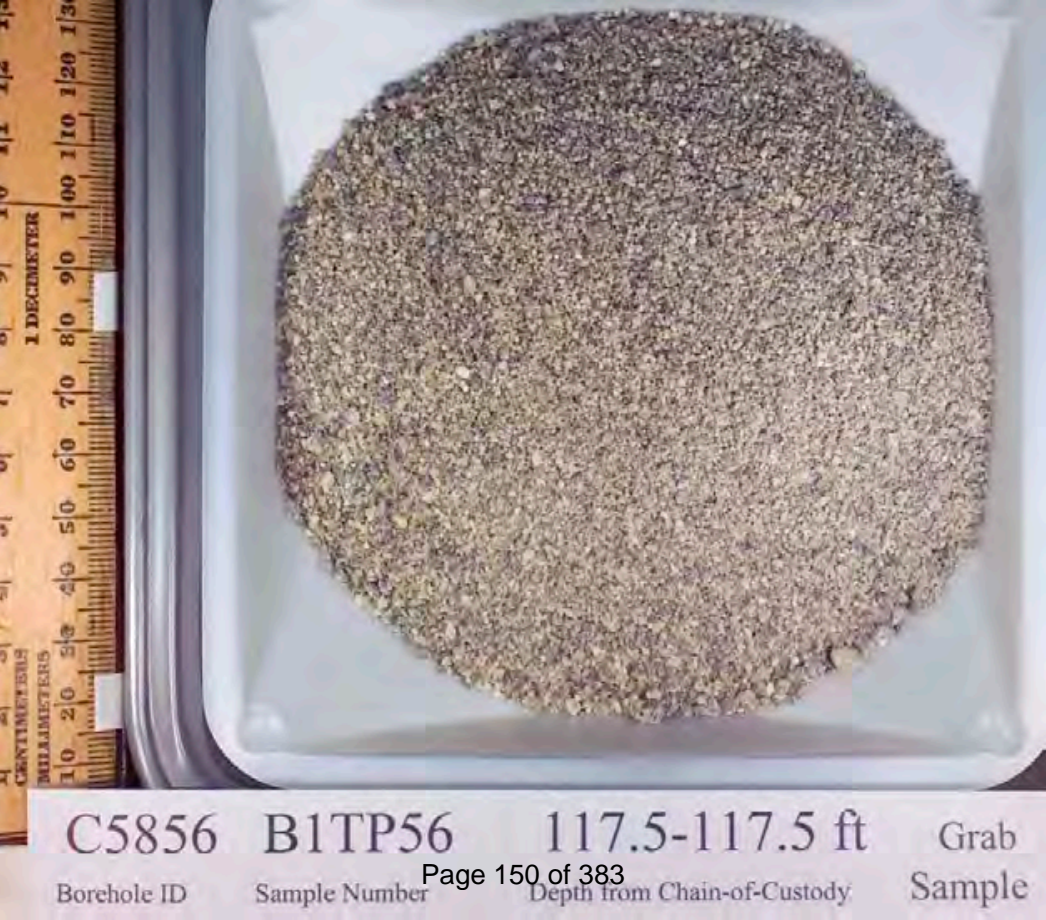




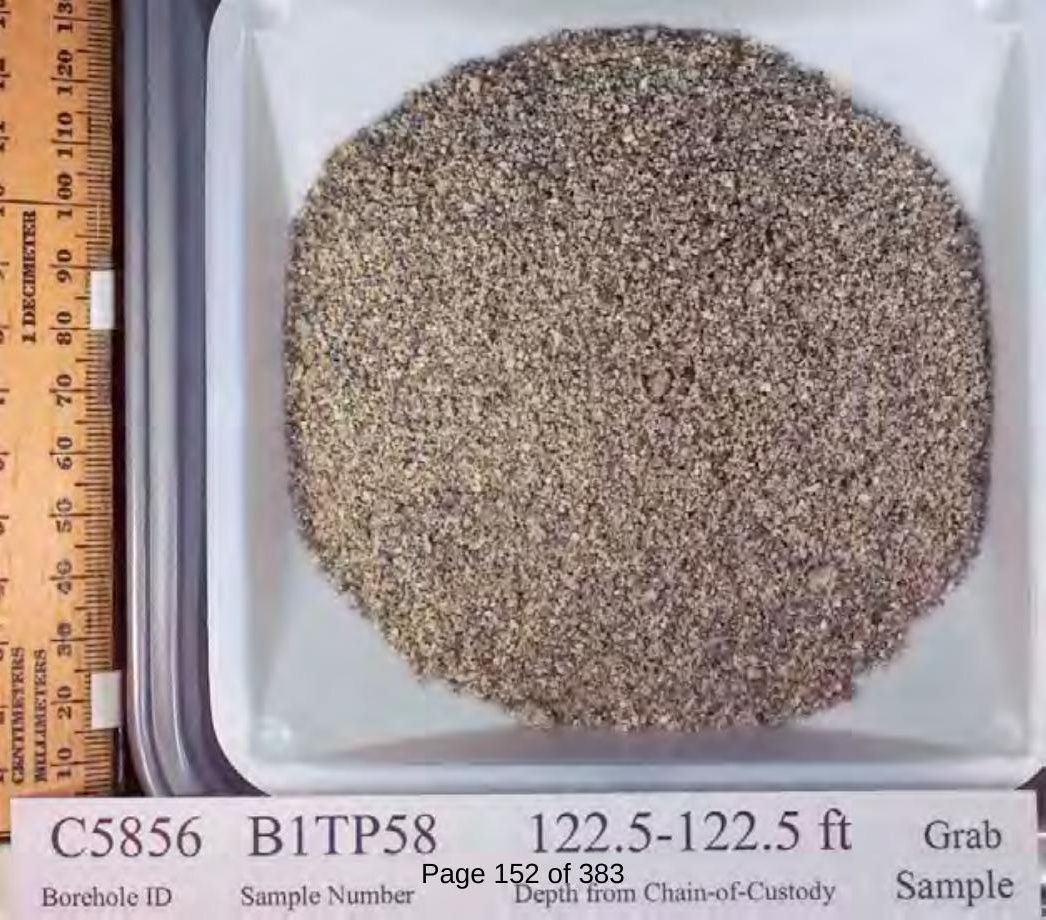




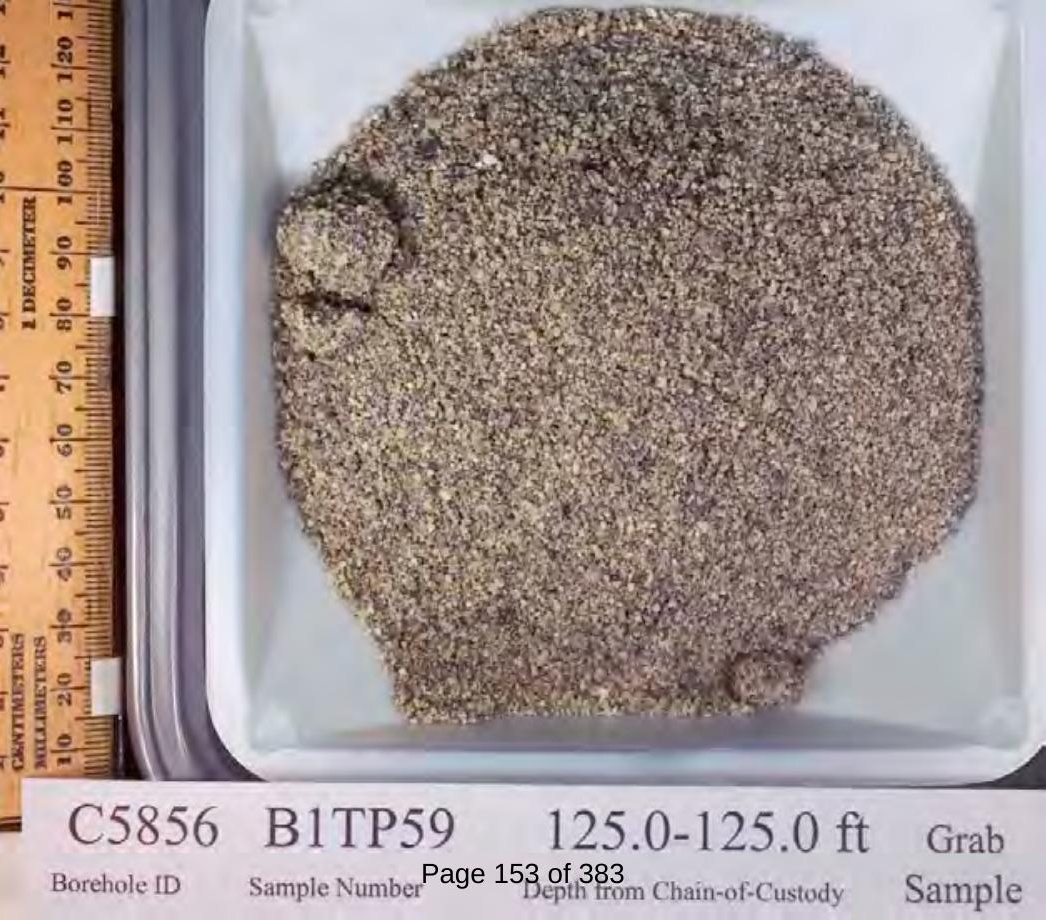




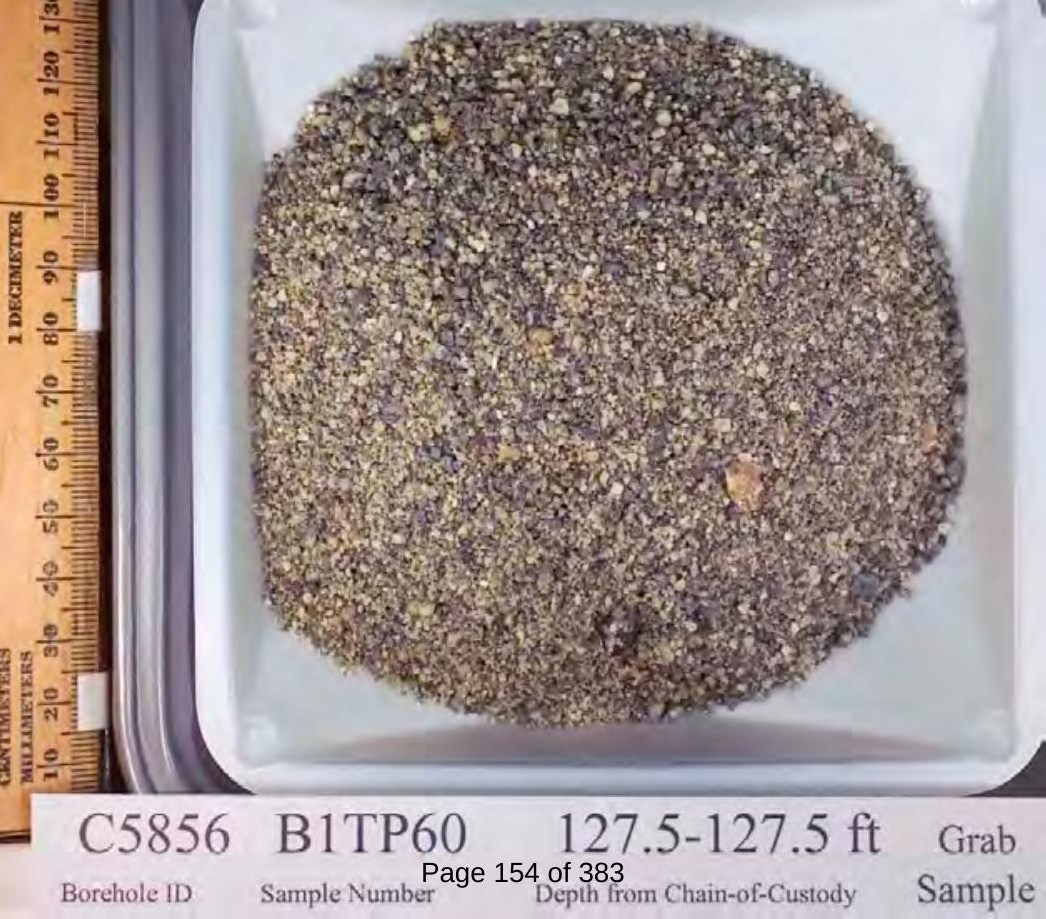




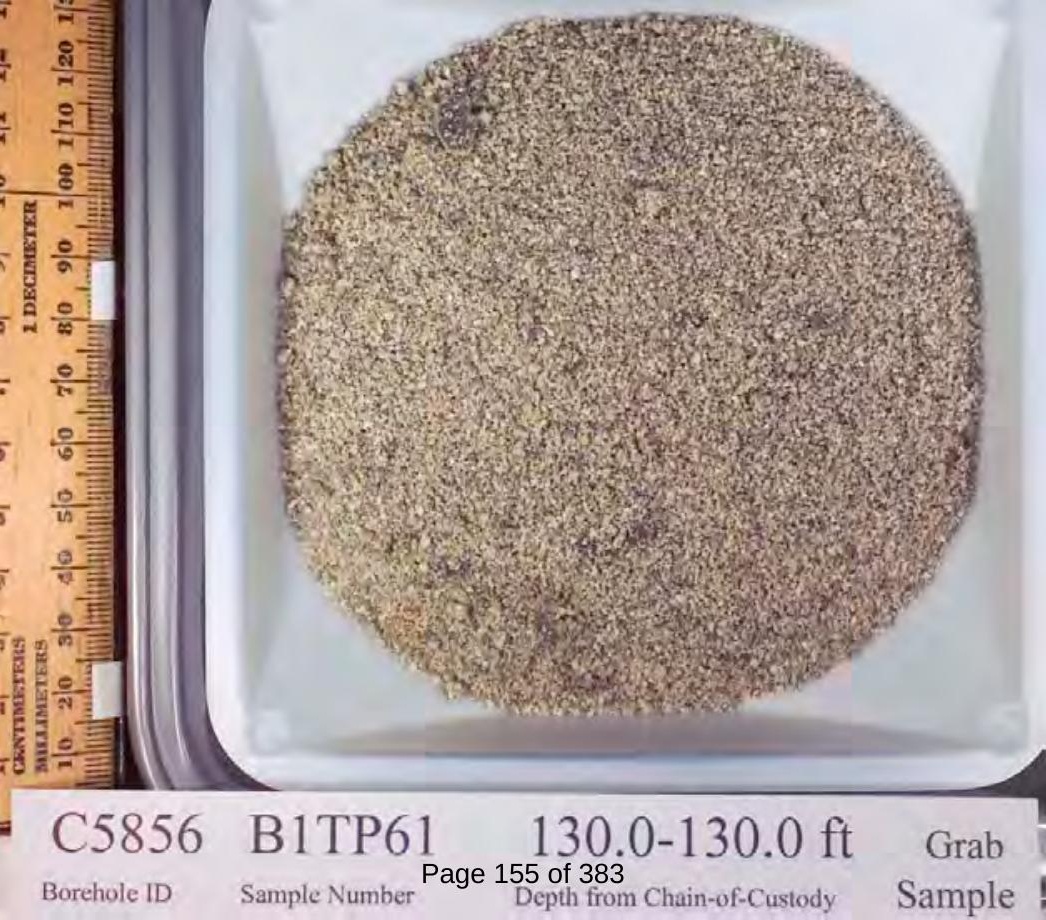




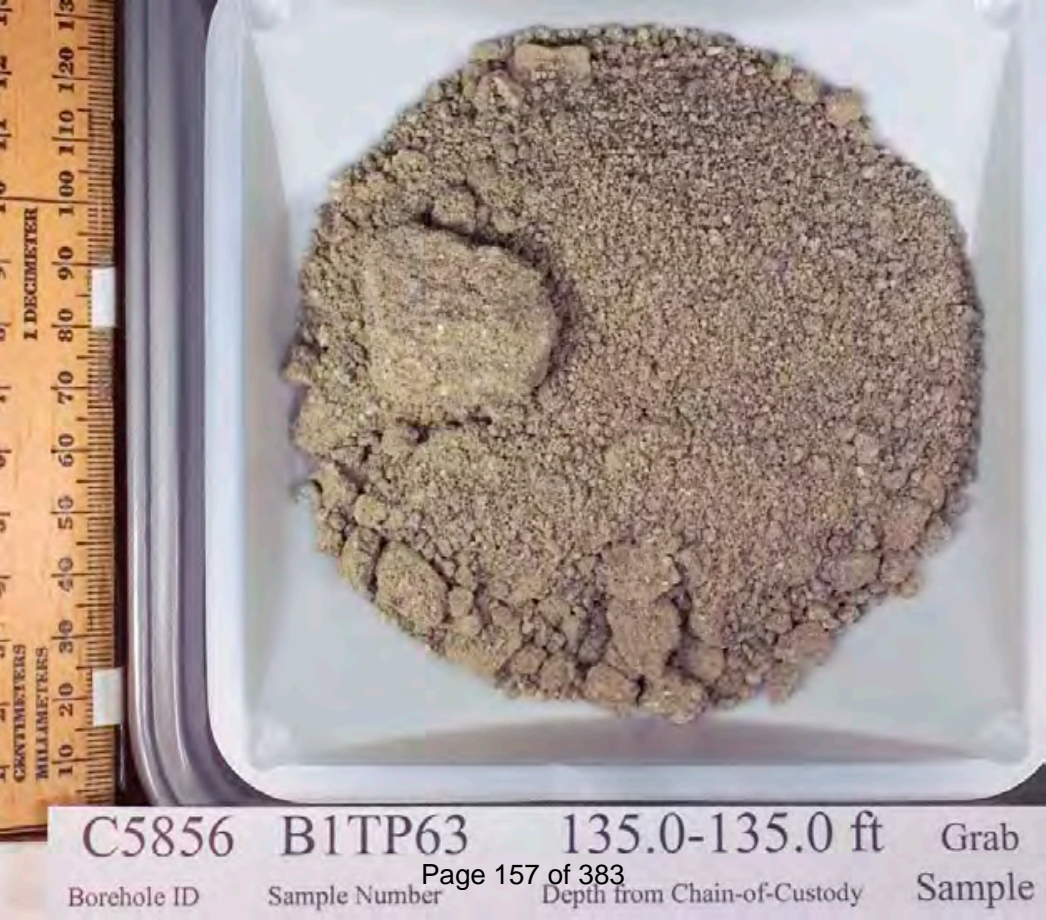




Q5ing 3.5.

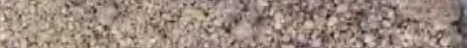

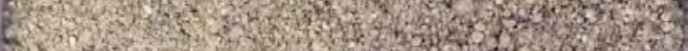

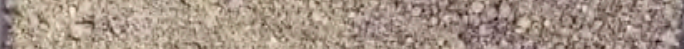

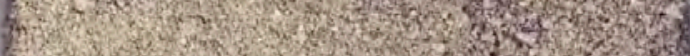

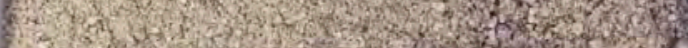

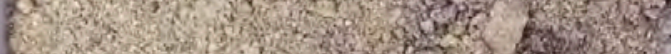

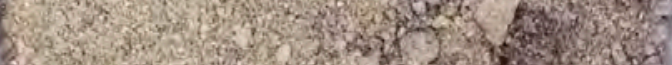
3 S.

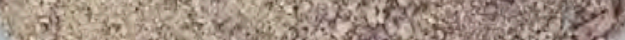

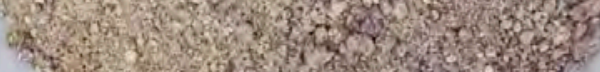
Py s.

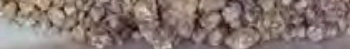

C5856

Borehole ID

\section{B1TP65} Pr
$140.0-140.0 \mathrm{ft}$ Sample Number
Page 159 of 383

Depth from Chain-of-Custody
Grab Sample 


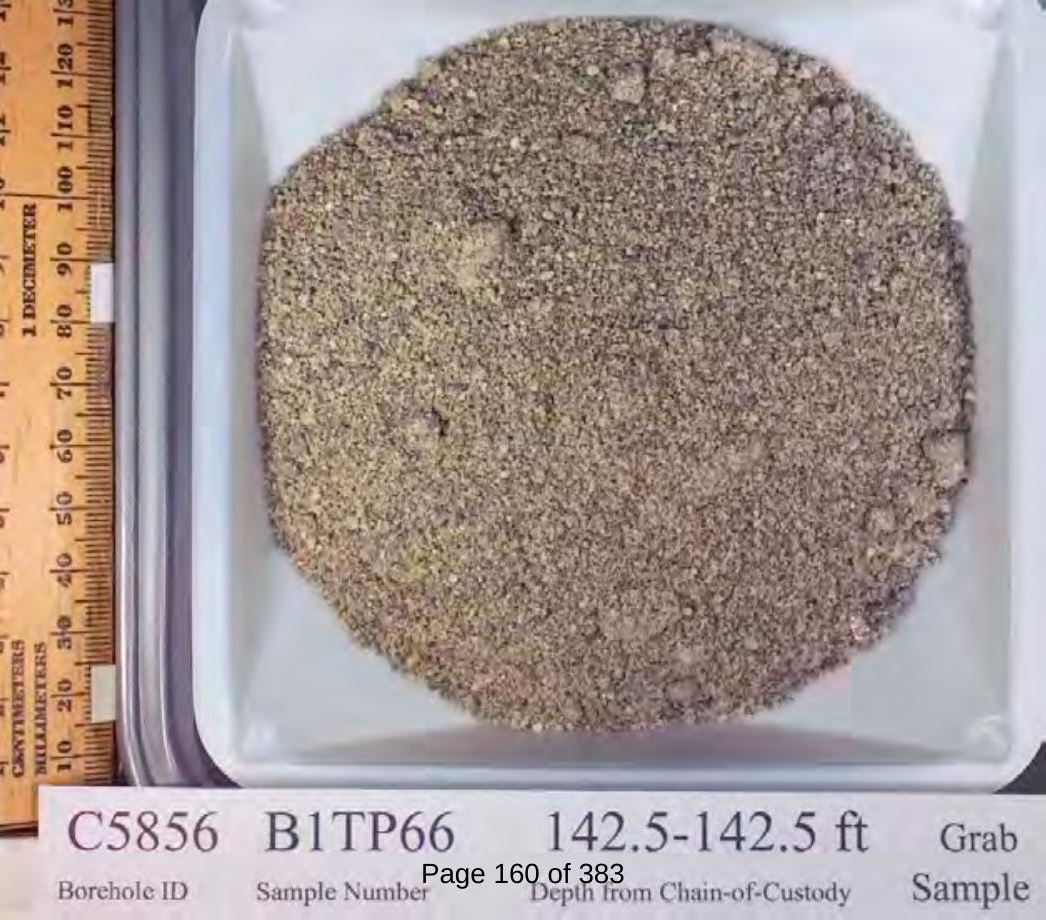




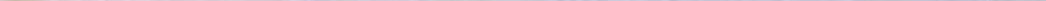




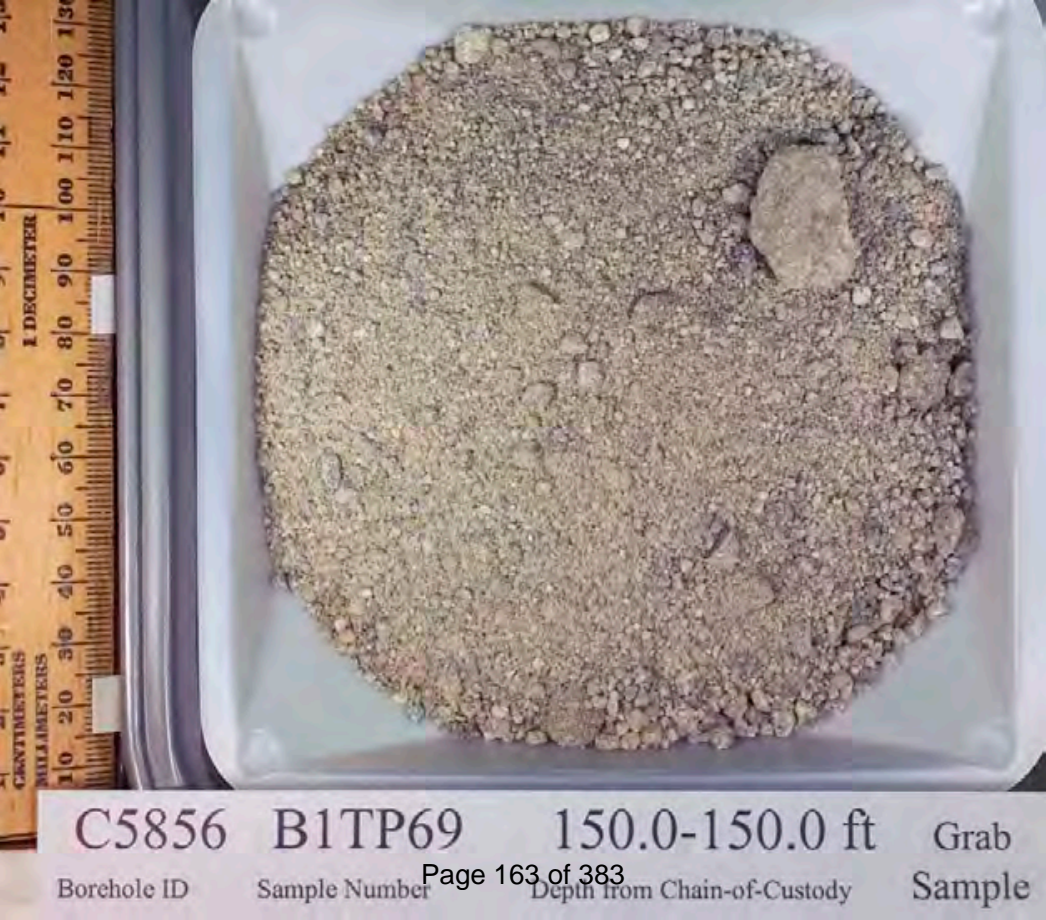




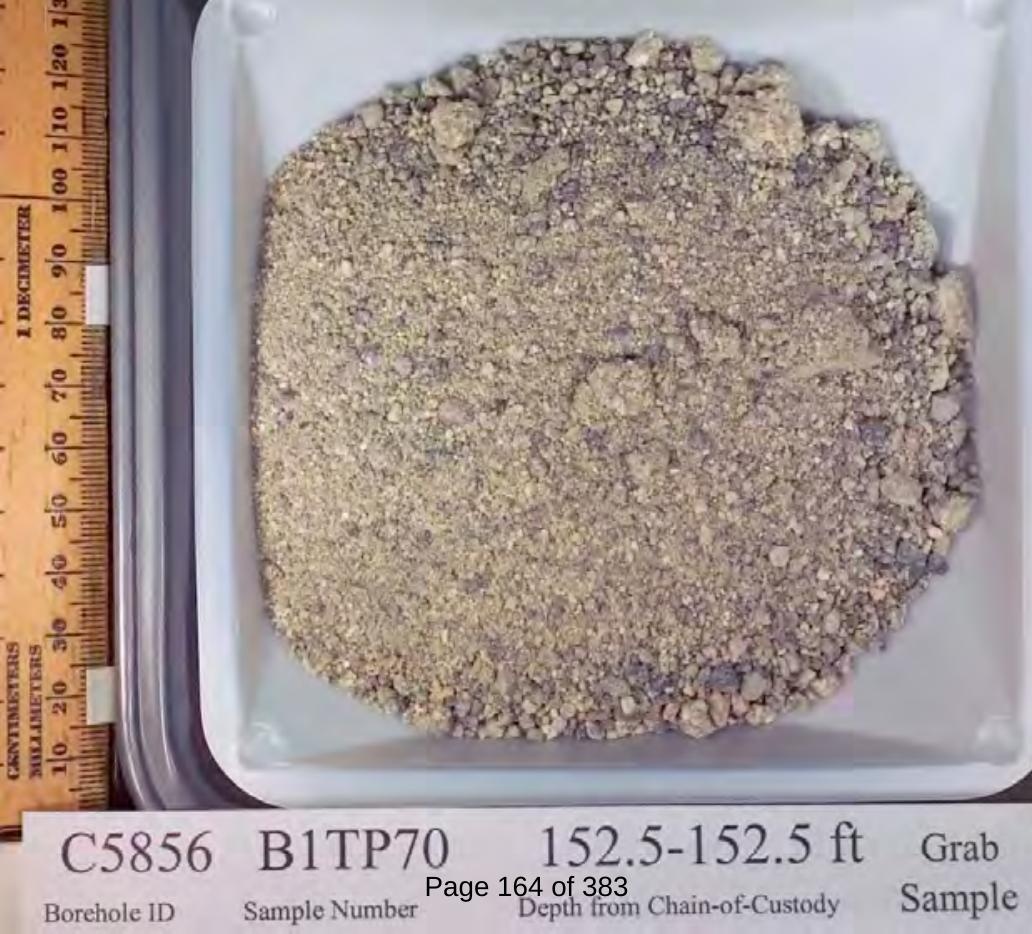




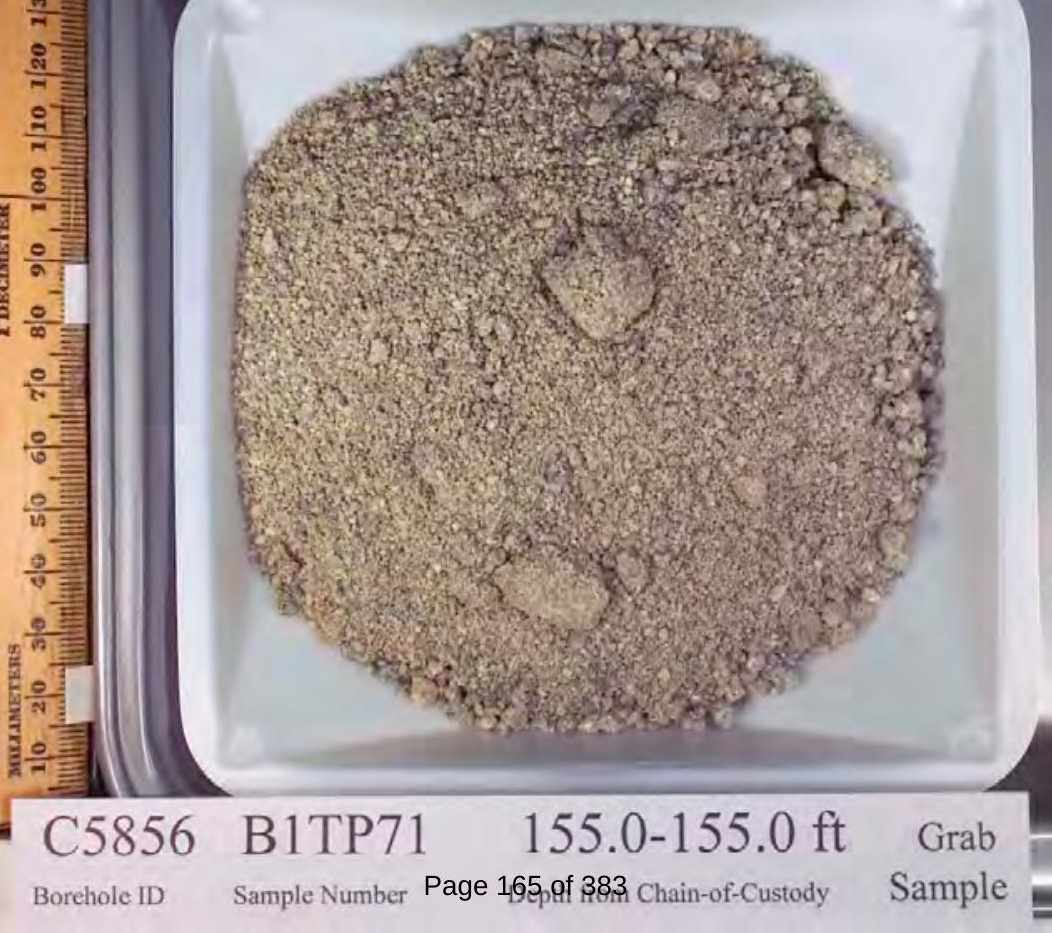




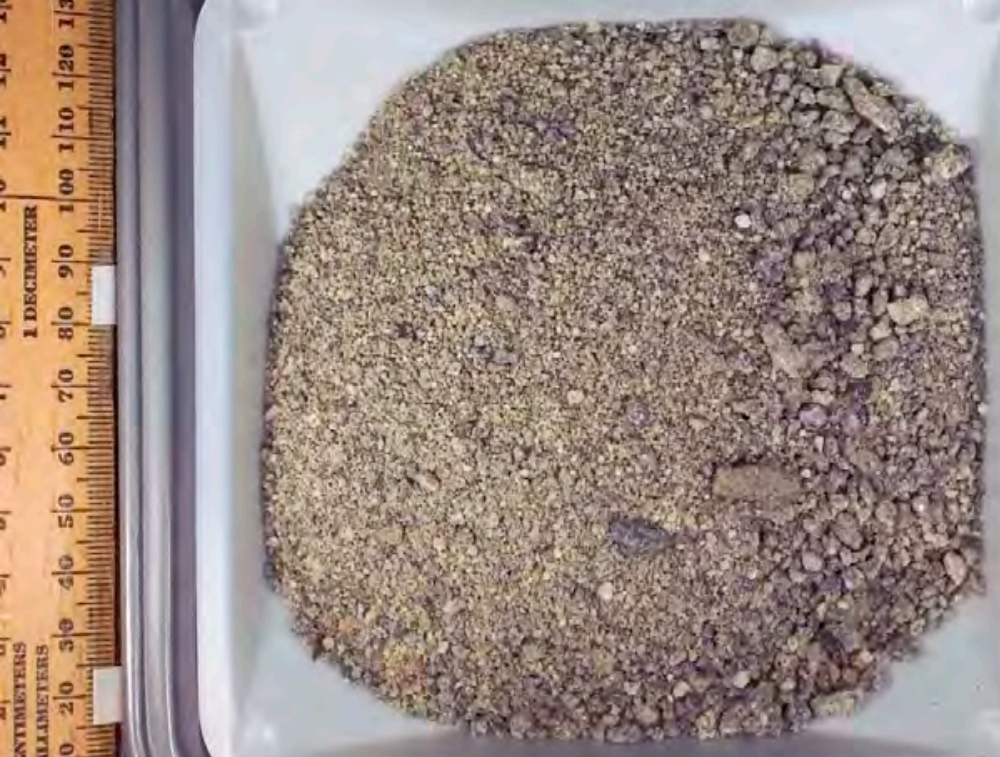
C5856
B1TP72
$157.5-157.5 \mathrm{ft}$

Grab

Borehole ID

Sample NumberPage $166^{\circ} \mathrm{ofl}^{3} 8^{\mathrm{m}}$ Chain-of-Custody Sample 


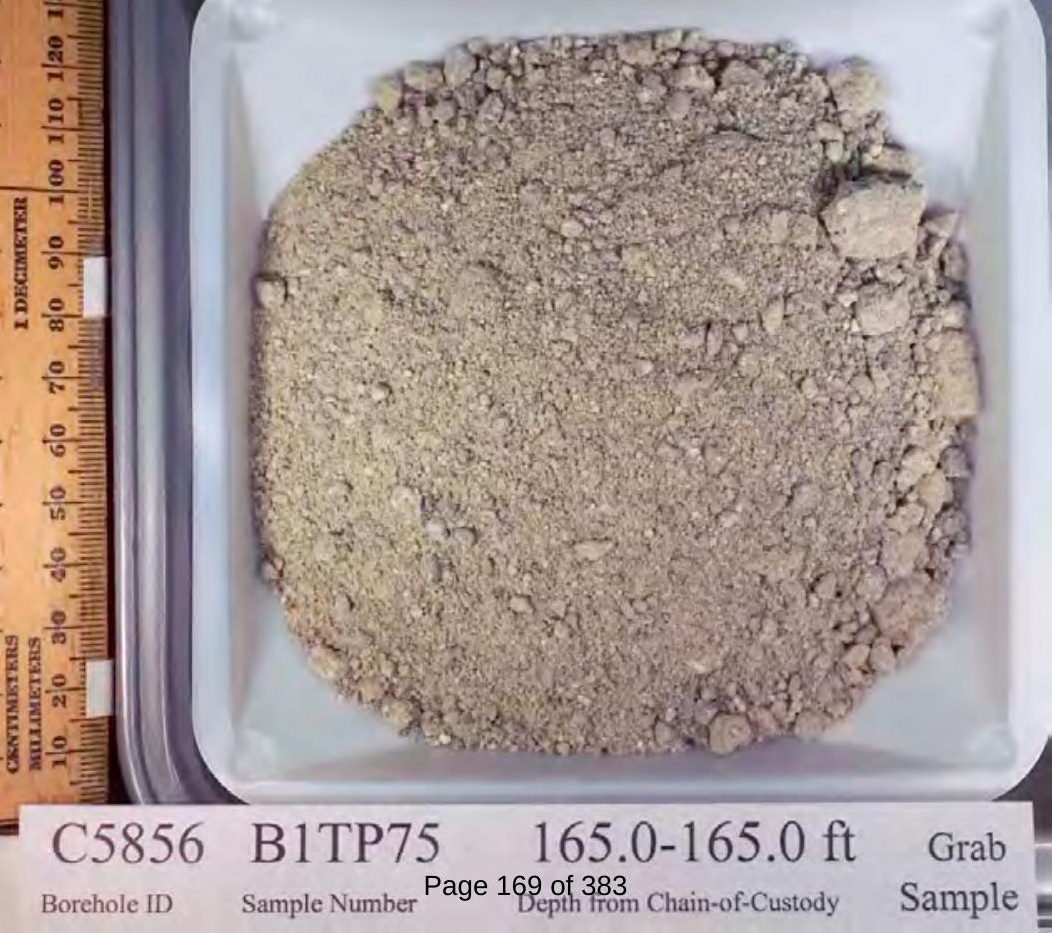




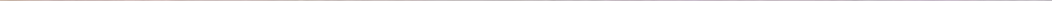




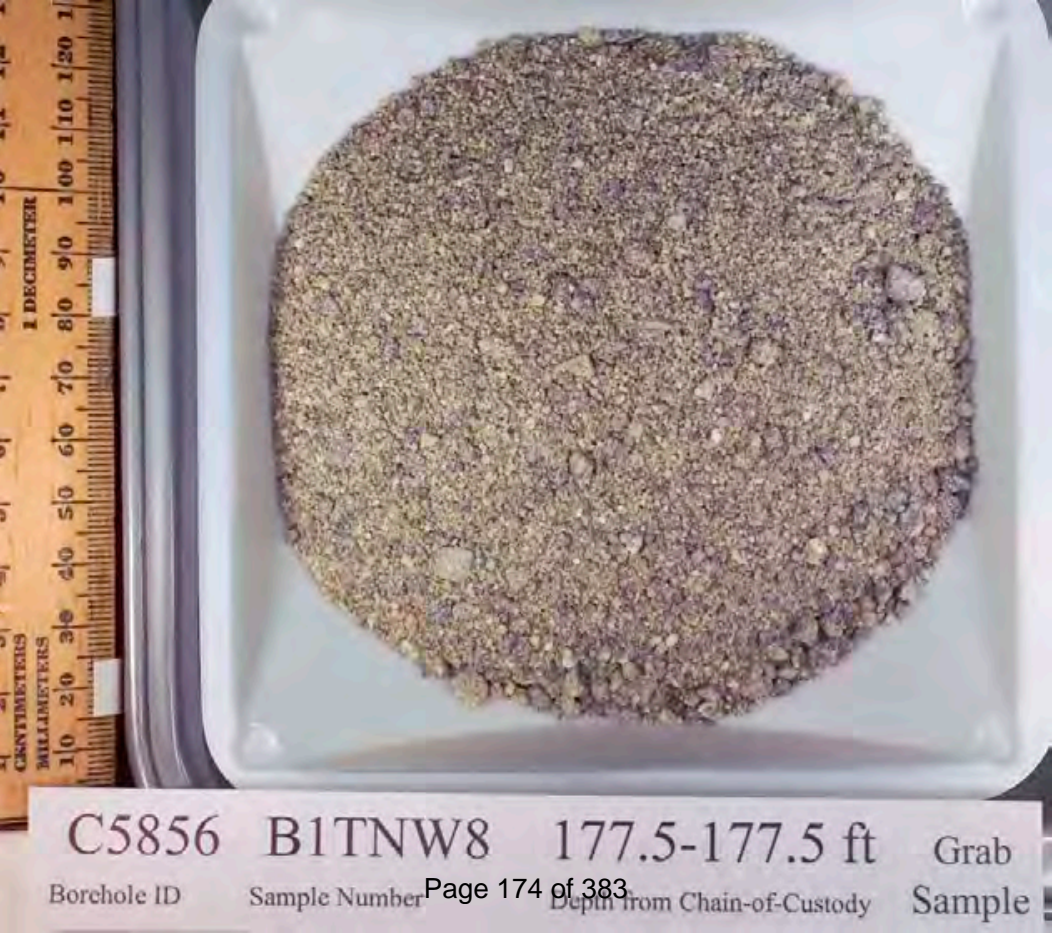




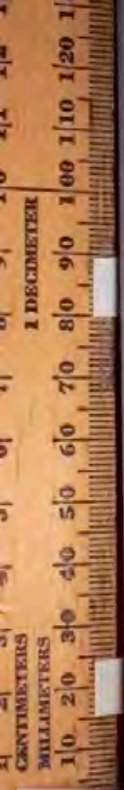

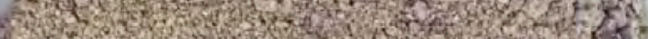

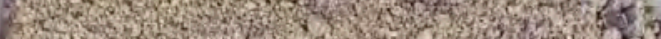

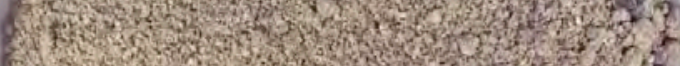

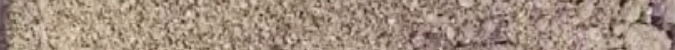

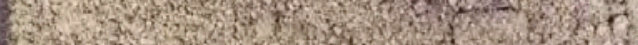

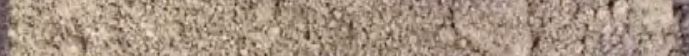

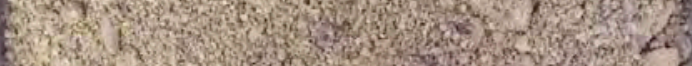

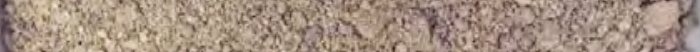

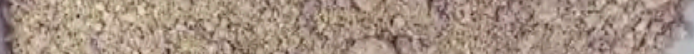
8.

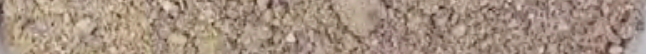

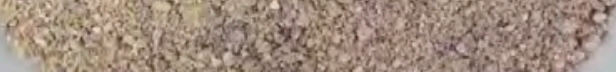

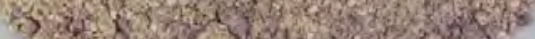

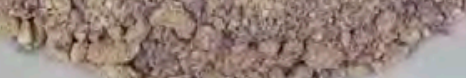

C5856 B1TP78 $180.0-180.0 \mathrm{ft}$

Borehole ID Sample Number Page 175 pf 383 Chain-of-Custody Sample 


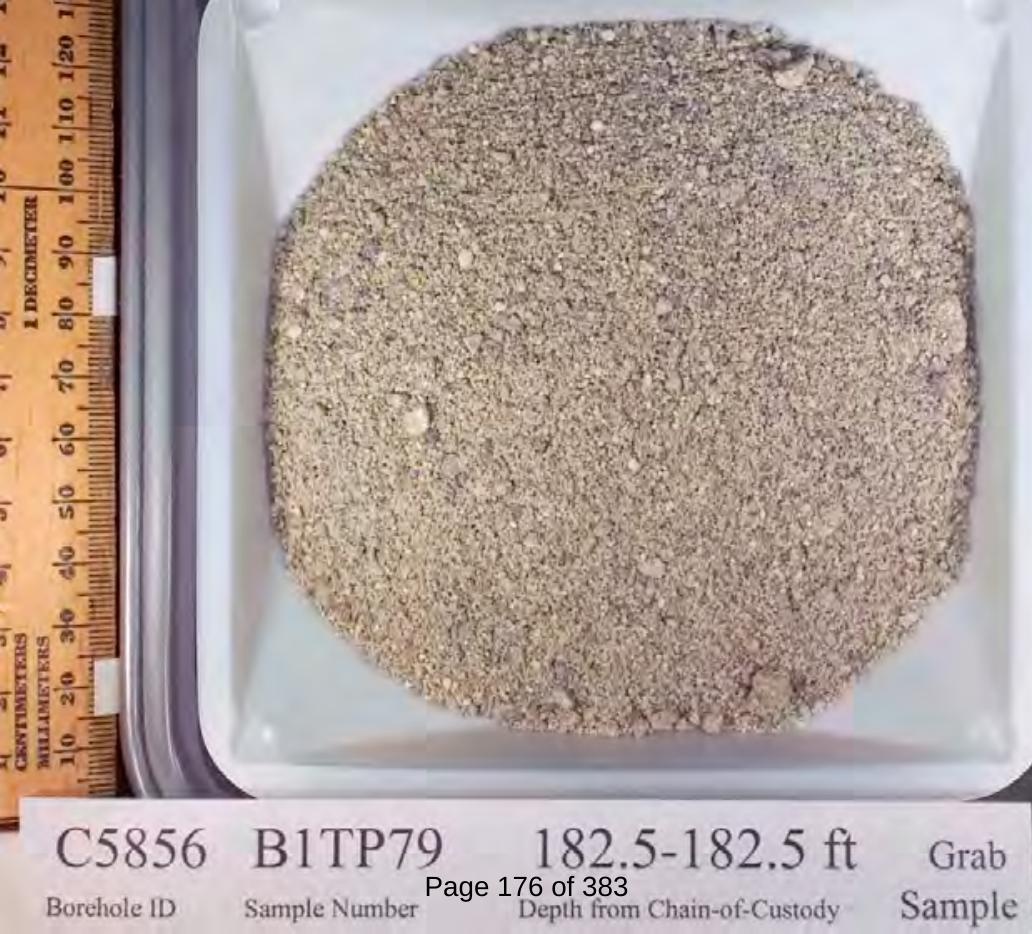




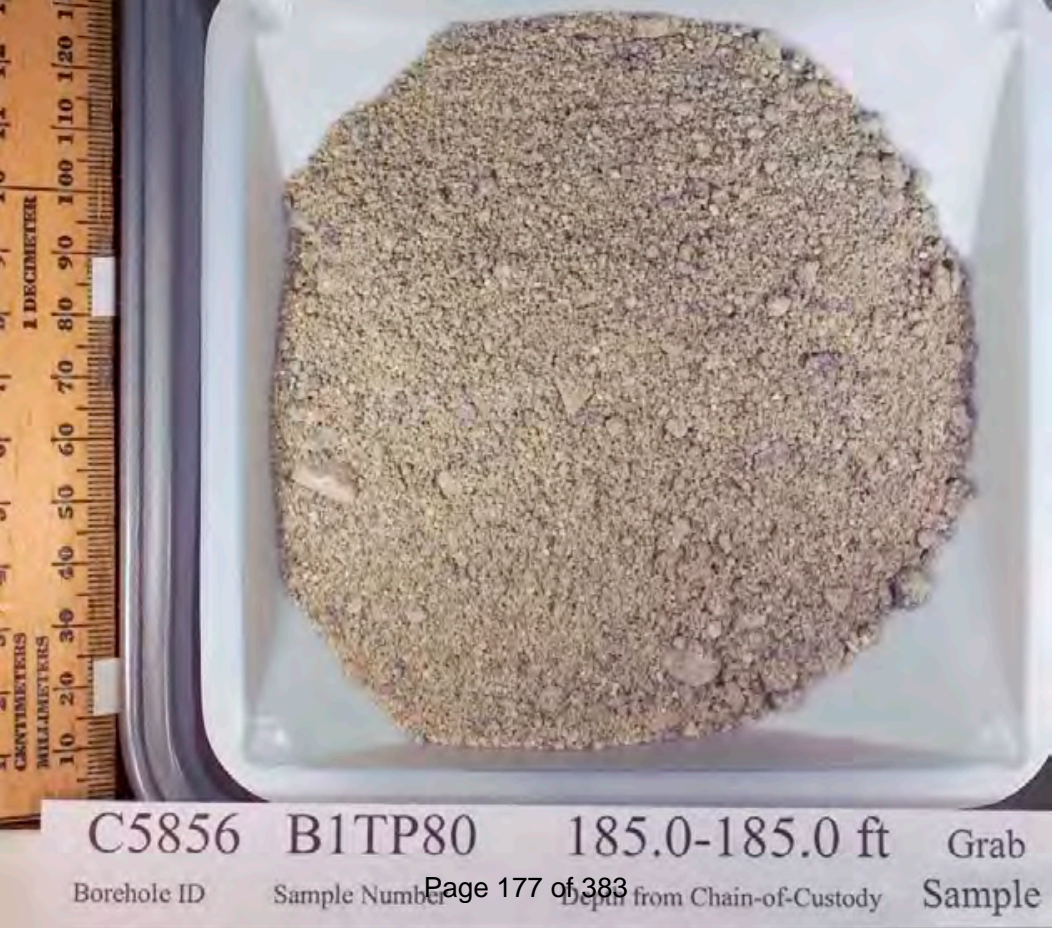




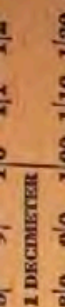
\$.15-2.

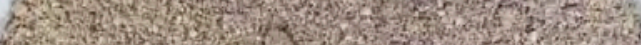

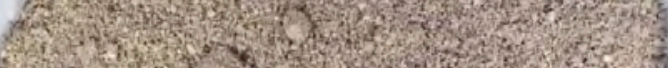

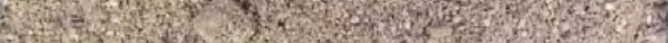

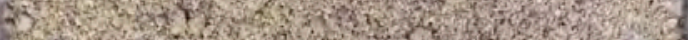

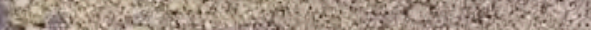

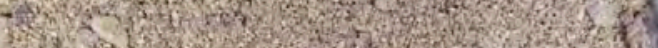
Hath

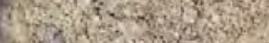

20. Whet

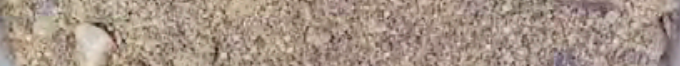

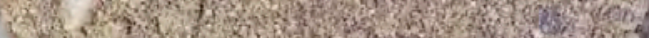
W.

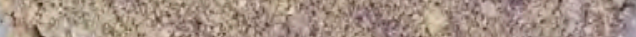
P.

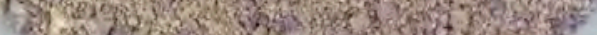

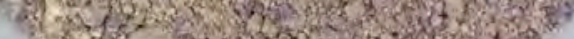
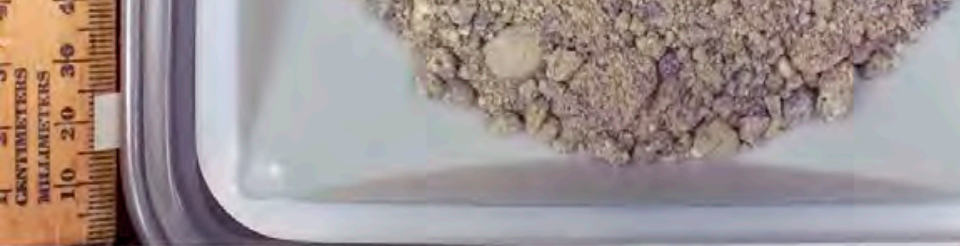

C5856 B1TP81

Borehole ID
Sample Number
$187.5-187.5 \mathrm{ft}$

Depth from Chain-of-Custody
Grab Sample 


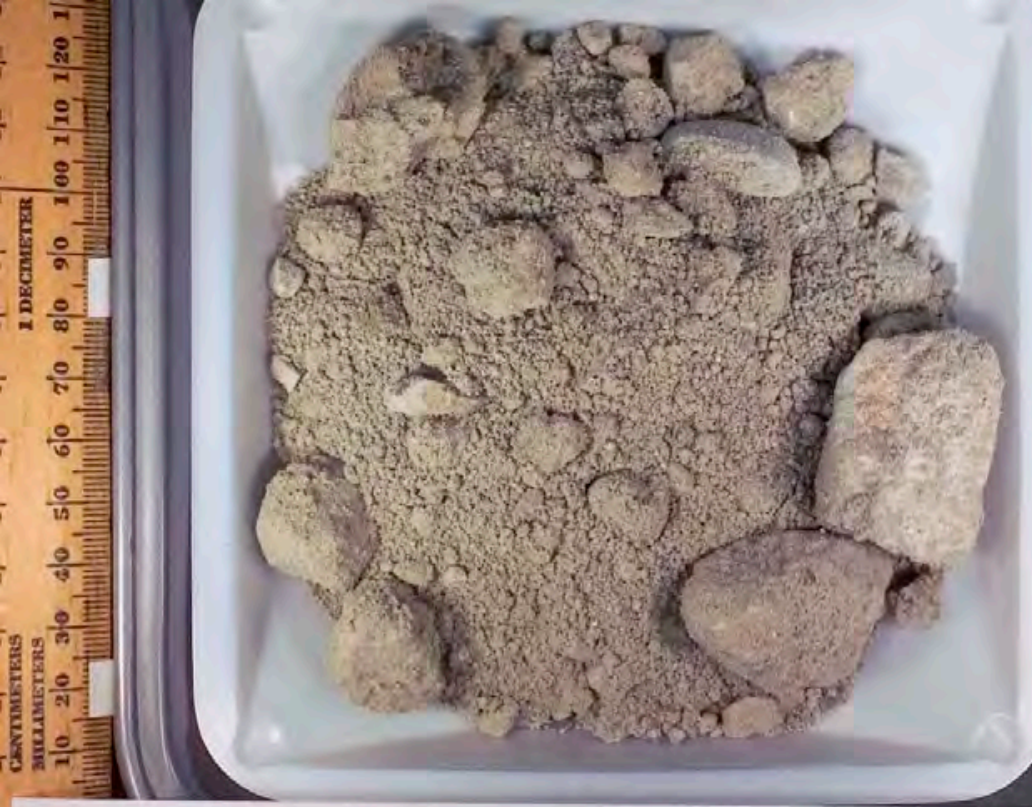

\section{C5856 B1TP84 195.0-195.0 ft \\ Borchole ID \\ Sample Number \\ Page 181 of 383 \\ Depth from Chain-of-Custody \\ Grab \\ Sample}


$\frac{5}{4}$

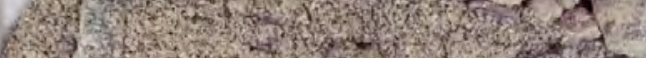

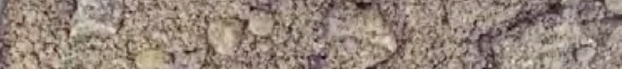
F. W. Q.

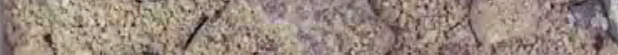

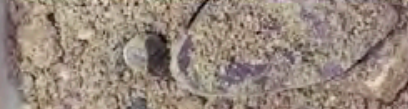

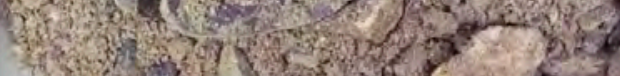

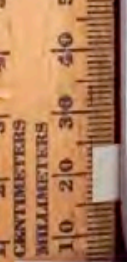
C5856 


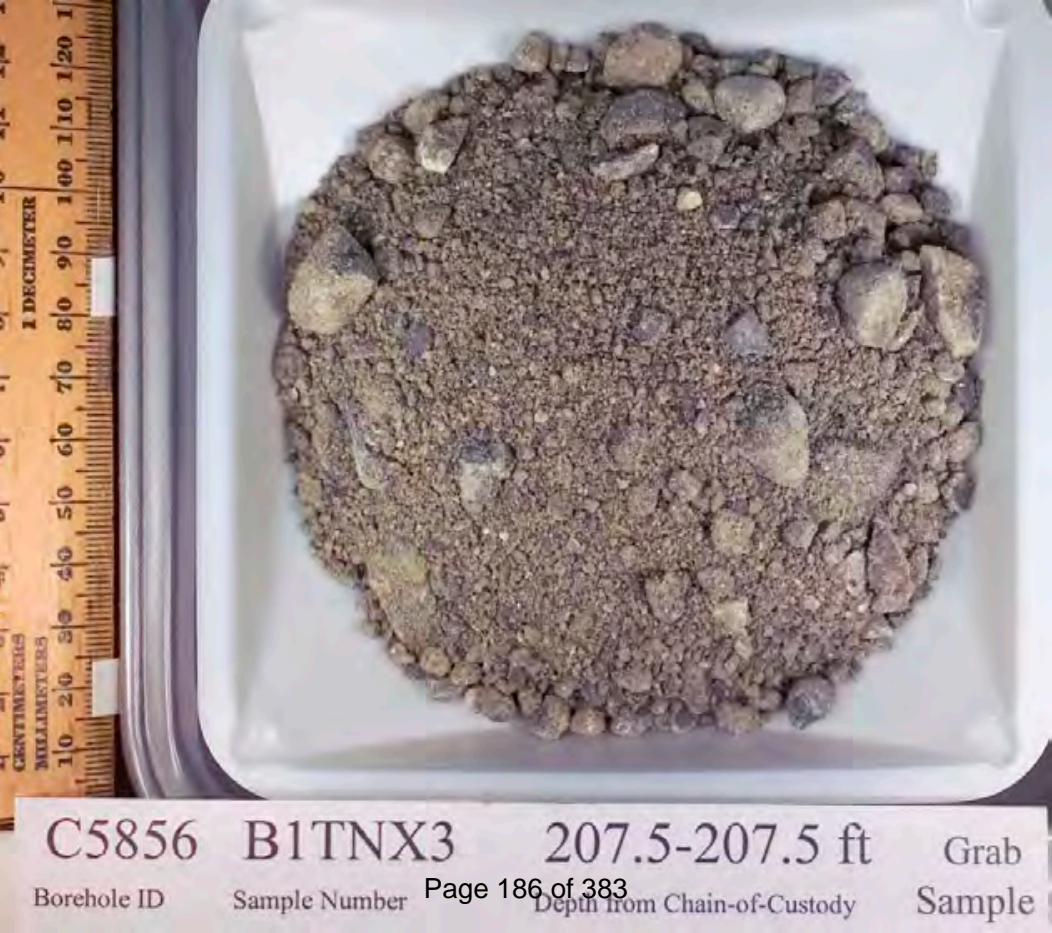




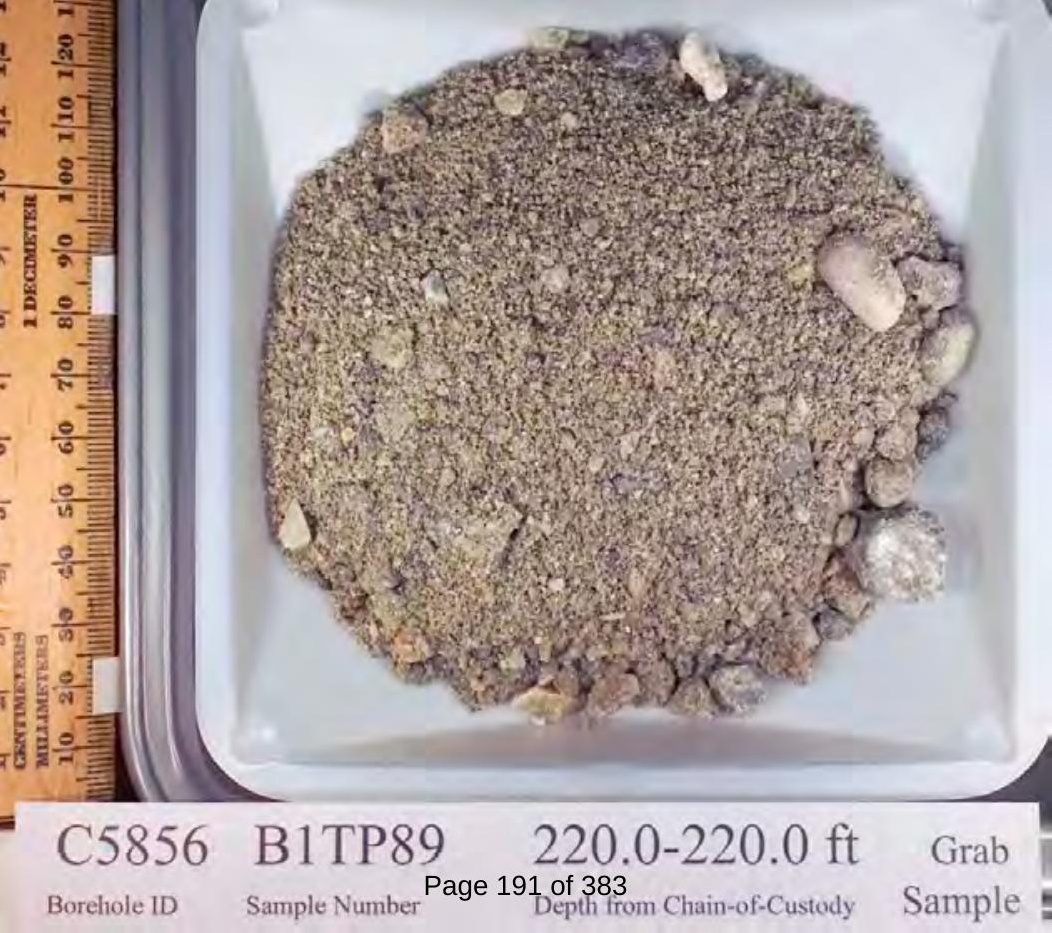




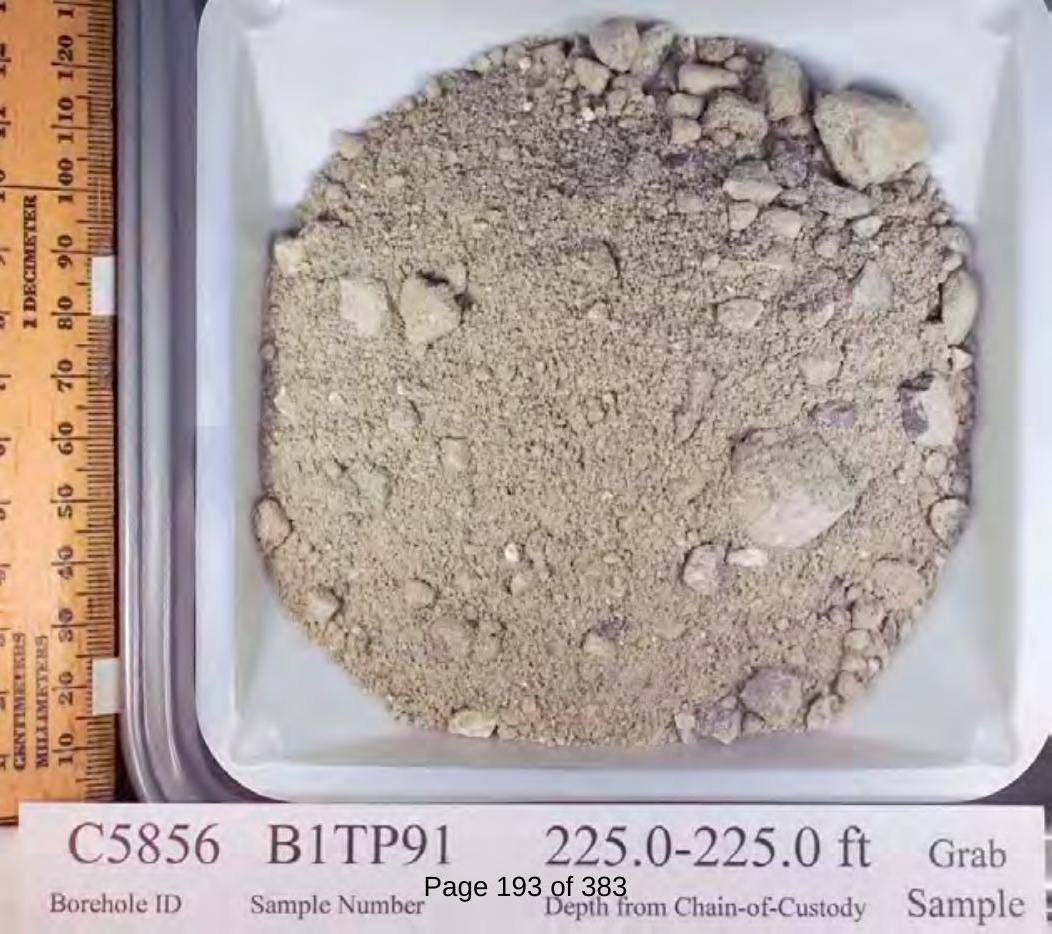




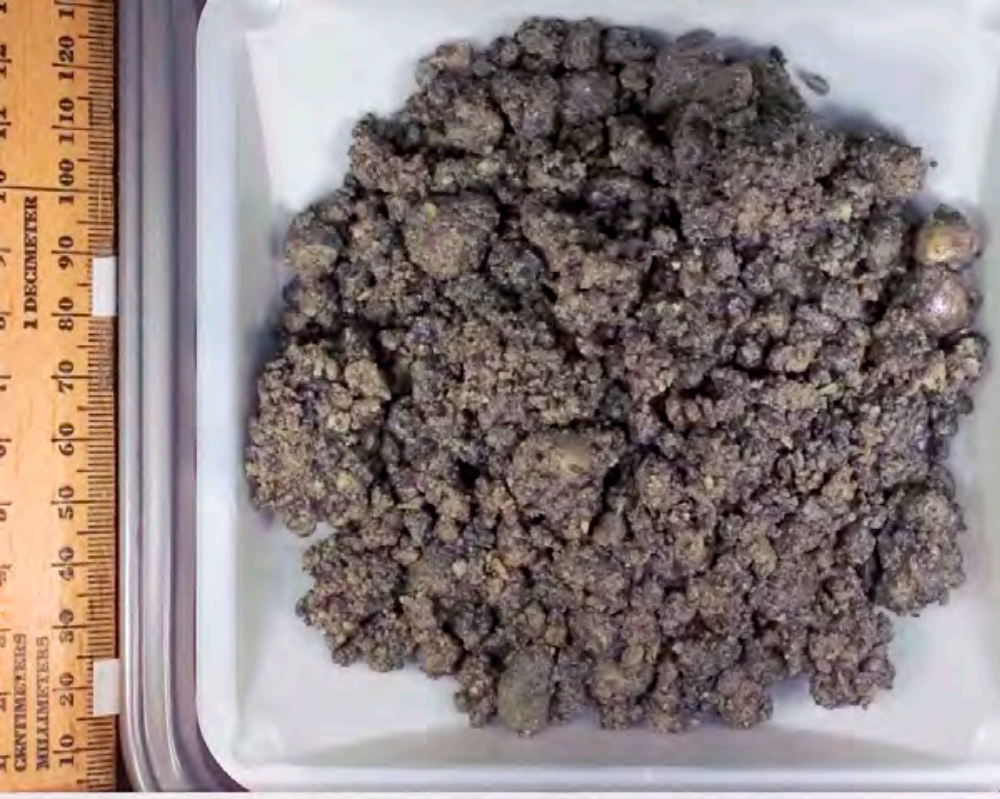

\section{C5856 B1TP94 \\ $232.5-232.5 \mathrm{ft}$ \\ Borehole ID \\ Sample Number \\ Page 196 of 383 Chain-of-Custody \\ Grab \\ Sample}




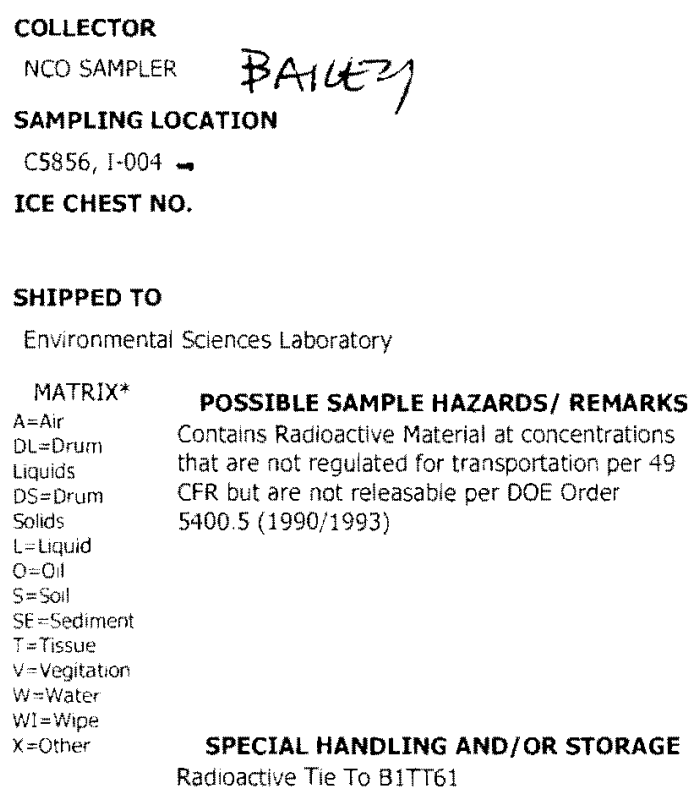

COMPANY CONTACT

TELEPHONE NO

PROJECT COOROINATOR

TRENT, STEVE

373-5869 WIORIG, OL

\section{PROJECT DESIGNATION}

\section{SAF NO.}

200-BP-5 OU Characterization for Wells 299-E33-341 and 299-E33-342 G F08-075

\section{FIELD LOGBOOK NO.}

ACTUAL SAMPLE DEPTH

$$
\text { HNF-W-488-1 }
$$
OFFSITE PROPERTY NO.

N/A

$$
17
$$

\section{COA}

123514 ES 10

BILL OF LADING/AIR BILL NO.
PRICE CODE $8 \mathrm{~N}$

AIR QUALITY TURNAROUND

45 Days / 45

\section{METHOD OF SHIPMENT}

\begin{tabular}{|c|c|c|c|}
\hline SAMPLE NO. & MATRIX* & SAMPLE DATE & SAMPLE TIME \\
\hline
\end{tabular}
GOVERNMENT VEHICLE

\section{LOT NO.}

\section{CHAIN OF POSSESSION}

RELINQUISHED BY/REMOVED FROM JKFALLI) JKBia RELINQUISHEO BY/REMOYED FOT mo 245 Rust RELINQUYGHED BY/REMOVER FROM D. Darath RELINQUISHED BY/REMOVEDEROM

RELINQUISHED BY/REMOVED FROM

RELINOUISHED BY/REMOVED FROM

RELINQUISHED BY/REMOVED FROM SECTION

FINAL SAMPLE DISPOSITION

\section{4}

\section{SIGN/ PRINT NAMES}

DATE/TIME

RECEIVED BY/STORED IN DATE/TIME, Y $0^{\text {RECEIVED RY/STORED IN }}$

Y. 30.0 DATE/TIME HA B RECEIVED BY/STORED IN

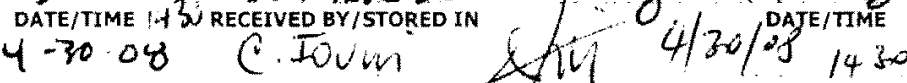

INSTRUCTIONS

SEE PAGE 2 FOR ALL SPECIAL INSTRUCTIONS
DATE/TIME

DATE/TIME

DATE/TIME

DATE/TIME
RECEIVED BY/STORED

RECEIVED BY/STORED IN

RECEIVED BY/STORED IN

RECEIVED BY/STORED IN 


\section{COLLECTOR}

NCO SAMPLER

COMPANY CONTACT

TRENT, STEVE

TELEPHONE NO.

373-5869

PROJECT COOROINATOR

WIDRIG, DL

PRICE CODE

$8 N$

SAF NO.

200-EP-5 OU Characterization for Wells 299-E33-341 and 299-E33-342 - G F08-075

C5856, 1-004

FIELD LOGBOOK NO.

ACTUAL SAMPLE DEPTH

COA

123514ES10

AIR QUALITY

DATA TURNAROUND

45 Days / 45

Days

OFFSITE PROPERTY NO.

$\mathrm{N} / \mathrm{A}$

BIIL OF LADING/AIR BILL. NO.

N/A

Environmental Sciences Laboratory

\section{SPECIAL INSTRUCTIONS}

** The 200 Area S\&GRP Characterization and Monitoring Sampling and Analysis GKI applies to this SAF.

** ESL is to determine unsaturated hydraulic conductivity using the method(s) best suited for the soil texture and type.

** ESL is to submit copies of all Chain of Custodies and associated sample login documentation to EIS-SDM within 24 hours of sample receipt. They may be sent via e-mail to ^CPP Sample Management.

(1)6020M_ICPMS_ASTM_AE (TAL) \{Cadmium, Chromium, Silver\} 6020M_ICPMS_ASTM_AE (Add-On) \{Arsenic, Lead, Molybdenum, Selenium\} 6020M_METALS_ICPMS_WE (TAL) \{Cadmium, Chromium, Silver\}

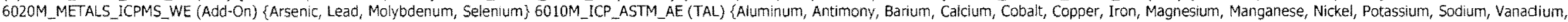

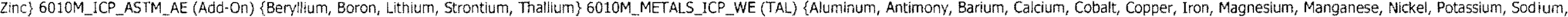

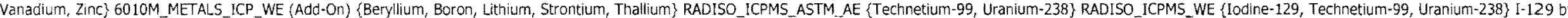

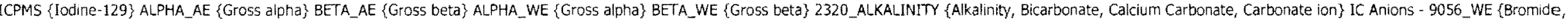

Chioride, Fluoride, Nitrate, Nitrite, Phosphate, Sulfate\} TOC - ASTME1915A \{Total Inorganic Carbon, Total carbon, Total organic carbon\} pH (Water) - 9045 WE; 


\section{COLLECTOR}

NCOSAMPLER BAILLZ

SAMPLING LOCATION

C5856, I-005

ICE CHEST NO.

\section{SHIPPED TO}

Environmental Sciences Laboratory

\begin{tabular}{|c|c|}
\hline \multicolumn{2}{|r|}{ POSSIBLE SAMPLE HAZARDS/ REMARKS } \\
\hline \multicolumn{2}{|r|}{ al at concentrations } \\
\hline $\begin{array}{l}\text { Le=ham } \\
\text { Liquids }\end{array}$ & that are not regulated for transportation per 49 \\
\hline DS $=$ Drum & CFR but are not releasable per DOE Order \\
\hline Solids & $5400.5(1990 / 1993)$ \\
\hline $\begin{array}{l}L=\text { Liquid } \\
0=0 \text { il }\end{array}$ & \\
\hline$S=501$ & \\
\hline$S E=$ Sedimen & \\
\hline $\begin{array}{l}T \text {-Tisstie } \\
V=V e g i t a t i o n\end{array}$ & \\
\hline$W=$ water & \\
\hline$w 1=$ Wipe & \\
\hline$x=0$ ther & $\begin{array}{l}\text { SPECIAL HANDLING AND/OR STORAGE } \\
\text { Radioactive Tie To } B 1 T T 61\end{array}$ \\
\hline
\end{tabular}

COMPANY CONTACT

TELEPHONE NO.

373-5869

PROJECT COORDINATOR

WIORIG, OL

SAF NO.

PROJECT DESIGNATION

Fo8-075

200-BP-5 OU Characterization for Wells 299-E33-341 and 299-E33-342FIELD LOGBOOK NO.

ACTUAL SAMPLE DEPTH

HNF- NJ-488-1

OFFSITE PROPERTY NO.

N/A

\section{COA}

123514 ES 510

BILL OF LADING / AIR BILL NO.

N/A

\begin{tabular}{cl} 
PRESERVATION & None \\
TYPE OF CONTAINER & $\begin{array}{l}\text { Split Spoon } \\
\text { Liner }\end{array}$ \\
NO. OF CONTAINER(S) & 2 \\
VOLUME & $1000 \mathrm{~g}$ \\
SAMPLE ANALYSIS & $\begin{array}{l}\text { SEE ITEM (1) IN } \\
\text { SPECIAL } \\
\text { INSTRUTIONS }\end{array}$ \\
\hline
\end{tabular}

PRICE CODE $\quad 8$ N

AIR QUALITY

\section{METHOD OF SHIPMENT}

GOVERNMENT VEHICLE
DATA
TURNAROUND

45 Days / 45

Days

SAMPLE DATE SAMPLE TIME

$4-24-01105$

\begin{tabular}{|c|c|c|c|}
\hline SAMPLE NO. & MATRIX* & SAMPLE DATE & SAMPLE TIME \\
\hline BITNV8 & SOIL & $9-0$ & 1105 \\
\hline
\end{tabular}

\section{CHAIN OF POSSESSION}

RELINQUISHED BY/REMOVED FROM

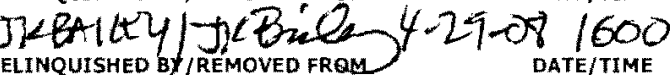
me $745 \wedge f \pm 1$ RELINQYSHED BY/REMOHED FROM L RELINQUISHED BY/REMOVED FROM $4-30.0 \%$ (

RECEIVED BY/STORED IN DATE/TIME

\section{SPECIAL INSTRUCTIONS}

** The 200 Area S\&GRP Characterization and Monitoring Sampling and Analysis GKI applies to this SAF.

** ESL is to determine unsaturated hydraulic conductivity using the method(s) best RECETEG BY/STORED IN

$4-30-* *$ ESL is to submit copies of all Chain of Custodies and associated sample login 4 DATE/TIME documentation to EIS-SDM within 24 hours of sample receipt. They may be sent via DATE/TIME/430 RECEIVED BY/STORED IN $4,30.06$ (. Dun DATESTIME RECEIVED BY/STORED $4 / 30 / 03 / 430$ e-mail to ^CPP Sample Management.

bate/TIme (1)Density; Particle Density - D854; Particle Size (Dry Sieve) - D422; Particle Size

DATE/TIME RECEIVED BY/STORED IN

DATE/TIME

(Hydrometer) - D422; Saturated Hydraulic Conductivity; CATIONEXCH_TR; KD -

RELINQUISHED BY/REMOVED FROM

DATE/TIME

RECEIVED BY/STORED IN

DATE/TIME

DATE/TIME
RECEIVED BY/STORED IN
DATE/TIME
DATE/TIME

DATE/TIME

FINAL SAMPLE DISPOSAL METHOD 


\section{COLLECTOR}

\section{BAlLEY}

\section{SAMPLING LOCATION}

C5856, I-006

ICE CHEST NO

\section{SHIPPED TO}

Environmental Sciences Laboratory

MATRIX* POSSIBLE SAMPLE HAZARDS/ REMARKS

$A=A i r \quad$ Contains Radioactive Material at concentrations

QL=Drum that are not regulated for transportation per 49

$D S=D$ rum CFR but are not releasabte per DOE Order

$\begin{array}{ll}\text { Solids } & 5400.5(1990 / 1993)\end{array}$

$2=$ Liquid
$0=0,1$

$0=0$ il
$S=S$ oil

SE=Sediment

$\hat{T=T}$ Tissue

$V=$ Vegitatic

$W=$ Water

$W_{x=\text { Wipe }}$

5400.5 (1990/1993)

SPECIAL HANDLING AND/OR STORAG Radioactive TIE TO B1TT62

SAMPLE NO

MATRIX

B1TP24

SOIL

COMPANY CONTACT

TRENT, STEVE

TELEPHONENO.

373-5869

PROJECT COORDINATOR

WIDRIG, DI

SAF NO.

PROJECT DESIGNATION

200-BP-5 oU Characterization for Wells 299-E33-341 and 299-E33-342-G

FIELD LOGBOOK NO.

ACTUAL SAMPLE DEPTH

Fo8-075

HNF-NS- 488 -

OFFSITE PROPERTY NO.

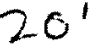

COA

$123514 E 510$

BILL OF LADING/AIR BILL NO.

N/A

N/A
PRICE CODE $\quad 8 \mathrm{~N}$

AIR QUALITY

METHOD OF SHIPMEN

GOVERNMENT VEHICLE
PRESERVATION None None

TYPE OF CONTAINER G/P Moisture

NO. OF CONTAINER(S) 1

VOLUME $\quad$ H 2009

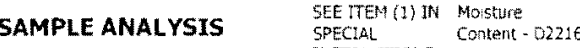

SAMPLE DATE SAMPLE TIME

$4-29 \cdot 0 R \quad 1105$

31394

\section{SIGN/ PRINT NAMES}

DATE/TIME

RELINQUISHED BY/REMOVED FROM JKEAlKY/OKERE RELINQQUISHED BY (REMOVED FROM mo 245 RP 41 RELINQYUSHED BY/REMOVED FROM D. Parel brenth REINQUISHED BYIREMOVED FROM

RELINQUISHED BY/REMOVED FROM

RELINQUISHED BY/REMOVED FROM

RELINQUISHED BY/REMOVED FROM $4-29-281600$ 4.30 .08 ATE/TIME

RECEIVED BY/STORED IN

UOT45 REF \#1 4-79-0 DATE/TIME

D. Dracher Sary DATE/TIME 430 $4,30-6$ DATE/TIME

DATE/TIME C I B /STORED IN C. DVum RECEIVED BY/STORED

4. $30-06$ $4 / 3 N / 3 / 430$ 舟 RECEIVED BY/STORED IN DATE/TIME

DATE/TIME DATE/TIME RECEIVED BY/STORED IN

DATE/TIME DATE/TIME

\section{SPECIAL INSTRUCTIONS}

SEE PAGE 2 FOR ALL SPECIAL INSTRUCTIONS 


\section{COLLECTOR}

NCO SAMPLER

\section{SAMPLING LOCATION}

C5856, I-006

ICE CHEST NO.

\section{SHIPPED TO}

Environmentat Sciences Laboratory

\section{COMPANY CONTACT}

TRENT, STEVE

TELEPHONE NO.

373-5869

\section{PROJECT DESIGNATION}

200-BP-5 OU Characterization for Welts 299-E33-341 and 299-E33-342-G FIELD LOGBOOK NO.

ACTUAL SAMPLE DEPTH

\section{OFFSITE PROPERTY NO.}

N/A
PROJECT COORDINATOR

WIDRIG, DL

\section{SAF NO.}

F08-075
PRICE CODE
DATA TURNAROUND

\section{METHOD OF SHIPMENT}

$123514 E S 10$

GOVERNMENT VEHICLE

BILL OF LADING/AIR BILL NO.

\section{SPECIAL INSTRUCTIONS}

** The 200 Area S\&GRP Characterization and Monitoring Sampling and Analysis GKI applies to this SAF,

** ESL is to determine unsaturated hydraulic conductivity using the method(s) best suited for the soll texture and type.

** ESL is to submit copies of all Chain of Custodies and associated sample login documentation to EIS-SDM within 24 hours of sample receipt. They may be Sent via e-mail to ACPP Sample Management.

(1)6020M_ICPMS_ASTM_AE (TAL) \{Cadmium, Chromium, Silver\}6020M_ICPMS_ASTM_AE (Add-On) \{Arsenic, Lead, Molybdenum, Selenium\} 6020M_METALS_ICPMS_WE (TAL) \{Cadmium, Chromium, Silver\}

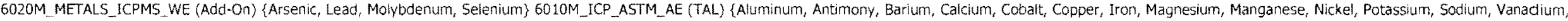

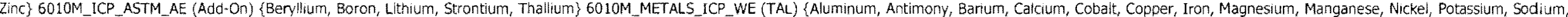

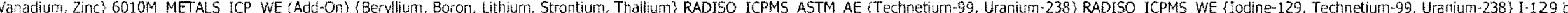

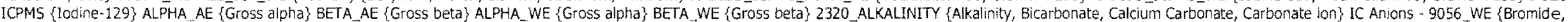

Chioride, Fluoride, Nitrate, Nitrite, Phosphate, Sulfate\} TOC - ASTME1915A \{Total Inorganic Carbon, Total carbon, Totai organic carbon\} pH (Water) - 9045 WE; 


\section{CHAIN OF CUSTODY/SAMPLE ANALYSIS REQUEST}

\section{TELEPHONE NO.}

$373-5869$

\section{TRENT, STEVE}

PROJECT DESIGNATION

200-BP-5 OU Characterization for Wells 299-E33-341 and 299-E33-342 - G FIELD LOGBOOK NO.

ACTUAL SAMPLE DEPTH

HNF-N-488-1

OFFSITE PROPERTY NO.

$$
22.51
$$

N/A

$\begin{array}{cll}\text { PRESERVATION } & \text { None } & \text { None } \\ \text { TYPE OF CONTAINER } & \text { G/p } & \begin{array}{l}\text { Moisture } \\ \text { Resistent Cont }\end{array} \\ & & \end{array}$

NO. OF CONTAINER(S)

VOLUME

$1 L$

2009

SAMPLE ANALYSIS

$$
\begin{aligned}
& \text { SEE IEM (1) INA Mostute } \\
& \text { SFECLAL } \\
& \text { NASTRUTTONS COntent - D2216: }
\end{aligned}
$$

SAMPLE DATE SAMPLE TIME

$4.29 .08 \quad 1305$
PAGE 1 OF 2

PROJECT COORDINATOR

WIDRIG, DL

SAF NO

F08-07

COA

$123514 E S 10$

BILL OF LADING/AIR BILL NO.

N/A

PRICE CODE $\quad 8 N$

DATA TURNAROUND

45 Days / 45 Days Radioactive Tie To BIVAR2

SAMPLE NO

MATRIX
SOIL

31394

\section{SIGN/ PRINT NAMES}

CHAIN OF POSSESSION

DATE/TIME

RELINQUISHED BY/REMOVED FROM

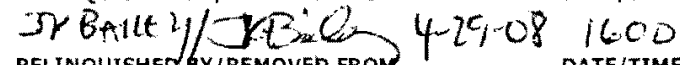

RELINQUISHED GY/REMOVEP FRON

$m o 745 R+1$ RELIN $Q Y I S H E O$ BY/REMOVED FROM D. Parahea PLR RELINQUTSHED BY/REMOVED FROM

RELINQUISHED BY/REMOVED FROM

RELINQUISHED BY/REMOVED FROM

RELINQUISHED BY/REMOVED FROM

\section{LABORATORY RECEIVED BY} SECTION

FINAL SAMPLE DISPOSAL METHOD

$4 \cdot 30.28$ DATE/TIME 43 3RECEIVEDBY/STORED IN 4.33 .08 DATE/TIME

DATE/TIME C.bun xist

RECEIVED BY/STOREO IN

\section{DATE/TIME}

$4-25-08 \quad 1600$ OCNA DATE/TIME $4.30 \cdot 08$ $43 \% 1 \% 81430$ DATE/TIME OATE/TIME RECEIVED BY/STORED IN DATE/TIME DATE/TIME RECEIVED BY/STORED IN

\section{SPECIAL INSTRUCTIONS}

SEE PAGE 2 FOR ALL SPECIAL INSTRUCTIONS 


\section{COLLECTOR}

COMPANY CONTACT

TRENT, STEVE

TELEPHONE NO.

PROJECT COORDINATOR

373-5869

WIDRIG, DL

PRICE CODE

$8 \mathrm{~N}$

DATA

\section{PROJECT DESIGNATION}

SAF NO.

SAF NO.
F08-075

C5856, I-007

200-BP-5 OU Characterization for Wells 299-E33-341 and 299-E33-342-G

FIELD LOGBOOK NO.

ACTUAL SAMPLE DEPTH

\section{COA}

AIR QUALITY

TURNAROUND

45 Days / 45

Days

\section{OFFSITE PROPERTY NO}

N/A

$123514 E S 10$

METHOD OF SHIPMENT

BILL OF LADING/AIR BILL NO

$N / A$

Environmental Sciences Laboratory

\section{SPECIAL INSTRUCTIONS}

** The 200 Area S\&GRP Characterization and Monitoring Sampling and Analysis GKI applies to this SAF.

** ESL is to determine unsaturated hydraulic conductivity using the method(s) best suited for the soil texture and type.

** ESL is to submit copies of all Chain of Custodies and associated sample login documentation to EIS-SDM within 24 hours of sample receipt. They may be sent via e-mail to ^CPP Sample Management.

(1)6020M ICPMS ASTM AE (TAL) \{Cadmium, Chromium, Silver\} 6020M_ICPMS_ASTM_AE (Add-On) \{Arsenic, Lead, Molybdenum, Selenium\}6020M_METALS_ICPMS_WE (TAL) \{Cadmium, Chromium, Siver\}

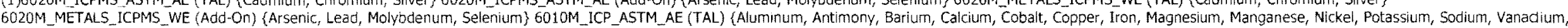

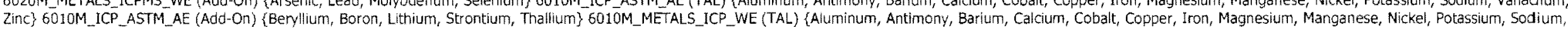

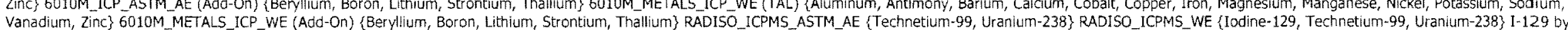

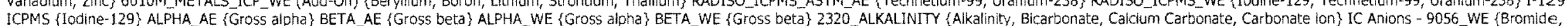
Chloride, Fiuoride, Nitrate, Nitrite, Phosphate, Sulfate\} TOC - ASTME1915A (Total Inorganic Carbon, Total carbon, Total organic carbon\} pH (Water) - 9045 _WE; 


\section{COLLECTOR}

nCo sampler Bailey

\section{SAMPLING LOCATION}

C5856, I.009

ICE CHEST NO.

\section{SHIPPED TO}

Envronmental Sciences Laboratory

MATRIX*

$A=$ Atr

SLE SAMPLE HAZARDS/REMARKS Contains Radioactive Material at concentrations that are not regulated for transportation per 49 CFR but are not releasable per DOE Order $5400.5(1990 / 1993)$

DS=Drum

Solidigu

$0=0$ il

$S=$ Sol

SE $=$ Sedimen

$T=$ Tissue

$\checkmark=$ vegtation

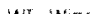

$x=$ Other

SPECIAL HANDLING AND/OR STORAGE Radioactive Tie To BlVAR4

\section{COMPANY CONTACT}

TRENT, STEVE

TELEPHONE NO.

373-5869

PROJECT COORDINATOR

WIDRIG, DL

\section{SAF NO.}

PROJECT DESIGNATION

200-8P-5 OU Characterization for Wells 299-E33-341 and 299-E33-342-G FIELD LOGBOOK NO.

ACTUAL SAMPLE DEPTH

HNF-NJ-488-1

OFFSITE PROPERTY NO.

$$
27.5^{\prime}
$$

N/A

\begin{tabular}{|c|c|c|}
\hline PRESERVATION & None & None \\
\hline TYPE OF CONTAINER & $G / P$ & $\begin{array}{l}\text { Moisture } \\
\text { Resistant Cont }\end{array}$ \\
\hline NO. OF CONTAINER(S) & 1 & 1 \\
\hline VOLUME & $1 \mathrm{~L}$ & 2009 \\
\hline SAMPLE ANALYSIS & $\begin{array}{l}\text { SEE TTEM (1) IN } \\
\text { SPECIAL } \\
\text { INSTRUCTONS }\end{array}$ & $\begin{array}{l}\text { Mosture } \\
\text { Content-D2216; }\end{array}$ \\
\hline
\end{tabular}

PRICE CODE $\quad 8$ N

AIR QUALITY

METHOD OF SHIPMENT

GOVERNMENT VEHICLE
DATA TURNAROUND

45 Days / 45 Days
SAMPLE DATE SAMPLE TIME
$4-29-08 \quad 1335$
SAMPLE NO.
MATRIX
B1TP27
SOIL

\section{SIGN PRINT NAMES 31314}

CHAIN OF POSSESSION

RELINQUISHED BY/REMOVED FROM JKBAllt J JKBOO RELINQUISHED BW/REMOVED FRONmo 245 Rft RELINRUISHED BY/REMMVVD FROM DELINQUISHED BY/REMOVED FRG

RELINQUISHED BY/REMOVED FROM

RELINQUISHED BY/REMOVED FROM

RELINQUISHED BY/REMOVED FROM

\begin{abstract}
LABORATORY RECEIVED BY
\end{abstract} SECTION

FINAL SAMPLE DISPOSAL METHOD DISPOSITION

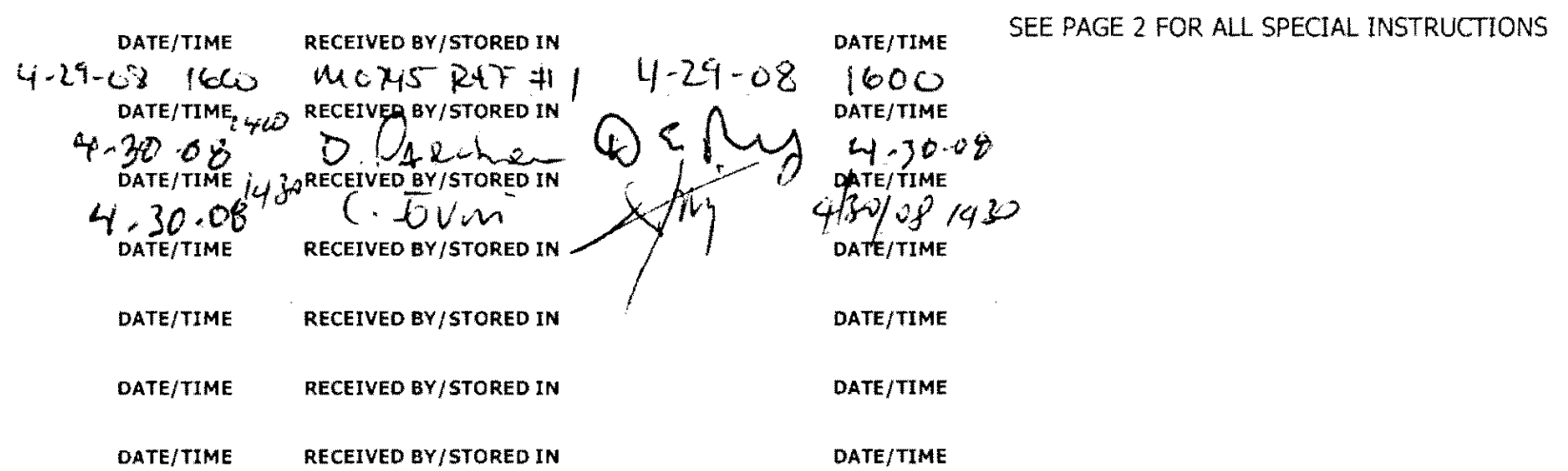




\section{COLLECTOR}

NCO SAMPLER

\section{SAMPLING LOCATION}

C5856, 1-009

ICE CMEST NO.

\section{SHIPPED TO}

Environmental Sciences Laboratory

\section{COMPANY CONTACT}

TRENT, STEVE

TELEPHONE NO.

PROJECT DESIGNATION

373-5869

FID

\section{OFFSITE PROPERTY NO.}

$N / A$
PROJECT COORDINATOR

WIDRIG $\mathrm{DL}$

\section{SAF NO.}

COA

123514 ES10

\section{PRICE CODE $\quad 8 \mathrm{~N}$}

AIR QUALITY TURNAROUND

45 Days / 45

Days

METHOD OF SHIPMENT

GOVERNMENT VEHICLE

\section{SPECIAL INSTRUCTIONS}

** The 200 Area S\&GRP Characterization and Monitoring Sampling and Analysis GKI applies to this SAF.

** ESL is to determine unsaturated hydraulic conductivity using the method(s) best suited for the soil texture and type.

** ESL is to submit copies of all Chain of Custodies and associated sample login documentation to EIS-SOM within 24 hours of sample receipt. They may be sent via e-mail to C CPP Sample Management.

(1)6020M_ICPMS ASTM AE (TAL) \{Cadmium, Chromium, Silver\}6020M_ICPMS_ASTM_AE (Add-On) \{Arsenic, Lead, Molybdenum, Selenium\} 6020M_METALS_ICPMS_WE (TAL) \{Cadmilum, Chromium, Silver\}

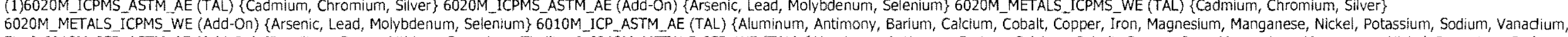

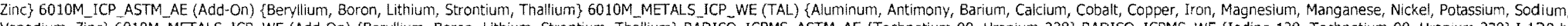

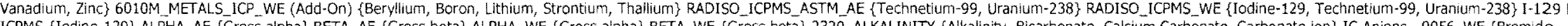

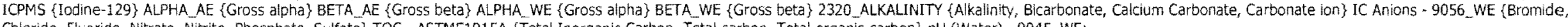
Chioride, Fluoride, Nitrate, Nitrite, Phosphate, Sulfate\} TOC - ASTME1915A \{Total Inorganic Carbon, Total carbon, Total organic carbon\} pH (Water) - 9045 _WE; 
CHAIN OF CUSTODY/SAMPLE ANALYSIS REQUEST

\section{TELEPHONE NO.}

373-5869

TRENT, STEVE

PROJECT DESIGNATION

200-BP-5 OU Characterization for Wells 299-E33-341 and 299-E33-342 - G FIELD LOGBOOK NO, ACTUAL SAMP
30

HNE-N-48 OFFSITE PROPERTY NO.

N/A

$\begin{array}{cll}\text { PRESERVATION } & \text { None } & \text { None } \\ \text { TYPE OF CONTAINER } & \text { G/F } & \begin{array}{l}\text { Moisture } \\ \text { Resistant Cont }\end{array} \\ \text { NO. OF CONTAINER(S) } & 1 & 1 \\ \text { VOLUME } & 11 & 2009 \\ \text { SAMPLE ANALYSIS } & \begin{array}{l}\text { SEE TEM (1) IN Mosture } \\ \text { SPEILA } \\ \text { INSTRLCTIONS }\end{array}\end{array}$

PAGE 1 OF 2

PROJECT COORDINATOR

WIDRIG, DL

PRICE CODE

$8 \mathrm{~N}$

\section{SAF NO.}

AIR QUALITY

COA

123514 ES10

METHOD OF SHIPMENT GOVERNMENT VEHICLE

\section{SHIPPED TO}

MATRIX*

POSSIBLE SAMPLE HAZARDS/ REMARKS Contains Radioactive Material at concentrations that are not regulated for transportation per 49 CFR but are not releasable per DOE Order $5400.5(1990 / 1993)$

Soldeds

$0=0.1$

$S=50$ il

$T=$ Tissue

$V=$ vegitation
$W=$ Water

WI $=$ Wipe

SPECIAL HANDLING AND/OR STORAGE Radioactive TIe To B1TP97

SAMPLE NO. MATRIX*

B1TP28

SOLL.

SAMPLE DATE SAMPLE TIME

$4-29.08 \cdot 1355$

\section{BILL OF LADING / AIR BILL NO.}

N/A

\section{CHAIN OF POSSESSION}

RELINQUISHED BY/REMOVED FROM

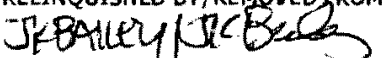
RELINQUISHED bY/REMOVED FROM mo $245 R P+1$ RELINQYSSHED BY REMOVED FROM D. Parehe 0 ReLinquisheO BY/REMOVED FROM

RELINQUISHED BY/REMOVED FROM

RELINQUISHED BY/REMOVED FROM

RELINOUISHED BY/REMOVED FROM

RECEIVED BY

\section{LABORATORY} SECTION

FINAL SAMPLE DISPOSAL METHOD
31394

\section{SIGN/ PRINT NAMES}

RECEIVED BY/STORED IN

WOXS REF $14.29-08^{\text {DATE/TIME }}$

$29-388_{\text {DATE/TIME }}^{\text {DATE/TIME }}$

RECEIVED BY/STORED IN

D. Rareh

Oegly

DATE/TIME M 3ORECEIVED BY/STORED IN

4.30 .06

DATE/TIME

DATE/TIME

C. bum

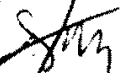

RECEIVED BY/STORED -

RECEIVED BY/STORED IN/

DATE/TIME

RECEIVED BY/STORED IN

RECEIVED BY/STORED IN
DATE/TIME

\section{DATE/TIME}

$4 \cdot 30 \cdot 28$

DATE/TIME

$4 / 30 / \mathrm{P}^{2} / 130$

GATE/TIME

DATE/TIME

DATE/TIME

DATE/TIME

\section{SPECIAL INSTRUCTIONS}

SEE PAGE 2 FOR ALL SPECIAL INSTRUCTIONS 


\section{COLLECTOR}

NCO SAMPLER

\section{SAMPLING LOCATION}

C5856, I-010

ICE CHEST NO.

\section{SHIPPED TO}

Environmental Sciences Laboratory

\section{COMPANY CONTACT}

TRENT, STEVE

TELEPHONE NO.

373-5869

PROJECT DESIGNATION

200-BP-5 OU Characterization for Wells 299-E33-341 and 299-E33-342-G

FIELD LOGBOOK NO.

ACTUAL SAMPLE DEPTH

OFFSITE PROPERTY NO.

N/A
PROJECT COOROINATOR

WIDRIG, DL

SAF NO.

FOP-075

COA

$123514 E S 10$

\section{PRICE CODE BN}

AIR QUALITY

METHOD OF SHIPMENT

\section{SPECIAL INSTRUCTIONS}

*** The 200 Area S\&GRP Characterization and Monitoring Sampling and Analysis GKI applies to this SAF.

** ESL is to determine unsaturated hydraulic conductivity using the method(s) best suited for the soll texture and type.

** ESL is to submit copies of all Chain of Custodies and associated sample login documentation to EIS-SDM within 24 hours of sample receipt. They may be sent via e-mail to ^CPP Sample Management.

(1)6020M_ICPMS_ASTM_AE (TAL) \{Cadmium, Chromium, Silver\}6020M_ICPMS_ASTM_AE (Add-On) (Arsenic, Lead, Molybdenum, Selenium\}6020M_METALS ICPMS WE (TAL) \{Cadmium, Chromium, Silver\}

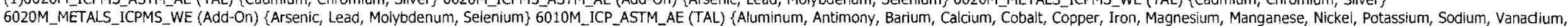

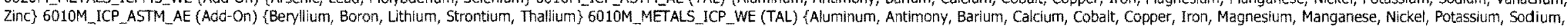

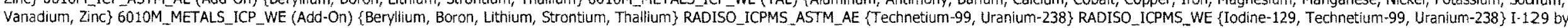

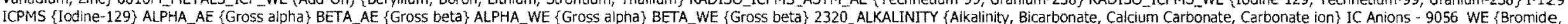
Chloride, Fivoride, Nitrate, Nitrite, Phosphate, Sulfate\} TOC - ASTME1915A \{Total Inorganic Carbon, Total carbon, Total organic carbon\} pH (Water) - 9045 .WE; 
COLLECTOR

NCOSAMPLER BALLEY

SAMPLING LOCATION

C5856, I-011

ICE CHEST NO.

SHIPPED TO

Environmental Sciences Laboratory

MATRIX* POSSIBLE SAMPLE HAZARDS/ REMARKS

$A=$ Air

$\mathrm{DL}=$ Drum

Liquids

Solids
Solin

Contains Radioactive Material at concentration

that are not regulated for transportation per 49

CFR but are not releasable per DOE Order

$5400.5(1990 / 1993)$

$S=5$ oil

$S E=$ Sediment

$T=T i s s u e$

$V=$ vegitation

$W=$ Water

$W I=$ Wipe

SPECIAL HANDLING AND/OR STORAGE Radioactive Tie To B1TP97

SAMPLE NO.

MATRIX*

B1TP29

SOIL

\section{COMPANY CONTACT}

TRENT, STEVE

TELEPHONE NO.

373-5869

PROJECT COORDINATOR

WIDRIG, DL

\section{SAF NO.}

200-8P-5 OU Characterization for Wells 299-E33-341 and 299-E33-342-G F08-075

\section{FIELD LOGBOOK NO.}

ACTUAL SAMPLE DEPTH

\section{COA}

HNF.N- $488-1$

OFFSITE PROPERTY NO.

$32.5^{\prime}$

$N / A$

$\begin{array}{cll}\text { PRESERVATION } & \text { None } & \text { None } \\ \text { TYPE OF CONTAINER } & \text { G/P } & \begin{array}{l}\text { Moisture } \\ \text { Resistant Cont }\end{array} \\ \text { NO. OF CONTAINER(S) } & 1 & 1 \\ \text { VOLUME } & \text { il } & 2009 \\ \text { SAMPLE ANALYSIS } & \begin{array}{l}\text { SEE TEM (1) IN Maistire } \\ \text { SPECIAL } \\ \text { INSTRUCHONS }\end{array}\end{array}$

SAMPLE DATE SAMPLE TIME

$4-29-08 \quad 1450$
PRICE CODE 8 N

AIR QUALITY

METHOD OF SHIPMENT

GOVERNMENT VEHICLE
DATA TURNAROUND

45 Days / 45 Days

CHAIN OF POSSESSION
RELINQUISHED BY/REMOVED FROM
RELINQUISHED BY/REMOVED FROM
RELINQUISHED BY/REMOVED FROM

SPECLAL INSTRUCTIONS

SEE PAGE 2 FOR ALL SPECIAL INSTRUCTIONS

$\begin{array}{ll}\text { LABORATORY } & \text { RECEIVED BY } \\ \text { SECTION } & \\ \text { FINAL SAMPLE } \\ \text { DISPOSITION }\end{array}$




\section{COLLECTOR}

NCO SAMPLER

\section{SAMPLING LOCATION}

C5856, I-011

ICE CHEST NO.

\section{SHIPPED TO}

Environmental Sciences Laboratory

\section{COMPANY CONTACT}

TRENT, STEVE

TELEPHONE NO.

373-5869

PROJECT DESIGNATION

200-BP-5 OU Characterization for Wells 299-E33-341 and 299-E33-342-G FIELD LOGBOOK NO.

ACTUAL SAMPLE DEPTH

\section{OFFSITE PROPERTY NO.}

N/A
PROJECT COORDINATOR

WIDRIG, DL

SAF NO.

FO8-075

COA

123514 ES 10

\section{PRICE CODE 8 N}

AIR QUALITY

METHOD OF SHIPMENT

GOVERNMENT VEHICLE

\section{SPECIAL INSTRUCTIONS}

** The 200 Area S\&GRP Characterization and Monitoring Sampling and Analysis GKI applies to this SAF.

** ESL is to determine unsaturated hydraulic conductivity using the method(s) best suited for the soil texture and type.

** ESL is to submit copies of all Chain of Custodies and associated sample login documentation to EIS-SDM within 24 hours of sample receipt. They may be sent via e-mail to ^CPP Sample Management.

(1)6020M_ICPMS ASTM AE (TAL) \{Cadmium, Chromium, Silver\} 6020M_ICPMS ASTM AE (Add-On) \{Arsenic, Lead, Molybdenum, Selenium\} 6020M_METALS_ICPMS_WE (TAL) \{Cadmium, Chromium, Silver\}

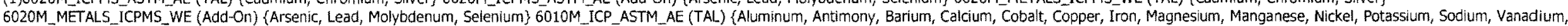

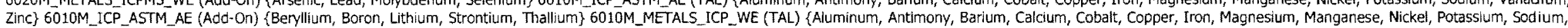

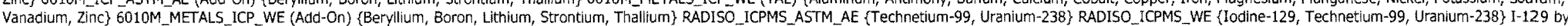

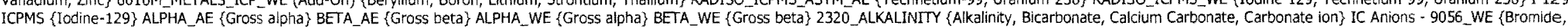
Chloride, Fluoride, Nitrate, Nitrite, Phosphate, Sulfate\} TOC - ASTME1915A \{Total Inorganic Carbon, Total carbon, Total organic carbon\} pH (Water) - 9045 -WE; 


\section{COLLECTOR}

nco sampler PatLey

SAMPLING LOCATION

C5856, I-012

ICE CHEST NO

SHIPPED TO

Environmental Sciences Laboratory

MATRIX*

$A=$ Air

OL=Drum

SIBLE SAMPLE HAZARDS/ REMARKS Contains Radioactive Material at concentrations that are not regulated for transportation per 49 CFR but are not releasabie per DOE Order $5400.5(1990 / 1993)$

Solids

$0=0$ it

$\mathrm{S}=5 \mathrm{Soll}$
$\mathrm{SE}=$ Sediment

$T=$ Tissue

$V=$ vegitation

$w I=W i p e$

$x=$ other

SPECIAL HANDLING AND/OR STORAGE Radioactive Tie To B1TP97
COMPANY CONTACT

TRENT, STEVE

TELEPHONE NO.

373-5869

PROJECT COORDINATOR

WIDRIG, OL

PROJECT DESIGNATION

SAF NO.

FO8-075

200-BP-5 OU Characterization for Wells 299-E33-341 and 299-E33-342-G FIELD LOGBOOK NO. ACTUAL SAMPLE DEPTH

\section{COA}

HNF - NJ- $488-1$ OFFSITE PROPERTY NO.

$$
351
$$

$\mathrm{N} / \mathrm{A}$

BILL OF LADING/AIR BILL NO.

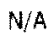

PRICE CODE BN

AIR QUALITY

METHOD OF SHIPMENT

GOVERNMENT VEHTCLE

$\begin{array}{lll}\text { PRESERVATION } & \text { None } & \text { None } \\ \text { GPE OF CONTAINER } & \text { G/P } & \begin{array}{l}\text { Moisture } \\ \text { Resistant Cont }\end{array} \\ \end{array}$

NO. OF CONTAINER(S)

1

VOLUME

it $\quad \overline{2} 00 \mathrm{~g}$

SAMPLE ANALYSIS

SEE TTEM (1) IN Mosture
SPECAL
Content - O2216;

SAMPLE DATE SAMPLE TIME

$4-27-08$ is 0

\section{CHAIN OF POSSESSION}

RELINOUISHED BY/REMOVED FROM JKGALLR RELINQUISHED JTC Baten $429-081603$ MO $74 \leq R \& A$ RELINQPISHED BY/REMOVED FROM Le S. $R$ RELINQUISHED BY/REMOVED FROM

RELINQUISHED BY/REMOVED FROM

RELINOUISHED BY/REMOVED FROM

RELINQUISHED BY/REMOVED FROM DATE/TIME

DATE/TIME

DATE/TIME

DATE/TIME

\section{4}

\section{SIGN/ PRINT NAMES}

RECEIVED BY/STORED IN

\section{SPECIAL INSTRUCTIONS}

(2E) $4-29-881600$

4-30-0

DATE/TIME / 3 QRECETVED BY/STORED IN

4-3020 C. Dum Sth 4/30/9/430

DATE/TIME RECEIVED BY/STORED IN DATE/TIME

RECEIVED BY/STORED IN

DATE/TIME

RECEIVED BY/STORED IN

DATE/TIME

RECEIVED BY/STORED IN

DATE/TIME

\section{LABORATORY RECEIVED BY} SECTION

FINAL SAMPLE DISPOSAL METHOD DISPOSITION 


\section{COMPANY CONTACT}

TRENT, STEVE

\section{TELEPHONE NO.}

373-5869

\section{PROJECT DESIGNATION}

PROJECT COORDINATOR

WIDRIG, DL

SAF NO.

200-BP-5 OU Characterization for Wells 299-E33-341 and 299-E33-342-G FO8-075

FIELD LOGBOOK NO.

ACTUAL SAMPLE DEPTH

PRICE CODE

$8 N$

AIR QUALITY

DATA

TURNAROUND

45 Days / 45

Days
ICE CHEST NO.

\section{SHIPPED TO}

Environmental Sciences Laboratory

\section{OFFSITE PROPERTY NO}

N/A

\section{COA}

$123514 E S 10$

METHOD OF SHIPMENT

\section{SPECIAL INSTRUCTIONS}

** The 200 Area S\&GRP Characterization and Monitoring Sampling and Analysis GKI applies to this SAF.

** ESL is to determine unsaturated hydraulic conductivity using the method(s) best suited for the soll texture and type.

** ESL is to submit copies of all Chain of Custodies and associated sample login documentation to EIS-SDM within 24 hours of sample receipt. They may be sent via e-mail to ACPP Sample Management.

(1)6020M_ICPMS_ASTM_AE (TAL) \{Cadmium, Chromium, Silver\} 6020M_ICPMS_ASTM_AE (Add-On) \{Arsenic, Lead, Molybdenum, Selenium\} 6020M_METALS ICPMS_WE (TAL) \{Cadmium, Chromium, Silver\}

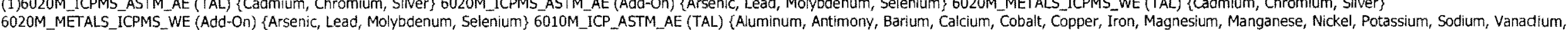

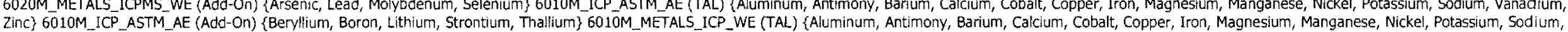

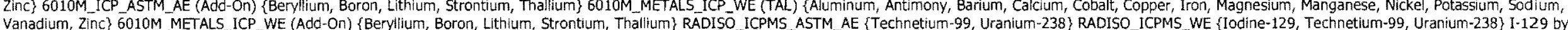

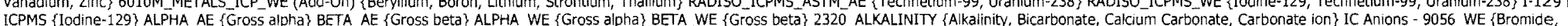
Chloride, Fluoride, Nitrate, Nitrite, Phosphate, Sulfate) TOC. ASTME1915A (Total Inorganic Carbon, Total carbon, Total organic Carbon\} pH (Water) - 9045 .WE: 


\section{COLLECTOR \\ nCo SAMPLER BAile}

\section{SAMPLING LOCATION}

C5856, I-013

ICE CHEST NO.

\section{SHIPPED TO}

Environmental Sciences Laboratory

MATRIX*

$A=$ Air

$\mathrm{DL}=\mathrm{Drum}$

Liquids

DS=Drum

$S$ Solids
$L=L$ Liquid

$0=0 i$
0

$\mathrm{O}=0$ if
$\mathrm{S}=\mathrm{Soil}$

$S E=$ Sediment

$T=$ Tissue

$\checkmark=$ vegitation

$W=$ water

$W I=W / p e$

$x=0$ other

SPECIAL HANDLING AND/OR STORAGE Radioactive Tie To: B1TP97

SSIBLE SAMPLE HAZARDS/ REMARKS Contains Radioactive Material at concentrations that are not regulated for transportation per 49 CFR but are not releasable per DOE Order $5400.5(1990 / 1993)$

\begin{tabular}{|c|c|c|c|c|}
\hline SAMPLE NO. & & MATRIX* & SAMPLE DATE & SAMPLE TIME \\
\hline ITNV9 & SOIL & & $4-29-08$ & 15,5 \\
\hline
\end{tabular}

\section{CHAIN OF POSSESSION}

RELINQUISHED BY/REMOVED FROM IKCAli2)/CKCBW RELINQUISHED BY/REMOVED FROMmo $745 R A A$ RELINQPISHED BY/REMONEP FROM is RELINQUISHED BYIREMOVED FROM

RELINQUISHED BY/REMOVED FROM

RELINQUISHED BY/REMOVED FROM

RELINQUISHED BY/REMOVED FROM

RECEIVED BY

\section{LABORATORY}

\section{SECTION}

FINAL SAMPLE DISPOSITION FIELD LOGBOOK NO.

\section{COMPANY CONTACT}

TRENT, STEVE TELEPHONE NO.

373-5869

PROJECT COORDINATOR

WIDRIG, DL

PRICE CODE

$8 N$

SAF NO.

200-BP-5 OU Characterization for Wells 299-E33-341 and 299-E33-342-G F08-075

ACTUAL SAMPLE DEPTH

$\mathrm{COA}$

HNF-N $-488-1$

$3^{2} .5^{1}$

$123514 E S 10$

BILL OF LADING/AIR BILL NO

OFFSITE PROPERTY NO.

N/A

$\begin{array}{lll}\text { PRESERVATION } & \text { None } & \text { None } \\ \text { TYPE OF CONTAINER } & \text { G/P } & \begin{array}{l}\text { Moisture } \\ \text { Resistant Cont }\end{array}\end{array}$

NO. OF CONTAINER(S)

VOLUME IL

SAMPLE ANALYSIS $\quad$ SEE TEM (1) IN Meisture

45 Days / 45

Days

METHOD OF SHIPMENT

GOVERNMENT VEHICLE 


\section{TELEPHONE NO.}

373-5869

TRENT, STEVE

\section{PROJECT DESIGNATION}

200-BP-5 OU Characterization for Wells 299-E33-341 and 299-E33-342-G FIELD LOGBOOK NO.

ACTUAL SAMPLE DEPTH

OFFSITE PROPERTY NO.

N/A
PROJECT COORDINATOR

WIDRIG, DL

\section{SAF NO.}

F08-075

COA

123514 ES 10
PRICE CODE

$\mathbf{8 N}$

METHOD OF SHIPMENT

GOVERNMENT VEHICLE

\section{SHIPPED TO}

Environmental Sclences Laboratory

\section{BILL OF LADING/AIR BILL NO.}

N/A

\section{SPECIAL INSTRUCTIONS}

** The 200 Area S\&GRP Characterization and Monitoring Sampling and Analysis GKI applies to this SAF.

(1)6020M_ICPMS_ASTM_AE (TAL) \{Cadmium, Chromium, Silver\} 5020M_ICPMS_ASTM_AE (Add-On) \{Arsenic, Lead, Molybdenum, Selenium\}6020M_METALS_ICPMS_WE (TAL) \{Cadmium, Chromium, Silver\}

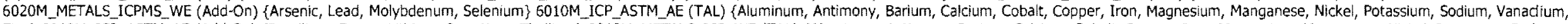

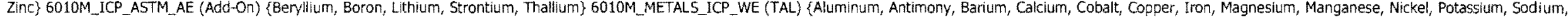

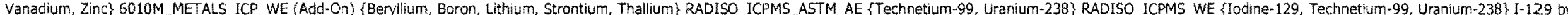

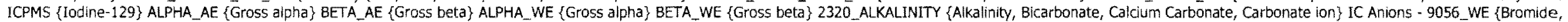

Chloride, Fluoride, Nitrate, Nitrite, Phosphate, Sulfate\} TOC - ASTME1915A \{Total Inorganic Carbon, Total carbon, Total organic carbon\} pH (Water) - 9045 WE 


\section{COLLECTOR \\ nCO SAMPLER BALlley}

SAMPLING LOCATION

C5856, I-001

ICE CHEST NO.

\section{SHIPPED TO}

Environmental Sciences Laboratory

\section{MATRIX* POSSIBLE SAMPLE HAZARDS/ REMARKS \\ A=Air Contains Radioactive Material at concentrations \\ $D L=$ Drum that are not regulated for transportation per 49 \\ $\begin{array}{ll}\text { Liguids } & \text { that are not regulated for transportation per } \\ D S=D \text { Drum } & \text { CFR but are not releasable per DOE Order }\end{array}$ \\ $\begin{array}{ll}\text { DS=Drum } & \text { CFR but are not releasab } \\ \text { Solids } & 5400.5(1990 / 1993)\end{array}$ \\ $L=$ Liquid
$0=0 !$ \\ $0=0$ il
$S=S$ Dil \\ $\mathrm{SE}=$ Sediment \\ $S E=$ Sediment
$T=$ Tissue \\ $Y=$ Vegsue
$W=$ Vegtion \\ $W=$ Water \\ $W I=$ Wipe \\ $x=$ Other \\ SPECIAL HANDLING AND/OR STORAGE Radioactive TIE TO BITP96}

\section{COMPANY CONTACT}

TRENT, STEVE

\section{TELEPHONE NO.}

373-5869

\section{PROJECT DESIGNATION}

200-BP-5 OU Characterization for Wells 299-E33-341 and 299-E33-342-G F08-075

\section{FIELD LOGBOOK NO.}

HNF - N- $488-1$

OFFSITE PROPERTY NO.

N/A

$$
15^{\prime}-17.5^{\prime}
$$

PROJECT COORDINATOR

WIDRIG, DL

COA

123514ES10

BILL OF LADIN
PRICE CODE 8 N

AIR QUALITY

METHOD OF SHIPMENT

GOVERNMENT VEHICLE

\begin{tabular}{cc} 
PRESERVATION & None \\
TYPE OF CONTAINER & $\begin{array}{l}\text { Split Spoon } \\
\text { Liner }\end{array}$ \\
NO. OF CONTAINER(S) & 2 \\
VOLUME & 10009 \\
SAMPLE ANALYSIS & \\
\hline SEE TTEM (1) IM \\
SPECIA \\
INSTRUTIONS
\end{tabular}

SAMPLE NO.
B1TNV6

SAMPLE DATE SAMPLE TIME

4-2908. 0930

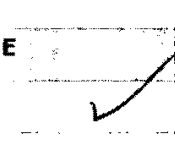

\section{CHAIN OF POSSESSION}

RELINQUISHED BY/REMOVEP FROM JK BAILY I SFBul RELINQUISHED BV/REMOVED FETM ho 745 R Fit RELINQYTSHED BY/REM GFE FROM D. $24 R$ RELINQUISHED BY/REMOVED FROM

RELINQUISHED BY/REMOVED FROM

RELINQUISHED BY/REMOVED FROM RELINQUISHED BY/REMOVED FROM

$\begin{array}{ll}\text { LABORATORY } & \text { RECEIVED BY } \\ \text { SECTION } & \\ \text { FINAL SAMPLE } \\ \text { DISPOSITION }\end{array}$

\section{SIGN/ PRINT NAMES}

DATE/TIME RECEIVED BY/STORED IN WOAT REF 081600

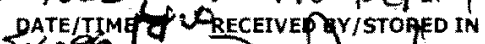
DATE/TIMA DATE/TIMES/ SBECEIVED BY/STORED IN 47000 (v.tinn DATE/TIME I4 RECEIVED BY/STORED DATE/TIME RECEIVED BY/STORED IN $/$

DATE/TIME RECEIVED BY/STORED IN

DATE/TIME

\section{SPECIAL INSTRUCTIONS}

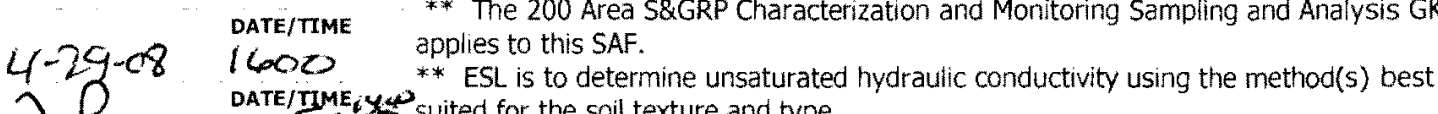

** The 200 Area S\&GRP Characterization and Monitoring Sampling and Analysis GKI

4-29-C8 1600 applies to this SAF.

1. DATE/TME 5 PATE/TMME documentation to EIS-SDM within 24 hours of sample receipt. They may be sent via $5 / 1 / 93 / 1$ - 2 -mail to $\wedge$ CPP Sample Management.

DÁte/TIme (1)Density; Particle Density - D854; Particle Size (Dry Sieve) - D422; Particle Size (Hydrometer) - D422; Saturated Hydraulic Conductivity; CATIONEXCH_TR; KD DATE/TIME Batch;

DATE/TIME

DATE/TIME 


\section{COLLECTOR}

nCo SAMPLER BAILEZ

SAMPLING LOCATION

C5856, 1-002

ICE CHEST NO.

\section{SHIPPED TO}

Environmental Sciences Laboratory

MATRIX*

$A=$ Air

$\mathrm{DL}=$ Drum

Liquids

DS $=$ Drum
solids

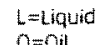

$0=0$ il
$5=5$ oil

$S E=$ Sediment

$\mathrm{SE}=$ Sediment
$\mathrm{T}=$ Tissue

$T=$ Tissue
$V=$ Vegitation

$W=W$ ater

$W=$ Wipe
$X=0$ ther

POSSIBLE SAMPLE HAZARDS/ REMARKS Contains Radioactive Material at concentrations that are not regulated for transportation per 49 CFR but are not releasable per DOE Order $5400.5(1990 / 1993)$

SPECIAL HANDLING AND/OR STORAGE Radioactive Tle To B1TP96

SAMPLE NO. MATRIX*

B1TP22

SOIL

\section{COMPANY CONTACT}

TRENT, STEVE

\section{TELEPHONE NO.}

$373-5869$

PROJECT COORDINATOR

WIDRIG, DL

SAF NO.

200-BP-5 OU Characterization for Wells 299-E33-341 and 299-E33-342-G F08-075 FIELO LOGBOOK NO.

ACTUAL SAMPLE DEPTH

COA

HNF. MJ-48E-1

$15^{1}$

OFFSITE PROPERTY NO.

123514 ES10

BILL OF LADING/AIR BILL NO

$\mathrm{N} / \mathrm{A}$

$\begin{array}{cll}\text { PRESERVATION } & \text { None } & \text { None } \\ \text { TYPE OF CONTAINER } & \text { G/P } & \begin{array}{l}\text { Moisture } \\ \text { Resistant Cont }\end{array} \\ \text { NO. OF CONTAINER(S) } & 1 & 1 \\ \text { VOLUME } & 11 & 2009 \\ \text { SAMPLE ANALYSIS } & \begin{array}{l}\text { SEE ITEM (1) IN } \\ \text { SPECCAL Moisture } \\ \text { INSTRUCTIONS }\end{array}\end{array}$

SAMPLE DATE SAMPLE TIME

$4-29.08$ OK35

N/A
PRICE COOE

8 N

AIR QUALITY

METHOD OF SHIPMENT

GOVERNMENT VEHICLE
DATA TURNAROUND

45 Days / 45

Days

\begin{abstract}
LLT NO.
CHAIN OF POSSESSION

RELINQUISHED BY/ IEMOVED FROM JK EAlley JKFEC RELINQUISHED BY/REMOVED FROM RELINQUISHED BY/REMOVED FROM RELINQUUSHED BY/REMOVED FROM RELINQUISHED BY/REMOVED FROM RELINQUISHED BY/REMOVED FROM

SIGN/PRINT NAMES
DATE/TIME
DATE/TIME

\section{PECIAL INSTRUCTIONS}

SEE PAGE 2 FOR ALL SPECIAL INSTRUCTIONS 


\section{COLLECTOR}

NCO SAMPLER

\section{SAMPLING LOCATION}

C5856, 1-002

ICE CHEST NO.

\section{SHIPPED TO}

Environmentai Sciences Laboratory

\section{COMPANY CONTACT}

TRENT, STEVE

TELEPHONE NO.

$373-5869$

PROJECT DESIGNATION

200-BP-5 OU Characterization for Wells 299-E33-341 and 299-E33-342-G FIELD LOGBOOK NO.

ACTUAL SAMPLE DEPTH

\section{OFFSITE PROPERTY NO}

PROJECT COORDINATOR

WIDRIG, DL

SAF NO.

Fo8-075

$\cos$

$123514 E S 10$

\section{PRICE CODE $\quad 8 \mathrm{~N}$}

AIR QUALITY
45 Days / 45

Days

\section{SPECIAL INSTRUCTIONS}

** The 200 Area SRGRP Characterization and Monitoring Sampling and Analysis GKI applies to this SAF.

** ESL is to determine unsaturated hydraulic conductivity using the method(s) best suited for the soll texture and type.

** ESL is to submit copies of all Chain of Custodies and associated sample login documentation to EIS-SDM within 24 hours of sample receipt. They may be sent via e-mail to CPP Sample Management.

(1)6020M_ICPMS_ASTM_AE (TAL) \{Cadmium, Chromium, Silver\} 6020M_ICPMS_ASTM_AE (Add-On) \{Arsenic, Lead, Molybdenum, Selenium\} 6020M_METALS_ICPMS_WE (TAL) \{Cadmium, Chromium, Silver\}

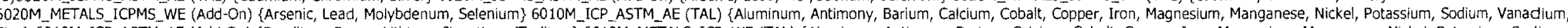

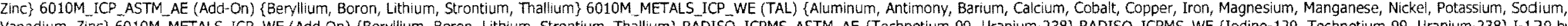

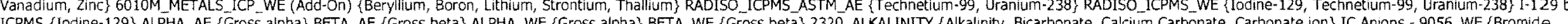

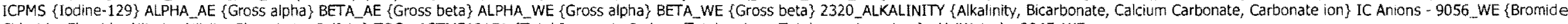
Chloride, Fluoride, Nitrate, Nitrite, Phosphate, Sulfate\} TOC-ASTME1915A \{Total Inorganic Carbon, Total carbon, Total organic carbon\} pH (Water)-9045 WE; 


\title{
COLLECTOR
}

NCOSAMPLER

BAlLEY

\section{SAMPLING LOCATION}

C5856, 1-003

ICE CHEST NO.

\section{COMPANY CONTACT}

TRENT, STEVE

\section{TELEPHONE NO.}

373-5869

PROJECT COORDINATOR

WIDRIG, DL

PRICE CODE

$8 \mathrm{~N}$

DATA

PROJECT DESIGNATION

SAF NO.

AIR QUALITY

TURNAROUNO

45 Days / 45

Days

200-BP-5 OU Characterization for Wells 299-E33-341 and 299-E33-342 - G F08-075

FIELD LOGBOOK NO.

ACTUAL SAMPLE DEPTH "COA

METHOD OF SHIPMENT

HNE. M. $488-1 \quad 17.5^{\prime}-20^{\prime} 123514$ ES10 Government VeHICLE

\section{SHIPPED TO}

Environmental Sciences Laboratory

\section{MATRIX* POSSIBLE SAMPLE HAZARDS/ REMARKS A=Air $\quad$ Contains Radioactive Material at concentrations uquids that are not regulated for transportation per 49 DS=Drum CFR but are not releasable per DOE Order Solids $\quad 5400.5(1990 / 1993)$ \\ $i=$ Liquid
$0=0$ il
$S=501$ \\ $0=0 \mathrm{il}$ \\ $S=S 0$ S
$S E=$ Sediment \\ $\mathrm{SE}=$ Sediment
$\mathrm{T}=\mathrm{Tissue}$ \\ $V=$ vegitation \\ $W=$ Water \\ $x=0$ ther \\ SPECIAL HANDLING AND/OR STORAGE Radioactive Tie To BITP96}

OFFSITE PROPERTY NO.

N/A

$\begin{array}{cl}\text { PRESERVATION } & \text { None } \\ \text { TYPE OF CONTAINER } & \begin{array}{l}\text { Split Spoon } \\ \text { Liner }\end{array} \\ \text { NO. OF CONTAINER(S) } & 2 \\ \text { VOLUME } & 10009 \\ \text { SAMPLE ANALYSIS } & \begin{array}{l}\text { SEE TEM (1) IN } \\ \text { SPECIAL } \\ \text { INSTRUTIONS }\end{array}\end{array}$

SAMPLE NO.

MATRIX*

SAMPLE DATE SAMPLE TIME

\begin{abstract}
B1TNV7
\end{abstract}
SOIL

BILL OF LADING/AIR BILL NO.

N/A

\section{CHAIN OF POSSESSION}

RELINQUISHED BY/REMOVED FROM

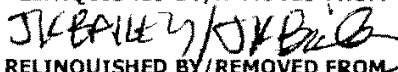
RELINQUISHED B $/$ REMOUED FROM mo 245 RELINQUISYED BY/REMOVED FPQM RELINQUISHED BY/REMOVED FROM

RELINQUISHED BY/REMOVED FROM

RELINQUISHED BY/REMOVED FROM

RELINQUISHED BY/REMOVED FROM

LABORATORY
SECTION
FINAL SAMPLE
DISPOSITION BY DISPOSAL METHOD

\section{SIGN/ PRINT NAMES}

RECEIVED BY/STORED IN DATE/TIME

\section{SPECIAL INSTRUCTIONS}

*** The 200 Area S\&GRP Characterization and Monitoring Sampling and Analysis GKI applies to this SAF

** ESL is to determine unsaturated hydraulic conductivity using the method(s) best 1600

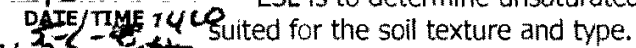

$4,32=08 *$ ESL is to submit copies of all Chain of Custodies and associated sample login $5 / P 9 T F^{\mathrm{TME}}$ documentation to EIS-SDM within 24 hours of sample receipt. They may be sent via

$\begin{array}{ll}\text { DATE/TIME } & \text { (1)Density; Particle Density - D854; Parti }\end{array}$

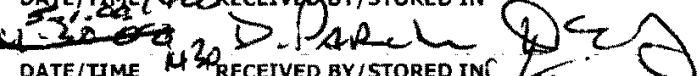
DATE/TIME
DATE/TIME

DATE/TIME RECEIVED BY/STORA IN

DATE/TIME

RECEIVED BY/STORED IN

RECEIVED BY/STORED IN

DATE/TIME
RECEIVED BY/STORED IN
DATE/TIME

(1) Density; Particle Density - D854; Partide Size (Dry Sieve) - D422; Particle Size

(Hydrometer) - D422; Saturated Hydraulic Conductivity; CATIONEXCH_TR; KD -

DATE/TIME

DATE/TIME
DATE/TIME
DATE/TIME 


\section{COLLECTOR \\ nco sampler Ballez?}

\section{SAMPLING LOCATION}

C5856, 1-008

ICE CHEST NO.

\section{SHIPPED TO}

Environmental Sciences Laboratory

MATRIX*

$A=A$ ir

$\mathrm{D}=$ Drum

Lquids

OS $=$ Drum
Solids
$t=$ Liquid

$0=0$ ol

$S=$ Soit
$S E=$ Sediment

$S E=$ Sediment
$T=$ Tissue

$v=$ vegitation

$W=$ water

WI $=$ Wipe

$x=$ other

POSSIBLE SAMPLE MAZARDS/ REMARKS Contains Radioactive Material at concentrations that are not regulated for transportation per 49 CFR but are not releasable per DOE Order

$5400.5(1990 / 1993)$

SPECIAL HANDLING AND/OR STORAGE Radioactive Tie To B1V4R3

\section{COMPANY CONTACT}

TRENT, STEVE

TELEPHONE NO.

$373-5869$

\section{PROJECT DESIGNATION}

200-BP-5 OU Characterization for Wells 299-E33-341 and 299-E33-342-G F08-075

\section{FIELD LOGBOOK NO.}

HNF-N- $488-1$

OFFSITE PROPERTY NO.

ACTUAL SAMPLE DEPTH

$$
251
$$

$N / A$

$\begin{array}{cll}\text { PRESERVATION } & \text { None } & \text { None } \\ \text { TYPE OF CONTAINER } & G / P & \begin{array}{l}\text { Moisture } \\ \text { Resistant Cont }\end{array} \\ \text { NO. OF CONTAINER(S) } & 1 & 1 \\ \text { VOLUME } & 11 & 2009 \\ \text { SAMPLE ANALYSIS } & \begin{array}{l}\text { SEE ITEM (1) in } \\ \text { SPECIAI Moisture } \\ \text { INSTRUCTIONS }\end{array}\end{array}$

SAMPLE DATE SAMPLE TIME

\section{$4-29-08 \quad 1320$}

\section{PROJECT COORDINATOR}

WIDRIG, DL

COA

123514 ES 10

BILL OF LADING/AIR BILL NO.

$N / A$
PRICE CODE 8 N

AIR QUALITY

METHOD OF SHIPMENT

GOVERNMENT VEHTCLE
SAMPLE NO.

B1TP26
SOIL
MATRIX*
31314

SIGN/ PRINT NAMES

DATE/TIME RECEIVED BY/STORED IN

RELINQUISHED BY/REMOVED FROM

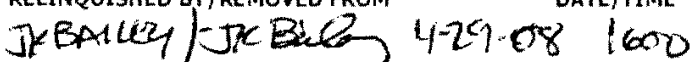
RELINQUTSHED GY/REMOVEDRETS MO $245 R$

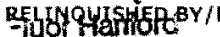

RELINQUISHED BY/REMOVED FROM OS

RELINQUISHED BY/REMOVED FROM

RELINQUISHED BY/REMOVED FROM

RELINQUUSHED BY/REMOVED FROM

LABORATORY RECEIVED BY SECTION

FINAL SAMPLE DISPOSAL METHOD DISPOSITION
WOTS REF \#। $4-29.08 \quad 1600$

DATE/TIME TORECEIVED BY/STORED IV / TATE/TIME I 200

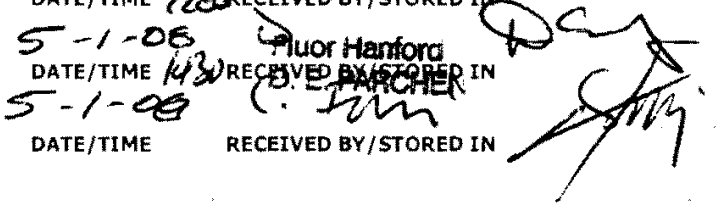

$5-1-0,5$

DATE/TIME

$\int / 1 / 001430$

DATE/TIME

DATE/TIME

DATE/TIME
DATE/TIME RECEIVED BY/STORED IN

DATE/TIME RECEIVED BY/STORED IN
SPECIAL INSTRUCTIONS

SEE PAGE 2 FOR ALL SPECIAL INSTRUCTIONS 


\section{TELEPHONE NO.}

$373-5869$

TRENT, STEVE

\section{PROJECT DESIGNATION}

SAF NO.

FIELD LOGBOOK NO.

ACTUAL SAMPLE DEPTH

COA

$123514 E 510$

\section{BILL OF LADING/AIR BILL NO.}

\section{OFFSITE PROPERTY NO.}

N/A
PRICE CODE

8N

METHOD OF SHIPMENT

GOVERNMENT VEHICLE

Environmental Solences Laboratory

\section{SPECIAL INSTRUCTIONS}

** The 200 Area S\&GRP Characterization and Monitoring Sampling and Analysis GKI applies to this SAF.

** ESL is to determine unsaturated hydraulic conductivity using the method(s) best suited for the soll texture and type.

** ESL is to submit copies of all Chain of Custodies and associated sample login documentation to EIS-SDM within 24 hours of sample receipt. They may be sent via e-mail to ^CPP Sample Management.

(1)6020M_ICPMS_ASTM_AE (TAL) \{Cadmium, Chromum, Silver\} 6020M_ICPMS_ASTM_AE (Add-On) \{Arsenic, Lead, Molybdenum, Selenium\} 6020M_METALS_ICPMS_WE (TAL) \{Cadmium, Chromium, Silver\}

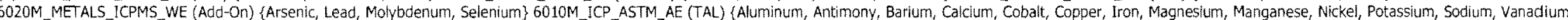

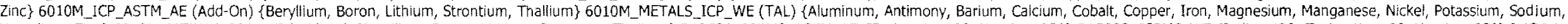

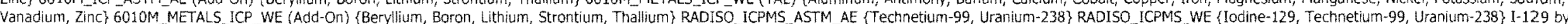

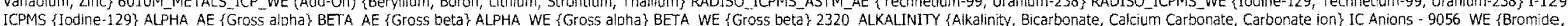

ICPMS \{lodine-129\} ALPHA_AE \{Gross alpha\} BETA_AE \{GrosS beta\} ALPHA_WE \{Gross alpha\} BETA_WE \{Gross beta\} 2320_ALKALINITY \{Alkalinity, Bicarbonate,
Chloride, Fluoride, Nitrate, Nitrite, Phosphate, Sulfate\} TOC-ASTME1915A \{Total Inorganic Carbon, Total carbon, Total organic carbon\} pH (Water)-9045 wE; 


\section{SAMPLING LOCATION}

C5856, I-014

ICE CHEST NO.

\section{SHIPPED TO}

Environmental Sciences Laboratory

MATRIX* POSSIBLE SAMPLE HAZARDS/ REMARKS
$A=A I T$

DL=Drum Contains Radioalte Mat transportation per 49

Liquids that are not regulated for transportation per 49

$\begin{array}{ll}\text { OS=Drum } & \text { CFR but are not rele } \\ \text { Solids } & 5400.5(1990 / 1993)\end{array}$

Lathouid

$\mathrm{O}=\mathrm{OH}$
$\mathrm{S}=\mathrm{Soit}$

$S E=$ Sediment

$T=T$ Tissue

$V=$ Vegtation

$W=$ Water

WI $=$ Wipe

SPECIAL HANDLING AND/OR STORAGE Radioactive Tie To: B1TP97

SAMPLE NO

MATRIX*

B1TNWO

SOIL

\section{COMPANY CONTACT}

TRENT, STEVE

\section{TELEPHONE NO.}

373-5869

PROJECT COORDINATOR

WIDRIG, DL

\section{SAF NO.}

200-BP-5 OU Characterization for Wells 299-E33-341 and 299-E33-342-G F08-075

FIELD LOGBOOK NO.

HNF-N-488-1

OFFSITE PROPERTY NO.

ACTUAL SAMPLE DEPTH

$$
40^{\circ}
$$

\section{COA}

123514ES10

BILL OF LADING/AIR BILL NO.

$N / A$

$\begin{array}{lll}\text { PRESERVATION } & \text { None } & \text { None } \\ \text { TYPE OF CONTAINER } & \text { G/P } & \begin{array}{l}\text { Moisture } \\ \text { Resistant Cont }\end{array}\end{array}$

No. OF CONTAINER(S)

vOLUME

IL

2009

SAMPLE ANALYSIS
PRICE CODE 8N

AIR QUALITY
DATA TURNAROUND

45 Days / 45

\section{METHOD OF SHIPMENT}

GOVERNMENT VEHICLE

\section{SAMPLE DATE SAMPLE TIME}

$4-30-08 \quad 0800$

LOT NO.

\section{CHAIN OF POSSESSION}

RELINQUISHED BY/REMOVED FROM RELINQUISHED A T/REMOVED GROM mo $245 R$ R ol RELINQUISHED BY/REMOVED FROM ivor trantord REIINOUISARGHFF REMOVED FROM

RELINQUISHED BY/REMOVED FROM

RELINQUISHED BY/REMOVED FROM

RELINQUISHED BY/REMOVED FROM

LABORATORY RECEIVED BY

SECTION

FINAL SAMPLE DISPOSAL METHOD

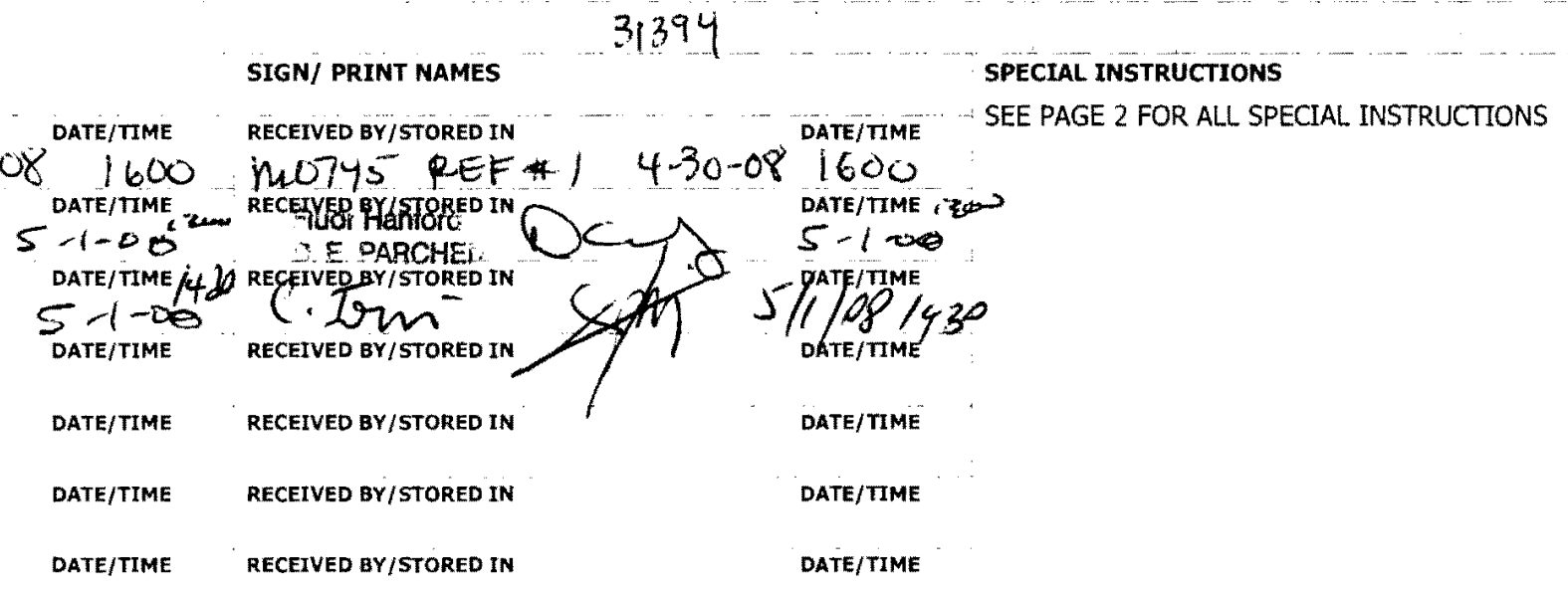




\section{COLLECTOR}

COMPANY CONTACT

TRENT, STEVE

TELEPHONE NO.

373-5869

PROJECT COORDINATOR

WIDRIG, DL

PRICE CODE

ATA

\section{SAMPLING LOCATION}

C5856, $1-014$

ICE CHEST NO.

\section{SHIPPED TO}

Environmental Sciences Laboratory

\section{PROJECT DESIGNATION}

200-BP-5 OU Characterization for Wells 299-E33-341 and 299-E33-342-G

FIELD LOGBOOK NO.

ACTUAL SAMPLE DEPTH

OFFSITE PROPERTY NO.

N/A
SAF NO.

F08-075

COA

123514 ES10
AIR QUALITY

\section{BILL OF LADING/AIR BILL NO.}

\section{SPECIAL INSTRUCTIONS}

** The 200 Area S\&GRP Characterization and Monitoring Sampling and Analysis GKI applies to this SAF.

(1)6020M_ICPMS ASTM AE (TAL) \{Cadmium, Chromium, Silver\} 6020M_ICPMS ASTM_AE (Add-On) \{Arsenic, Lead, Molybdenum, Selenium\} 6020M METALS ICPMS WE (TAL) \{Cadmium, Chromium, Silver\}

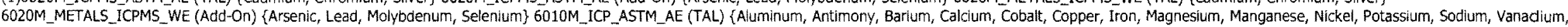

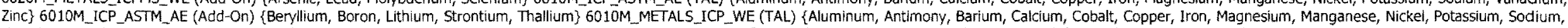

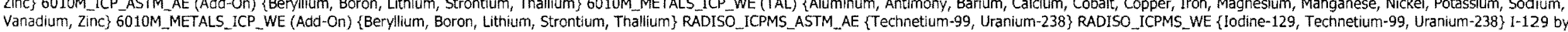

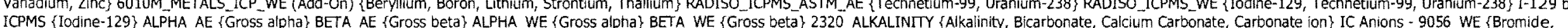

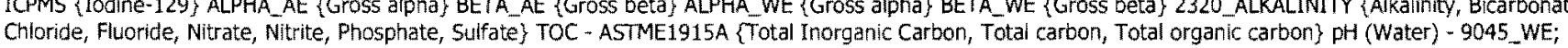




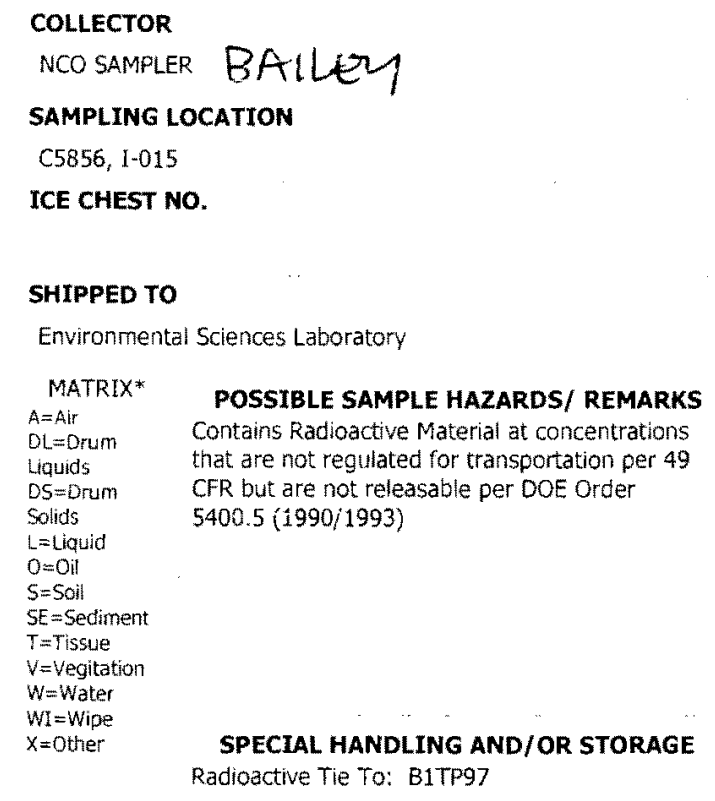

SHIPPED TO

Environmental Sciences Laboratory

MATRIX*

$A=$ Air

$\mathrm{DL}=\mathrm{Drum}$

Liquids

DS =idrum
Solds

Solids

$0=0$ il
$5=5$ oil

$S=S \mathrm{Sil}$
$\mathrm{SE}=\mathrm{Sediment}$
$\mathrm{T}=$ Tissue

$V=$ Vegitation

WI =Wipe

$x=$ other

POSSTBLE SAMPLE HAZARDS/ REMARKS Contains Radioactive Material at concentrations that are not regulated for transportation per 49 CFR but are not releasable per DOE Order $5400.5(1990 / 1993)$

SPECIAL HANDLING AND/OR STORAGE Radioactive Tie To: B1TP97

\section{COMPANY CONTACT}

TRENT, STEVE

TELEPHONE NO.

PROJECT COORDINATOR

WIORIG, DL

PRICE CODE $\quad 8 \mathrm{~N}$

\section{PROJECT DESIGNATION}

SAF NO.

200-BP-5 OU Characterization for Wells 299-E33-341 and 299-E33-342 - G FO8-075

\section{FIELD LÖGBOOK NO. ACTUAL SAMPLE DEPTH COA}

AIR QUALITY

METHOD OF SHIPMENT

$123514 E S 10$ GOVERNMENT VEHICLE

BILL OF LADING/AIR BILL NO.

OFFSITE PROPERTY NO.

$$
42.51
$$

$N / A$

\begin{tabular}{|c|c|c|}
\hline PRESERVATION & None & None \\
\hline TYPE OF CONTAINER & G/P & $\begin{array}{l}\text { Moisture } \\
\text { Resistant Cont }\end{array}$ \\
\hline NO. OF CONTAINER(S) & 1 & 1 \\
\hline VOLUME & IL & 2009 \\
\hline SAMPLE AN & $\begin{array}{l}\text { SEE TIEM (1) IN } \\
\text { SPEEILL } \\
\text { INSTRUCTIONS }\end{array}$ & $\begin{array}{l}\text { Moisture } \\
\text { Content - D2: }\end{array}$ \\
\hline
\end{tabular}

SAMPLE DATE SAMPLE TIME

$4-30-08 \quad 02=0$

\section{CHAIN OF POSSESSION}

RELINQUISHED BY/REMOVEQ FROM

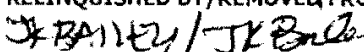
4.30 .081600 on $07 \times 5$ Ref

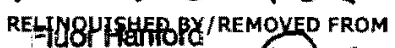
D. E. PARCHEN USO

RELINQUISHED BY/REMOVED FROM

RELINQUISHED BY/REMOVED FROM

RELINQUISHED BY/REMOVED FROM

LABORATORY RECEIVED BY
SECTION
$\begin{gathered}\text { FINAL SAMPLE } \\ \text { DISPOSITION }\end{gathered}$

MATRIX* OIL

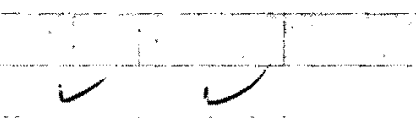

\section{4}

\section{SIGN/ PRINT NAMES}

DATE/TIME

\section{SPECIAL. INSTRUCTIONS}

SEE PAGE 2 FOR ALL SPECIAL INSTRUCTIONS $5-1-\infty$

RECTD BY/STORED IN

MOTYS REF

$\checkmark=$ PARCHE DATE/TIME 1430 RECEIVEDEY/STORED IN $5.1-08$ DATE/TIME

DATE/TIME

cigrn

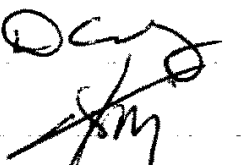

ATE/TIME $C$

$5 . c 008$

DATE/TIME

$\$ 1 / 1001430$

DATE/TIME

RECEIVED BY/STORED IN

DATE/TME

DATE/TIME

DATE/TIME

DATE/TIME 


\section{COLLECTOR}

COMPANY CONTACT

TELEPHONE NO.

PROJECT COORDINATOR

373-5869

WIDRIG, DL

\section{SAMPLING LOCATION}

TRENT, STEVE

SAF NO.

PRICE CODE

\section{PROJECT DESIGNATION}

AIR QUALITY

TURNAROUND

200-BP-5 OU Characterization for Wells 299-E33-341 and 299-E33-342-G

FIELD LOGBOOK NO.

ACTUAL SAMPLE DEPTH

\section{COA}

METHOD OF SHIPMENT

123514 ES10

GOVERNMENT VEHICLE

OFFSITE PROPERTY NO

N/A

\section{BILL OF LADING/AIR BILL NO}

Environmental Sciences Laboratory

$\mathrm{N} / \mathrm{A}$

\section{SPECIAL INSTRUCTIONS}

** The 200 Area S\&GRP Characterization and Monitoring Sampling and Analysis GKI applies to this SAF.

(1)6020M_ICPMS_ASTM_AE (TAL) \{Cadmium, Chromium, Silver\} 6020M_ICPMS_ASTM_AE (Add-On) \{Arsenic, Lead, Molybdenum, Selenium\} 6020M_METALS_ICPMS_WE (TAL) \{Cadmium, Chromium, Silver\}

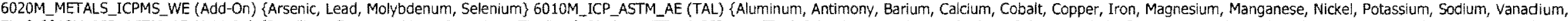

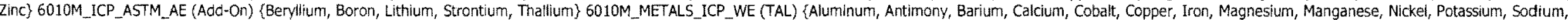

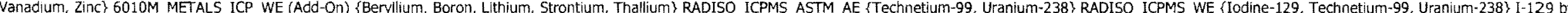

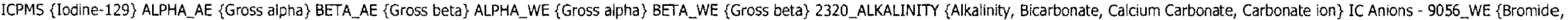

Chloride, Fluoride, Nitrate, Nitrite, Phosphate, Sulfate\} TOC - ASTME1915A \{Total Inorganic Carbon, Total carbon, Total organic carbon\} pH (Water) - 9045 WE; 


\section{COLLECTOR}

NCO SAMPLER BAlLE?

\section{SAMPLING LOCATION}

C5856, I-016

ICE CHEST NO.

\section{SHIPPED TO}

Environmental Sciences Laboratory

\begin{tabular}{|c|c|}
\hline MATRIX* & \\
\hline$A=A i r$ & Contains Radioactive Material at concentrations \\
\hline $\begin{array}{l}\mathrm{DL}=\mathrm{Crum} \\
\mathrm{Linutids}\end{array}$ & that are not regulated for transportation per 49 \\
\hline$D S=D r u m$ & CFR but are not releasable per DOE Order \\
\hline Solids & $5400.5(1990 / 1993)$ \\
\hline $\begin{array}{l}\text { L=Liquid } \\
\mathrm{D}=\mathrm{Oit}\end{array}$ & \\
\hline $5=5$ oil & \\
\hline$S E=$ Sediment & \\
\hline$T=T$ issue & \\
\hline $\begin{array}{l}V=\text { vegitation } \\
W=\text { Water }\end{array}$ & \\
\hline WI=Wipe & - - \\
\hline$x=$ other & $\begin{array}{l}\text { SPECIAL HANDLING AND/OR STORAGE } \\
\text { Radioactive Tie To: B1TPG7 }\end{array}$ \\
\hline
\end{tabular}

\section{COMPANY CONTACT}

TELEPHONE NO.

PROJECT COORDINATOR

373-5869 WIDRIG, DL

\section{PROJECT DESIGNATION}

SAF NO.

RICE CODE

200-BP-5 OU Characterization for Wells 299-E33-341 and 299-E33-342-G F08-075

FIELD LOGBOOK NO.

ACTUAL SAMPLE DEPTH

COA

HNA $-C D-488-1$

OFFSITE PROPERTY NO.

$$
45
$$

123514 ES 10

METHOD OF SHIPMENT

GOVERNMENT VEHICLE

BILI OF LADING/AIR BILL NO.

N/A

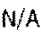

SAMPLE NO. MATRIX*

B1TNW2

SAMPLE DATE SAMPLE TIME

$4-30-08 \quad 0853$

\begin{tabular}{|c|c|c|}
\hline PRESERVATION & None & None \\
\hline TYPE OF CONTAINER & G/P & $\begin{array}{l}\text { Moisture } \\
\text { Resistant Cont }\end{array}$ \\
\hline NO. OF CONTAINER(S) & 1 & 1 \\
\hline VOLUME & $1 \mathrm{~L}$ & 2009 \\
\hline SAMPLE ANALYSIS & $\begin{array}{l}\text { SEE TTEM (1) IN } \\
\text { SPECIAL } \\
\text { INSTRUCTONS }\end{array}$ & $\begin{array}{l}\text { Moisture } \\
\text { Content- D2z }\end{array}$ \\
\hline
\end{tabular}

Lot nJo.

CHAIN OF POSSESSION

RELINQUISHED BY/REMOVED FROM IKBAlLC) $\mid<$ BiD RELINQUISHED BY/REMOKED FRON mo $775 R$ R RELINQUISHED BY REMOVEDEROM $D_{\text {RELINQUISHED BY/REMOVED FROM }}$

RELINQUISHED BY/REMOVED FROM

RELINQUISHED BY/REMOVED FROM

RELINQUISHED GY/REMOVED FROM

RECEIVED BY

\section{LABORATORY}

\section{SECTION}

FINAL SAMPLE DISPOSITION
DISPOSAL METHOD

\section{4}

\section{SIGN/ PRINT NAMES}

DATE/TIME

RECEIVED BY/STORED IN

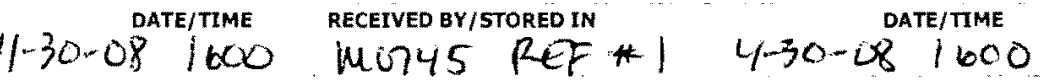
DATE/TIME I I $\omega$ RECEIVEP BY/STORED IN DATE/TIME * 30\%

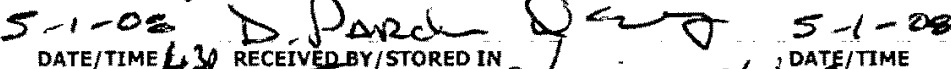

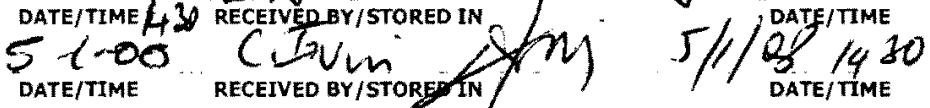
DATE/TIME

DATE/TMME

RECEIVED BY/STORED IN

DATE/TIME

DATE/TIME

RECEIVED BY/STORED IN

DATE/TIME

DATE/TIME
SPECIAL INSTRUCTIONS

SEE PAGE 2 FOR ALL SPECIAL. INSTRUCTIONS 


\section{COLLECTOR}

COMPANY CONTACT

TELEPHONE NO.

$373-5869$

PROJECT COORDINATOR

TRENT, STEVE

WIDRIG, DL

PRICE CODE

DATA

\section{SAMPLING LOCATION}

C5856, I-016

ICE CHEST NO.

\section{SHIPPED TO}

Environmental Sciences Laboratory

\section{PROJECT DESIGNATION}

SAF NO.

AIR QUALITY

\section{METHOD OF SHIPMENT \\ GOVERNMENT VEHICLE}

\section{OFFSITE PROPERTY NO.}

N/A
ACTUAL SAMPIE DEPTH

\section{BILL OF LADING / AIR BILL NO.}

N/A

\section{SPECIAL INSTRUCTIONS}

*** The 200 Area S\&GRP Characterization and Monitoring Sampling and Analysis GKI applies to this SAF.

(1)6020M_ICPMS_ASTM_AE (TAL) \{Cadmium, Chromium, Silver\} 6020M_ICPMS_ASTM_AE (Add-On) \{Arsenic, Lead, Molybdenum, Selenium\} 6020M_METALS_ICPMS_WE (TAL) \{Cadmium, Chromium, Silver\}

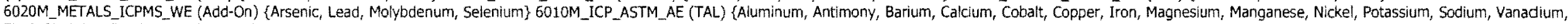

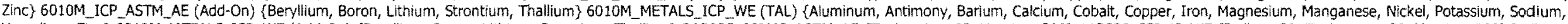

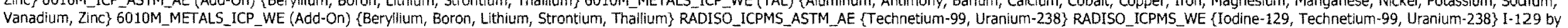

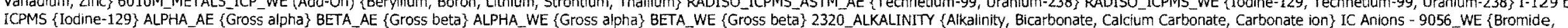

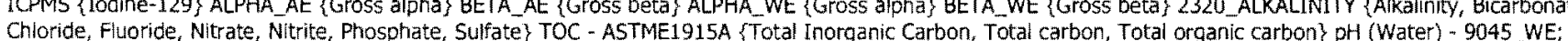




\section{PROJECT DESIGNATION}

\section{SAF NO.}

200-BP-5 OU Characterization for Wels 299-E33-341 and 299-E33-342-G FO8-075

ICE CHEST NO.

\section{SHIPPED TO}

Environmental Sciences Laboratory

\section{MATRIX* POSSIBLE SAMPLE HAZARDS/ REMARKS \\ A $=$ Air $\quad$ Contains Radioactive Matera \\ $\mathrm{DL}=$ Drum that are not regulated for transportation per 49 \\ Liquids $\quad$ that are not regulated for transportation per \\ $D S=$ Drum \\ $\zeta=L$ iquid \\ $\mathrm{O}=\mathrm{Oil}$ \\ $S=50 i 1$ \\ $T=$ Tissue \\ $v=$ vegitation \\ $W=$ Water \\ $w !=$ wipe \\ SPECIAL HANDLING AND/OR STORAGE Radioactive Tie To: B1TP9? \\ tare noteasable per DOE Order $5400.5(1990 / 1993)$}

SAMPLE NO. MATRIX*

B1TNW3

SOIL
COA

HNF-N- $-48 ?$ -

ACTUAL SAMPLE DEPTH

$$
47.51
$$

123514ES10

\section{METHOD OF SHIPMENT}

BIL OF LADING/AIR BILL NO.

OFFSITE PROPERTY NO.

N/A

A

\begin{tabular}{|c|c|c|}
\hline PRESERVATION & None & None \\
\hline TYPE OF CONTAINER & G/P & $\begin{array}{l}\text { Molsture } \\
\text { Resistant Con }\end{array}$ \\
\hline NO. OF CONTAINER(S) & 1 & 1 \\
\hline VOLUME & IL & $200 \mathrm{~g}$ \\
\hline SAMPLE ANALYSIS & $\begin{array}{l}\text { SEE ITEM (1) IN } \\
\text { SPECIAL } \\
\text { INSTRUCTIONS }\end{array}$ & $\begin{array}{l}\text { Moisture } \\
\text { Content. }\end{array}$ \\
\hline
\end{tabular}

SAMPLE dATE SAMPLE TIME

$4-30.08 \quad 0915$

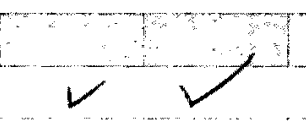

\section{CHAIN OF POSSESSION}

RELINQUISHED BY/REMOVED FROM I BAILY UCBSil RELINQUISHED EY/REMOVEREROM mo $245 R+A$ GEUNRYSYHOE BY/RETOYED FROM EELINQUISHED BY/REMOVED FROM

RELINQUISHED BY/REMOVED FROM

RELINQUISHED BY/REMOVED FROM

RELINQUISHED BY/REMOVED FROM

LABORATORY RECEIVED BY SECTION

FINAL SAMPLE DISPOSAL METHOD

\section{$313 \times 4$}

SIGN/ PRINT NAMES

DATE/TIME RECEIVED BY/STORED IN

iMOT4S REF

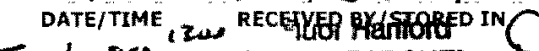

$5-1-08$ $\checkmark$ FARCHEN DATE/TIME 43 RECEIVEQRY/STORED IN $5-1-08$ C. bum DATE/TIME

DATE/TIME

RECEIVED BY/STORED IN

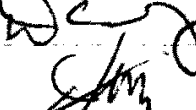

DATE/TIME

\section{SPECIAL INSTRUCTIONS}

SEE PAGE 2 FOR ALL SPECIAL INSTRUCTIONS
DATE/TIME

RECEIVED BY/STORED IN

DATE/TIME RECEIVED BY/STORED IN DATE/TIME

1000

DATE/TIME ; $\mathrm{SO}=$

$5-2-08$

5 paje/time

$5 / 1 / 0814+0$

DATE/TIME

DATE/TIME

DATE/TIME

DATE/TIME RECEIVED BY/STORED IN DATE/TIME 


\section{PROJECT DESIGNATION}

WIDRIG, DL

AIR QUALITY

TURNAROUND

200-BP-5 OU Characterization for Wells 299-E33-341 and 299-E33-342-G F08-075

C5856, I-017

FIELD LOGBOOK NO.

ACTUAL SAMPLE DEPTH

COA

METHOD OF SHIPMENT

5 Days / 45

Days

\section{OFFSITE PROPERTY NO.}

N/A

123514 ES10

GOVERNMENT VEHICLE

\section{SHIPPED TO}

Environmental Sciences Laboratory

\section{SPECIAL INSTRUCTIONS}

** The 200 Area S\&GRP Characterization and Monitoring Sampling and Analysis GKI applies to this SAF.

(1)6020M_ICPMS_ASTM_AE (TAL) \{Cadmium, Chromium, Silver\} 6020M_ICPMS_ASTM_AE (Add-On) \{Arsenic, Lead, Molybdenum, Selenium\} 6020M_METALS_ICPMS_WE (TAL) \{Cadmium, Chromium, Silver\}

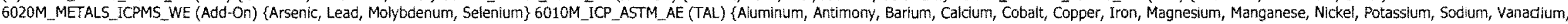

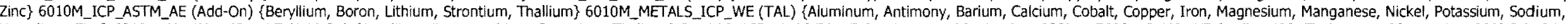

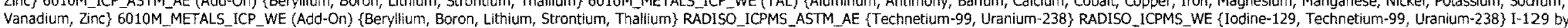

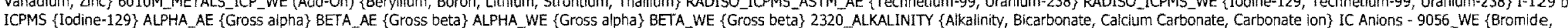

Chloride, Fluoride, Nitrate, Nitrite, Phosphate, Sulfate\} TOC - ASTME1915A \{Total Inorganic Carbon, Total carbon, Total organic carbon\} pH (Water) -9045_WE; 


\section{PROJECT DESIGNATION}

\section{SAF NO.}

C5856,1-018

200-BP-5 OU Characterization for Wells 299-E33-341 and 299-E33-342-G F08-075

ICE CHEST NO.

\section{FIELD LOGBOOK NO.}

ACTUAL SAMPLE DEPTH

\section{COA}

HNE-N- $488-1$

$50^{\prime}$

$123514 E S 10$

METHOD OF SHIPMENT

45 Days / 45 Days

\section{SHIPPED TO}

Environmental Sciences Laboratory
MATRIX*
$A=$ Air
$\mathrm{Dt}=$ Drum
POSSIBLE SAMPLE HAZARDS/ REMARKS
D5=Drum CFR but are not releasable per DOE Order
Solids $\quad 5400.5(1990 / 1993)$

OFFSITE PROPERTY NO.

BILL OF LADING/AIR BILL NO.

GOVERNMENT VEHICLE

$N / A$

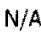

$L=$ Liquid
$O=0$ il

$S=S$ oil

SE $=$ Sediment

$T=$ Tissue

$V=$ Vegitation

$W I=$ Wipe

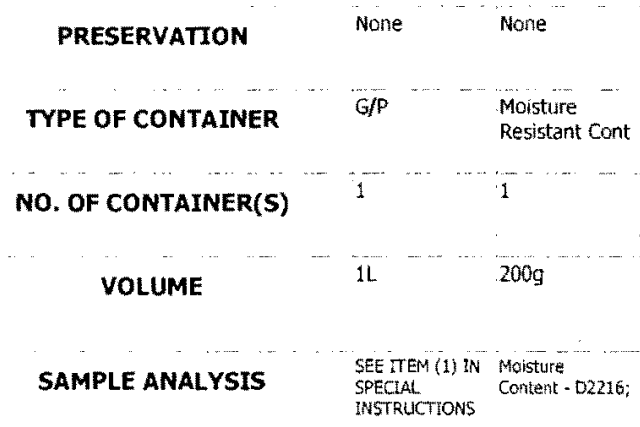

Radioactive Tie To: B1TP9?

SAMPLE DATE SAMPLE TIME

SAMPLE NO.

MATRIX*

B1TNW4

SOIL

$4-30-0 P \quad 0930$

\section{4}

\section{SIGN/ PRINT NAMES}

DATE/TIME RECEIVED BY/STORED IN

RELINQUISHED BY/REMOVED FROM

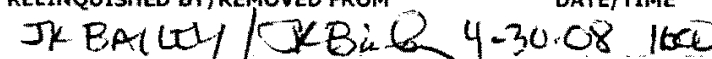
RELINQUISHED BY/REMOVED FRQM DSATE/TIME I ZOORECETVED BY/STORED IN morres $R$ RELINQUISHED BY REMOVEP FROM $\therefore=$ DARCHE (bor RELINQUISHED BY IREMOVED FROM

RELINQUISHED BY/REMOVED FROM

RELINQUISHED BY/REMOVED FROM

RELINQUISHED BY/REMOVED FROM $5-1$ - OS tuor Hanforo DATE/TIME ADREgEIVABEAFEORED IN $5-1-08$ DATE/TIME

DATE/TIME

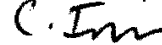

RECEIVED BY/STORED

DATE TIME

RECEIVED BY/STORED IN

RECEIVED EY/STORED IN

DATE/TIME 


\section{COLLECTOR}

\section{NCO SAMPLER}

\section{COMPANY CONTACT}

\section{TELEPHONE NO.}

$373-5869$

PROJECT COORDINATOR

WIDRIG, DL

PRICE CODE

8N

DATA

\section{PROJECT DESIGNATION}

\section{SAF NO.}

AIR QUALITY

TURNAROUND

200-BP-5 OU Characterization for Wells 299-E33-341 and 299-E33-342-G F08-075

FIELD LOGBOOK NO.

ACTUAL SAMPLE DEPTH

COA

$123514 \mathrm{ES} 10$

METHOD OF SHIPMENT

GOVERNMENT VEHICLE

OFFSITE PROPERTY NO.

$N / A$

BILL OF LADING/AIR BILL NO.

N/A

\section{SPECIAL INSTRUCTIONS}

Environmental Sciences Laboratory

** The 200 Area S\&GRP Characterization and Monitoring Sampling and Analysis GKI applies to this SAF.

(1)6020M_ICPMS_ASTM_AE (TAL) (Cadmium, Chromium, Silver\}6020M_ICPMS_ASTM_AE (Add-On) \{Arsenic, Lead, Molybdenum, Selenium\}6020M_METALS_ICPMS_WE (TAL) \{Cadmium, Chromium, Silver\}

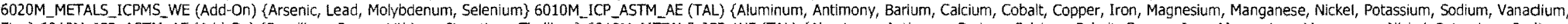

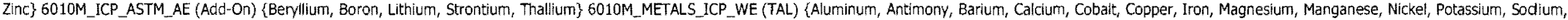

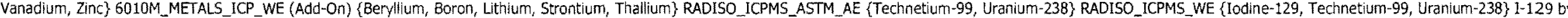

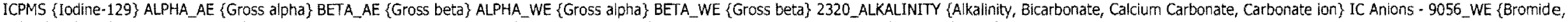
Chloride, Fluoride, Nitrate, Nitrite, Phosphate, Sulfate\} TOC - ASTME1915A \{Total Inorganic Carbon, Total carbon, Total organic carbon\} pH (Water) - 9045 WE; 


\section{ICE CHEST NO.}

\section{SHIPPED TO}

Environmental Sciences Laboratory

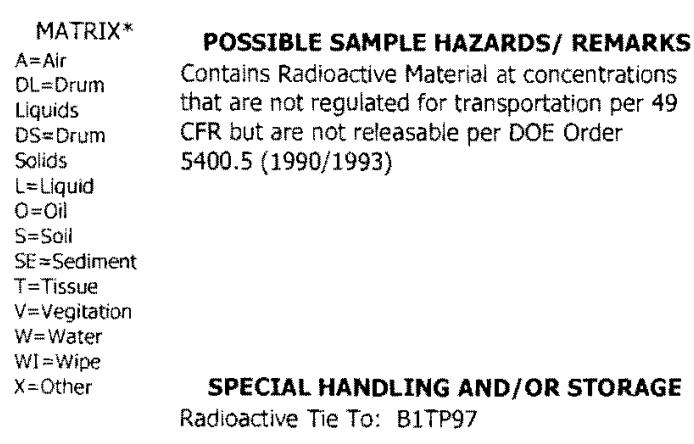

POSSIBLE SAMPLE HAZARDS/ REMARKS Contains Radioactive Material at concentrations that are not requlated for transportation per 49 CFR but are not releasable per DOE Order $5400.5(1990 / 1993)$

HNF-N- $488-1$

COA

OFFSITE PROPERTY NO.

$$
52.51
$$

$123514 E S 10$

METHOD OF SHIPMENT

GOVERNMENT VEHICLE

BILL OF LADING /AIR BILL NO.
N/A

\begin{tabular}{|c|c|c|}
\hline PRESERVATION & None & None \\
\hline TYPE OF CONTAINER & $\mathrm{G} / \mathrm{P}$ & $\begin{array}{l}\text { Moisture } \\
\text { Resistant Cont }\end{array}$ \\
\hline NO. OF CONTAINER(S) & 1 & 1 \\
\hline VOLUME & 1L" & 2009 \\
\hline SAMPLE ANALYSIS & $\begin{array}{l}\text { SEE TTEM (1) IN } \\
\text { SPECIAL } \\
\text { INSTRUCTIONS }\end{array}$ & $\begin{array}{l}\text { Mossture } \\
\text { Content - D Z Z }\end{array}$ \\
\hline
\end{tabular}

SAMPLE NO. MATRIX*

B1TNW5

SAMPLE DATE SAMPLE TIME

$4-30.08 \quad 1025$

\section{4}

SIGN/ PRINT NAMES

RECEIVED BY/STORED IN

\section{SPECIAL INSTRUCTIONS}

DATE/TIME

\section{0}

DATE/TIME , ZNO

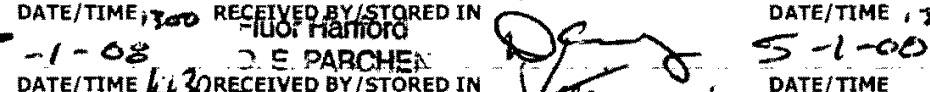

DATE/TTME 4 30RECEIVED BY/STORED IN DTT DATE/TIME

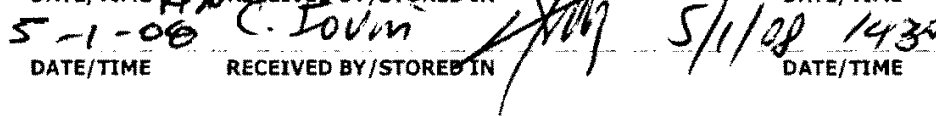

DATE/TIME RECEIVED BY/STORED IN

DATE/TIME

DATE/TIME RECEIVED BY/STORED IN

DATE/TIME

DATE/TIME 


\section{COLLECTOR}

NCO SAMPLER

SAMPLING LOCATION

C5856, I-019

ICE CHEST NO.

\section{SHIPPED TO}

Environmental Sciences Laboratory

\section{COMPANY CONTACT}

TRENT, STEVE

PROJECT DESIGNATION

200-BP-5 OU Characterization for Wells 299-E33-341 and 299-E33-342-G

FIELD LOGBOOK NO.

ACTUAL SAMPLE DEPTH

OFFSITE PROPERTY NO

N/A

\section{TELEPHONE NO.}

373-5869
PROJECT COORDINATOR

WIDRIG, DL

SAF NO.

COA

123514ES10

\section{PRICE CODE}

AIR QUALITY
DATA TURNAROUND

45 Days / 45

Days

\section{SPECIAL INSTRUCTIONS}

** The 200 Area S\&GRP Characterization and Monitoring Sampling and Analysis GKI applies to this SAF

(1)6020M_ICPMS_ASTM_AE (TAL) \{Cadmium, Chromium, Silver\} 6020M_ICPMS_ASTM_AE (Add-On) \{Arsenic, Lead, Molybdenum, Selenium\} 6020M METALS ICPMS WE (TAL) \{Cadmium, Chromium, Silver\}

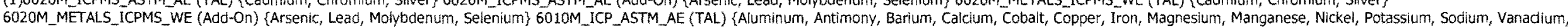

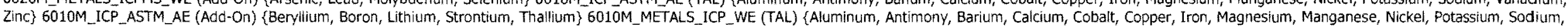

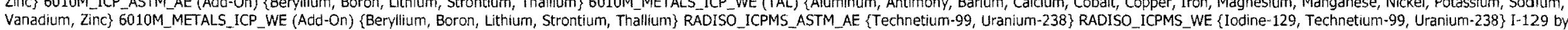

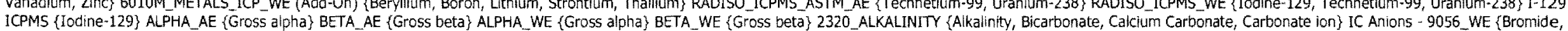
Chloride, Fluoride, Nitrate, Nitrite, Phosphate, Sulfate\} TOC - ASTME1915A \{Total Inorganic Carbon, Total carbon, Total organic carbon\} pH (Water) - 9045 .WE; 


\section{COLLECTOR}

nCo SAMPLER Ballet

SAMPLING LOCATION

C5856, I-020

ICE CHEST NO.

\section{SHIPPED TO}

Environmental Sciences Laboratory

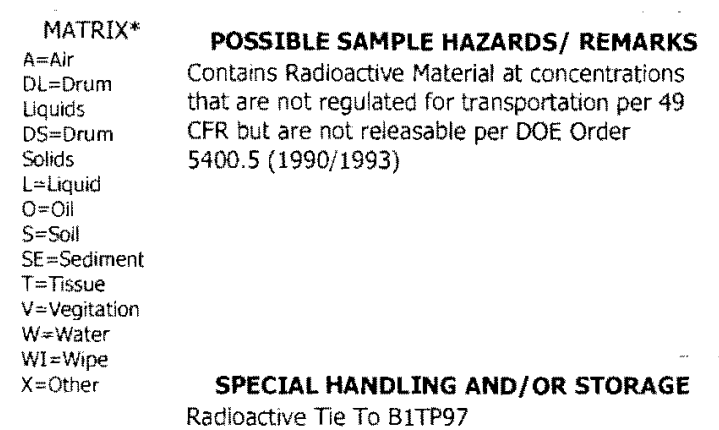

SAMPLE NO. MATRIX*

B1TT72 SOIL

\section{COMPANY CONTACT}

TRENT, STEVE

TELEPHONE NO.

$373-5869$

PROJECT DESIGNATION

200-BP-5 OU Characterization for Walls 299-E33-341 and 299-E33-342-G F08-075

FIELD LOGBOOK NO.

ACTUAL SAMPLE DEPTH

HNF-N-488-1

OFFSITE PROPERTY NO.

$55^{\prime}-57.5^{\prime}$

N/A

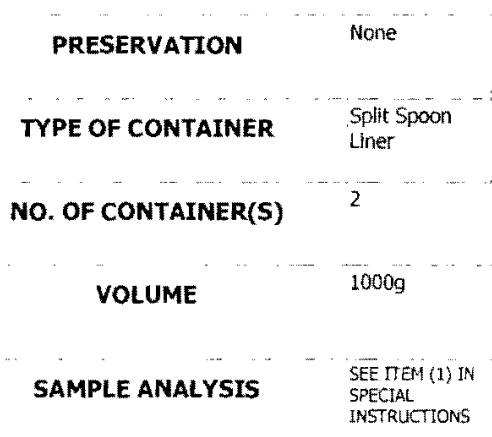

SAMPLE DATE SAMPLE TIME

$4-30-08.1100$

\section{PROJECT COORDINATOR}

WIDRIG, DL.

SAF NO.
F08-075

COA

$123514 E 510$

BILL OF LADING / AIR BILL NO

N/A
PRICE CODE

DATA TURNAROUND

45 Days / 45 Days
METHOD OF SHIPMENT

GOVERNMENT VEHICLE 


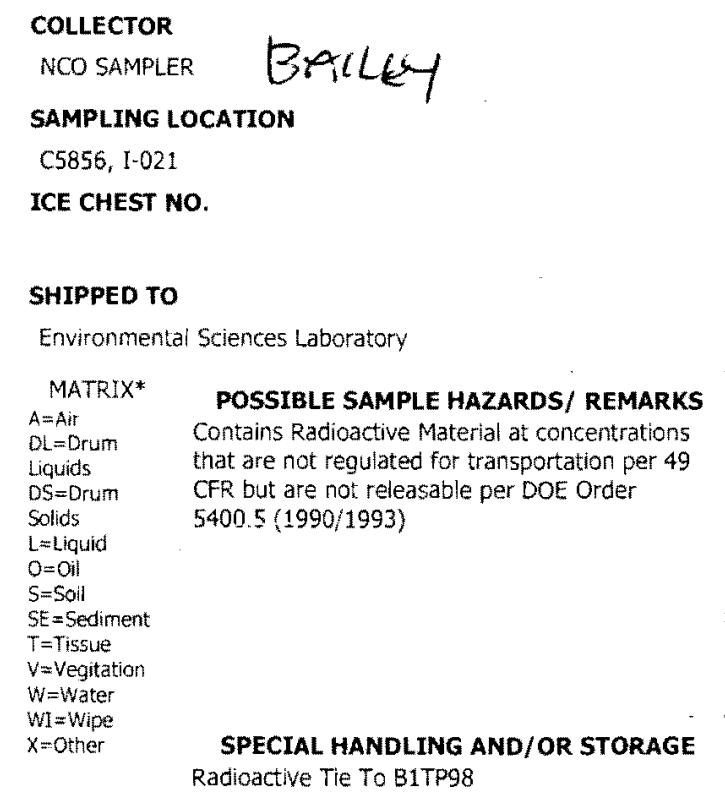

SHIPPED TO

Environmental Sciences Laboratory
MATRIX* POSSIBLE SAMPLE HAZARDS/ REMARKS A $=$ Air
$D L=D$ rum DS=Drum CFR but are not releasable per DOE Order $\sum_{L=L \text { iquid }} \quad 5400.5(1990 / 1993)$
$L=$ Liquid
$0=0 \mathrm{il}$
$\mathrm{S}=\mathrm{OII}$
$\mathrm{Sol}$
$S=S a$ Sediment
$T=T$ issue
$T=T$ issue
$V=V$ Vegitation
$W=$ water
WI $x$ Wipe
$x=$ Other
SPECIAL HANDLING AND/OR STORAGE Radioactive TIe To B1TP98 Liquids that are not regulated for transportation per

\section{COMPANY CONTACT}

TRENT, STEVE

TELEPHONE NO.

PROJECT COORDINATOR

WIDRIG, DL.

PRICE CODE

DATA

PROJECT DESIGNATION

SAF NO.

200-BP-5 OU Characterization for Wells 299-E33-341 and 299-E33-342-G F08-075

FIELD LOGBOOK NO.

HNF-N. Y $88-1$

ACTUAL SAMPLE DEPTH

COA

OFFSITE PROPERTY NO

$$
55
$$

123514ES10

AIR QUALITY

TURNAROUND

45 Days / 45

Days

$N / A$

\begin{tabular}{|c|c|c|}
\hline PRESERVATION & None & None \\
\hline TYPE OF CONTAINER & $\mathrm{G/P}$ & $\begin{array}{l}\text { Moisture } \\
\text { Resistant Cont }\end{array}$ \\
\hline NO. OF CONTAINER(S) & 1 & 1 \\
\hline VOLUME & $1 \mathrm{~L}$ & 2009 \\
\hline SAMPLE ANALYSIS & $\begin{array}{l}\text { SEE ITEM (1) IN } \\
\text { SPECTAL } \\
\text { INSTRUCTONS }\end{array}$ & $\begin{array}{l}\text { Moisture } \\
\text { Content - D22 }\end{array}$ \\
\hline
\end{tabular}

GOVERNMENT VEHTCLE

BILL OF LADING/AIR BILL NO.

N/A

SAMPLE NO.
BITP31 SOIL MATRIX*

SAMPLE DATE SAMPLE TIME

$4-3008 \quad 1043$

\section{4}

\section{SIGN/ PRINT NAMES}

RECEIVED BY/STORED IN

WOT45 REF H 430 -OR DATE/TMM

RECEIVED BY+STORED IN DATE/TIME

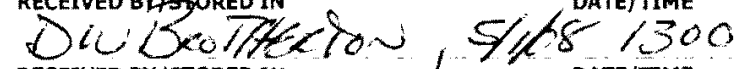

RECEIVEREY/STOREDIN

Ci IO W

RECEIVEO BY / STORED

RECEIVED BY/STORED IN

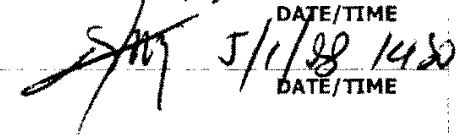

DATE/TMME

DATE/TIME

RECEIVED BY/STORED IN

DATE/TIME

RELINQUISHED BY/REMOVED FROM

DATE/TIME

RECEIVED BY/STORED IN

DATE/TIME

DATE/TTME

RELINQUISHED BY/REMOVED FROM

\section{SPECIAL INSTRUCTIONS}

SEE PAGE 2 FOR ALL SPECIAL INSTRUCTIONS

RECEIVED BY

LABORATORY
SECTION

FINAL SAMPLE DISPOSAL METHOD

DISPOSITION

TITLE

DATE/TIME

DISPOSED BY

DATE/TIME 


\section{COLLECTOR}

NCO SAMPLER

\section{SAMPLING LOCATION}

C5856, 1-021

ICE CHEST NO.

\section{SHIPPED TO}

Environmental Sciences Laboratory

\section{COMPANY CONTACT}

TRENT, STEVE

TELEPHONE NO.

373-5869

\section{PROJECT DESIGNATION}

200-BP-5 OU Characterization for Wells 299-E33-341 and 299-E33-342 - 6

\section{FIELD LOGBOOK NO.}

ACTUAL SAMPLE DEPTH

\section{OFFSITE PROPERTY NO.}

N/A
PROJECT COORDINATOR

WIDRIG, DL

SAF NO.

F08-075

123514ES 10

\section{BILL OF LADING/AIR BILL NO.}

\section{SPECIAL INSTRUCTIONS}

** The 200 Area S\&GRP Characterization and Monitoring Sampling and Analysis GKI applies to this SAF.

** ESL is to determine unsaturated hydraulic conductivity using the method(s) best suited for the soil texture and type.

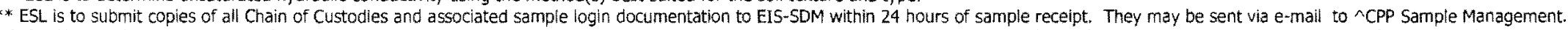

(1)6020M_ICPMS_ASTM_AE (TAL) \{Cadmium, Chromium, Siver\} 6020M_ICPMS_ASTM_AE (Add-On) \{Arsenic, Lead, Molybdenum, Selenium\} 6020M_METALS_ICPMS WE (TAL) \{Cadmium, Chromium, Silver\}

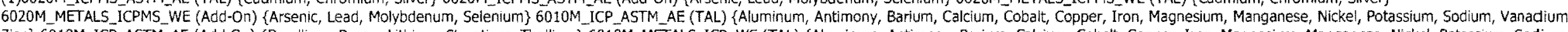

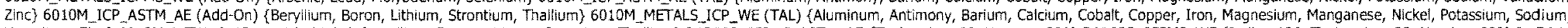

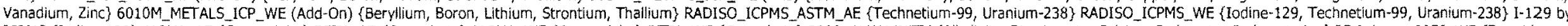

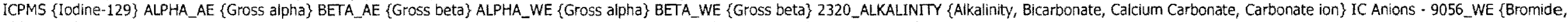

Chioride, Fluoride, Nitrate, Nitrite, Phosphate, Sulfate\} TOC - ASTME1915A (Total Inorganic Carbon, Total carbon, Total organic carbon\} pH (Water) - 9045 WE; 


\section{COLLECTOR \\ nco sampler Ballery}

SAMPLING LOCATION

C5856, I-022

ICE CHEST NO.

\section{SHIPPED TO}

Environmental Sciences Laboratory

MATRIX*
A=Air
DL=Drum
Liquids
DS $=$ Drum
Solids
$L=$ Liquid
$D=0$ il
$S=$ Soil
$S E=S$ Sediment
$T=$ Tissue
$V=$ Vegitation
$W=$ Water
$W=$ Wipe
$X=0$ ther

POSSIBLE SAMPLE HAZARDS/ REMARKS Contains Radicactive Material at concentrations that are not regulated for transportation per 49 CFR but are not releasable per DOE Order $5400.5(1990 / 1993)$ per DOE Order

\section{SPECIAL HANDLING AND/OR STORAGE Radioactive Tle To B1TP98}

\begin{abstract}
SAMPLE NO. MATRIX*
B1TT73
\end{abstract}

\section{COMPANY CONTACT}

TRENT, STEVE

\section{TELEPHONE NO.}

\section{PROJECT DESIGNATION}

200-BP-5 OU Characteriza FIELD LOGBOOK NO.

$$
H N F-N-488-1
$$

OFFSITE PROPERTY NO.

N/A

\begin{tabular}{|c|c|}
\hline PRESERVATION & None \\
\hline TYPE OF CONTAINER & $\begin{array}{l}\text { Split Spoon } \\
\text { Liner }\end{array}$ \\
\hline NO. OF CONTAINER(S) & 2 \\
\hline VOLUME & 10009 \\
\hline SAMPLE ANALYSIS & $\begin{array}{l}\text { SEE TTEM (1) IN } \\
\text { SPECIAL } \\
\text { INSTRUCTOONS }\end{array}$ \\
\hline
\end{tabular}

SAMPLE DATE SAMPLE TIME

$4.30-08 \quad 1230$
373.5869

\section{PRICE CODE $8 \mathrm{~N}$}

AIR QUALITY -

WIDRIG, D

\section{SAF NO.}

ACTUAL SAMPLE DEPTH COA

123514ES10

BILL OF LADING/AIR BILL NO.

METHOD OF SHIPMENT

GOVERNMENT VEHICLE
SOIL

\section{N/A}

DATA TURNAROUND

45 Days / 45

Days

\section{CHAIN OF POSSESSION}

REIINQUISHED BY/REMOVED FROM

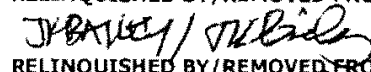
MELINQUISHED BY/REMOVEYTROM RELINQUISHED BY/REMOVED FROM Fluar Hantord $2 \mathrm{C}_{2}$

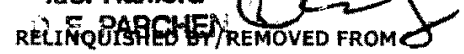

RELINQUISHED BY/REMOVED FROM

RELINQUISHED BY/REMOVED FROM

RELINQUISHED BY/REMOVED FROM

LABORATORY RECEIVED BY SECTION

FINAL SAMPLE DISPOSAL METHOD
DATE/TIME OQ IECO $5-1-0 \in 3$ DATE/TIME $k$ Y 5-1-06 DATE/TIME

DATE/TIME

DATE/TIME

DATE/TIME

\section{SIGN/ PRINT NAMES}

RECEIVED BY/STOREO IN WO 745 PEF I RECEIVED BY/STOR
GOO Hantord
D

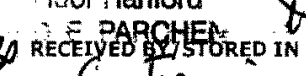
C.Inm RECEIVED BY/STORED IN

RECEIVED BY/STORED IN

RECEIVED BY/STORED IN

RECEIVED BY/STORED IN

\section{SPECLAL INSTRUCTIONS}

DATE/TIME 1600 applies to this SAF.

Area S\&GRP Characterization and Monitoring Sampling and Analysis GKI pplies to this SAF. $\mathrm{DATE} / \mathrm{TMME}$, sw suited for the soil texture and type.

$5-1-03$ ** ESL is to submit copies of all Chain of Custodies and associated sample login DATE/TIME documentation to EIS-SDM within 24 hours of sample receipt. They may be sent via $5 / 1 / 02 / 4$ lo - mail to ^ CPp Sample Management.

Gafe/fime (1)Density; Particle Density - 0854; Particle Size (Dry Sieve) - D422; Particle Size

(Hydrometer) - D422; Saturated Hydraulic Conductivity; CATIONEXCH_TR; KD -

DATE/TIME Batch;

DATE/TIME

DATE/TIME
ITTLE

DISPOSED BY
DATE/TIME 


\section{COLLECTOR}

nCo sampler Ballet

SAMPLING LOCATION

C5856, 1-023

ICE CHEST NO.

\section{SHIPPED TO}

Environmental Sciences Laboratory

\begin{tabular}{|c|c|}
\hline MATRIX* & POSSIBLE SAMPLE HAZARDS/ REMARKS \\
\hline $\begin{array}{l}A=A i r \\
D L=\text { Drum }\end{array}$ & Contains Radioactive Material at concentrations \\
\hline Lquids & that are not regulated for transportation per 49 \\
\hline $\begin{array}{l}\text { DS=Drum } \\
\text { Solids }\end{array}$ & $\begin{array}{l}\text { CFR but are not releasable per DOE Order } \\
5400.5(1990 / 1993)\end{array}$ \\
\hline $\begin{array}{l}L=\text { Liquid } \\
O=O i l\end{array}$ & \\
\hline$S=$ Soll & \\
\hline $\begin{array}{l}\text { SE }=\text { Sedinent } \\
T=T \text { Tissue }\end{array}$ & \\
\hline$V=$ Vegitation & \\
\hline $\begin{array}{l}W=\text { Water } \\
\text { WI }=\text { Wipe }\end{array}$ & \\
\hline$x=$ other & $\begin{array}{l}\text { SPECIAL HANDLING AND/OR STORAGE } \\
\text { adioactive Tie To B1TP98 }\end{array}$ \\
\hline
\end{tabular}
Radioactive Tie To B1TP98

SAMPLE NO.

MATRIX*
SOIL

\section{COMPANY CONTACT}

TRENT, STEVE

PROJECT DESIGNATION

\section{TELEPHONE NO.}

373-5869
PROJECT COORDINATOR

WIDRIG DL

SAF NO.

\section{FIELD LOGBOOK NO.}

HNJF, NO- 488 -

OFFSITE PROPERTY NO.

N/A

COA

123514 ES10

METHOD OF SHIPMENT

GOVERNMENT VEHICLE

OF LADING/AIR BILI NO

\begin{tabular}{|c|c|c|}
\hline PRESERVATION & None & None \\
\hline TYPE OF CONTAINER & $\mathrm{G} / \mathrm{P}$ & $\begin{array}{l}\text { Moisture } \\
\text { Resistant Cont }\end{array}$ \\
\hline NO. OF CONTAINER(S) & 1 & 1 \\
\hline VOLUME & $1 \mathrm{~L}$ & $200 \mathrm{~g}$ \\
\hline SAMPLE ANALYSIS & $\begin{array}{l}\text { SEE ITEM (1) IN } \\
\text { SPECTAL } \\
\text { INSTRUCTIONS }\end{array}$ & $\begin{array}{l}\text { Moisture } \\
\text { Content - }\end{array}$ \\
\hline
\end{tabular}

SAMPLE DATE SAMPLE TIME

$4-30-01 \quad 1223$

\section{4}

\section{SIGN/ PRINT NAMES}

RECEIVED BY/STORED IN

MoT45 LEF +1

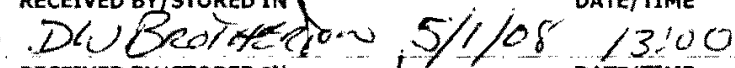

DATEITIME

$5 / 1 / 08 / 3100$

$5 \%$ i O $\delta^{\text {DATE/TIME }}+30$

Mo>4S TEP

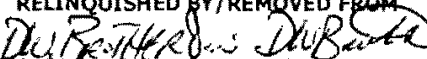

REIINQUISHED BY/REMOVED FROM

DATE/TIME

RELINQUISHED BY/REMOVED FROM

DATE/TIME

DATE/TIME

DATE/TIME
RECEIVED BY/STORED IN

RECEIVED BY/STORED

RECEIVED BY/STORED IN

RECEIVED BY/STORED IN

RECEIVED BY/STORED IN

\section{SPECIAL INSTRUCTIONS}

SEE PAGE 2 FOR ALL SPECIAL INSTRUCTIONS
RELINOUISHED BY/REMOVED FROM

LABORATORY RECEIVED BY

$$
\text { SECTION }
$$

FINAL SAMPLE DISPOSAL METHOD 


\section{COLLECTOR}

NCO SAMPLER

SAMPLING LOCATION

C5856, 1-023

ICE CHEST NO.

\section{SHIPPED TO}

Environmental Sciences Laboratory

\section{COMPANY CONTACT}

TRENT, STEVE

PROJECT DESIGNATION

TELEPHONE NO.

373-5869

200-BP-5 OU Characterization for Weils 299-E33-341 and 299-E33-342 - G

FIELD LOGBOOK NO.

ACTUAL SAMPLE DEPTH

OFFSITE PROPERTY NO

N/A
PROJECT COORDINATOR

WIDRIG, DL

SAF NO.

F08-075

COA

123514 ES10
PRICE CODE

$8 \mathrm{~N}$

AIR QUALITY

Days

METHOD OF SHIPMENT

GOVERNMENT VEHICLE

\section{SPECIAL INSTRUCTIONS}

** The 200 Area 5\&GRP Characterization and Monitoring Sampling and Analysis GKI applies to this SAF.

** ESL is to determine unsaturated hydraulic conductivity using the method(s) best suited for the soil texture and type.

** ESL is to submit copies of all Chain of Custodies and associated sample login documentation to EIS-SDM within 24 hours of sample receipt. They may be sent via e-mail to ACPP Sample Management.

(1)6020M_ICPMS_ASTM_AE (TAL) (Cadmium, Chromium, Silver\} 6020M_ICPMS_ASTM AE (Add-On) \{Arsenic, Lead, Molybdenum, Selenium\} 6020M_METALS ICPMS WE (TAL) \{Cadmium, Chromium, Silver\}

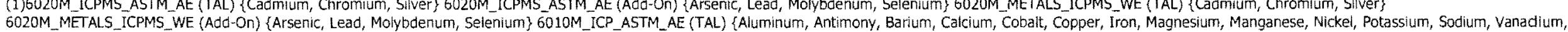

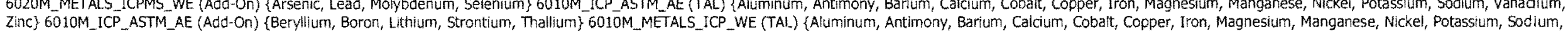

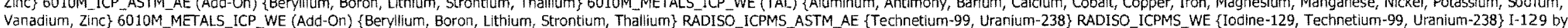

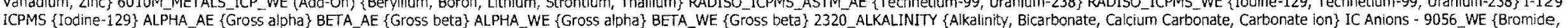
Chioride, Fluoride, Nitrate, Nitrite, Phosphate, Sulfate\} TOC - ASTME1915A \{Total Inorganic Carbon, Total carbon, Total organic carbon\} pH (Water) -9045_WE; 


\section{COLLECTOR \\ nco sampler PAlley}

SAMPLING LOCATION

C5856, 1-024

ICE CHEST NO.

\section{SHIPPED TO}

Environmental Sciences Laboratory

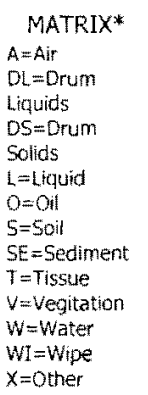

POSSIBLE SAMPLE HAZARDS/ REMARKS Contains Radioactive Material at concentrations that are not regulated for transportation per 49 CFR but are not releasable per DOE Order $5400.5(1990 / 1993)$

SPECIAL HANDLING AND/OR STORAGE Radioactive Tie To B1TP98

SAMPLE NO.

MATRIX*

B1TT74

SOIL

\section{COMPANY CONTACT}

TRENT, STEVE

TELEPHONE NO.

$373-5869$

PROJECT COORDINATOR

WIDRIG, DL

SAF NO.

PROJECT DESIGNATION

200-BP-5 OU Characterization for Wells 299-E33-341 and 299-E33-342-G F08-075

FIELD LOGBOOK NO.

ACTUAL SAMPLE DEPTH

COA

HNF-N-488-1

OFFSITE PROPERTY NO.

N/A

$$
60^{\prime}-62.5^{\prime}
$$

23514ES10

BILL OF LADING/AIR BIUL NO.
PRICE CODE $\quad 8 \mathrm{~N}$ TURNAROUND

45 Days / 45

Days
PRESERVATION

TYPE OF CONTAINER Split Spoon

NO. OF CONTAINER(S)

VOLUME

10009

SAMPLE ANALYSIS

\section{METHOD OF SHIPMENT}

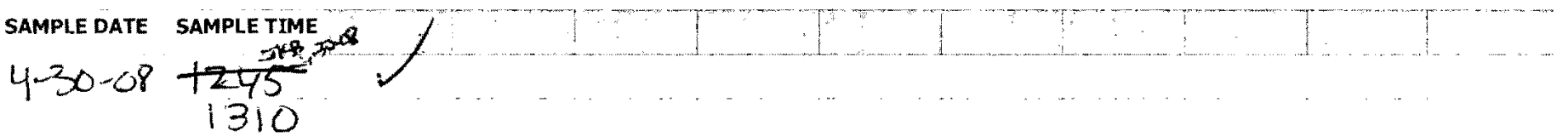

\section{CHAIN OF POSSESSION}

RELINQUISHED BY/REMOVED FROM TE BAILY OKK BCL Mo Z45 R $P$ t REINQUISHED BY/REMOVED FROM Guor Hantord

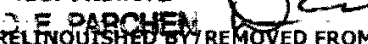

RELINQUISHED BY/REMOVED FROM

RELINQUISHED BY/REMOVED FROM

RELINQUISHED BY/REMOVED FROM

RECEIVED BY

\section{ABORATOR}

$$
\text { SECTION }
$$

RECEIVED BYISTORED IN

M0745 REF \#/ 430 408 1600 DATE/TIME,

\section{$5-1$-OS} DATE PARCHEN DATE/TIME T\$KRECEYYED BY/STORED $5-1-2 S$

DATE/TIME

$$
\text { E: }
$$

Loz

DATE/TIME

RECEIVED BY/STORED IN

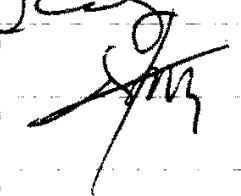

DATE/TIME

RECEIVED BY/STORED IN

DATE/TIME

DATE/TIME

DATE/TIME

\section{SPECIAL INSTRUCTIONS}

** The 200 Area S\&GRP Characterization and Monitoring Sampling and Analysis GKI applies to this SAF.

** ESL is to determine unsaturated hydraulic conductivity using the method(s) best 1300 suited for the soil texture and type.

$-1 \rightarrow *$ ESL is to submit copies of all Chain of Custodies and associated sample login DATE/TIME documentation to EIS-SDM within 24 hours of sample receipt. They may be sent via $5 / 1 / 091432$-mail to ^ CPP Sample Management.

(1)Density; Particle Density - D854; Particle Size (Dry Sieve) - D422; Particle Size

(Hydrometer) - D422; Saturated Hydraulic Conductivity; CATIONEXCH_TR; KD -

DATE/TIME Batch; 


\section{COLLECTOR}

nco sampler Bfilty

\section{SAMPLING LOCATION}

C5856, I-025

ICE CHEST NO.

\section{SHIPPED TO}

Environmental Sciences Laboratory

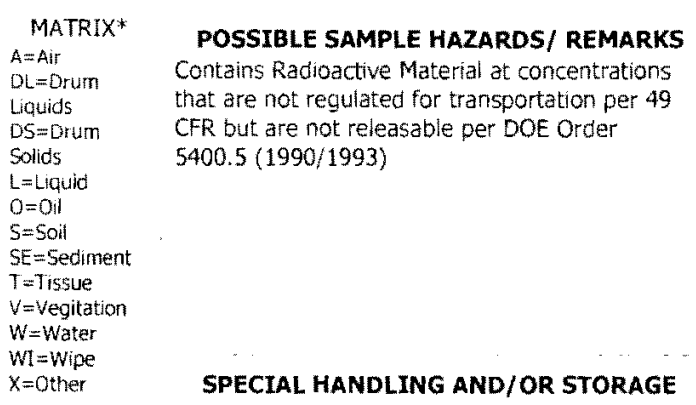

MATRIX*

\section{CHAIN OF POSSESSION}

RELINQUISHED BY/REMOVED FROM TLBALEY JLBEO $4-3008160$ RELINOUISHED BY/KEMOVED FROM D OATE/TIME Mi 745 pef $5 / 108$ RELINQUISHED BY/ AEMOVED FROM DE Brothleton Dis RELINQUISHED BY/REMOVED FROM DATE/TIME $5 / 108142$

RELINQUISHED BY/REMOVED FROM DATE/TIME RELINQUISHED BY/REMOVED FROM DATE/TIME DATE/TIME

\section{TELEPHONE NO.}

373-5869

TRENT, STEVE
PROJECT COOROINATOR WIDRIG, DL

\section{SAF NO.}

200-BP-5 OU Characterization for Wells 299-E33-341 and 299-E33-342-G F08-075

FIELD LOGBOOK NO.

ACTUAL SAMPLE DEPTH

123514 ES10

BILL OF LADING/AIR BILL NO.

HNF,N.488-1 $60^{\prime} \quad 123514$ ES10 GOVERNMENT VEHICLE

OFFSITE PROPERT
PRICE CODE $\quad 8 N$

AIR QUALITY

$\mathrm{N} / \mathrm{A}$

$\begin{array}{lll}\text { PRESERVATION } & \text { None } & \text { None } \\ \text { TYPE OF CONTAINER } & \text { G/P } & \begin{array}{l}\text { Moisture } \\ \text { Resistant Cont }\end{array}\end{array}$

NO. OF CONTAINER(\$) 1

VOLUME

1. 200

SAMPLE ANALYSIS SEE TEM (1) IN Moisture
SPECLAL
IMSTRUCTONS

SAMPLE DATE SAMPLE TIME

$4.30 .08 \quad 1245$

3) 394

SIGN / PRINT NAMES

RECEIVED BY/STORED IN MOT45 RE 4

RECEIVEO BY/STOREDIN

Dhergar

RECEVEQTE/STORER IN Cinin

RECEIVED BY/STORED IN

RECEIVEO BY/STORED IN

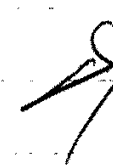

$5 / 108$ 13,10 DATE/TIME $5 / 100$ bate/TIME

DATE/TIME

DATE/TIME

DATE/TIME

\section{RECEIVED BY/STORED IN}

RECEIVED BY/STORED IN

\section{SPECIAL INSTRUCTIONS}

SEE PAGE 2 FOR ALL SPECIAL INSTRUCTIONS
RELINOUISHED BY/REMOVED FROM

\section{LABORATORY} SECTION

FINAL SAMPLE DISPOSITION
RECEIVED BY

DISPOSAL METHOD 
COLECTOR

Fluor Hanford Inc.

NCO SAMPLER

SAMPLING LOCATION

C5856, I-025

ICE CHEST NO

SHIPPED TO

Envirommental Sciences Laboratory

\section{SPECIAL INSTRUCTIONS}

** The 200 Area $5 \& G 8 p$ C

** ESL is to determine unsaturated hydraulic conductivity Samping and Analysis GKI applies to this SAF.

(1)6020 M ICFM it copies of all Chain of Custodies and associng the method(s) best suited for the soil texture and type.

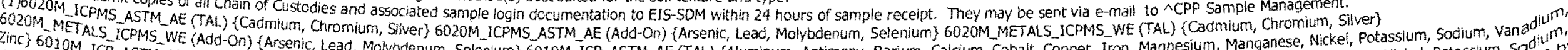

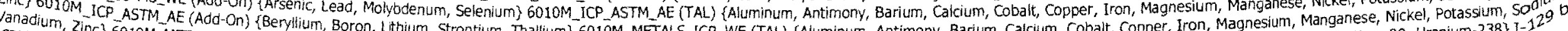

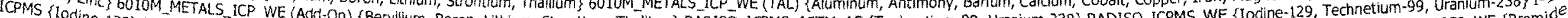

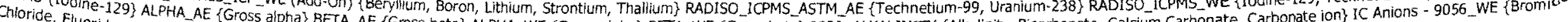

Cide, Fluoride, Nitrate, Nitrite, Phosphate, Suifate $\}$ TOC -ASTS beta\} ALPHA_WE \{Gross alpha\} BETA_WE \{Gross beta\} 2320_ALXALINITV \{Alkalinity, Bicarbonate,

\section{F08-075-026}

PROJECT DESIGNATION

200-BP-5 OU Characterization for Wells 299-E33-341 and 299-E33-342-G

\section{OFFSITE PROPERTY NO.}

ACTUAL SAMPLE DEPTH
PROJECT COORDINATOR

WDRIG, OL

\section{SAF NO.}

COA

$123514 E 510$
DATA $N$ D TURNARO 45 Days $/ 4$

\section{METHOD OF SHIPMENT}

GOVERNMENT VEHICLE

\section{DING/AIR BILL NO.}

\section{COMPANY CONTACT}




\section{COLLECTOR}

NCO SAMPLER BAILEZ

SAMPLING LOCATION

C5856, 1-026

ICE CHEST NO.

\section{SHIPPED TO}

Environmental Sciences Laboratory

MATRIX*

$A=A$ Air
$D L=D r u m$

$\mathrm{O}=\mathrm{Drum}$
Liguids

LS $=$ Drum

Solids

$\mathrm{L}=\mathrm{Liquid}$
$\mathrm{O}=\mathrm{O}$ il

$S=5$ oil

SE $=$ Sediment

$T=T$ Tissue
$V=$ Veutration
$W$

$V=$ vegltation
$W=$ water

$W=$ Wipe

$x=0$ ther

POSSIBLE SAMPLE HAZARDS/ REMARKS Contains Radioactive Material at concentrations that are not regulated for transportation per 49 CFR but are not releasable per DOE Order $5400.5(1990 / 1993)$

SPECIAL HANDIING AND/OR STORAGE Radioactive Tie To B1TP98
SAMPLE NO.

B1TT75
MATRIX*

SOIL

\section{TELEPHONE NO.}

373-5869
COMPANY CONTACT

TRENT, STEVE

PROJECT DESIGNATION

200-BP-5 OU Characterization for Wells 299-E33-341 and 299-E33-342-G FIELD LOGBOOK NO.

\section{ACTUAL SAMPLE DEPTH}

HNF-N-488-

N/A

\section{PRESERVATION}

TYPE OF CONTAINER Split Spoon

NO. OF CONTAINER(S)

VOLUME

$62.5^{\prime}-65,0$

PROJECT COORDINATOR

WIDRIG, OL

SAF NO.

F08-075

COA

123514ES 10

BILL ÓF LADING/AIR BILL NO

N/A
OFFSITE PROPERTY NO.

\section{METHOD OF SHIPMENT}

GOVERNMENT VEHICLE
SAMPLE DATE SAMPLE TIME

$430.08 \quad 1342$
None"

2

$1000 \mathrm{~g}$

SEE TEM (1) IN

SPECIAL
INSTRUCTIONS

\section{CHAIN OF POSSESSION}

RELINQUISHED BY/REMOVED FROM JKEAILU/CJPBCElO 4.30 .081600 RELINQUISHED B//REMGVD FhOM

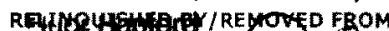
D. E. PARCHEN ( 2 RELINQUISHED BY/REMOVED FROM O

RELINQUISHED BY/REMOVED FROM

RELINQUISHED BY/REMOVED FROM

RELINQUISHED BY/REMOVED FROM

LABORATORY RECEIVED BY
SECTION
FINAL SAMPLE DISPOSAL METHOD
DISPOSIYION
$5-1-00$ $5-1$-OO I 30 E PARCHEN $5-1+0$ DATE/TIME

DATE/TIME

DATE/TIME

DATE/TIME MO 7L REF \#

\section{SIGN/ PRINT NAMES}

RECEIVED BY/STORED IN E PAACHEX

C. TO WM RECEIVED BY/STORED

RECEIVED BY/STORED IN

RECEIVED BY/STORED IN

RECEIVED BY/STORED IN

\section{SPECIAL INSTRUCTIONS}

** The 200 Area S\&GRP Characterization and Monitoring Sampling and Analysis GKI applies to this SAF.

** ESL is to determine unsaturated hydraulic conductivity using the method(s) best 1600 DATE/TIME, 30ssuited for the soil texture and type.

$5-1-O \&, * *$ ESL is to submit copies of all Chain of Custodies and associated sample login - DATE/TIME documentation to EIS-SDM within 24 hours of sample receipt. They may be sent via $\nu_{\text {e-mail to }}^{\text {^CPP Sample Management. }}$

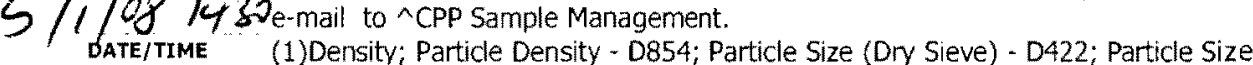

(Hydrometer) - D422; Saturated Hydraulic Conductivity; CATIONEXCH_TR; KD -

DATE/TIME Batch

DATE/TIME

DATE/TIME
TTLE

DISPOSED BY
DATE/TIME

DATE/TIME 


\section{TELEPHONE NO.}

$373-5869$

TRENT, STEVE

\section{PROJECT DESIGNATION}

200-BP-5 OU Characterization for Wells 299-E33-341 and 299-E33-342 - G FIELD LOGBOOK NO.

$$
\text { HNF.N-48R-1 }
$$

OFFSITE PROPERTY NO.

$N / A$ UAL SAMPLE DEPTH

$$
62.5^{\prime}
$$

\section{PROJECT COORDINATÓ} WIDRIG, DL

\section{SAF NO.}

COA

$123514 E S 10$

BILL OF LADING/AIR BILL NO.
Environmental Sciences Laboratory

MATRIX*

$A=$ Air

$D \mathrm{~L}=\mathrm{Drum}$

Liquids

DS=Drum

Solids

$L=L$ iquid
$0=0$ il

$S=S$ Sol
$S E=$ Sediment

$S E=$ Sediment
$T=$ Tissue

$\forall=$ vegitation

$w=$ water

WI $=$ Wipe

$X=0$ ther Contains Radioactive Material at concentrations
SPECIAL HANDLING AND/OR STORAGE Radioactive Tie To B1TP98

POSSIB SAMPLE HAZARDS/ REMARKS that are not regulated for transportation per 49 CFR but are not releasable per DOE Order $5400.5(1990 / 1993)$

SAMPLE NO.

MATRIX*

SOLL

\section{CHAIN OF POSSESSION}

RELINQUISHED BY/REMPVED FROM JKEAILU/ $\angle K E \times C O$ y 30.08 DATE/TIME RELINQUISHE BYIREMOVEOROM N10 $>45$ AEF Z RELINOUYSHED BY(REMOVEDFROM

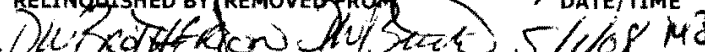
RELINOUISHED BY/REMOVED FROM

REINOUISHED BY/REMOVED FROM DATE/TIME

RELINQUISHED BY/REMOVED FROM

DATE/TIME

RELINQUISHED BY/REMOVED FROM

DATE/TIME

\begin{tabular}{|c|c|c|}
\hline PRESERVATION & None & None \\
\hline TYPE OF CONTAINER & G/P & $\begin{array}{l}\text { Moisture } \\
\text { Resistant Cont }\end{array}$ \\
\hline NO. OF CONTAINER(S) & $i$ & i $\cdots$ \\
\hline VOLUME & $1 \mathrm{~L}$ & $200 \mathrm{~g}$ \\
\hline SAMPLE ANALYSIS & $\begin{array}{l}\text { SEE TTEM (I) IN } \\
\text { SPECIAL } \\
\text { INSTRUTIONS }\end{array}$ & $\begin{array}{l}\text { Moisture } \\
\text { Content-Dis16: }\end{array}$ \\
\hline
\end{tabular}

SAMPLE DATE SAMPLE TIME

$4-30-08 \quad 1324$
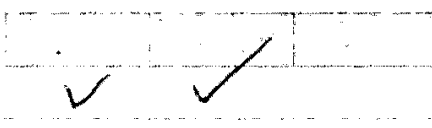

\section{4}

\section{SIGN/ PRINT NAMES}

RECEIVED BY/STORED IN - 12 Date/TMme RECEIVER QY STORED IN Xul RECEIVERRY/STORED IN C:192 RECEIVED BY/STORED IN

RECEIVED BY/STORED IN

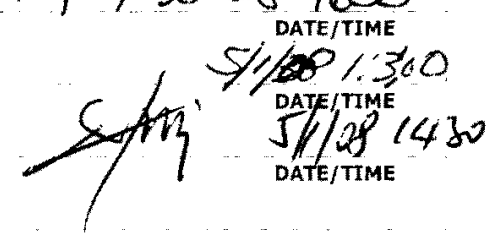

\section{SPECIAL INSTRUCTIONS}

SEE PAGE 2 FOR ALL SPECIAL INSTRUCTIONS

LABORATORY
SECTION
FINAL SAMPLE
DISPOSITION

\section{RECEIVED BY/STORED IN}

RECEIVED BY/STORED IN

\section{DATE/TIME}

DATE/TIME

DATE/TIME 
PROJECT COORDINATOR

NCO SAMPLER

TRENT, STEVE

$373-5869$

WIDRIG, DL

PROJECT DESIGNATION

SAF NO.

200-BP-5 OU Characterization for Wells 299-E33-341 and 299-E33-342 - G F08-075

C5856, I-027

ICE CHEST NO.

FIEID LOGBOOK NO.

ACTUAL SAMPLE DEPTH

COA

$123514 E S 10$

\section{SHIPPED TO}

Environmental Sciences Laboratory

\section{OFFSITE PROPERTY NO.}

N/A
PRICE CODE 8 N

AIR QUALITY
DATA TURNAROUND

45 Days / 45

Days

METHOD OF SHIPMENT

GOVERNMENT VEHICLE

\section{SPECIAL INSTRUCTIONS}

** The 200 Area S\&GRP Characterization and Monitoring Sampling and Analysis GKI applies to this SAF.

** ESL is to determine unsaturated hydraulic conductivity using the method(s) best suited for the soil texture and type.

** ESL is to submit copies of all Chain of Custodies and associated sample login documentation to EIS-SDM within 24 hours of sample receipt. They may be sent via e-mail to ACPP Sample Management.

(1)6020M_ICPMS_ASTM_AE (TAL) \{Cadmium, Chromium, Silver\} 6020M_ICPMS_ASTM_AE (Add-On) \{Arsenic, Lead, Molybdenum, Selenium\} 6020M_METALS_ICPMS_WE (TAL) \{Cadmium, Chromium, Silver\}

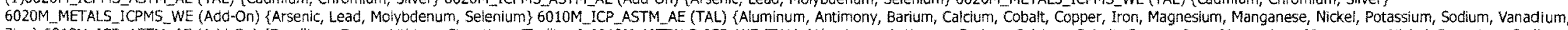

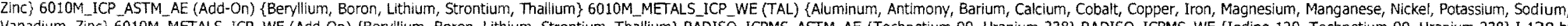

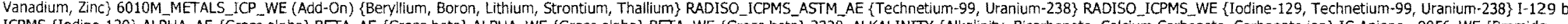

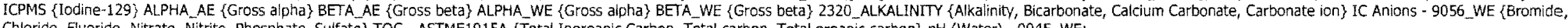

Chloride, Fluoride, Nitrate, Nitrite, Phosphate, Sulfate\} TOC - ASTME1915A \{Total Inorganic Carbon, Total carbon, Total organic Carbon\} pH (Water) - 9045 _WE; 


\section{COLLECTOR \\ nCo sampler Batlet?}

\section{SAMPLING LOCATION}

C5856, I-028

ICE CHEST NO.

\section{SHIPPED TO}

Environmental Sciences Laboratory

\section{MATRIX* POSSIBLE SAMPLE HAZARDS/ REMARKS $A=A 1 r$
$\mathrm{~L}=\mathrm{Drum}$$\quad$ Contains Radioactive Material at concentrations Liquids that are not regulated for transportation per 49 $\mathrm{DS}=\mathrm{Drum}$ Solids $L=$ Liquid $\mathrm{O}=\mathrm{OI}$
$\mathrm{S}=\mathrm{S}$ - $S E=S e d i m e n t$ $T=$ Tissue $T=1$ Issue
$V=$ Vegitation \\ $V=$ Vegitation
$W=$ Water \\ W! $=$ Wipe \\ $x=O$ ther \\ SPECIAL HANDLING AND/OR STORAGE $5400.5(1990 / 1993)$} Radioactive Tie To B1TP98

SAMPLE NO.

MATRIX*

\section{COMPAMY CONTACT}

TRENT, STEVE

\section{PROJECT DESIGNATION}

200-8P-5 OU Charaterzenon for FIELD LOGBOOK NO. 330

HNENN 55488 .

OFFSITE PROPERTY NO.

N/A

PRESERVATION

TYPE OF CONTAINER $\begin{aligned} & \text { Split Spoon } \\ & \text { Liner }\end{aligned}$

NO. OF CONTAINER(S)

volume

$1000 \mathrm{~g}$

SAMPLE ANALYSIS

SEE TEM (1) IN SEE TEM (1) IN
SPECAA
WSTRLTKTONS

\section{TELEPHONE NO.}

373-5869

PROJECT COORDINATOR

WIDRIG, DL

\section{SAF NO.}

F08-075

\section{ACTUAL SAMPLE DEPTH COA}

123514 ES10

BILL OF LADING/AIR BILL NO.
PRICE CODE $8 \mathrm{~N}$

AIR QUALITY

\section{METHOD OF SHIPMENT}

GOVERNMENT VEHICLE

\footnotetext{
BITT76 SOIL
}

SAMPLE DATE SAMPLE TIME

$4-30.08 \quad 1415$

\section{CHAIN OF POSSESSION}

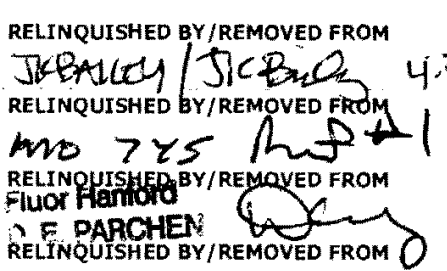

RELINQUISHED BY/REMOVED FROM

RELINQUISHED BY/REMOVED FROM

RELINQUISHED BY/REMOVED FROM

LABORATORY RECEIVEDBY

$$
\text { SECTION }
$$

FINAL SAMPLE DISPOSAL METHOD
RECEIVED BY/STORED IN

MOTYS REF DATE/TIME 5,104 DATE/TIME A 3orECEVBARYHSAED IN $5-1-00$ ( Jun DATE/TIME

DATE/TIME

DATE/TIME

DATE/TIME RECEIVEO BY/STOREDTN

RECEIVEO BY/STORED IN

RECEIVED BY/STORED IN

RECEIVED BY/STORED IN

\section{SPECIAL INSTRUCTIONS}

DATE/TIME

$4-30-0816000$ DATE/TIME * The 200 Area S\&G

applies to this SAF.

** ESL is to determine unsaturated hydraulic conductivity using the method(s) best

$51-5 \% * *$ ESL is to submit copies of all Chain of Custodies and associated sample login 5 DATE/ZIME documentation to EIS-SDM within 24 hours of sample receipt. They may be sent via

/1/08/430 e-mail to ^CPP Sample Management.

DATE/TIME

(1)Density; Particle Density - D854; Particle Size (Dry Sieve) - D422; Particle Size

(Hydrometer) - D422; Saturated Hydraulic Conductivity; CATIONEXCH TR; KD -

DATE/TIME Batch;

DATE/TIME

DATE/TIME 


\section{COLLECTOR \\ nCo sampler BAiley}

SAMPLING LOCATION

C5856, 1-029

ICE CHEST NO.

\section{SHIPPED TO}

Environmental Sciences Laboratory

\begin{tabular}{|c|c|}
\hline MATRIX* & POSSIBLE SAMPLE HAZARDS/ REMARKS \\
\hline $\begin{array}{l}A=A i r \\
D L=0 \text { rum }\end{array}$ & Contains Radioactive Material at concentrations \\
\hline Lquids & that are not reguiated for transportation per 49 \\
\hline DS=Drum & CFR but are not releasable per DOE Order \\
\hline $\begin{array}{l}\text { Solids } \\
L=\text { Liquid }\end{array}$ & $5400.5(1990 / 1993)$ \\
\hline $\begin{array}{l}0=0 \mathrm{il} \\
S=\text { Soil }\end{array}$ & \\
\hline $\begin{array}{l}S=\text { Sol } \\
\mathrm{SE}=\text { Sediment }\end{array}$ & \\
\hline$T=T$ issue & \\
\hline$V=$ Vegitation & \\
\hline$W=$ Wipe & \\
\hline$x=$ Other & $\begin{array}{l}\text { SPECIAL HANDLING AND/OR STORAGE } \\
\text { Radioactive Tle TO B1TP98 }\end{array}$ \\
\hline
\end{tabular}

\section{COMPANY CONTACT}

TRENT, STEVE

TELEPHONE NO

PROJECT COORDINATOR

WIDRIG, DL

\section{SAF NO.}

200-BP-5 OU Characterization for Wells 299-E33-341 and 299-E33-342-G F08-075

FIELD LOGBOOK NO.

ACTUAL SAMPLE DEPTH

COA

HNF-N-488-1

$65^{-1}$

123514 ES 10

BILL OF LADING/AIR BILL NO.

OFFSITE PROPERTY NO.

N/A

\begin{tabular}{|c|c|c|}
\hline PRESERVATION & None & None \\
\hline TYPE OF CONTAINER & $G / P$ & $\begin{array}{l}\text { Mossture } \\
\text { Resistant Cont }\end{array}$ \\
\hline NO. OF CONTAINER(S) & 1 & 1 \\
\hline vOLUME & $\overline{1 L} \cdot \cdots \cdot$ & $200 \mathrm{~g}$ \\
\hline SAMPLE ANALYSIS & $\begin{array}{l}\text { SEE TEM (1) IN } \\
\text { SPETCA } \\
\text { INSTRUCTONS }\end{array}$ & $\begin{array}{l}\text { Moisture - } \\
\text { Content - 02216; }\end{array}$ \\
\hline
\end{tabular}

SAMPLE DATE SAMPLE TIME

$4-300 \% 1355$
PRICE CODE

DATA TURNAROUND

45 Days / 45

Days
SAMPLE NO. MATRIX*

B1TP35 SOLL

\section{CHAIN OF POSSESSION}

RELINQUISHED BY/REMOVED FROM JKEALEय OTERL 4.30081600 DATE/TIME No745 Ppet RELINQUSSHED BY REMOVED FPG 1) to trothen w 205 RELINQUISHED BY/REMOVED FROM

RELINQUISHED BY/REMOVED FROM

RELINQUISHED BY/REMOVED FROM

RELINQUISHED BY/REMOVED FROM

LABORATORY
SECTION
FINAL SAMPLE
DISPOSITION

\section{SIGN/ PRINT NAMES}

RECEIVED BY/STORED IN

M074S REF \#14.30.08 UATE/TIME

RECEIVEDBY/STOKEDIA DATE/TIME

S/1/or $/ 3: 10$ 57 OAT 1430 DATE/TIME c) $13,2=t 2$ RECEIVED BY/STORED IN C:.5 RECETVED BY/STORED IN RECEIVED BY /STORED IN DATE/TIME DATE/TIME DATE/TIME
RECEIVED BYISTORED IN

RECEIVED BY/STORED IN

\section{SPECIAL INSTRUCTIONS}

SEE PAGE 2 FOR ALL SPECIAL INSTRUCTIONS

METHOD OF SHIPMENT GOVERNMENT VEHICLE 


\section{CHAIN OF CUSTODY/SAMPLE ANALYSIS REQUEST}

TELEPHONE NO.

373-5869

TRENT, STEVE

PROJECT DESIGNATION

200-BP-5 OU Characterization for Wells 299-E33-341 and 299-E33-342 - G FIELD LOGBOOK NO.

ACTUAL SAMPLE DEPTH

OFFSITE PROPERTY NO.

N/A
F08-075-030

PAGE 2 OF 2

PROJECT COORDINATOR

WIDRIG, DI

SAF NO.

F08-075

COA

123514 ES10

\section{SHIPPED TO}

Environmentai Sciences Laboratory

\section{BILL OF LADING/AIR BILL NO.}

N/A

\section{SPECIAL INSTRUCTIONS}

** The 200 Area S\&GRP Characterization and Monitoring Sampling and Analysis GKI applies to this SAF.

** ESL is to determine unsaturated hydraulic conductivity using the method(s) best suited for the soil texture and type.

** ESL is to submit copies of all Chain of Custodies and associated sample login documentation to EIS-SOM within 24 hours of sample receipt. They may be sent via E-mail to ^CPP Sample Management.

(1)6020M_ICPMS ASTM_AE (TAL) \{Cadmium, Chromium, Silver\} 6020M_ICPMS_ASTM_AE (Add-On) \{Arsenic, Lead, Molybdenum, Selenium\} 6020M_METALS ICPMS WE (TAL) \{Cadmium, Chromium, Silver\}

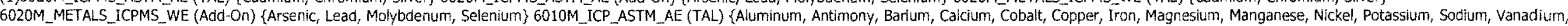

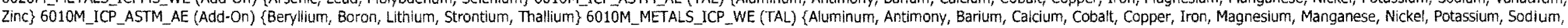

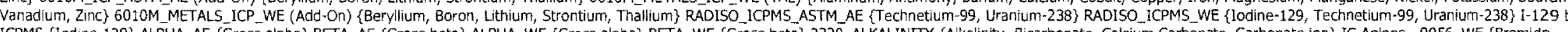

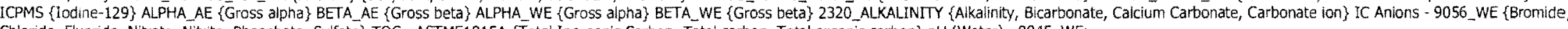

Chioride, Fluoride, Nitrate, Nitrite, Phosphate, Sulfate\} TOC-ASTME1915A \{Total Inorganic Carbon, Total carbon, Total organic carbon\} pH (Water) - 9045 . WE; 


\section{COLLECTOR}

NCO SAMPLER BAllezy

SAMPLING LOCATION

C5856, I-030

ICE CHEST NO.

\section{SHIPPED TO}

Environmental Sciences Laboratory

MATRIX* POSSIBLE SAMPLE HAZARDS/ REMARKS A $=$ Air

$\mathrm{DL}=\mathrm{Drum}$

Contains Radioactive Material at concentrations that are not regulated for transportation per 49 CFR but are not releasable per DOE Order

$5400.5(1990 / 1993)$

Solids

$\mathrm{L}=\mathrm{Liquid}$
$\mathrm{O}=\mathrm{OH}$

$S=S$ oil

SE $=$ Sediment

T=Tissue

$V=$ Vegitatio

$W=$ water

WI=Wipe

SPECIAL HANDLING AND/OR STORAGE Radioactive Tie To 81 TP98

MATRIX*

B1TT77

SOIL

\section{COMPANY CONTACT}

TRENT, STEVE

373-5869

\section{PROJECT DESIGNATION}

PROJECT COOROINATOR

WIDRIG, $D L$

\section{SAF NO.}

200-BP-5 OU Characterization for Wells 299-E33-341 and 299-E33-342 - G F08-075 FIELD LOGBOOK NO.

ACTUAL SAMPLE DEPTH COA

HNF, N- $488-1$

OFFSITE PROPERTY NO.

$67.5^{-1}-70.0^{\prime}$

123514ES10

GOVERNMENT VEHICL

BILL OF LADING/AIR BILL NO.

N/A
$N / A$

\begin{tabular}{|c|c|}
\hline PRESERVATION & None \\
\hline TYPE OF CONTAINER & $\begin{array}{l}\text { Split Spoon } \\
\text { Liner }\end{array}$ \\
\hline NO. OF CONTAINER(S) & 2 \\
\hline vOLUME & \\
\hline SAMPLE ANALYSIS & $\begin{array}{l}\text { SEE TTEM (1) IN } \\
\text { SPEIAL } \\
\text { INSTRUTIONS }\end{array}$ \\
\hline
\end{tabular}

PRICE CODE 8 N DATA

TURNAROUND

45 Days / 45

Days

SAMPLE NO.

SAMPLE DATE SAMPLE TIME

$4-30.081438$

\section{CHAIN OF POSSESSION}

RELINOUISHED BY/REMOVED FROM TEPALUK KEO RELINQUISHED BV/REMOVED FROM

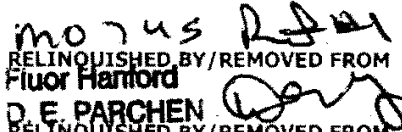

RELINQUISHED BY/REMOVED FROM

RELINQUISHED BY/REMOVED FROM

RELINQUISHED BY/REMOVED FROM

LABORATORY RECEIVED BY SECTION

FINAL SAMPLE DISPOSAL METHOD

SIGN/ PRINT NAMES
40.08 DATE/TIME 1600 RECEIVED BY/STORED IN
DATE/TIME, 200 RECEIVED BY/STOREOIN
DATE/TIME
DATE/TIME

** The 200 Area S\&GRP Characterization and Monitoring Sampling and Analysis GKI applies to this SAF.

** ESL is to determine unsaturated hydraulic conductivity using the method(s) best $16 x$ $5-\mathrm{L}-34$ suited for the soil texture and type.

TATE/TIME documentation to EIS-SDM within 24 hours of sample receipt. They may be sent via $3 / 1081432$-mail to ${ }^{\wedge}$ CPP Sample Management.

date/TIme (1)Density; Particle Density - D854; Particle Size (Dry Sieve) - D422; Particle Size (Hydrometer) - D422; Saturated Hydraulic Conductivity; CATIONEXCH_TR; KD DATE/TIME Batch;

DATE/TIME

DATE/TIME
DATE/TIME

DATE/TIME 


\section{COLLECTOR}

NCO SAMPLER BAllt27

\section{SAMPLING LOCATION}

C5856, I-031

ICE CHEST NO.

\section{SHIPPED TO}

Environmental Sciences Laboratory

\section{MATRIX* POSSIBLE SAMPLE HAZARDS/ REMARKS \\ A=Air Contains Radioactive Material at concentrations \\ $\begin{array}{ll}\text { DL }=\text { Drum } & \text { Contains Radioactive Material at concentrations } \\ \text { that are not regulated for transportation per } 49\end{array}$ \\ $\begin{array}{ll}\text { Liquids } & \text { that are not regulated for transportation per } \\ \text { DS=Drum } & \text { CFR but are not releasable per DOE Order }\end{array}$ \\ $\begin{array}{ll}\text { DS=Drum } & \text { CFR but are not releasat } \\ \text { Solids } & 5400.5(1990 / 1993)\end{array}$ \\ L=Liquid \\ $0=0$ in
$S=S c i l$ \\ $S=$ Soil \\ $T=$ Tissue \\ $T=-$ Tissue
$V=$ Vegitation \\ $\gamma=$ Water
$W=W$ \\ $W !=W$ ipe \\ $x=0$ ther \\ SPECIAL HANDLING AND/OR STORAGE} Radioactive Tie To B1TP98

\section{COMPANY CONTACT}

TRENT, SIEVE

TELEPHONE NO.

PROJECT COORDINATOR

WIDRIG, OL

PRICE CODE

DATA

\section{PROJECT DESIGNATION}

\section{SAF NO.}

AIR QUALITY

RNAROUND

200-BP-5 OU Characterization for Weils 299-E33-341 and 299-E33-342-G F08-075

FIELD LOGBOOK NO.

ACTUAL SAMPLE DEPTH

COA

$$
\text { HNF, NJ-488-1 67.5' }
$$

$123514 E S 10$

METHOD OF SHIPMENT

OFFSITE PROPERTY NO.

BILL OF LADING/AIR BILL NO.

N/A

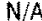

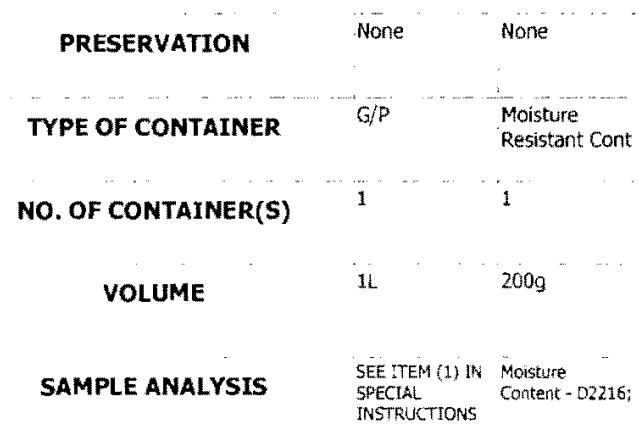

SAMPLE DATE SAMPLE TIME

$4-30$ - or 1430

SAMPLE NO.
BITP36 SOIL

\section{CHAIN OF POSSESSION}

RELINQUISHED BY/REMOVED FROM

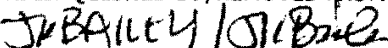
RELINQUISHED BY REMOVED FROM LIO 745 TO RELINQUYSHED BV/REMOVER FRQY

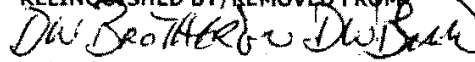
RELINQUISHED BY/REMOVED FROM

RELINQUISHED BY/REMOVED FROM

RELINQUISHED BY/REMOVED FROM

RELINOUISHED BY/REMOVED FROM

RECEIVED BY

\section{LABORATORY} SECTION

FINAL SAMPLE DISPOSAL METHOD
MATRIX*

IL




\section{COLLECTOR}

NCO SAMPLER

\section{SAMPLING LOCATION}

C5856, I-031

ICE CHEST NO.

\section{SHIPPED TO}

Environmentai Sciences Laboratory

\section{COMPANY CONTACT}

TRENT, STEVE

TELEPHONE NO.

373-5869

PROJECT DESIGNATION

200-BP-5 OU Characterization for Wells 299-E33-341 and 299-E33-342-G

FIELD LOGBOOK NO.

ACTUAL SAMPLE DEPTH

\section{OFFSITE PROPERTY NO.}

$\mathrm{N} / \mathrm{A}$
PROIECT COORDINATOR

WIDRIG, DL

SAF NO.

SAF NO.
F08.075

$\mathrm{COA}$

123514 ES10
PRICE CODE

AIR QUALITY
DATA TURNAROUND

45 Days / 45

Days

\section{SPECIAL INSTRUCTIONS}

** The 200 Area S\&GRP Characterization and Monitoring Sampling and Analysis GKI apolies to this SAF.

** ESL is to determine unsaturated hydraulic conductivity using the method(s) best suited for the soll texture and type

** ESL is to submit copies of all Chain of Custodies and associated sample login documentation to EIS-SDM within 24 hours of sample receipt. They may be sent via e-mail to $A$ CPP Sample Management.

(1)6020M_ICPMS_ASTM_AE (TAL) \{Cadmium, Chromium, Silver\} 6020M_ICPMS_ASTM_AE (Add-On) \{Arsenic, Lead, Molybdenum, Selenium\}6020M_METALS_ICPMS_WE (TAL) \{Cadmium, Chromium, Silver\}

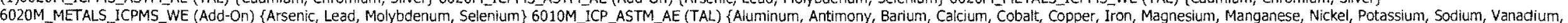

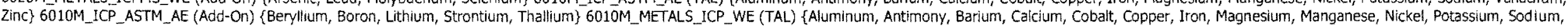

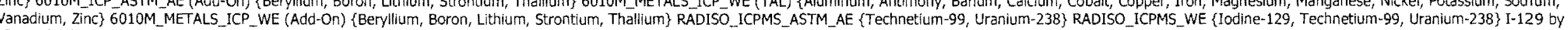

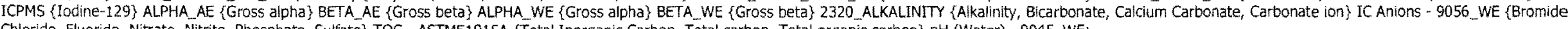
Chloride, Fluoride, Nitrate, Nitrite, Phosphate, Sulfate\} TOC - ASTME1915A (Total Inorganic Carbon, Total carbon, Total organic carbon\} pH (Water) -9045 WE; 


\section{COLLECTOR}

NCO SAMPLER BAlLtZ

\section{SAMPLING LOCATION}

C5856, 1 -033

ICE CHEST NO.

\section{COMPANY CONTACT}

TELEPHONE NO.

PROJECT COOROINATOR

WIDRIG, DL

PRICE CODE

TRENT, STEVE

\section{SAF NO.}

AIR QUALITY

200-BP-5 OU Charatterization for Wells 299-E33-341 and 299-E33-342-G Fo8-075

FIELD LOGBOOK NO.

ACTUAL SAMPLE DEPTH

COA

$H N E, N-48 \%-1$

$70^{\prime}$

$123514 E S 10$

METHOD OF SHIPMENT

GOVERNMENT VEHICLE

BILL OF LADING/AIR BILL. NO.

N/A

$\mathrm{N} / \mathrm{A}$

Environmental Sciences Laboratory

MATRIX* POSSIBLE SAMPLE HAZARDS/ REMARKS

$A=$ Air $\quad$ Contains Radioactive Material at concentrations

$D L=D r u m$ that are not regulated for transportation per 49

Liquids that are not regulated for transportation per 49

$\begin{array}{ll}\text { DS=Drum } & \text { CFR but are not reieasab } \\ \text { Solids } & 5400.5(1990 / 1993)\end{array}$

Leliquid

$0=0$ if

$S=$ Soil
$S E=$ Sediment

$\mathrm{T}=\mathrm{Tis}$ isue

$V=$ vegitation

$W=$ Water

$W t=$ Wipe

SPECIAL HANDLING AND/OR STORAGE Radioactive Tie To B1TP99

$\begin{array}{lll}\text { PRESERVATION } & \text { None } & \text { None } \\ \text { TPE OF CONTAINER Gosture } & & \\ \text { Resistant Cont }\end{array}$

NO. OF CONTAINER(S) "1

VOLUME $\quad 1 \mathrm{~L} \quad 2009$

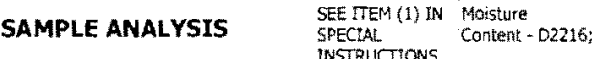

SAMPLE DATE SAMPLE TIME

$4-30.04$, 450

31394

SIGN/ PRINT NAMES

RECEIVED BY/STORED IN H) 4008 Date/TIME

DATE/TIME

RELINQUISHED BY/REMOVED FROM JEBAILEY/JTCBID DATE/TIME DATE/TIME Mli45 FOE RELINQUISHED BY/REMGVED FROM $5 / 10$ O 143

RELINQUISHED BY/REMOVED FROM

RELINQUISHED BY/REMOVED FROM

DATE/TIME $x+5$ RECEIVED BY/STORED IN CiTeCrun" RECEIVED BY/STORED IY RECEIVED BY/STORED IN DATE/TIME RECEIVED BY/STORED IN DATE/TIME
RECEIVED BY/STORED IN
RELINQUISHED BY/REMOVED FROM

\section{PPECIAL INSTRUCTIONS}

SEE PAGE 2 FOR ALL SPECIAL INSTRUCTIONS

RECEIVED BY 


\section{COLLECTOR}

NCO SAMPLER

\section{SAMPLING LOCATION}

C5856, 1-033

ICE CHEST NO.

\section{SHIPPED TO}

Environmental Sciences Laboratory

\section{COMPANY CONTACT}

TRENT, STEVE

PROJECT DESIGNATION

200-BP-5 OU Characterization for Wells 299-E33-341 and 299-E33-342-G

FIELD LOGBOOK NO.

ACTUAL SAMPLE DEPTH

\section{OFFSITE PROPERTY NO.}

N/A

\section{TELEPHONE NO.}

373-5869

ACTUALA SA
PROJECT COORDINATOR

WIDRIG, DL

SAF NO.

F08-075

COA

$123514 E S 10$
PRICE CODE 8 N

AIR QUALITY

\section{METHOD OF SHIPMENT}

GOVERNMENT VEHICLE
DATA TURNAROUND

45 Days / 45

Days

\section{SPECIAL INSTRUCTIONS}

** The 200 Area S\&GRP Characterization and Monitoring Sampling and Anaiysis GKI applies to this SAF.

** ESL is to determine unsaturated hydraulic conductivity using the method(s) best suited for the soil texture and type.

** ESL is to submit copies of all Chain of Custodies and associated sample login documentation to EIS-SDM within 24 hours of sample receipt. They may be sent via e-mail to ^CPP Sample Management.

(1)6020M_ICPMS_ASTM_AE (TAL) \{Cadmium, Chromium, Silver\} 6020M_ICPMS_ASTM_AE (Add-On) \{Arsenic, Lead, Molybdenum, Selenium\} 6020M_METALS_ICPMS_WE (TAL) \{Cadmium, Chromium, Silver\}

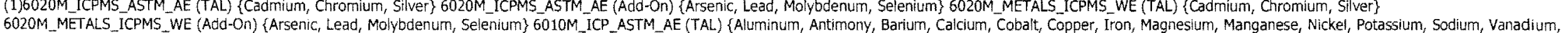

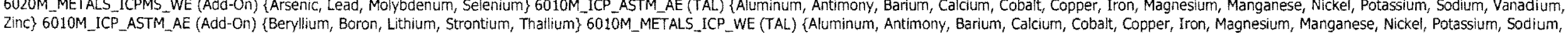

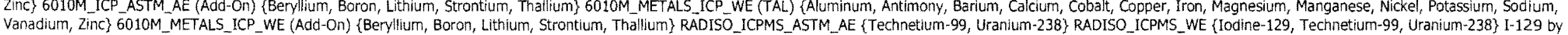

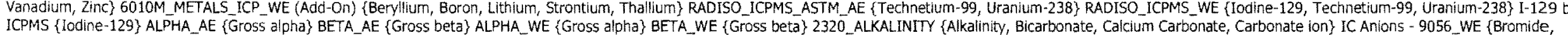
ICPMS \{lodine-129\} ALPHA_AE \{Gross alpha\} BETA_AE \{Gross beta\} ALPHA_WE \{Gross alpha\} BETA_WE \{Gross beta\} 2320_ALKALINITY \{Alkalinity, Bicarbonate,
Chioride, Fluoride, Nitrate, Nitrite, Fhosphate, Sulfate\} TOC - ASTME1915A \{Total Inorganic Carbon, Total carbon, Total organic carbon\} pH (Water) - 9045 _WE; 


\section{COLLECTOR}

nco sampler Ballegl

\section{SAMPLING LOCATION}

C5856, $\mathrm{I}-032$

ICE CHEST NO.

\section{SHIPPED TO}

Environmental Sciences Laboratory

\section{MATRI** \\ $A=A$ Ar \\ $\mathrm{DL}=$ Drum \\ Liquids
DS=Drum \\ Solids \\ POSSIBLE SAMPLE HAZARDS/ REMARKS Contains Radioactive Material at concentrations that are not regulated for transportation per 49 CFR but are not releasable per DOE Order $5400.5(1990 / 1993)$}

Solids

le Liquid
$0=01$

$\mathrm{S}=\mathrm{Soil}$

$S=$ Soil
$S E=$ Sedinent

$T=$ Tissue

$V=$ vegitation

$W=$ Water

WI $=$ Wipe

SPECIAL HANDLING AND/OR STORAGE Radioactive TIE To B1TP98

SAMPLE NO

MATRIX*

B1TT78

SOIL

\section{COMPANY CONTACT}

TRENT, STEVE

TELEPHONE NO.

373-5869

PROJECT COORDINATOR

WIDRIG, DL

\section{SAF NO.}

\section{PROJECT DESIGNATION}

F08-075

200-BP-5 OU Characterization for Wells 299-E33-341 and 299-E33-342 - G

FIELD LOGBOOK NO.

ACTUAL SAMPLE DEPTH

HNE-N-48Z - $1 \operatorname{cg} 2670^{\prime}-72.5$

OFFSITE PROPERTY NO.

$123514 E S 10$

GOVERNMENT VEHICLE

BILL OF LADING/AIR BILL NO.

METHOD OF SHIPMENT
N/A

\section{PRESERVATION}

None

TYPE OF CONTAINER Split Spoon

NO. OF CONTAINER(S)

VOLUME

SAMPLE ANALYSIS SEE TEM (1) IN
PRICE CODE $\quad$ BN

DATA TURNAROUND

45 Days / 45

SAMPLE DATE SAMPLE TIME

$5-1.08 .0830$

\section{SIGN/ PRINT NAMES}

\section{CHAIN OF POSSESSION}

RELINQUISHED BY/REMOVED FROM JKExilery ot Bedo RELINQUISHED BY/REMOVEP FROM MO 745 REF RELINQUISHED BY/BEXVUEP FROM $O$
JOSh Herick RELPS Hanfor EY $/$ REMOVED FRoh

RELINQUISHED BY/REMOVED FROM

RELINQUISHED BY/REMOVED FROM

RELINQUISHED BY/REMOVED FROM

LABORATORY RECEIVED BY SECTION

FINAL SAMPLE DISPOSAL METHOD
DATE/TTME RECEIVED BY/STORED IN

5.1 .08 i600 mo745 RE IE/ 5.1 .081600

\section{RECEYYEP BY/STORED IN}

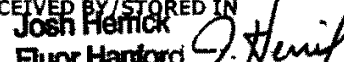

DATE/TIME

5.6 .0813

DATE/TIME

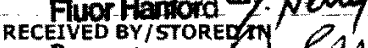

OF 1300 SSTIH $5.6 .08^{\text {DATE/TIME }}$

RECEIVED BY/STORED IN

\section{DATE/TIME}

DATE/TIME

RECEIVED BY/STORED IN

DATE/TIME

RECEIVED BY/STORED IN

DATE/TIME

DATE/TIME

DATE/TIME
RECEIVED BY/STORED IN
DATE/TIME

\section{SPECIAL INSTRUCTIONS}

** The 200 Area S\&GRP Characterization and Monitoring Sampling and Analysis GKI applies to this SAF.

** ESL is to determine unsaturated hydraulic conductivity using the method(s) best suited for the soil texture and type.

** ESL is to submit copies of all Chain of Custodies and associated sample login documentation to EIS-SDM within 24 hours of sample receipt. They may be sent via e-maif to ^CPP Sample Management.

(1)Density; Particle Density - D854; Particle Size (Dry Sieve) - D422; Particle Size (Hydrometer) - D422; Saturated Hydraulic Conductivity; CATIONEXCH_TR; KD Batch; 


\section{SAMPLING LOCATION}

C5856, I-034

ICE CHEST NO.

\section{SHIPPED TO}

Environmental Sciences Laboratory

MATRIX* POSSIBLE SAMPLE HAZARDS/ REMARKS

$A=$ Air $\quad$ Contains Radioative Material at concentrations

$\mathrm{DL}=\mathrm{Drum} \quad \mathrm{COntans}$ Radion

Liquids that are not regulated for transportation per

$\begin{array}{ll}D S=D \text { Dum } & \text { CFR but are not releasab } \\ \text { Solids } & 5400.5(1990 / 1993)\end{array}$

$l=$ Liquid
$0=0$ OI

$S=5$ oil

$S E=$ Sediment

$T=$ Tissue

$\checkmark=$ vegitatio

$W$-water

$W !=$ Wipe

SPECIAL HANDLING AND/OR STORAGE Radioactive TIE TO B1TP99

SAMPLE NO

MATRIX*

B1TT79

SOLL

\section{COMPANY CONTACT}

TRENT, STEVE

TELEPHONE NO

373-5869

\section{PROJECT DESIGNATION}

PROJECT COORDINATOR

WIORIG, DL

\section{SAF NO.}

200-BP-5 OU Characterization for Wells 299-E33-341 and 299-E33-342-G F08-075

FIELD LOGBOOK NO.

ACTUAL SAMPLE DEPTH

HNE-NJ-488-7 pg26 $72.5^{\prime}-75^{\prime}$
OFFSITE PROPERTY NO.

$\mathrm{N} / \mathrm{A}$

$123514 E S 10$

AIR QUALITY

DATA

TURNAROUND

45 Days / 45

\section{METHOD OF SHIPMENT}

GOVERNMENT VEHICIE

BILL OF LADING/AIR BILL NO.
N/A

\begin{tabular}{|c|c|}
\hline PRESERVATION & None \\
\hline TYPE OF CONTAINER & $\begin{array}{l}\text { Split Spoon } \\
\text { Liner }\end{array}$ \\
\hline NO. OF CONTAINER(S) & 2 \\
\hline VOLUME & 10009 \\
\hline SAMPLE ANALYSIS & $\begin{array}{l}\text { SEE TEM (1) IN } \\
\text { SPECIAL } \\
\text { INSTRUCTIONS }\end{array}$ \\
\hline
\end{tabular}

SAMPLE DATE SAMPLE TIME

$5-1-08 \quad 0918$

\section{SIGN/ PRINT NAMES}

\section{CHAIN OF POSSESSION}

RELINQUISHED GY/REMOVFD FROM JRBAILY JIC BuE MO 745 REF EI RELINOUISHED BY / REMOVEP FROM Josh Herrick Henn

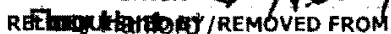

RELINQUISHED BY/REMOVED FROM RELINQUISHED BY/REMOVED FROM RELINQUISHED BY/REMOVED FROM $5-1-08 \quad 1600$ $5.6 .05 \quad 1300$ DATE/TIME

DATE/TIME

RECEIVED BY/STORED IN WOTS RET

RECEIVED BY/STORED IN

DATE/TIME

RECEIVED BY/STORED IN

DATE/TIME
RECEIVED BY/STORED IN

WOTUS RET
RECEIVED BY/STOREP
Josh Harrick

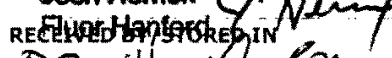

\section{SPECIAL INSTRUCTIONS}

DATE/TIME

** The 200 Area S\&GRP Characterization and Monitoring Sampling and Analysis GKI applies to this SAF.

** ESL is to determine unsaturated hydraulic conductivity using the method(s) best suited for the soil texture and type.

** ESL is to submit copies of all Chain of Custodies and associated sample login documentation to EIS-SDM within 24 hours of sample receipt. They may be sent via e-mail to ${ }^{\wedge} \mathrm{CPP}$ Sample Management.

(1)Density; Particle Density - D854; Particle Size (Dry Sieve) - D422; Particle Size (Hydrometer) - D422; Saturated Hydraulic Conductivity; CATIONEXCH_TR; KD Batch;
LABORATORY RECEIVED BY SECTION

FINAL SAMPLE DISPOSAL METHOD

\section{DATE/TIME}

68

DATE/TIME

DATE/TIN

DATE/TIME

DATE/TIME

DATE/TIME
TITLE

DATE/TIME

DISPOSED BY

DATE/TIME 


\section{NCO SAMPLER BAllEY}

\section{SAMPLING LOCATION}

C5856, I-035

ICE CHEST NO.

\section{SHIPPED TO}

Environmental Sciences Laboratory

MATRIX*

$A=A$ ir

$D L=D r u m$

Liquids
DS $=$ Drum

DS $=$ Drun
Solvels

Soldels

$0=0$ in

$\mathrm{S}=$ Soli
$\mathrm{SE}=$ Sedment

$\mathrm{S}=\mathrm{T}=\mathrm{Sedin}$
$\mathrm{Tis}$ sue

$V=$ Vegitation

$W=$ Water

$W ?=$ Wipe

SPECIAL HANDLING AND/OR STORAGE Radioactive Tie To B1TP99

SAMPLE NO.

MATRIX*

B1TP38

SOIL

\section{CHAIN OF POSSESSION}

RELINQUISHED BY /REMOVED, FROM JKBAILY/CJLBUEO RELINQUISHEP BY/REMOVED FROR MUIUT RE

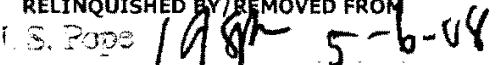
RELINQUISHED WY/LEMOVED FROM

RELINQUISHED BY/REMOVED FROM

RELINQUISHED BY/REMOVED FROM

RELINQUISHED BY/REMOVED FROM

\section{LABORATORY}

RECEIVED BY

SECTION

FINAL SAMPLE

DISPOSAL METHOD

\section{TELEPHONE NO}

$373-5869$

TRENT, STEVE

PROJECT DESIGNATION

200-BP-5 OU Characterization for Wells 299-E33-341 and 299-E33-342-G FIELD LOGBOOK NO. ACTUAL SAMPLE DEPTH

HNF. N- $488-1$ pg $26 \quad 72.5^{-1}$
OFFSITE PROPERTY No.

N/A

\begin{tabular}{cll} 
PRESERVATION & None & None \\
TYPE OF CONTAINER & G/F & $\begin{array}{l}\text { Moisture } \\
\text { Resistant Cont }\end{array}$ \\
NO. OF CONTAINER(S) & 1 & 1 \\
VOLUME & $1 \mathrm{~L}$ & 2009 \\
SAMPLE ANALYSIS & $\begin{array}{l}\text { SEE MEM (1) IN } \\
\text { SFELIAL Moisture } \\
\text { INSTRUCTIONS }\end{array}$ \\
\hline
\end{tabular}

SAMPLE DATE SAMPLETIME

$5 \cdot 1.080903$

\section{4}

\section{SIGN/ PRINT NAMES}

DATE/TIME

1600

DATE

DATE/TIME

1310

DATE/TIME

DATE/TIME

DATE/TIME

DATE/TIME
PROJECT COORDINATOR

WIORIG, OL

\section{SAF NO.
F08-075}

COAA

$123514 E S 10$

BILL OF LADING/AIR BILL NO.
PRICE CODE 8 N

AIR QUALITY

METHOD OF SHIPMENT

GOVERNMENT VEHICLE

RECEIVED BY/STORED IN
WAOTY5 PEF $15-1.08$

SPECIAL INSTRUCTIONS

SEE PAGE 2 FOR ALL SPECIAL INSTRUCTIONS 


\section{COLLECTOR}

\section{COMPANY CONTACT}

TRENT, STEVE

\section{TELEPHONE NO.}

373-5869

\section{PROJECT DESIGNATION}

200-BP-5 OU Characterization for Wells 299-E33-341 and 299-E33-342-G

FIEĹD LOGBOOK NO.

ACTUAL SAMPLE DEPTH

\section{OFFSITE PROPERTY NO.}

N/A
PROJECT COORDINATOR

WIDRIG, DL

\section{SAF NO.}

FO8-075

AIR QUALITY

\section{METHOD OF SHIPMENT}

$123514 E \$ 10$

GOVERNMENT VEHICLE

\section{BILL OF LADING/AIR BILL NO.}

N/A

\section{SPECIAL INSTRUCTIONS}

Environmental Sciences Laboratory

** The 200 Area S\&GRP Characterization and Monitoring Sampling and Analysis GKI applies to this SAF

** ESL is to determine unsaturated hydraulic conductivity using the method(s) best suited for the soil texture and type.

** ESL is to submit copies of all Chain of Custodies and associated sample login documentation to EIS-SOM within 24 hours of sample receipt. They may be sent via e-mail to ^CPP Sample Management.

(1)6020M_ICPMS_ASTM_AE (TAL) \{Cadmium, Chromium, Silver\} 6020M_ICPMS_ASTM_AE (Add-On) \{Arsenic, Lead, Molybdenum, Seienium\} 6020M_METALS_ICPMS_WE (TAL) \{Cadmium, Chromium, Silver\}

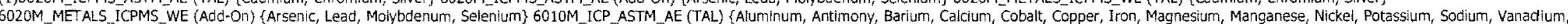

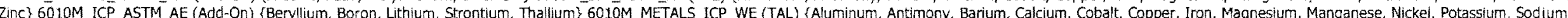

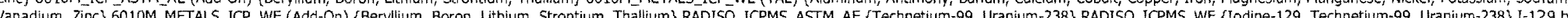

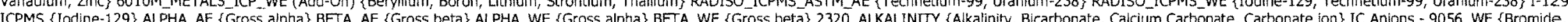

Chloride, Fluoride, Nitrate, Nitrite, Phosphate, Sulfate\} TOC - ASTME1915A \{Total Inorganic Carbon, Total carbon, Total organic carbon\} pH (Water) - 9045 WE; 


\section{CHAIN OF CUSTODY/SAMPLE ANALYSIS REQUEST}

TELEPHONE NO.

$373-5869$

TRENT, STEVE

PROJECT DESIGNATION

200-BP-5 OU Characterization for Wells 299-E33-341 and 299-E33-342-G F08-075

FIELD LOGBOOK NO.

ACTUAL SAMPLE DEPTH

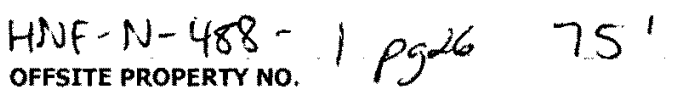

N/A

Environmental Sciences Laboratory

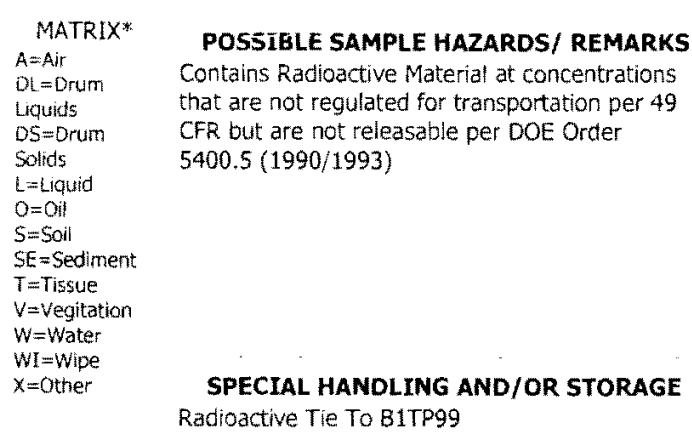

POSSIBLE SAMPLE HAZARDS/ REMARKS Contains Radioactive Material at concentrations that are not regulated for transportation per 49 CFR but are not releasable per DOE Order $5400.5(1990 / 1993)$

\begin{abstract}
SAMPLE NO.
\end{abstract}
MATRIX*

B1TP39 SOIL

LOTNO.

\section{CHAIN OF POSSESSION}

RELINQUISHED BY/REMOVED FROM

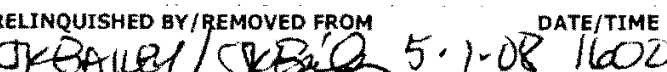
RELINQUTSHED E/ /REMOVED FROM MU-748 (CEFH 5.6 .08 OJSO RELINQUISHED ITI/EMOVED FROM DATE/TIME

RELINQUISHED BY/REMOVED FROM

RELINQUISHED BY/REMOVED FROM

DATE/TIME

DATE/TIME

DATE/TIME

\begin{tabular}{|c|c|c|}
\hline PRESERVATION & None & None \\
\hline TYPE OF CONTAINER & G/P & $\begin{array}{l}\text { Moisture } \\
\text { Resistant Cont }\end{array}$ \\
\hline NO. OF CONTAINER(S) & 1 & $i$ \\
\hline VOLUME & IL & 2009 \\
\hline SAMPLE ANALYSIS & $\begin{array}{l}\text { SEE TEM (1) IN } \\
\text { SPECAL (1) } \\
\text { INSTRUCTIONS }\end{array}$ & $\begin{array}{l}\text { Moisture } \\
\text { Content - D2 }\end{array}$ \\
\hline
\end{tabular}

SAMPLE DATE SAMPLE TIME

$5 * 1-08.0930$

$$
\begin{aligned}
& 28688 \\
& 3+394
\end{aligned}
$$

\section{SIGN/ PRINT NAMES}

RECEIVED BY/STORED IN

MO745 REF 5.1 .08

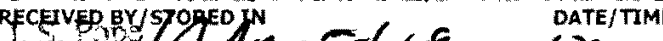

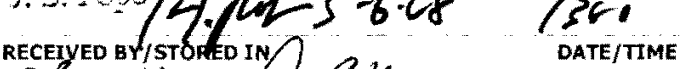
DSWmith the 5.6 .081310 DATE/TIME

RECEIVED BY/STORED IN

RECEIVED BY/STORED IN

DATE/TIME

RECEIVED BY/STORED IN
RELINQUISHED BY/REMOVED FROM
RECEIVED BY

LABORATORY

DISPOSAL METHOD

FINAL SAMPLE

DISPOSAL METHOD
COA

123514 ES 10

BILL OF LADING/AIR BILL NO.
METHOD OF SHIPMENT

GOVERNMENT VEHICLF 


\section{COLLECTOR}

NCO SAMPLER

\section{SAMPLING LOCATION}

C5856, I-036

ICE CHEST NO.

\section{SHIPPED TO}

Environmental Sciences Laboratory

\section{COMPANY CONTACT}

TRENT, STEVE

TELEPHONE NO.

$373-5869$

PROJECT DESIGNATION

200-BP-5 OU Characterization for Welts 299-E33-341 and 299-E33-342-G FIELD LOGBOOK NO.

ACTUAL SAMPLE DEPTH

\section{OFFSITE PROPERTY NO}

N/A
PRICE CODE

\section{N}

DATA WIDRIG, DL

SAF NO.

AIR QUALITY

F08-075

coA

$123514 E S 10$

\section{METHOD OF SHIPMENT}

GOVERNMENT VEHICLE

\section{SPECIAL INSTRUCTIONS}

** The 200 Area S\&GRP Characterization and Monitoring Sampling and Analysis GKI applies to this SAF.

** ESL is to determine unsaturated hydraulic conductivity using the method(s) best suited for the soil texture and type.

** ESL is to submit copies of all Chain of Custodies and associated sample login documentation to EIS-SDM within 24 hours of sample receipt. They may be sent via e-mail to $A$ CPP Sample Management.

(1)6020M ICPMS ASTM AE (TAL) \{Cadmium, Chromium, Silver\} 6020M_ICPMS ASTM AE (Add-On) \{Arsenic, Lead, Molybdenum, Selenium\} 6020M METALS ICPMS WE (TAL) \{Cadmium, Chromlum, Silver\}

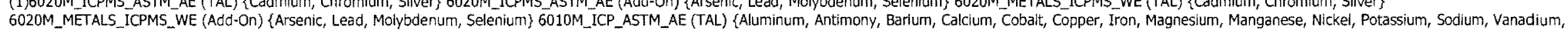

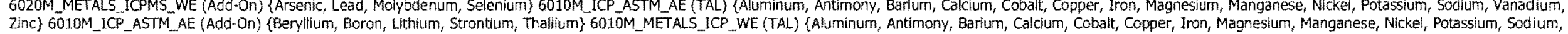

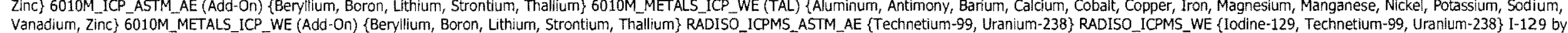

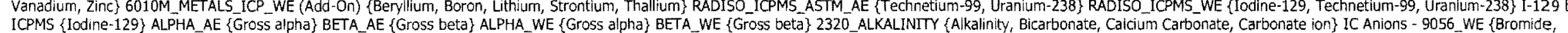
ICPMS \{Iodine-129\} ALPHA_AE \{Gross alpha\} BETA_AE \{Gross beta\} ALPHA_WE \{Gross alpha\} BETA_WE \{Gross beta\} 2320_ALKALINITY \{Alkalinity, Bicarbonate,
Chloride, Fluoride, Nitrate, Nitrite, Phosphate, Sulfate\} TOC - ASTME1915A (Total Inorganic Carbon, Total carbon, Total organic carbon\} pH (Water) - 9045 -WE; 


\section{COLLECTOR \\ NCO SAMPLER BALlCEY}

\section{SAMPLING LOCATION}

C5856, 1-037

ICE CHEST NO.

\section{SHIPPED TO}

Environmental Sciences Laboratory

MATRIX*

$A=A$ ir

$\mathrm{DL}=$ Drum

Liquids

DS=Drum
Solids

Solids
$L=$ Liquild

$0=0$ it

$\mathrm{S}=\mathrm{SOH}$
$\mathrm{SE}=$ Sediment

$T=T$ Tissue

$\checkmark=$ vegitation

$W=$ Water

$W=$ Wipe
$x=$ other

POSSIBLE SAMPLE HAZARDS/ REMARKS Contains Radioactive Material at concentrations Sportation per 49

$5400.5(1990 / 1993)$

SPECIAL HANDLING AND/OR STORAGE Radioactive Tie To B1TP9g

SAMPLE NO. MATRIX*

B1TP40

SOIL

\section{CHAIN OF POSSESSION}

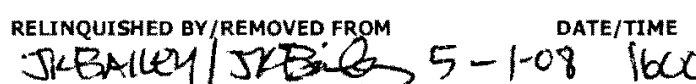
RELINQUISHEQ BY/REMOVED FROM Mo-74s ReF \#1 5.6.8 1300 RELINQUISHED BY/REMONED FROM DATE/TIME

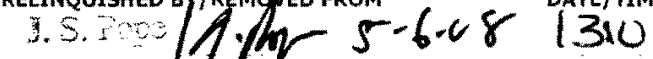
RELINQUISHED 6 / REg GOOUED FROM DATE/TIME

RELINQUISHED BY/REMOVED FROM DATE/TIME

RELINQUISHED BY/REMOVED FROM DATE/TIME

RELINQUISHED BY/REMOVED FROM

DATE/TIME

\section{COMPANY CONTACT}

TRENT, STEVE

TELEPHONE NO.

373-5869

PROJECT COORDINATOR

WIDRIG, DL

\section{SAF NO.}

F08-075

200-BP-5 OU Characterization for Wells 299-E33-341 and 299-E33-342-G FIELD LOGBOOK NO. ACTUAL SAMPLE DEPTH

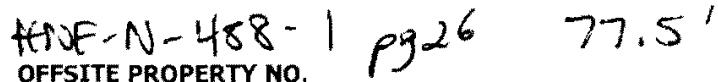

N/A

123514ES 10

BILL OF LADING/AIR BILL NO.
N/A

\begin{tabular}{|c|c|c|}
\hline PRESERVATION & None & None \\
\hline TYPE OF CONTAINER & GP & $\begin{array}{l}\text { Moisture } \\
\text { Resistant Cont }\end{array}$ \\
\hline NO. OF CONTAINER(S) & 1 & $1 \cdots$ \\
\hline VOLUME & $1 \mathrm{~L}$ & 2009 \\
\hline SAMPLE ANALYSIS & $\begin{array}{l}\text { SEE TEMM (I) IN } \\
\text { SECCAL } \\
\text { INSTRUCTONS }\end{array}$ & $\begin{array}{l}\text { Moisture } \\
\text { Content - D22 }\end{array}$ \\
\hline
\end{tabular}

SAMPLE DATE SAMPLE TIME

$5 \cdot 1-08$ 10 45

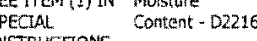

\section{SIGN/ PRINT NAMES}

RECEIVED BY/STORED IN

DATE/TIME

RECEIVED BY/STORED \# $5 * 1-07$ 160D

j. S. PoDe $/ 12-5.68$ RECEIVED QV/sTorepoN DSomith ift ift $5.6 .08^{\text {DATE/TIME }} 130$ RECEIVED BY/STORED IN

RECEIVED BY/STORED IN

RECEIVED GY/STORED IN

RECEIVED BY/STORED IN

\section{DATE/TME}

DATE/TIME

DATE/TIME

DATE/TIME
SPECIAL INSTRUCTIONS

SEE PAGE 2 FOR ALL SPECIAL INSTRUCTIONS

\section{SECTION}




\section{COLLECTOR}

NCO SAMPLER

SAMPLING LOCATION

C5856, I-037

ICE CHEST NO.

\section{SHIPPED TO}

Environmentai Sciences Laboratory

\section{COMPANY CONTACT}

TRENT, STEVE

PROJECT DESIGNATION

200-BP-5 OU Characterization for Wells 299-E33-341 and 299-E33-342-G FIELD LOGBOOK NO.

ACTUAL SAMPLE DEPTH

\section{OFFSITE PROPERTY NO}

N/A
PROJECT COORDINATOR

WIORIG, DL

SAF NO.

F08-075

COA

123514 ES 10

\section{PRICE CODE 8 N}

AIR QUALITY

METHOD OF SHIPMENT

\section{SPECIAL INSTRUCTIONS}

** The 200 Area S\&GRP Characterization and Monitoring Sampling and Analysis GKI applies to this SAF.

** ESL is to determine unsaturated hydraulic conductivity using the method(s) best suited for the soll texture and type.

** ESL is to submit copies of atl Chain of Custodies and associated sample login documentation to EIS-SDM within 24 hours of sample receipt. They may be sent via e-mail to $A$ CPP Sample Management.

(1)6020M_ICPMS_ASTM_AE (TAL) \{Cadmium, Chromium, Silver\} 6020M_ICPMS_ASTM_AE (Add-On) \{Arsenic, Lead, Molybdenum, Selenium\} 6020M_METALS_ICPMS_WE (TAL) \{Cadmium, Chromium, Silver\}

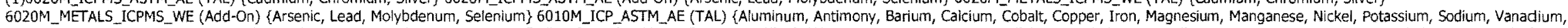

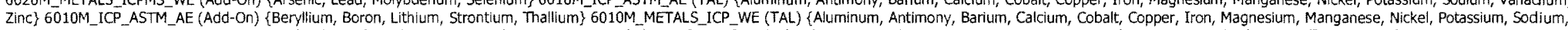

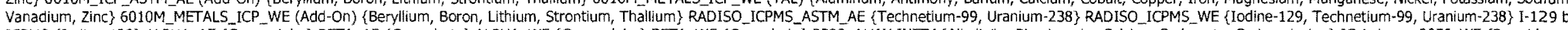

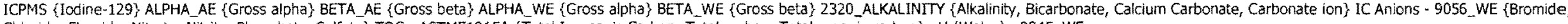
Chioride, Fluoride, Nitrate, Nitrite, Phosphate, Sulfate\} TOC - ASTME1915A \{Total Inorganic Carbon, Total carbon, Total organic carbon\} pH (Water) - 9045_WE; 


\section{CHAIN OF CUSTODY/SAMPLE ANALYSIS REQUEST}

\section{TELEPHONE NO.}

373-5869

\section{COMPANY CONTACT}

TRENT, STEVE

\section{PROJECT DESIGNATION}

200-BP-5 OU Characterization for Wells 299-E33-341 and 299-E33-342-G FIELD LOGBOOK NO. WE 5 - 1 - ACTUAL SAMPLE DEPTH HNF-N $=588$ 488-1 $80^{\circ}$ OFFSITE PROPERTY NO. $\quad$ Pg 20

N/A

Environmental Sciences Laboratory

\section{MATRIX*}

$A=A i r$
DL $=$ Drum

Al. $=$ Drum

Liquids

DSEDrum
Solids

SIBLE SAMPLE HAZARDS/ REMARKS Contains Radioactive Material at concentrations that are not reculated for transportation per 49 CFR but are not releasable per DOE Order $5400.5(1990 / 1993)$

$l=L i q u i d$
$0=0$ il

$S=S$ oil

$\mathrm{SE}=\mathrm{Sediment}$

$T=$ Tissue

$V=$ vegitation

$W=$ Water

$W I=$ Wipe

SPECIAL HANDLING AND/OR STORAGE Radioactive Tie To B1TP99

SAMPLE NO. MATRIX

B1TP41

SOIL

\section{CHAIN OF POSSESSION}

RELINQUISHED BY/REMOVED FROM

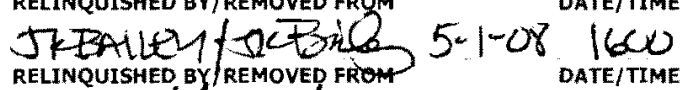
WH-7YSlREF-fi 5.6 .681300 RELINQUISHEb/BY/REYPOVD FRON DATE/TIME

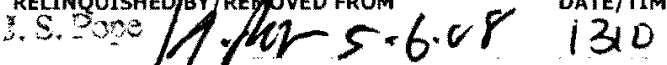
RELINQUISH L O EY REMOVED FROM DATE/TIME

RELINQUISHED GY/REMOVED FROM

DATE/TIME

RELINQUISHED BY/REMOVED FROM

DATE/TIME

RELINQUISHED BY/REMOVED FROM

DATE/TIME

\begin{tabular}{lll} 
PRESERVATION & None & None \\
TYPE OF CONTAINER & GP & $\begin{array}{c}\text { Moisture } \\
\text { Resistant Cont }\end{array}$ \\
\hline
\end{tabular}

NO. OF CONTAINER(S) 11

VOLUME il 2009

$\begin{array}{lll}\text { SAMPLE ANALYSIS } & \text { SEE TEM (1) IN Mosture } \\ \text { SPECIRA } \\ \text { TNSTRUTTONIS }\end{array}$
28688

SIGN/ PRINT NAMES

RECEIVED BY/STORED IN

MOTS DEt $15.1-08$ DATE/TIME

8. 1600

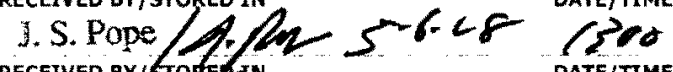
BECEIVED ByifToreg, RECEIVED BY/STORED IN

DATE/TIME

RECEIVED BY/STORED IN

DATE/TIM

DATE/TIME

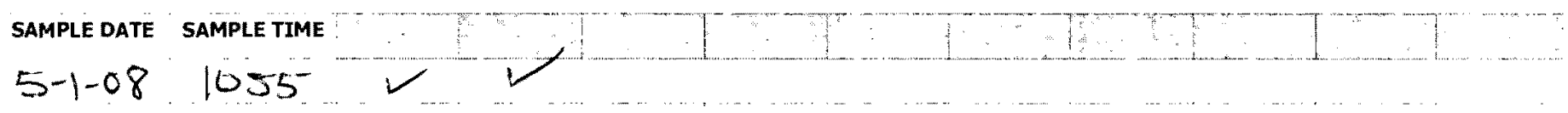

F08-075-039

PAGE 1 OF 2

PROJECT COOROINATOR

WIDRIG, DL

SAF NO.

COA

123514 ES10

BILL OF LADING/AIR BILL NO

\section{LABORATORY RECEIVED BY SECTION




\section{COLLECTOR}

COMPANY CONTACT

TRENT, STEVE

TELEPHONE NO.

PROJECT COORDINATOR

WIDRIG, DL

PRICE CODE

8N

DATA

\section{PROJECT DESIGNATION}

SAF NO.

AIR QUALITY

TURNAROUND

200-BP-5 OU Characterization for Wells 299-E33-341 and 299-E33-342-G F08-075

FIELD LOGBOOK NO.

ACTUAL SAMPLE DEPTH

COA

123514 ES10

METHOD OF SHIPMENT

OFFSITE PROPERTY NO.

N/A

BILL OF LADING/AIR BILL NO

$N / A$

\section{SHIPPED TO}

Environmental Sciences Laboratory

OVERNMENT VEHICLE

\section{SPECIAL INSTRUCTIONS}

** The 200 Area S\&GRP Characterization and Monitoring Sampling and Analysis GKI applies to this SAF.

** ESL is to determine unsaturated hydraulic conductivity using the method(s) best suited for the soil texture and type.

** ESL is to submit copies of all Chain of Custodies and associated sample login documentation to EIS-SDM within 24 hours of sample receipt. They may be sent via e-mail to ^CPP Sample Management.

(1)6020M_ICPMS_ASTM_AE (TAL) \{Cadmium, Chromium, Silver\} 6020M_ICPMS_ASTM_AE (Add-On) \{Arsenic, Lead, Molybdenum, Selenium\} 6020M_METALSICPMS WE (TAL) \{Cadmium, Chromium, Silver\}

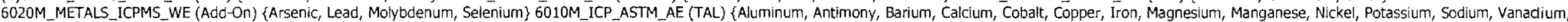

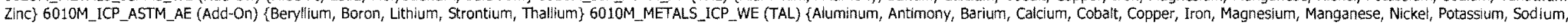

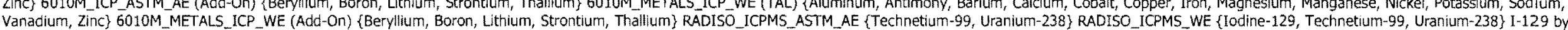

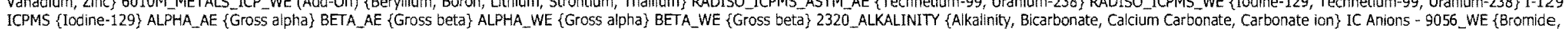

ICPMS \{lodine-129\} ALPHA_AE \{Gross alpha\} BEIA_AE \{Gross beta\} ALPHA_WE \{Gross alpha\} BETA_WE \{Gross beta\} 2320_ALALINITY \{Alkalinity, BiCarbona.
Chloride, Fluoride, Nitrate, Nitrite, Phosphate, Sulfate\} TOC - ASTME1915A \{Total Inorganic Carbon, Total carbon, Total organic carbon\} pH (Water) 9045 WVE; 


\section{COLLECTOR}

NCO SAMPLER D Commoth

C5856, 1-039

ICE CHEST NO.

\section{SHIPPED TO}

Environmental Sciences Laboratory

MATRIX* POSSIBLE SAMPLE HAZARDS/ REMARKS

$A=A i r \quad$ Contains Radioactive Material at concentrations

$\mathrm{OL=Drum} \quad$ that are not regulated for transportation per 49

DS=Drum CFR but are not releasable per DOE Order

Solids $\quad 5400.5(1990 / 1993)$

$L=$ Liquid
$\mathrm{O}=0 \mathrm{il}$

$S=$ Soif

$S=$ Soif
SE $=$ Sediment

$T=T$ Tissue

$\checkmark=V$ egitation

$W=$ Water

WI $=$ Wipe

SPECIAL HANDLING AND/OR STORAGE Radioactive Tie To B1TP99

MATRIX*

B1TP42

SOIL

\section{COMPANY CONTACT}

TRENT, STEVE

TELEPHONE NO.

373-5869

PROJECT COORDINATOR

WIDRIG, DL

SAF NO.

PROJECT DESIGNATION

F08-075

200-BP-5 OU Characterization for Wells 299-E33-341 and 299-E33-342 - G

FIELD LOGBOOK NO. ACTUAL SAMPLE DEPTH

HNF-N-488-1 pq $26 \quad 82 . \mathrm{s}^{\prime}$
OFFSITE PROPERTY NO.

$N / A$

123514 ES10

\section{BILL OF LADING/AIR BILL NO.}

\section{N/A}

PRICE CODE $\quad$ SN

AIR QUALITY

METHOD OF SHIPMENT

GOVERNMENT VEHICLE

TYPE OF CONTAINER G/P $\quad \begin{aligned} & \text { Moisture } \\ & \text { Resistant Cont }\end{aligned}$

NO. OF CONTAINER(S)

VOLUME

$\begin{array}{ll}\text { SAMPLE ANALYSIS } & \text { SEE ITEM (1) IN Moisture } \\ \text { SPECIAL } & \text { Content-02216 }\end{array}$

SAMPLE DATE SAMPLE TIME

$5-1-8$ 12235

SPECIAL INSTRUCTIONS

SIGN/ PRINT NAMES

031394

\section{CHAIN OF POSSESSION}

RELINQUISHED BY/REMOYE EOM DCoun a.t's Col?

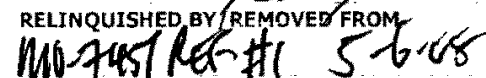
RELINCUISHEP BY/RFMOVED FRO J.5. 20I RELINQUISHEG/B/IREMOVED FROM

RELINQUISHED BY/REMOVED FROM

RELINQUISHED BY/REMOVED FROM

RELINQUISHED BY/REMOVED FROM

DATE/TIME DO RECIVED BY/STORED IN
DATE/TIME
DATE/TIME
DATE/TIME

SEE PAGE 2 FOR ALL SPECIAL INSTRUCTIONS

\footnotetext{
LABORATORY RECEIVED BY SECTION 


\section{COLLECTOR}

NCO SAMPLER

\section{SAMPLING LOCATION}

C5856, I-039

ICE CHEST NO.

\section{SHIPPED TO}

Environmental Sciences Laboratory

\section{COMPANY CONTACT}

TRENT, STEVE

PROJECT DESIGNATION

TELEPHONE NO.

373-5869

FIELD LOGBOOK NO.

OFFSITE PROPERTY NO.

$\mathrm{N} / \mathrm{A}$
PROJECT COORDINATOR

WIDRIG, DL

\section{SAF NO.}

FO8-075

COA

123514 ES10
PRICE CODE

$8 N$

AIR QUALITY

METHOD OF SHIPMENT

GOVERNMENT VEHICLE

\section{SPECIAL INSTRUCTIONS}

** The 200 Area S\&GRP Characterization and Monitoring Sampling and Analysis GKI applies to this SAF.

** ESL is to determine unsaturated hydraulic conductivity using the method(s) best suited for the soll texture and type.

** ESL is to submit copies of all Chain of Custodies and associated sample login documentation to EIS-SDM within 24 hours of sample receipt. They may be sent via e-mail to ACPP Sample Management.

(1)6020M_ICPMS_ASTM_AE (TAL) \{Cadmium, Chromium, Silver\}6020M_ICPMS_ASTM_AE (Add-On) \{Arsenic, Lead, Molybdenum, Selenium\} 6020M_METALS_ICPMS_WE (TAL) \{Cadmilum, Chromium, Silver\}

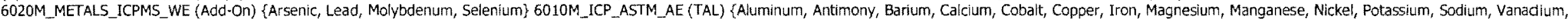

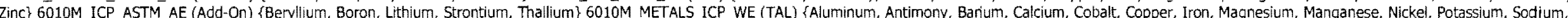

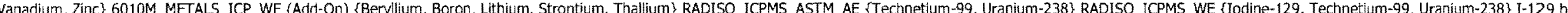

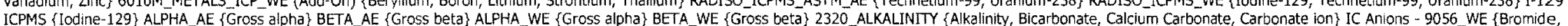

Chloride, Fiuoride, Nitrate, Nitrite, Phosphate, Suffate\} TOC - ASTME1915A \{Total Inorganic Carbon, Total carbon, Total organic carbon\} pH (Water) - 9045 WE; 


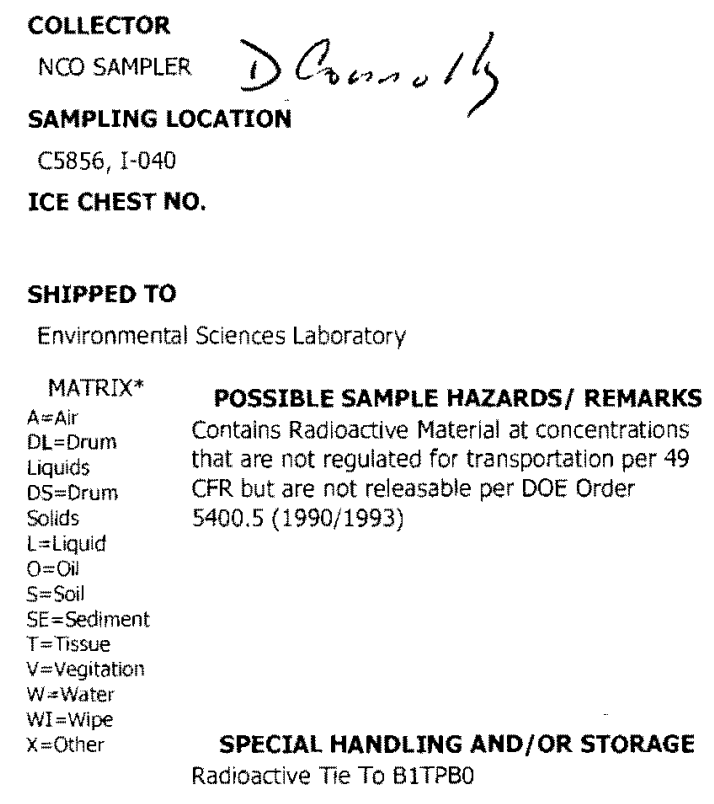

MATRIX*

\section{COMPANY CONTACT}

TRENT, STEVE TELEPHONE NO.

$$
\text { 373-5869 }
$$

\section{PROJECT COORDINATOR} WIDRIG, DL

PRICE CODE $\mathbf{8 N}$

\section{SAF NO.}

200-EP-5 OU Characterization for Wells 299-E33-341 and 299-E33-342 - G F08-075 FIELD LOGBOOK NO.

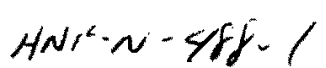
OFFSITE PROPERTY NO. ACTUAL SAMPLE DEPTH

\section{COA}

123514ES10

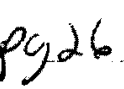
$85 ?$

N/A

$\begin{array}{cll}\text { PRESERVATION } & \text { None } & \text { None } \\ \text { TYPE OF CONTAINER } & \text { G/P } & \begin{array}{c}\text { Moisture } \\ \text { Resistant Cont }\end{array} \\ \text { NO. OF CONTAINER(S) } & 1 & 1 \\ \text { VOLUME } & \text { 1L } & 2009 \\ \text { SAMPLE ANALYSIS } & \begin{array}{l}\text { SEE TEM (1) IN Mosture } \\ \text { SPECILL } \\ \text { INSTRCTIONS }\end{array}\end{array}$

BILL OF LADING/AIR BILLL NO.

\section{SAMPLE DATE SAMPLE TIME} $5 \cdot 1-8 \quad 1245$

\section{CHAIN OF POSSESSION}

RELINQUISHED BY/REMOVEF EROM DConnol/s MO-745 REF. HI TEDBY/REMOVED FROM $=0009 \cdot 62-5.6 .08$ RELINQUSSM LD BY/REMOVED FROM

RELINQUISHED BY/REMOVED FROM

RELINQUISHED BY/REMOVED FROM

RELINQUISHED BY/REMOVED FROM

LABORATORY RECEIVED BY SECTION

FINAL SAMPLE DISPOSITION
DISPOSAL METHOD

Sign/ PRINT NAMES




\section{COLLECTOR}

NCO SAMPLER

\section{SAMPLING LOCATION}

C5856, 1-040

ICE CHEST NO.

\section{SHIPPED TO}

Environmental Sciences Laboratory

\section{COMPANY CONTACT}

TRENT, STEVE

\section{PROJECT DESIGNATION}

\section{TELEPHONE NO.}

373-5869

200-BP-5 OU Characteriza

FIEL.D LOGBOOK NO.

OFFSITE PROPERTY NO.

N/A
PRÖJECT COORDINATOR

WIDRIG, DL

\section{SAF NO.}

F08-075

\section{COA}

$123514 E S 10$
PRICE CODE

AIR QUALITY

METHOD OF SHIPMENT GOVERNMENT VEHICLE

\section{SPECIAL INSTRUCTIONS}

** The 200 Area S\&GRP Characterization and Monitoring Sampling and Analysis GKI apples to this SAF

** ESL is to determine unsaturated hydraulic conductivity using the method(s) best suited for the soil texture and type.

** ESL is to submit copies of all Chain of Custodies and associated sample login documentation to EIS-SOM within 24 hours of sample receipt. They may be sent via e-mail to ^CPP Sample Management.

(1)6020M_ICPMS ASTM AE (TAL) \{Cadmium, Chromium, Silver\} 6020M_ICPMS_ASTM_AE (Add-On) \{Arsenic, Lead, Molybdenum, Selenium\} 6020M_METALS ICPMS_WE (TAL) \{Cadmium, Chromium, Silver\}

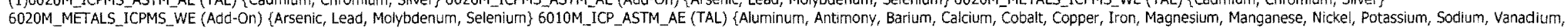

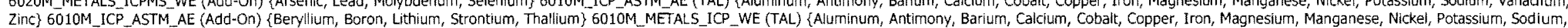

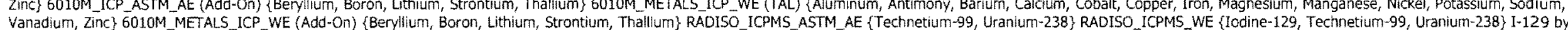

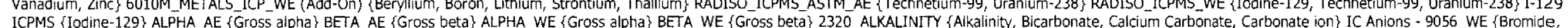

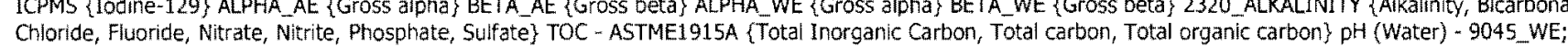




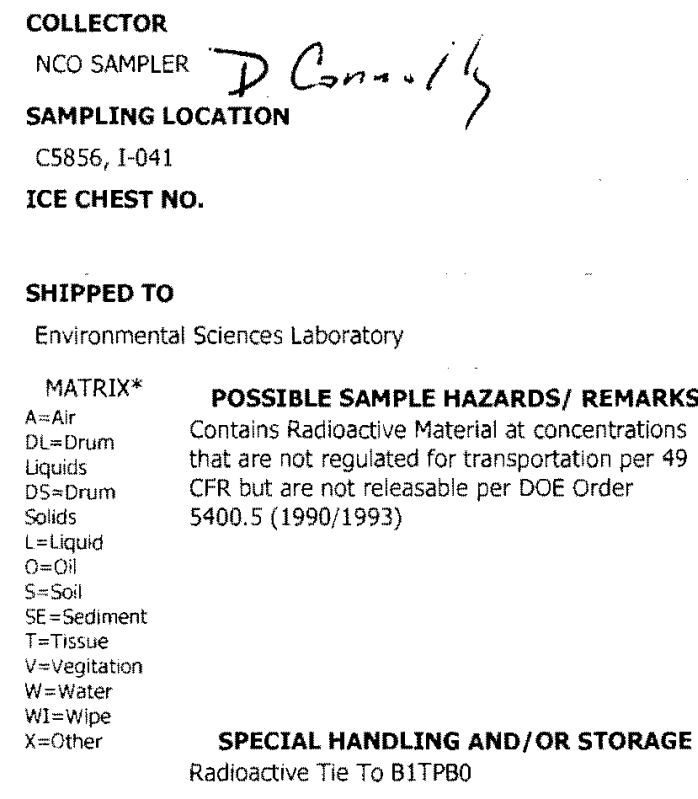

\section{COMPANY CONTACT}

TRENT, STEVE

TELEPHONE NO.

373-5869

PROJECT COORDINATOR

WIDRIG, DL

SAF NO.

PROJECT DESIGNATION

200-BP-5 OU Characterization for Wells 299-E33-341 and 299-E33-342-G FO8-075

FIELD LOGBOOK NO.

ACTUAL SAMPLE DEPTH

\section{COA}

HNF N.4F. Ag $26 \quad 87.5 \%$

$123514 \mathrm{ES} 10$

BILL OF LADING/AIR BILLL NO

N/A

N/A

DATA TURNAROUND

45 Days / 45 Days

\begin{tabular}{llll} 
PRESERVATION & None & None \\
TYPE OF CONTAINER & G/P & $\begin{array}{l}\text { Moisture } \\
\text { Resistant Cont }\end{array}$ \\
\hline
\end{tabular}

NO. OF CONTAINER(S)

VOLUME H. H 2009

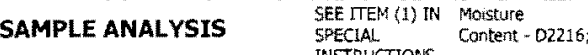

\section{SAMPLE DATE SAMPLE TIME}

$5 \cdot 1.8 / 300$

\section{8}

\section{SIGN/ PRINT NAMES}

RECEIVED BY/STORED IN

\section{SPECIAL INSTRUCTIONS}

DATE/TIME - SEE PAGE 2 FOR ALL SPECIAL INSTRUCTIONS

RELINQUISHED BY/REMOVEDFROM
RELINQUISHED BY/REMOVED FROM
RELINQUISHED BY/REMOVED FROM
RELINQUISHED BY/REMOVED FROM

LABORATORY RECEIVED BY SECTION

FINAL SAMPLE DISPOSAL METHOD DISPOSITION 


\section{COLLECTOR}

NCO SAMPLER

\section{SAMPLING LOCATION}

C5856, I-041

ICE CHEST NO.

\section{SHIPPED TO}

Environmental Sciences Laboratory

\section{COMPANY CONTACT}

TRENT, STEVE

TELEPHONE NO.

$373-5869$
PROJECT COORDINATOR

WIDRIG, DL

\section{SAF NO.}

200-BP-5 OU Characterization for Wells 299-E33-341 and 299-E33-342-G : F08-075
ACTUAL SAMPLE DEPTH

PRICE CODE

$8 N$

DATA

\section{FIELD LOGBOOK NO.}

OFFSITE PROPERTY NO.

N/A

\section{AIR QUALITY}

\section{SPECIAL INSTRUCTIONS}

** The 200 Area S\&GRP Characterization and Monitoring Sampling and Analysis GKI applies to this SAF.

** ESL is to determine unsaturated hydraulic conductivity using the method(s) best suited for the soil texture and type.

** ESL is to submit copies of all Chain of Custodies and associated sample login documentation to EIS-SDM within 24 hours of sample receipt. They may be sent via e-mail to ^CPP Sample Management.

(1)6020M_ICPMS_ASTM_AE (TAL) \{Cadmium, Chromium, Silver\} 6020M_ICPMS_ASTM_AE (Add-On) \{Arsenic, Lead, Molybdenum, Selenium\} 6020M_METALS ICPMS_WE (TAL) \{Cadmium, Chromium, Silver\}

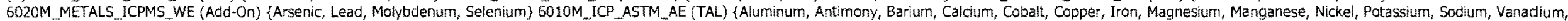

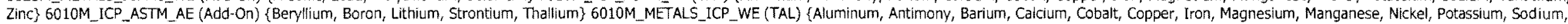

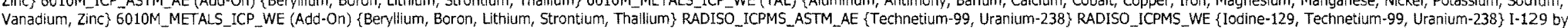

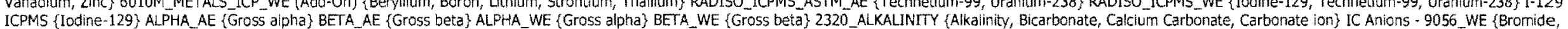

Chloride, Fluoride, Nitrate, Nitrite, Phosphate, Sulfate\} TOC - ASTME1915A \{Total Inorganic Carbon, Total carbon, Total organic carbon\} pH (Water)-9045 WE; 


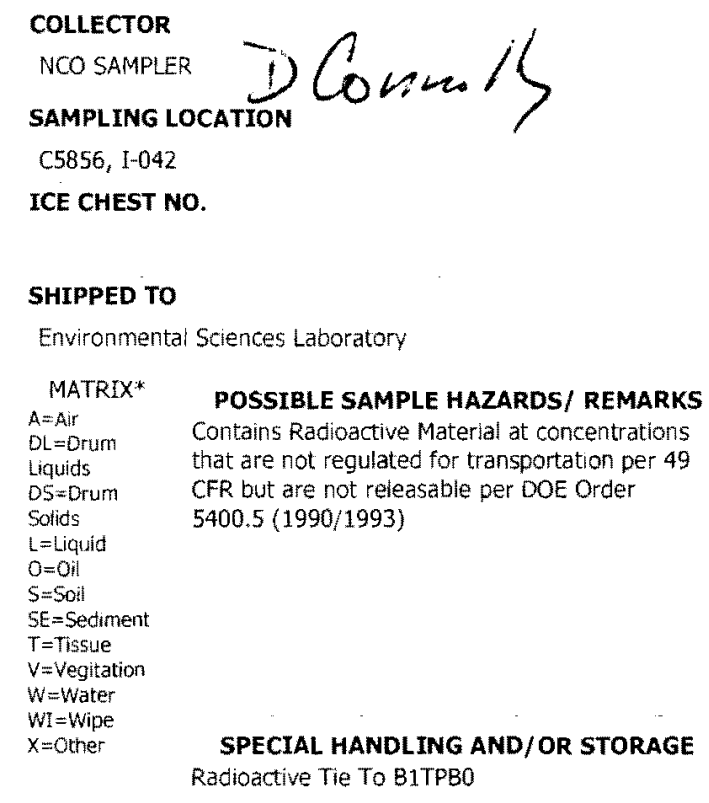

\section{SHIPPED TO}

Environmental Sciences Laboratory

MATRIX**

$A=A$ ir

$\mathrm{DL}=\mathrm{Drum}$

Liquids
$05 *$ Drum

DS*Crum
Solids

$L=L i q u i d$

$0=0$ in
$S=S \mathrm{i} i$

$S=S 0: 1$
$S E=S e d i m e n t$

$S E=$ Sediment
$\mathrm{T}=$ Tissue

$V=$ Vegitation

$W=$ Water

$W I=$ Wipe

SPECIAL HANDLING AND/OR STORAGE Radioactive Tie To B1TPBO

PSSIBLE SAMPLE HAZAROS/ REMARKS Contains Radioactive Material at concentrations that are not regulated for transportation per 49 CFR but are not releasable per DOE Order 5400.5 (1990/1993)

\section{SAF NO.}

PRICE CODE

TRENT, STEVE
PROJECT DESIGNATION

F08-075

200-BP-5 OU Characterization for Wells 299-E33-341 and 299-E33-342FIELD LOGBOOK NO. ACTUAL SAMPLE DEPTH

AIR QUALITY

HNF-N- 4 Ff.

SAMPLE NO

MATRIX*

B1TP45

SOIL

SAMPLE TIME

$5 \cdot 1.8 \quad 1340$

\section{8}

SIGN/ PRINT NAMES

DATE/TIME

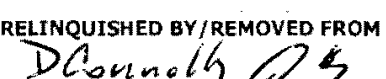

Dovnoly 5

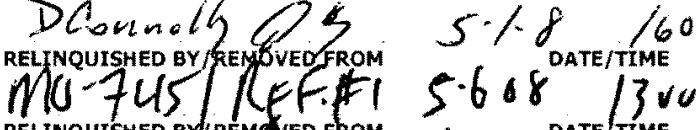

RELINQUISHED BWIREMg VIED FROM

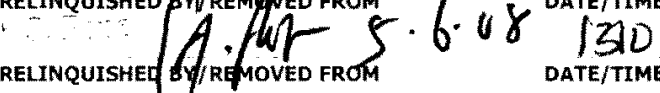

RELINQUISHED BY/REMOVED FROM

DATE/TIME

DATE/TIME

DATE/TIME

RELINQUISHED EY/REMOVED FROM

RECEIVED BY/STORED IN

RELINQUISHED BY/REMOVED FROM RECEIVED BY/STOREYIN

RECEIVED BY/STORED IN

RECEIVED BY/STORED IN

\section{COA}

$$
90^{\prime}
$$

123514 ES10

METHOD OF SHIPMENT

GOVERNMENT VEHICLE
$N / A$

N/A Mo 745 Refl $5.10 / 600$

SPECIAL INSTRUCTIONS

SEE PAGE 2 FOR ALL SPECIAL. INSTRUCTIONS DATE/TIME

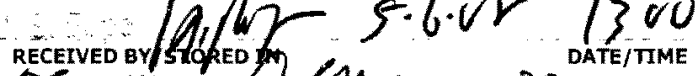
Bomith $6244-5.681310$ RECEIVED BY/STORED IN

\section{DATE/TIME}

DATE/TIME

DATE/TIME 
COMPANY CONTACT

NCO SAMPLER

TRENT, STEVE

TELEPHONE NO.

373-5869

PROJECT COORDINATOR

WIDRIG, DL

PRICE CODE

\section{PROJECT DESIGNATION}

SAF NO.

AIR QUALITY

URNAROUND

45 Days / 45

Days

200-BP-5 OU Characterization for Welis 299-E33-341 and 299-E33-342-G

FIELD LOGBOOK NO.

ACTUAL SAMPLE DEPTH

COA

METHOD OF SHIPMENT

123514ES10 GOVERNMENT VEHICLE

\section{OFFSITE PROPERTY NO.}

$N / A$

\section{BILL OF LADING/AIR BILL NO}

N/A

Environmental Sciences Laboratory

\section{SPECIAL INSTRUCTIONS}

** The 200 Area S\&GRP Characterization and Monitoring Sampling and Analysis GKI applies to this SAF.

** ESL is to determine unsaturated hydraulic conductivity using the method(s) best suited for the soll texture and type.

** ESL is to submit copies of all Chain of Custodies and associated sample login documentation to EIS-SDM within 24 hours of sample receipt. They may be sent via e-mail to ^CPP Sample Management.

(1)6020M ICPMS ASTM_AE (TAL) \{Cadmium, Chromium, Silver\} 6020M_ICPMS_ASTM_AE (Add-On) \{Arsenic, Lead, Molybdenum, Selenium\} 6020M_METALSICPMS_WE (TAL) \{Cadmium, Chromlum, Silver\}

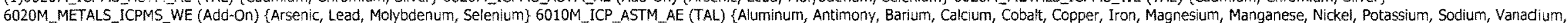

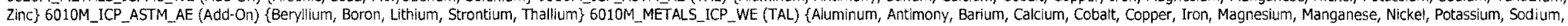

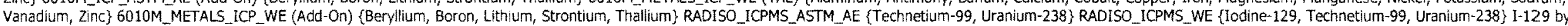

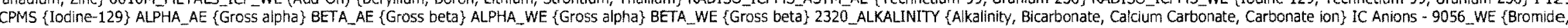
Chloride, Fiuoride, Nitrate, Nitrite, Phosphate, Sulfate\} TOC - ASTME1915A (Total Inorganic Carbon, Total carbon, Total organic carbon) pH (Water) - 9045 WE; 


\section{COLLECTOR \\ NCO SAMPLER DConn/S}

SAMPLING LOCATION

C5856, 1-043

ICE CHEST NO.

SHIPPED TO

Environmental Sciences Laboratory

MATRIX*

$A=$ Air

DL.=Drum

Liquids

DS=Drum
Solids

$\mathrm{L}=\mathrm{Linu}$

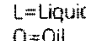

$S=5$ ill

SE $=$ Sediment

T=Tissue

$V=$ Vegitation

$W=$ water

WI $=$ Wipe

SPECIAL HANDLING AND/OR STORAGE Radioactive TIe To B1TPBO

DARLE HAZARDS/ REMARKS Contains Radioactive Material at concentrations that are not regulated for transportation per 49 CFR but are not releasable per DOE Order $5400.5(1990 / 1993)$

SAMPLE NO

MATRIX*

B1TP46

SOIL

\section{CHAIN OF POSSESSION}

RELINQUISHED BY/REMONED FROM DC nall $5.1 .8^{\text {DATE/TME }} 1600$ MU.745 Ref $\$ 115.6 .08$ Date/Tume RELINQUISHED WYIPEMOYED FROM RELINQUISHED BY, KEITOVED FROM

RELINQUISHED BY/REMOVED FROM

RELINQUISHED BY/REMOVED FROM

RELINQUISHED BY/REMOVED FROM

RECEIVED BY

\section{LABORATORY} SECTION

FINAL SAMPLE DISPOSITION

\section{COMPANY CONTACT}

TRENT, STEVE TELEPHONE NO.

\section{PROJECT COORDINATOR} WIDRIG, DL

PRICE CODE

$\mathbf{8 N}$

\section{SAF NO.}

200-BP-5 OU Characterization for Wells 299-E33-341 and 299-E33-342-G F08-075

FIELD LOGBOOK NO. ACTUAL SAMPLE DEPTH

\section{COA}

AIR QUALITY

$$
92.5^{* i}
$$

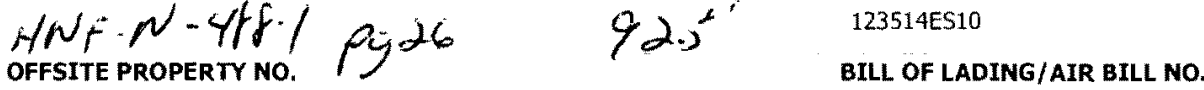

METHOD OF SHIPMENT

GOVERNMENT VEHICLE

N/A

N/A

DATA

URNAROUND

45. Days / 45

\begin{tabular}{|c|c|c|}
\hline PRESERVATION & None & None \\
\hline YPE OF CONTAINER & $G / P$ & $\begin{array}{l}\text { Moisture } \\
\text { Resistant Cont }\end{array}$ \\
\hline 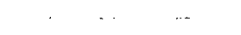 & $\ldots$ & - \\
\hline
\end{tabular}

NO. OF CONTAINER(S)

VOLUME

ii $\quad 2009$

SAMPLE ANALYSIS
SEE ITEM (1) IN Moisture
SPECEAL
INSTRUTTONS

SAMPLE DATE SAMPLE TIME

$5-1 \%$. 1355

028688

\section{SIGN/ PRINT NAMES}

RECEIVED BY/STORED IN

Mo 745 Refl

orTOREOIV 5.64 DATELIME

RECEIYED BY STOREO IN DATE/TIME

Somith 5.6081310

DATE/TIME

DATE/TIME

DATE/TIME

RECEIVED BY/STORED IN

DATE/TIME

DATE/TIME

RECEIVED BY/STORED IN

DATE/TIME

DATE/TIME
RECEIVED BY/STORED IN

\section{SPECIAL INSTRUCTIONS}

SEE PAGE 2 FOR ALL SPECIAL INSTRUCTIONS 


\section{COLLECTOR}

NCO SAMPLER

\section{SAMPLING LOCATION}

C5856, I-043

ICE CHEST NO.

\section{SHIPPED TO}

Environmental Sciences Laboratory

\section{COMPANY CONTACT}

TRENT, STEVE

PROJECT DESIGNATION

200-BP-5 OU Characterization for Wells 299-E33-341 and 299-E33-342-G

FIELD LOGBOOK NO.

ACTUAL SAMPLE DEPTH

\section{OFFSITE PROPERTY NO}

N/A

\section{TELEPHONE NO.}

373-5869

\section{SPECIAL INSTRUCTIONS}

** The 200 Area S\&GRP Characterization and Monitoring Sampling and Analysis GKI applies to this SAF.

** ESL is to determine unsaturated hydraulic conductivity using the method(s) best suited for the soll texture and type.

** ESL is to submit copies of all Chain of Custodies and associated sample login documentation to EIS-SOM within 24 hours of sample receipt. They may be sent via e-mail to $A$ CPP Sample Management.

(1)6020M_ICPMS ASTM_AE (TAL) \{Cadmium, Chromium, Silver\} 6020M_ICPMS_ASTM_AE (Add-On) \{Arsenic, Lead, Molybdenum, Selenium\} 6020M_METALS_ICPMS_WE (TAL) \{Cadmium, Chromium, Silver\}

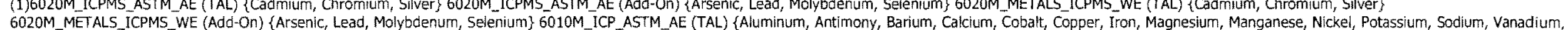

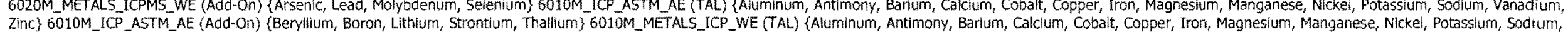

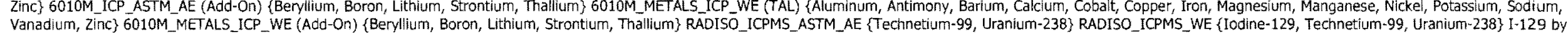

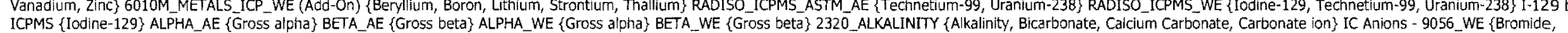
ICPMS \{Iodine-129\} ALPHA_AE \{Gross alpha\} BETA_AE \{Gross beta\} ALPHA_WE \{Gross alpha\} BETA_WE \{Gross beta\} 2320_ALKALINITY \{Alkalinity, Bicarbonat
Chioride, Fluoride, Nitrate, Nitrite, Phosphate, Sulfate\} TOC-ASTME1915A \{Total Inorganic Carbon, Total carbon, Total organic carbon\} pH (Water) -9045_WE; 


\section{Fluor Hanford Inc.}

\section{COLLECTOR \\ NCO SAMPLER \\ SAMPLING LOCATION}

C5856, I-045

ICE CHEST NO.

\section{SHIPPED TO}

Environmental Sciences Laboratory

MATRIX*

$A=A i r$

$\mathrm{DL}=\mathrm{Drum}$

Lquids
DS=Drum

Solids

$L=L$ iquid

$0=0 i l$
$5=5011$

$S=S$ ofl
$S E=$ Sediment

$T=$ Tissue

$V=$ Vegitation

$W=$ Water

WI $=$ Wipe

POSSIBLE SAMPLE HAZARDS/ REMARKS Contains Radioactive Material at concentrations that are not regulated for transportation per 49 CFR but are not releasable per DOE Order

$5400.5(1990 / 1993)$

SPECIAL HANDLING AND/OR STORAGE Radioactive Tie To BITPBO

SAMPLE NO.

MATRIX*

B1TP47

SOIL

\section{CHAIN OF POSSESSION \\ DCments \&}

RELINQUISHED BY/REMOVERFROM RELINQUISHED GY REMOVED FROM WU FUS REF. F RELINQUISHEP OY REMOYED FROM RELINQUISHES B $/$ REMPVED FROM

$5 \cdot c \cdot 08 / 3 d$ - G. u. 1310

RELINQUISHED BY/REMOVED FROM DATE/TIME

RELINQUISHED BY/REMOVED FROM

DATE/TIME

DATE/TIME

RELINQUISHED BY/REMOVED FROM

DATE/TIME
CHAIN OF CUSTODY/SAMPLE ANALYSIS REQUEST

\section{TELEPHONE NO.}

373-5869

PROJECT COORDINATOR

WIDRIG, DL

SAF NO.

PROJECT DESIGNATION

F08-075

200-BP-5 OU Characterization for Wells 299-E33-341 and 299-E33-342 - G FIELD LOGBOOK NO. ACTUAL SAMPLE DEPTH

\section{$\operatorname{COA}$}

HNH-w-4 ffy pga6 $95 \%$

N/A

$123514 E S 10$

BILL OF LADING/AIR BILL NO.

N/A

\begin{tabular}{lll} 
PRESERVATION & None & None \\
TYPE OF CONTAINER & G/P & $\begin{array}{l}\text { Moisture } \\
\text { Resistant Cont }\end{array}$ \\
\hline
\end{tabular}

NO. OF CONTAINER(S)

VOLUME

il 2009

SAMPLE ANALYSIS

SAMPLE DATE SAMPLE TIME

$5 \cdot 1 \cdot 14 / 2$

SIGN/ PRINT NAMES

\section{8}

RECEIVED BY/STORED IN DATE/TIME n=745 Ket/ s.F 1600 RECEIVED BY/STORED Iy 5.6 .08 DATE/TIME

RECEIVED BY/STORED IN

DATE/TIME

RECETVED BY/STORED IN

DATE/TIME

RECEIVED BY/STORED IN

DATE/TIME

\section{SPECIAL INSTRUCTIONS}

SEE PAGE 2 FOR ALL SPECIAL INSTRUCTIONS

\section{LABORATORY RECEIVED BY}




\section{COLLECTOR}

NCO SAMPLER

\section{SAMPLING LOCATION}

C5856, I-045

ICE CHEST NO.

\section{SHIPPED TO}

Environmental Sciences Laboratory

\section{COMPANY CONTACT}

TRENT, STEVE

PROJECT DESIGNATION

\section{TELEPHONE NO.}

$373-5869$

200-BP-5 OU Characterization for Wells 299-E33-341 and 299-E33-342-G F08-075

FIELD LOGBOOK NO.

ACTUAL SAMPLE DEPTH

COA

123514ES10
PRICE CODE

$\mathbf{8 N}$

TURNAROUND

45 Days / 45

Days

\section{OFFSITE PROPERTY NO.}

$N / A$
AIR QUALITY

METHOD OF SHIPMENT

GOVERNMENT VEHICLE

\section{SPECIAL INSTRUCTIONS}

** The 200 Area S\&GRP Characterization and Monitoring Sampling and Analysis GKI applies to this SAF.

** ESL is to determine unsaturated hydraulic conductivity using the method(s) best suited for the soll texture and type.

** ESL is to submit copies of all Chain of Custodies and associated sample login documentation to EIS-SDM within 24 hours of sample receipt. They may be sent via e-mail to ACPP Sample Management.

(1)6020M ICPMS ASTM AE (TAL) \{Cadmium, Chromium, Silver\} 6020M ICPMS_ASTM_AE (Add-On) (Arsenic, Lead, Molybdenum, SElenium\} 6020M METALSICPMS WE (TAL) \{Cadmium, Chromium, Silver\}

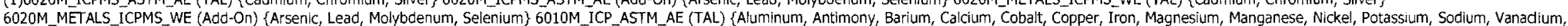

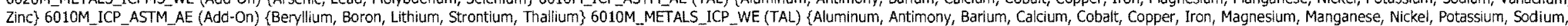

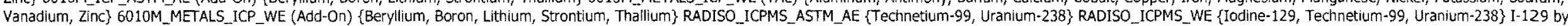

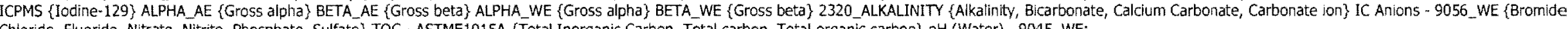
Chloride, Fluoride, Nitrate, Nitrite, Phosphate, Sulfate\} TOC - ASTME1915A \{Total Inorganic Carbon, Total carbon, Total organic carbon\} pH (Water) - 9045 -WE; 


\section{COLLECTOR \\ NCO SAMPLER

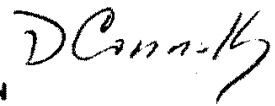 \\ SAMPLING LOCATION}

C5856, I-047

ICE CHEST NO

\section{SHIPPED TO}

Environmental Sciences Laboratory

MATRIX*
A=Air
$D L=$ Drum
Liquids
DS=Drum
Solids
L=Liquid
$O=$ Ol
$S=$ Sol
$S E=$ Sediment
$T=$ Tisste
$V=$ Vegitation
$W=$ Water
WI=Wipe
$X=$ Other

POSSIBLE SAMPLE HAZARDS/ REMARKS Contains Radioactive Material at concentrations that are not regulated for transportation per 49 CFR but are not releasable per DOE Order $5400.5(1990 / 1993)$

SPECIAL HANDLING AND/OR STORAGE Radioactive Tie TO B1TPBO

MATRIX*

B1TP48

SOIL

\section{COMPANY CONTACT}

TRENT, STEVE

TELEPHONE NO.

373-5869

\section{PROJECT DESIGNATION}

PROJECT COORDINATOR

WIDRIG DL

SAF NO

200-BP-5 OU Characterization for Wells 299-E33-341 and 299-E33-342-G FO8-075 FIELD LOGBOOK NO.

HNF"NAYfd / pg L 16 ACTUAL SAMPLE DEPTH 97.8

N/A

COA

123514 ES 10

BILL OF LADING/AIR BILL NO

N/A

\begin{tabular}{|c|c|c|}
\hline PRESERVATION & None & None \\
\hline TYPE OF CONTAINER & $\mathrm{G} / \mathrm{P}$ & $\begin{array}{l}\text { Moisture } \\
\text { Resistant Cont }\end{array}$ \\
\hline NO. OF CONTAINER(S) & 1 & $i^{\cdots}$ \\
\hline VOLUME & $1 \mathrm{~L}$ & $200 \mathrm{~g}$ \\
\hline SAMPLE ANALYSIS & $\begin{array}{l}\text { SEE TTEM (1) IN } \\
\text { SPECAN } \\
\text { INSTRUCIONS }\end{array}$ & $\begin{array}{l}\text { Meisture } \\
\text { Content - D2216; }\end{array}$ \\
\hline
\end{tabular}

SAMPLE DATE SAMPLE TIME

$5 \%(144)^{\circ}$

\section{CHAIN OF POSSESSION}

RELINQUISHED BY/REMOVER FROM

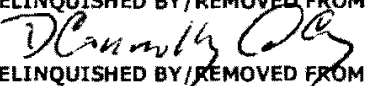

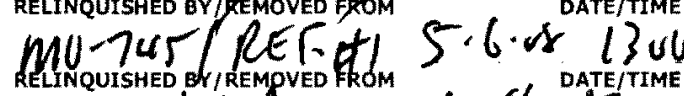
S DATE/TIME

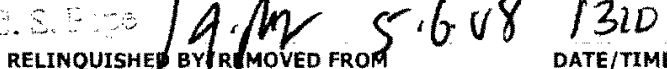

RELINQUISHED BY/REMOVED FROM DATE/TIME

RELINQUISHED BY/REMOVED FROM

DATE/TIME

RELINQUISHED BY/REMOVED FROM

DATE/TIME

DATE/TIME
$0.266+8$

SIGN/ PRINT NAMES

SPECIAL INSTRUCTIONS

RECEIVED BY/STORED IN DATE/TIME SEE PAGE 2 FOR ALL SPECIAL INSTRUCTIONS

Mo74j Ret ( 5./P lioo

RECEIVED BY/STORED WN $5 \cdot 6.08$ DATE/TIME

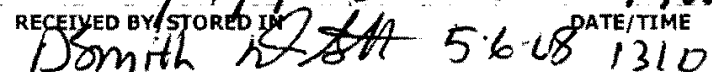

RECEIVED BY/STORED IN

RECEIVED BY/STORED IN

DATE/TIME

RECEIVED BY/STORED IN

DATE/TIME

RECEIVED BY/STORED IN

DATE/TIME
LABORATORY RECEIVED BY

SECTION

FINAL SAMPLE DISPOSAL METHOD 


\section{COLLECTOR}

NCO SAMPLER

\section{SAMPLING LOCATION}

C5856, I-047

ICE CHEST NO.

\section{SHIPPED TO}

Environmental Sciences Laboratory

\section{COMPANY CONTACT}

TRENT, STEVE

PROJECT DESIGNATION

\section{TELEPHONE NO.}

373-5869

200-BP-5 OU Characterization for Wells 299-E33-341 and 299-E33-342-G

FIELD LOGBOOK NO.

ACTUAL SAMPLE DEPTH

\section{OFFSITE PROPERTY NO.}

N/A

\section{PROJECT COORDINATOR}

WIDRIG, DL

\section{SAF NO.}

F08-075

COA

123514 ES10

\section{PRICE CODE}

8N

DATA

AIR QUALITY

TURNAROUND

45 Days / 45

Days

\section{METHOD OF SHIPMENT}

GOVERNMENT VEHICLE

\section{BILL OF LADING/AIR BILL NO.}

$\mathrm{N} / \mathrm{A}$

\section{SPECIAL INSTRUCTIONS}

** The 200 Area S\&GRP Characterization and Monitoring Sampling and Analysis GKI applies to this SAF.

** ESL is to determine unsaturated hydraulic conductivity using the method(s) best suited for the soil texture and type.

** ESL is to submit copies of all Chain of Custodies and associated sample login documentation to EIS-SDM within 24 hours of sample receipt. They may be sent via e-mail to ^CPP Sample Management.

(1)6020M_ICPMS_ASTM_AE (TAL) \{Cadrnium, Chromlum, Silver\} 6020M_ICPMS ASTM AE (Add-On) \{Arsenic, Lead, Molybdenum, Selenium\} 6020M_METALS_ICPMS WE (TAL) \{Cadmium, Chromium, Silver\}

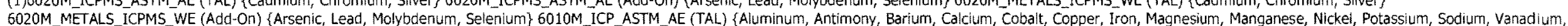

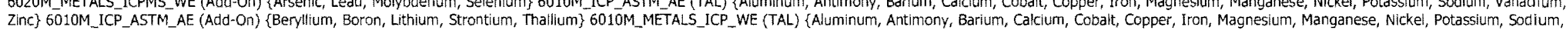

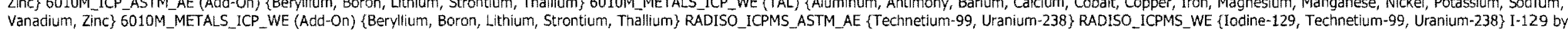

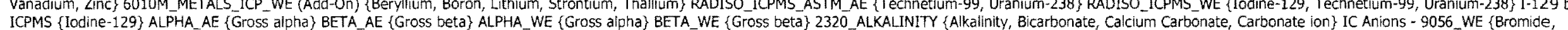
Chloride, Fluoride, Nitrate, Nitrite, Phosphate, Sulfate\} TOC - ASTME1915A \{Total Inorganic Carbon, Total Carbon, Total organic carbon\} PH (Water) - 9045 _WE; 


\section{COLLECTOR \\ nCO SAMPLER DCuituol/7 \\ SAMPLING LOCATION}

C5856, I-049

ICE CHEST NO.

\section{SHIPPED TO}

Environmental Sciences Laboratory

MATRIX*

$A=$ Air

DL. $=$ Drum

Liquids

DS $=$ Drum

L=Liquid

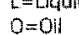

$5=5$ ol

$S E=$ Sediment

$T=T$ Tissue

$v=$ venitation

$W=$ Water

$W I=$ Wipe

SPECIAL HANDLING AND/OR STORAGE Radioactive Tie To B1TPB1 Contains Radioactive Material at concentrations that are not regulated for transportation per 49 FR but are not releasable per DOE Order $5400.5(1990 / 1993)$

SAMPLE NO.

MATRIX*

\section{COMPANY CONTACT}

TELEPHONE NO.

PROJECT COORDINATOR

SAF NO.

PROJECT DESTGNATION

$373 \cdot 5869$

F08-075

200-BP-5 OU Characterization for Wells 299-E33-341 and 299-E33-342 - G

FIELD LOGBOOK NO.

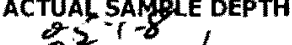

$\mathrm{COA}$

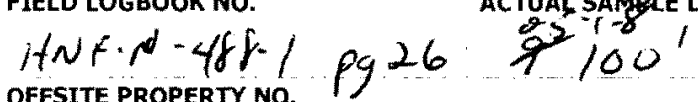

AIR QUALITY $[\quad 45$ Days / 45

N/A

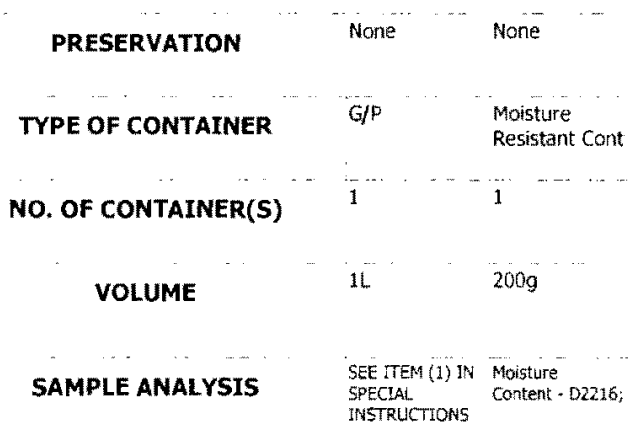

SAMPLE DATE SAMPLE TIME

$5 \%$. 1505
$123514 E S 10$

BILL OF LADING/AIR BILL NO.

GOVERNMENT VEHICLE
SOIL

B1TP49

\section{CHAIN OF POSSESSION}

RELINQUISHED BY/REMOXED FROM $D C+i n \cdot 16, C$ RELINOUISHEP B /REMCVEd FROM $J / \%$ / MU-745 ReF t $5 \cdot 6.0 Y$ 1300 RELINQUISH O GY/RYMOVED FROM RELINQUISHEG /Y/ REMOVED FROM

RELINQUISHED BY/REMOVED FROM

RELINQUISHED BY/REMOVED FROM

RELINQUISHED BY/REMOVED FROM

RECEIVED BY

\section{LABORATORY}

SECTION

FINAL SAMPLE DISPOSAL METHOD

DISPOSITION
DATE/TIME

DATE/TIME

DATE/TIME

DATE/TIME

\section{8}

\section{SIGN/ PRINT NAMES}

- $-\ldots . . .$.

RECEIVED BY/STORED IN

Mu?45 Ret I

RECETyE BY foked

Domith 48 fat 5.6 .081320

RECEIVED BY/STORED IN

RECEIVED BY/STORED IN

DATE/TIME

RECEIVED BY/STORED IN

DATE/TIME

RECEIVED BY/STORED IN

DATE/TIME

\section{SPECIAL INSTRUCTIONS}

\section{8}

\section{N/A}

\section{....}


COMPANY CONTACT

TRENT, STEVE

TELEPHONE NO.

373.5869

PROJECT COORDINATOR

WIDRIG, DL

PRICE CODE

$\mathbf{8 N}$

DATA

\section{PROJECT DESIGNATION}

SAF NO.

AIR QUALITY

TURNAROUN

45 Days / 45

Days
C5856, 1.049

ICE CHEST NO.

\section{SHIPPED TO}

Environmental Sciences Laboratory
FIELD LOGBOOK NO.

ACTUAL SAMPLE DEPTH

COA

123514 ES10
METHOD OF SHIPMENT

GOVERNMENT VEHICLE
OFFSITE PROPERTY NO.

N/A

\section{BILL OF LADING/AIR BILL NO}

N/A

\section{SPECIAL INSTRUCTIONS}

** The 200 Area S\&GRP Characterization and Monitoring Sampling and Analysis GKI applies to this SAF.

** ESL is to determine unsaturated hydraulic conductivity using the method(s) best suited for the soil texture and type.

** ESL is to submit copies of all Chain of Custodies and associated sample login documentation to EIS-SDM within 24 hours of sample receipt. They may be sent via e-mail to aCPP Sample Management.

(1)6020M_ICPMS_ASTM_AE (TAL) \{Cadmium, Chromium, Silver\} 6020M_ICPMS_ASTM_AE (Add-On) \{Arsenic, Lead, Molybdenum, Selenium\} 6020M_METALS_ICPMS_WE (TAL) \{Cadmium, Chromium, Silver\}

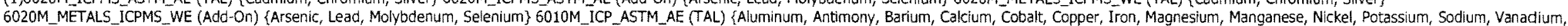

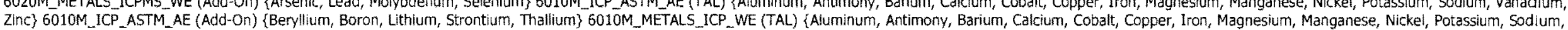

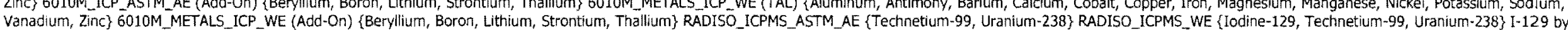

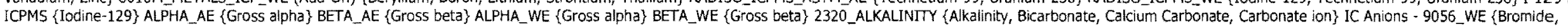
Chloride, Fluoride, Nitrate, Nitrite, Phosphate, Sulfate\} TOC - ASTME1915A \{Total Inorganic Carbon, Total carbon, Total organic carbon\} pH (Water) - 9045 .WE; 


\section{COLLECTOR}

nCo sampler BAlley

SAMPLING LOCATION

C5856, I-051

ICE CHEST NO.

SHIPPED TO

Environmental Sciences Laboratory

\section{MATRIX* \\ $A=$ Air \\ $\mathrm{DL}=\mathrm{Drum}$ \\ DS=Drum \\ Solids \\ $L=$ Liquid \\ $0=0 \mathrm{il}$
$\mathrm{S}=5 \mathrm{il}$ \\ SE $=$ Sediment \\ $T=$ Tisste \\ $v=$ vegitation \\ WT=wipe \\ WI $=$ Wipe \\ POSSIBLE SAMPLE HAZARDS/ REMARKS Contains Radioactive Material at concentrations that are not regulated for transportation per 49 CFR but are not releasable per DOE Order $5400.5(1990 / 1993)$ \\ SPECIAL HANDLING AND/OR STORAGE} Radioactive Tie To B1TPB1

\section{COMPANY CONTACT}

TRENT, STEVE

$$
\text { 373-5869 }
$$

PROJECT COORDINATOR

WIDRIG, DL

PROJECT DESIGNATION

SAF NO.

200-BF-5 OU Characterization for Wells 299-E33-341 and 299-E33-342-G F08-075 FIELD LOGBOOK NO.

HNF-N-48S-1

OFFSITE PROPERTY NO.

\section{ACTUAL SAMPLE DEPTH}

COA

123514 ES 10

BILL OF LADING/AIR BILL NO.

N/A
N/A

\begin{tabular}{cll} 
PRESERVATION & None & None \\
TYPE OF CONTAINER & G/P & $\begin{array}{l}\text { Moisture } \\
\text { Resistant Cont }\end{array}$ \\
NO. OF CONTAINER(5) & 1 & 1 \\
VOLUME & $1 \mathrm{~L}$ & $200 \mathrm{~g}$ \\
SAMPLE ANALYSIS & $\begin{array}{l}\text { SEE TEM (1) IN Moisture } \\
\text { SPECLL } \\
\text { INSTRUTIONS }\end{array}$ \\
\hline
\end{tabular}

PRICE CODE 8 N

AIR QUALITY

METHOD OF SHIPMENT

GOVERNMENT VEHICLE

SAMPLE DATE SAMPLE TIME

$5.8 .08 \quad 0820$

\section{SPECIAL INSTRUCTIONS}

SIGN/ PRINT NAMES

DATE/TIME

RELINOUISHED BY/REMOVED FROM JKBALCY OKC E $=0$ RELINQUISHED BY/REMOVED FRQM MU-7US REF A 3 REUNQUISHER BY/REMAVED $F$ ROM Ln. OUNG RELINOUISHED BY/REMOVED FROM

RELINQUISHED BY/REMOVED FROM MAY O $92003 / 1030$

RECEIVD BY/STORED IN
WOTUS REF

\section{8}

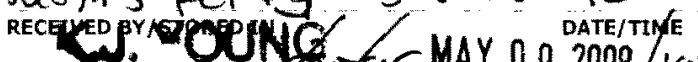
RECEIVED BY/STOREo IN $519108 / 40)$ DATE/TIME DATE/TIME RECEIVED BY/S IORED IN Lin $5 / 9 / 9 / 40$ DATE/TIME DATE/TIME
RECEIVED BY/STORED IN

DATE/TIME

RECEIVED BY/STORED IN

RECEIVED BY/STORED IN
RELINQUISHED BY/REMOVED FROM
SEE PAGE 2 FOR ALL SPECIAL INSTRUCTIONS

RECEIVED BY

LABORATORY

DISPOSAL METHOD

FINAL SAMPLE

DISPOSAL METHOD

DISPOSITION 


\section{COLLECTOR}

NCO SAMPLER

\section{SAMPLING LOCATION}

C5856, I-051

ICE CHEST NO.

\section{SHIPPED TO}

Environmentai Sciences Laboratory

\section{COMPANY CONTACT}

TRENT, STEVE

\section{PROJECT DESIGNATION}

\section{TELEPHONE NO.}

373-5869

200-BP-5 OU Characteriz

FIELD LOGBOOK NO.

\section{OFFSITE PROPERTY NO}

$\mathrm{N} / \mathrm{A}$
WIDRIG, DL

SAF NO.

F08-075

COA

123514 ES10
PRICE CODE

AIR QUALITY

$\mathbf{8 N}$

DATA

TURNAROUND

45 Days / 45

Days

\section{SPECIAL INSTRUCTIONS}

** The 200 Area S\&GRP Characterization and Monitoring Sampling and Analysis GKI applies to this SAF.

** ESL is to determine unsaturated hydraulic conductivity using the method(s) best suited for the soil texture and type.

*** ESL is to submit copies of all Chain of Custodies and associated sample login documentation to EIS-SDM within 24 hours of sample receipt. They may be sent via e-mail to ^CPP Sample Management.

(1)6020M_ICPMS_ASTM_AE (TAL) \{Cadmium, Chromium, Silver\} 6020M_ICPMS_ASTM_AE (Add-On) \{Arsenic, Lead, Molybdenum, Selenium\} 6020M_METALS_ICPMS_WE (TAL) \{Cadmium, Chromium, Silver\}

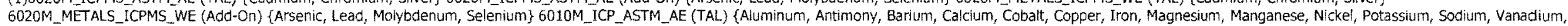

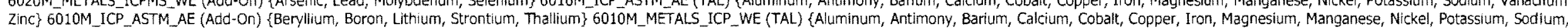

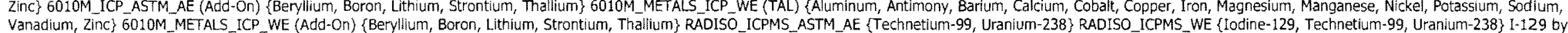

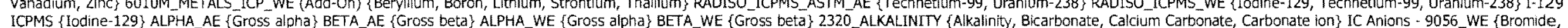

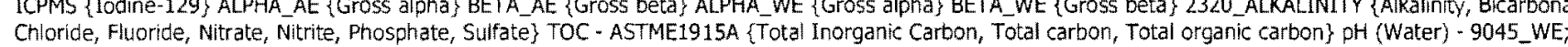


COLLECTOR

NCO SAMPLER BAllel

SAMPLING LOCATION

C5856, I-053

ICE CHEST NO.

\section{SHIPPED TO}

Environmental Sciences Laboratory

MATRIX*

$A=$ Air

$\mathrm{DL}=\mathrm{Drum}$

Liquids

Solids

Solids

$0=0$ il

SE=Soil

$T=T$ Tssue

$V=v e g i t a t i o n$

$W=$ water

WI $=$ Wipe

SPECIAL HANDLING AND/OR STORAGE Radioactive Tie To B1TPB1

SAMPLE HAZARDS/ REMARKS Contains Radioactive Material at concentrations

that are not regulated for transportation per 49

CFR but are not releasable per DOE Order

$5400.5(1990 / 1993)$

SAMPLE NO.

MATRIX*

B1TP51

SOIL

\section{TELEPHONE NO.}

373-5869

PROJECT COORDINATOR

WIORIG, DL

\section{SAF NO.}

PROJECT DESIGNATION

F08-075

200-BP-5 OU Characterization for Wells 299-E33-341 and 299-E33-342-G FIELD LOGBOOK NO.

ACTUAL SAMPLE DEPTH

HNE-TJ-488-1 1051

COA

$123514 E S 10$

BILL OF LADING/AIR BILL NO.

OFFSITE PROPERTY NO.

$\mathrm{N} / \mathrm{A}$
PRICE CODE BN

AIR QUALITY

METHOD OF SHIPMENT

GOVERNMENT VEHICLE
N/A

\begin{tabular}{|c|c|c|}
\hline PRESERVATION & None & None \\
\hline TYPE OF CONTAINER & GPP & $\begin{array}{l}\text { Moisture } \\
\text { Resistant Cont }\end{array}$ \\
\hline
\end{tabular}

VOLUME

1

2009

SAMPLE ANALYSIS
DATA TURNAROUND

45 Days / 45
LOT NO. CHAIN OF POSSESSION

RELINQUISHED BY/REMOVED FROM JKBAILCY JKEZO $5.8 .8 / 600$ RELINQUISHED BY/REMOVED FROM - MAY OATE/THE MO-745 REF \#3 RELINQUISHED BY/REMOVED FROM 5 Doterime

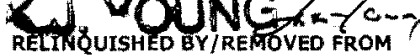

RELINQUISHED BY/REMOVED FROM

RELINQUISHED EY/REMOVED FROM

RELINOUISHED BY/REMOVED FROM

LABORATORY RECEIVED BY
SECTION
FINAL SAMPLE
DISPOSITION

SAMPLE DATE SAMPLE TIME

5-8.08.0845
SEE ITEM (D) IN MOisture
SPECTAL
INSTRUCTON 


\section{COLLECTOR}

NCO SAMPLER

\section{SAMPLING LOCATION}

C5856, I-053

ICE CHEST NO.

\section{SHIPPED TO}

Environmentai Sciences Laboratory

\section{COMPANY CONTACT}

TRENT, STEVE

TELEPHONE NO.

373-5869

\section{PROJECT DESIGNATION}

200-BP-5 OU Characterization for Wells 299-E33-341 and 299-E33-342-G FIELD LOGBOOK NO.

ACTUAL SAMPLE DEPTH

\section{OFFSITE PROPERTY NO.}

N/A
PROJECT COORDINATOR

WIDRIG, DL

SAF NO.

F08-075

123514 ES10

PRICE CODE

\section{AIR QUALITY}

$8 N$

DATA TURNAROUND

45 Days / 45

METHOD OF SHIPMENT GOVERNMENT VEHICLE

\section{SPECIAL INSTRUCTIONS}

** The 200 Area S\&GRP Characterization and Monitoring Sampling and Analysis GKI applies to this SAF.

** ESL is to determine unsaturated hydraulic conductivity using the method(s) best suited for the soil texture and type.

** ESL is to submit copies of all Chain of Custodies and associated sample login documentation to EIS-SDM within 24 hours of sample receipt. They may be sent via e-mail to ${ }^{\wedge}$ CPP Sample Management.

(1)6020M ICPMS_ASTM AE (TAL) \{Cadmium, Chromium, Silver\} 6020M ICPMS_ASTM_AE (Add-On) \{Arsenic, Lead, Molybdenum, Setenium\} 6020M METALS ICPMS WE (TAL) \{Cadmium, Chromium, Silver\}

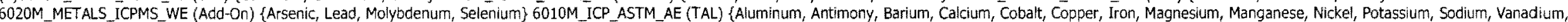

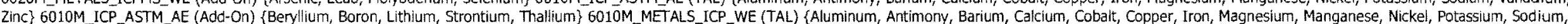

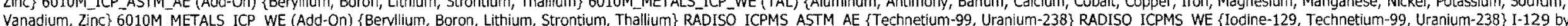

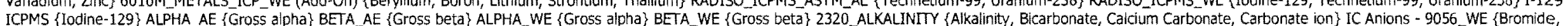
Chloride, Fluoride, Nitrate, Nitrite, Phosphate, Sulfate\} TOC - ASTME1915A \{Total Inorganic Carbon, Total carbon, Total organic carbon\} pH (Water) - 9045_WE; 


\section{COLLECTOR}

\section{nCo sampler PAILE'?}

\section{SAMPLING LOCATION}

C5856, $1-055$

ICE CHEST NO.

\section{SHIPPED TO}

Environmental Sciences Laboratory

MATRIX*
$A=$ Air
DL=Drum
Liguids
$D S=$ Drum
Solids
$L=$ Liquid
$O=$ Oi
$S=5$ oil
$S E=$ Sediment
$T=$ Tissue
$V=$ Vegitation
$W=$ Water
WI=Wipe
$X=$ Other

POSSIBLE SAMPLE HAZARDS/ REMARKS

Contains Radioactive Material at concentrations that are not regulated for transportation per 49

CFR but are not releasable per DOE Order

$5400.5(1990 / 1993)$

SPECIAL HANDLING AND/OR STORAGE Radioactive Tie TO BITPB1

\section{COMPANY CONTACT}

TRENT, STEVE TELEPHONE NO.

373-5869

\section{PROJECT DESIGNATION}

200-BP-5 OU Characterization for Wells 299-E33-341 and 299-E33-342-G FIELD LOGBOOK NO.

$H N F-N-488-1$ OFFSITE PROPERTY NO.

$N / A$

\begin{tabular}{|c|c|c|}
\hline PRESERVATION & None & None \\
\hline TYPE OF CONTAINER & G/P & $\begin{array}{l}\text { Moisture } \\
\text { Resistant Cont }\end{array}$ \\
\hline NO. OF CONTAINER(S) & ... & 1 \\
\hline volume & 11 & 2009 \\
\hline SAMPLE ANAI & $\begin{array}{l}\text { SEE TTEM (1) IN } \\
\text { SPECTAL INTONS } \\
\text { INSTRUCTONS }\end{array}$ & $\begin{array}{l}\text { Moistura } \\
\text { Content - } 02215\end{array}$ \\
\hline
\end{tabular}

PROJECT COORDINATOR WIDRIG, DL.

SAF NO.

F08-075

COA

123514 ES 10

BILL OF LADING/AIR BILL NO.

N/A
PRICE CODE $8 \mathrm{~N}$

AIR QUALITY []

METHOD OF SHIPMENT

GOVERNMENT VEHICLE

\section{DATA} TURNAROUND

45 Days / 45 Days
SAMPLE DATE SAMPLE TIME
$5-8-7 \quad 0910$
SAMPLE NO.
MATRIX
B1TP52
SOIL
CHAIN OF POSSESSION
RELINQUISHED BY/REMOVED FROM IKBAILEY IVBICK 5-8.8'/ RELINQUISHED BYKREMOVED FROM DATE/TIME Mo-745 Ruf 3 lo30/MAY P ? 2008 RELINQUISHED BY/REMOVED From RELINQUISHED BYIREMOVED FROM
RELINQUISHED BY/REMOVED FROM DATE/TIME
RELINQUISHED BY/REMOVED FROM DATE/TIME
RELINQUISHED BY/REMOVED FROM
DATE/TIME

\section{4}
SIGN/ PRINT NAMES
RECEIVED BY/STORED IN MOT45 RET \#3 $5 \times 8$ / 160 RECEIVERYSTRREIN / MAY Q9 $2008 / 1030$

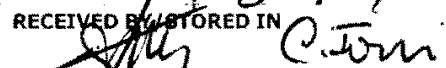 RECEIVG BYISTORED IN $5 / 9.99 \mathrm{OP} 1400$ . RECEIVED BY/STORED IN DATE/TIME RECEIVED BY/STORED IN DATE/TIME RECEIVED BY/STORED IN
DATE/TIME

\section{SPECIAL INSTRUCTIONS}

SEE PAGE 2 FOR ALL SPECIAL INSTRUCTIONS

LABORATORY RECEIVED BY

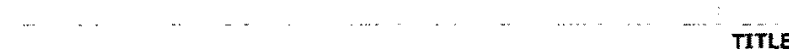

DATE/TIME 


\section{COLLECTOR}

NCO SAMPLER

SAMPLING LOCATION

C5856, I-055

ICE CHEST NO.

\section{SHIPPED TO}

Environmental Sciences Laboratory

\section{COMPANY CONTACT}

TRENT, STEVE

PROJECT DESIGNATION

TELEPHONE NO.

373-5869

200-BP-5 OU Characterization for Wels 299-E33-341 and 299-E33-342 - G

FIELD LOGBOOK NO.

ACTUAL SAMPLE DEPTH

\section{OFFSITE PROPERTY NO.}

PROJECT COORDINATOR

WIDRIG, $\mathrm{DL}$

SAF NO.

F08-075

$\operatorname{COA}$

$123514 \mathrm{ES} 10$

\section{PRICE CODE $\quad 8 N$}

AIR QUALITY

\section{METHOD OF SHIPMENT}

GOVERNMENT VEHICLE

\section{SPECIAL INSTRUCTIONS}

** The 200 Area S\&GRP Characterization and Monitoring Sampling and Analysis GKI applies to this SAF.

** ESL is to determine unsaturated hydraulic conductivity using the method(s) best suited for the soll texture and type.

** ESL is to subrnit copies of all Chain of Custodies and associated sample login documentation to EIS-SDM within 24 hours of sample receipt. They may be sent via e-mail to ^ CPP Sample Management.

(1)6020M ICPMS_ASTM AE (TAL) \{Cadmium, Chromium, Silver\} 6020M_ICPMS_ASTM_AE (Add-On) (Arsenic, Lead, Molybdenum, Selenium) 6020M METALS ICPMS WE (TAL) (Cadmium, Chromium, Silver)

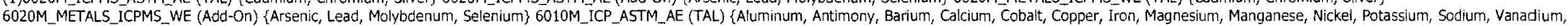

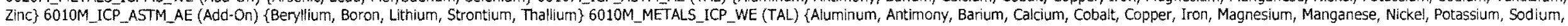

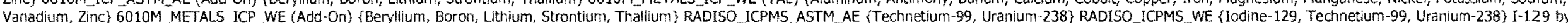

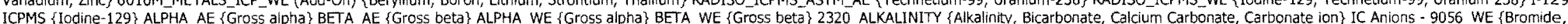
Chioride, Fluoride, Nitrate, Nitrite, Phosphate, Sulfate\} TOC-ASTME1915A (Total Inorganic Carbon, Total carbon, Total organic carbon\} pH (Water) - 9045 _WE; 


\section{COLLECTOR}

NCOSAMPLER BAILEY

SAMPLING LOCATION

C5856, $1-057$

ICE CHEST NO.

SHIPPED TO

Environmental Sciences Laboratory

MATRIX* POSSIBLE SAMPLE HAZARDS/ REMARKS

$A=$ Air $\quad$ Contains Radioactive Material at concentrations

$\mathrm{Ol}=\mathrm{Drum} \quad$ that are not regulated for transportation per 49

Liquids $\quad$ CFR but are not releasable per DOE Order

Solids $\quad 5400.5(1990 / 1993)$

$\mathrm{L}=\mathrm{Liquid}$
$\mathrm{O}=\mathrm{O}$ il

$5=5$ oil

SE=Sediment

$\mathrm{T}=$ Tissue

$V=$ Vegitation

$W=$ Water

Wi $=$ wipe

SPECIAL HANDLING AND/OR STORAGE Radioactive Tie To BITPB1

SAMPLE NO

MATRIX*

B1TP53

SOL

\section{COMPANY CONTACT}

TRENT, STEVE

TELEPHONE NO.

$373-5869$

PROJECT COORDINATOR

WIDRIG, DL

SAF NO.

PROJECT DESIGNATION

200-BP-5 OU Characterization for Wells 299-E33-341 and 299-E33-342-G F08-075

FIELD LOGBOOK NO.

HNF-N-488-1

OFFSITE PROPERTY NO.

N/A

\begin{tabular}{|c|c|c|}
\hline PRESERVATION & None & None \\
\hline TYPE OF CONTAINER & $G / P$ & $\begin{array}{l}\text { Moisture } \\
\text { Resistant Cont }\end{array}$ \\
\hline NO. OF CONTAINER(S) & 1 & 1 \\
\hline VOLUME & $1 i$ & 2009 \\
\hline SAMPLE ANALYYSIS & $\begin{array}{l}\text { SEE TTEM (I) IN } \\
\text { SPECAL } \\
\text { INSTRUCTIONS }\end{array}$ & $\begin{array}{l}\text { Moisture } \\
\text { Content - D22 }\end{array}$ \\
\hline
\end{tabular}

SAMPLE DATE SAMPLE TIME

$5-8-8$ 0955 V V

\section{4} SAMPLE DEPTH

COA

123514 ES10

BILL OF LADING/AIR BILL NO.

\section{CHAIN OF POSSESSION}

RELINQUISHED BY/REMOVED FROM JKBALL4/JKEZO2 $58-8$ DATE/TME 1600 RELINQUISHED \&Y/REMOVED MAY M O DATE/TIYIE MO-745 REF 3 MAY O $92008 / \mathrm{k} 30$ K. RELWOMSHER BWREMOVED FROM

RELINQUISHED BY TREMOVED FRÓM

RELINQUISHED BY/REMOVED FRÖM

RELINQUISHED BY/REMOVED FROM

RELINQUISHED BY/REMOVED FROM
DATE/TIME

1400

DATE/TIME

DATE/TIME

DATE/TIME

DATE/TIME
SIGN/ PRINT NAMES

RECEIVED BY/STORED IN

SPECIAL INSTRUCTIONS

WUTS REF $\# 3$ 5-8-8 1600

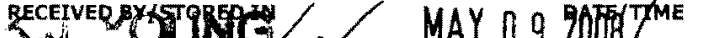

RECEIVED BY/STÓBEO IN

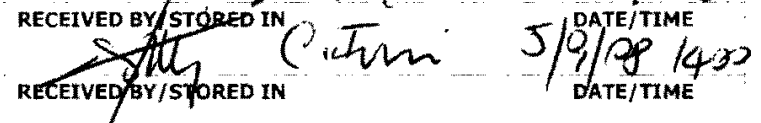

RECEIVED BY/STORED IN

RECEIVED BY/STORED IN

RECEIVED BY/STORED IN
DATE/TIME

DATE/TTME

DATE/TIME

LABORATORY
SECTION
FINAL SAMPLE
DISPOSITION




\section{COLLECTOR}

\section{NCO SAMPLER}

\section{COMPANY CONTACT}

TRENT, STEVE

\section{TELEPHONE NO.}

373-5869

PROJECT COORDINATOR

WIDRIG, DL

PRICE CODE

$8 N$

DATA

\section{PROJECT DESIGNATION}

\section{SAF NO.}

AIR QUALITY

TURNAROUND

200-BP-5 OU Characterization for Wells 299-E33-341 and 299-E33-342-G F08-075

FIELD LOGBOOK NO.

ACTUAL SAMPLE DEPTH

COA

123514 ES10

METHOD OF SHIPMENT

GOVERNMENT VEHICLE

OFFSITE PROPERTY NO.

N/A

\section{BILL OF LADING/AIR BILL NO}

N/A

\section{SPECIAL INSTRUCTIONS}

** The 200 Area S\&GRP Characterization and Monitoring Sampling and Analysis GKI applies to this SAF.

* ESL is to deternine unsaturated hydraulic conductivity using the method(s) best suited for the soil texture and type.

** ESL is to submit copies of all Chain of Custodies and associated sample login documentation to EIS-SDM within 24 hours of sample receipt. They may be sent via e-mail to ^CPP Sample Management.

(1)6020M_ICPMS_ASTM_AE (TAL) \{Cadmium, Chromium, Silver\} 6020M_ICPMS_ASTM_AE (Add-On) \{Arsenic, Lead, Molybdenum, Selenium\} 6020M_METALS_ICPMS WWE (TAL) \{Cadmium, Chromium, Silver\}

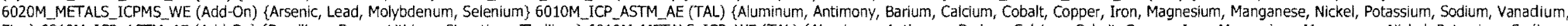

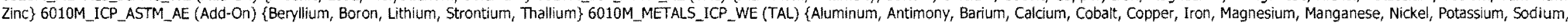

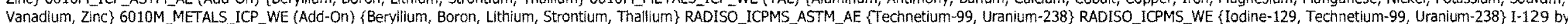

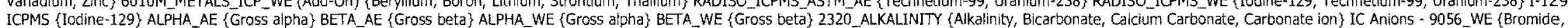

ICPMS \{lodine-129\} ALPHA_AE \{GrosS alpha\} BETA_AE \{Gross beta\} ALPHA_WE \{Gross alpha\} BETA_WE \{Gross beta\} 2320_ALKALINIT \{Alkalinity, BiCarbonat
Chloride, Fluoride, Nitrate, Nitrite, Phosphate, Sulfate\} TOC - ASTME1915A (Total Inorganic Carbon, Total carbon, Total organic carbon\} pH (Water) - 9045 WE; 


\section{COLLECTOR}

nco sampler Ballez

\section{SAMPLING LOCATION}

C5856, I-059

ICE CHEST NO.

\section{SHIPPED TO}

Environmental Sciences Laboratory

MATRIX* POSSIBLE SAMPLE HAZARDS/ REMARKS

$A=A i r \quad$ Contains Radioactive Material at concentrations

$\mathrm{DL}=\mathrm{Drum}$
Liquids

DS=Drum CFR but are not releasable per DOE Order

$\begin{array}{ll}\text { Solids } & 5400.5(1990 / 1993)\end{array}$

$\mathrm{L}=$ Liquid

$\mathrm{O}=\mathrm{Oil}$

$S=$ Soil
$S E=$ Sediment

$T=$ Tissue

$v=$ vegitation

W=Water

$\underset{x=0 \text { ther }}{w}$

SPECIAL HANDLING AND/OR STORAGE Radioactive Tie To B1TPB1

SAMPLE NO

MATRIX*

B1TP54

SOIL

\section{COMPANY CONTACT}

TRENT, STEVE

373-5869

PROJECT COORDINATOR

WIDRIG, DL

SAF NO.

PROJECT DESIGNATION

200-BP-5 OU Characterization for Wells 299-E33-341 and 299-E33-342-G F08-075

FIELD LOGBOOK NO.

ACTUAL SAMPLE DEPTH

HNF- $-45-48-1$

OFFSITE PROPERTY NO.

$112.5^{\prime}$

N/A

$\begin{array}{ccc}\text { PRESERVATION } & \text { None } & \text { None } \\ \text { TYPE OF CONTAINER } & \text { G/p } & \begin{array}{c}\text { Moisture } \\ \text { Resistant Cont }\end{array} \\ \text { NO. OF CONTAINER(S) } & 1 & 1 \\ \text { VOLUME } & 11 & 2009 \\ \text { SAMPLE ANALYSIS } & \begin{array}{l}\text { SEE ITEM (D) IN Mosture } \\ \text { SPELIA } \\ \text { INSTRUCTIONS }\end{array}\end{array}$

SAMPLE DATE SAMPLE TIME

$5 \cdot 8-8,1115$

\section{$\mathrm{COA}$}

$123514 \mathrm{ES} 10$

\section{ILL OF LADING/AIR BILL NO.}

PRICE CODE $\quad$ 8N

AIR QUALITY

\section{METHOD OF SHIPMENT}

GOVERNMENT VEHICLE

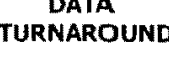

45 Days / 45

Days
LOT NO.

CHAIN OF POSSESSION

RELINQUISHED BY/REMOVED FROM DATE/TIME JK EAILU/ 4 CE:C O 5.8 .81600 MO-74S REP \#3

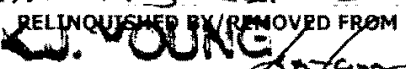

RELINQUISHED BY/REMOVED FROM

RELINQUISHED BY/REMOVED FROM 56108 DATE/TIME DATE/TIME

\section{RELINQUISHED BY/REMOVED FROM}

DATE/TIME

DATE/TIME

RELINQUISHED BY/REMOVED FROM

DATE/TIME

\section{4}

SIGN/ PRINT NAMES

RECEIVED BY/STORED IN

\section{SPECIAL INSTRUCTIONS}

RECEVERAYSTSORESE

RECEIVED a Sayor DAY P Q $2000 / 1030$

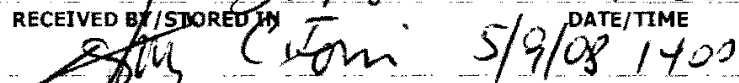

RECEIVEP BY/ŞTORED IN

DATE/TIME

RECEKUED BY/STORED IN

DATE/TIME

RECEIVED BY/STORED IN

DATE/TIME

DATE/TIME

RECEIVED QY/STORED IN

\footnotetext{
LABORATORY RECEIVED BY

SECTION 


\section{CHAIN OF CUSTODY/SAMPLE ANALYSIS REOUEST}

\section{TELEPHONE NO.}

$373-5869$

TRENT, STEVE

PROJECT DESIGNATION

200-BP-5 OU Characterization for Wells 299-E33-341 and 299-E33-342-G

FIELD LOGBOOK NO. ACTUAL SAMPLE DEPTH

\section{OFFSITE PROPERTY NO}

N/A
F08-075-060

PAGE 2 OF 2

DATA TURNAROUND

45 Days / 45

Days

\section{SHIPPED TO}

Environmental Sciences Laboratory

\section{SPECIAL INSTRUCTIONS}

** The 200 Area S\&GRP Characterization and Monitoring Sampling and Analysis GKI applies to this SAF.

** ESL is to determine unsaturated hydraulic conductivity using the method(s) best suited for the soil texture and type.

** ESL is to submit copies of all Chain of Custodies and associated sample login documentation to EIS-SDM within 24 hours of sample receipt. They may be sent via e-mail to ^CPP Sample Management.

(1)6020M ICPMS ASTM AE (TAL) \{Cadmium, Chromium, Silver\} 6020M ICPMS ASTM AE (Add-On) (Arsenic, Lead, Molybdenum, Selenium\} 6020M METALS ICPMS WE (TAL) \{Cadmium, Chromium, Siver\}

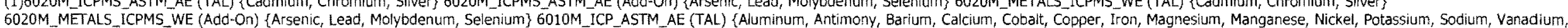

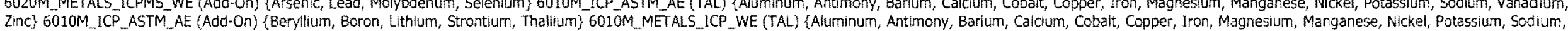

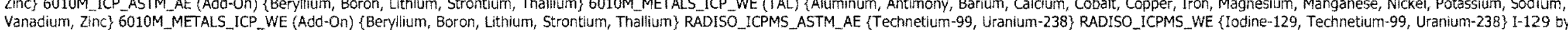

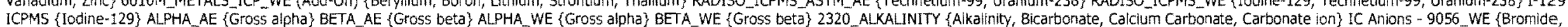
Chloride, Fluoride, Nitrate, Nitrite, Phosphate, Sulfate\} TOC - ASTME1915A (Total Inorganic Carbon, Total carbon, Total organic carbon) pH (Water) - 9045 _.WE; 


\section{COLLECTOR}

nco sampler Ballezy

\section{SAMPLING LOCATION}

C5856, 1-061

ICE CHEST NO.

\section{SHIPPED TO}

Environmentâl Sciences Laboratory

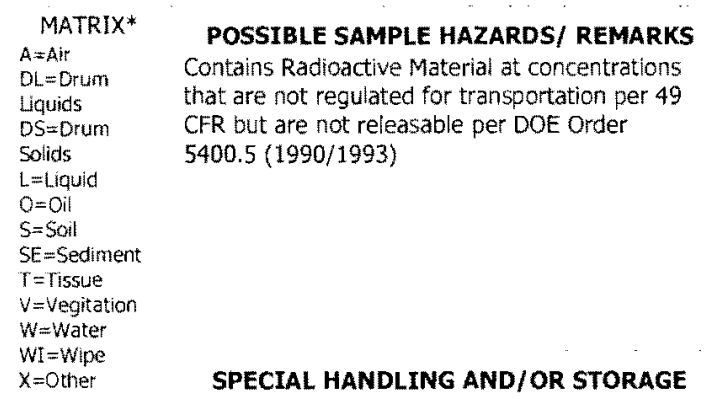
Radioactive Tie To B1TPB2

\section{COMPANY CONTACT}

TRENT, STEVE

TELEPHONE NO.

373-5869
PROJECT COORDINATOR

WIDRIG, DL

\section{PROJECT DESIGNATION}

200-BP-5 OU Characterization for Wells 299-E33-341 and 299-E33-342-G F08-075

FIELD LOGBOOK NO.

ACTUAL SAMPLE DEPTH

$$
\text { HNF-N-488-1 }
$$

OFFSITE PROPERTY NO.

$$
1151
$$

$N / A$

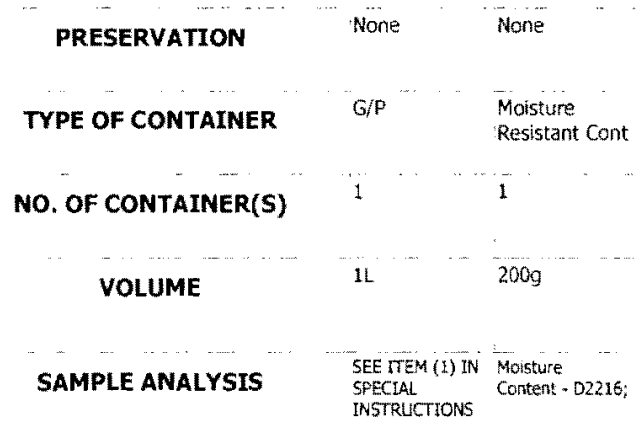

SAMPLE DATE SAMPLE TIME

$5-8-8 \quad 1400$

\section{COA}

123514ES10
PRICE CODE TURNAROUND

45 Days / 45

Days

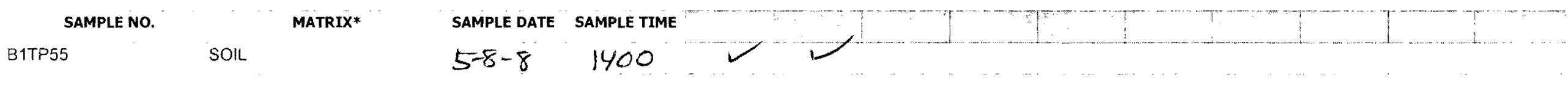

$\operatorname{Lot} N D$

CHAIN OF POSSESSION

RELINOUISHED BY/REMOVED FROM 5.CBALEA KJEE $6358-81600$ RELINOUISHED BY/REMOVED FROM DATE/TTME MO-74S Ref\$3 RELINQUISHED BY/REMOVED FROA

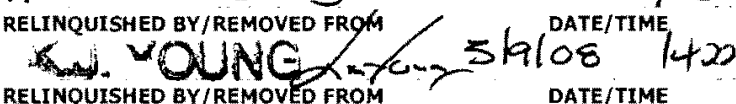

RELINQUISHED EY/REMOVED FROM

RELINQUISHED BY/REMOVED FROM

RELINQUISHED BY/REMOVED FROM ATE/TIME

DATE/TIME

DATE/TIME

DATE/TIME

\section{4}

\section{SIGN/ PRINT NAMES}

\section{SPECIAL INSTRUCTIONS}

RECEIVED BY/STORED IN

WOTL REF *3 5-8-8 l600

\section{LABORATORY RECEIVED BY}

SECTION

FINAL SAMPLE DISPOSAL METHOD

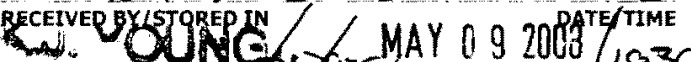

RECEIVED BYISTORED IN

OF LADING/AIR BILL NO.

\section{METHOD OF SHIPMENT}

GOVERNMENT VEHICLE

$\mathrm{N} / \mathrm{A}$

$\cdots$




\section{COLLECTOR}

TELEPHONE NO.

PROJECT COORDINATOR

COMPANY CONTACT

TRENT, STEVE

373-5869

PRICE CODE

BN

DATA

PROJECT DESIGNATION

SAF NO.

AIR QUALITY

OUND

200-BP-5 OU Characterization for Wells 299-E33-341 and 299-E33-342-G

C5856, I-061

FIELD LOGBOOK NO.

ACTUAL SAMPLE DEPTH

COA

Days

OFFSITE PROPERTY NO.

N/A

123514ES10

METHOD OF SHIPMENT

GOVERNMENT VEHICLE

BILL OF LADING/AIR BILI, NO

$\mathrm{N} / \mathrm{A}$

Environmental Sciences Laboratory

\section{SPECIAL INSTRUCTIONS}

** The 200 Area S\&GKP Characterization and Monitoring Sampling and Analysis GKI applies to this SAF.

** ESL is to determine unsaturated hydraulic conductivity using the method(s) best suited for the soll texture and type.

** ESL is to submit copies of all Chain of Custodies and associated sample login documentation to EIS-SDM within 24 hours of sample receipt. They may be sent via e-mail to ACPP Sample Management.

(1)6020M_ICPMS_ASTM_AE (TAL) \{Cadmium, Chromium, Silver\} 6020M_ICPMS_ASTM_AE (Add-On) \{Arsenic, Lead, Molybdenum, Selenium\}6020M_METALS_ICPMS_WE (TAL) \{Cadmium, Chromium, Silver\}

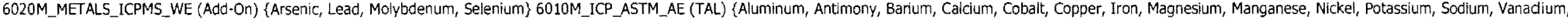

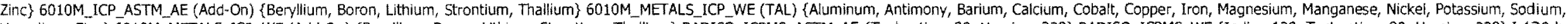

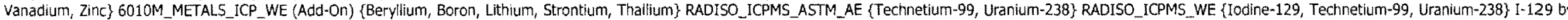

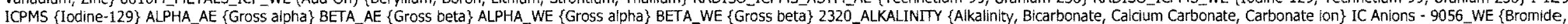

Chloride, Fluoride, Nitrate, Nitrite, Phosphate, Sulfate) TOC - ASTME1915A (Total Inorganic Carbon, Total carbon, Total organic carbon\} pH (Water) - 9045 WE; 


\section{COLLCTOR}

NCO SAMPLER BAILE'

SAMPLING LOCATION

C5856, 1-063

ICE CHEST NO.

\section{SHIPPED TO}

Environmenta! Sciences Laboratory

\begin{tabular}{|c|c|}
\hline \multicolumn{2}{|r|}{ OSSIBLE SAMPLE HAZARDS/ REMARKS } \\
\hline$A=$ Air & Contains Radioactive Material at concentrations \\
\hline $\begin{array}{l}\text { DL=Drum } \\
\text { Liquids }\end{array}$ & that are not regulated for transportation per 49 \\
\hline$D S=$ Drum & CFR but are not releasable per DOE Order \\
\hline $\begin{array}{l}\text { Sollds } \\
L=\text { Liguid }\end{array}$ & $5400.5(1990 / 1993)$ \\
\hline $0=0$ il & \\
\hline $\begin{array}{l}5=50 i l \\
S E=\text { Sediment }\end{array}$ & \\
\hline $\begin{array}{l}T=\text { Tissue } \\
V=\text { Vegitation }\end{array}$ & \\
\hline$W=$ Water & \\
\hline$W I=W i p e$ & \\
\hline$x=$ other & $\begin{array}{l}\text { SPECIAL HANDLING AND/OR STORAGE } \\
\text { Radioactive Tie To B1TPB2 }\end{array}$ \\
\hline
\end{tabular}

\section{COMPANY CONTACT}

TRENT, STEVE

TELEPHONE NO.

373-5869

PROJECT COORDINATOR

WIDRIG, DL

PROJECT DESIGNATION

SAF NO.

200-BP-5 OU Characterization for Wells 299-E33-341 and 299-E33-342-G FIELD LOGBOOK NO. ACTUAL SAMPLE DEPTH

FO8-075

HNE-N-488-1 $117.5^{\prime}$ OFFSITE PROPERTY NO.

\section{COA}

123514 ES10

BILL OF LADING/AIR BILL NO

N/A

\begin{tabular}{ccc} 
PRESERVATION & None & None \\
TYPE OF CONTAINER & G/P & $\begin{array}{l}\text { Moisture } \\
\text { Resistant Cont }\end{array}$ \\
\hline NO. OF CONTAINER(S) & 1 & 1 \\
\hline
\end{tabular}

NO. OF CONTAINER(S) 1

VOLUME

$\begin{array}{ll}\text { SAMPLE ANALYSIS } & \text { SEE TIEM (1) IN Moisture } \\ \text { SPEIAL } & \text { Content - D2216; } \\ \text { INSTRKTIONS }\end{array}$

SAMPLE DATE SAMPLE TIME $5-8-8 \quad 1425$

$\begin{array}{ll}\text { SAMPLE NO. } & \text { MATRIX* } \\ \text { B1TP56 } & \text { SOIL }\end{array}$

PRICE CODE

8N

DATA TURNAROUND

45 Days / 45 


\section{COLLECTOR}

NCO SAMPLER

\section{SAMPLING LOCATION}

C5856, $\mathrm{I}-063$

ICE CHEST NO.

\section{SHIPPED TO}

Environmental Sciences Laboratory

\section{COMPANY CONTACT}

TRENT, STEVE

\section{PROJECT DESIGNATION}

200-BP-5 OU Characterization for Wells 299-E33-341 and 299-E33-342-G FIELD LOGBOOK NO.

ACTUAL SAMPLE DEPTH

\section{OFFSITE PROPERTY NO}

N/A
PROJECT COORDINATOR

WIDRIG, DL

SAF NO.

FO8-075

COA

$123514 E S 10$
PRICE CODE $\quad 8 \mathrm{~N}$

\section{AIR QUALITY}

METHOD OF SHIPMENT GOVERNMENT VEHICLE

\section{SPECIAL INSTRUCTIONS}

** The 200 Area S\&GRP Characterization and Monitoring Sampling and Analysis GKI applies to this SAF.

* ESL is to determine unsaturated hydraulic conductivity using the method(s) best suited for the soil texture and type.

** ESL is to submit copies of all Chain of Custodies and associated sample login documentation to EIS-SDM within 24 hours of sample receipt. They may be sent via e-mall to ^CPP Sample Management.

(1)6020M ICPMS ASTM AE (TAL) \{Cadmium, Chromium, Silver\} 6020M ICPMS ASTM AE (Add-On) \{Arsenic, Lead, Molybdenum, Selenium) 6020M METALS ICPMS WE (TAL) \{Cadmium, Chromium, Silver\}

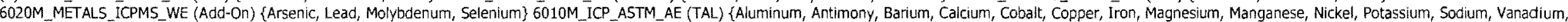

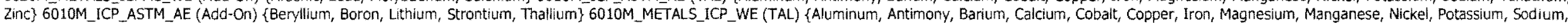

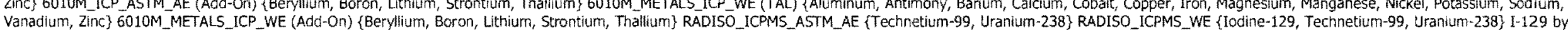

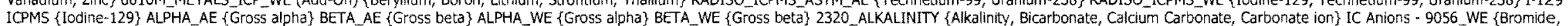

Chloride, Fluoride, Nitrate, Nitrite, Phosphate, Sulfate\} TOC - ASTME1915A (Total Inorganic Carbon, Total carbon, Totai organic carbon\} pH (Water) - 9045 -WE; 


\section{COLLECTOR}

nCO SAMPLER BEILEZ

\section{SAMPLING LOCATION}

C5856, I-D64

ICE CHEST NO.

\section{SHIPPED TO}

Environmental Sciences Laboratory

\section{MATRIX* POSSIBLE SAMPLE HAZARDS/ REMARKS $A=A i r \quad$ Contains Radioactive Material at concentrations $O L=D r u m \quad$ that are not regulated for transportation per 49 LS Lidds $\quad$ CFR but are not releasable per DOE Order \\ Solids $\quad 5400.5(1990 / 1993)$ \\ $L=$ Lquid
$\mathrm{O}=\mathrm{O}$ \\ $5=5$ oil \\ $\mathrm{SE}=$ Sediment \\ $T=$ Tissue \\ $V=$ Vegitatio \\ Wh $=$ Wipe \\ SPECIAL HANDLING AND/OR STORAGE Radioactive TIE TO BITPB2}

MATRIX*

B1TP57

SOIL

\section{CHAIN OF POSSESSION}

RELINOUISHED BY/REMOVED FROM 0 S. DATE/TIME RELINQUISHED BY/REMOVED FRQM DATE/TIME M0.745 REF E $5-14.0$ R.PFISTR BY/REMOVED EROM RELINQUISHED BY/REMOVED FROM

RELINQUISHED BY/REMOVED FROM

RELINQUISHED BY/REMOVED FROM

RELINQUISHED BY/REMOVED FROM $14.0 \mathrm{~s}$ i) 30 DATE/TMME 1145 DATE/TIME DATE/TIME DATE/TIME DATE/TIME

\section{COMPANY CONTACT}

TRENT, STEVE

TELEPHONE NO.

$373-5869$

PRÖJECT COORDINATOR

WIDRIG, DL

SAF NO.

F08-07

200-BP-5 OU Characterization for Wells 299-E33-341 and 299-E33-342 FIELD LOGBOOK NO. ACTUAL SAMPLE DEPTH

$$
\text { HNF-N - } 488-1
$$$$
120^{\prime}
$$

\section{$\mathrm{COA}$}

123514ES10

BILL OF LADING/AIR BILL NO.

N/A

N/A

$\begin{array}{lll}\text { PRESERVATION } & \text { None } & \text { None } \\ \text { TYPE OF CONTAINER } & \text { G/P } & \begin{array}{l}\text { Moisture } \\ \text { Reistant Cont }\end{array} \\ \end{array}$

NO. OF CONTAINER(S) 1 i

VOLUME

11.2009

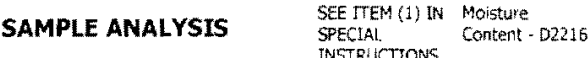

\section{$\begin{array}{lll}\text { PRICE CODE } & 8 \mathrm{~N} & \text { DATA } \\ \text { TURNAROUND }\end{array}$ \\ 45 Days / 45 \\ Days}

SAMPLE DATE SAMPLE TIME

$5-9-8 \quad 0750$

LABORATORY RECEIVED BY
SECTION
FINAL SAMPLE
DISPOSITION

SPECIAL INSTRUCTIONS

RECEIVED BY/STORED IN

MOTHS REF \#, 5-9-8 1500

R.pFrspe/tis/lo 5.14 .081130 RECETYEQ BYSTORED IN RECEI/ED BY/STORED IN RECEIVED BY/STORED IN - DATE/TIME RECEIVED BY/STORED IN DATE/TIME RECEIVED BY/STORED IN

\section{SEE PAGE 2 FOR ALL SPECIAL INSTRUCTIONS}




\section{COLLECTOR}

NCO SAMPLER

\section{SAMPLING LOCATION}

C5856, I-064

ICE CHEST NO.

\section{SHIPPED TO}

Environmental Sciences Laboratory

\section{COMPANY CONTACT}

TRENT, STEVE

\section{PROJECT DESIGNATION}

TELEPHONE NO.

373-5869

200-BP-5 OU Characterizat

FIELD LOGBOOK NO.

OFFSITE PROPERTY NO.

N/A
PRICE CODE $8 \mathrm{~N}$ WIDRIG, DL

\section{SAF NO.}

FO8-075

$\operatorname{COA}$

123514 ES10
AIR QUALITY
METHOD OF SHIPMENT

GOVERNMENT VEHICLE
DATA

TURNAROUND

\section{SPECIAL INSTRUCTIONS}

** The 200 Area S\&GRP Characterization and Monitoring Sampling and Anaiysis GKI applies to this SAF.

** ESL is to determine unsaturated hydraulic conductivity using the method(s) best sulted for the soll texture and type.

** ESL is to submit copies of all Chain of Custodies and associated sample login documentation to EIS-SDM within 24 hours of sample recelpt. They may be sent via e-mail to ^CPP Sample Management.

(1)6020M_ICPMS_ASTM_AE (TAL) \{Cadmium, Chromium, Silver\} 6020M_ICPMS_ASTM_AE (Add-On) \{Arsenic, Lead, Molybdenum, Selenium\} 6020M_METALS_ICPMS WE (TAL) \{Cadmiurn, Chromium, Silver\}

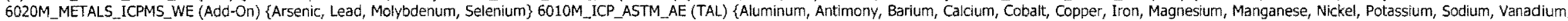

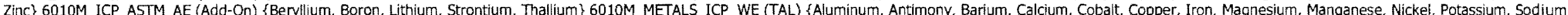
(TAL) SAlT

Chloride, Fiuoride, Nitrate, Nitrite, Phosphate, Sulfate\} TOC.ASTME1915A \{Total Inorganic Carbon, Total carbon, Total organic carbon\} pH (Water) - 9045 _WE; 


\section{COLLCTOR}

NCO SAMPLER BALLZ

SAMPLING LOCATION

C5856, I-065

ICE CHEST NO

\section{SHIPPED TO}

Environmental Sciences Laboratory

\begin{tabular}{|c|c|}
\hline$A=A i r$ & POSSIBLE SAMPLE HAZARDS/ REMARKS \\
\hline $\mathrm{DL}=$ Drum & Contains Radioactive Material at concentrations \\
\hline Liquids & that are not regulated for transportation per 49 \\
\hline & CFR but are not releasable per DOE Order \\
\hline $\begin{array}{l}\text { Solids } \\
L=\text { Liquid }\end{array}$ & $5400.5(1990 / 1993)$ \\
\hline $0=$ oil & \\
\hline$S E=S e d i m e n$ & \\
\hline $\begin{array}{l}T=T \text { issue } \\
V=\text { Vegitatio }\end{array}$ & \\
\hline Water & \\
\hline$x=$ other & SPECTAL HANDI TNG ANDIOD STOPAGE \\
\hline
\end{tabular}
Radicactive Tie TO B1TPB2

SAMPLE NO. MATRIX*

B1TP58 SOIL

\section{COMPANY CONTACT}

TRENT, STEVE

\section{TELEPHONE NO.}

373-5869

\section{PROJECT DESIGNATION}

200-8P-5 OU Characterization FIELD LOGBOOK NO.

HNF-NJ- $48 \%$

OFFSITE PROPERTY NO.

$\mathrm{N} / \mathrm{A}$

\begin{tabular}{|c|c|c|}
\hline PRESERVATION & None & None \\
\hline TYPE OF CONTAINER & $\bar{G} / \mathrm{P}$ & $\begin{array}{l}\text { Moisture } \\
\text { Resistant Cont }\end{array}$ \\
\hline No. OF CONTAINER(S) & 1 & $1 \ldots$ \\
\hline VOLUME & $1 \mathrm{~L}$ & 2009 \\
\hline SAMPLE ANALYSIS & $\begin{array}{l}\text { SEE TEM (1) IN } \\
\text { SPECIAL } \\
\text { INSTRUCTONS }\end{array}$ & $\begin{array}{l}\text { Moisture } \\
\text { Contert - D22 }\end{array}$ \\
\hline
\end{tabular}

SAMPLE DATE SAMPLE TIME

$5-9-8 \quad 0755$

\section{PROJECT COORDINATOR}

WIDRIG, DL

SAF NO.
F08 075

COA

123514 ES10

BILL OF LADING/AIR BILL NO

N/A
METHOD OF SHIPMENT

GOVERNMENT VEHICLF

\section{$3) 394$}

\section{SIGN/ PRINT NAMES}

RECEIVED BY/STORED IN

RELINQUISHED BY/REMOVED FROM

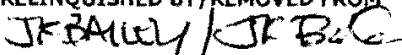
RELINOUISHED GYIREMOVED FROM $11074 \leq R\left(-F^{*}\right)$ $5 \cdot 14 \cdot 0$ RELINQUISHED BY/REMOVED FROM R. $P F i s+R / P=14.08$ RELINQUTSHED BY/REMOKED FROM

RELINQUISHED BY/REMOVED FROM

RELINQUISHED BY/REMOVED FROM

RELINQUISHED BY/REMOVED FROM

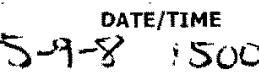

11.45 DATE/TIME

DATE/TIME

DATE/TIME

DATE/TIME
MOTU5 REF 1 5-9-8 DATE/TIME

RECEIVED BY/STORED IN

R.PFists /RE 5.14 .08 1130

RECEIYED BYSSTORED IN 2 DATE/TIME

RECEYUED BN/STORED IN

RECEIVEO BY/STORED IN DATE/TIME

RECEIVED BY/STORED IN

DATE/TIME

RECEIVED BY/STORED IN

\section{SPECIAL INSTRUCTIONS}

SEE PAGE 2 FOR ALL SPECIAL INSTRUCTIONS

LABORATORY RECEIVED BY
SECTION
FINAL SAMPLE
DISPOSITION




\section{COLLECTOR}

NCO SAMPLER

\section{SAMPLING LOCATION}

C5856, $1-065$

ICE CHEST NO.

\section{SHIPPED TO}

Environmental Sciences Laboratory

\section{COMPANY CONTACT}

TRENT, STEVE

\section{TELEPHONE NO.}

$373-5869$

\section{PROJECT DESIGNATION}

200-BP-5 OU Characte

ACTUAL SAMPLE DEPTH

\section{OFFSITE PROPERTY NO.}

N/A
PRICE CODE

WIDRIG, DL

SAF NO.

FO8-

123514ES 10
AIR QUALITY

METHOD OF SHIPMENT GOVERNMENT VEHICLE

\section{SPECIAL INSTRUCTIONS}

** The 200 Area S\&GRP Characterization and Monitoring Sampling and Analysis GKI applies to this SAF.

* ESL is to determine unsaturated hydraulic conductivity using the method(s) best suited for the soil texture and type.

** ESL is to submit coples of all Chain of Custodies and associated sample login documentation to EIS-SDM within 24 hours of sample receipt. They may be sent via e-mail to ^CPP Sample Management.

(1)6020M ICPMS ASTM AE (TAL) (Cadmium, Chromium, Silver\} 6020M ICPMS_ASTM_AE (Add-On) \{Arsenic, Lead, Molybdenum, Selenium) 6020M METALS_ICPMS WE (TAL) fCadmium, Chromlum, Siver\}

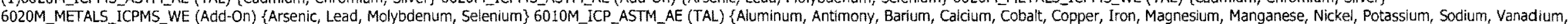

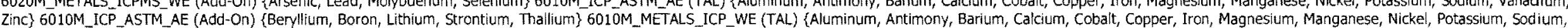

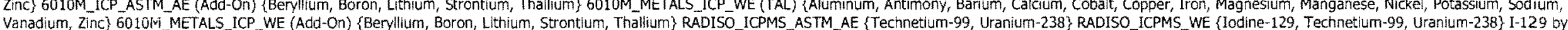

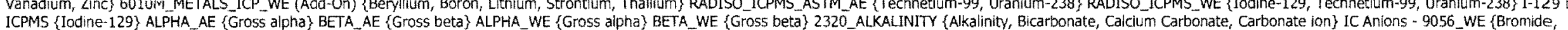
Chloride, Fluoride, Nitrate, Nitrite, Phosphate, Sulfate\} TOC - ASTME1915A \{Total Inorganic Carbon, Total carbon, Total organic carbon\} pH (Water)-9045_WE; 


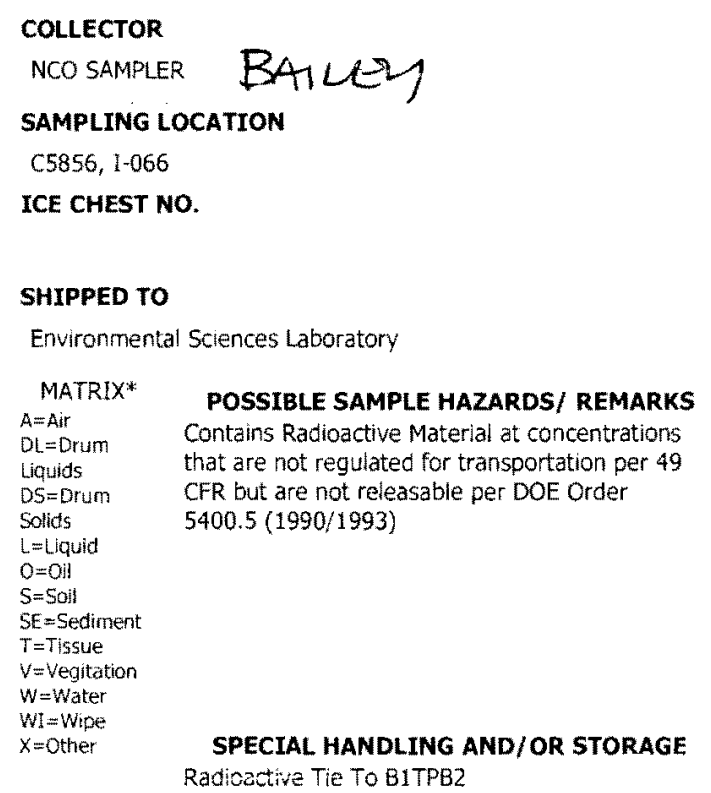

SAMPLE NO. "MATRIX

B1TP59 SOIL

\section{CHAIN OF POSSESSION}

RELINQUISHED BY/REMOVED FROM

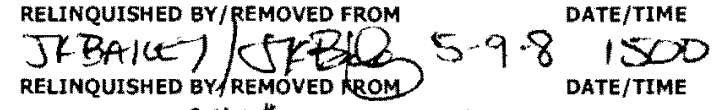
MO74SREF" S.14.0\% RELINQUISHED BY/REMOVED FROM

RELINQUISHED BY/REMOVED FROM 5.14 .08 DATE/TIME RELINQUISHED GY/REMOYED FROM

RELINQUISHED BY/REMOVED FROM

RELINQUISHED BY/REMOVED FROM

RELINQUISHED BY/REMOVED FROM

RECEIVED BY

\section{LABORATORY} SECTION

FINAL SAMPLE DISPOSAL METHOD DISPOSITION
DATE/TIME

DATE/TIME

DATE/TIME

DATE/TIME

\section{COMPANY CONTACT}

TRENT, STEVE

TELEPHONE NO.

373-5869

PROJECT COORDINATOR

WIDRIG, DL

\section{SAF NO.}

200-BP-5 OU Characterization for Wells 299-E33-341 and 299-E33-342-G F08-075 FIELD LOGBOOK NO.

ACTUAL SAMPLE DEPTH

\section{COA}

HNF $-N-488-1$ 1251

$123514 E S 10$

BILL OF LADING/AIR BILL NO.

SITE PROPERTY NO

N/A

\begin{tabular}{|c|c|c|}
\hline PRESERVATION & None & None \\
\hline TYPE OF CONTAINER & $G / P$ & $\begin{array}{l}\text { Moisture } \\
\text { Resistant Cont }\end{array}$ \\
\hline NO. OF CONTAINER(S) & 1 & 1 \\
\hline VOLUME & $1 \mathrm{~L}$ & 2009 \\
\hline SAMPLE ANAL & $\begin{array}{l}\text { SEE TREM (1) IN } \\
\text { SPECAL. } \\
\text { INSTRUCTIONS }\end{array}$ & $\begin{array}{l}\text { Mousture } \\
\text { Content - 02216; }\end{array}$ \\
\hline
\end{tabular}

SAMPLE DATE SAMPLE TIME

$5-1-8 \quad 0830$

\section{4}

\section{SIGN/ PRINT NAMES}

RECEIVED BY/STORED IN DATE/TIME RECEIVED BY/STIRED IN DATE/TIME R.PFISTR /2DOP 5.14 .08 1130 RECEIVED BY/STORED IN C.Tom SATh $5 / 141081145$ RECEIVED BY/STOREDIN DATE/TIME RECEIVED BY/STORED IN RECEIVED BY/STORED IN DATE/TIME RECEIVED BY/STORED IN

DATE/TIME

\section{SPECIAL INSTRUCTIONS}

SEE PAGE 2 FOR ALL SPECIAL INSTRUCTIONS

\section{PRICE CODE}

8N

AIR QUALITY

METHOD OF SHIPMENT

GOVERNMENT VEHICLE 


\section{COLLECTOR}

NCO SAMPLER

\section{SAMPLING LOCATION}

C5856, I-066

ICE CHEST NO.

\section{SHIPPED TO}

Environmental Sciences Laboratory

\section{COMPANY CONTACT}

TRENT, STEVE

\section{PROJECT DESIGNATION}

\section{TELEPHONE NO.}

373-5869

FIELD LOGBOOK NO.

\section{OFFSITE PROPERTY NO}

N/A
PROJECT COORDINATOR

WIDRIG, DL

\section{SAF NO.}

F08-075

COA
PRICE CODE

ATR QUALITY

$8 N$

DATA TURNAROUND

45 Days / 45

\section{SPECIAL INSTRUCTIONS}

** The 200 Area S\&GRP Characterization and Monitoring Sampling and Analysis GKI applies to this SAF.

* ESL is to determine unsaturated hydraulic conductivity using the method(s) best suited for the soil texture and type.

** ESL is to submit copies of all Chain of Custodies and associated sample login documentation to EIS-SDM within 24 hours of sample receipt. They may be sent via e-mail to ACPP Sample Management.

(1)6020M_ICPMS_ASTM_AE (TAL) \{Cadmium, Chromium, Silver\} 6020M_ICPMS_ASTM_AE (Add-On) \{Arsenic, Lead, Molybdenum, Selenium\} 6020M_METALS_ICPMS_WE (TAL) \{Cadmium, Chromium, Silver\}

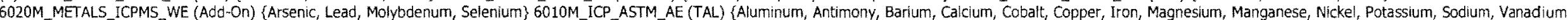

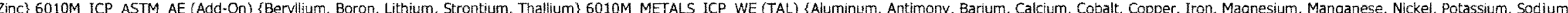

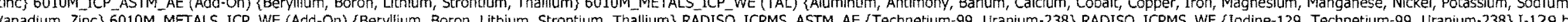

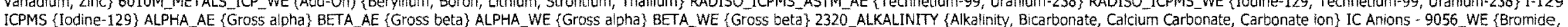

Chloride, Fluoride, Nitrate, Nitrite, Phosphate, Sulfate\} TOC - ASTME1915A \{Total Inorganic Carbon, Total carbon, Total organic carbon\} pH (Water) - 9045 WE: 


\section{COLLECTOR}

NCO SAMPLER BAILES

SAMPLING LOCATION

C5856, I-067

ICE CHEST NO.

\section{SHIPPED TO}

Environmental Sciences Laboratory

\section{MATRIX* POSSIBLE SAMPLE HAZARDS/ REMARKS $A=A i r \quad$ Contains Radioactive Material at concentrations DL-Dnum that are not regulated for transportation per 49 Liquids that are not regulated for transportation per 49 $\begin{array}{ll}\text { Solios } & 5400.5(1990 / 1993)\end{array}$ \\ $l=$ Liquid
$0=0 i$ \\ $0=0$ il
$S=5$ oil
$S$ \\ $S E=$ Sediment \\ $T=$ Tissue \\ $V=$ vegitatio \\ $x=$ water \\ whather \\ SPECIAL HANDLING AND/OR STORAGE} Radioactive Tie TO B1TPB?
SAMPLE NO

B1TP60
MATRIX*

SOIL

\section{CHAIN OF POSSESSION}

RELINQUISHED BY/REMOVED FROM RELINQUISHED of / REMOVED FROM 110745 REF' $5-14-08.1130$ RELINQUISHED BY/REMOVED FROM DATE/TIME

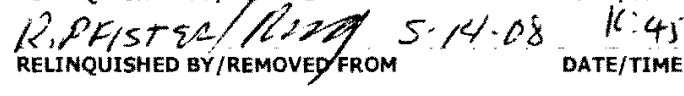

RELINQUISHED BV/REMOVED FROM

DATE/TIME

RELINQUISHED BY/REMOVED FROM

DATE/TIME

RELINQUISHED BY/REMOVED FROM

DATE/TIME

\section{COMPANY CONTACT}

TEIEPHONE NO.

373-5869

PROJECT COORDINATOR

WIDRIG, DL

SAF NO.

PROJECT DESIGNATION

200-BP-5 OU Characterization for Wells 299-E33-341 and 299-E33-342 - G FIELD LOGBOOK NO.

ACTUAL SAMPLE DEPTH

HNF-N-488-1 127.5

OFFSITE PROPERTY NO.

$\mathrm{N} / \mathrm{A}$

COA

$123514 E S 10$

BILL OF LADING/AIR BILL NO.

N/A

$\begin{array}{ccc}\text { PRESERVATION } & \text { None } & \text { None } \\ \text { TYPE OF CONTAINER } & \text { G/P } & \begin{array}{c}\text { Moisture } \\ \text { Resistant Cont }\end{array} \\ \text { NO. OF CONTAINER(S) } & 1 & 1 \\ \text { VOLUME } & 11 & 2009 \\ \text { SAMPLE ANALYSIS } & \begin{array}{l}\text { SEE TEEM (1) IN Masture } \\ \text { SPECAL } \\ \text { INGTRUCTONS }\end{array}\end{array}$

SAMPLE DATE SAMPLE TIME

$5-9-8 \quad 0840$

\section{4}

\section{SIGN/ PRINT NAMES}

RECEIVED QY/STORED IN

DATE/TIME

RECEIVED BYITORED \# $5-9-8$ 1500

C.PFIsTCR/LEP 5.14 .08 il30 RECEIVED BY/STORED IN

C.Ion: RECEIVED BY/STOREO IN $7 / 4$ J/14/08 //45

RECEIVED BY/STORED IN

DATE/TIME

RECEIVED BY/STORED IN

DATE/TIME

RECEIVED BY/STORED IN

\section{SPECIAL INSTRUCTIONS}

SEE PAGE 2 FOR AL SPECIAL INSTRUCTIONS

LABORATORY RECEIVED BY SECTION

FINAL SAMPLE DISPOSAL METHOD DISPOSITION 


\section{COLLECTOR}

NCO SAMPLER

SAMPLING LOCATION

C5856, I-067

ICE CHEST NO.

SHIPPED TO

Environmental Sciences Laboratory

\section{SPECIAL INSTRUCTIONS}

** The 200 Area S\&GRP Characterization and Monitoring Sampling and Analysis GKI applies to this SAF.

** ESL is to determine unsaturated hydraulic conductivity using the method(s) best suited for the soil texture and type.

** ESL is to submit copies of all Chain of Custodies and associated sample login documentation to EIS-SDM within 24 hours of sample receipt. They may be sent via e-mail to $\wedge$ CPP Sample Management.

(1)6020M_ICPMS_ASTM_AE (TAL) \{Cadmium, Chromium, Silver\} 6020M_ICPMS_ASTM_AE (Add-On) \{Arsenic, Lead, Molvbdenum, Selenium\} 6020M_METALS_ICPMS_WE (TAL) \{Cadmium, Chromium, Silver\}

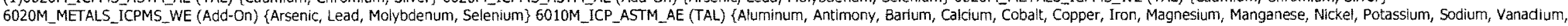

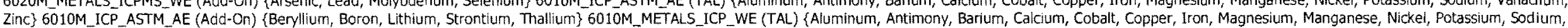

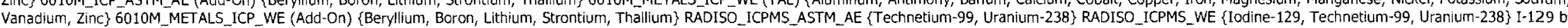

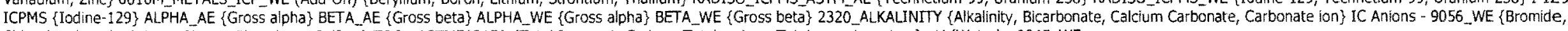
Chloride, Fiuoride, Nitrate, Nitrite, Phosphate, Sulfate\} TOC - ASTME1915A \{Total Inorganic Carbon, Total carbon, Total organjC carbon\} pH (Water) - 9045_WE;
WIDRIG, DL

SAF NO.

F08-075

123514 ES10
DATA TURNAROUND

45 Days / 45

Days

\section{OFFSITE PROPERTY NO.}

DIDING / AIR BILL NO

N/A
METHOD OF SHIPMENT

GOVERNMENT VEHICLE

\section{NiA}

200-BP-5 OU Characterization for Wells 299-E33-341 and 299-E33-342 - G

FIELD LOGBOOK NO. ACTUAL SAMPLE DEPTH 


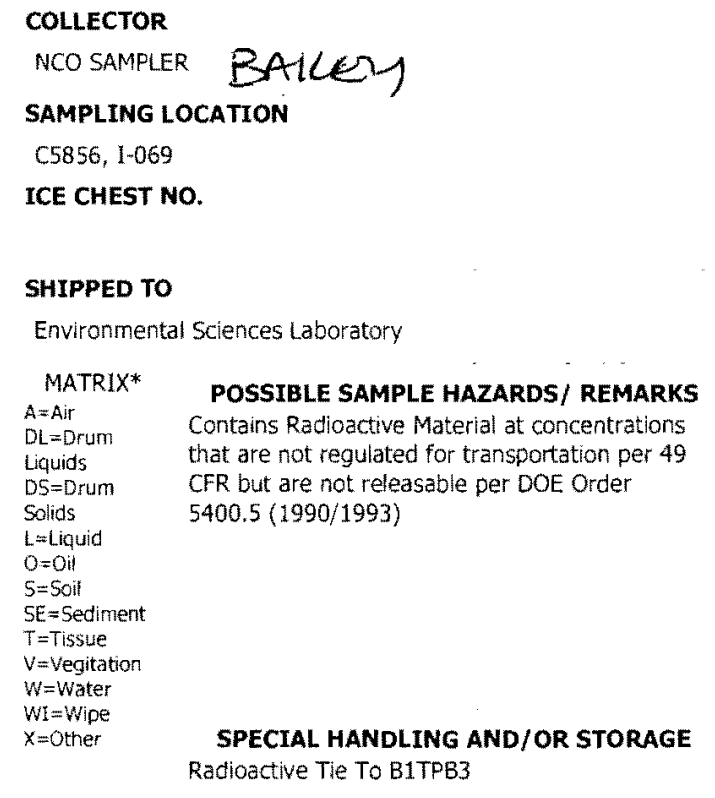

\section{COMPANY CONTACT}

TELEPHONE NO.

PROJECT COORDINATOR

PROJECT DESIGNATION

$373-5869$

WIDRIG, DL

PRICE CODE

DATA

200-BP-5 OU Characterization for Wells 299-E33-341 and 299-E33-342-G F08-075

FIELD LOGBOOK NO.

AIR QUALITY

\section{METHOD OF SHIPMENT}

123514 ES10 GOVERNMENT VEHICLE

HNE-N-488-1 $130^{\prime} \quad 123514 E 510$

N/A

N/A

\begin{tabular}{llll} 
PRESERVATION & None & None \\
TYPE OF CONTAINER & G/P & $\begin{array}{l}\text { Moisture } \\
\text { Resistant Cont }\end{array}$ \\
\hline NO OF CONTATNER(S) & 1 & 1 &
\end{tabular}

VOLUME $\quad 11-2009$

SAMPLE ANALYSIS SEE TEM (1) IN Maisture

SAMPLE DATE SAMPLE TIME

$5-9-8 \quad 0900$
SAMPLE NO.
MATRIX*

\begin{abstract}
B1TP61
\end{abstract}
SOIL

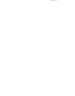

\section{CHAIN OF POSSESSION}

RELINQUISHED BY/REMOVED FROM JxEAlley Oreath RELINOUISHED BY/REMOVED RELINOUSSHED BYIREMOVED FROM $5 / 15 / 08$ O O:'

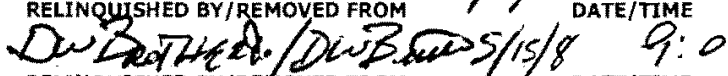
RELINQUISHED BY/REMOVED FROM DATE/TIME

RELINQUISHED BY/REMOVED FROM DATE/TIME

RELINQUISHED BY/REMOVED FROM DATE/TIME

RELINQUISHED BY/REMOVED FROM

DATE/TIME

\section{4}

RECEIVED BY/STORED IN

MoT15 REF $=159.8,1500$

RECEIVED BY/STORED IN

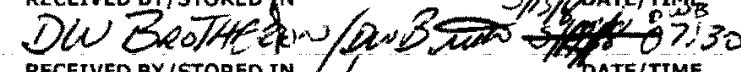
RECEIVED BY/STORED IV DOTE/TIME RECEIVED BYISTORED IN $5 / \mathrm{s} / \mathrm{g}$ POT

DATE/TTME

RECEIVED BY/STORED IN

DATE/TIME

RECEIVED BY/STORED IN

DATE/TIME

RECEIVED BY/STORED IN

\section{SPECIAL INSTRUCTIONS}

SEE PAGE 2 FOR ALL SPECIAL INSTRUCTIONS

\section{LABORATORY RECEIVED BY SECTION}

FINAL SAMPLE DISPOSAL METHOD 


\section{COLLECTOR}

NCO SAMPLER

SAMPLING LOCATION

C5856, J-069

ICE CHEST NO.

\section{SHIPPED TO}

Environmental Sciences Laboratory

\section{COMPANY CONTACT}

TRENT, STEVE

TELEPHONE NO.

373-5869

PROJECT DESIGNATION

200-BP-5 OU Characterization for Wells 299-E33-341 and 299-E33-342-G

FIELD LOGBOOK NO.

ACTUAL SAMPLE DEPTH

\section{OFFSITE PROPERTY NO.}

$N / A$
PRICE CODE

\section{N}

WIDRIG, DL

SAF NO.

AIR QUALITY

METHOD OF SHIPMENT

GOVERNMENT VEHICLE

\section{SPECIAL INSTRUCTIONS}

** The 200 Area S\&GRP Characterization and Monitoring Sampling and Analysis GKI applies to this SAF.

** ESL is to determine unsaturated hydraulic conductivity using the method(s) best suited for the soil texture and type.

** ESL is to submit copies of all Chain of Custodies and associated sample login documentation to EIS-SDM within 24 hours of sample receipt. They may be sent via e-mail to ^CPP Sample Management.

(1)6020M ICPMS ASTM AE (TAL) \{Cadmium, Chromium, Silver\} 6020M ICPMS ASTM AE (Add-On) \{Arsenic, Lead, Molybdenum, Selenium\} 6020M METALS ICPMS WE (TAL) \{Cadmium, Chromium, Silver\}

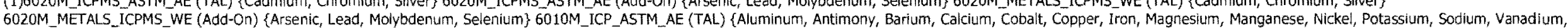

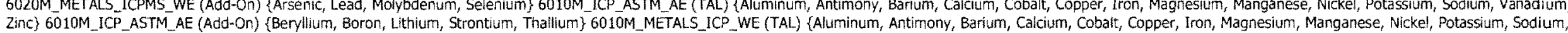

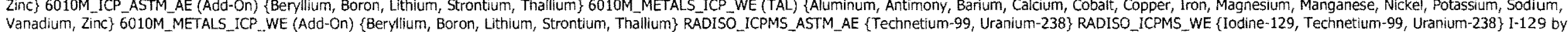

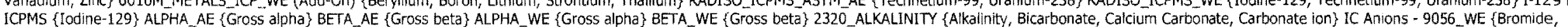
Chloride, Fluoride, Nitrate, Nitrite, Phosphate, Sulfate\} TOC - ASTME1915A (Total Inorganic Carbon, Total carbon, Total organic carbon\} pH (Water) - 9045 _WE; 


\section{COLLECTOR}

nCO SAMPLER Bftlety

\section{SAMPLING LOCATION}

C5856, $1-071$

ICE CHEST NO.

\section{SHIPPED TO}

Environmental Sciences Laboratory

MATRIX*

$A=$ Air

$\mathrm{DL}=$ Drum

Lquids
DS $=$ Drum

DS $=$ Drum
Solids

Solids
$L=$ Liguid
$0=0$ i

$0=\mathrm{Oi}$
$5=501$

$S=S O i l$
$S E=$ Sediment

$T=$ Tissue

$v=$ vegitation

$W=$ Water

WI $=$ Wipe

SPECIAL HANDLING AND/OR STORAGE Radioactive Tie To B1TPB3 Contains Radioactive Material at concentrations that are not regulated for transportation per 49 CFR but are not releasable per DOE Order

$5400.5(1990 / 1993)$

SAMPLE NO.

MATRIX*

B1TP62

SOIL

\section{CHAIN OF POSSESSION}

RELINQUISHED BY/REMOVED FROM JK EAllY JKEIQ RELINQUISHED BY/REMOVED FROU Mo.745 Pef t

RELIMOUISHED BY/REMOVER FROY $\$ / L / 8$

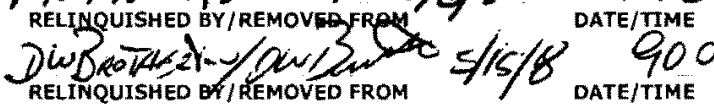

RELINQUISHED BY/REMOVED FROM

RELINQUISHED BY/REMOVED FROM

RELINQUISHED BY/REMOVED FROM

LABORATORY
SECTION
FINAL SAMPLE
DISPOSITION

\section{COMPANY CONTACT}

TRENT, STEVE

TELEPHONE NO.

373-5869

PROJECT COORDINATOR

WIDRIG, DL

PROJECT DESIGNATION

SAF NO.

200-BP-5 OU Characterization for Wells 299-E33-341 and 299-E33-342-G FIELD LOGBOOK NO.

ACTUAL SAMPLE DEPTH

$$
\text { HNE }=-N \cdot 488-1
$$$$
132.51
$$

F08-075

OFFSITE PROPERTY NO.

COA

123514 ES 10

BILL OF LADING/AIR BILL NO.

N/A

\begin{tabular}{|c|c|c|}
\hline PRESERVATION & None & None \\
\hline TYPE OF CONTAINER & $G / P$ & $\begin{array}{l}\text { Moisture } \\
\text { Resstrtart Cont }\end{array}$ \\
\hline NO. OF CONTAINER(S) & 1 & \\
\hline VOLUME & it. & 2009 \\
\hline SAMPLE ANALYSIS & $\begin{array}{l}\text { SEE TEM (1) IN } \\
\text { SPECIAL } \\
\text { INSTRUCTIONS }\end{array}$ & $\begin{array}{l}\text { Moisture } \\
\text { Content - D2216; }\end{array}$ \\
\hline
\end{tabular}

SAMPLE DATE SAMPLE TIME

$5=9-8 \quad 0920$
N/A$$
\text { (5) }
$$

PRICE CODE 8 N

\section{METHOD OF SHIPMENT}

GOVERNMENT VEHICLE 


\section{COLLECTOR}

NCO SAMPLER

\section{SAMPLING LOCATION}

C5856, I-071

ICE CHEST NO.

\section{SHIPPED TO}

Environmental Sciences Laboratory

\section{COMPANY CONTACT}

TRENT, STEVE

\section{PROJECT DESIGNATION}

200-BP-5 OU Characterization for Wells 299-E33-341 and 299-E33-342 - G

FIELD LOGBOOK NO.

ACTUAL SAMPLE DEPTH

\section{OFFSITE PROPERTY NO}

N/A

\section{TELEPHONE NO.}

$373-5869$
PROJECT COORDINATOR

WIDRIG, DL

SAF NO.

F08-075

COA

123514 ES10
PRICE CODE

8N

METHOD OF SHIPMENT

GOVERNMENT VEHICLE

\section{SPECIAL INSTRUCTIONS}

** The 200 Area S\&GRP Characterization and Monitoring Sampling and Analysis GKI applies to this SAF.

** ESL is to determine unsaturated hydraulic conductivity using the method(s) best suited for the soil texture and type.

** ESL is to submit copies of all Chain of Custodies and associated sample login documentation to EIS-SDM within 24 hours of sample receipt. They may be sent via e-mail to ^CPP Sample Management.

(1)6020M_ICPMS_ASTM_AE (TAL) \{Cadmium, Chromium, Silver\} 6020M_ICPMS_ASTM_AE (Add-On) \{Arsenic, Lead, Molybdenum, Selenium\} 6020M_METALS_ICPMS_WE (TAL) \{Cadmium, Chromium, Silver\}

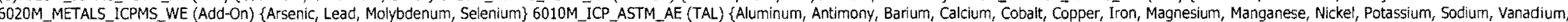

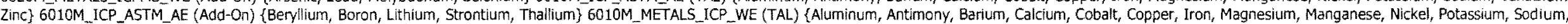

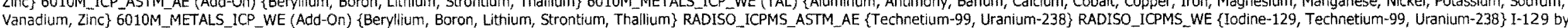

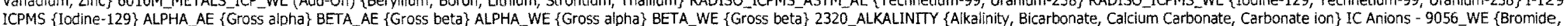
Choride, Fluoride, Nitrate, Nitrite, Phosphate, Sulfate\} TOC - ASTME1915A (Total Inorganic Carbon, Total carbon, Total organic carbon) pH (Water) - 9045 _WE; 
COLLECTOR

nCo sampler Ballet)

SAMPLING LOCATION

C5856, I-073

ICE CHEST NO.

SHIPPED TO

Environmental Sciences Laboratory

MATRIX* POSSIBLE SAMPLE HAZARDS/ REMARKS

A A Air Contains Radioactive Material at concentrations

$D L=D r u m \quad$ that are not regulated for transportation per 49

DS=Drum CFR but are not releasable per DOE Order

Solids $5400.5(1990 / 1993)$

$i=$ Liquid
$0=0$ id

$S=5$ oil

$S=50$ il
$S E=S e d i m e n t$

$T=$ Tissue

$V=V e g i t a t i o n$

W=Water

WI $=$ Wipe

SPECIAL HANDLING AND/OR STORAGE Radioactive Tie To B1TPB3

SAMPLE NO.

MATRIX*

B1TP63

SOIL

\section{COMPANY CONTACT}

TELEPHONE NO.

PROJECT COORDINATOR

WIDRIG, DL

\section{SAF NO.}

PROJECT DÉSIGNATIONON

373-5869

F08-075

200-BP-5 OU Characterization for Wells 299-E33-341 and 299-E33-342 FIELD LOGBOOK NO.

ACTUAL SAMPLE DEPTH

\section{COA}

HNE-NO- 488-1

1351

123514 ES 10

BILL OF LADING/AIR BILL NO.

$\begin{array}{lc}\text { OFFSITE PROPERTY NO. } & \text { BILL } \\ \text { N/A } & \text { N/A }\end{array}$

$\begin{array}{ll}\text { N/A } & \text { BILL } \\ \text { NITE PROPERTY NO. }\end{array}$

\begin{tabular}{|c|c|c|}
\hline PRESERVATION & None & None \\
\hline TYPE OF CONTAINER & $G / P$ & $\begin{array}{l}\text { Moisture } \\
\text { Resistant Cont }\end{array}$ \\
\hline NO. OF CONTAINER(S) & 1 & - \\
\hline VOLUME & IL & 2009 \\
\hline SAMPLE ANALYSIS & $\begin{array}{l}\text { SEE TEM (1) IN } \\
\text { SFECIAL } \\
\text { INSTRUCTONS }\end{array}$ & $\begin{array}{l}\text { Moisture } \\
\text { Content- } 0222\end{array}$ \\
\hline
\end{tabular}

SAMPLE DATE SAMPLE TIME

$5-9-8 \quad 10 / 3$
PRICE CODE $\quad \mathbf{8 N}$

DATA TURNAROUND

45 Days / 45 Days

METHOD OF SHIPMENT

GOVERNMENT VEHICLE

\section{CHAIN OF POSSESSION}

RELINQUISHED BY/REMOVED FROM JHBALEY YCBIC

DATE/TIME

M0745 REF \#/ $5 / 15 / 08$ 07:30

RELINQUISHED BY/REMOVED FROM

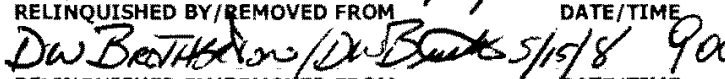

RELINOUISHED BY/REMOVED FROM

RELINQUISHED BY/REMOVED FROM

RELINQUISHED BY/REMOVED FROM

RELINQUISHED BY/REMOVED FROM

LABORATORY RECEIVED BY

SECTION

FINAL SAMPLE DISPOSAL METHOD

$\begin{array}{llc}\text { DATE/TIME } & \text { RECEIVEO BY/STORED IN } \\ \text { DATE/TIME } & \text { RECEIVED BY/STORED IN } & \text { DATE/TIME } \\ \text { DATE/TIME } & \text { RECEIVED BY/STORED IN } & \text { DATE/TIME }\end{array}$

\section{SPECIAL INSTRUCTIONS}

SIGN/ PRINT NAMES

MECEIVED BY/STORED IN

SEE PAGE 2 FOR ALL SPECIAL INSTRUCTIONS

RECEIVED BY/STORED IN DATE/TIME

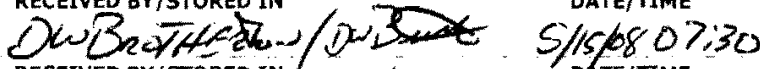

RECEIVED BYISTORED IN

C.TOVWh

$5 / 15108900$

DATE/TIME

TITLE

DATE/TIME

DISPOSED BY

DATE/TIME 


\section{COLLECTOR}

NCO SAMPLER

COMPANY CONTACT

TRENT, STEVE

\section{TELEPHONE NO.}

373-5869

\section{PROJECT DESIGNATION}

200-BP-5 OU Characterization for Wells 299-E33-341 and 299-E33-342-G

FIELD LOGBOOK NO.

ACTUAL SAMPLE DEPTH

\section{OFFSITE PROPERTY NO.}

N/A
PROJECT COORDINATOR

WIDRIG, DL

SAF NO.

F08.075

\section{COA}

$123514 \mathrm{ES} 10$

\section{PRICE CODE}

8N

AIR QUALITY

METHOD OF SHIPMENT GOVERNMENT VEHICLE

\section{SHIPPED TO}

Environmenta! Sciences Laboratory
BILL OF LADING/AIR BILL NO.

$N / A$

\section{SPECIAL INSTRUCTIONS}

** The 200 Area S\&GRP Characterization and Monitoring Sampling and Analysis GKI applies to this SAF.

** ESL is to determine unsaturated hydraulic conductivity using the method(s) best suited for the soil texture and type.

** ESL is to submit coples of all Chain of Custodies and associated sample login documentation to EIS-SDM within 24 hours of sample receipt. They may be sent via e-mail to ^CPP Sample Management.

(1)6020M_ICPMS_ASTM_AE (TAL) \{Cadmium, Chromium, Silver\}6020M_ICPMS_ASTM_AE (Add-On) (Arsenic, Lead, Molybdenum, Selenium\} 6020M_METALS_ICPMS_WE (TAL) \{Cadmium, Chromium, Silver\}

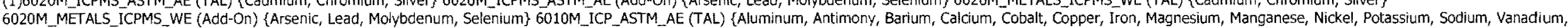

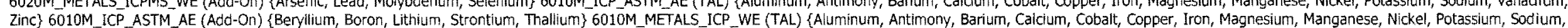

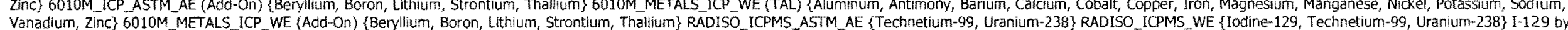

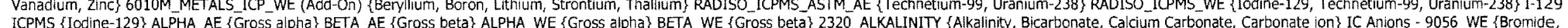
Chloride, Fluoride, Nitrate, Nitrite, Phosphate, Sulfate $\}$ TOC - ASTME1915A \{Total Inorganic Carbon, Total carbon, Total organic carbon\} pH (Water) - 9045 _WE: 
COLLECTOR

nco sampler Pfilet

\section{SAMPLING LOCATION}

C5856, I-075

ICE CHEST NO.

\section{SHIPPED TO}

Environmental Sciences Laboratory

MATRIX*

$A=$ Air
DL $=$ Drum

Liquids

DS=Drum

Solids
$1=$ Liouid

$0=0$ il

$0=0$ il
$S=5$ in

$\mathrm{SE}=\mathrm{S}=$ Sediment

$T=T$ issue

$V=$ vegitatio

$W=$ Water

Wi= Wipe

SPECIAL HANDLING AND/OR STORAGE Radioactive Tie To B1TPB3

PSTBLE SAMPLE HATARDS/ REMARKS Contains Radioactive Material at concentrations that are not regulated for transportation per 49

CFR but are not releasable per DOE Order

$5400.5(1990 / 1993)$

SAMPLE NO

MATRIX*

B1TP64

SOIL

\section{CHAIN OF POSSESSION}

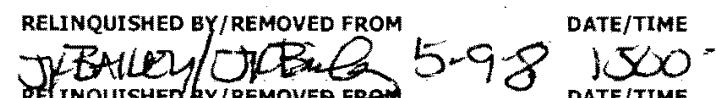
RELINQUISHEO BY REMOVEQ EROA NOT45 MEF Z $5 / 15 / 8$ DATE/TIME

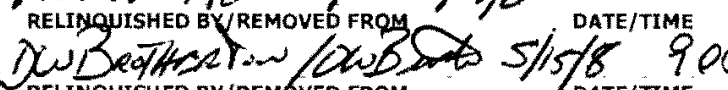

RELINOUISHED BY/REMUVED FROM

DATE/TIME

RELINQUISHED BY/REMÖVED FROM

DATE/TIME

RELINQUISHED BY/REMOVED FROM

DATE/TIME

RELINQUISHED BY/REMOVED FROM

DATE/TIME

\section{COMPANY CONTACT}

TRENT, STEVE

TELEPHONE NO.

$373-5869$

PROJECT COORDINATOR

WIDRIG, DL

SAF NO.

PROJECT DESIGNATION

200-8P-5 OU Characterization for Wells 299-E33-341 and 299-E33-342-C FIELD LOGBOOK NO.

ACTUAL SAMPLE DEPTH

HNE,N-488-1 137.5

OFFSITE PROPERTY NO.

COA

123514 ES10

GIL OF LADING/AIR BILL NO.
N/A

\begin{tabular}{ccc} 
PRESERVATION & None & None \\
TYPE OF CONTAINER & G/P & $\begin{array}{l}\text { Moisture } \\
\text { Resistant Cont }\end{array}$ \\
\hline
\end{tabular}

NO. OF CONTAINER(S)

vOLUME

11

2009

SAMPLE ANALYSIS

SEE TEEM (1) IN Mosture
SPECIAL
DATA TURNAROUND

45 Days / 45 Days

\section{SAMPLE DATE SAMPLE TIME}

$5-9-8 \quad 1038$

SPECIAL INSTRUCTIONS

\section{SIGN/ PRINT NAMES}

\section{4}

RECEIVED BY/STORED IN ET $508^{\text {DATE/TIME }}$ SEE PAGE 2 FOR ALL SPECIAL INSTRUCTIONS

DYISTOREO IN

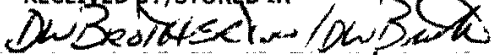
RECEIVED BY/STORED IN C. Tovmi RECEIVED BY/STORED RECEIVED BYISTORED

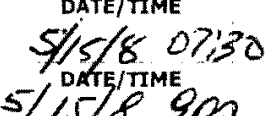
$5 / 15 / 890$

\section{RECEIVED BY/STORED IN}

DATE/TIME

\section{LABORATORY RECEIVED BY}




\section{COLLECTOR}

NCO SAMPLER

\section{COMPANY CONTACT}

TRENT, STEVE

\section{TELEPHONE NO.}

PROJECT COORDINATOR

\section{PROJECT DESIGNATION}

373.5869

WIDRIG, DL

PRICE CODE

$\mathbf{8 N}$

DATA

TURNAROUND

\begin{tabular}{l|l:cccc}
45 Days $/ 45$ \\
Days
\end{tabular}

200-BP-5 OU Characterization for Wells 299-E33-341 and 299-E33-342-G F08-075
FIELD LOGBOOK NO.
ACTUAL SAMPLE DEPTH

C5856, I-075

ICE CHEST NO.

\section{OFFSITE PROPERTY NO.}

$123514 E 510$

N/A

BILL OF LADING/AIR BILL NO.

Environmental Sciences Laboratory

$\mathrm{N} / \mathrm{A}$

\section{SPECIAL INSTRUCTIONS}

** The 200 Area S\&GRP Characterization and Monitoring Sampling and Analysis GKI applies to this SAF.

** ESL. is to determine unsaturated hydraulic conductivity using the method(s) best suited for the soil texture and type.

** ESL. is to submit copies of all Chain of Custodies and associated sample login documentation to EIS-SDM within 24 hours of sample receipt. They may be sent via e-mail to ${ }^{\wedge}$ CPP Sample Management.

(1)6020M_ICPMS_ASTM_AE (TAL) \{Cadmium, Chromium, Silver\} 6020M_ICPMS_ASTM_AE (Add-On) \{Arsenic, Lead, Molybdenum, Selenium\} 6020M_METALSIICPMS_WE (TAL) \{Cadmium, Chromium, Silver\}

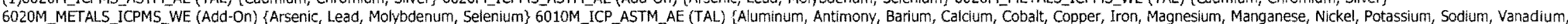

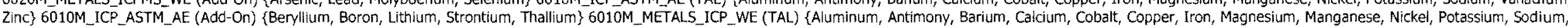

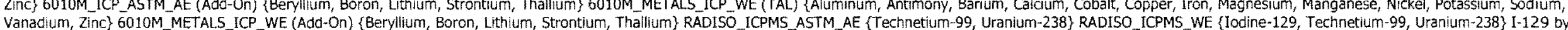

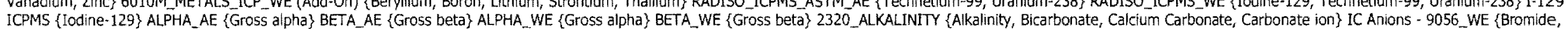
Chloride, Fiuoride, Nitrate, Nitrite, Phosphate, Sulfate\} TOC - ASTME1915A \{Total Inorganic Carbon, Total carbon, Total organic carbon\} pH (Water) - 9045 .WE: 


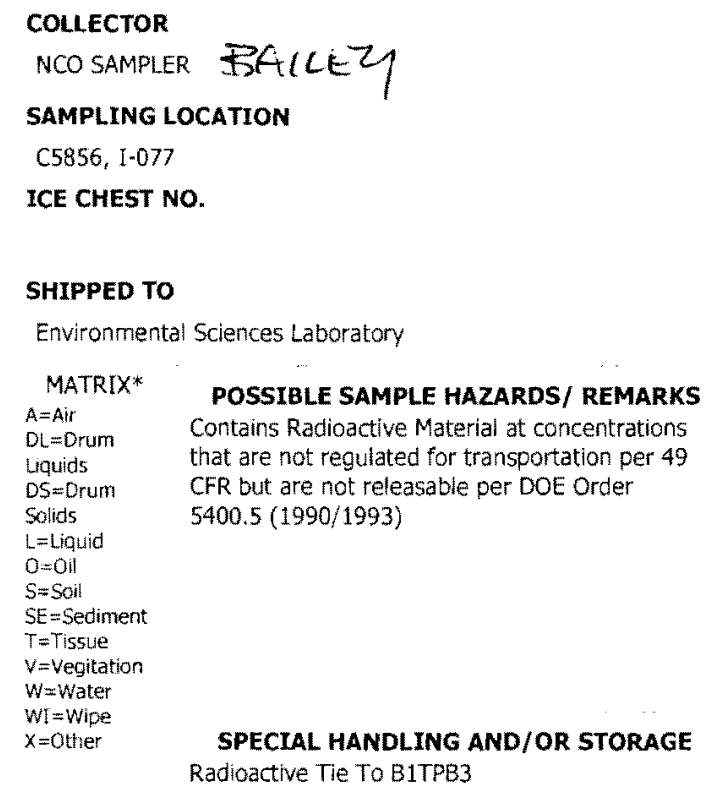

POSSIBLE SAMPLE HAZARDS/ REMARKS Contains Radioactive Material at concentrations that are not regulated for transportation per 49 CFR but are not releasable per DOE Order $5400.5(1990 / 1993)$

\section{SAMPLE NO.}

MATRIX*

B1TP65

SOIL

\section{CHAIN OF POSSESSION}

RELINQUISHED BY/REMOVED FROM RELINOUISHED BY/REMOVED FROM MO745 PEF $45 / 5 / 8$

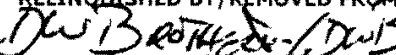
RELINOUISHED BY/REMOVED FROM

RELINQUISHED BY/REMOVED FROM

RELINQUISHED BY/REMOVED FROM

RELINQUISHED BY/REMOVED FROM

LABORATORY RECEIVED BY SECTION

FINAL SAMPLE DISPOSAL METHOD DISPOSITION
DATE/TIME

DATE/TIME

DATE/TIME

\section{COMPANY CONTACT}

TRENT, STEVE

TELEPHONE NO.

373-5869

PROJECT COORDINATOR WIDRIG, DL

\section{SAF NO.}

PROJECT DESIGNATION

F08-075

200-BP-5 OU Characterization for Wells 299-E33-341 and 299-E33-342-G FIELD LOGBOOK NO.

ACTUAL SAMPLE DEPTH

COA

$H N F-N-488-1$

OFFSITE PROPERTY NO.

$$
140
$$

123514 ES 10

BILL OF LADING/AIR BILL ND.

$\mathrm{N} / \mathrm{A}$

\section{$N / A$}

PRICE CODE BN

AIR QUALITY

OD OF SHIPMENT

GOVERNMENT VEHICLE
PRESERVATION

TYPE OF CONTAINER

NO. OF CONTAINER(S)

VOLUME

SAMPLE ANALYSIS
None None

Moisture

Resistant cont

1

2009
SEE TTEM (1) IN MOisture
SPECIAL
TWSTPUCIONS COitent-D2216;

SAMPLE DATE SAMPLE TIME

$5-9-8 \quad 1105$

\section{4}

\section{SIGN/ PRINT NAMES}

RECEIVED BY/STORED IN

MOT4S REF \#1 5.7-8 1500 DATE/TIME $5 / \angle 5 / 8900$ ECEIYED BY/STORED IN WOROthex-10us RECEIVED BY/STORED IN C.IOUn: Eceived aristor - Recenved aYistóne
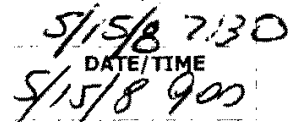

Date/time

\section{SPECIAL INSTRUCTIONS}

SEE PAGE 2 FOR ALL SPECIAL INSTRUCTIONS 


\section{COLLECTOR}

NCO SAMPLER

\section{COMPANY CONTACT}

TRENT, STEVE

TELEPHONE NO.

PROJECT COORDINATOR

WIDRIG, DL

PRICE CODE

$\mathbf{8 N}$

DATA

\section{PROJECT DESIGNATION}

SAF NO.

AIR QUALITY

200-BP-5 OU Characterization for Wells 299-E33-341 and 299-E33-342 - G

FIELD LOGBOOK NO.

ACTUAL SAMPLE DEPTH

COA

METHOD OF SHIPMENT

OFFSITE PROPERTY NO.

\section{BILL OF LADING/AIR BILL NO.}

GOVERNMENT VEHICLE

\section{SHIPPED TO}

Environmental Sciences Laboratory
N/A

N/A

\section{SPECIAL INSTRUCTIONS}

** The 200 Area S\&GRP Characterization and Monitoring Sampling and Analysis GKI applies to this SAF.

** ESL is to determine unsaturated hydraulic conductivity using the method(s) best suited for the soil texture and type.

** ESL is to submit copies of all Chain of Custodies and associated sample login documentation to EIS-SDM within 24 hours of sample receipt. They may be sent via e-mail to ACPP Sample Management.

(1)6020M_ICPMS_ASTM_AE (TAL) \{Cadmium, Chromium, Silver\} 6020M_ICPMS_ASTM_AE (Add-On) \{Arsenic, Lead, Molybdenum, Selenium\} 6020M_METALS_ICPMS_WE (TAL) \{Cadmium, Chromium, Silver\}

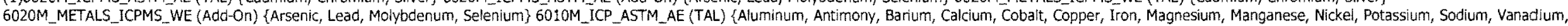

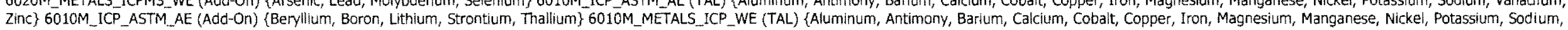

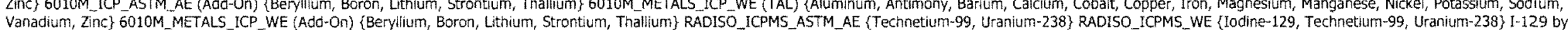

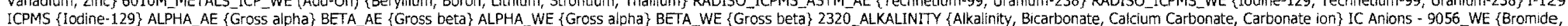
Chloride, Fluoride, Nitrate, Nitrite, Phosphate, Sulfate\} TOC - ASTME1915A (Total Inorganic Carbon, Total carbon, Total organic carbon\} pH (Water) -9045_WE; 


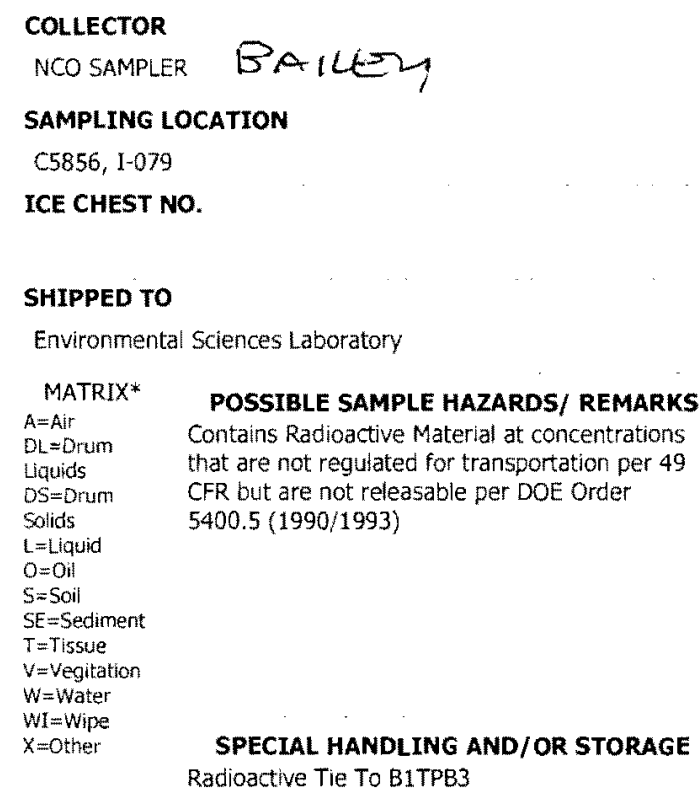

Radioactive Tie TO B1TPB3

POSSIBLE SAMPLE HAZARDS/ REMARKS Contains Radicactive Material at concentrations that are not regulated for transportation per 49 CFR but are not releasable per DOE Order $5400.5(1990 / 1993)$

SAMPLE NO. MATRIX*

BTTP66 SOIL

\section{COMPANY CONTACT}

TRENT, STEVE

TELEPHONE NO.

PROJECT COORDINATOR

PRICE CODE

DATA TURNAROUND

\section{PROJECT DESIGNATION}

373-5869

WIDRIG, DL

AIR QUALITY

45 Days / 45

200-BP-5 OU Characterization for Wells 299-E33-341 and 299-E33-342 - G F08-075 FIELD LOGBOOK NO. ACTUAL SAMPLE DEPTH

COA

HNF-N $-488-1$ 142.51

123514ES10

\section{METHOD OF SHIPMENT}

GOVERNMENT VEHICLE

OFFSITE PROPERTY NO.

BILL OF LADING /AIR BILL NO.

$\mathrm{N} / \mathrm{A}$

N/A

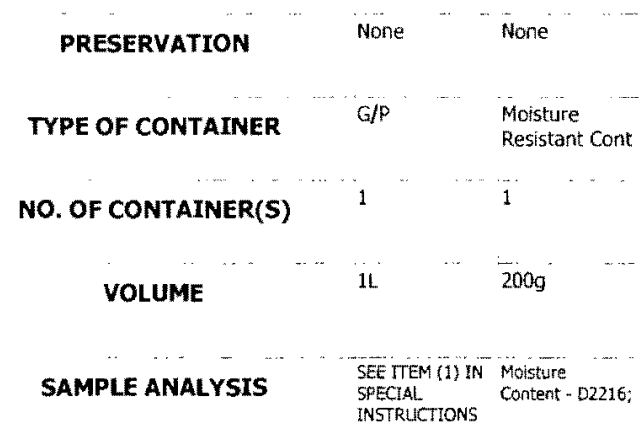

SAMPLE DATE SAMPLE TIME $5-9-8 \quad 1240$

\section{CHAIN OF POSSESSION}

RELINQUISHED BY/REMOVED FROM

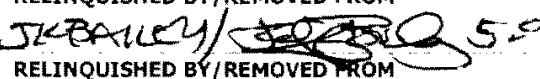
Mo745/PE $/$ I $5 / 15 / 08$ DuBrotasedin/Dw50 RELINQUISHED BY/REM 6VED FROM

RELINQUISHED BY/REMOVED FROM RELINQUISHED BY/REMOVED FROM RELINQUISHED BY/REMOVED FROM RECEIVED BY LABORATORY SECTION

\section{4}

\section{SIGN/ PRINT NAMES}

DATE/TIME

RECEIVED BY/STORED IN

$-8.500$

MOT45 REF

RECEIVED BY/STORER IN

$0>30$

DU Bratased. $5 / 15 / 08$ Date/TIME 900

RECEIVED BY/STORED IN C.IOU W

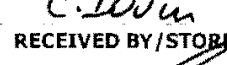

DATE/TIME

RECEIVED BY/STORED IN

DATE/TIME

RECEIVED BY/STORED IN

DATE/TIME
RECEIVED BY/STORED IN

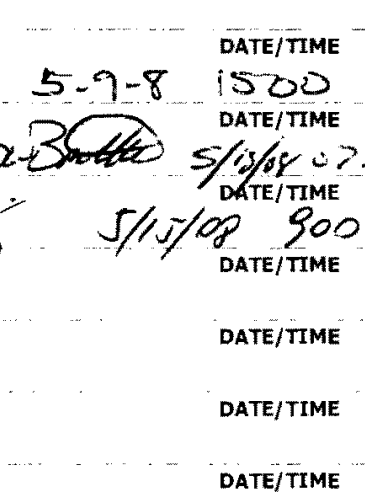

DATE/TIME

DATE/TIME

\section{SPECIAL INSTRUCTIONS}

SEE PAGE 2 FOR ALL SPECIAL INSTRUCTIONS 


\section{COLLECTOR}

COMPANY CONTACT

TRENT, STEVE 373-5869

TELEPHONE NO.

PROJECT COORDINATOR

WIDRIG, DL

PRICE CODE

$\mathbf{8 N}$

DATA

PROJECT DESIGNATION

SAF NO.

AIR QUALITY

TURNAROUND

GNATION

FIELD LOGBOOK NO. "ACTUAL SAMPLE DEPTH

COA

METHOD OF SHIPMENT

ICE CHEST NO.

OFFSITE PROPERTY NO

$N / A$

123514 ES 10

GOVERNMENT VEHICLE

\section{SHIPPED TO}

Environmental Sciences Laboratory

\section{BILL OF LADING/AIR BILL NO}

N/A

\section{SPECIAL INSTRUCTIONS}

** The 200 Area S\&GRP Characterization and Monitoring Sampling and Analysis GKI applies to this SAF.

** ESL is to determine unsaturated hydraulic conductivity using the method(s) best suited for the soil texture and type.

** ESL is to submit copies of all Chain of Custodies and associated sample login documentation to EIS-SDM within 24 hours of sample receipt. They may be sent via e-mail to ^CPP Sample Management.

(1)6020M ICPMS ASTM AE (TAL) \{Cadmium, Chromium, Silver\} 6020M ICPMS ASTM AE (Add-On) \{Arsenic, Lead, Molybdenum, Selenium\} 6020M_METALS_ICPMS WE (TAL) \{Cadmium, Chromium, Silver\}

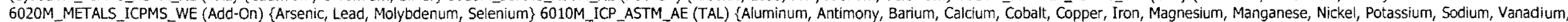

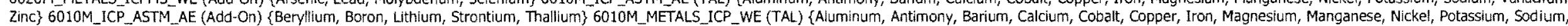

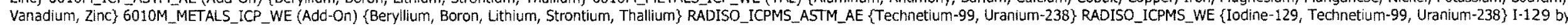

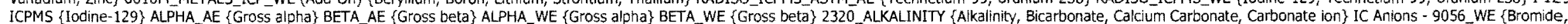
Chloride, Fluoride, Nitrate, Nitrite, Phosphate, Sulfate\} TOC - ASTME1915A \{Total Inorganic Carbon, Total carbon, Total organic carbon\} pH (Water) - 9045_WE; 


\section{COLLECTOR}

nCO SAMPLER BALLT?

\section{SAMPLING LOCATION}

C5856, I- -081

ICE CHEST NO.

\section{SHIPPED TO}

Environmental Sciences Laboratory

\begin{tabular}{|c|c|}
\hline MATRIX* & POSSIBLE SAMPLE HAZARDS/ REMARKS \\
\hline $\begin{array}{l}A=\text { Air } \\
D L=D \text { rum }\end{array}$ & Contains Radioactive Material at concentrations \\
\hline Liquids & that are not regulated for transportation per 49 \\
\hline$D S=D$ rum & CFR but are not releasable per DOE Order \\
\hline $\begin{array}{l}\text { Solids } \\
L=L \text { Liquite }\end{array}$ & $5400.5(1990 / 1993)$ \\
\hline $\begin{array}{l}0=0 \text { il } \\
5=50 \text { i }\end{array}$ & \\
\hline $\begin{array}{l}S=\text { Soil } \\
S E=\text { Sedinent }\end{array}$ & \\
\hline $\begin{array}{l}\text { SE }=\text { Sediment } \\
T=\text { Tisste }\end{array}$ & \\
\hline$V=V$ Vegitation & \\
\hline $\begin{array}{l}W=\text { Water } \\
W I=\text { Wipe }\end{array}$ & . \\
\hline$x=0$ ther & SPECIAL HANDLING AND/OR STORAGE \\
\hline & Radioactive Tie To B1TPB4 \\
\hline
\end{tabular}

SAMPLE NO. MATRIX*

B1TP67 SOIL

\section{CHAIN OF POSSESSION}

RELINQUISHED GY/REMOVED FROM JHEXILY UTER ND-745 PEF tE/ $5 / 15 / 08$ RELINOUISHED BY/REMOVED FROM DUBROTHEST. /OW RELINQUISHED BY/REYIOVED FROM

RELINQUISHED BY/REMOVED FROM

RELINQUISHED BY/REMOVED FROM

RELINQUISHED BY/REMOVED FROM

LABORATORY
SECTION
FINAL SAMPLE
DISPOSITION

\section{COMPANY CONTACT}

TRENT, STEVE

TELEPHONE NO.

373-5869

PROJECT COORDINATOR

WIDRIG, DL

SAF NO.

200-BP-5 OU Characterization for Wells 299-E33-341 and 299-E33-342-G F08-075

FIELD LOGBOOKK NO.

ACTUAL SAMPLE DEPTH

COA

HNF-N-488-1

OFFSITE PROPERTY NO.

$145^{\prime}$

$123514 E S 10$

BILL OF LADING/AIR BILL NO.

N/A

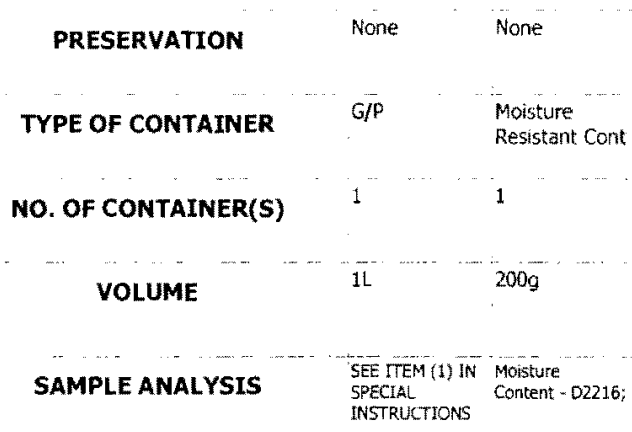

SAMPLE DATE SAMPLE TIME

$5-9.8 \quad 1300$
N/A
SIGN/ PRINT NAMES

RECEIVED BY/STORED IN

WOT45 REF

RECEIVED BYISTORED IN

DEC

RECEIVED BY/STORED IN

P. Joveri

DATE/TIME syigloy go

DATE/TIME

RECEIVED BY/STORED IN

3174

DATE/TIME

DATE/TIME
RECEIVED BY/STORED IN

RECEIVED BY/STORED IN

\section{SPECIAL INSTRUCTIONS}

DATE/TIME

SEE PAGE 2 FOR ALL SPECIAL INSTRUCTIONS

PRICE CODE

METHOD OF SHIPMENT

GOVERNMENT VEHICLE 


\section{COLLECTOR}

NCO SAMPLER

\section{SAMPLING LOCATION}

C5856, I-081

ICE CHEST NO.

\section{SHIPPED TO}

Environmental Sciences Laboratory

\section{COMPANY CONTACT}

TRENT, STEVE

TELEPHONE NO.

PROJECT DESIGNatIon

373-5869

200-BP-5 OU Characterization for Wells 299-E33-341 and 299-E33-342 - G

FIELD LOGBOOK NO.

ACTUAL SAMPIE DEPTH

\section{OFFSITE PROPERTY NO}

N/A
PROJECT COORDINATOR WIDRIG, DL

SAF NO.

F08-075

$\operatorname{COA}$

$123514 E S 10$
PRICE CODE BN

AIR OUALITY
DATA TURNAROUND

45 Days / 45 Days

\section{SPECIAL INSTRUCTIONS}

** The 200 Area S\&GRP Characterization and Monitoring Sampling and Analysis GKI applies to this SAF.

** ESL is to determine unsaturated hydraulic conductivity using the method(s) best suited for the soil texture and type.

** ESL is to submit coples of all Chain of Custodies and associated sample login documentation to EIS-SDM within 24 hours of sample receipt. They may be sent via e-mail to ^CPP Sample Management.

(1)6020M_ICPMS_ASTM_AE (TAL) \{Cadmium, Chromium, Silver\} 6020M_ICPMS_ASTM_AE (Add-On) \{Arsenic, Lead, Molybdenum, Selenium) 6020M_METALS_ICPMS_WE (TAL) \{Cadmium, Chromium, Silver\}

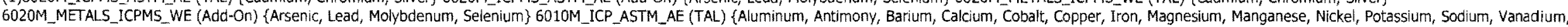

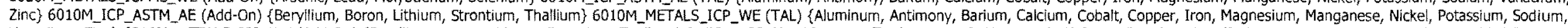

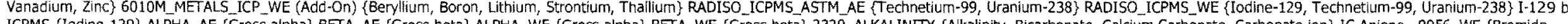

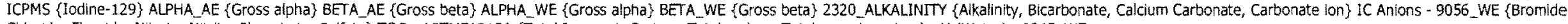
Chloride, Fluoride, Nitrate, Nitrite, Phosphate, Sulfate\} TOC - ASTME1915A \{Total Inorganic Carbon, Total carbon, Total organic carbon\} pH (Water) - 9045 _WE; 


\section{CHAIN OF CUSTODY/SAMPLE ANALYSIS REQUEST}

\section{TELEPHONE NO.}

373.5869

TRENT, STEVE

PROJECT DESIGNATION

200-BP-5 OU Characterization for Wells 299-E33-341 and 299-E33-342 - G FIELD LOGBOOK NO. ACTUAL SAMPLE DEPTH

C5856, I-083

ICE CHEST NO.

\section{SHIPPED YO}

Environmental Sciences Laboratory

MATRIX*

$A=$ Air

$\mathrm{DL}=\mathrm{Drum}$

Lquids

DS = Drum
Solids

$L=$ Liquid

$0=0$ il
$S=5$ oil

SE $=$ Sediment

$T=T i s s u e$

$V=$ Vegitation

$W=$ Water

$W I=$ Wipe
$x=0$ ther

SPECIAL HANDLING AND/OR STORAGE Radioactive Tie TO B1TPB4 Contains Radioactive Material at concentrations that are not regulated for transportation per 49 CFR but are not releasable per DOE Order $5400.5(1990 / 1993)$

SAMPLE NO.

MATRIX*

B1TP68

SOIL
147.51

OFFSITE PROPERTY NO.

N/A

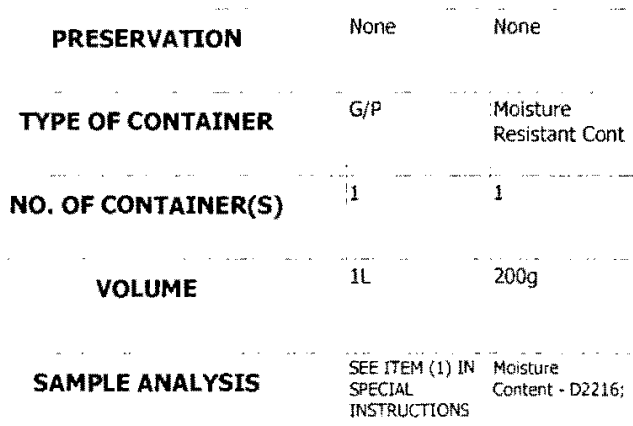

SAMPLE DATE SAMPLE TIME

$5-9-8 \cdot 1325$
PROJECT COORDINATOR

PRICE CODE

$\mathbf{8 N}$

DATA TURNAROUND

45 Days / 45 Days

\section{SAF NO.}

F08-07

COA

$123514 \mathrm{ES} 10$

BILL OF LADING/AIR BILL NO.

\section{CHAIN OF POSSESSION}

RELINQUISHED BY/REMOVED FROM DATE/TIME

RELINQUISHED BY IREMOVED HNOM DATE/TIME Mo 745 PE $=2 / 5 / 15 / 0 y$ DE

RELINOUISHED BY/REMOVED FROM

RELINQUUISHED BY/REMOVED FROM

RELINQUISHED BY/REMOVED FROM

RELINQUISHED BY/REMOVED FROM

RECEIVED BY

\section{LABORATORY}

\section{SECTION}

FINAL SAMPLE DISPOSITION

SIGN/ PRINT NAMES

DATE/TIME

DATE/TIME
SPECIAL INSTRUCTIONS

SEE PAGE 2 FOR ALL SPECIAL INSTRUCTIONS 


\section{COLLECTOR}

\section{NCO SAMPLER}

COMPANY CONTACT

\section{TELEPHONE NO}

TRENT, STEVE

373-5869

PROJECT COORDINATOR

\section{PROJECT DESIGNATION}

WIDRIG, DL

\section{SAF NO.
F08-075}

200-BP-5 OU Characterization for Wells 299-E33-341 and 299-E33-342 *G

FIELD LOGBOOK NO.

ACTUAL SAMPLE DEPTH

COA

123514 ES10

PRICE CODE $8 \mathrm{~N}$

DATA

TURNAROUND

45 Days / 45

Days

OFFSITE PROPERTY NO

N/A

BILL OF LADING/AIR BILL NO.

N/A

\section{SPECIAL INSTRUCTIONS}

Environmental Sciences Laboratory

** The 200 Area S\&GRP Characterization and Monitoring Sampling and Analysis GKI applies to this SAF.

** ESL is to determine unsaturated hydraulic conductivity using the method(s) best suited for the soil texture and type.

** ESL is to submit copies of all Chain of Custodies and associated sample login documentation to EIS-SDM within 24 hours of sample receipt. They may be sent via e-mail to ^CPP Sample Management.

(1)6020M_ICPMS_ASTM_AE (TAL) \{Cadmium, Chromium, Silver\} 6020M_ICPMS_ASTM_AE (Add-On) \{Arsenic, Lead, Molybdenum, Selenium\} 6020M_METALS_ICPMS_WE (TAL) \{Cadmium, Chromium, Silver\}

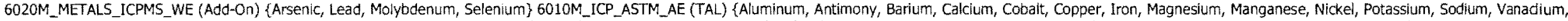

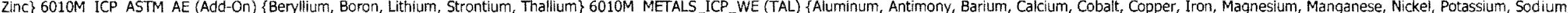

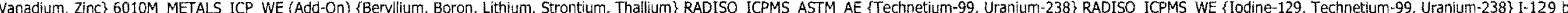

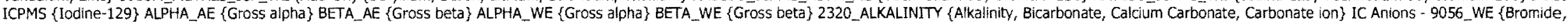

Chloride, Fluoride, Nitrate, Nitrite, Phosphate, Sulfate\} TOC - ASTME1915A \{Total Inorganic Carbon, Total carbon, Total organic carbon\} pH (Water) - 9045 WE; 


\section{COLLECTOR}

COMPANY CONTACT

TELEPHONE NO.

PROJECT COORDINATOR

WIDRIG, OL

PRICE CODE

FIELD LOGBOOK NO.

HNF-N $-488-1$

\section{COA}

\section{METHOD OF SHIPMENT}

GOVERNMENT VEHICLE OFFSITE PROPERTY NO.

SHIPPED TO

Environmental Sciences Laboratory

\section{MATRIX* POSSIBLE SAMPLE HAZARDS/ REMARKS $A=A$ Air $\quad$ Contains Radioactive Material at concentrations Biquids that are not regulated for transportation per 49 DS=Drum $\quad$ CFR but are not releasable per DOE Order Solids $\quad 5400.5(1990 / 1993)$ \\ $L=$ Liquid \\ $\mathrm{S}=$ Sol
$\mathrm{SE}=$ Sediment \\ $T=$ Tissue \\ $v=$ vegitation \\ WI $=$ Wipe \\ SPECIAL HANDLING AND/OR STORAGE Radicactive TIe TO B1TPB4}

N/A

\section{BILL OF LADING / AIR BILL NO}

\begin{tabular}{|c|c|c|}
\hline PRESERVATION & None & None \\
\hline TYPE OF CONTAINER & $G / P^{\circ}$ & $\begin{array}{l}\text { Moisture } \\
\text { Resistant Cont }\end{array}$ \\
\hline NO. OF CONTAINER(S) & 1 & 1 \\
\hline VOLUME & 1L & 2009 \\
\hline SAMPLE ANALYS. & $\begin{array}{l}\text { SEE ITEM (1) IN } \\
\text { SPECIAL } \\
\text { INSTRUTTIONS }\end{array}$ & $\begin{array}{l}\text { Moisture } \\
\text { Cortent - D2216; }\end{array}$ \\
\hline
\end{tabular}

SAMPLE DATE SAMPLE TIME

\section{CHAIN OF POSSESSION}

RELINQUISHED BY/REMOVED FROM

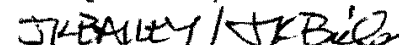
RELINQUISHED BX/REMOVED FRQM MO 745 PE t 519 TYW

RELINQUISHED BY/REMOVED FROM

RELINQUISHED BY/REMOVED FROM

RELINQUISHED BY/REMOVED FROM

RELINQUISHED BY/REMOVED FROM

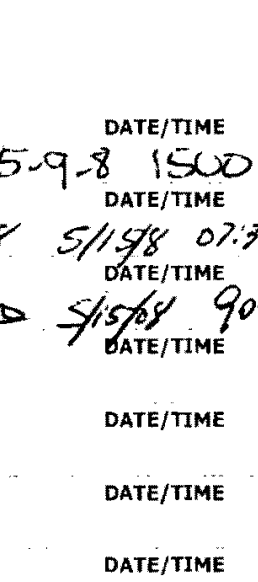

\section{SIGN/ PRINT NAMES}

RECEIVED BY/STORED IN MOT4S REFH RECEYYED BY/ST RRED IN

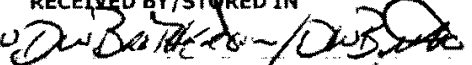

RECEIVED BY/STORED C. $40 \mathrm{din}$ RECEIVED BY/STORE RECEIVED BY/STORED KN 31394 RECEIVED BY/STOREDIN RECEIVED BY/STORED IN

\section{PECIAL INSTRUCTIONS}

SEE PAGE 2 FOR ALL SPECIAL INSTRUCTIONS

\section{LABORATORY RECEIVED BY}

SECTION 


\section{COLLECTOR}

NCO SAMPLER

\section{SAMPLING LOCATION}

C5856, I-085

ICE CHEST NO.

\section{SHIPPED TO}

Environmental Sciences Laboratory

\section{COMPANY CONTACT}

TRENT, STEVE

\section{TELEPHONE NO.}

373-5869

\section{PROJECT DESIGNATION}

200-8P-5 OU Characterization for Wells 299-E33-341 and 299-E33-342 - G

FIELD LOGBOOK NO.

ACTUAL SAMPLE DEPTH

\section{OFFSITE PROPERTY NO}

NiA
PROJECT COORDINATOR

WIDRIG, DL

SAF NO.

F08-075

COA

123514 ES 10

\section{PRICE CODE}

IR QUALITY

$8 \mathrm{~N}$

DATA TURNAROUND

Days

\section{METHOD OF SHIPMENT}

\section{BILL OF LADING/AIR BILL NO.}

$\mathrm{N} / \mathrm{A}$

\section{SPECIAL INSTRUCTIONS}

** The 200 Area S\&GRP Characterization and Monitoring Sampling and Analysis GKI applies to this SAF.

** ESL is to determine unsaturated hydraulic conductivity using the method(s) best suited for the soil texture and type.

** ESL is to submit copies of all Chain of Custodles and associated sample login documentation to EIS-SDM within 24 hours of sample receipt. They may be sent via e-mail to ACPP Sample Management.

(1)6020M_ICPMS ASTM_AE (TAL) \{Cadmium, Chromium, Silver\} 6020M_ICPMS_ASTM_AE (Add-On) \{Arsenic, Lead, Molybdenum, Selenium\} 6020M_METALS_ICPMS WE (TAL) \{Cadmium, Chromium, Silver\}

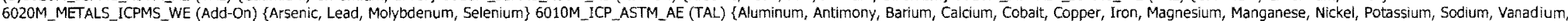

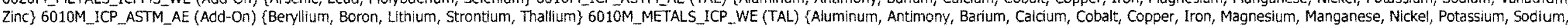

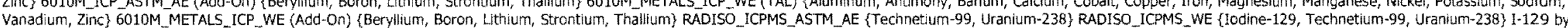

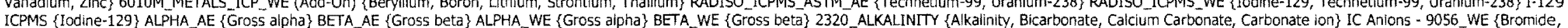
Chioride, Fluoride, Nitrate, Nitrite, Phosphate, Sulfate\} TOC - ASTME1915A (Total Inorganic Carbon, Total carbon, Total organic carbon\} pH (Water) - 9045 WE: 


\section{COLLECTOR}

nCO SAMPLER EAILEY

\section{SAMPLING LOCATION}

C5856, I-087

ICE CHEST NO.

SHIPPED TO

Environmental Sciences Labcratory

MATRIX* POSSIBLE SAMPLE HAZARDS/ REMARKS

AmAir Contains Radicactive Material at concentrations

DL=Drum that are not reguiated for transportation par 49

DS $=$ Drum CFR but are not releasable per DOE Order

Solids $\quad 5400.5(1990 / 1993)$

$L=$ Liquid

$\mathrm{S}=$ Soll

$S E=$ Sediment

$\mathrm{T}=$ Tissue

$V=$ Vegitatio

$W=$ water

$W=W=$ Wipe
$X=0$ ther

SPECIAL HANDLING AND/OR STORAGE Radioactive Tie To B1TPBA

\section{COMPAMY CONTACT}

TRENT, STEVE

$373-5869$

PROJECT COORDINATOR

WIDRIG, DL

\section{PROJECT DESIGNATION}

FIELD LOGBOOK NO.

HNF - N- $488-1$

OFFSITE PROPERTY NO.

N/A

\begin{tabular}{|c|c|c|}
\hline PRESERVATION & None & None \\
\hline TYPE OF CONTAINER & Gip & $\begin{array}{l}\text { Moisture } \\
\text { Resistant Cont }\end{array}$ \\
\hline NO. OF CONTAINER(S) & $i^{\prime} \cdots$ & 1 \\
\hline VOLUME & $I L$ & 2009 \\
\hline SAMPLE ANALYSIS & $\begin{array}{l}\text { SEE TEMM (I) IN } \\
\text { SPECIAL } \\
\text { INSTRUCIONS }\end{array}$ & $\begin{array}{l}\text { Mosture } \\
\text { Content - D2216; }\end{array}$ \\
\hline
\end{tabular}

BILL OF LADING/AIR BILL NO. N/A
INSTRUCTIONS Content - D2216
PRICE CODE

$8 N$
SAMPLE DATE SAMPLE TIME
$512-8 \quad 0925$
SAMPLE NO.
MATRIX*
B1TP70
SOIL

\section{CHAIN OF POSSESSION}
RELINQUISHED BY/REMOVED FROM

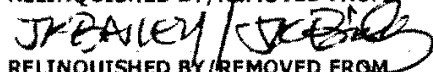 DATE/TIME 190745 REF $5 / 58$ 08000 RELINQYISHED QY/REMOVED FROM

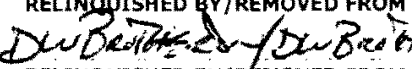
RELINQUISHED BY/REMOVED FROM
RELINQUISHED BY/REMOVED FROM
RELINQUISHED BY/REMOVED FROM
RELINQUISHED BY/REMOVED FROM

\section{LABORATORY}

RECEIVED BY

SECTION

FINAL SAMPLE DISPOSAL METHOD

DISPOSITION

\section{ACTUAL SAMPLE DEPTH \\ COA \\ METHOD OF SHIPMENT}

123514 ES 10

GOVERNMENT VEHIC.E 


\section{COLLECTOR}

NCO SAMPLER

\section{COMPANY CONTACT}

TRENT, STEVE

TELEPHONE NO.

PROJECT COORDINATOR

TRENT STEVE $\quad 373-5869$

WIDRIG, DL

PRICE CODE

8N

DATA

PROJECT DESIGNATION

SAF NO.

AIR QUALITY

TURNAROUND

FIELD LOGBOOK NO.

ACTUAL SAMPLE DEPTH

COA

METHOD OF SHIPMENT

ICE CHEST NO.

\section{OFFSITE PROPERTY NO}

N/A

$123514 E S 10$

GOVERNMENT VEHICLE

\section{SHIPPED TO}

Environmental Sciences Laboratory

\section{BILL OF LADING/AIR BILL NO}

N/A

\section{SPECIAL INSTRUCTIONS}

** The 200 Area S\&GRP Characterization and Monitoring Sampling and Analysis GKI applies to this SAF.

** ESL is to determine unsaturated hydraulic conductivity using the method(s) best suited for the soll texture and type.

** ESL is to submit copies of all Chain of Custodies and associated sample login documentation to EIS-SDM within 24 hours of sample receipt. They may be sent via e-mail to ${ }^{\wedge}$ CPP Sample Management.

(1)6020M ICPMS ASTM AE (TAL) \{Cadmium, Chromium, Silver\} 6020M_ICPMS ASTM AE (Add-On) \{Arsenic, Lead, Molybdenum, Selenium\} 6020M METALS ICPMS WE (TAL) \{Cadmium, Chromium, Silver\}

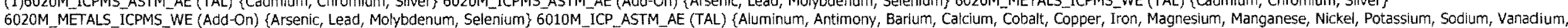

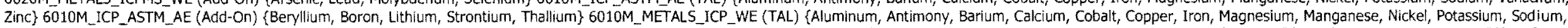

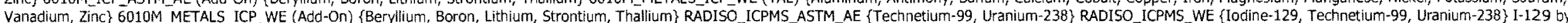

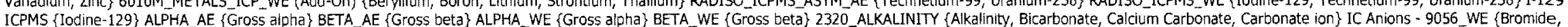
Chloride, Fuoride, Nitrate, Nitrite, Phosphate, Sulfate\} TOC - ASTME1915A \{Total Inorganic Carbon, Total carbon, Total organic carbon\} pH (Water) - 9045 _WE; 


\section{COLLECTOR \\ nCo SAMPLER BaILt?}

SAMPLING LOCATION

C5856, I-089

ICE CHEST NO.

\section{SHIPPED TO}

Environmental Sciences Laboratory

MATRIX*

$\mathrm{A}=\mathrm{Air}$
$\mathrm{DL}=\mathrm{Drum}$

$\mathrm{DL}=$ Drum
Lquids

DS=Drum
Solids

Sollds
$L=$ Liquid

$0=01$

$S E=$ Sediment

$T=$ Tissue

$V=$ Vegitation

$W=$ water

WI $=$ Wipe

SPECIAL HANDLING AND/OR STORAGE Radioactive Tie To B1TPB4 Contains Radioactive Material at concentrations that are not regulated for transportation per 49 CFR but are not releasable per DOE Order $400.5(1990 / 1993)$

SAMPLE NO

MATRIX*

B1TP71

SOIL

\section{CHAIN OF POSSESSION}

RELINQUISHED BY/REMOVED FROM

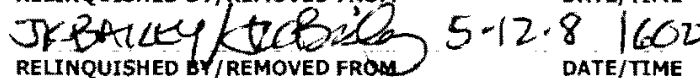
$5-12.8$ DATE/TIME Mo745 T2 $5 / 1 / 5$

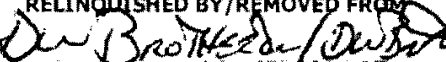
RELINQUISHED BY/REMOVED FROM 08.00 silify $9: 00$

RELINQUISHED BY/REMOVED FROM DATE/TIME

RELINQUISHED GY/REMOVED FROM DATE/TIME RELINQUISHED BY/REMOVED FROM DATE/TIME

\section{COMPANY CONTACT}

TRENT, STEVE TELEPHONE NO.

373-5869

\section{PROJECT DESIGNATION}

200-8P-5 OU Characterization for Wells 299-E33-341 and 299-E33-342-G FIELD LOGBOOK NO. ACTUAL SAMPLE DEPTH

HNF-NJ-488-1

OFFSITE PROPERTY NO.

$$
1551
$$

N/A

\begin{tabular}{ccl} 
PRESERVATION & None & None \\
TYPE OF CONTAINER & G/P & $\begin{array}{l}\text { Molsture } \\
\text { Resistant Cont }\end{array}$ \\
\hline
\end{tabular}

NO. OF CONTAINER(S)

$\begin{array}{llll}\text { VOLUME } & \text { IL } & 2009\end{array}$

$\begin{array}{lll}\text { SAMPLE ANALYSIS } & \text { SEE TIEM (1) IN Moisture } \\ \text { SPECIAL } & \text { Content - D2Z16; }\end{array}$

SAMPLE DATE SAMPLE TIME

$5-12-8 \quad 1025$

\section{4}

\section{SIGN/ PRINT NAMES}

RECEIVED BY/STORED IN

UO245 REF I $542.8^{\text {DATE/TIME }}$ L600

RECETYED BY/STORED IN

RECEIVED BY/STORED IN

P. $\sqrt{10} \mathrm{um}$. RECEIVED BY/STOREDIN/N

$5 / 15 / \log$ S.os

DATE/TIME

RECEIVED BY/STORED IN

DATE/TIME

RECEIVED BY/STORED IN

DATE/TIME

RECEIVED BY/STORED IN
SPECIAL INSTRUCTIONS

SEE PAGE 2 FOR ALL SPECIAL INSTRUCTIONS

LABORATORY RECEIVED BY
SECTION
FINAL SAMPLE
DISPOSITION




\section{COLLECTOR}

NCO SAMPLER

\section{SAMPLING LOCATION}

C5856, I-089

ICE CHEST NO.

\section{SHIPPED TO}

Environmental Sciences Laboratory

\section{TELEPHONE NO.}

373-5869

\section{COMPANY CONTACT}

TRENT, STEVE

\section{PROJECT DESIGNATION}

200-BP-5 OU Characterization for Wells 299-E33-341 and 299-E33-342-G FIELD LOGBOOK NO. ACTUAL SAMPLE DEPTH

\section{OFFSITE PROPERTY NO.}

N/A

\section{PRÓJECT CŌORDINATOR}

WIDRIG, DL

\section{SAF NO.}

F08-075

\section{COA}

123514ES10
PRICE CODE SN

\section{AIR QUALITY}

METHOD OF SHIPMENT

\section{SPECIAL INSTRUCTIONS}

** The 200 Area S\&GRP Characterization and Monitoring Sampling and Analysis GKI applies to this SAF.

** ESL is to determine unsaturated hydraulic conductivity using the method(s) best suited for the soll texture and type.

** ESL is to submit copies of all Chain of Custodies and associated sample login documentation to EIS-SDM within 24 hours of sample receipt. They may be sent via e-mail to $A$ CPP Sample Management.

(1)6020M_ICPMS_ASTM_AE (TAL) \{Cadmium, Chromium, Silver\}6020M_ICPMS_ASTM_AE (Add-On) \{Arsenic, Lead, Molybdenum, Selenium\} 6020M_METALS_ICPMS_WE (TAL) \{Cadmium, Chromium, Silver\}

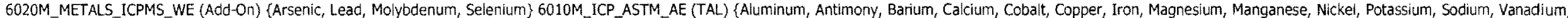

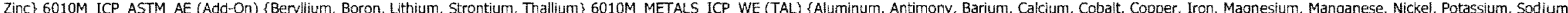

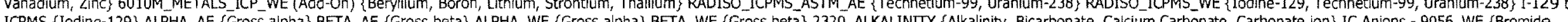

Chloride, Fluoride, Nitrate, Nitrite, Phosphate, Sulfate $\}$ TOC - ASTME1915A \{Total Inorganic Carbon, Total carbon, Total organic carbon\} pH (Water) - 9045 -WE; 


\section{COLLECTOR}

NCO SAMPLER BAILET

\section{SAMPLING LOCATION}

C5856, I-091

ICE CHEST NO.

\section{SHIPPED TO}

Environmental Sclences Laboratory

\section{MATRIX*}

$A=A$ Air

$\mathrm{DL}=\mathrm{Drum}$

Liquids

DS=Drum
Solids

$\mathrm{L}=$ Liquid

$0=\mathrm{Oin}$
$\mathrm{S}=\mathrm{Sol}$

$S E=$ Sediment

$\mathrm{SE}=$ Sediment
$T=$ Tissue

$\forall=$ vegitation

$W=$ Water

WI $=$ Wipe

$x=$ other

SPECIAL HANDLING AND/OR STORAGE Radioactive Tie To B1TPBA Pontans Radioactive Material at concentrations that are not regulated for transportation per 49 CFR but are not releasable per DOE Order $5400.5(1990 / 1993)$

SAMPLE NO

MATRIX*
SOIL

\section{COMPANY CONTACT}

TRENT, STEVE

\section{PROJECT DESIGNATION}

TELEPHONE NO.

373-5869

PROJECT COORDINATOR

WIDRIG, OL

SAF NO

200-BP-5 OU Characterization for Wells 299-E33-341 and 299-E33-342 - G F08-075

FIELD LOGBOOK NO.

ACTUAL SAMPLE DEPTH

COA

HNE-N-48?-1 157.51

OFFSITE PROPERTY NO.

123514ES10

BILL OF LADING/AIR BILL NO
N/A

\begin{tabular}{ccc} 
PRESERVATION & None & None \\
TYPE OF CONTAINER & & \\
\hline & & $\begin{array}{c}\text { Moisture } \\
\text { Resistant Cont }\end{array}$
\end{tabular}

NO. OF CONTAINER(S)

VOLUME

$11 \quad 2009$

SAMPLEANALYSIS $\mathbf{8 N}$

AIR QUALIT

METHOD OF SHIPMENT

GOVERNMENT VEHICLE
DATA TURNAROUND

45 Days / 45 Days

SAMPLE DATE SAMPLE TIME

$5.12 .8 \quad 1105$ L

\section{CHAIN OF POSSESSION}

RELINQUISHED BY/REMOVED FROM

JKBAILEY KECOPDC 5.12 .8160

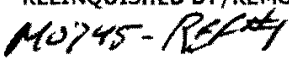

RELINOUISHED BY/REMOVED $5 / 15 / 5$

Duberteryours

RELINQUISHED BY/REMOVED FROM

RELINQUISHED BY/REMOVED FROM

RELINQUISHED BY/REMOVED FROM

RELINQUISHED BY/REMOVED FROM

\begin{tabular}{l} 
LABORATORY \\
SECTION \\
$\begin{array}{c}\text { FINAL SAMPLE } \\
\text { DISPOSITION }\end{array}$ \\
\hline
\end{tabular}

DATE/TIME

DATE/TIME

\section{SPECIAL INSTRUCTIONS}

RECEIVED BY/STORED IN

MO745 REF $+5-12.8 \quad 1600$

RECEIVED BY/STORED IN DATE/TIME

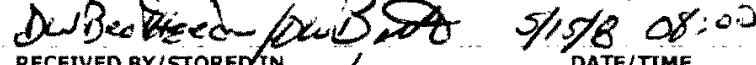

RECEIVED BY/STOREDIN LT DATE/TIME

RECEIVED BYISTOREOIN

RECEIVED BY/STORED IN

DATE/TIME

RECEIVED BY/STORED IN

DATE/TIME 


\section{COLLECTOR}

NCO SAMPLER

\section{SAMPLING LOCATION}

C5856, I-091

ICE CHEST NO.

\section{SHIPPED TO}

Environmental Sciences Laboratory

\section{COMPANY CONTACT}

TRENT, STEVE

\section{PROJECT DESIGNATION}

F08-075

FIELD LOGBOOK NO.

\section{TELEPHONE NO.}

373-5869

PROJECT COORDINATOR

WIORIG, DL

$\operatorname{COA}$

ACTUAL SAMPLE DEPTH

123514ES10
PRICE CODE

AIR QUALITY

DATA TURNAROUND

45 Days / 45

\section{OFFSITE PROPERTY NO}

BILL OF LADING/AIR BILL NO

N/A

\section{SPECLAL INSTRUCTIONS}

** The 200 Area S\&GRP Characterization and Monitoring Sampling and Analysis GKI applies to this SAF.

** ESL is to determine unsaturated hydraulic conductivity using the method(s) best suited for the soil texture and type.

** ESL is to submit copies of all Chain of Custodles and associated sample login documentation to EIS-SDM within 24 hours of sample receipt. They may be sent via e-mail to ^CPP Sample Management.

(1)6020M_ICPMS_ASTM_AE (TAL) \{Cadmium, Chromium, Siver\} 6020M_ICPMS_ASTM_AE (Add-On) \{Arsenic, Lead, Molybdenum, Selenium\} 6020M_METALS_ICPMS_WE (TAL) \{Cadmium, Chromium, Siver\}

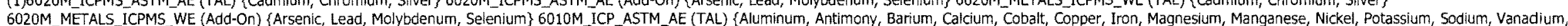

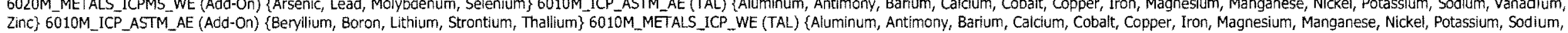

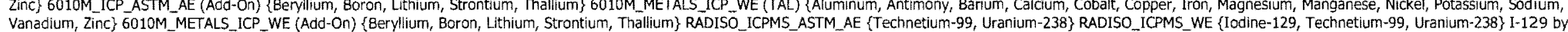

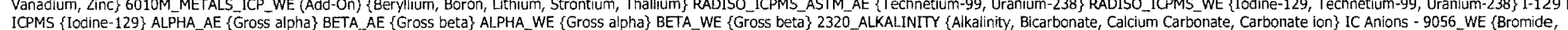

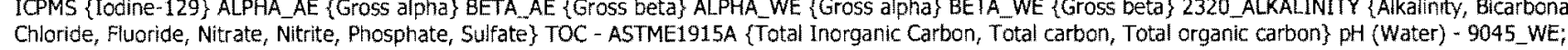




\section{CHAIN OF CUSTODY/SAMPLE ANALYSIS REQUEST}

TELEPHONE NO.

373-5869

TRENT, STEVE

PROJECT DESIGNATION

200-BP-5 OU Characterization for Wells 299-E33-341 and 299-E33-342-G FIELD LOGBOOK NO.

HNE-NS- $488-1$

OFFSITE PROPERTY NO.

N/A

MATRIX* POSSIBLE SAMPLE HAZARDS/ REMARKS

$A=$ Air Contains Radioactive Material at concentrations

$D L=D$ rum that are not regulated for transportation per 49

LS L $=$ Drum CFR but are not releasable per DOE Order

solids $\quad 5400.5(1990 / 1993)$

$\mathrm{L}=$ Liquid

$S=50$ il

$\mathrm{SE}=$ Sediment

$T=$ Tissue

$V=$ vegitation

$W=$ water

WI $=$ Wipe

SPECIAL HANDLING AND/OR STORAGE Radioactive Tie To B1TPB5

PRESERVATION None None

TYPE OF CONTAINER G/P $\quad \begin{aligned} & \text { Moisture } \\ & \text { Resistant Cont }\end{aligned}$

NO. OF CONTAINER(S)

VOLUME

2009

SAMPIE ANALYSIS

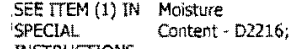

MATRIX

B1TP73

SOIL

\section{CHAIN OF POSSESSION}

RELINQUISHED BY/REMOVED FROM JXPALEY KTPLC?

RELINQUISHED BY/KEMOVED FROM M0.745 AEF $5 / 15 / 8$

RELINQUISHED BY/REMOVED FRGM D) 0 ondted Daba

RELINQUISHED BY/REGMOVED FROM

RELINQUISHED BY/REMOVED FROM

RELINQUISHED BY/REMOVED FROM

RELINQUISHED BY/REMOVED FROM

RECEIVED BY

LABORATORY

SECTION

FINAL SAMPLE

DISPOSAL METHOD

F08-075-094

PAGE 1 OF

PRICE CODE

8 N

DATA TURNAROUND

45 Days / 45 Days

SAMPLE DATE SAMPLE TIME

$5-12-8 \quad 1130$

$5 / 6 \% 8$ Q:O0

$$
31394
$$

SIGN/ PRINT NAMES

DATE/TIME $5-12-8 \times 00$ DATE/TIME

RECEIVED BY/STORED IN

DATE/TTME

MOT45 \&EF \#/ $512-8$ 1600

RECEYYED BY/STORED IN DATE/TIME

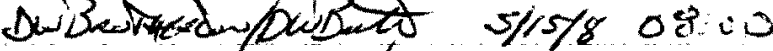

RECEIVED BY/STOREG IN

C. 10 vin. RECEIVED BY/STORED

DATE/TIME

DATE/TIME

the $5 / 15 / 2500$

Date/TIME

DATE/TIME

DATE/TIME
RECEIVED BY /STORED IN

RECEIVED BY/STORED IN

DATE/TIME

RECEIVED BY/STORED IN

\section{SPECIAL INSTRUCTIONS}

SEE PAGE 2 FOR ALL SPECIAL INSTRUCTIONS 


\section{COLLECTOR}

\section{COMPANY CONTACT}

TRENT, STEVE

TELEPHONE NO.

373-5869

\section{PROJECT DESIGNATION}

200-BP-5 OU Characterization for Wells 299-E33-341 and 299-E33-342 -

FIELD LOGBOOK NO.

ACTUAL SAMPLE DEPTH

\section{OFFSITE PROPERTY NO.}

N/A
PROJECT COORDINATOR

WIDRIG, DL

PRICE CODE TURNAROUND

45 Days / 45

45 Days $/ 45$
Days

METHOD OF SHIPMENT

COA

GOVERNMENT VEHICLE

\section{BILL OF LADING/AIR BILL NO.}

N/A

\section{SPECIAL INSTRUCTIONS}

** The 200 Area S\&GRP Characterization and Monitoring Sampling and Analysis GKI applies to this SAF.

** ESL is to determine unsaturated hydraulic conductivity using the method(s) best suited for the soil texture and type.

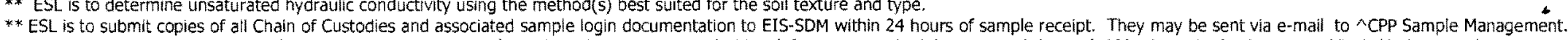

(1)6020M_ICPMS_ASTM_AE (TAL) \{Cadmium, Chromium, Silver\} 6020M_ICPMS_ASTM_AE (Add-On) \{Arsenic, Lead, Molybdenum, Selenium) 6020M_METALS_ICPMS_WE (TAL) \{Cadmium, Chromium, Silver\}

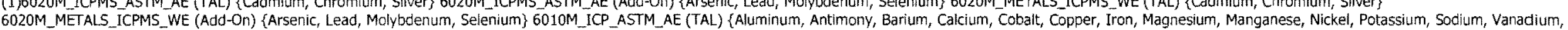

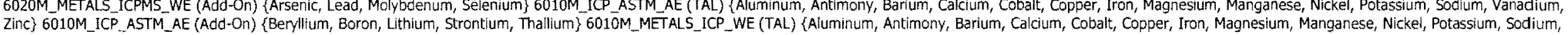

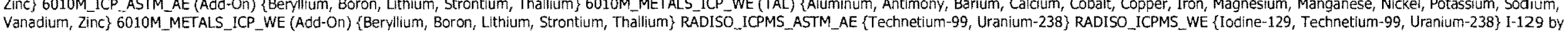

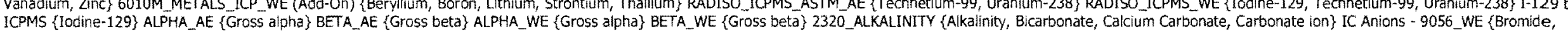
Chioride, Fluoride, Nitrate, Nitrite, Phosphate, Sulfate\} TOC - ASTME1915A \{Total Inorganic Carbon, Total carbon, Total organic carbon\} pH (Water) - 9045 WE; 


\section{COLLECTOR}

NCO SAMPLER MA What

SAMPLING LOCATION

C5856, I-095

ICE CHEST NO.

\section{SHIPPED TO}

Environmental Sciences Laboratory

\section{MATRIX* POSSIBLE SAMPLE HAZARDS/ REMARKS}

$A=A i \quad$ Contains Radioactive Material at concentrations

that are not regulated for transportation per 49

DS=Drum $\quad$ CFR but are not releasable per DOE Order

Solids $\quad 5400.5(1990 / 1993)$

$L=$ Liguid

$\mathrm{O}=\mathrm{Oi}$

$S E=$ Sediment

$T=$ Tissue

$V=$ vegitation

$W=$ Water

$W I=$ Wipe

SPECIAL HANDLING AND/OR STORAGE Radioactive Tie To BITPB5

\section{COMPANY CONTACT}

TRENT, STEVE

TELEPHONE NO.

373-5869

\section{PROJECT DESIGNATION}

200-BP-5 OU Characterization for Wells 299-E33-341 and 299-E33-342-G F08-075

FIELD LOGBOOK NO.

ACTUAL SAMPLE DEPTH

$$
\text { HNF }-N-488^{\circ}-1
$$$$
162.5^{\prime \prime}
$$

OFFSITE PROPERTY NO.

$N / A$

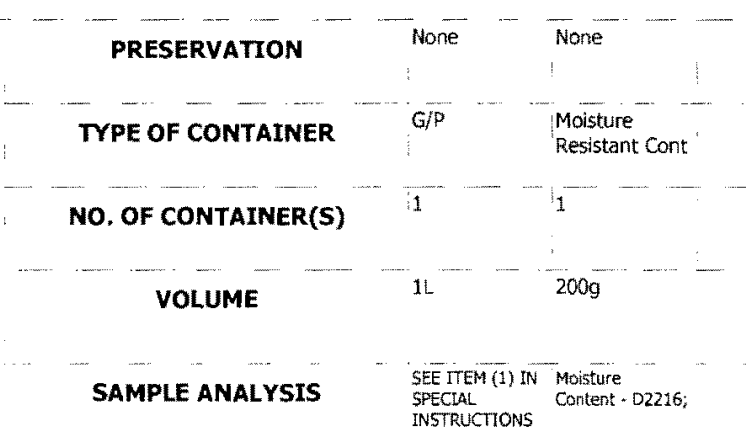

123514 ES10

N/A

\section{SAMPLE DATE SAMPLE TIME \\ 5.081349}

PRICE CODE

\section{METHOD OF SHIPMENT}

GOVERNMENT VEHICLE

\section{BILL OF LADING/AIR BILL NO}

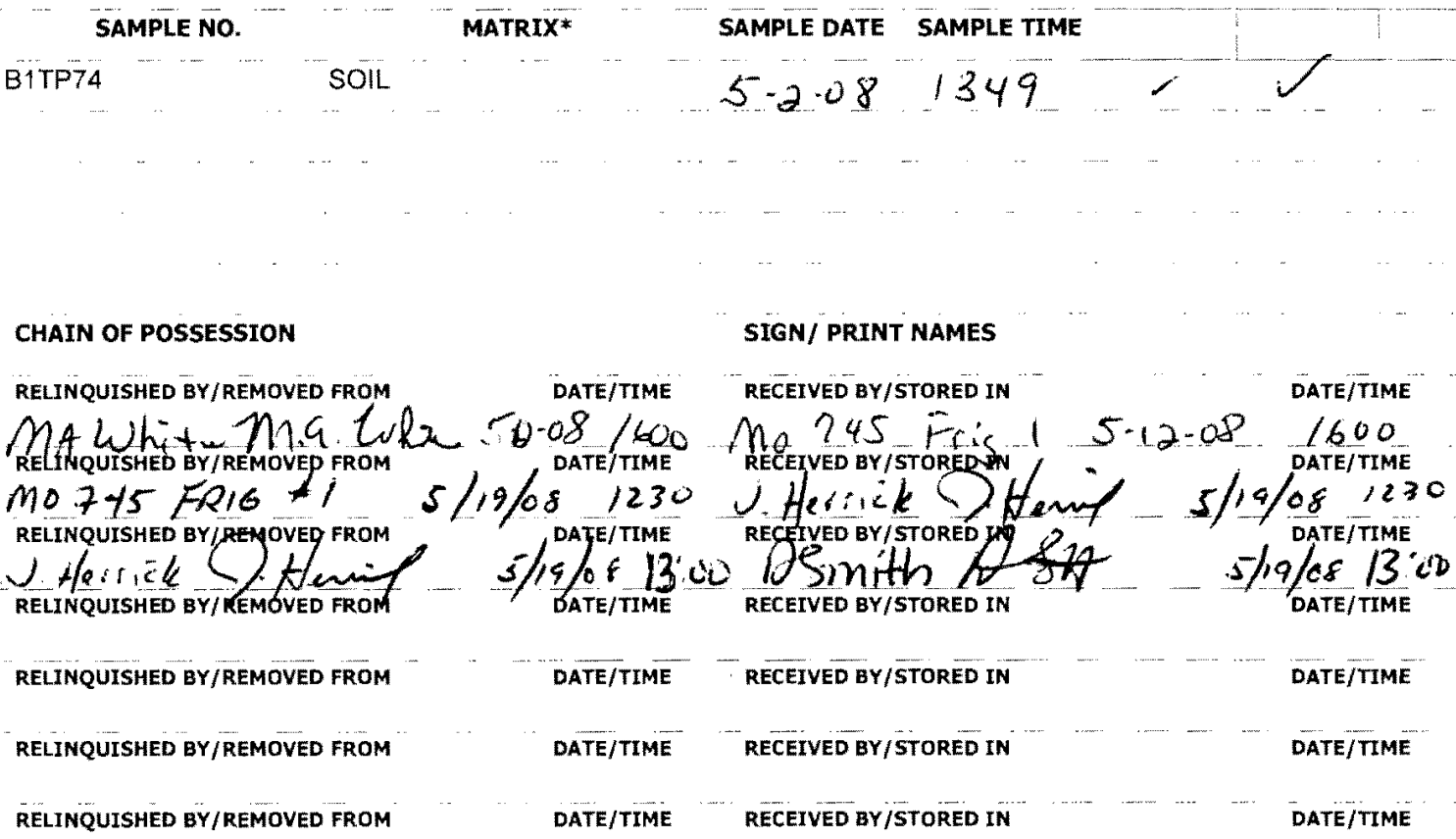

SPECIAL INSTRUCTIONS

SEE PAGE 2 FOR ALL SPECIAL INSTRUCTIONS

\section{LABORATORY RECEIVED BY \\ SECTION




\section{COLLECTOR}

NCO SAMPLER

\section{COMPANY CONTACT}

TRENT, STEVE

PROJECT DESIGNATION

TELEPHONE NO.

$373-5869$

200-BP-5 OU Characterization for Wells 299-E33-341 and 299-E33-342-G

FIELD LOGBOOK NO.

ACTUAL SAMPLE DEPTH

OFFSITE PROPERTY NO.

$\mathrm{N} / \mathrm{A}$

Environmental Sciences Laboratory

\section{SPECIAL INSTRUCTIONS}

** The 200 Area S\&GRP Characterization and Monitoring Sampling and Analysis GKI applies to this SAF.

** ESL is to determine unsaturated hydraulic conductivity using the method(s) best suited for the soll texture and type.

** ESL is to submit copies of all Chain of Custodies and associated sample login documentation to EIS-SDM within 24 hours of sample receipt. They may be sent via e-mail to ${ }^{\wedge}$ CPP Sample Management.

(1)6020M_ICPMS_ASTM_AE (TAL) \{Cadmium, Chromium, Silver\} 6020M_ICPMS_ASTM_AE (Add-On) \{Arsenic, Lead, Molybdenum, Selenium\} 6020M_METALS_ICPMS_WE (TAL) \{Cadmium, Chromium, SIlver\}

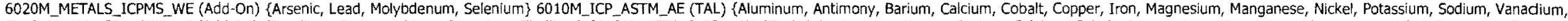

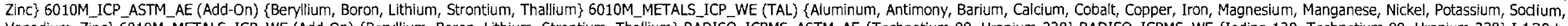

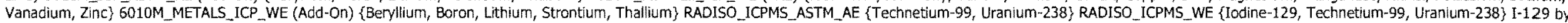

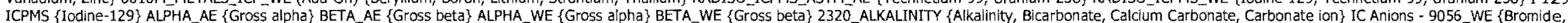

Chloride, Fluoride, Nitrate, Nitrite, Phosphate, Sulfate\} TOC - ASTME1915A \{Total Inorganic Carbon, Total carbon, Total organic carbon\} pH (Water) - 9045 _WE; 
CHAIN OF CUSTODY/SAMPLE ANALYSIS REQUEST

\section{TELEPHONE NO.}

373-5869

nCO SAMPLER Ma Whito

SAMPLING LOCATION

C5856, I-097

ICE CHEST NO.

\section{SHIPPED TO}

Environmental Sciences Laboratory

\section{MATRIX*}

$A=A$ ir

DL=Drum

Liquids
DS=Drum

DS=Drum
Solids

$L=$ Liquid

$0=0 i$

SE $\approx$ Sediment

$S E=$ Sediment
$T=T$ Tissue

$V=$ Vegitation

$W=$ Water

WI=Wipe

$x=$ Other

POSSIBLE SAMPLE HAZARDS/ REMARKS Contains Radioactive Material at concentrations that are not regulated for transportation per 49 CFR but are not releasable per DOE Order $5400.5(1990 / 1993)$

\section{SPECIAL HANDLING AND/OR STORAGE} Radioactive Tie TO B1TPBS

SAMPLE NO.

MATRIX*

B1TP75

SOLL

\section{CHAIN OF POSSESSION}

RELINQUISHED BY/REMOVED FROM

DATE/TIME

Mawhitu Ma. Whate 5.12.03/60:

REINOUISHED BY/REMOVED FROM DATE/TIME

Molis Fis $5 / 15 / 0$ \% 08100

RELINQUISHED BY/REMOVED FROMT DATE/TIME

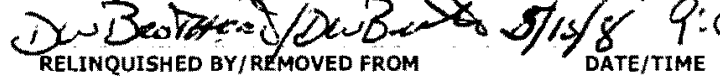

RELINOUISHED BY/REMOVED FROM

DATE/TIME

RELINQUISHED BY/REMOVED FROM

RELINQUISHED BY/REMOVED FROM

DATE/TMME

DATE/TIME

TRENT, STEVE

\section{PROJECT DESIGNATION}

200-BP-5 OU Characterization for Wells 299-E33-341 and 299-E33-342-G F08-075 FIELD LOGBOOK NO.

ACTUAL SAMPLE DEPTH

COA

HNF.N. 488.1

$165^{\prime}$

OFFSITE PROPERTY NO.

N/A

\section{PRESERVATION}

TYPE OF CONTAINER G

NO. OF CONTAINER(S)

VOLUME $\quad$ II $\quad 2009$

SEE TEM (1) IN Moisture
SAMPLE ANALYYSIS
SPECAL
INSTRUTHONS Content- D2216;

SAMPLE DATE SAMPLE TIME

$5 \cdot 12 \cdot 08 \quad 1436$

\section{SIGN/ PRINT NAMES}

RECEIVED BY/STORED IN

Mu 745 Frif

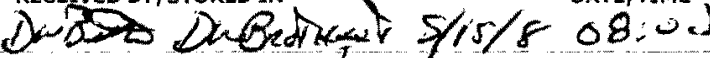

RECEIVED BY/STORED IN / D DATE/TIME

Ton $5 / 15 / 300$

RECEIVED BY/STORE IN $\$$ DATE/TIME

DATE/TIME

RECEIVED BY/STORED IN

DATE/TIME

RECEIVED BY/STORED IN
RECEIVED BY/STORED IN

PROIECT COORDINATOR

WIDRIG, DL

PAGE 1 OF 2

SAF NO.

123514 ES10

BILL OF LADING/AIR BILL NO.

\section{SPECIAL INSTRUCTIONS}

SEE PAGE 2 FOR ALL SPECIAL INSTRUCTIONS

\footnotetext{
LABORATORY RECEIVED BY SECTION

FINAL SAMPLE DISPOSAL METHOD

DISPOSITION
} 


\section{COLLECTOR}

NCO SAMPLER

\section{COMPANY CONTACT}

TRENT, STEVE

\section{TELEPHONE NO.}

$373-5869$

PROJECT COORDINATOR

\section{PROJECT DESIGNATION}

WIDRIG, DL

PRICE CODE

$\mathbf{8 N}$

DATA

SAMPLING LOCATION

200-BP-5 OU Characterization for Wells 299-E33-341 and 299-E33-342-G

FIELD LOGBOOK NO.

ACTUAL SAMPLE DEPTH

SAF NO.

AIR QUALITY

TURNAROUND

45 Days / 45

Days

\section{OFFSITE PROPERTY NO.}

$N / A$
COA

$123514 E S 10$
METHOD OF SHIPMENT GOVERNMENT VEHICLE

\section{SHIPPED TO}

Environmental Sciences Laboratory

\section{BILL OF LADING/AIR BILL NO.}

N/A

\section{SPECIAL INSTRUCTIONS}

** The 200 Area S\&GRP Characterization and Monitoring Sampling and Analysis GKI applies to this SAF.

** ESL is to determine unsaturated hydraulic conductivity using the method(s) best suited for the soil texture and type.

** ESL is to submit copies of all Chain of Custodies and associated sample login documentation to EIS-SDM within 24 hours of sample receipt. They may be sent via e-mail to ^CPP Sample Management.

(1)6020M_ICPMS_ASTM_AE (TAL) \{Cadmium, Chromium, Silver\} 6020M_ICPMS_ASTM_AE (Add-On) \{Arsenic, Lead, Molybdenum, Selenium\} 6020M_METALSIICPMS_WE (TAL) \{Cadmium, Chromium, Silver\}

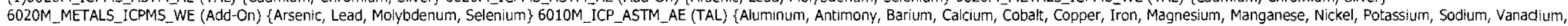

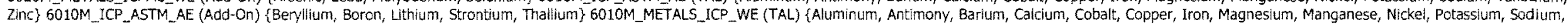

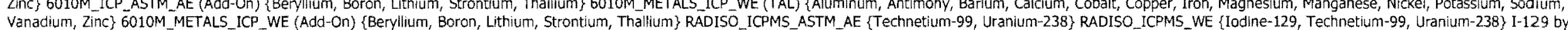

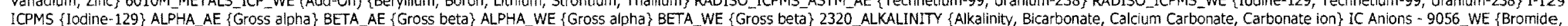
Chloride, Fiuoride, Nitrate, Nitrite, Phosphate, Sulfate\} TOC-ASTME1915A \{Total Inorganic Carbon, Total carbon, Total organic carbon) pH (Water) - 9045 _WE; 
CHAIN OF CUSTODY/SAMPLE ANALYSIS REQUEST

\section{TELEPHONE NO.}

$373-5869$
F08-075-100

PRICE CODE

WIDRIG, OL

PROJECT DESIGNATION

200-BP-5 OU Characterization for Wells 299-E33-341 and 299-E33-342 - G FIELD LOGBOOK NO. ACTUAL SAMPLE DEPTH

\section{SAF NO.}

COA

$H N F \cdot N-489-1$ $16.35^{\prime}$ FFSITE PROPERTY NO.

$N / A$

$123514 \mathrm{ES} 10$

BILL OF LADING/AIR BILL NO.

\section{Environmental Sciences Laboratory}

MATRIX* POSSIBLE SAMPLE HAZARDS/ REMARKS $A=A$ ir $\quad$ POSSIBLE SAMPLE HAZARDS/ REMARKS

DL=Drum that are not regulated for transportation per 49
Liqu

$D S=D r u m \quad C F R$ but are not releasable per DOE Orde

Solids $\quad 5400.5(1990 / 1993)$

$0=0$ il
$S=50$ il

$S=$ SE $=$ Sediment
$T=$ Tissue

$V=$ Vegitation

$W=$ Water
$W I=$ Wipe

$X=0$ ther

SPECIAL HANDLING AND/OR STORAGE Radioactive Tie To B1TPB5

\begin{tabular}{|c|c|c|}
\hline PRESERVATION & None & None \\
\hline TYPE OF CONTAINER & G/P & $\begin{array}{l}\text { Moisture } \\
\text { Resistart Cont }\end{array}$ \\
\hline NO. OF CONTAINER(S) & 1 & 1 \\
\hline VOLUME & 1L & 2009 \\
\hline SAMPLE ANALYSIS & $\begin{array}{l}\text { SEE TTEM (1) IN } \\
\text { SPECIAL } \\
\text { INSTRUCTIONS }\end{array}$ & $\begin{array}{l}\text { Moisture } \\
\text { Content - } 02216 \text {; }\end{array}$ \\
\hline
\end{tabular}

\section{SAMPLE NO. MATRIX}

SOIL

SAMPLE DATE SAMPLE TIME

$5-13-03 \quad 0 p+7$

PAGE 1 OF 2 DATA TURNAROUND 45 Days / 45 Days

\section{CHAIN OF POSSESSION}

RELINQUISHED BY/REMOVED FROM

Ma whit Yhatho.

REINOUISHED BY/REMOVED FROM

Mo745 NAO $5 / 152$

TELINOYYSHED BY/REMOVED EROM

DLUX SratrEef-z/DLd

RELINQUISHED BY/REMOVED FROM

RELINQUISHED BY/REMOVED FROM

RELINQUISHED BY/REMOVED FROM

RECEIVED BY

LABORATORY

SECTION

FINAL SAMPLE

DISPOSITION

\section{$10 T^{\#}$}

DATE/TIME

$8 / 600$ of -003 DATE/TIME DATE/TIME

DATE/TIME

DATE/TIME

DATE/TIME

\subsection{4 \\ SIGN/ PRINT NAMES}

RECEIVED BY/STORED IN DATE/TIME

\section{SPECIAL INSTRUCTIONS}

Mo 745 Fris

Dubedfeerofondas RECEIVED BYISTORED IN

\section{C.Iorn}

RECEIVED BY/STORED

$15 / 8$ o8:icis

RECEIVED BY/STORED IN

RECEIVED BY/STORED IN

RECEIVED BY/STORED IN 


\section{COLLECTOR}

NCO SAMPLER

\section{SAMPLING LOCATION}

C5856, I-099

ICE CHEST NO.

\section{SHIPPED TO}

Environmental Sciences Laboratory

\section{COMPANY CONTACT}

TRENT, STEVE

PROJECT DESIGNATION

200-BP-5 OU Characterization for Wells 299-E33-341 and 299-E33-342-G FIELD LOGBOOK NO.

ACTUAL SAMPLE DEPTH

\section{OFFSITE PROPERTY NO.}

N/A
PROJECT COORDINATOR

WIDRIG, DL

\section{SAF NO.}

F08-075

COA

123514 ES10
PRICE CODE

$8 \mathrm{~N}$

AIR QUALITY

METHOD OF SHIPMENT

GOVERNMENT VEHICLE

\section{SPECIAL INSTRUCTIONS}

** The 200 Area S\&GRP Characterization and Monitoring Sampling and Analysis GKI applies to this SAF.

** ESL is to determine unsaturated hydraulic conductivity using the method(s) best suited for the soil texture and type.

** ESL is to submit copies of all Chain of Custodies and associated sample login documentation to EIS-SDM within 24 hours of sample receipt. They may be sent via e-mail to ${ }^{2}$ CPP Sample Management.

(1)6020M_ICPMS_ASTM_AE (TAL) \{Cadmium, Chromium, Silver\} 6020M_ICPMS_ASTM_AE (Add-On) \{Arsenic, Lead, Molybdenum, Selenium\} 6020M_METALS_ICPMS_WE (TAL) \{Cadmium, Chromium, Silver\}

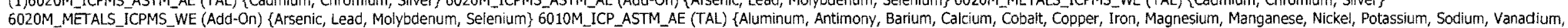

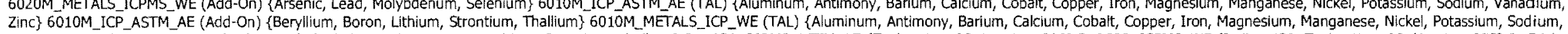

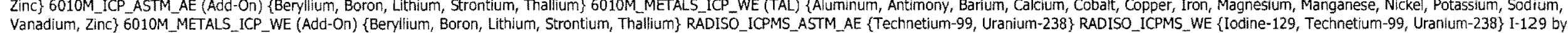

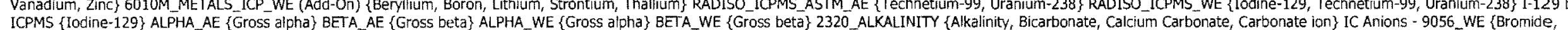
ICPMS \{lodine-129\} ALPHA_AE \{GrosS alpha\} BETA_AE \{Gross beta\} ALPHA_WE \{GrosS alpha\} BETA_WE \{Gross beta\} 2320_ALKALINITY \{Alkalinity, Bicarbona.
Chloride, Fluoride, Nitrate, Nitrite, Phosphate, Sulfate\} TOC - ASTME1915A \{Total Inorganic Carbon, Total carbon, Total organic carbon\} pH (Water) -9045_WE; 


\section{CHAIN OF CUSTODY/SAMPLE ANALYSIS REQUEST}

\section{TELEPHONE NO.}

373-5869

TRENT, STEVE

\section{PROJECT DESIGNATION}

200-BP-5 OU Characterization for Wells 299-E33-341 and 299-E33-342-G FIELD LOGBOOK NO. ACTUAL SAMPLE DEPTH

$$
H N=-N-498 \cdot 1
$$$$
170^{\prime}
$$

OFFSITE PROPERTY NO.

N/A

Environmental Sclences Laboratory

\section{MATRIX* POSSIBLE SAMPLE HAZARDS/REMARKS $A=$ Air
$\mathrm{OL}=\mathrm{Drum}$$\quad$ Contains Radioactive Material at concentrations Liquids that are not regulated for transportation per 49 DS=Drum CFR tuit are not releasable per DOE Order Solids $\quad 5400.5(1990 / 1993)$ \\ $L=$ Liquid \\ $0=$ iil \\ $\mathrm{SE}=$ Sediment \\ $T=$ Tissue \\ $V=$ Vegitation
$W=$ water
$W$ \\ $W=$ water \\ $W=$ Wipe
$x=$ Other \\ SPECIAL HANDLING AND/OR STORAGE Radloactive TIE TO B1TPB5}

SAMPLE NO. MATRIX*

B1TP77

SOLL

\section{CHAIN OF POSSESSION}

LOT

RELINQUISHED BY/REMOVED FROM

DATE/TIME

\section{SIGN/ PRINT NAMES}

\section{4}

Ma whitu Matol RELINQUISHED BY/REMOVEQ FROM Po: 715 Hes 51308.000 RECEIVED BY/STORED IN MO 745 Fri

5-13.08 1600

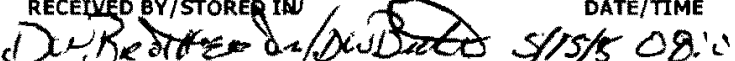

RELINQUISHED EY/REMOVED FROM

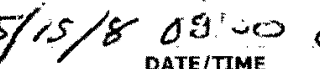
DATEITME

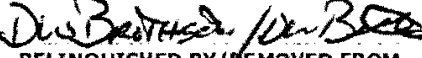
$515 / 8$ Q:00

RELINQUISHED BY/REMOVED FROM DATE/TIME

RELINQUISHED BY/REMOVED FROM

DATE/TIME

RELINQUISHED BY/REMOVED FROM

DATE/TIME

RECEIVED BY/STORED IN

$$
\text { C. } \frac{100 \mathrm{in}}{}
$$

RECEIVED BY/STOR

RECEIVED BY/STORED IN

\section{DATE/TTME}

$5 / 1 / 2089: 00$

DATE/TIME

DATE/TIME

RECEIVED BY/STORED IN

DATE/TIME

DATE/TIME

RECEIVED BY/STORED IN

DATE/TIME

RELINQUISHED BY/REMOVED FROM

\section{SPECIAL INSTRUCTIONS}

SEE PAGE 2 FOR ALL SPECIAL INSTRUCTIONS

RECEIVED BY

SECTION

FINAL SAMPLE 


\section{COLLECTOR}

NCO SAMPLER

\section{SAMPLING LOCATION}

C5856, I 1100

ICE CHEST NO.

\section{SHIPPED TO}

Environmental Sciences Laboratory

\section{COMPANY CONTACT}

TRENT, STEVE

TELEPHONE NO.

373-5869

\section{PROJECT DESIGNATION}

200-BP-5 OU Characterization for Wells 299-E33-341 and 299-E33-342-G

\section{FIELD LOGBOOK NO.}

ACTUAL SAMPLE DEPTH

\section{OFFSITE PROPERTY NO}

$N / A$

\section{PRICE CODE BN}

AIR QUALITY
WIDRIG, DL

SAF NO.

F08-075

COA

123514ES10
DATA TURNAROUND

45 Days / 45

Days

\section{SPECIAL INSTRUCTIONS}

** The 200 Area S\&GRP Characterization and Monitoring Sampling and Analysis GKI applies to this SAF.

** ESL is to determine unsaturated hydraulic conductivity using the method(s) best suited for the soil texture and type.

** ESL is to submit copies of all Chain of Custodies and associated sample login documentation to EIS-SDM within 24 hours of sample receipt. They may be sent via e-mall to $\wedge$ CPP Sample Management.

(1)6020M_ICPMS_ASTM_AE (TAL) \{Cadmium, Chromium, Silver\} 6020M_ICPMS_ASTM_AE (Add-On) \{Arsenic, Lead, Molybdenum, Selenium\} 6020M_METALS_ICPMS_WE (TAL) \{Cadmium, Chromium, Silver\}

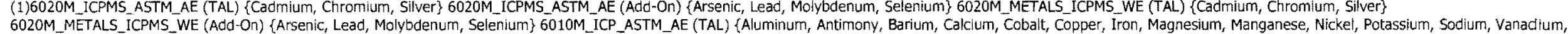

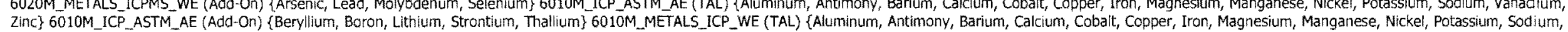

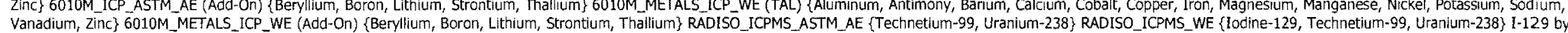

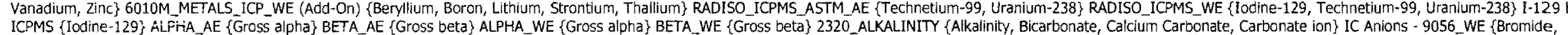
ICPMS \{lodine-129\} ALFriA_AE \{Gross alpha\} BETA_AE \{Gross beta\} ALPHA_WE \{Gross alpha\} BETA_WE \{Gross beta\} 2320_ALKALINITY \{Alkalinity, Bicarbona:
Chloride, Fluoride, Nitrate, Nitrite, Phosphate, Sulfate\} TOC. ASTME1915A \{Total Inorganic Carbon, Total carbon, Total organic carbon\} pH (Water) -9045_WE; 


\section{COLLECTOR}

NCO SAMPLER Mr whit -

SAMPLING LOCATION

C5856, 1-105

ICE CHEST NO.

\section{SHIPPED TO}

Environmental Sciences Laboratory

MATRIX*

A-Air

$\mathrm{DL}=\mathrm{Drum}$

Lquids
DS $=$ Drum

Solids

Solids
$L=$ Liquid
$0=0 \mathrm{i}$

$0=0$ il
$S=S$ oil

$S=S$ oil
$S E=$ Sediment

$T=T$ issue

$V=$ Vegitation

$W=$ Water

WI $=$ Wipe

SPECIAL HANDLING AND/OR STORAGE Radioactive Tie To B1TPBG

STELE SAMPLE HAZARDS/ REMARKS Contains Radioactive Material at concentrations that are not regulated for transportation per 49 CFR but are not rejeasable per DOE Order $5400.5(1990 / 1993)$

MATRIX*

B1TP78

SOIL

\section{COMPANY CONTACT}

TRENT, STEVE

TELEPHONE NO.

373-5869

\section{PROJECT DESIGNATION}

200-BP-5 QU Characterization for Wells 299-E33-341 and 299-E33-342-G FIELD LOGBOOK NO.

$$
\text { ACTUAL SAMPLE DEPTH }
$$$$
80)^{\prime}
$$

$4 N F-N-48 D$

OFFSITE PROPERTY NO.

N/A

$\begin{array}{ccc}\text { PRESERVATION } & \text { None } & \text { None } \\ \text { TYPE OF CONTAINER } & \text { G/P } & \begin{array}{l}\text { Moisture } \\ \text { Resistant Cont }\end{array} \\ \text { NO. OF CONTAINER(S) } & 1 & 1 \\ \text { VOLUME } & 11 & 2009 \\ \text { SAMPLE ANALYSIS } & \begin{array}{l}\text { SEE TIEM (1) IN Moisture } \\ \text { SPECAL } \\ \text { INSTRUCTIONS }\end{array}\end{array}$

SAMPLE DATE SAMPLE TIME

$5.13-080955$
PRICE CODE

$8 N$

DATA TURNAROUND

45 Days / 45

AIR QUALITY

\section{CHAIN OF POSSESSION}

RELINQUISHED BY/REMOVED FROM Mol TS TRO1 DATE/TIME RELINQUISHEt BYYREMOVED FROM $5.13-08$ DATE/TIME Mo745 Friy/ 5.20.08 1200 RELINQUISHED BY/REMUVED FROM Mawhit Ma100\% 5.2008 RELINQUISHED BY/REMOVED FROM

RELINQUISHED BY/REMOVED FROM

RELINQUISHED BY/REMOVED FROM

RELINQUISHED BY/REMOVED FROM DATE/TIME

DATE/TIME

DATE/TIME

DATE/TIME

\section{LTS $5 \cdot 3 \cdot 07$}

RECEIVED BY/STORED IN

\section{SPECIAL INSTRUCTIONS}

SEE PAGE 2 FOR ALL SPECIAL INSTRUCTIONS

LABORATORY RECEIVED BY

RECEIVEO BY/STORED IN

Fo8-075

123514 ES 10

METHOD OF SHIPMENT

GOVERNMENT VEHICLE

BILL. OF LADING/AIR BILL NO.

N/A

Ma whit That wete 5-2003/200

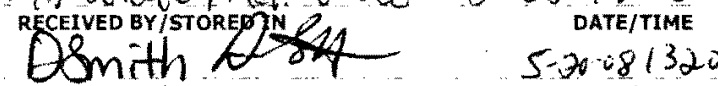

bimith

$-20,81320$

RECEIVED BY/STORED IN

DATE/TIME

RECEIVED BY/STORED IN

DATE/TIME

RECEIVED BY/STORED IN

DATE/TIME 


\section{CHAIN OF CUSTODY/SAMPLE ANALYSIS REQUEST}

\section{TELEPHONE NO.}

$373-5869$

\section{TRENT, STEVE}

PROJECT DESIGNATION

200-BP-5 OU Characterization for Wells 299-E33-341 and 299-E33-342-G FIELD LOGBOOK NO.

ACTUAL SAMPLE DEPTH

\section{OFFSITE PROPERTY NO}

N/A

\section{F08-075-103}

PAGE 2 OF 2

DATA TURNAROUND

45 Days / 45

Days

AIR QUALITY

\section{$\cos$}

123514 ES10

\section{METHOD OF SHIPMENT}

GOVERNMENT VEHICLE
SHIPPED TO

Environmental Sciences Laboratory
BILL OF LADING/AIR BILL NO.

N/A

\section{SPECIAL INSTRUCTIONS}

** The 200 Area S\&GRP Characterization and Monitoring Sampling and Analysis GKI applies to this SAF.

** ESL is to determine unsaturated hydraulic conductivity using the method(\$) best suited for the soil texture and type.

** ESL is to submit copies of all Chain of Custodies and associated sample login documentation to EIS-SDM within 24 hours of sample receipt. They may be sent via e-mail to $A$ CPP Sample Management.

(1)6020M_ICPMS_ASTM_AE (TAL) \{Cadmium, Chromium, Silver\} 6020M_ICPMS_ASTM_AE (Add-On) \{Arsenic, Lead, Molybdenum, Selenium\} 6020M_METALS_ICPMS_WE (TAL) (Cadmium, Chromium, Silver\}

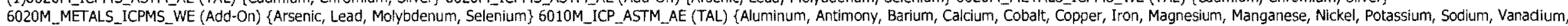

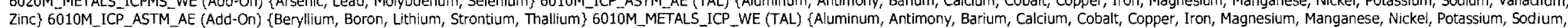

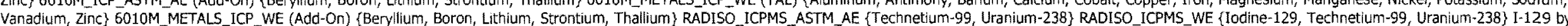

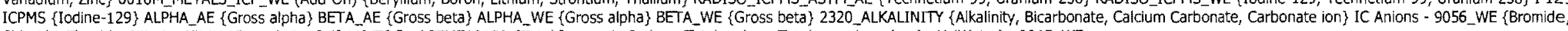

Chloride, Fluoride, Nitrate, Nitrite, Phosphate, Sulfate\} TOC - ASTME1915A \{Total Inorganic Carbon, Total carbon, Totai organic carbon\} pH (Water) - 9045 -WE 
NCO SAMPLER Malukt

\section{SAMPLING LOCATION}

C5856, i-107

ICE CHEST NO.

\section{SHIPPED TO}

Environmental Sciences Laboratory

\section{MATRIX* POSSIBLE SAMPLE HAZARDS/ REMARKS \\ $A=A i r \quad$ Contains Radioactive Material at concentrations \\ jouids that are not regulated for transportation per 49 \\ DS=Drum CFR but are not releasable per DOE Order \\ Solids $\quad 5400.5(1990 / 1993)$ \\ $L=$ Liquir \\ $0=0$ il
$S=S$ il \\ $\mathrm{SE}=$ Sediment \\ $T=$ Tissue
$V=$ vegitation \\ $V=$ vegitation
$W=$ water \\ $W=$ water
$W 1=W$ ipe \\ $x=$ other \\ SPECIAL HANDLING AND/OR STORAGE} Radioactive Tie To BITPBE

\section{SAMPLE NO.}

MATRIX*

B1TP79

SOIL

CHAIN OF POSSESSION
RELINQUISHED BY/REMOVED FROM
RA Whit
RELINQUISHED BY/REMOVED FROM
RELINQUISHED BY/REMOVED FROM
REUINQUISHED BY/REMOVED FROM
RELINQUISHED BY/REMOVED FROM
RELINQUISHED BY/REMOVED FROM
LABORATORY RECEIVED BY
SECTION
FINAL SAMPLE DISPOSAL METHOD
DISPOSITION

$$
405
$$

DATE/TIME $5.13 .08 / 600$ 8 DATE/TIME 1320

DATE/TIME

DATE/TIME

DATE/TIME

\section{TELEPHONE NO.}

373-5869

TRENT, STEVE

\section{Project}

WIDRIG, DL

SAF No.

200-BP-5 OU Characterization for Welis 299-E33-341 and 299-E33-342-G F08-075

FIELD LOGBOOK NO.

ACTUAL SAMPLE DEPTH

COA

$$
\text { HNF-N-488-1 182.5 }
$$

OFFSITE PROPERTY NO.

$\mathrm{N} / \mathrm{A}$

\section{BILL OF LADING/AIR BILL NO}

$N / A$

PRICE CODE $\quad$ 8N

METHOD OF SHIPMENT GOVERNMENT VEHICLE

\section{PRESERVATION}

TYPE OF CONTAINER

GP

Mesistare

NO. OF CONTAINER(S)

VOLUME

11. $\quad 2000$

SAMPLE ANALYSIS SEE ITEM (1) IN Moisture
SPECIAL
INSTRUCTONS COntent - D2216

SAMPLE DATE SAMPLE TIME

$5 \cdot 13.08 \cdot 1025$

\section{SIGN/ PRINT NAMES}

0.31394

RECEIVED BY/STORED IN

Mo 745 Fris

Mathein Ma wht 520.081200 REGEIVEDAY/STORFEIN O DATE/TIME

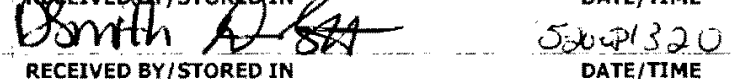

RECEIVED BY/STORED IN

RECEIVED BY/STORED IN

RECEIVED BY/STORED IN
DATE/TIME

DATE/TIME

DATE/TIME

\section{SPECIAL INSTRUCTIONS}

SEE PAGE 2 FOR ALL SPECIAL INSTRUCTIONS 


\section{COLLECTOR}

NCO SAMPLER

\section{SAMPLING LOCATION}

C5856, $1-107$

ICE CHEST NO.

\section{SHIPPED TO}

Enviromental Sciences Laboratory

\section{COMPANY CONTACT}

TRENT, STEVE

PROJECT DESIGNATION

200-BP-5 OU Characteriz

\section{FIELD LOGBOOK NO.}

\section{ELEPHONE NO.}

373-5869

\section{PROJECT COORDINATOR}

WIDRIG, DL

\section{SAF NO.}

F08-075

\section{METHOD OF SHIPMENT}

\section{OFFSITE PROPERTY NO.}

123514 ES10

ACTUAL SAMPLE DEPTH

\section{BILL OF LADING/AIR BILL NO}

\section{SPECIAL INSTRUCTIONS}

** The 200 Area S\&GRP Characterization and Monitoring Sampling and Analysis GKI applies to this SAF.

* ESL is to determine unsaturated hydraulic conductivity using the method(s) best suited for the soil texture and type.

** ESL is to submit copies of all Chain of Custodies and associated sample login documentation to EIS-SOM within 24 hours of sample receipt. They may be sent via e-mail to ^CPP Sample Management.

(1)6020M ICPMS ASTM AE (TAL) (Cadmium, Chromium, Silver\} 6020M ICPMS ASTM AE (Add-On) \{Arsenic, Lead, Molybdenum, Selenium\} 6020M METALS_ICPMS WE (TAL) \{Cadmium, Chromium, Silver\}

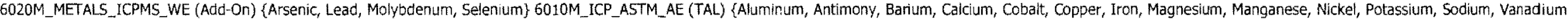

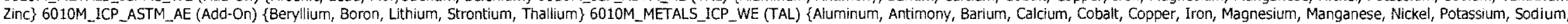

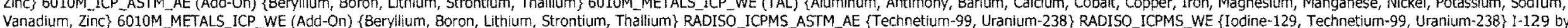

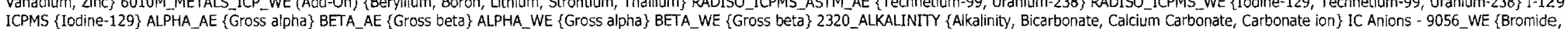

ICPMS \{Iodine-129\} ALPHA_AE \{GrosS alpha\} BETA_AE \{GroSS beta\} ALPHA WE \{Gross alpha\} BETA_WE \{Gross beta\} 2320_ALKALINITY \{Alkalinity, Bicarbonate
Chloride, Fluoride, Nitrate, Nitrite, Phosphate, Sulfate\} TOC - ASTME1915A (Total Inorganic Carbon, Total carbon, Total organic carbon\} pH (Water) - 9045 WE; 


\section{CHAIN OF CUSTODY/SAMPLE ANALYSIS REQUEST}

\section{TELEPHONE NO.}

373-5869

\section{COMPANY CONTACT}

TRENT, STEVE

PROJECT DESIGNATION

200-BP-5 OU Characterization FIELD LOGBOOK NO.

$$
\text { TNF-N-488-1 }
$$

OFFSITE PROPERTY NO

N/A

Environmental Sciences Laboratory

MATRIX*

$A=$ Air

$\mathrm{DL}=\mathrm{Drum}$

biquids

DS:Drum
Solids

POSSIBLE SAMPLE HAZARDS/ REMARKS Contains Radioactive Material at concentrations that are not regulated for transportation per 49 CFR but are not releasable per DOE Order $5400.5(1990 / 1993)$

$0=011$

$S=5$ oil

$\mathrm{SE}=$ Sediment
$\mathrm{T}=$ Tissue

$T=$ rissue
$V=$ Vegitation

$W=$ water

$W 1=$ Wipe

$x=$ other

SPECLAL HANDLING AND/OR STORAGE Radioactive Tie To B1TPB6
SAMPLE NO.

MATRIX*
SOIL

\section{$10 T^{4}$}

\section{CHAIN OF POSSESSION}

RELINQUISHED BY/REMOVED FROM

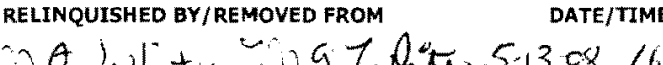
RELINQUISHEO BY/REM bVED FROM Mo 745 Fri 5 $20-08 / 200$ RELINQUISHED BY/REMOVED FROM DATE/TIME

RELINQUISHED BY/REMOVED FROM DATE/TIME RELINQUISHED BY/REMOVED FROM DATE/TIME DATE/TIME

RELINOUISHED BY/REMOVED FROM MA Whitenglulut 520081320

\section{4}

RECEIVED BY/STORED IN

\section{SIGN/ PRINT NAMES}

F08-075-107

PAGE 1 OF 2

PROJECT COORDINATOR

WIDRIG, DL

PRICE CODE

$8 N$

DATA TURNAROUND 45 Days / 45 Days

AIR QUALITY
SAMPLE DATE SAMPLE TIME

$5-1308 / 302$

COA

METHOD OF SHIPMENT

123514 ES 10

GOVERNMENT VEHICLE

GILL OF LADING/AIR BILL NO N/A

Mo?45 Fri $15-13-08 / 600$

RECEIVED BY/STORED IN

Mawht Mawhio 52008 /200

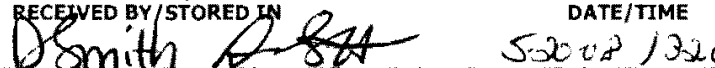

DATE/TIME

\section{SPECIAL INSTRUCTIONS}

SEE PAGE 2 FOR ALL SPECIAL INSTRUCTIONS

RECEIVED BY

TITLE

DATE/TIME 


\section{COMPANY CONTACT}

TRENT, STEVE

TELEPHONE NO.

PROJECT DESIGNATION

373-5869

PROJECT COORDINATOR

WIDRIG, DL

PRICE COD

AIR QUALITY

DATA TURNAROUND

45 Days / 45 Days
C5AMPL, [-109

ICE CHEST NO.

\section{SHIPPED TO}

Environmental Sciences Laboratory
200-BP-5 OU Characterization for Wells 299-E33-341 and 299-E33-342-G

FIELD LOGBOOK NO.

ACTUAL SAMPLE DEPTH

\section{OFFSITE PROPERTY NO}

METHOD OF SHIPMENT

$\mathrm{COA}$

$123514 E S 10$

\section{GOVERNMENT VEHICLE}

\section{SPECIAL. INSTRUCTIONS}

** The 200 Area S\&GRP Characterization and Monitoring Sampling and Analysis GKI applies to this SAF.

** ESL is to determine unsaturated hydraulic conductivity using the method(s) best suited for the soll texture and type.

** ESL is to submit copies of all Chain of Custodies and associated sample login documentation to EIS-SDM within 24 hours of sample receipt. They may be Sent via e-mail to ${ }^{\wedge}$ CPP Sample Management.

(1)6020M ICPMS ASTM AE (TAL) \{Cadmium, Chromium, Silver\} 6020M_ICPMS ASTM_AE (Add-On) \{Arsenic, Lead, Molybdenum, Selenium\} 6020M_METALS ICPMS WE (TAL) \{Cadmium, Chromium, Silver\}

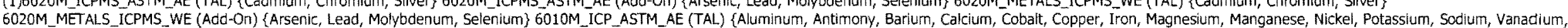

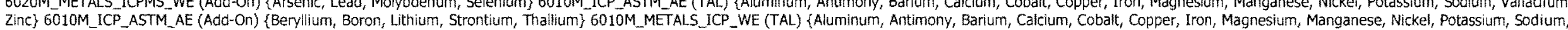

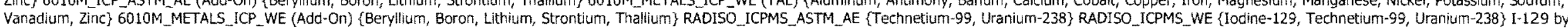

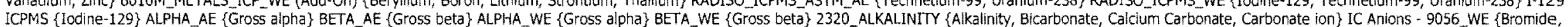
Chloride, Fluoride, Nitrate, Nitrite, Phosphate, Sulfate\} TOC - ASTME1915A (Total Inorganic Carbon, Total carbon, Total organic carbon\} pH (Water) - 9045 _WE; 


\section{COLLECTOR}

NCO SAMPLER MA WUitite SAMPLING LOCATION

C5856, I-111

ICE CHEST NO.

\section{SHIPPED TO}

Environmental Sciences Laboratory

MATRIX*

$A=$ Air

$\begin{aligned} & A=\text { Air } \\ & D L\end{aligned}=$ Drum

Lquids

DS=Drum
Solids

Solids

$O=0$ ii
$S=5$ it

$S=S$ onl
$S E=$ Sediment:
$T=$ Tissue

$T=1$ issue
$Y=$ Vegitation

$W=$ Water

WI $=$ Wipe

$x=$ Other

POSSIBLE SAMPLE HAZARDS/ REMARKS Contains Radioactive Material at concentrations that are not regulated for transportation per 49 CFR but are not releasable per DOE Order $5400.5(1990 / 1993)$

\section{SPECIAL HANDLING AND/OR STORAGE} Radioactive Tie To B1TPB6

\section{SAMPLE NO. MATRIX*}

B1TP81

SOIL

\section{TELEPHONE NO.}

373-5869
PROJECT COORDINATOR

WIDRIG, DL

\section{SAF NO.}

200-BP-5 OU Characterization for Wells 299-E33-341 and 299-E33-342-G F08-075 FIELD LOGBOOK NO.

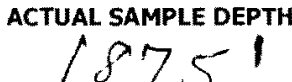

COA

123514 ES10

BILL OF LADING/AIR BILL NO.

\section{OFPSITE PROPERTY NO.}

N/A

SAMPLE DATE SAMPLE TIME

$5 \cdot 13 \cdot 09 \cdot 133 y$
PRICE CODE

$8 \mathrm{~N}$ MO 745 F $15+3-08^{\circ}$ DATE/TIME

$$
\text { LUTH }
$$

\section{CHAIN OF POSSESSION}

RELINQUISHED BY/REMOVED FROM/

\section{SIGN/ PRINT NAMES}

RECEIVED BY/STORED IN
Maluhite 729.2 RELINQUISHED BY/REMOVEO FROM

5.3331600

\section{4} DATE/TIME

Mo 745 Frid 5.30 .08 1 100 Malukire Mawhit RELINQUISHED BY/REMOVED FROM $5.2008 / 320$

RELINOUISHED BY/REMOVED FROM

DATE/TIME

RELINQUISHED BY/REMOVED FROM

DATE/TIME

DATE/TIME RECEIVED BY/STORED IN RECEIVED BY/STORED IN

RELINQUISHED BY/REMOVED FROM

LABORATORY
SECTION
FINAL SAMPLE
DISPOSITION

Ma. Wibt MA white $5.2008 / 200$

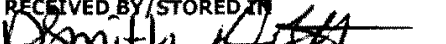
$5-20.08 \quad 1320$ DATE/TIME RECEIVED BY/STORED IN

DATE/TTME DATE/TIME

\section{SPECIAL INSTRUCTIONS}

METHOD OF SHIPMENT GOVERNMENT VEHICLE
N/A 


\section{COLLECTOR}

NCO SAMPLER

\section{SAMPLING LOCATION}

C5856, I-111

ICE CHEST NO.

\section{SHIPPED TO}

Environmental Sciences Laboratory

\section{COMPANY CONTACT}

TRENT, STEVE

TELEPHONE NO.

373-5869

\section{PROJECT DESIGNATION}

200-BP-5 OU Characterization for Wells 299-E33-341 and 299-E33-342-G FIELD LOGBOOK NO.

ACTUAL SAMPLE DEPTH

\section{OFFSITE PROPERTY NO}

N/A
PROJECT COORDINATOR

WIDRIG, DL

SAF NO.

F08-075

COA

123514 ES10
PRICE CODE $\quad \mathbf{8 N}$

AIR QUALITY

\section{METHOD OF SHIPMENT}

GOVERNMENT VEHICLE

\section{SPECIAL INSTRUCTIONS}

** The 200 Area S\&GRP Characterization and Monitoring Sampling and Analysis GKI applies to this SAF.

** ESL is to determine unsaturated hydraulic conductivity using the method(s) best suited for the soil texture and type.

** ESL is to submit coples of all Chain of Custodies and associated sample login documentation to EIS-SOM within 24 hours of sample receipt. They may be sent via e-mail to ^CPP Sample Management.

(1)6020M_ICPMS ASTM AE (TAL) \{Cadmium, Chromiurn, Silver\} 6020M ICPMS_ASTM AE (Add-On) \{Arsenic, Lead, Molvbdenum, Selenium\} 6020M METALS ICPMS WE (TAL) \{Cadmium, Chromium, 5ilver\}

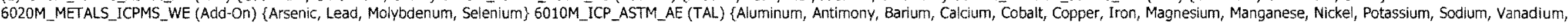

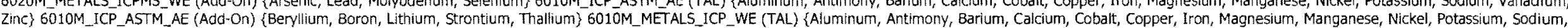

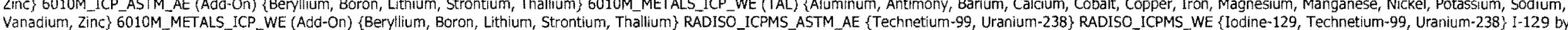

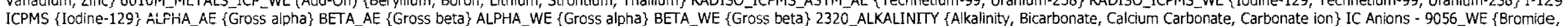

Chloride, Fluoride, Nitrate, Nitrite, Phosphate, Sulfate\} TOC - ASTME1915A (Total Inorganic Carbon, Total carbon, Total organic carbon\} pH (Water) - 9045 _WE; 
CHAIN OF CUSTODY/SAMPLE ANALYSIS REQUEST

\section{COLLECTOR}

NCO SAMPLER MA Luhutw

SAMPLING LOCATION

C5856, I-113

ICE CHEST NO.

\section{SHIPPED TO}

Environmental Sciences Laboratory

\section{MATRIX* POSSIBLE SAMPLE HAZARDS/ REMARKS $A=A i r$
$D L=D r u m$$\quad$ Contains Radioactive Material at concentrations $\mathrm{DL}=\mathrm{Drum}$
Liquids DS=Drum CFR but are not releasabie per DOE Order Sotids $\quad 5400.5(1990 / 1993)$ \\ $0=0$ it \\ $\mathrm{S}=5$ oil \\ $S E=$ Sediment \\ $V=V$ tissue \\ $V=$ vegtation
$W=$ Water \\ WI $=$ Wipe \\ $X=$ other \\ SPECIAL HANDLING AND/OR STORAGE Radicactive Tie To BITPB6}

SAMPLE NO.

MATRIX*
SOIL

\section{COMPANY CONTACT}

TRENT, STEVE

PROJECT DESIGNATTON

FIELD LOGBOOK NO.

ACTUAL SAMPLE DEPTH

$4 N F-N-488$.

OFFSITE PROPERTY NO.

N/A

\begin{tabular}{|c|c|c|}
\hline PRESERVATION & None & None \\
\hline TYPE OF CONTAINER & $\mathrm{G} / \mathrm{P}$ & $\begin{array}{l}\text { Moisture } \\
\text { Resistant Cont }\end{array}$ \\
\hline NO. OF CONTAINER(S) & 1 & 1 \\
\hline VOLUME & $1 \mathrm{LL}$ & 2009 \\
\hline SAMPLE ANAL & $\begin{array}{l}\text { SEE ITEM (1) IN } \\
\text { SPECLAL } \\
\text { INSTRUCTIONS }\end{array}$ & $\begin{array}{l}\text { Moisture } \\
\text { Content } 02216 \text {; }\end{array}$ \\
\hline
\end{tabular}

$$
190^{\prime}
$$

SAMPLE DATE SAMPLE TIME

$5-13-08 / 411$
F08-075-111

PAGE 1 OF 2
PROJECT COORDINATOR

WIDRIG, DL.

SAF NO.

F08-075

COA

123514 ES10

BILL OF LADING/AIR BILL NO.
METHOD OF SHIPMENT

GOVERNMENT VEHICLE

\section{LOT}

\section{CHAIN OF POSSESSION}

RELINQUISHED BY/REMOVED FROM

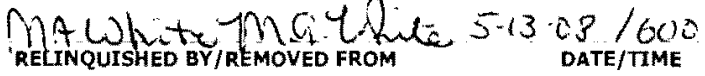
Mo 345 Frid $5 \cdot 20$ as $/ 200$ Mre. Wiric Ma White 5 to.e8 1320

RELINQUISHED BY/REMOVED FROM

DATE/TIME

RELINQUISHED BY/REMOVED FROM

RELINQUISHED BY/REMOVED FROM

DATE/TIME

DATE/TIME

LABORATORY RECEIVED BY
SECTION
FINAL SAMPLE
DISPOSITION




\section{COLLECTOR}

\section{NCO SAMPLER}

\section{SAMPLING LOCATION}

ICE CHEST NO.

\section{SHIPPED TO}

Environmental Sciences Laboratory
C5856, [-113

\section{COMPANY CONTACT}

TRENT, STEVE

\section{PROJECT DESIGNATION}

200-BP-5 OU Characterization for Wells 299-E33-341 and 299-E33-342-G FIELD LOGBOOK NO.

$$
\text { HNF-N-488.1 }
$$
OFFSITE PROPERTY NO.

N/A

\section{TELEPHONE NO.}

373-5869

ACTUAL SAMPLE DEPTH

\section{SPECIAL INSTRUCTIONS}

** The 200 Area S\&GRP Characterization and Monitoring Sampling and Analysis GKI applies to this SAF.

** ESL is to determine unsaturated hydraulic conductivity using the method(s) best suited for the soil texture and type.

** ESL is to submit copies of all Chain of Custodies and associated Sample login documentation to EIS-SDM within 24 hours of sample receipt. They may be sent via e-mail to ACPP Sample Management.

(1)6020M_ICPMS_ASTM_AE (TAL) \{Cadmium, Chronium, Silver\}6020M_ICPMS_ASTM_AE (Add-On) \{Arsenic, Lead, Molybdenum, Selenium\} 6020M_METALS_ICPMS_WE (TAL) \{Cadmium, Chromium, Silver\}

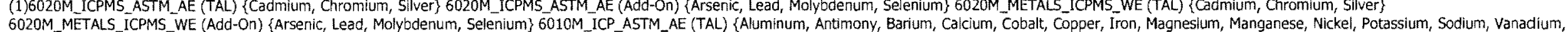

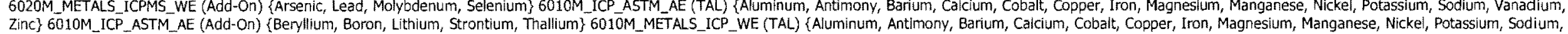

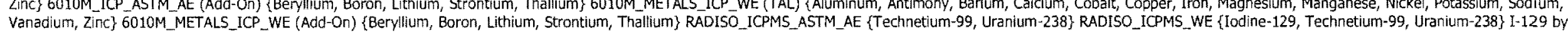

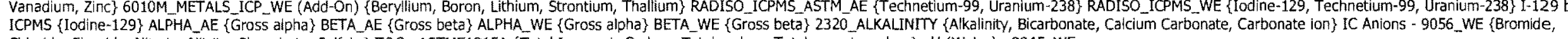
Chloride, Fluoride, Nitrate, Nitrite, Phosphate, Sulfate\} TOC - ASTME1915A \{Total Inorganic Carbon, Total carbon, Total organic carbon\} pH (Water) - 9045 _WE; 
CHAIN OF CUSTODY/SAMPLE ANALYSIS REQUEST

\section{TELEPHONE NO.}

373-5869

NCO SAMPLER

SAMPLING LOCATION

C5856, I-115

ICE CHEST NO.

SHIPPED TO

Environmental Sciences Laboratory

MATRIX*

$A=A$ Ar

$\mathrm{DL}=$ Drum

Lquids

OS=Drum
Solids

Solds

$0=0 \mathrm{il}$
$\mathrm{l}$

$0=0$ il
$S=5$ oil

$S E=$ Sediment

$T=T$ issue

$V=$ vegitation

$W=$ Water

WI $=$ Wipe

$x=$ Other

TRENT, STEVE

PROJECT DESIGNATION

200-BP-5 OU Characterization for Wells 299-E33-341 and 299-E33-342-G FIELD LOGBOOK NO. ACTUAL SAMPIE DEPTH

HNF.N. 488 , OFFSITE PROPERTY NO

$$
192.5
$$

N/A

COA

N/A

\begin{tabular}{|c|c|c|}
\hline PRESERVATION & None & None \\
\hline TYPE OF CONTAINER & GP & $\begin{array}{l}\text { Moisture } \\
\text { Resistant Cont }\end{array}$ \\
\hline NO. OF CONTAINER(S) & : & it \\
\hline VOLUME & $11 \mathrm{~L}$ & 2009 \\
\hline SAMPL & $\begin{array}{l}\text { SEE TIEM (1) IN } \\
\text { SPECISL } \\
\text { INSTRUTTONS }\end{array}$ & $\begin{array}{l}\text { Moisture } \\
\text { Content-02216; }\end{array}$ \\
\hline
\end{tabular}

SPECIAL HANDLING AND/OR STORAGE Radioactive Tie To BITPB6

POSSIBLE SAMPLE HAZARDS/ REMARKS Contains Radioactive Material at concentrations that are not regulated for transportation per 49 CFR but are not releasable per DOE Order $5400.5(1990 / 1993)$

SAMPLE NO. MATRIX

\section{PROJECT COORDINATOR}

WIDRIG, DL

F08 075

$123514 E S 10$

BILL OF LADING/AIR BILL NO.

SAMPLE DATE SAMPLE TIME
PRICE CODE

DATA TURNAROUND

45 Days / 45 Days

\section{METHOD OF SHIPMENT}

GOVERNMENT VEHICLE
$5 / 16 / 08 \quad 0705$
B1TP83
SOIL

\section{CHAIN OF POSSESSION}

RELINQUISHED BY/REMONED FROM $\checkmark$ terick homile no?45 Fríl 5 RELINQUISHED BY/REMEVED FROM TU\&

RELINQUISHED BY/REMOVED FROM

RELINQUISHED BY/REMOVED FROM

RELINQUISHED BY/REMOVED FROM

LABORATORY
SECTION
FINAL SAMPLE
DISPOSITION

Lot 2 STGN/PRINT NAMES 31384

\section{SIGN/ PRINT NAMES}

$5 / 16 / 081500$ MO MO 745 FRig Al

$5 / 16 / 08$ Date/TIME

RECEIVED BY/STORED IN

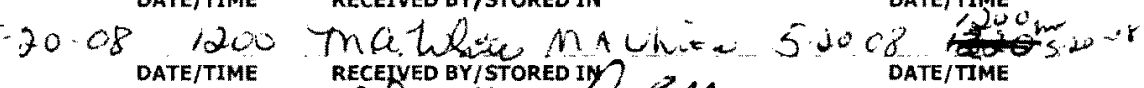

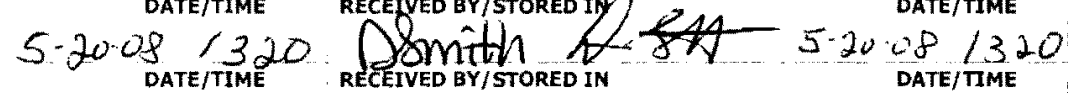

DATE/TIME RECEIVED BY/STORED IN

DATE/TIME

DATE/TIME
RECEIVED BY/STORED IN

RECEIVED BY/STORED IN

\section{SPECIAL INSTRUCTIONS}

SEE PAGE 2 FOR ALL SPECIAL INSTRUCTIONS 


\section{COLLECTOR \\ NCO SAMPLER}

\section{SAMPLING LOCATION}

C5856, I"115

ICE CHEST NO.

\section{SHIPPED TO}

Environmental Sciences Laboratory

\section{COMPANY CONTACT}

TRENT, STEVE

TELEPHONE NO.

373-5869

\section{PROJECT DESIGNATION}

200-BP-5 OU Characterization for Wells 299-E33-341 and 299-E33-342-G FIELD LOGBOOK NO.

HNE.U.480 OFFSITE PROPERTY NO.
ACTUAL SAMPLE DEPTH

192.5

\section{PROJECT COORDINATOR}

WIDRIG, DL

SAF NO.
PRICE CODE

$8 \mathrm{~N}$

AIR QUALITY
COA
DATA

TURNAROUND

45 Days / 45

Days

\section{SPECIAL INSTRUCTIONS}

** The 200 Area S\&GRP Characterization and Monitoring Sampling and Analysis GKI applies to this SAF.

** ESL is to determine unsaturated hydraulic conductivity using the method(s) best suited for the soil texture and type.

** ESL is to submit copies of all Chain of Custodies and associated sample login documentation to EIS-SDM within 24 hours of sample receipt. They may be sent via e-mail to $A$ CPP Sample Management.

(1)6020M ICPMS ASTM AE (TAL) \{Cadmium, Chromium, Silver\} 6020M ICPMS ASTM AE (Add-On) (Arsenic, Lead, Molybdenum, Selenium) 6020M METALS ICPMS WE (TAL) \{Cadmium, Chromium, Silver\}

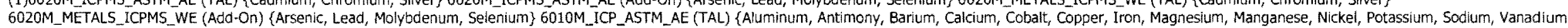

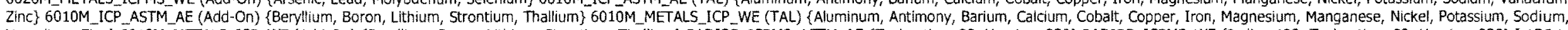

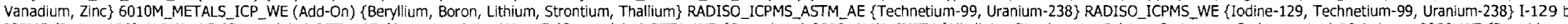

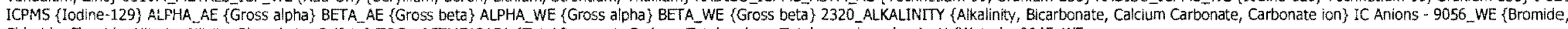

Chloride, Fluoride, Nitrate, Nitrite, Phosphate, Sulfate\} TOC - ASTME1915A (Total Inorganic Carbon, Total carbon, Total organic carbon\} pH (Water) - 9045 WE; 
CHAIN OF CUSTODY/SAMPLE ANALYSIS REQUEST

\section{TELEPHONE NO.}

$373-5869$

TRENT, STEVE

PROJECT DESIGNATION

200-BP-5 OU Characterization for Wells 299-E33-341 and 299-E33-342-G FIELD LOGBOOK NO.

HUF. N -1881

ACTUAL SAMPLE DEPTH

$$
195^{\circ}
$$

OFFSITE PROPERTY NO.

N/A

Environmental Sciences Laboratory

\section{MATRIX*}

$A=A$ ir

DL=Drum

Liquids

DS=Drum
Solids

Solids
$L=$ Liquid

$O=0$ il
$\mathrm{S}=\mathrm{S}$ cil

$\mathrm{SE}=$ Sediment

$T=$ Tissue

$V=$ vegitation

$W=$ Water

WI $=$ Wipe

$x=$ Other

POSSIBLE SAMPLE HAZARDS/ REMARKS Contains Radioactive Materiai at concentrations that are not regulated for transportation per 49 CFR but are not releasable per DOE Order $5400.5(1990 / 1993)$

SPECIAL HANDLING AND/OR STORAGE Radioactive Tie To B1Tा5?
PRESERVATION

TYPE OF CONTAINER Gip Moisture

NO. OF CONTAINER(S)

VOLUME 2009

$\begin{array}{lll}\text { SEE TTEM (1) IN Moisture } \\ \text { SAMPLE ANALYSIS } & \text { SPECIAL } \\ \text { INSTRUTTONS } & \text { Content-02216; }\end{array}$
COA

N/A
F08-075-114

PAGE 1 OF 2

PRICE CODE $\quad 8 N$

DATA

45 Days / 45

Days

AIR QUALITY
SAMPLE NO.
MATRIX*
B1TP84
SOIL
SAMPLE DATE SAMPLE TIME
$5 / 14 / 08$
080

$.0807 x$

WIDRIG, DL

F08-075

METHOD OF SHIPMENT

$123514 E S 10$

GOVERNMENT VEHICLE

iILL of LADING/AIR BILL No.

\begin{abstract}
CHAIN OF POSSESSION
RELINQUISHED BY/REMQVED GROM.

MO 745 Fris

REYINQUISHED BU/REMOVF KROM

RELINQUISHED BY/REMOVED FROM

RELINQUISHED BY/REMOVED FROM

RELINQUISHED BY/REMOVED FROM

LABORATORY
SECTION
FINAL SAMPLE
DISPOSITION
\end{abstract}

Lot \pm 031364

RELINQUISHED BY/REMQVED GROM

RELINQUISHED BY/REMQVED GROM

SIGN/ PRINT NAMES

\#1

$15 / 16 / 08 / 7 C O$

SPECIAL INSTRUCTIONS

SEE PAGE 2 FOR ALL SPECIAL INSTRUCTIONS
RELINQUISHED BY/REMOVED FROM
DATE/TIME

DATE/TIME

DATE/TIME

DATE/TIME

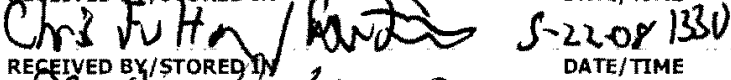

fomith 5.22.08\%1400

RECEIVED BY/STORED IN

DATE/TTME

DATE/TIME

DATE/TIME

DATE/TMME
TITLE

DATE/TIME

DISPOSED BY
DATE/TIME 


\section{COLLECTOR}

NCO SAMPLER

\section{SAMPLING LOCATION}

C5856, I-116

ICE CHEST NO.

\section{SHIPPED TO}

Environmental Sciences Laboratory

\section{COMPANY CONTACT}

TRENT, STEVE

PROJECT DESIGNATION

200-BP-5 OU Characterization for Wells 299-E33-341 and 299-E33-342-G FIELD LOGBOOK NO.

$$
\text { HNF-N } 488
$$
OFFSITE PROPERTY NO.

N/A

\section{TELEPHONE NO.}

373-5869

ACTUAL SAMPLE DEPTH

$$
195
$$

\section{SPECIAL INSTRUCTIONS}

** The 200 Area 58GRP Characterization and Monitoring Sampling and Analysis GKI applies to this SAF.

** ESL is to determine unsaturated hydraulic conductivity using the method(S) best suited for the soil texture and type.

** ESL is to submit copies of all Chain of Custodies and associated sample login documentation to EIS-SOM within 24 hours of sample receipt. They may be sent via e-mail to ^CPP Sample Management.

(1)6020M_ICPMS_ASTM_AE (TAL) \{Cadmium, Chromium, Silver\} 6020M_ICPMS_ASTM_AE (Add-On) \{Arsenic, Lead, Molybdenum, Selenium\} 6020M_METALS_ICPMS_WE (TAL) \{Cadmium, Chromium, Silver\}.

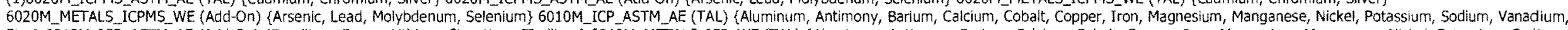

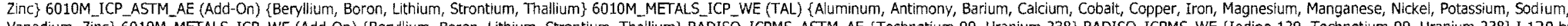

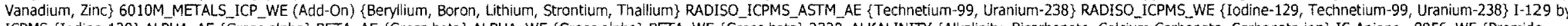

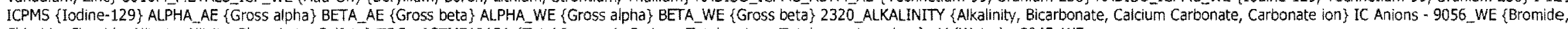

Chlorice, Fluoride, Nitrate, Nitrite, Phosphate, Sulfate\} TOC - ASTME1915A \{Total Inorganic Carbon, Total carbon, Total organic carbon\} pH (Water) - 9045 _WE; 
CHAIN OF CUSTODY/SAMPLE ANALYSIS REQUEST

\section{TELEPHONE NO.}

$373-5869$

\section{COMPANY CONTACT}

TRENT, STEVE

PROJECT DESIGNATION

200-BP-5 OU Characterization for Wells 299-E33-341 and 299-E33-342-G

FIELD LOGBOOK NO.

ACTUAL SAMPLE DEPTH

HNF.N. 488 ,

OFFSITE PROPERTY NO.

$$
210^{\circ}
$$

N/A

MATRIX*

$A=$ Air

$D L=$ Drum

Liquids

DS=Drum

Solids

$L=$ Liquid
$0=0$ il

$0=0$ il
$S=5$ il

$\mathrm{SE}=$ Sediment

$T=T$ Tissue

$V=$ vegitation

$W=$ water

WI $=$ Wipe

SPECIAL HANDLING AND/OR STORAGE Radioactive TIE TO B1TT58

PSIBLE SAMPLE HAZARDS/ REMARKS Contains Radioactive Material at concentrations that are not regulated for transportation per 49 CFR but are not releasable per DOE Order $5400.5(1990 / 1993)$

SAMPLE NO

MATRIX*

B1TP85

SOIL

\section{CHAIN OF POSSESSION}

RELINQUISHED BY/REMOVED FROM -j. Herrick Pew Hew

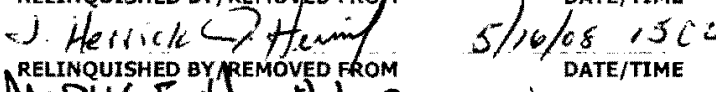
MoRAS Frillset) $5-22-08$ 1330 PELINQUISHE BY/REMTIED FRgM

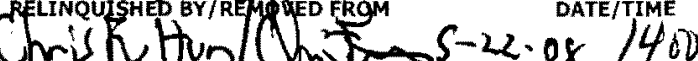
RELINOUISHED BY/REMOVED FROM

RELINQUISHED BY/REMOVED FROM DATE/TIME DATE/TIME DATE/TIME

\begin{tabular}{|c|c|c|}
\hline PRESERVATION & None & None \\
\hline TYPE OF CONTAINER & $\mathrm{G} / \mathrm{P}$ & $\begin{array}{l}\text { Moisture } \\
\text { Resistant Cont }\end{array}$ \\
\hline NO. OF CONTAINER(S) & 1 & i \\
\hline VOLUME & iL & 2009 \\
\hline SAMPLE ANALYSIS & $\begin{array}{l}\text { SEE ITEM (1) IN } \\
\text { SPECIAL } \\
\text { INSTRCTIONS }\end{array}$ & $\begin{array}{l}\text { Moisture } \\
\text { Content- Dz216; }\end{array}$ \\
\hline
\end{tabular}

SAMPLE DATE SAMPLE TIME

$3 / 16 / 28$ 1313

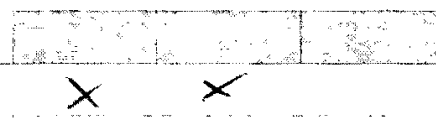

RELINQUISHED BY/REMOVED FROM

\section{WIDRIG, DL}

AIR QUALITY $\square$

ROUND

45 Days / 45 Days

F08-075

\section{$\operatorname{COA}$}

$123514 E S 10$

METHOD OF SHIPMENT

GOVERNMENT VEHICLE

OFIL OF LADING/AIR BILL NO

\section{Lat +031304}

SIGN/ PRINT NAMES

RECEIVED BY/STORED IN Mo 745 ERiE

RECEIVED BY/STORED IN

SPECIAL INSTRUCTIONS

SEE PAGE 2 FOR ALL SPECIAL INSTRUCTIONS 


\section{COLLECTOR}

NCO SAMPLER

\section{SAMPLING LOCATION}

C5856, $\mathrm{i}-123$

ICE CHEST NO.

\section{SHIPPED TO}

Environmental Sciences Laboratory

\section{COMPANY CONTACT}

TRENT, STEVE

PROJECT DESIGNATION

TELEPHONE NO.

373-5869

200-BP-5 OU Characterization for Wells 299-E33-341 and 299-E33-342 - G FIELD LOGBOOK NO.

ACTUAL SAMPLE DEPTH

HNF.N 488 OFFSITE PROPERTY NO.

N/A
PROJECT COORDINATOR

WIDRIG, DL

SAF NO.

F08-075

$\operatorname{COA}$

$123514 E S 10$
PRICE CODE

$\mathbf{8 N}$

AIR QUALITY

\section{BILL OF LADING/AIR BILL NO.}

N/A

\section{SPECIAL INSTRUCTIONS}

** The 200 Area S\&GRP Characterization and Monitoring Sampling and Analysis GKI applies to this SAF.

** ESL is to determine unsaturated hydraulic conductivity using the method(s) best suited for the soll texture and type.

** ESL is to submit conies of all Chain of Custodies and associated sample login documentation to ElS-SOM within 24 hours of sample receipt. They may be sent via e-mail to ACPP Sample Management.

(1)6020M_ICPMS_ASTM_AE (TAL) \{Cadmium, Chromium, Silver\} 6020M_ICPMS_ASTM_AE (Add-ON) \{Arsenic, Lead, Molybdenum, Selenium\} 6020M_METALS_ICPMS_WE (TAL) \{Cadmium, Chromium, Silver\}

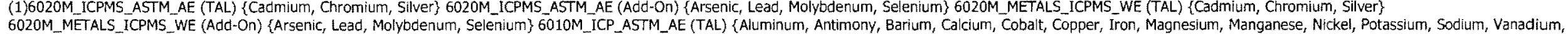

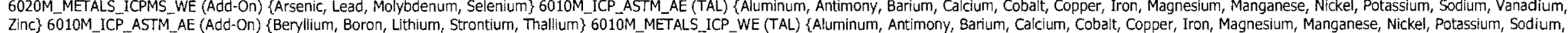

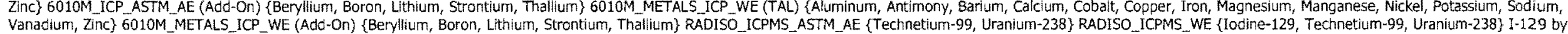

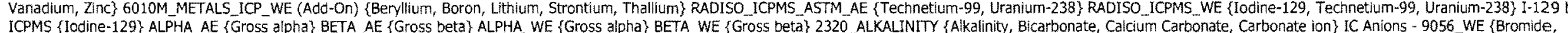
ICPMS \{Iodine-129\} ALPHA_AE \{Gross alpha\} BETA_AE \{Gross beta\} ALPHA_WE \{Gross alpha\} BETA_WE \{Gross beta\} 2320_ALKALINITY \{Alkalinity, Bicarbona
Chloride, Fluoride, Nitrate, Nitrite, Phosphate, Sulfate\} TOC - ASTME1915A (Total Inorganic Carbon, Total carbon, Total organic carbon\} pH (Water) - 9045 WEE; 
CHAIN OF CUSTODY/SAMPLE ANALYSIS REQUEST

\section{TELEPHONE NO.}

373-5869

COMPANY CONTACT

TRENT, STEVE

PROJECT DESIGNATION

200-BP-5 OU Characterization for Wells 299-E33-341 and 299-E33-342-G FIELD LOGBOOK NO. DG 34 ACTUAL SAMPLE DEPTH

HNF. N. Y88, OFFSITE PROPERTY NO.

N/A

Environmental Sciences Laboratory

MATRIX*

A $=$ Air
DL $=$ Drum

DLA $=$ Drum
Liquids

D $5=$ Drum

Solids

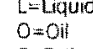

$S=50 i 1$

$S E=$ Sediment

$y=$ Tessue

$W=$ Water

$W=$ Water
$W I=$ Wipe

$x=0$ ther

POSSIBLE SAMPLE HAZARDS/ REMARKS Contains Radioactive Material at concentrations that are not regulated for transportation per 49 CFR but are not releasable per DOE Order $5400.5(1990 / 1993)$

SPECIAL HANDLING AND/OR STORAGE Radioactive Tie To B1TT58

COA

N/A

$\begin{array}{clc}\text { PRESERVATION } & \text { None } & \text { None } \\ \text { TYPE OF CONTAINER } & \text { G/P } & \begin{array}{c}\text { Moisture } \\ \text { Resistant Cont }\end{array} \\ \text { NO. OF CONTAINER(S) } & 1 & 1 \\ \text { VOLUME } & 11 & 200 \mathrm{~g} \\ \text { SAMPLE ANALYSIS } & \begin{array}{l}\text { SEE ETEM (1) IN } \\ \text { SPCAL Moisture } \\ \text { INSTRUCTIONS }\end{array}\end{array}$

SAMPLE NO. MATRIX*

Soll

SAMPLE DATE SAMPLE TIME

$5 / 16 / 05 / 410 \quad x \quad x$

METHOD OF SHIPMENT

123514 ES10

BILL OF LADING/AIR BILL NO.

\section{CHAIN OF POSSESSION}

$$
20+* 31344
$$

\section{SIGN/ PRINT NAMES}

RECEIVED BY/STORED IN $5 / 16 / 0515 \%$ MO 745 FRIG $\#$

\section{SPECIAL INSTRUCTIONS}

DATE/TIME $5 / 10 / 08 / 560$

SEE PAGE 2 FOR ALL SPECIAL INSTRUCTIONS

J. He:ricl 2 He

REINOUISHED BY REḾ⿴囗十 VD FROM PATE/TIME T/10/08 1360 Mo Tus Fridsel S-22.08 1330

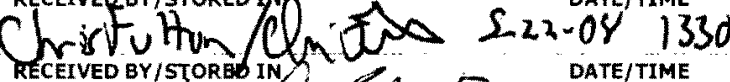

REIINQUISHED BY/REMOYQPFROM

DATE/TIME

Shristu trun

1-22. OP 1400 J8mith

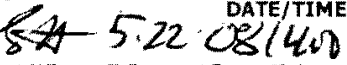

DATE/TIME

DATE/TIME

RELINQUISHED BY/REMOVED FROM

DATE/TIME

RECEIVED BY/STORED IN

DATE/TIME

RELINQUISHED BY/REMOVED FROM

DATE/TIME

RECEIVED BY/STORED IN

DATE/TIME

RELINQUISHED BY/REMOVED FROM

DATE/TIME

RECEIVED BY/STORED IN

DATE/TIME

LABORATORY RECEIVED BY
SECTION
FINAL SAMPLE
DISPOSITION




\section{COLLECTOR}

COMPANY CONTACT

TRENT, STEVE

\section{TELEPHONE NO.}

PROJECT COORDINATOR

373-5869

WIDRIG, DL

\section{SAMPLING LOCATION}

PROJECT DESIGNATION

SAF NO.

200-BP-5 OU Characterization for Wells 299-E33-341 and 299-E33-342 - G

\section{C5856, I-125}

ICE CHEST NO.

\section{FIELD LOGBOOK NO.}

ACTUAL SAMPLE DEPTH

\section{SHIPPED TO}

Environmental Sciences Laboratory

$$
\text { HNF.N.488) }
$$

$212.5^{\prime}$

\section{OFFSITE PROPERTY NO.}

$N / A$

\section{COA}

METHOD OF SHIPMENT

GOVERNMENT VEHICLE

\section{SPECIAL INSTRUCTIONS}

** The 200 Area S\&GRP Characterization and Monitoring Sampling and Analysis GKI applies to this SAF.

** ESL is to determine unsaturated hydraulic conductivity using the method(s) best suited for the soil texture and type.

**ESL is to submit copies of all Chain of Custodies and associated sample login documentation to EIS-SDM within 24 hours of sample receipt. They may be sent via e-mall to ^CPP Sample Management.

(1)6020M_ICPMS ASTM AE (TAL) \{Cadmium, Chromium, Silver\} 6020M_ICPMS ASTM AE (Add-OD) \{Arsenic, Lead, Molybdenum, Selenium\} 6020M METALS ICPMS WE (TAL) (Cadmium, Chromium, Silver\}

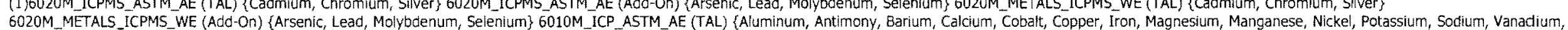

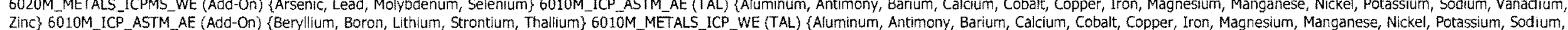

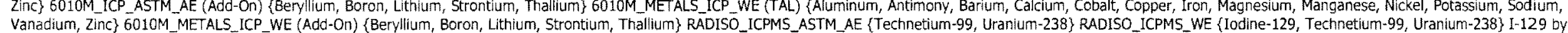

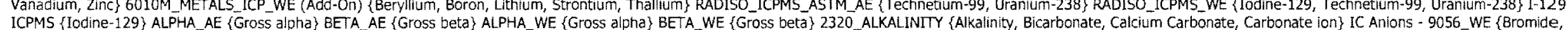
ICPMS \{lodine-129\} ALPHA_AE \{Gross alpha\} BETA_AE \{Gross beta\} ALPHA_WE \{Gross aipha\} BETA_WE \{Gross beta\} 2320_ALALINITY \{Alkalinity, Bicarbona-
Chloride, Fluoride, Nitrate, Nitrite, Phosphate, Sulfate\} TOC-ASTME1915A \{Total Inorganic Carbon, Total carbon, Total organic carbon\} pH (Water) -9045_WE; 
CHAIN OF CUSTODY/SAMPLE ANALYSIS REQUEST

\section{TELEPHONE NO.}

373-5869

\section{COMPANY CONTACT}

TRENT, STEVE

PROJECT DESIGNATION

200-BP-5 OU Characterization for Wells 299-E33-341 and 299-E33-342 - G

FIELD LOGBOOK NO.

ACTUAL SAMPLE DEPTH

HNF-N- $488=1$

2151

OFFSITE PROPERTY NO.

N/A

Environmental Sciences Laboratory

MATRIX*

$A=$ Air

$\mathrm{DL}=\mathrm{Drum}$

Liquids

DS $=$ Drum
Solids

Solids
$L=$ Liquid

$0=0$ :

$S E=$ Sediment

$T=$ Tissue

$V=$ vegitation

$W=$ Water

WI $=$ Wipe

$x=0$ ther

POSSIBLE SAMPLE HAZARDS/ REMARKS Contains Radioactive Material at concentrations that are not regulated for transportation per 49 CFR but are not releasable per DOE Order $5400.5(1990 / 1993)$

\section{SPECIAL HANDLING AND/OR STORAGE} Radioactive Tie To B1TT58

\begin{tabular}{ccc} 
PRESERVATION & None & None \\
TYPE OF CONTAINER & G/P & $\begin{array}{l}\text { Moisture } \\
\text { Resistant Cont }\end{array}$ \\
NO. OF CONTAINER(S) & 1 & 1 \\
\hline VOLUME & $1 \mathrm{~L}$ & $200 \mathrm{~g}$ \\
\hline SAMPLE ANALYSIS & $\begin{array}{l}\text { SEE ITEM (1) IN Mosture } \\
\text { SPECAL } \\
\text { INSTRUCTIONS }\end{array}$
\end{tabular}

N/A
MATRIX*

SOIL

SAMPLE DATE SAMPLE TIME

$5-20-8 \quad 0805$

\section{F08-075-120}

PAGE 1 OF 2

PROJECT COORDINATOR

WIDRIG, DL

PRICE CODE

$8 \mathrm{~N}$

DATA TURNAROUND

45 Days / 45 Days
B1TP87

$$
\text { SOIL }
$$

\section{CHAIN OF POSSESSION}

RELINQUISHED BY/REMOVED FROM

JKEALl4, JJEC $5-20-8$ 1600

Mo 74 Frilse +15

RELINQUISHED BYIREMOYER FROY DATE/TTME

Chrif t

RELINQUISHED BY/REMQVED FROM

RELINQUISHED BY/REMOVED FROM

DATE/TIME

RELINQUISHED BY/REMOVED FROM

DATE/TIME

RELINQUISHED BY/REMOVED FROM

DATE/TIME

DATE/TIME

\section{SAF NO.}

COA

$123514 E S 10$

METHOD OF SHIPMENT

GOVERNMENT VEHICLE

BILL OF LADING/AIR BILL NO

LABORATORY
SECTION
FINAL SAMPLE
DISPOSITION

TITLE

DATE/TIME

\section{SIGN/ PRINT NAMES}

RECEIVED BY/STORED IN

MOPUS REE $415-20-8 \quad 1600$
Chrisfitio fo to $5-22.081330$

\section{SPECIAL INSTRUCTIONS}

SEE PAGE 2 FOR ALL SPECIAL INSTRUCTIONS
Comith

RECEIVED BY/STORED IN

DATE/TIME

RECEIVED BY/STORED IN

DATE/TIME

RECEIVED BY/STORED IN

DATE/TIME

RECEIVED BY/STORED IN

DATE/TIME 


\section{COLLECTOR}

NCO SAMPLER

\section{SAMPLING LOCATION}

C5856, I-127

ICE CHEST NO.

\section{SHIPPED TO}

Environmental Sciences Laboratory

\section{COMPANY CONTACT}

TRENT, STEVE

PROJECT DESIGNATION

200-BP-5 OU Characterization for Wells 299-E33-341 and 299-E33-342-G

FIELD LOGBOOK NO.

ACTUAL SAMPLE DEPTH

\section{OFFSITE PROPERTY NO}

N/A

\section{PRICE CODE}

\section{$\mathbf{8 N}$}

WIDRIG, DL

SAF NO.
F08-075

COA

AIR QUALITY
METHOD OF SHIPMENT

GOVERNMENT VEHICLE

\section{SPECIAL INSTRUCTIONS}

** The 200 Area S\&GRP Characterization and Monitoring Sampling and Analysis GKI applies to this SAF.

** ESL is to determine unsaturated hydraulic conductivity using the method(s) best suited for the soil texture and type.

** ESL is to submit copies of all Chain of Custodies and associated sample login documentation to EIS-SDM within 24 hours of sample receipt. They may be sent via e-mail to $\wedge$ CPP Sample Management.

(1)6020M_ICPMS_ASTM_AE (TAL) \{Cadmium, Chromium, Siver\} 6020M_ICPMS_ASTM_AE (Add-On) \{Arsenic, Lead, Molybdenum, Selenium\} 6020M_METALS_ICPMS_WE (TAL) \{Cadmium, Chromium, Silver\}

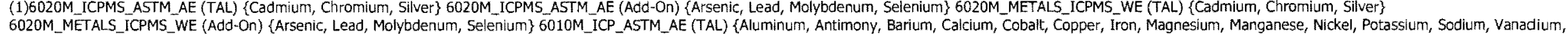

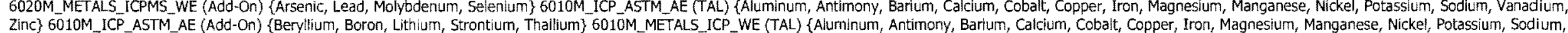

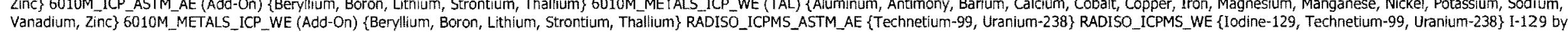

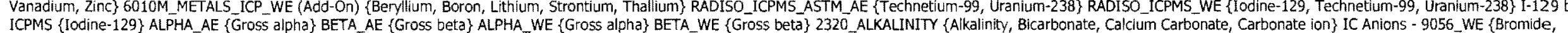
ICPMS \{Iodine-129\} ALPHA_AE \{Gross alpha\} BETA_AE \{Gross beta\} ALPHA_WE \{Gross alpha\} BETA_WE \{Gross beta\} 2320_ALKALINITY \{Alkalinity, Bicarbonat
Chloride, Fluoride, Nitrate, Nitrite, Phosphate, Sulfate\} TOC-ASTME1915A \{Total Inorganic Carbon, Total carbon, Total organic carbon\} pH (Water) - 9045 WE; 
CHAIN OF CUSTODY/SAMPLE ANALYSIS REQUEST

\section{TELEPHONE NO.}

373-5869

nco SAMPLer Balle?

C5856, I-129

ICE CHEST NO.

\section{SHIPPED TO}

Environmental Sciences Laboratory

MATRIX*

$A=A$ Air

$\mathrm{OL}=$ Drum

Liquids
DS=Drum

DS=Drum
Solids

Solids
$L=$ Liquid

$0=0$ il

$S E=$ Sediment

$T=T i s s u e$

$T=T$ Vegitation
$V=$.

$W=$ water

$W I=$ Wipe

$X=0$ ther

POSSIBLE SAMPLE HAZARDS/ REMARKS Contains Radioactive Material at concentrations that are not regulated for transportation per 49 CFR but are not releasable per DOE Order $5400.5(1990 / 1993)$

SPECIAL HANDLING AND/OR STORAGE Radioactive Tie To B1TT58

SAMPLE NO. MATRIX*

B1TP88

SOIL

\section{CHAIN OF POSSESSION}

RELINQUISHED BY/REMOVED FROM

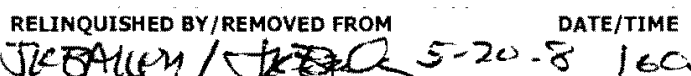

RELINQUISHEP BY/REMOVER FROH DATE/TIME

Mo Y4s tridge

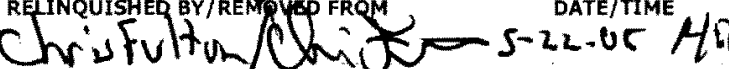

DATE/TIME

RELINQUISHED BY/REMOVED FROM

DATE/TIME

DATE/TIME

DATE/TIME

TRENT, STEVE

FIELD LOGBOOK NO.

ACTUAL SAMPLE DEPTH

COA

HNF-N-48 - -1

$2,7.51$

OFFSITE PROPERTY NO.

$N / A$

\begin{tabular}{|c|c|c|}
\hline PRESERVATION & None & None \\
\hline TYPE OF CONTAINER & $G / P$ & $\begin{array}{l}\text { Molsture } \\
\text { Resistant Cont }\end{array}$ \\
\hline No. OF CONTAINER(S) & 1 & $i_{1} \cdots$ \\
\hline VOLUME & $1 \mathrm{~L}$ & $200 \mathrm{~g}$ \\
\hline SAMPLE ANALYSIS & $\begin{array}{l}\text { SEE ITEM (1) IN } \\
\text { SPEELA } \\
\text { INSTRUCTONS }\end{array}$ & $\begin{array}{l}\text { Moisture } \\
\text { Contert-D2215; }\end{array}$ \\
\hline
\end{tabular}

SAMPLE DATE SAMPLE TIME

$5-20-8 \quad 0950$

RELINQUISHED BY/REMOVED FROM

RELINQUISHED BY/REMOVED FROM
SAMPLING LOCATION

REINQUISHED BY/RE OVED FR

PROJECT COOROINATOR

WIDRIG, DL

SAF NO.

200-BP-5 OU Characterization for Wells 299-E33-341 and 299-E33-342 - G F08-075

$123514 \mathrm{ES} 10$

BILL OF LADING/AIR BILL NO.

\section{2}

SIGN/ PRINT NAMES

We 0745 REF

REGEIVED BY/STPRED IN

RECEIYED BY/STORED

Somith

RECEIVED BY/STORED IN

RECEIVED BY/STORED IN

RECEIVED BY/STORED IN

RECEIVED BY/STORED IN
RECETVED BY/STORED IN

\section{F08-075-122}

PAGE 1 OF 2

PRICE CODE $\quad 8$ N

DATA TURNAROUND

45 Days / 45

Days
RECEIVED BY

TITLE

DATE/TIME

ABORATORY

SECTION

DISPOSAL METHOD

DATE/TIME
SPECIAL INSTRUCTIONS

DATE/TIME

SEE PAGE 2 FOR ALL SPECIAL INSTRUCTIONS 


\section{COLLECTOR}

\section{NCO SAMPLER}

\section{SAMPLING LOCATION}

C5856, $1-129$

ICE CHEST NO.

\section{SHIPPED TO}

Environmental Sciences Laboratory

\section{COMPANY CONTACT}

TRENT, STEVE

PROJECT DESIGNATION

200-BP-5 OU Characterization for Wells 299-E33-341 and 299-E33-342-G FIELD LOGBOOK NO.

ACTUAL SAMPLE DEPTH

\section{OFFSITE PROPERTY NO}

$\mathrm{N} / \mathrm{A}$

\section{TELEPHONE NO.}

373-5869

DATA

PRICE CODE 8N TURNAROUND

AIR QUALITY

45 Days / 45

Days

\section{SPECIAL INSTRUCTIONS}

** The 200 Area S\&GRP Characterization and Monitoring Sampling and Analysis GKI applies to this SAF.

*** ESL is to determine unsaturated hydraulic conductivity using the method(s) best suited for the soll texture and type.

** ESL is to submit copies of all Chain of Custodies and associated sample login documentation to EIS-SDM within 24 hours of sample receipt. They may be sent via e-mail to $\wedge$ CPP Sample Management.

** ESL is to submit copies of all Chain of Custodies and associated sample login documentation to EIS-SDM within 24 hours of sample receipt. They may be sent via e-mail to
(1)6020M ICPMS ASTM AE (TAL) \{Cadmium, Chromium, Silver\} 6020M ICPMS ASTM AE (Add-On) \{Arsenic, Lead, Molybdenum, Selenium\} 6020M METALS ICPMS WE (TAL) \{Cadmium, Chromium, Silver\}

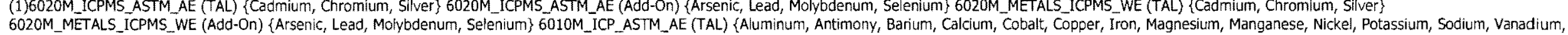

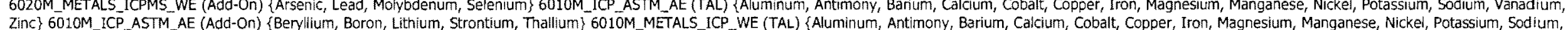

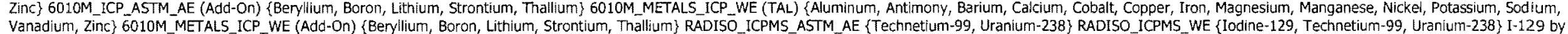

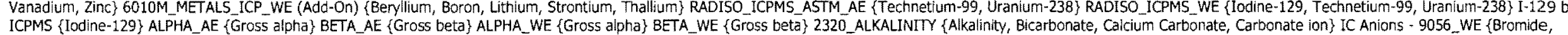
ICPMS \{lodine-129\} ALPHA_AE \{Gross aipha\} BETA_AE \{Gross beta\} ALPHA_WE \{Gross alpha\} BETA_WE \{Gross beta\} 2320_ALKALINIT \{Alkalinity, Bicarbonate,
Chloride, Fluoride, Nitrate, Nitrite, Phosphate, Sulfate\} TOC.ASTME1915A \{Total Inorganic Carbon, Total carbon, Total organic carbon\} pH (Water)-9045_WE; 


\section{CHAIN OF CUSTODY/SAMPLE ANALYSIS REQUEST}

\section{TELEPHONE NO.}

373-5869

TRENT, STEVE

PROJECT DESIGNATION

FIELD LOGBOOK NO.

ACTUAL SAMPLE DEPTH

$$
220^{\prime}
$$

$$
\text { HNF-N-48 - } 1
$$

OFFSITE PROPERTY NO.

$N / A$

$$
\begin{aligned}
& \text { POSSIBLE SAMPLE HAZARDS/ REMARKS } \\
& \text { Contains Radioactive Material at concentrations } \\
& \text { that are not regulated for transportation per } 49 \\
& \text { CFR but are not releasable per DOE Order }
\end{aligned}
$$
$5400.5(1990 / 1993)$

N/A

Li=Liquid

$0=0$ il

$S=$ Soil
$S E=$ Sediment

$T=T$ Tssue

$V=$ Vegitation
$W=$ Water

WI $=$ Wipe

$x=0$ ther

SPECIAL HANDLING AND/OR STORAGE Radioactive TIE To B1TT58

SAMPLE NO. MATRIX*

B1TP89

SOIL

$5-20-8 \quad 1025$

\section{8}

SIGN/ PRINT NAMES

DATE/TIME RECEIVED BY/STORED IN

DATE/TTME

T4S REF * I 5-20-8 1600 CheEIVEPBySTORED DATE/TIME $1>30$

DATE/TIME MOTUS Fridse A S.22.08

Chris Futtun

DATE/TIME

EEgED BYISTOR

Domith th

DATE/TIME

DATE/TIME

RECEIVED BY/STORED IN

RELINQUISHED BY/REMOVED FROM

DATE/TIME

RECEIVED BY/STORED IN

DATE/TIME

RECEIVED BY/STORED IN
METHOD OF SHIPMENT
BILL OF LADING/AIR BILL NO

\section{SPECIAL INSTRUCTIONS}


CHAIN OF CUSTODY/SAMPLE ANALYSIS REQUEST

TELEPHONE NO.

\section{COMPANY CONTACT}

TRENT, STEVE

TELPHONE

PROJECT DESIGNATION

200-BP-5 OU Characterization for Wells 299-E33-341 and 299-E33-342 - G FIELD LOGBOOK NO.

ACTUAL SAMPLE DEPTH

ICE CHEST NO.

\section{SHIPPED TO}

Environmental Sciences Laboratory

\section{OFFSITE PROPERTY NO}

N/A
F08-075-124

PAGE 2 OF 2

DATA TURNAROUND

45 Days / 45

Days

\section{SPECIAL INSTRUCTIONS}

** The 200 Area S\&GRP Characterization and Monitoring Sampling and Analysis GKI applies to this SAF.

** ESL is to determine unsaturated hydraulic conductivity using the method(s) best sulted for the soll texture and type.

* ESL is to submit copies of all Chain of Custodies and associated sample login documentation to EIS-SDM within 24 hours of sample receipt. They may be sent via e-mall to ACPP Sampie Management.

1)6020M_ICPMS_ASTM_AE (TAL) \{Cadmium, Chromium, Silver\} 6020M_ICPMS_ASTM_AE (Add-On) \{Arsenic, Lead, Molybdenum, Selenium\} 6020M METALS_ICPMS WE (TAL) \{Cadmium, Chromium, Silver\}

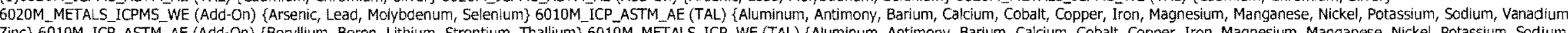

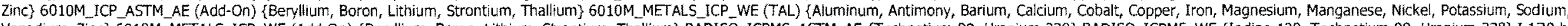

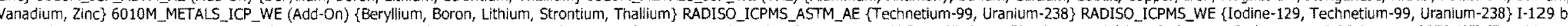

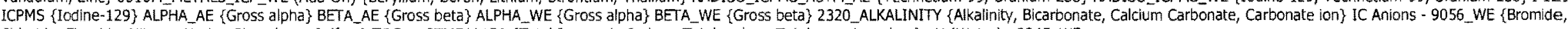

Chloride, Fiuoride, Nitrate, Nitrite, Phosphate, Sulfate\} TOC - ASTME1915A \{Total Inorganic Carbon, Total carbon, Total organic carbon\} pH (Water) - 9045 WE; 
CHAIN OF CUSTODY/SAMPLE ANALYSIS REQUEST

NCO SAMPLER BAILEY

\section{SAMPLING LOCATION}

C5856, I-133

ICE CHEST NO.

\section{SHIPPED TO}

Environmental Sciences Laboratory

\section{MATRIX*}

$A=$ Air

$\mathrm{A}=\mathrm{Ail}$
$\mathrm{L}=\mathrm{Drum}$
Licuids

Liquids
DS=Drum

DS=Drum
Solids

$L=L$ lquid

$0=011$

$\mathrm{SE}=$ Sediment

SE= Sediment
TeTissue

$V=$ vegitation

$V=$ vegitation
$W=$ Water

Wi=Wipe

$X=0$ ther

POSSIGLE SAMPLE HAZARDS/ REMARKS Contains Radioactive Material at concentrations that are not regulated for transportation per 49 CFR but are not releasable per DOE Order $5400.5(1990 / 1993)$

SPECIAL HANDLING AND/OR STORAGE Radioactive Tie TO B1TT58

\section{TELEPHONE NÖ.}

373-5869

\section{COMPANY CONTACT}

TRENT, STEVE

PROJECT DESIGNATION

$200-B P-5$ OU

FIELD LOG̈BOOK NO.

$$
\text { HNF-N } 488-1
$$

OFFSITE PROPERTY NO.

N/A

PRESERVATION
TYPE OF CONTAINER
NO. OF CONTAINER(S)

SAMPLE DATE SAMPLE TIME

$5-20-8 \quad 113 c$
9-E33-341 and 299-E33-342-

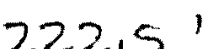

\section{N/A}

\author{
SAMPLE NO. \\ SOIL \\ B1TP9O
}

MATRIX*
AIR QUALITY

\section{SAF NO.}

METHOD OF SHIPMENT

\section{\begin{tabular}{l|l}
$123514 E S 10$ & GOVERNMENT VEHICLE
\end{tabular}}

BILL OF LADING/AIR BILL NO.

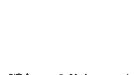




\section{COLLECTOR}

NCO SAMPLER

\section{SAMPLING LOCATION}

C5856, I-133

ICE CHEST NO.

\section{SHIPPED TO}

Environmental Sciences Laboratory

\section{COMPANY CONTACT}

TRENT, STEVE

PROJECT DESIGNATION

200-BP-5 OU Characterization for Wells 299-E33-341 and 299-E33-342-G FIELD LOGBOOK NO.

ACTUAL SAMPLE DEPTH

\section{OFFSITE PROPERTY NO}

N/A
PROJECT COORDINATOR WIORIG, OL

\section{SAF NO.}

FO8-075

\section{$\cos$}

\section{$123514 \mathrm{ES} 10$}

PRICE CODE 8 N

AIR QUALTTY

\section{METHOD OF SHIPMENT}

GOVERNMENT VEHICLE

\section{SPECIAL INSTRUCTIONS}

** The 200 Area S\&GRP Characterization and Monitoring Sampling and Analysis GKI applies to this SAF.

** ESL is to determine unsaturated hydraulic conductivity using the method(s) best sulted for the soil texture and type.

** ESL is to submit copies of all Chain of Custodies and associated sample login documentation to EIS-SDM within 24 hours of sample receipt. They may be sent via e-mail to $\wedge$ CPP Sample Manacement.

(1)6020M_ICPMS_ASTM_AE (TAL) \{Cadmium, Chromium, Silver\} 6020M_ICPMS_ASTM_AE (Add-ON) \{Arsenic, Lead, Molybdenum, Selenium\} 6020M_METALS_ICPMS_WE (TAL) \{Cadmilum, Chromlum, Silver\}

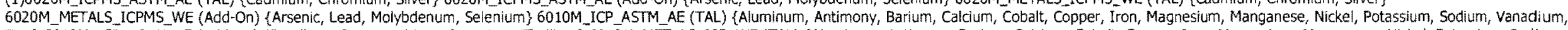

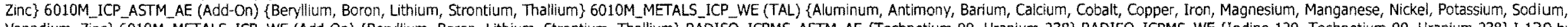

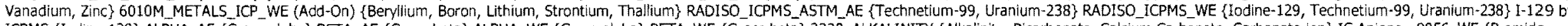

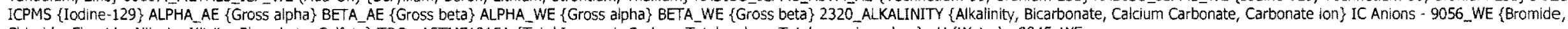
Chioride, Fluoride, Nitrate, Nitrite, Phosphate, Sulfate\} TOC - ASTME1915A \{Total Inorganic Carbon, Total carbon, Total organic carbon\} pH (Water) - 9045 .WE;

$\mathrm{N} / \mathrm{A}$ 


\section{CHAIN OF CUSTODY/SAMPLE ANALYSIS REQUEST}

\section{TELEPHONE NO.}

373-5869

TRENT, STEVE

PROJECT DESIGNATION

200-BP-5 OU Characterization for Wells 299-E33-341 and 299-E33-342 - G FIELD LOGBOOK NO.

HNF-N.48 OFFSITE PROPERTY NO

N/A

POSSIBLE SAMPLE HAZARDS/ REMARKS Contains Radioactive Material at concentrations that are not regulated for transportation per 49 CFR but are not releasable per DOE Order $5400.5(1990 / 1993)$

(1)

225

$\mathrm{N} / \mathrm{A}$

Solids

L=Liquid

$S=S$ oil

SEESediment

$T=$ Tissue
$V=$ Vegitation

$V=$ Vegitation

$W=$ Water

$x=$ Other

SPECIAL HANDLING AND/OR STORAGE Radioactive TIE To B1TT59

SAMPLE NO.

B1TP91
MATRIX*
F08-075-128

PRICE CODE

WIDRIG, DL

SAF NO.

F08-07

COA

AIR QUALITY

METHOD OF SHIPMENT

GOVERNMENT VEHICLE

123514 ES10

PAGE 1 OF

BILL OF LADING / AIR BILL NO.

\section{CHAIN OF POSSESSION}

RELINQUISHED BY/REMOVED FROM T2.54144 אJK E REL $74 \mathrm{~s}^{-} \mathrm{Ke} f$ RELINQUISHED BY/REMOVER FROM $D_{\text {RELINQUISHED BY/REMOVED FRGM }}$

RELINQUISHED BY/REMOVED FROM

RELINQUISHED BY/REMOVED FROM

RELINQUISHED BY/REMOVED FROM

DATE/TIME $5-20-8 ; 600$ $5.25 \cdot \rho^{\text {DATE/TIME }}$ $2+2 / 23$ $5-28 \cdot 8$ Date/Time DATE/TIME DATE/TIME DATE/TIME

\section{PRESERVATION}

TYPE OF CONTAINER

NO. OF CONTAINER(S)

VOLUME

SAMPLE ANALYSIS i1.

2009

SAMPLE DATE SAMPLE TIME

\section{$5-25-8 \quad 1435$}

\section{LABORATORY RECEIVED BY SECTION \\ FINAL SAMPLE} DISPOSITION

\section{8}

\section{SIGN/ PRINT NAMES}

RECEIVED BY/STORED IN

MOTL DATE/TIME

NEO TLS DEF \& $520-81600$

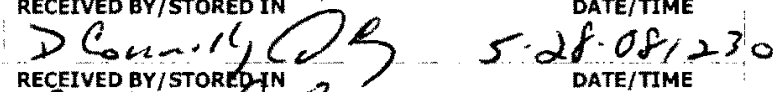

RECEIVED BY/STOREOIN 2

Domith

$3+8$ of 1300

RECEIVED BY/STORED IN

DATE/TIME

DATE/TIME

DATE/TIME

\section{SPECIAL INSTRUCTIONS}

SEE PAGE 2 FOR ALL SPECIAL INSTRUCTIONS 


\section{COLLECTOR}

NCO SAMPLER

\section{SAMPLING LOCATION}

C5856, 1-135

\section{ICE CHEST NO.}

\section{SHIPPED TO}

Environmental Sciences Laboratory

\section{COMPANY CONTACT}

TRENT, STEVE

$373-5869$

\section{PROJECT DESIGNATION}

200-BP-5 OU Characterization for Wells 299-E33-341 and 299-E33-342 - G FIELD LOGBOOK NO.

ACTUAL SAMPLE DEPT

\section{OFFSITE PROPERTY NO.}

N/A
PRICE CODE $\quad$ SN

AIR QUALITY

SAF NO.

\section{METHOD OF SHIPMENT}

$123514 \mathrm{ES} 10$

\section{BILL OF LADING/AIR BILL NO.}

N/A

** The 200 Area S\&GRP Characterization and Monitoring Sampling and Analysis GKI applies to this SAF.

** ESL is to determine unsaturated hydraulic conductivity using the method(s) best suited for the soil texture and type.

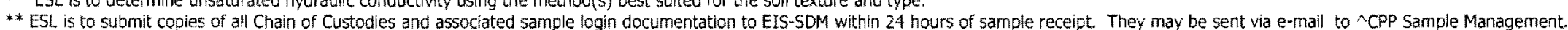

(1)6020M_ICPMS ASTM_AE (TAL) \{Cadmium, Chromium, Silver) 6020M_ICPMS_ASTM_AE (Add-On) \{Arsenic, Lead, Molybdenum, Selenium\} 6020M_METALS ICPMS_WE (TAL) \{Cadmium, Chromium, Silver)

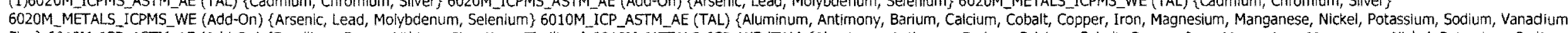

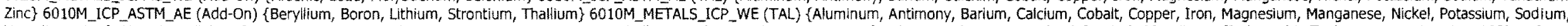

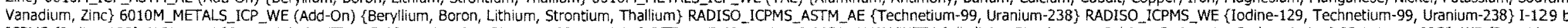

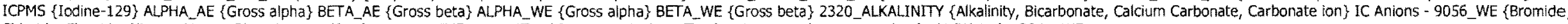
Chloride, Fluoride, Nitrate, Nitrite, Phosphate, Sulfate\} TOC - ASTME1915A \{Total Inorganic Carbon, Total carbon, Total organic carbon\} pH (Water) - 9045 WE; 


\section{CHAIN OF CUSTODY/SAMPLE ANALYSIS REQUEST}

\section{COMPANY CONTACT}

TRENT, STEVE

PROJECT DESIGNATION

200-BP-5 OU Characterization for Wells 299-E33-341 and 299-E33-342 - G FIELD LOGBOOK NO.

$H N^{1} F-N-488$

ACTUAL SAMPLE DEPTH

$2.77 .5^{\prime}$

Environmental Sciences Laboratory

MATRIX*

A $=$ Air
$\mathrm{DL}=$ Drumn

Liquids

DS=Drum
Solids

Solids

$0=0$ il
$5=5$ oil

$\mathrm{SE}=$ Sediment

$S E=$ Sedime
$T=T i s s u e$

$T=$ Vegitation
$V=$ The

$W=$ Water

WI $=$ Wipe

$x=0$ ther

POSSIBLE SAMPLE HAZARDS/ REMARKS Contains Radioactive Material at concentrations that are not regulated for transportation per 49 CFR but are not releasable per DOE Order $5400.5(1990 / 1993)$

SPECLAL HANDLING AND/OR STORAGE Radioactive Tie TO B1TT59

\begin{abstract}
B1TP92
\end{abstract}
SAMPLE NO. MATRIX*
F08-075-131

PAGE 1 OF 2

PRICE CODE 8 N

$\mathrm{N} / \mathrm{A}$

OFFSITE PROPERTY NO.

\begin{tabular}{|c|c|c|}
\hline PRESERVATION & None & None \\
\hline TYPE OF CONTAINER & $\mathrm{G} / \mathrm{P}$ & $\begin{array}{l}\text { 'Moisture } \\
\text { Resistant Cont }\end{array}$ \\
\hline NO. OF CONTAINER(S) & 1 & 1 \\
\hline VOLUME & $1 \mathrm{~L}$ & 2009 \\
\hline SAMPLE ANALYSIS & $\begin{array}{l}\text { SEE TEM (1) IN } \\
\text { SPETAAL } \\
\text { NNSTRUTIONS }\end{array}$ & $\begin{array}{l}\text { Moisture } \\
\text { Content - D2216; }\end{array}$ \\
\hline
\end{tabular}

SAMPLE DATE SAMPLE TIME

$5-22-081120 \times x$

\section{CHAIN OF POSSESSION}

RELINQUISHED BY/REMONEP FROM-

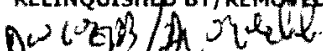

RELINQUISHED BY/REMOVED FROM Ho $4 / 3 / / 1 / 2 x=2$ RELINQUISHED BY/REMGVER FROM

RELINQUISHED BY/REMOVED FROM

RELINQUISHED BY/REMOVED FROM

RELINQUISHED BY/REMOVED FROM

LABORATORY RECEIVEDBY

SECTION

FINAL SAMPLE DISPOSAL METHOD

DISPOSITION

SAF NO.
FO8-075

COA

123514 ES 10

METHOD OF SHIPMENT

BILL OF LADING/AIR BILL NO

N/A 


\section{COLLECTOR}

\section{NCO SAMPLER}

\section{SAMPLING LOCATION}

C5856, I-137

ICE CHEST NO.

\section{SHIPPED TO}

Environmental Sciences Laboratory

\section{COMPANY CONTACT}

TRENT, STEVE

\section{PROJECT DESIGNATION}

TELEPHONE NO.

373-5869

200-BP-5 OU Characterization for Wells 299-E33-341 and 299-E33-342 - G FIELD LOGBOOK NO.

ACTUAL SAMPLE DEPTH

\section{OFFSITE PROPERTY NO.}

$N / A$
PROJECT COORDINATOR

WIDRIG, DL

SAF NO.

FO8-075

\section{$\mathrm{COA}$}

$123514 E S 10$
PRICE CODE $\quad 8 N$

AIR QUALITY

\section{METHOD OF SHIPMENT}

GOVERNMENT VEHICLE

\section{SPECIAL INSTRUCTIONS}

** The 200 Area S\&GRP Characterization and Monitoring Sampling and Analysis GKI applies to this SAF.

** ESL is to determine unsaturated hydraulic conductivity using the method(s) best suited for the soil texture and type.

*** ESL is to submit coples of all Chain of Custodies and associated sample login documentation to EIS-SDM within 24 hours of sample receipt. They may be sent via e-mail to $\wedge$ CPP Sample Management.

(1)6020M ICPMS ASTM AE (TAL) \{Cadmium, Chromium, Silver\}6020M ICPMS ASTM AE (Add-On) \{Arsenic, Lead, Molybdenum, Selenium\} 6020M METALS ICPMS WE (TAL) (Cadmium, Chromium, Silver\}

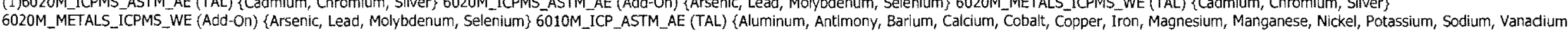

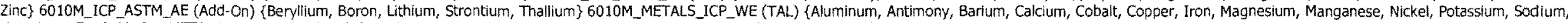

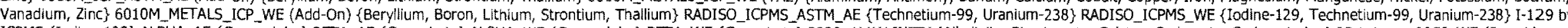

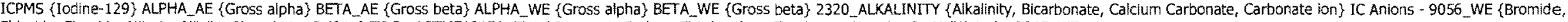
Chloride, Fluoride, Nitrate, Nitrite, Phosphate, Sulfate\} TOC - ASTME1915A \{Total Inorganic Carbon, Total carbon, Total organic carbon\} pH (Water) - 9045 WE; 
CHAIN OF CUSTODY/SAMPLE ANALYSIS REQUEST

\section{TELEPHONE NO.}

$373-5869$

NCO SAMPLER WEBb

SAMPLING LOCATION

C5856, I- 138

ICE CHEST NO.

\section{SHIPPED TO}

Environmentai Sciences Laboratory

\section{MATRIX*}

$A=A$ ir

$A=A i r$
$\mathrm{DL}=$ Drum

Liquids

DS=Drum
Solids

Solids

$0=0$ il

$S=S$ oil
$S E=S$ Sdiment
$T=T$ Tissue

$v=$ vegitation

$V=V$ egitat

WI $=$ wipe

$x=$ other

POSSIBLE SAMPLE HAZARDS/ REMARKS Contains Radioactive Material at concentrations that are not regulated for transportation per 49 CFR but are not releasable per DOE Order $5400.5(1990 / 1993)$

SPECIAL HANDLING AND/OR STORAGE Radioactive Tie To B1TT59
TRENT, STEVE

PROJECT DESIGNATION

200-8P-5 OU Characterz FIELD LOGBOOK NO.

HNF-N-4881

OFFSITE PROPERTY NO.

$N / A$

\section{PRESERVATION}

TYPE OF CONTAINER

NO. OF CONTAINER(S)

VOLUME
SAMPLE ANALYSIS

\section{ACTUAL SAMPLE DEPTH}

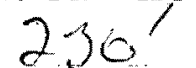

SEE rTEM (a) IN MOsture

SPECIAL Content - D2216:
AIR QUALITY
SAMPLE NO. MATRIX*

B1TP93
SOIL

\section{SAF NO}

METHOD OF SHIPMENT GOVERNMENT VEHICLE

\section{BILL OF LADING/AIR BILL NO}

SAMPLE DATE SAMPLE TIME

$5-22+6 / 411 \times x$

\section{SIGN/ PRINT NAMES}

RECEIVED BY/STORED IN "

RELINQUISHED BY/REMOVED FROM

713 Feros 2

DATE/TE

RELINQUISHED BY/REMOVED FROM

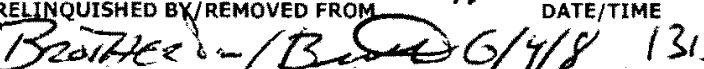

RELINQUISHED BY/REMOVED FROM

RELINOUISHED BY/REMOVED FROM

DATE/TIME

RECEXYED BY/STORED IN

RELINQUISHED BY/REMOVED FROM

DATE/TIME

RECEIVED BY/STORED IN

EIYED BYISTORED IN

Clined

RELINQUISHED BY/REMOVED FROM

DATE/TIME

RECEIVED BY/STORED IN

\section{SPECIAL INSTRUCTIONS}

DATE/T/ME

$5-7 \pm-08 / / 6 c e$

DATETIME

$6 / 4 / 811: 30$

GDAERTM

$6 / 412313 / 5$

DATE/TIME

DATE/TIME

DATE/TIME

DATE/TIME 


\section{COLLECTOR}

NCO SAMPLER

\section{SAMPLING LOCATION}

C5856, I-138

ICE CHEST NO.

\section{SHIPPED TO}

Environmental Sciences Laboratory

\section{COMPANY CONTACT}

TRENT, STEVE

TELEPHONE NO.

PROJECT DESIGNATION

$373-5869$

SAF NO.

F08-075

FIELD LOGBOOK NO.

ACTUAL SAMPLE DEPTH

\section{COA}

123514ES10
PRICE CODE

$\mathbf{8 N}$

DATA

TURNAROUND

45 Days / 45

Days

\section{OFFSITE PROPERTY NO.}

N/A

\section{BILL OF LADING/AIR BILL NO.}

N/A

\section{SPECIAL INSTRUCTIONS}

** The 200 Area S\&GRP Characterization and Monitoring Sampling and Analysis GKI applies to this SAF.

** ESL is to determine unsaturated hydraulic conductivity using the method(s) best suited for the soil texture and type.

** ESL is to submit copies of all Chain of Custodies and associated sample login documentation to EIS-SDM within 24 hours of sample receipt. They may be sent via e-mail to ^CPP Sample Management.

(1)6020M ICPMS ASTM AE (TAL) \{Cadmium, Chromium, Silver\},6020M ICPMS ASTM AE (Add-On) \{Arsenic, Lead, Molybdenum, Selenium) 6020M METALS ICPMS WE (TAL) \{Cadmium, Chromium, Silver\}

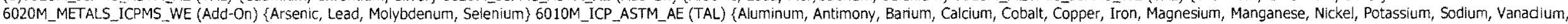

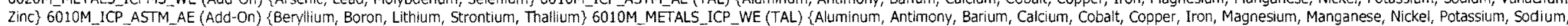

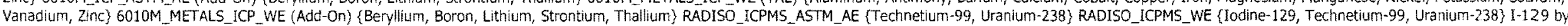

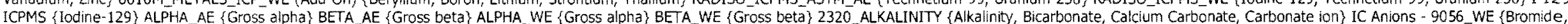
Chloride, Fluoride, Nitrate, Nitrite, Phosphate, Sulfate\} TOC-ASTME1915A (Total Inorganic Carbon, Total carbon, Total organic carbon\} pH (Water) - 9045..WE; 


\section{COLLECTOR}

NCO SAMPLER LUEIZ?

\section{COMPANY CONTACT}

TELEPHONE NO.

PROJECT COORDINATOR

WIDRIG, DL

PRICE CODE

DATA

PROJECT DESIGNATION

SAF NO.

C5856, 1-139

ICE CHEST NO.

\section{SHIPPED TO}

Environmental Sciences Laboratory

MATRIX*

$A=A$ ir

$\mathrm{DL}=$ Drum

Liquids
DS $=$ Drum

DS $=$ Drum
Solds

POSSIBLE SAMPLE HAZARDS/ REMARKS Contains Radioactive Material at concentrations that are not regulated for transportation per 49 CFR but are not releasable per DOE Order $5400.5(1990 / 1993)$

$0=0$ il
$5=5$ il

Stail

$T=$ Tissue

$T=1$ issue
$\mathrm{V}=$ vegitation

$W=$ water

$W]=$ Wipe

$x=$ Other

SPECIAL HANDLING AND/OR STORAGE Radioactive Tie To B1T559

200-BP-5 OU Characterization for Wells 299-E33-341 and 299-E33-342-G F08-075 FIELD LOGBOOK NO. ACTUAL SAMPLE DEPTH

COA

$$
\text { Hnf }-n^{\prime}-4881 \quad 232.5^{\prime}
$$

$123514 \mathrm{ES} 10$

AIR QUALITY

OFFSITE PROPERTY NO.

BILL OF LADING/AIR BILL NO

N/A

$N / A$

METHOD OF SHIPMENT

GOVERNMENT VEHICLE

\begin{tabular}{|c|c|c|}
\hline PRESERVATION & None & None \\
\hline TYPE OF CONTAINER & $G P$ & $\begin{array}{l}\text { Motsture } \\
\text { Resistant Cont }\end{array}$ \\
\hline YO. OF CONTAINER(S) & 1 & 1 \\
\hline VOLUME & $1 \mathrm{t}$ & 2009 \\
\hline SAMPLE ANALYSIS & $\begin{array}{l}\text { SEE TTEM (1) IN } \\
\text { SPECIAL } \\
\text { INSTRUCTONS }\end{array}$ & $\begin{array}{l}\text { Moisture } \\
\text { Content-D2216; }\end{array}$ \\
\hline
\end{tabular}

SAMPLE DATE SAMPLE TIME

$5+2-1455 \times x$

\section{SIGN / PRINT NAMES}

CHAIN OF POSSESSION

RELINQUISHE 6 BY/REMOVER,FROM

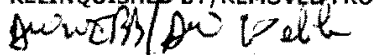

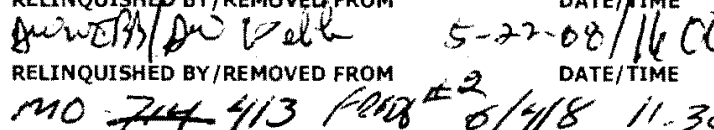
RELINQUISHED BY/REMOVED FIOM DATE/TIME

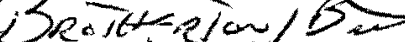
RELINQUISHED BY/REMOVED FROM $6 / 4 / 813 / 5$

RELINQUISHED BY/REMOVED FROM

DATE/TIME

DATE/TIME

DATE/TIME

RECEIVED BY/STORED IN

RELINQUISHED BY/REMOVED FROM $C, I$

RELINQUISHED BY/REMOVED FROM RECEIVED BY/STORED

RECEIVED BY/STORED IN RECEIVED BY/STORED IN

RECEIVED BY/STORED IN

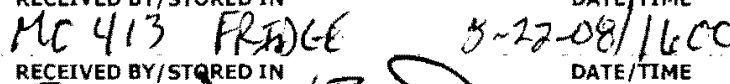
RECEIVED BY/STQREOIN $P \longrightarrow$ DATE/TIME

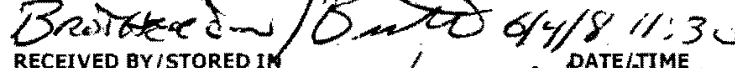
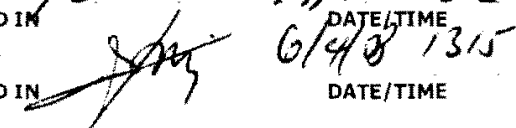

DATE/TIME

DATE/TIME

LABORATORY RECEIVED BY SECTION

FINAL SAMPLE DISPOSALMETHOD

DATE/TIME

DATE/TIME 


\section{COLLECTOR}

NCO SAMPLER

\section{SAMPLING LOCATION}

C5856, I-139

ICE CHEST NO.

\section{SHIPPED TO}

Environmental Sciences Laboratory

\section{COMPANY CONTACT}

TRENT, STEVE

TELEPHONE NO.

373-5869

\section{PROJECT DESIGNATION}

200-BP-5 OU Characterization for Wells 299-E33-341 and 299-E33-342 - G FIELD LOGBOOK NO.

ACTUAL SAMPLE DEPTH

\section{OFFSITE PROPERTY NO.}

$N / A$
PROJECT COORDINATOR

WIDRIG, DL.

SAF NO.

F08-075

\section{COA}

123514 ES10

\section{PRICE CODE SN}

AIR QUALITY

\section{METHOD OF SHIPMENT}

GOVERNMENT VEHICLE

BILL OF LADING/AIR BILL NO

$N / A$

\section{SPECIAL INSTRUCTIONS}

** The 200 Area S\&GRP Characterization and Monitoring Sampling and Analysis GKI applies to this SAF

** ESL is to determine unsaturated hydraulic conductivity using the method(s) best suited for the sol texture and type.

** ESL is to submit copies of all Chain of Custodies and associated sample login documentation to EIS-SDM within 24 hours of sample receipt. They may be sent via e-mail to ^CPP Sample Management.

(1)6020M_ICPMS_ASTM_AE (TAL) \{Cadmium, Chromium, Silver\} 6020M_ICPMS_ASTM_AE (Add-On) \{Arsenic, Lead, Molybdenum, Selenium\} 6020M_METALS_ICPMS WE (TAL) \{Cadmium, Chromium, Silver\}

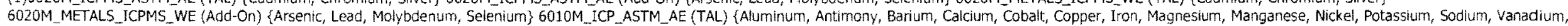

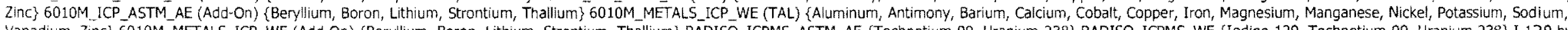

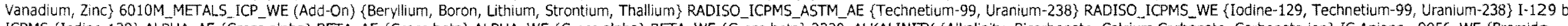

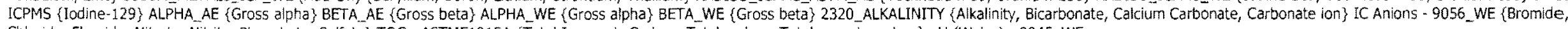

Chloride, Fluoride, Nitrate, Nitrite, Phosphate, Sulfate\} TOC - ASTME1915A \{Total Inorganic Carbon, Total carbon, Total organic carbon\} pH (Water) * 9045 _WE: 
SAMPLING Locatión

C5856, I-10]

ICE CHEST NO.

\section{SHIPPED TO}

Environmental Sciences Laboratory

MATRIX*

$A=$ Air

$\mathrm{DL}=\mathrm{Drum}$

Licuids

DS $=$ Drum
Solids

$L=$ Liquid

$0=0$ il
$\mathrm{S}=\mathrm{Sol}$

$\mathrm{S}-\mathrm{Sol}$
$\mathrm{SE}=\mathrm{Sediment}$

$T=$ Tissue

$V=$ Vegitation

$W=$ water

$W 1=$ Wipe
$X=$ other

POSSIBLE SAMPLE HAZARDS/ REMARKS Contains Radioactive Material at concentrations that are not requlated for transportation per 49 CFR but are not releasable per DOE Order $5400.5(1990 / 1993)$

SPECIAL HANDLING AND/OR STORAGE Radloactive TIe To: B1TPB5

\section{COMPANY CONTACT}

TRENT, STEVE

TELEPHONE NO.

373.5869

PROJECT COORDINATOR

WIDRIG, DL

PRICE CODE

DATA

\section{SAF NO.}

F08.075

200-BP-5 OU Characterization for Wells 299-E33-341 and 299-E33-342-G

FIELD LOGBOOK NO.

ACTUAL SAMPLE DEPTH

\section{COA}

AIR QUALITY

TURNAROUND

45 Days / 45 Days

$H N=-2-488.172 .5^{\prime}$

OFFSITE PROPERTY NO

$123514 \mathrm{ES} 10$

METHOD OF SHIPMENT

BILL OF LADING/AIR BILL NO.

N/A

N/A
SAMPLE NO.
MATRIX*

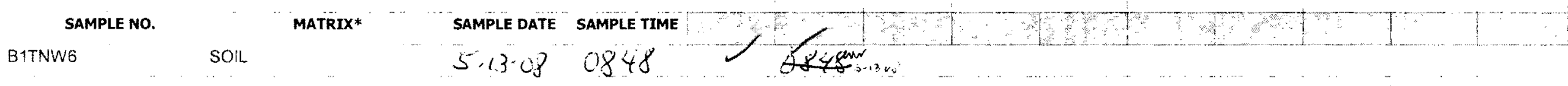
$5.13080848 \cdots$

\section{CHAIN OF POSSESSION}

RELINQUISHED BY/REMOVED FROM

Mathitr ha.t.

RELINQUISHED BY/REMOVED FROM M0 1045 PEPXI RELINQUISHED BY/REMOVED FROM $\$ / / 58$

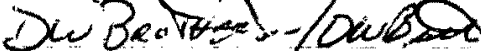
RELINQUISHED BY/REMOLIED FROM

RELINQUISHED BY/REMOVED FROM

RELINQUISHED BY/REMOVED FROM

RELINOUISHED BY/REMOVED FROM

LABORATORY RECEIVED BY
SECTION

FINAL SAMPLE DISPOSALMETHOD

DISPOSITION

$\begin{array}{ccc}\text { PRESERVATION } & \text { None } & \text { None } \\ \text { TYPE OF CONTAINER } & \text { G/P } & \begin{array}{l}\text { Moisture } \\ \text { Resistant Cont }\end{array} \\ \text { NO. OF CONTAINER(S) } & 1 & 1 \\ \text { VOLUME } & 1 \mathrm{~L} & 2009 \\ \text { SAMPLE ANALYSIS } & \begin{array}{l}\text { SEE IEEM (1) iN } \\ \text { SPECLAL Moistire } \\ \text { TWSTRUCTIONS }\end{array}\end{array}$

\section{C.T SIGN/ PRINT NAMES -.}

DATE/TIME RECEIVED BY/STOREDIN DATE/TIME

-OQ woo Mo.745 Fris $15.13-081600$

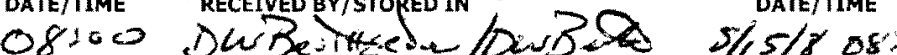

DATE/TIME RECEIVED BY/STORED IN

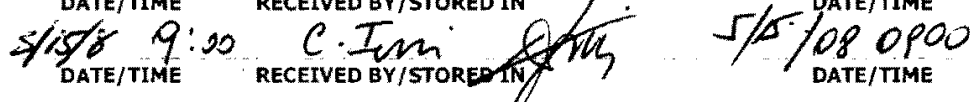

DATE/TIME

RECEIVED BY/STOREDIN

DATE/TME

DATE/TIME

RECEIVED BY/STORED IN

DATE/TIME

DATE/TIME

\section{SEE PAGE 2 FOR ALL SPECIAL INSTRUCTIONS}

TITLE

DATE/TIME

DISPOSED BY

DATE/TIME 


\section{COLLECTOR}

NCO SAMPLER

\section{SAMPLING LOCATION}

C5856, I-101

ICE CHEST NO.

\section{SHIPPED TO}

Environmental Sciences Laboratory

\section{COMPANY CONTACT}

TRENT, STEVE

PROJECT DESIGNATION

200-BP-5 Cu Characterization for Wells 299-E33-341 and 299-E33-342-G FIELD LOGBOOK NO.

\section{OFFSITE PROPERTY NO}

N/A
ACTUAL SAMPLE DEPTH

\section{TELEPHONE NO.}

373-5869

$-1$

\section{SPECIAL INSTRUCTIONS}

** The 200 Area S\&GRP Characterization and Monitoring Sampling and Analysis GKI applies to this SAF.

(1)6020M_ICPMS_ASTM_AE (TAL) \{Cadmium, Chromium, Silver\} 6020M_ICPMS_ASTM_AE (Add-On) \{Arsenic, Lead, Molybdenum, Selenium\} 6020M_METALS_ICPMS WE (TAL) \{Cadmium, Chromium, Silver\}

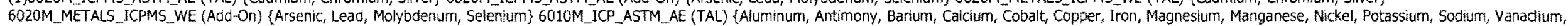

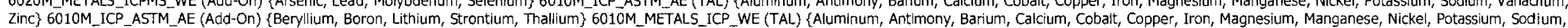

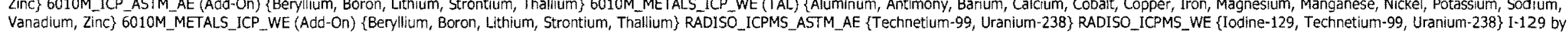

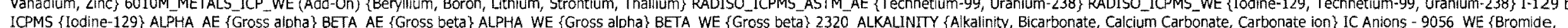
Chloride, Fiuoride, Nitrate, Nitrite, Phosphate, Sulfate\} TOC-ASTME1915A \{Total Inorganic Carbon, Total carbon, Total organic carbon\} pH (Water) -9045_WE: 


\section{CHAIN OF CUSTODY/SAMPLE ANALYSIS REQUEST}

\section{TELEPHONE NO.}

373-5869

TRENT, STEVE

PROJECT DESIGNATION

200-BP-5 OU Characterization for Wells 299-E33-341 and 299-E33-342-G FIELD LOGBOOK NO.

GNF-N.488-1

OFFSITE PROPERTY NO.

N/A

$\mathrm{N} / \mathrm{A}$
F08-075-158

PRICE CODE

PROJECT COORDINATOR

WIORIG, DL

SAF NO.

FOB-075
C5856, 1-102

\section{SHIPPED TO}

Envirommental Sciences Laboratory

MATRIX* POSSIBLE SAMPLE HAZARDS/ REMARKS

A=Air Contains Radioactive Material at concentrations

$D L=D r u m \quad$ that are not regulated for tuansportation per 49

Liquids that are not regulated for transportation per

$\begin{array}{ll}\text { DS=Dram } & \text { CrR but are not rele } \\ \text { Solids } & 5400.5(1990 / 1993)\end{array}$

$L=$ Liquid

$S=$ Soil

$\mathrm{SE}=$ Sediment

$\mathrm{T}=\mathrm{T}$ issue

$V=$ vegitation

$W I=$ Wipe

SPECIAL HANDLING AND/OR STORAGE Radioactive Tie TO: BITPB5

\begin{tabular}{cll} 
PRESERVATION & None & None \\
\hline TYPE OF CONTAINER & G/P & $\begin{array}{l}\text { Moisture } \\
\text { Resistant Cont }\end{array}$ \\
NO. OF CONTAINER(S) & 1 & 1 \\
VOLUME & $1 \mathrm{~L}$ & 2009 \\
\hline SAMPLE ANALYSIS & $\begin{array}{l}\text { SEE IEM (1) IN MDisture } \\
\text { SPECAL } \\
\text { INSTRUCTIONS }\end{array}$ \\
\hline
\end{tabular}

\section{COA}

$123514 E S 10$
DATA TURNAROUND

45 Days / 45 Days

OF LADING/AIR BILL NO

METHOD OF SHTPMENT

GOVERNMENT VEHICLE

\begin{abstract}
SAMPLE NO. MATRIX*
\end{abstract}
SOIL

$$
\text { LOTH }
$$

CHAIN OF POSSESSION

RELINQUISHED BY/REMOVED FROM DATE/TIME

M9 Whit May Dit 5-1308 1600 A.745 Ref

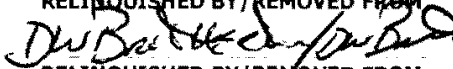

REIINOUISHED BY/RENOVED FROM

RELINQUISHED BY/REMOVED FROM

RELINQUISHED BY/REMOVED FROM

RELINQUISHED BY/REMOVED FROM

$5 \% 578$ 9:0

DATE/TIME

DATE/TIME

DATE/TIME

DATE/TIME

SAMPLE DATE SAMPLE TIME

$5.13 .08 \quad 0927$

\section{LABORATORY RECEIVED BY} SECTION

FINAL SAMPLE DISPOSITION
DISPOSAL METHOD

\section{SIGN/ PRINT NAMES \\ 031394}

RECEIVED BY/STORED IN

DATE/TIME

Mo 475 Frig $151308 / 600$

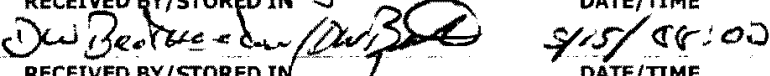

RECEIVED BY/STORED IN

C. $10 \mathrm{vin}$

RECEIVED BY/STORED

RECEIVED BY/STOREO IV

$\sigma / 1, \% / \mathscr{O}$ j:OS

DATE/TIME

DATE/TIME

RECEIVED BY/STORED IN

DATE/TIME

RECEIVED BY/STORED IN
SPECIAL INSTRUCTIONS

SEE PAGE 2 FOR ALL SPECIAL. INSTRUCTIONS 


\section{COLLECTOR}

NCO SAMPLER

\section{SAMPLING LOCATION}

C5856, I-102

ICE CHEST NO.

\section{SHIPPED TO}

Environmental Sciences Laboratory

\section{COMPANY CONTACT}

TRENT, STEVE

PROJECT DESIGNATION

200-BP-5 OU Characterization for Weils 299-E33-341 and 299-E33-342-G FIELD LOGBOOK NO.

\section{OFFSITE PROPERTY NO}

N/A
ACTUAL SAMPLE DEPTH

TELEPHONE NO.

373-5869

PROJECT COORDINATOR

WIDRIG, DL

\section{SAF NO.}

F08-075

COA

$123514 E S 10$

\section{PRICE CODE}

$8 \mathrm{~N}$

AIR QUALITY TURNAROUND

45 Days / 45

Days

\section{SPECIAL INSTRUCTIONS}

** The 200 Area S\&GRP Characterization and Monitoring Sampling and Analysis GKI applies to this SAF.

(1)6020M_ICPMS_ASTM_AE (TAL) \{Cadmium, Chromium, Silver\} 6020M_ICPMS_ASTM_AE (Add-On) \{Arsenlc, Lead, Molybdenum, Selenium\} 6020M_METALS ICPMS WE (TAL) \{Cadmium, Chromium, Silver\}

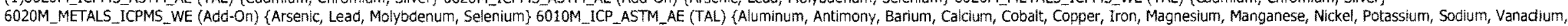

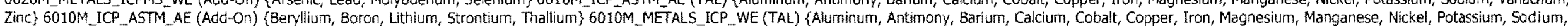

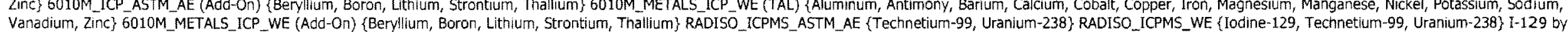

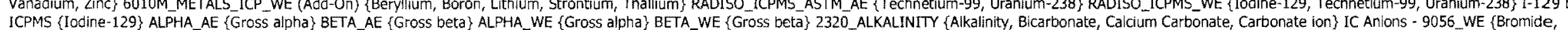
ICPMS \{Iodine-129\} ALPHA_AE \{Gross alpha\} BETA_AE \{Gross beta\} ALPHA_WE \{Gross alpha\} BETA_WE \{Gross beta\} 2320_ALKALINIT \{Alkalinity, Bicarbona.
Chloride, Fluoride, Nitrate, Nitrite, Phosphate, Sulfate\} TOC-ASTME1915A (Total Inorganic Carbon, Total carbon, Total organic carbon\} pH (Water) - 9045 WE; 


\section{COLLECTOR}

NCO SAMPLER Malukite

SAMPLING LOCATION

C5856, I-103

ICE CHEST NO.

\section{SHIPPED TO}

Environmental Sciences Laboratory

MATRIX*

$A=$ Air

$D L=$ Orum

tiquids

DS= Drum

Solids

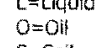

$S=S$ oil

SEE Sediment
$\mathrm{T}=$ Tissue

$V=$ Vecitation

$W=$ Water

$W=$ Wipe

$\mathrm{X}=$ other

\section{POSSIBLE SAMPLE HAZARDS/ REMARKS} Contains Radioactive Material at concentrations that are not regulated for transportation per 49 CFR but are not releasable per DOE Order $5400.5(1990 / 1993)$

SPECIAL HANDLING AND/OR STORAGE Radioactive Tie To: BITPB5

SAMPLE NO.
B1TNW8

\section{COMPANY CONTACT}

TRENT, STEVE

TELEPHONE NO.

373-5869

PROJECT COORDINATOR

WIDRIG, DL

PROJECT DESIGNATION

PRICE CODE

DATA

TURNAROUND

AIR QUALITY

FIELD LOGBOOK NO.

$$
\begin{aligned}
& \text { HNF-N- } 488.1 \\
& \text { OFFSITE PROPERTY NO. }
\end{aligned}
$$

COA

123514 ES10

METHOD OF SHIPMENT

GOVERNMENT VEHICLE
N/A

PRESERVATION
TYPE OF CONTAINER

SAMPLE DATE SAMPLE TIME

$5-13-080937$

\section{CHAIN OF POSSESSION}

RELINQUISHED BY/REMOVED FROM Ma Whit Maci, is lat MO 745 FRIG RELINQUISHED BY / RE HOVEE FROM J. Herick () Him RELINQUISHED BY/RTMOVED FROM

RELINQUISHED BY/REMOVED FROM

RELINQUISHED BY/REMOVED FROM

RELINQUISHED BY/REMOVED FROM

LABORATORY
SECTION
FINAL SAMPLE
DISPOSITION

DATE/TIME 5.13-0P /60O $5 / 14 \%$ o8 1230 D TTE/TIME $5 / 19 / 0$ or 130

DATE/TIME

DATE/TIME

DATE/TIME
RECEIVED BY /STORED IN

\section{SIGN/ PRINT NAMES}

\section{4}

Mo $245 F_{\text {is }} 15-13-0.1600$

3 Hewick $5 / 19 / 051230$

REgEIYED BY/STORED IE]

RECEIVED BY/STORED IN

DATE/TIME

RECEIVED BY/STORED IN

RECEIVED BY/STORED IN

RECEIVED BY/STORED IN

\section{SPECIAL INSTRUCTIONS}

SEE PAGE 2 FOR ALL SPECIAL INSTRUCTIONS 


\section{CHAIN OF CUSTODY/SAMPLE ANALYSIS REQUEST}

TELEPHONE NO.

$373-5869$

TRENT, STEVE

PROJECT DESIGNATION

200-BP-5 OU Characterization for Wells 299-E33-341 and 299-E33-342 - G

FIELD LOGBOOK NO.

ACTUAL SAMPLE DEPTH

OFFSITE PROPERTY NO.

N/A
F08-075-159

PAGE 2 OF 2

TURNAROUND

45 Days / 45

Days
WIDRIG, DL

SAF NO.

123514 ES10
PRICE CODE 8 N

AIR QUALITY

METHOD OF SHIPMENT

GOVERNMENT VEHICLE

\section{SHIPPED TO}

Environmental Sciences Laboratory

\section{BILL OF LADING/AIR BILL NO}

$N / A$

\section{SPECIAL INSTRUCTIONS}

** The 200 Area S\&GRP Characterization and Monitoring Sampling and Analysis GKI applies to this SAF

(1)6020M_ICPMS_ASTM_AE (TAL) \{Cadmium, Chromium, Silver\} 6020M_ICPMS_ASTM_AE (Add-On) \{Arsenic, Lead, Molybdenum, Selenium\} 6020M_METALS_ICPMS_WE (TAL) \{Cadmium, Chromium, Silver\}

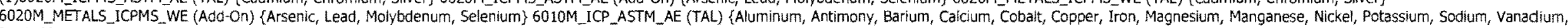

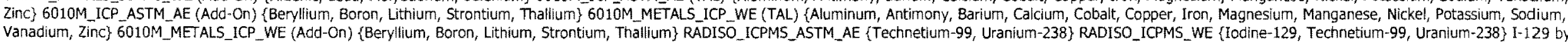

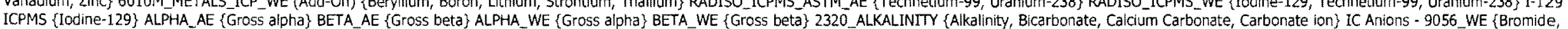
Chloride, Fluoride, Nitrate, Nitrite, Phosphate, Sulfate\} TOC - ASTME1915A \{Total Inorganic Carbon, Total carbon, Total organic carbon\} pH (Water) - 9045_WE: 


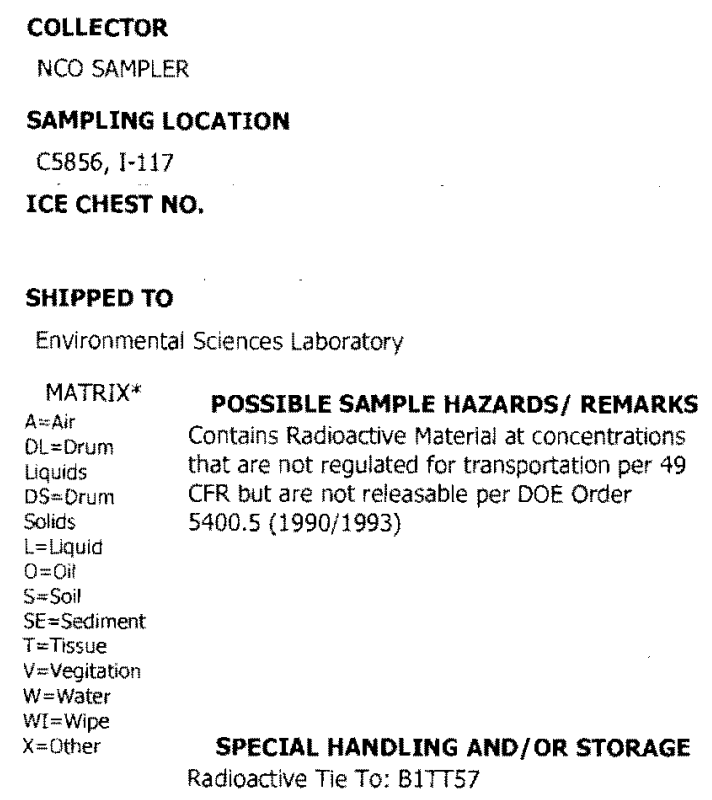

SHIPPED TO

Environmentai Sciences Laboratory

\section{PROJECT DESIGNATION}

373.5869

\section{SAF NO.}

200-BP-5 OU Characterization for Wells 299-E33-341 and 299-E33-342-G FO8-075

FIELD LOGBOOK NO. PEN 34 ACTUAL SAMPLE DEPTH

COA

HNF N-488, 197. OFFSITE PROPERTY NO.

123514 ES10

N/A

BILL OF LADING/AIR BILL NO.

METHOD OF SHIPMENT

GOVERNMENT VEHICLE

\begin{tabular}{ccc} 
PRESERVATION & None & None \\
TYPE OF CONTAINER & G/P & $\begin{array}{c}\text { Moisture } \\
\text { Resistant Cont }\end{array}$ \\
NO. OF CONTAINER(S) & 1 & 1 \\
VOLUME & 11 & 2009 \\
\hline SAMPLE ANALYSIS & $\begin{array}{c}\text { SEE IEM (1) IN Masture } \\
\text { SPELIAL } \\
\text { INSTRUCTONS }\end{array}$ \\
\hline Contert - D2216;
\end{tabular}

SAMPLE NO. MATRIX*

B1TNWG

SOIL

SAMPLE DATE SAMPLE TIME

$5 / 16 / 08 \quad 6830 \quad x \quad x$

\section{CHAIN OF POSSESSION}

RELINQUISHED BY/REMOVED FROM ). Herrick Hemi RELINQUISHED BY REMOVED FRO ON PDTHFring) RELINQUISHED BY/RAMOYED FROM Chris futtin

RELINQUISHED BY/REMOVED FROM

RELINQUISHED BY/REMOVED FROM RELINQUISHED BY/REMOVED FROM

\begin{tabular}{l} 
LABORATORY \\
SECTION \\
$\begin{array}{c}\text { FINAL SAMPLE } \\
\text { DISPOSITION }\end{array}$ \\
\hline
\end{tabular}

$$
\text { Lot } 031344
$$

\section{SIGN/ PRINT NAMES}

RECEIVED BY/STORED IN $5 / 4$ os 1.500 MO 745 FRIDG
DATE/TIME $5 / 16 / 08 \quad 150$

\section{SPECIAL INSTRUCTIONS}

SEE PAGE 2 FOR ALL SPECIAL INSTRUCTIONS

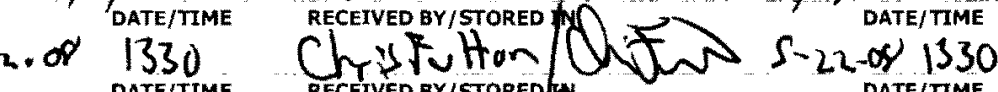

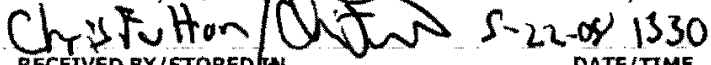 DATE/TIME}

S2L- OP 140 DATE/TIME

Arom ith it RECEIVED BY/STORED IN $68 / 400$ DATE/TIME DATE/TIME

RECEIVED BY/STORED IN DATE/TIME DATE/TIME RECEIVED BY/STORED IN RECEIVED BY/STORED IN
DATE/TIME DATE/TIME 


\section{COMPANY CONTACT}

TRENT, STEVE

TELEPHONE NO.

\section{PROJECT DESIGNATION}

$373-5869$

\section{SAMPLING LOCATION}

C5856, I-117

ICE CHEST NO.

\section{SHIPPED TO}

Environmental Sciences Laboratory
200-BP-5 OU Characterization for Wells 299-E33-341 and 299-E33-342-G

FIELD LOGBOOK NO.

ACTUAL SAMPLE DEPTH

\section{HN \\ OFFSITE PROPERTY NO.}

197.5

N/A
PROJECT COORDINATOR

WIDRIG, DL

SAF NO.

F08-075

COA

123514ES10

\section{RICE CODE 8 N}

AIR QUALITY
DATA TURNAROUND

45 Days / 45 Days

\section{SPECIAL INSTRUCTIONS}

** The 200 Area S\&GRP Characterization and Monitoring Sampling and Analysis GKI applies to this SAF.

(1)6020M_ICPMS ASTM_AE (TAL) \{Cadmium, Chromium, Silver\} 6020M_ICPMS_ASTM_AE (Add-On) \{Arsenic, Lead, Molybdenum, Selenium\} 6020M_METALS ICPMS WE (TAL) \{Cadmium, Chromium, Silver\}

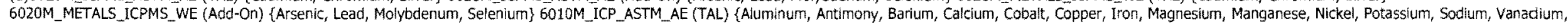

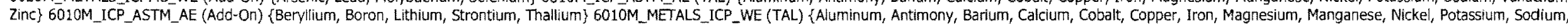

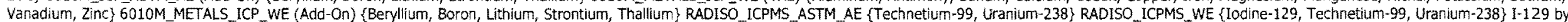

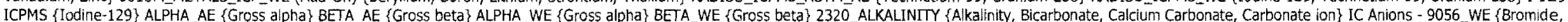
Chloride, Fluoride, Nitrate, Nitrite, Phosphate, Sulfate\} TOC - ASTME1915A \{Total Inorganic Carbon, Total carbon, Total organic carbon\} pH (Water) - 9045 _WE; 
CHAIN OF CUSTODY/SAMPLE ANALYSIS REQUEST

\section{TELEPHONE NO.}

373-5869

TRENT, STEVE

PROJECT DESIGNATION

200-BP-5 Ou Characterization for Wells 299-E33-341 and 299-E33-342-G F08-075

FIELD LOGBOOK NO.

HNF.N-48*1

OFFSITE PROPERTY NO.

ACTUAL SAMPLE DEPTH

$$
200
$$

N/A

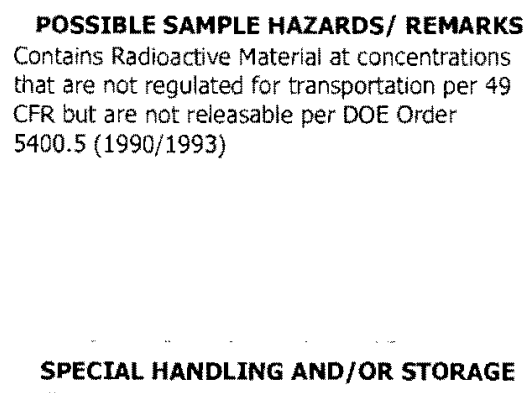

POSSIBLE SAMPLE HAZARDS/ REMARKS Contains Radioactive Material at concentrations that are not regulated for transportation per 49 that are not regulated for transportation per 49
CFR but are not releasable per DOE Order $5400.5(1990 / 1993)$

SPECIAL HANDLING AND/OR STORAGE

$D S=$ orum

Solids

$\mathrm{C}=\mathrm{OI}$
$\mathrm{S}=\mathrm{S}$

$S=$ Soll
$S E=$ Sediment

SE=Tissue

$T=7$ issue
$V=V$ egitation
$W=V$ W

$W=$ Water
$W I=$ Wipe

Wi= Wipe
$x=$ other Radioactive Tie To: B1TT57

COA

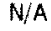

MATRIX*
SOIL

PRESERVATION
TYPE OF CONTAINER

SAMPLE DATE SAMPLE TIME

$5 / 16 / 0807 / 2 \times+$
PAGE 1 OF 2

PRICE CODE

TURNAROUND

45 Days / 45

Days

\begin{abstract}
BITNXO
\end{abstract}

\section{CHAIN OF POSSESSION}

RELINQUISHED BY/REMOVED FROM

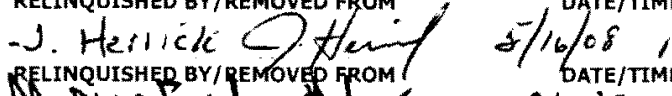
- 1. Herick Pflem f $\$ / 16 / 08$, D. 145 Fidse $5-22.08^{1330}$ RELINQUISHED BY/REMPOEP FROM DATE/TTME

Chrisfultor Sou

RELINQUISHED BY/REMOVED FROM

RELINQUISHED BY/REMOVED FRÖM

RELINQUISHED BY/REMOVED FROM

$$
\begin{aligned}
& \text { LABORATORY } \\
& \text { SECTION }
\end{aligned}
$$

INAL SAMPLE

DISPOSITION

\section{RECEIVED BY}

DISPOSAL METHOD
DATE/TTME

DATE/TIME

DATE/TIME

$$
\cot 031397
$$

\section{SIGN/ PRINT NAMES}

RECEIVED BY/STORED IN Mo.745 FRID6

\section{SPECIAL INSTRUCTIONS}

SEE PAGE 2 FOR ALL SPECIAL INSTRUCTIONS IVEDBYSTOREDII Chisfution Whitos S.22.0\% 1330 RECEIVED BY/STORES WN DATE/TTME Domith fI JA $5: 22.08140$ RECEIVED QY/STORED IN

BILL. OF LADING/AIR BILL NO.

RECEIVED BY/STORED IN

DATE/TIME

RECEIVED BY/STORED IN

DATE/TIME

RECEIVED BY/STORED IN

DATE/TIME 


\section{COLLECTOR}

NCO SAMPLER

\section{COMPANY CONTACT}

TRENT, STEVE

TELEPHONE NO.

PROJECT DESIGNATION

$373-5869$

PROJECT COORDINATOR

WIDRIG, DL

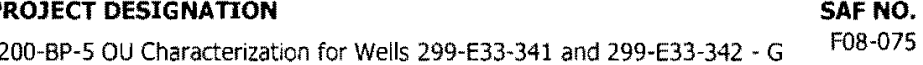

\section{FIELD LOGBOOK NO.}

ACTUAL SAMPLE DEPTH

ICE CHEST No.

\section{SHIPPED TO}

Environmental Sciences Laboratory

\section{UNF-N Y 58 OFFSITE PROPERTY NO.}

N/A

\section{$200^{\circ}$}

$\operatorname{COA}$

123514 ES10
PRICE CODE BN

AIR OUALITY

DATA TURNAROUND

45 Days / 45

Days

\section{SPECIAL INSTRUCTIONS}

*** The 200 Area S\&GRP Characterization and Monitoring Sampling and Analysis GKI applies to this SAF.

(1)6020M ICPMS_ASTM AE (TAL) \{Cadmium, Chromium, Silver) 6020M ICPMS ASTM_AE (Add-On) \{Arsenic, Lead, Molybdenum, Selenium\} 6020M METALS ICPMS WE (TAL) \{Cadmium, Chromium, Silver\}

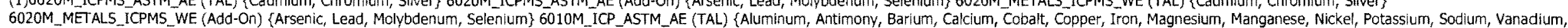

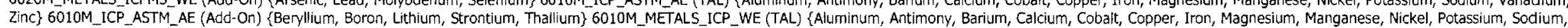

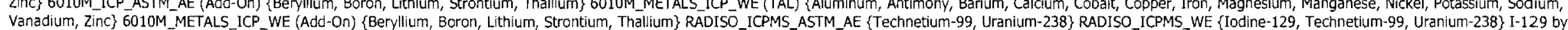

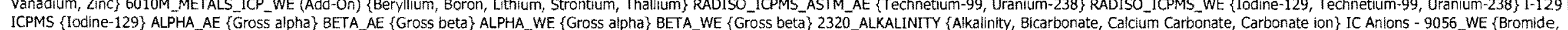
ICPMS \{lodine-129\} ALPHA AE \{Gross alpha\} BETA AE \{Gross beta\} ALPHA WE \{Gross alpha\} BETA_WE \{Gross beta\} 2320_ALKALINITY \{Alkalinity, Bicarbona
Chloride, Fluoride, Nitrate, Nitrite, Phosphate, Sulfate\} TOC. ASTME1915A (Total Inorganic Carbon, Total carbon, Total organic carbon\} pH (Water) -9045_WE; 


\section{TELEPHONE NO.}

373-5869

TRENT, STEVE

PROJECT DESIGNATION

200-BP-5 OU Characterization for Wells 299-E33-341 and 299-E33-342 - C FIELD LOGBOOK NO. ACTUAL SAMPLE DEPTH HNF.n.YSE) OFFSITE PROPERTY NO.

$$
202.5^{\circ}
$$

N/A

\begin{tabular}{|c|c|c|}
\hline PRESERVATION & None & None \\
\hline TYPE OF CONTAINER & G/P & $\begin{array}{l}\text { Moisture } \\
\text { Resistant Cont }\end{array}$ \\
\hline NO. OF CONTAINER(S) & 1 & 1 \\
\hline VOLUME & 12 & $200 \mathrm{~g}$ \\
\hline SAMPLE ANALYSIS & $\begin{array}{l}\text { SEE TEM (1) HA } \\
\text { SPECIAL } \\
\text { INSTRUTIONS }\end{array}$ & $\begin{array}{l}\text { Mossture } \\
\text { Content - D22 }\end{array}$ \\
\hline
\end{tabular}

\section{CHAIN OF CUSTODY/SAMPLE ANALYSIS REQUEST}

PAGE 1 OF 2

Environmental Sciences Laboratory

\section{MATRIX* POSSIBLE SAMPLE HAZARDS/ REMARKS $A=A i r \quad$ Contains Radioactive Material at concentrations Liquids that are not regulated for transportation per 49 DS=Drum CFR but are not releasable per DOE Order solids $\quad 5400.5(1990 / 1993)$ \\ $\mathrm{D}=\mathrm{Oi}$ il \\ $S=$ Soil \\ $\mathrm{SE}=$ Sediment \\ $T=T$ Issue
$V=V$ egitation
$W=W$ at \\ $W=$ Water
WI $=$ Wuipe
$x$ \\ $x=0$ ther \\ SPECIAL HANDLING AND/OR STORAGE Radioactive Tie To: B1TT5?}

PROJECT COORDINATOR

WIDRIG, DL

SAF NO.

$\cos$

123514 ES10

BILL OF LADING/AIR BILL NO

N/A
METHOD OF SHIPMENT

\footnotetext{
SAMPLE DATE SAMPLE TIME

$5 / 16 / 08$ c 935

MATRIX*

$$
\text { B1TNX1 }
$$

SAMPLE NO.

SOIL

$\therefore=$
}

\section{CHAIN OF POSSESSION}

RELINQUISHED BY/REMOYED FROM J.tenck S.tum RELINQuISHEPBY/AMOVEDFROM $5 / \%$ of 15

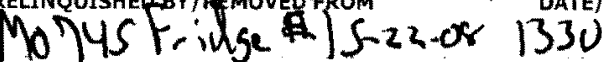
RELINQUTSHED BY/REMOYFP FRQRQR

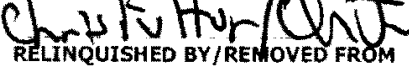

RELINQUISHED BY/REMOVED FROM

RELINQUISHED BY/REMOVED FROM

RELINQUISHED BY/REMOVED FROM

$$
\begin{aligned}
& \text { LABORATORY RECEIVED BY } \\
& \text { SECTION }
\end{aligned}
$$

FINAL SAMPLE DISPOSAL METHOD DISPOSITION

\section{DATE/TIME}

\section{$5-22-08^{\prime} / 4$} DATE/TIME

DATE/TIME

DATE/TIME

DATE/TIME

$$
\text { Lut }+031394
$$

\section{SIGN/ PRINT NAMES}

RECEIVED BY/STORED IN MO 745 Fe,06 4 DATE/TIME AEEIVEO

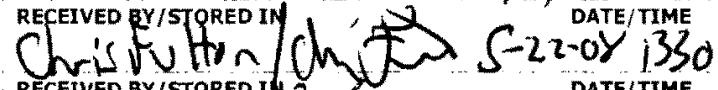
RECEIVED BY/STORED IN/ DATE/TIME

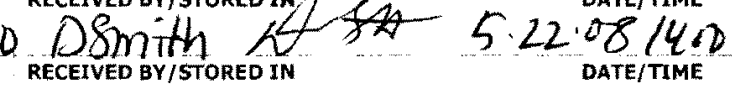
DATE/TIME

RECEIVED BY/STORED IN

DATE/TIME RECEIVED BY/STORED IN DATE/TIME

\section{SPECIAL INSTRUCTIONS}

SEE PAGE 2 FOR ALL SPECIAL INSTRUCTIONS
TITLE

DISPOSED BY
DATE/TIME

DATE/TIME 


\section{COLLECTOR}

NCO SAMPLER

SAMPLING LOCATION

C5856, I-119

ICE CHEST NO.

\section{SHIPPED TO}

Environmental Sciences Laboratory

\section{COMPANY CONTACT}

TRENT, STEVE

TELEPHONE NO.

$373-5869$

PROJECT DESIGNATION

200-BP-5 OU Characterization for Wells 299-E33-341 and 299-E33-342-G FIELD LOGBOOK NO.

ACTUAL SAMPLE DEPTH

HNF.N.488/ $202.5^{\circ}$

OFFSITE PROPERTY NO.

N/A
PROJECT COORDINATOR

WIDRIG, DL

SAF NO.

FD8-075

COA

$123514 E S 10$
PRICE CODE 8 N

AIR QUALITY

METHOD OF SHIPMENT

GOVERNMENT VEHICLE

\section{SPECIAL INSTRUCTIONS}

** The 200 Area S\&GRP Characterization and Monitoring Sampling and Analysis GKI applies to this SAF.

(1)6020M_ICPMS_ASTM_AE (TAL) \{Cadmium, Chromium, Silver\} 6020M_ICPMS_ASTM_AE (Add-On) \{Arsenic, Lead, Molybdenum, Selenium\}6020M_METALS_ICPMS WE (TAL) \{Cadmium, Chromium, Silver\}

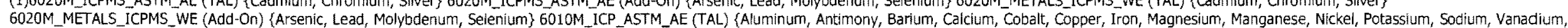

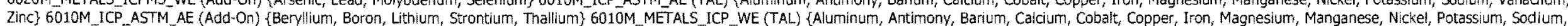

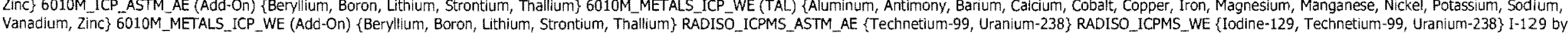

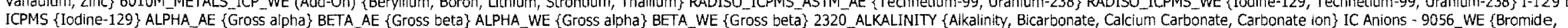
Chioride, Fluoride, Nitrate, Nitrite, Phosphate, Sulfate\} TOC - ASTME1915A \{Total Inorganic Carbon, Total carbon, Total organic carbon\} pH (Water) - 9045 WE; 
NCO SAMPLER

\section{SAMPLING LOCATION}

C5856, $1-120$

ICE CHEST NO.

\section{SHIPPED TO}

Environmental Sciences Laboratory

\section{MATRIX* POSSIBLE SAMPLE HAZARDS/ REMARKS $A=A i r \quad$ Contains Radioactive Material at concentrations Liquids that are not regulated for transportation per 49 DS=Drum CFR but are not releasable per DOE Order Solids $\quad 5400.5(1990 / 1993)$ \\ L= Liquid \\ $0=0$ il \\ SE $=$ Sediment \\ $V=$ Vegitation \\ $W=$ water \\ $W I=$ Wipe \\ $x=0$ ther \\ SPECIAL HANDLING AND/OR STORAGE Radioactive TIE TO: B1TTS7}

\section{TELEPHONE NO.}

373-5869

\section{COMPANY CONTACT}

TRENT, STEVE

PROJECT DESIGNATION

200-BP-5 OU Characterization for Wells 299-E33-341 and 299-E33-342-G FIELD LOGBOOK NO.

HNF-N. 4851

OFFSITE PROPERTY NO

ACTUAL SAMPLE DEPTH

$N / A$

\begin{tabular}{|c|c|c|}
\hline PRESERVATION & None & None \\
\hline TYPE OF CONTAINER & $G / P$ & $\begin{array}{l}\text { Moisture } \\
\text { Resistant Cont }\end{array}$ \\
\hline NO. OF CONTAINER(S) & 1 & 1 \\
\hline VOLUME & $1 \mathrm{~L}$ & 2000 \\
\hline SAMPLE ANALYSIS & $\begin{array}{l}\text { SEE TEEM (1) N } \\
\text { SPECARL } \\
\text { INSTRUCTONS }\end{array}$ & $\begin{array}{l}\text { Mosture } \\
\text { Content-D2216; }\end{array}$ \\
\hline
\end{tabular}

$205^{-1}$

NSTRUCTIONS
F08-075-163

PROJECT COOROINATOR

WIDRIG, DL

\section{SAF NO.}

F08-075

COA

$123514 \mathrm{ES} 10$

BILL OF LADING/AIR BILL NO
PRICE CODE

PAGE 1 OF 2

AIR QUALITY

METHOD OF SHIPMENT

GOVERNMENT VEHICLE

$\begin{array}{lll}\text { SAMPLE NO. } & \text { MATRIX* } & \text { SAMPLE DATE SAMPLE TIME } \\ \text { B1TNX2 } & \text { SOIL } & 5.16 .08\end{array}$

\section{CHAIN OF POSSESSION}

RELINQUISHED BY/REMOVED FROM

RELINQUISHED BY/REMOVED FROM

Molys frilse 15

RELINQUISHED BYIRET

ChrifuHu

RELINQUISHED BY/REMOVED FROM

RELINQUISHED BY/REMOVED FROM

RELINQUISHED BY/REMOVED FROM

LABORATORY RECEIVED BY
SECTION
FINAL SAMPLE
DISPOSITION

Lot 031394

\section{SIGN/ PRINT NAMES}

DATE/TIME RECEIVED BY/STORED IN $5 / 16 / 081500 m i$ 745 Faik

$5 \cdot 16 \cdot 08,1045$

DATE/TIME

DATE/TIME

RECEIVED BY/STORED IN

DATE/TME

DATE/TIME

RECEIVED BY/STORED IN

DATE/TTME

DATE/TIME RECEIVED BY/STORED IN

DATE/TIME

DATE/TIME RECEIVED BY/STORED IN

DATE/TIME
DATE/TIME 


\section{COLLECTOR}

NCO SAMPLER

\section{SAMPLING LOCATION}

C5856, I-120

ICE CHEST NO

\section{SHIPPED TO}

Environmental Sciences Laboratory

\section{SPECIAL INSTRUCTIONS}

*** The 200 Area S\&GRP Characterization and Monitoring Sampling and Analysis GKI applies to this SAF.

(1)6020M ICPMS ASTM AE (TAL) \{Cadmium, Chromium, Silver\} 6020M ICPMS ASTM AE (Add-On) (Arsenic, Lead, Molybdenum, Selenium\} 6020M METALS ICPMS WE (TAL) (Cadmium, Chromium, Silver\}

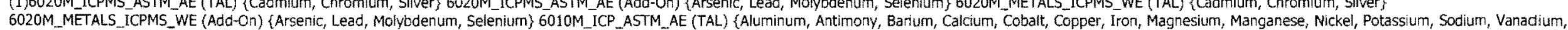

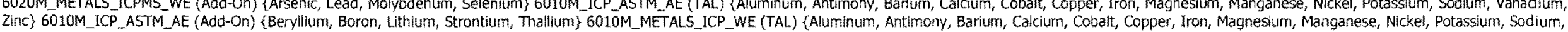

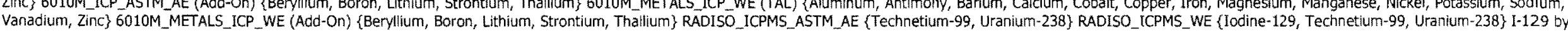

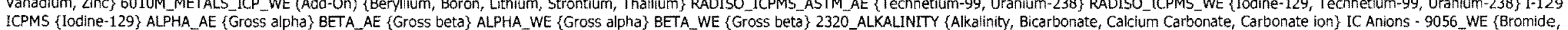

ICPMS \{lodine-129\}. ALPHA_AE \{Gross alpha\} BETA_AE \{Gross beta\} ALPHA_WE \{Gross alpha\} BETA_WE \{Gross beta\} 2320_ALKALINITY \{Alkalinity, Bicarbonate,
Chloride, Fluoride, Nitrate, Nitrite, Phosphate, Sulfate\} TOC - ASTME1915A \{Total Inorganic Carbon, Total carbon, Total organic carbon\} pH (Water) - 9045_WE;

\section{COMPANY CONTACT}

PROJECT DESIGNATION

\section{FIELD LOGBOOK NO.}

ACTUAL SAMPLE DEPTH

\section{OFFSITE PROPERTY NO.}

$$
265^{\circ}
$$

$123514 E S 10$

\section{PRICE CODE BN}

AIR QUALITY

METHOD OF SHIPMENT
GOVERNMENT VEHICLE

FIAIR BILL NO 


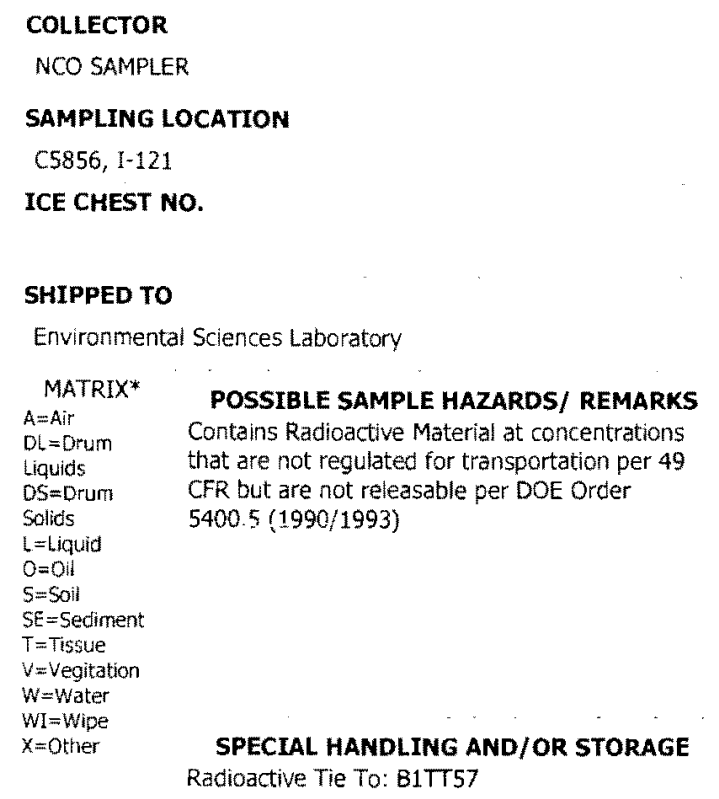

SAMPLING LOCATION

C5856, I-121

ICE CHEST NO.

\section{SHIPPED TO}

Environmental Sciences Laboratory

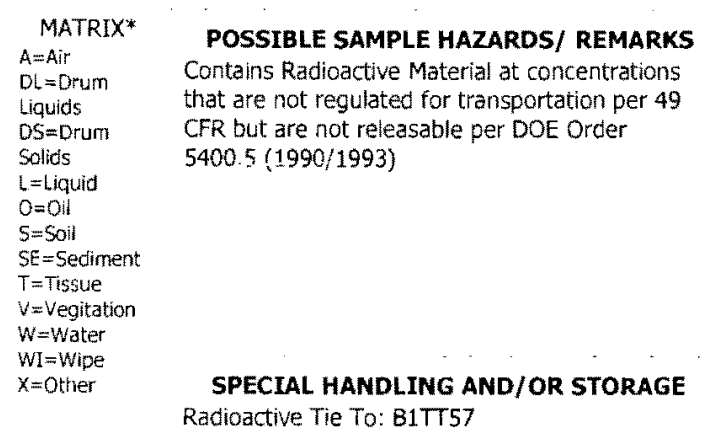

\section{PROJECT DESIGNATION}

373-5869

\section{SAF NO.}

200-BP-5 OU Characterization for Wells 299-E33-341 and 299-E33-342-G F08-075 FIELD LOGBOOK NO. ACTUAL SAMPLE DEPTH

COA

$$
\text { HNF-N-488 } 1 \quad 207.5^{\circ}
$$
OFFSITE PROPERTY NO.

123514ES10

METHOD OF SHIPMENT

N/A

BILL OF LADING/AIR BILL NO

$N / A$

\begin{tabular}{|c|c|c|}
\hline PRESERVATION & None & None \\
\hline TYPE OF CONTAINER & G/P & $\begin{array}{l}\text { Moisture } \\
\text { Resistant Cont }\end{array}$ \\
\hline NO. OF CONTAINER(S) & $\cdots$ & $1^{-\cdots}$ \\
\hline VOLUME & $1 \mathrm{~L}$ & 2009 \\
\hline SAMPLE ANALYSIS & $\begin{array}{l}\text { SEE TEM (1) } \\
\text { SPECIAL } \\
\text { INSTRLCTIONS }\end{array}$ & $\begin{array}{l}\text { Moisture } \\
\text { Content-D2215: }\end{array}$ \\
\hline
\end{tabular}

SAMPLE NO. MÁTRIX*

B1TNX3 SOLL

SAMPLE DATE SAMPLE TIME

$5.16 .081122 \quad x \quad x$

\section{CHAIN OF POSSESSION}

RELINQUISHED BY/REMOVED FROM $\checkmark$ Herick $Y$. Hen RELINQUISHED Or/KEMPVED EROM Mo Nus fride id । RELINQUZSHEQ GY/REM O \& FPRPM Chrisfu tron

RELINQUISHED BY/REMOVED FROM RELINQUISHED BY/REMOVED FROM RELINQUISHED BY/REMOVED FROM

LABORATORY
SECTION
FINAL SAMPLE
DISPOSITION
DATE/TIME

$$
\cot t 31394
$$

\section{SIGN/ PRINT NAMES}

RECEIVED BY/STORED IN

\section{SPECIAL INSTRUCTIONS}

DATE/TIME

$5.16 .08 / 500 \mathrm{MO} 745$ Fig $2 / 5-16081500$

DATE/TIME

DATE/TIME RECEIVED BY/STORED D DATE/TIME

$5-22.08140$ OBmith $/ 245.2208 / 42$

DATE/TIME

RECEIVED BY/STORED IN

DATE/TIME

DATE/TIME RECEIVED BY/STORED IN $\cdots \cdots \cdots$

DATE/TIME

RECEIVED BY/STORED IN

DATE/TIME

DATE/TIME

RECEIVED BY/STORED IN

DATE/TIME 


\section{COLLECTOR}

NCO SAMPLER

\section{SAMPLING LOCATION}

C5856, 1-121

ICE CHEST NO.

\section{SHIPPED TO}

Environmental Sciences Laboratory

\section{COMPANY CONTACT}

TRENT, STEVE

373-5869

\section{PROJECT DESIGNATION}

200-BP-5 OU Characterization for Wells 299-E33-341 and 299-E33-342-G

\section{FIELD LOGBOOK NO.}

$$
4 N F \cdot N-48 B \text { I }
$$

\section{OFFSITE PROPERTY NO.}

N/A
PROJECT COORDINATOR

WIORIG, DL

SAF NO.

F08-075

$\operatorname{COA}$

123514ES10

\section{PRICE CODE $\quad 8 N$}

AIR QUALITY

\section{METHOD OF SHIPMENT}

GOVERNMENT VEHICLE

\section{SPECIAL INSTRUCTIONS}

** The 200 Area S\&GRP Characterization and Monitoring Sampling and Analysis GKI applies to this SAF.

(1)6020M_ICPMS ASTM AE (TAL) \{Cadmium, Chromium, Silver\} 6020M ICPMS ASTM_AE (Add-On) \{Arsenic, Lead, Molybdenum, Selenium\} 6020M METALS ICPMS WE (TAL) \{Cadmium, Chromium, Silver\}

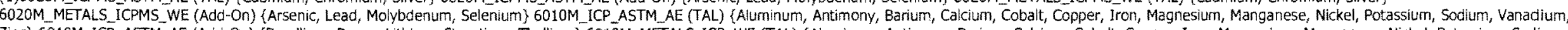

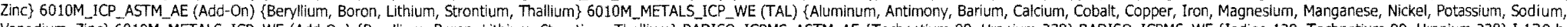

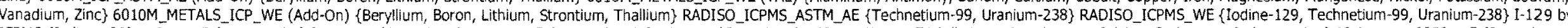

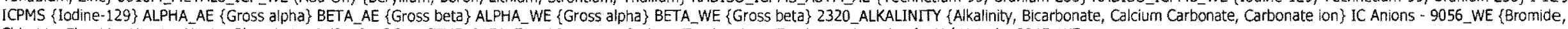

Chloride, Fluoride, Nitrate, Nitrite, Phosphate, Sulfate\} TOC - ASTME1915A \{Total Inorganic Carbon, Total carbon, Total organic carbon\} pH (Water) - 9045 WE 